\title{
PHYSICIANS ABSTRACTS
}

\section{Van Bekkum Award}

\section{1}

Improved cGvHD/relapse-free survival after HLA-identical sibling PBSC transplantation with ATG. A prospective multicenter randomized EBMT-labelled phase III trial (ATGfamilystudy)

C. Solano ${ }^{1, *}$, F. Bonifazi ${ }^{2}$, C. Wolschke ${ }^{3}$, F. Patriarca ${ }^{4}$, M. Pini ${ }^{5}$ A. Nagler ${ }^{6}$, M. Selleri', G. Messina ${ }^{8}$, W. Bethge, P. Herrera Fuentes ${ }^{10}$, R. Duarte ${ }^{11}, N$. Cascavilla ${ }^{12}$ M. Cimminiello ${ }^{13}$, S. Guidi ${ }^{14}$, J. Finke ${ }^{15}$, A. Gallamini ${ }^{16}$, C. Ferrà $^{17}$, J. Sierra ${ }^{18}$, D. Russo ${ }^{19}$, M. Petrini' ${ }^{20}, G$. Milone ${ }^{21}$, F. Benedetti ${ }^{22}$, M. Heinzelmann ${ }^{3}$, V. Liso ${ }^{23}$, M. Jurado ${ }^{24}$, E. M. Pogliani ${ }^{25}$, F. Narni' ${ }^{26}$, A. Völp ${ }^{27}$, G. Bandini' ${ }^{28}$, F. Ayuk ${ }^{3}$, T. Ruutu' ${ }^{29}$, N. Kröger $^{3}$ on behalf of Chronic Malignancies Working Party of the EBMT (CMWP)

${ }^{1}$ Hospital Clinico Valencia, Valencia, Spain, ${ }^{2}$ S. Orsola-Malpighi Hospital-Institute of Hematology and Medical Oncology, Bologna University, Bologna, Italy, ${ }^{3}$ Department of Stem Cell Transplantation, UNIVERSITY Medical Center HAMBURG, Hamburg, Germany, ${ }^{4}$ Policlinica Universario, Udine, ${ }^{5}$ Hematology and Marrow Transplant, A.O. SS Antonio e Biagio w C. Arrigo, Alessandria, Italy, ${ }^{6}$ Hematology Division, Chaim Sheba Medical Center, Tel Hashomer, Israel, "Universita Frederico II di Napoli, Naples, ${ }^{8}$ Hematology and Stem Cell Transplant Unit, Azienda Ospedaliera BMM, Reggio Calabria, Italy, ${ }^{9}$ Medical Department, Hematology and Oncology, University of Tübingen, Tübingen, Germany, ${ }^{10}$ Hospital Ramon y Cajal, Madrid, ${ }^{11}$ Instituto Catalan de Oncologoa, Barcelona, Spain, ${ }^{12}$ Hematology, IRCCS San Giovanni Rotondo, San Giovanni Rotondo, ${ }^{13}$ Hematology and Stem Cell Transplant Unit, Azienda Ospedaliera Regionale San Carlo, Potenza, ${ }^{4}$ Hematology, Ospedale di Careggi, Firenze, Italy, ${ }^{15}$ Hematology, University of Freiburg, Freiburg, Germany, ${ }^{16}$ Research, Innovation \& Statistics, A. Lacassagne Cancer Center, Nice, France, ${ }^{17}$ Hematology, University Hospital Germans Trias $i$ Pujol, Badalona, ${ }^{18}$ Hematology, Hospital de la Santa Creu i Sant Pau, Barcelona, Spain, ${ }^{19}$ Hematology, University, Brescia, ${ }^{20}$ Hematology, University of Pisa, Pisa, ${ }^{21}$ Hematology and Stem Cell Transplant Unit, Ospedale Ferrarotto, Catania, ${ }^{22} \mathrm{Hematol}-$ ogy, Ospedale Policlinico GB Rossi, Verona, ${ }^{23}$ Hematology, University of Bari, Bari, Italy, ${ }^{24}$ Hematology, Hospital Virgen des las Nieves, Granada, Spain, ${ }^{25}$ Ospedale San Gerardo, Monza, ${ }^{26}$ Medical and Surgical Sciences, University of Modena and Reggio Emilia, Modena, Italy, ${ }^{27}$ Psy Consult, Frankfurt, Germany, ${ }^{28}$ Hematology, BolognaUniversity, Bologna, Italy, ${ }^{29}$ University Central Hospital, Helsinki, Finland

Introduction: The incidence of CGvHD after allogeneic SCT is higher when peripheral blood stem cells are used as stem cell source. There is a strong need for preventing CGvHD after ASCT without increasing the risk of relapse.

Materials (or patients) and methods: We performed a multicenter, multinational, open-label, randomized study comparing anti-lymphocyte globulin (ATG-Fresenius ${ }^{\mathbb{R}}$ ) $10 \mathrm{mg} / \mathrm{kg}$ on day $-3,-2$ and -1 with no ATG in patients with AML $(n=110)$ or ALL $(n=45)$ in $1^{\text {st }}$ complete remission (CR; $n=139)$ or $2^{\text {nd }}$ CR $(n=16)$ who received peripheral blood stem cells from their HLA-identical sibling after standard TBI
(12 Gy)/Ccclophosphamide $(120 \mathrm{mg} / \mathrm{kg}$ ) or busulfan $(16 \mathrm{mg} /$ $\mathrm{kg}) / \mathrm{Cy}(120 \mathrm{mg} / \mathrm{kg})$ based myeloablative conditioning regimen. Standard GvHD prophylaxis consisted of cyclosporine A and a short course of MTX $\left(10 \mathrm{mg} / \mathrm{m}^{2}\right.$ on day $+1,+3,+6$ and $+11)$. The primary study aim was to compare the cumulative incidence of cGvHD at 2 years after ASCT.

Results: Out of 161 randomized patients from 27 centers and 4 nations 6 were withdrawn before conditioning and ASCT due to leukemia progression, or cancellation of the donor. 155 patients were analyzed for safety and efficacy; 83 were randomized to ATG and 72 to non-ATG. The treatment groups were comparable regarding recipient and donor age and sex CMV serostatus, disease (AML vs ALL), $1^{\text {st }}$ or $2^{\text {nd }}$ CR. The median time to leukocyte $(>1.0 \times 10 \mathrm{e} 9 / \mathrm{l})$ and platelet $(>20 \mathrm{x}$ $10 \mathrm{e} / \mathrm{l})$ engraftment was significantly delayed in the ATG group (18 vs. 15 days, $P<0.001$ and 20 vs 13 days, $P<0.001$ ). The incidence of acute GvHD grade I-IV was $25 \%$ for the ATG arm and $35 \%$ for the non-ATG arm $(P=0.32)$ and for severe grade III/IV acute GvHD $2 \%$ and $8 \%$, respectively $(P=0.2)$. The cumulative incidence of cGvHD at 2 years was $32 \%(95 \% \mathrm{Cl} 22$ $47 \%)$ in the ATG and 69\% (95\% Cl 51-74\%) in the non-ATG arm $(P<0.0001)$. Regarding limited and extensive $\mathrm{CGvHD}$ the $\mathrm{Cl}$ at 2 years was $26 \%$ vs $53 \%(P=0.002)$ and $19 \%$ vs $53 \%$ $(P<0.001)$, respectively. There was no difference in infectious complications, CMV and EBV reactivation between both arms. The cumulative incidence of therapy related mortality at 2 years was $14 \%$ (95\% Cl 8-24\%) for the ATG arm and 12\% (95\% $\mathrm{Cl} 6-22 \%)$ for the non-ATG arm $(P=0.60)$, resulting in 2 year relapse-free and overall survival of $59 \%(95 \% \mathrm{Cl} 48-69 \%)$ and $74 \%(95 \% \mathrm{Cl} 63-82 \%)$ for the ATG group and of $65 \%(95 \% \mathrm{Cl}$ $51-75 \%)$ and $78 \%(95 \% \mathrm{Cl} 66-86 \%)$ for the non-ATG group $(P=0.46$ and $P=0.21$, respectively). Chronic GvHD free survival at 2 years was $50 \%$ for the ATG and $23 \%$ for the non ATG arm $(P<0.001)$. A composite endpoint $C G v H D$ and relapse-free survival at 2 years was in favor for the ATG treated patients ( $30 \%$ vs. $17 \%, P=0.005)$.

Conclusion: This is the first randomized CGvHD prevention study providing evidence that ATG-Fresenius ${ }^{\circledR}(3 \times 10 \mathrm{mg} / \mathrm{kg})$ is highly effective in preventing limited and extensive CGvHD in HLA-identical sibling PBSC transplantation when used within a myeloablative preparative conditioning regimen. The use of ATG-Fresenius did not result in an obvious increase of infectious complications and relapse, resulting in similar overall survival rates, but improved cGVHD/relapse free survival.

Disclosure of Interest: C. Solano: None declared, F. Bonifazi: None declared, C. Wolschke: None declared, F. Patriarca: None declared, M. Pini: None declared, A. Nagler: None declared, M. Selleri: None declared, G. Messina: None declared, W. Bethge: None declared, P. Herrera Fuentes: None declared, R. Duarte: None declared, N. Cascavilla: None declared, M. Cimminiello: None declared, S. Guidi: None declared, J. Finke: None declared, A. Gallamini: None declared, C. Ferrà: None declared, J. Sierra: None declared, D. Russo: None declared, M. Petrini: None declared, G. Milone: None declared, F. Benedetti: None declared, M. Heinzelmann: None declared, V. Liso: None declared, M. Jurado: None declared, E. M. Pogliani: None declared, F. Narni: None declared, A. Völp: None declared, G. Bandini: None declared, F. Ayuk: None declared, T. Ruutu: None declared, N. Kröger Funding from: Neovii. 


\section{2}

Exploring Limiting Factors in the Prediction of Allogeneic HSCT Related Mortality: An In-Silico Machine Learning Analysis of the Acute Leukemia Working Party (ALWP) Registry of the EBMT

R. Shouval 1,* , M. Labopin ${ }^{2}$, R. Unger ${ }^{3}$, S. Giebel' ${ }^{4}$, F. Ciceri ${ }^{5}$, C. Schmid ${ }^{6}$, J. Esteve, , F. Baron ${ }^{8}$, N. C. Gorin', B. N. Savani ${ }^{10}$ M. Mohty ${ }^{11}$, A. Nagler ${ }^{12}$

${ }^{1}$ Division of Hematology and Bone Marrow Transplantation, The Chaim Sheba Medical Center, Tel-Hashomer, Ramat-Gan, Israel, ${ }^{2}$ EBMT Paris Office, Hospital Saint Antoine, Paris, France, ${ }^{3}$ The Mina and Everard Goodman Faculty of Life Sciences, Bar-llan University, Ramat-Gan, Israel, ${ }^{4}$ Department of Bone Marrow Transplantation and Oncohematology, Maria Sklodowska-Curie Memorial Cancer Center and Institute of Oncology, Gliwice Branch, Gliwice, Poland, ${ }^{5}$ Hematology and BMT Unit, San Raffaele Scientific Institute, Milan, Italy, ${ }^{6}$ Department of Hematology and Oncology, Klinikum Augsburg, Ludwig-MaximiliansUniversity, Munich, Germany, ${ }^{7}$ Hematology Department, IDIBAPS, Hospital Clínic, Barcelona, Spain, ${ }^{8} 17$-Hematology \& GIGA research, University of Liège, Liège, Belgium, ${ }^{9} 4-E B M T$ Paris Office, Hospital Saint Antoine, Paris, France, ${ }^{10}$ Hematology \& Stem Cell Transplantation Section, Vanderbilt University Medical Center, Nashville, TN, United States, ${ }^{11}$ AP-HP, Hématologie Clinique et Thérapie Cellulaire, Hôpital Saint-Antoine, Paris, France, ${ }^{12}$ Division of Hematology and Bone Marrow Transplantation, The Chaim Sheba Medical Center, Tel-Hashomer, Ramat-Gan, Israel

Introduction: The establishment of large transplant registries and introduction of novel statistical techniques have paved the way for large scale data analysis. Nevertheless, contemporary tools for risk prediction of transplant related mortality (TRM) following allogeneic (allo) hematopoietic stem cell transplantation (HCT) are of limited clinical use, owing to a sub-optimal predictive accuracy.

Apart from inherent procedural uncertainty, methodological factors impeding prediction might be attributed to the statistical methodology used, number and quality of features collected, or simply the population size. Using an in-silico approach (i.e. iterative computerized simulations), based on machine learning (ML) algorithms, we aimed to define prediction limiting factors of day 100 TRM and rank variable contribution.

$M L$ is a field of artificial intelligence dealing with the construction and study of systems that can learn from data, rather than follow explicitly programmed instructions. Commonly applied in complex data scenarios, such as financial settings, it may be suitable for outcome prediction of HCT.

Materials (or patients) and methods: Study population was a cohort of 28,236 adult acute leukemia allo-HCT recipients from the EBMT-ALWP. Twenty four variables were analyzed, including recipient, donor and transplant characteristics. Study design involved two phases. The first, focused on development and comparison of several ML based prediction models of day 100 TRM. In the second phase, a repetitive computerized simulation was applied. Factors necessary for optimal prediction were explored: algorithm type, size of data set, number of included variables, and performance in specific subpopulations. Models were assessed and compared on the basis of the area under the receiver operating characteristic curve (AUC).

Results: Six ML based prediction models for day 100 TRM were developed on the entire dataset. Optimal AUCs ranged from $0.65-0.68$. Depending on the algorithm used for prediction model development, the in-silico experimental system yielded the following results: Predictive performance plateaued on a population size ranging from $n=5647-8471$; A range of 6-12 ranked variables, selected by a separate feature selection algorithm, were necessary for optimal prediction; Disease status and donor type were consistently top ranking variables. Predictive performance of models developed for specific subpopulations, ranged from 0.59 to 0.67 for patients in second complete remission and patients receiving reduced intensity conditioning respectively.

Conclusion: We present a novel experimental system for assessment of prediction boundaries in HCT. The present approach has clinical implications. We show that predictive performance of day 100 TRM is unlikely to improve with the data routinely gathered on HCT recipients, as different algorithms reach approximately the same performance. In addition, an exhaustive search for variable importance, reveal that few variables "carry the weight" with regard to predictive influence. Predictive performance converged when sampling more than 5000 patients, reflecting the importance of large registry studies. Overall, it seems we have reached a point of predictive saturation. Improving predictive performance will likely require additional types of input like genetic, biologic and procedural factors.

Disclosure of Interest: None declared.

\section{Presidential Symposium}

0003

Myeloablative, reduced toxicity versus standard conditioning in AML: a randomized clinical trial from Gruppo Italiano Trapianto di Midollo Osseo (GITMO) A. Grassi ${ }^{1, *}$, C. Micò ${ }^{7}$, E. Oldani ${ }^{2}$, C. Boschini ${ }^{2}$, A. Busca ${ }^{3}$, B. Benedetto ${ }^{3}$, I. Cavattoni ${ }^{4}$, S. Santarone ${ }^{5}$, R. Raimondi', M. Montanari ${ }^{7}$, G. Milone ${ }^{8}$, P. Chiusolo ${ }^{9}$, G. Specchia ${ }^{10}$, S. Guidi ${ }^{11}$, F. Patriarca ${ }^{12}$, A. Bacigalupo ${ }^{13}$, A. M. Risitano ${ }^{14}$, G. Saporiti $^{15}$, M. Pini ${ }^{16}$, E. M. Pogliani ${ }^{17}$, W. Arcese ${ }^{18}$, G. Marotta ${ }^{19}$ A. M. Carella ${ }^{20}$, A. Nagler ${ }^{21}$, P. Corradini ${ }^{22}, D$. Russo ${ }^{23}$ E. P. Alessandrino ${ }^{24}$, G. F. Torelli ${ }^{25}$, N. Mordini ${ }^{26}$, R. Scimè ${ }^{27}$, A. Bosi ${ }^{28}$, A. Masciulli ${ }^{29}$, R. M. Marfisi ${ }^{30}$, A. Rambaldi ${ }^{1}$ Hematology and BMT Unit, AZIENDA OSPEDALIERA PAPA GIOVANNI XXIII, ${ }^{2}$ Hematology and BMT Unit, Ospedale Papa Giovanni XXIII, Bergamo, ${ }^{3}$ Bone Marrow Transplantation Unit, azienda ospedaliera-universitaria città della salute, Torino, ${ }^{4}$ Bone Marrow Transplantation Unit, Ospedale Civile Hematology and Bone Marrow Transplant Unit, Bolzano, ${ }^{5}$ Bone Marrow Transplantation Unit, Ospedale Civile BMT Center Pescara, Pescara, ${ }^{6}$ Hematology and Bone Marrow Transplant Unit, Ospedale San Bortolo, Vicenza, ${ }^{7}$ Hematology, Azienda Ospedali Riuniti, Ancona, ${ }^{8}$ Hematology and BMT Unit, Ospedale Ferrarotto, Catania, ${ }^{9}$ Division of Hematology, Policlinico A. Gemelli, Roma, ${ }^{10}$ Department of Emergency and Organ Transplantation, Section of Hematology with Transplantation, Medical School, University of Bari, Bari, "11 Department of Hematology, BMT Unit, AOU Careggi, University of Firenze, Firenze, ${ }^{12}$ Hematology and Bone Marrow Transplant Unit, Azienda Ospedaliera -Universitaria, Udine, ${ }^{13}$ Hematology and Oncology Department, IRCCS AOU San Martino - IST, Genova, ${ }^{14}$ Hematology, Federico II University of Naples, Napoli, ${ }^{15}$ Hematology - BMT Unit, Fondazione IRCCS Ca' Granda Ospedale Maggiore Policlinico, University of Milan, Milano, ${ }^{16}$ Hematology, AO SS. Antonio e Biagio e C. Arrigo, Alessandria, ${ }^{17}$ Hematology and BMT Unit, Ospedale San Gerardo, University of Milano Bicocca, Monza, ${ }^{18}$ Hematology DivisionStem Cell Transplant Unit, University of Rome Tor Vergata, Roma, ${ }^{19}$ Hematology, Azienda Ospedaliera Universitaria Senese, Siena,

${ }^{20}$ Hematoloy-BMT Unit, IRCCS, Casa sollievo della sofferenza, San Giovanni Rotondo (Fg), Italy, ${ }^{21}$ Hematology and BMT Unit, Chaim Sheba Medical Center, Tel Hashomer, Israel, ${ }^{22}$ Hematology-Bone Marrow Transplantation Unit, Fondazione IRCCS Istituto Nazionale Tumori and University of Milano, Milano, ${ }^{23} B M T$ Unit, Spedali Civili, Brescia, ${ }^{24}$ BMT Unit, IRCCS Fondazione Policlinico San Matteo \& University, Pavia, ${ }^{25}$ Cellular Biotechnologies \& Hematology, Sapienza University, Roma ${ }^{26}$ Hematology, Azienda Ospedaliera S. Croce e Carle, Cuneo, ${ }^{27}$ Hematology and BMT 
Unit, Ospedali Riuniti Villa Sofia-Cervello, Palermo, ${ }^{28}$ Hematology and BMT Unit, AOU Careggi, University of Firenze, Firenze, ${ }^{29}$ Fondazione Mario Negri Sud, Santa Maria Imbaro, ${ }^{30}$ Fondazione Mario Negri Sud, S. Maria Imbaro, Italy

Introduction: The combination of a myeloablative dose of intravenous (iv) busulfan with cyclophosphamide (BuCy2) is the standard conditioning regimen for allogeneic hematopoietic stem cell transplantation in AML. In patients older than 40 years, it can be associated to high non relapse mortality (NRM). The same myeloablative dose of busulfan combined to fludarabine (BuFlu) may be associated to a lower NRM.

Materials (or patients) and methods: The standard conditioning with iv busulfan at a dose of $0.8 \mathrm{mg} / \mathrm{kg} / 6 \mathrm{~h}$ for 4 consecutive days for a total dose of $12.8 \mathrm{mg} / \mathrm{kg}$, in combination with cyclophosphamide at the dose of $60 \mathrm{mg} / \mathrm{kg} / \mathrm{day}$ for 2 consecutive days for a total dose of $120 \mathrm{mg} / \mathrm{kg}$ (BUCY2 arm) was randomly compared to the same dose of busulfan combined with fludarabine at the dose of $40 \mathrm{mg} / \mathrm{m}^{2} /$ day for 4 consecutive days, for a total dose of $160 \mathrm{mg} / \mathrm{m} 2$ (BUFLU arm). Eligible were patients with a diagnosis of AML in 1st or 2nd complete remission (CR) with an age $\geq 40$ and $\leq 65$ years, and the availability of an HLA compatible sibling or unrelated donor. The GvHD prophylaxis was based on conventional Cyclosporine $\mathrm{A}$ and Methotrexate. In case of unrelated donors, ATG was given at a total dose of $5 \mathrm{mg} / \mathrm{kg}$. The primary study end-point was the one-year NRM using an intent-to-treat analysis.

Results: 252 patients were assessed for eligibility: 125 were randomized to BuCy2 (121 received the allocated intervention, 3 withdrew consent and 1 relapsed before conditioning) while 127 were randomized to BuFlu (124 received the allocated intervention and 3 relapsed before conditioning). Patients were stratified according to donor type and remission (1st vs. 2nd or more). The main clinical and transplant characteristics were well balanced between the randomization arms. The median age was 51 years, $85 \%$ of patients was in 1 st remission and the ELN risk subgroups were good (11\%), intermediate-1 $(49 \%)$, intermediate-2 $(16 \%)$ and adverse $(25 \%)$. The donor was a sibling related $(45 \%)$ or matched unrelated $(55 \%)$ while the stem cell graft was the peripheral blood in the majority of cases. On an intent to treat basis, at 1 year, the NRM in the BUCY2 arm was $17.2 \%$ vs. $7.9 \%$ in the BUFLU (Gray Test $P=0.03$ ). At 2 years and throughout the study, the same significantly different NRM was observed between study arms being respectively $18.2 \%$ vs. $8.9 \%$ and $19 \%$ vs. 9.7\% (Gray Test $P=0.05$ ) (Figure 1). By forest plots analysis the experimental treatment was better in all strata and particularly in patients in CR1. A non-significant lower incidence of relapse was documented in the BUCY2 vs. the BUFLU arm being $22.1 \%$ vs. $25.2 \%$ at 1 year, respectively (Gray test 0.47 ) and no difference could be detected by forest plot analysis in any strata. At 4 year, in the BuFlu and the BuCy2 arm respectively, the leukemia free survival was $51 \%$ vs. $42 \%$ and the overall survival $55 \%$ vs. $54 \%$. The overall (grade II-IV) cumulative incidence of acute GVHD was slightly higher in the BuCy2 arm and this difference was significant $(P=0.0083)$ when only grade III and IV were considered.

Conclusion: The conditioning regimen based on Busulfan and Fludarabine was associated with a lower non-relapse mortality and less acute GvHD (grade III-IV), with a similar incidence of relapse and comparable LFS and OS. This myeloablative, albeit reduced toxicity program is a valid alternative for older AML patients.

Disclosure of Interest: A. Grassi Conflict with: Pierre Fabre, Pharma, C. Micò: None declared, E. Oldani: None declared, C. Boschini: None declared, A. Busca: None declared, B. Benedetto: None declared, I. Cavattoni: None declared, S. Santarone: None declared, R. Raimondi: None declared, M. Montanari: None declared, G. Milone: None declared, P. Chiusolo: None declared, G. Specchia: None declared, S. Guidi: None declared, F. Patriarca: None declared, A. Bacigalupo: None declared, A. M. Risitano: None declared, G. Saporiti: None declared, M. Pini: None declared, E. M. Pogliani: None declared,

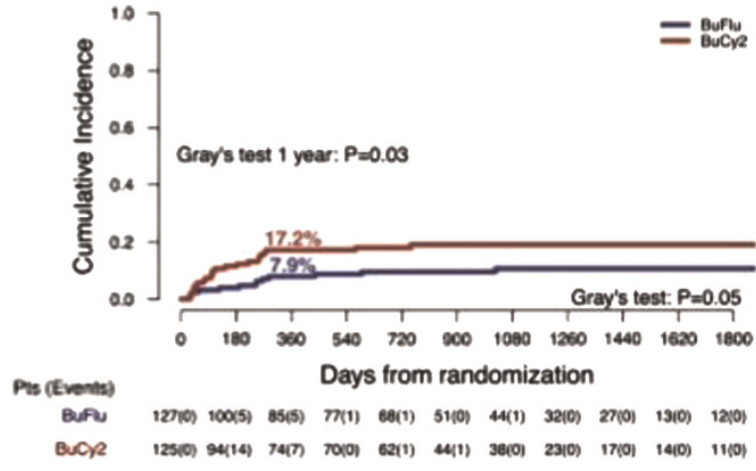

W. Arcese: None declared, G. Marotta: None declared, A. M. Carella: None declared, A. Nagler: None declared, P. Corradini: None declared, D. Russo: None declared, E. P. Alessandrino: None declared, G. F. Torelli: None declared, N. Mordini: None declared, R. Scimè: None declared, A. Bosi: None declared, A. Masciulli: None declared, R. M. Marfisi: None declared, A. Rambaldi Conflict with: Pierre Fabre, Pharma.

\section{4}

Related T-Cell Depleted HLA-Haploidentical Stem Cell Transplantation (TCD-Haplo) versus Umbilical Cord Blood Transplantation (UCBT) in Pediatric Patients with Acute Leukemia, a Eurocord, CBC-CTIWB, PDWP-EBMT Study F. Locatelli $i^{1, *}$, M. Labopin ${ }^{2}$, G. Michel ${ }^{3}$, R. Handgretinger ${ }^{4}$, T. O'Brien ${ }^{5}$, T. Klingebiel ${ }^{6}, M$. Ayas ${ }^{7}$, A. Bertaina ${ }^{8}$, M. Zecca $^{9}$, J.-H. Dalle 10 I. Capolsini ${ }^{11}$, C. D. de Heredia ${ }^{12}$, J. M. Cornish ${ }^{13}$, M. A. Diaz ${ }^{14}$, M. Berger ${ }^{15}$, E. Lanino ${ }^{16}$, C. Messina ${ }^{17}$,

S. Santarone ${ }^{18}$, C. Kenzey ${ }^{19}$, F. Giannotti ${ }^{19}$, C. Peters ${ }^{20}$, V. Rocha ${ }^{19,21}$, E. Gluckman ${ }^{19}$, P. Bader ${ }^{22}$, A. Ruggeri' ${ }^{2,19}$

${ }^{1}$ University of Pavia, Bambino Gesu' Children Hospital, Pavia, Italy, ${ }^{2}$ Service d'hématologie clinique et de thérapie cellulaire, Hôpital Saint-Antoine, AP-HP, Paris, ${ }^{3}$ Department of Hematology, Hôpital La Timone, Marseille, France, ${ }^{4}$ Department of Pediatric Oncology, University Children's Hospital, Tuebingen, Germany, ${ }^{5}$ Sydney Children's Hospital Centre for Children's Cancer, Randwick NSW, Australia, ${ }^{6}$ Department of Pediatric Oncology, University Hospital Frankfurt, University of Frankfurt, Frankfurt, Germany, ${ }^{7}$ Section of Pediatric SCT, King Faisal Specialist Hospital \& Research Centre-Riyadh, Riyadh, Saudi Arabia, ${ }^{8}$ Department of Pediatric Hematology-Oncology, Istituto di Ricovero e Cura a Carattere Scientifico (IRCCS), Bambino Gesù Children's Hospital, Roma, ${ }^{9}$ Department of Pediatric Onco-Hematology, IRCCS, Policlinico San Matteo Foundation, Pavia, Italy, ${ }^{10}$ Department of Pediatric Hematology, Immunology and Bone Marrow Transplantation, AP-HP, Robert-Debré Hospital and Paris Diderot University, Paris, France, ${ }^{11}$ Pediatric Oncology and Hematology Section, Santa Maria della Misericordia Hospital, Perugia, Italy, ${ }^{12}$ Hospital Universitari Vall d'Hebron - Area Materno Infantil, Barcelona, Spain, ${ }^{13}$ Department of Paediatric Bone Haematology/Oncology/Bone Marrow Transplantation, Paediatric Stem Cell Transplantation, Women and Children's Services, Bristol Royal Hospital for Children, Bristol, United Kingdom, ${ }^{14}$ Hospital Infantil Universitario Niño Jesús, Madrid, Spain, ${ }^{15}$ Pediatric OncoHematology and Stem Cell Transplant Division, Regina Margherita Children's Hospital, Turin, "I6 Itituto Giannina Gaslini, Genova, ${ }^{17}$ Department of Paediatrics "Salus Pueri", University of Padua, Padua, ${ }^{18}$ Bone Marrow Transplant Center, Spirito Santo Hospital, Pescara, Italy, ${ }^{19}$ Hôpital Saint-Louis AP-HP, Eurocord, Paris, France, ${ }^{20}$ St. Anna Kinderspital and Children's Cancer Research Institute, Medical University of Vienna, Vienna, Austria, ${ }^{21}$ Oxford University Hospitals NHS Trust, Oxford, United Kingdom,

${ }^{22}$ Department of Stem Cell Transplantation and Immunology, Hospital for Children and Adolescents, Frankfurt am Main, Germany

Introduction: TCD-Haplo and UCBT have been used effectively in the treatment of children with acute leukemia in need 
of an allograft and lacking a standard donor. To date, there are no study comparing the outcomes of these transplant approaches in this population.

Materials (or patients) and methods: We performed a retrospective registry based of children with either ALL or AML who received a TCD-Haplo or single UCBT after myeloablative conditioning regimen. Method of T-cell depletion was available for 169 TCD-Haplo grafts: CD34 + positive selection $n=120$, negative depletion $n=49$ (CD3/CD19 $n=19$; TCR alpha/beta + cell $n=30$ ). Transplants were performed from 2001 to 2012 in EBMT centers; 1180 patients (pts) received UCBT (AML $n=400 ;$ ALL $n=780$ ) and 215 TCD-Haplo (AML $n=83$; ALL $n=132$ ). Median follow-up was 49 months for TCD-Haplo and 33, for UCBT. Compared to TCD-Haplo. UCBT recipients were younger (median age 5.9 vs 10.3 years, $P=<0.0001$ ), were transplanted more often in CR1 (40\%vs24\%, $P=<0.001$ ) and less frequently in advanced disease $(9 \%$ vs $20 \%, P=<0.001)$. Median interval from diagnosis to transplant was 572 days for TCD-Haplo and 372 days for UCBT $(P<0.001)$. Conditioning regimen was mainly TBI-based in $88 \%$ of TCD-Haplo vs $82 \%$ of UCBT.

Results: Acute GVHD (grade II-IV) incidence was 18\% and 37\% $(P<0.001)$ and chronic GVHD was $14 \%$ and $16 \%,(P=0.40)$ for TCD-Haplo and UCBT, respectively. Univariate analysis in ALL pts $(n=912)$ showed no significant difference in the 2-year leukemia-free survival (LFS) $34 \%$ vs $41 \%(P=0.313)$ between TCD-Haplo and UCBT, with a cumulative incidence $(\mathrm{Cl})$ of nonrelapse mortality (NRM) $24 \%$ vs $30 \%(P=0.162)$ and relapse incidence (RI) $42 \%$ vs $29 \%(P=0.007)$ respectively. For pts in CR1, 2-year RI was $20 \%$ vs $22 \%, P=0.741$; NRM $23 \%$ vs $24 \%$, $P=0.887$; and 2 - year LFS $57 \%$ versus $55 \%, P=0.682$, for TCDHaplo and UCBT, respectively. For pts in CR2, 2-year RI was $41 \%$ vs $31 \%, P=0.120$; NRM $27 \%$ vs $33 \%, P=0.173$; and 2 year LFS $33 \%$ vs $36 \%, P=0.940$, for TCD-Haplo and UCBT respectively. In adv disease, 2-year LFS was $6 \%$ vs $11 \%$, $P=0.147$ for TCD-Haplo and UCBT.

AML Pts $(n=483)$ receiving TCD-Haplo had lower LFS: $30 \%$ vs $45 \%$ for UCBT $(P=<0.0001)$, RI $40 \%$ vs $28 \% \quad(P<0.001)$ respectively, with no difference in $\mathrm{Cl}$ of NRM (30\% vs $27 \%$; $P=0.621$ ). According to disease status: for AML pts in CR1 the 2 -year RI was $25 \%$ vs $16 \%$, $(P=0.438)$, NRM $54 \%$ vs $17 \%$ $(P<0.001)$ and 2 -year LFS $21 \%$ vs $67 \%(P=<0.001)$ in TCD Haplo and UCBT respectively. For AML pts in CR2, 2-year RI was $28 \%$ vs $25 \%$, $(P=0.639)$, NRM was $40 \%$ vs $24 \%(P=0.075)$, and 2 -year LFS was $33 \%$ vs $51 \%(P=0.037)$, for TCD-Haplo and UCBT respectively. For AML pts with adv disease 2-year LFS was $12 \%$ for TCD-Haplo and $16 \%$ for UCBT $(P=0.754)$.

In multivariate analysis for pts with ALL, disease status was the only factor associated with better LFS (HR 4.84, $P<0.0001$ ). UCBT was associated with a lower risk of RI (HR 0.69, $P=0.029$ ).

For AML, UCBT was associated with lower RI (HR 0.52, $P=0.007)$, lower NRM $(\mathrm{HR}=0.577 ; P=<0.019)$, and better LFS (HR 0.575; $P=<0.001$ ) when compared to TCD-Haplo. Adv disease was associated with lower LFS and higher RI.

Conclusion: In conclusion, children with ALL have comparable LFS after either UCBT or TCD-Haplo. By contrast, in children with AML, UCBT is associated with lower RI and NRM than TCD-Haplo, translating into better LFS.

Disclosure of Interest: None declared.
0005

Haplotransplants versus other alternative donors for allogeneic stem cell transplantation in Non Hodgkin Lymphoma (NHL): A retrospective analysis of the EBMT Lymphoma Working Party

S. Dietrich ${ }^{1, *}$, H. Finel ${ }^{2}$, C. Martinez ${ }^{1}$, J. Tischer ${ }^{1}$, D. Blaise ${ }^{1}$

N. Milpied ${ }^{1}$, A. Bacigalupo ${ }^{1}$, P. Corradini', M. Mohty', M. Sanz', A. Velardi ${ }^{1}$, A. Hausmann ${ }^{1}$, S. Montoto ${ }^{1}$, O. Hermine',

N. Schmitz', H. Schouten ${ }^{1}$, A. Sureda ${ }^{1}$, A. Tanase ${ }^{1}$, S. Robinson ${ }^{1}$, P. Dreger ${ }^{1}$

${ }^{1}$ Lymphoma Working Party, ${ }^{2}$ EBMT Lymphoma Working Party, Paris, France

Introduction: Allogeneic stem cell transplantation (SCT) can provide long-term disease control in selected patients with relapsed refractory NHL. Restricted availability of a matched sibling donor limits its use especially for patients with rapidly progressing disease in whom unrelated donor search cannot be awaited. Because data of alternative donor transplants in $\mathrm{NHL}$ is sparse, we aimed to compare outcome of haploidentical and cord blood transplants with conventional relatedand matched unrelated donor transplats for NHL.

Materials (or patients) and methods: Information of patients with mantle cell lymphoma (MCL), DLBCL, T-cell lymphoma (TCL) and follicular lymphoma (FL) who received an SCT from a sibling donor (SIB), 10/10 matched unrelated donor (MUD), haplo-identical donor (HAPLO) or cord blood (CORD) between 2007 and 2012 was downloaded from the EBMT database.

Results: 2798 patients with NHL were identified in the EBMT database meeting the inclusion criteria. 2065 received a transplant from a SIB, 447 from a MUD, 167 from CORD (18 $\mathrm{MCL}, 36 \mathrm{DLBCL}, 43 \mathrm{FL}, 70 \mathrm{TCL})$ and 119 from a HAPLO donor (16 MCL, $30 \mathrm{DLBCL}, 22 \mathrm{FL}, 51 \mathrm{TCL})$. Active disease $(P<0.01)$ and Karnofsky Index (KI) below $80 \%$ was also more common in the HAPLO group $(P=0.02)$. Other variables were balanced.

Median follow-up after SCT for all patients was 27 months $(\mathrm{Cl}$ 25 to 29). Relapse incidence after conventional transplants (SIB, MUD) and alternative donor transplants (HAPLO, CORD) was not significantly different within the whole group (HAPLO: HR 1.2 95\% Cl 0.9-1.8 $P=0.23$; CORD: HR 1.1 95\% Cl 0.7-1.4, $P=0.74)$ and across all studied disease entities. In contrast, NRM incidence was not significantly different between SIB and MUD, but significantly higher with alternative donor transplants (HAPLO: HR $1.895 \% \mathrm{Cl} 1.3-1.8, P<0.001$; CORD: HR 1.9 $95 \% \mathrm{Cl} 1.5-2.5, P<0.001)$. With the exception of FL where MUD in addition to HAPLO and CORD transplants had a significantly higher NRM incidence than SIB transplants.

Because patients who received a HAPLO transplant had more commonly active disease at transplant and worse $\mathrm{Kl}$, we performed multivariate modeling of relapse- and NRM incidences adjusting for the above mentioned covariates. No different relapse incidences between donor groups was observed. NRM incidence in contrast, was significantly higher in MUD (reference SIB, HR 1.36 Cl 1.1-1.8, $P=0.033$ ) and CORD (reference SIB, HR 2.5 Cl 1.7-3.6, $P=0.001$ ) but not in HAPLO transplants.

Most interestingly, acute GVHD incidence was significantly increased in MUD compared to SIB $(P=0.003)$ transplants but not in HAPLO $(P=0.08)$ or CORD $(P=0.34)$ transplants. Multivariate adjustment for diagnosis ( $M C L, D L B C L, F L, T C L)$, remission prior to $\mathrm{SCT}, \mathrm{KI}(\mathrm{KI}<90 \%$ vs. $>90 \%)$ and conditioning intensity (RIC vs. MAC) did not reveal worse OS for HAPLO but a worse OS for CORD (HR 1.6 Cl 1.3-2.0, $P<0.0003$ ).

Conclusion: Alternative donor transplants are a valuable option if no suitable SIB or MUD donor is available. Relapse incidence after alternative donor transplants was not significantly different from conventional transplants. Higher NRM incidence in CORD donor but not in HAPLO donor transplants suggests HAPLO transplants as valuable option if no well matched donor is available.

Disclosure of Interest: None declared. 


\section{6}

RIC vs. MAC followed by allogeneic stem cell transplantation for patients with MDS or secondary AML: A prospective, randomized phase III study of the CMWP of the EBMT (RICMAC-Trial)

N. Kröger, ${ }^{1, *}$, R. Brand ${ }^{2}$, D. Niederwieser ${ }^{3}$, U. Platzbecker ${ }^{4}$,

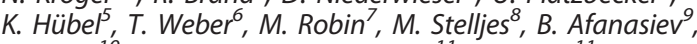
D. Heim ${ }^{10}$, G. Lambertenghi Deliliers ${ }^{11}$, F. Onida ${ }^{11}$, P. Dreger ${ }^{12}$, M. Pini ${ }^{13}$, S. Guidi ${ }^{14}$, L. Volin ${ }^{15}$, M. Gramatzki ${ }^{16}$,W. Bethge ${ }^{17}$, X. Poire ${ }^{18}$, G. Kobbe $e^{19}$, M. van Os ${ }^{20}$, S. lacobelli'i', T. de Witte 22 on behalf of Chronic Malignancies Working Party of the EBMT ${ }^{1}$ Department of Stem Cell Transplantation, University Medical Center Hamburg, Hamburg, Germany, ${ }^{2}$ Medical Statistics and Bioinformatics, Leiden University Medical Center, Leiden, Netherlands, ${ }^{3}$ University Hospital Leipzig, Leipzig, ${ }^{4}$ University Hospital Dresden, Dresden, ${ }^{5}$ University of Cologne, Cologne, ${ }^{6}$ Hematology/ Oncology, University Hospital Halle (Saale), Halle, Germany, 'Hematology and Bone Marrow Transplantation, Saint-Louis Hospital, Paris, France, ${ }^{8}$ Hematology/Oncology, University of Muenster, Münster, Germany, ${ }^{9}$ Department of Stem Cell Transplantation, Pavlov State Medical University, St. Petersburg, Russian Federation, ${ }^{10}$ Hematology, University Hospital Basel, Basel, Switzerland, "University of Milan, Milan, Italy, ${ }^{12}$ Hematology, University of Heidelberg, Heidelberg, Germany, ${ }^{13}$ Hematology and Bone Marrow Transplantation, A.O. SS Antonio e Biagio e C. Arrigo, Alessandria, ${ }^{14}$ Hematology, Ospedale de Careggi, Firenze, Italy, ${ }^{15}$ Helsinki University Cebtral Hospital, Helsinki, Finland, ${ }^{16}$ Department of Stem Cell Transplantation, University Hospital Schleswig-Holstein and University of Kiel, Kiel, ${ }^{17}$ Hematology/Oncology, University of Tuebingen, Tuebingen, Germany, ${ }^{18}$ Hematology, Cliniques Universitaires Saint-Luc, Brussels, Belgium, ${ }^{19}$ University od Duesseldorf, Duesseldorf, Germany, ${ }^{20}$ Medical Statistics and Bioinformatics, Leiden University, Leiden, Netherlands, ${ }^{21}$ Biostatistica e Bioinformatica, University of Tor Vergata, Rome, Italy, ${ }^{22}$ University Medical Centre St. Radboud, Nijmegen, Netherlands

Introduction: Retrospective studies in MDS/sAML suggest that reducing the intensity of the conditioning regimen prior to allogeneic stem cell transplantation reduces the risk of nonrelapse mortality but is associated with a higher risk of relapse, but prospective randomized studies for MDS are lacking so far. Materials (or patients) and methods: Within the Chronic Malignancies Working Party (CMWP) of EBMT, we performed a prospective randomized trial comparing a busulfan based (Busulfan $8 \mathrm{mg} / \mathrm{kg}$ orally or equivalent dosis intravenously (iv) plus fludarabin $180 \mathrm{mg} / \mathrm{m}^{2}$ ) reduced intensity conditioning regimen (RIC) and a standard myeloablative busulfan (Busulfan $16 \mathrm{mg} / \mathrm{kg}$ orally or equivalent dosis iv plus cyclophosphamide $120 \mathrm{mg} / \mathrm{kg}$ ) based regimen (MAC) in patients with MDS or sAML (<20\% blasts). Between May 2004 and December 2012, 129 patients were included from 18 centers and 7 nations and 127 could be analysed. Major inclusion criteria were: MDS (according to FAB: RA, RARS, RAEB, RAEB-t), CMML, and SAML, blasts less than $20 \%$, matched related or unrelated donor (HLA $8 / 8,1$ mismatch was allowed), age 18 - 60 years (for unrelated) and 18 - 65 years (for HLA-identical sibling). Included patients were stratified according related vs unrelated donor and blast count $<$ or $>$ than $5 \%$. The primary endpoint of the study was 1 year non-relapse mortality.The median age of the patients was 51.4 years (r.19-64y). Donors were HLA-identical sibling $(n=33)$, matched unrelated $(n=74)$ and mismatched related or unrelated $(n=20)$. The patients were well distributed in both arms regarding age, gender, IPSS risk profile, number of blasts at transplantation, donor source and mismatch donor. Results: Graft failure occurred in 1 after MAC and 2 after RIC. Median time to leukocyte ( $>1.0 \times 10 \mathrm{e} / \mathrm{L}$ ) occurred after 14days after MAC and 15days after RIC and and platelet (>20x10e9/L) engraftment occurred after 14 days after MAC and 15 days after RIC, respectively. Acute GvHD II-IV was noted in $28 \%$ after RIC and $31 \%$ after MAC. Chronic GvHD was seen in $64 \%$ after RIC and $63 \%$ after MAC. The cumulative incidence (CI) of non-relapse mortality (NRM) after 1 year was 17\% (95\%
$\mathrm{Cl} 8-26 \%)$ after $\mathrm{RIC}$ and $26 \%(95 \% \mathrm{Cl} 15-37 \%)$ after $\mathrm{MAC}$ $(P=0.17)$. The $\mathrm{Cl}$ of NRM at 1 year after HLA-identical sibling transplantation was lower than after unrelated transplantation after RIC ( $0 \%$ vs $23 \%, P=0.06)$ as well after MAC $(17 \%$ vs $32 \%$; $P=0.18)$ The $\mathrm{Cl}$ of relapse at 2 years was $18 \%(95 \% \mathrm{Cl} 8-27 \%)$ after RIC and 15\% (95\% CI 5-24\%) after MAC $(P=0.5)$, resulting in a 2 year relapse-free and overall survival of $62 \%(95 \% \mathrm{Cl} 50-$ $75 \%)$ and $76 \%(95 \% \mathrm{Cl} 65-87 \%)$ after RIC and 57\% (95\% Cl 44 $70 \%)$ and $62 \%(95 \% \mathrm{Cl} 49-75 \%)$ after MAC $(P=0.49$ and $P=0.06$, respectively).

Conclusion: This prospective randomized trial of EBMT provide evidence that RIC resulted in at least similar 2 year relapse-free and overall survival as in MAC for patients with MDS and SAML and less than $20 \%$ blasts.

Disclosure of Interest: N. Kröger: None declared, R. Brand: None declared, D. Niederwieser: None declared, U. Platzbecker: None declared, K. Hübel: None declared, T. Weber: None declared, M. Robin: None declared, M. Stelljes: None declared, B. Afanasiev: None declared, D. Heim: None declared, G. Lambertenghi Deliliers: None declared, F. Onida: None declared, P. Dreger: None declared, M. Pini: None declared, S. Guidi: None declared, L. Volin: None declared, M. Gramatzki: None declared, W. Bethge: None declared, X. Poire: None declared, G. Kobbe Funding from: Celgene, Amgen, Astellas, Novartis, Conflict with: Medav, Neovii, M. van Os: None declared, S. lacobelli: None declared, T. de Witte: None declared.

\section{7}

Allogeneic Transplantation in Patients with HIV-Infection: A Pair Matched Cohort Study by the European Society for Blood and Marrow Transplantation

R. F. F. Duarte ${ }^{1, *}$, M. Labopin ${ }^{2}$, M. Badoglio ${ }^{2}$, J. Y. Cahn ${ }^{2}$, V. Leblond ${ }^{3}$, N. Milpied ${ }^{4}$, A. Peniket ${ }^{5}$, J. Passweg ${ }^{6}$, C. Richard ${ }^{7}$, N. H. Russell ${ }^{8}$, G. Socié, ${ }^{9}$ A. van Biesen ${ }^{10}$, P. Bader ${ }^{11}$, S. Cesaro ${ }^{12}$, P. Dreger ${ }^{13}$, N. Krogër $^{14}$, A. Nagler ${ }^{15}$, A. Sureda ${ }^{7}$, M. Mohty ${ }^{16}$

${ }^{1}$ Catalan Institute of Oncology, Barcelona, Spain, ${ }^{2}$ EBMT Paris Office, ${ }^{3}$ Hopital Pitié-Salpêtrière, Paris, ${ }^{4} \mathrm{CHU}$ Bordeaux Hôpital Haut-leveque, Pessac, France, ${ }^{5}$ Cancer and Haematology Centre Churchill Hospital, Oxford, United Kingdom, ${ }^{6}$ Basel University Hospital, Basel, Switzerland, ${ }^{7}$ Hospital Marqués de Valdecilla, Santander, Spain, ${ }^{8}$ Nottingham City Hospital, Nottingham, United Kingdom, ${ }^{9}$ Hospital St. Louis, Paris, France, ${ }^{10}$ EBMT Leyden Office, Leyden, Netherlands, ${ }^{11}$ Goethe-Universität, Frankfurt, Germany, ${ }^{12}$ Policlinico G.B. Rossi, Verona, Italy, ${ }^{13}$ University of Heidelberg, Heidelberg, ${ }^{14}$ University Hospital Eppendorf, Hamburg, Germany, ${ }^{15}$ Chaim Sheba Medical Center, Tel-Hashomer, Israel, ${ }^{16}$ Hospital Saint Antoine, Paris, France

Introduction: The introduction of highly active antiretroviral therapy (HAART) in 1996 changed the natural history of HIVinfection. Nonetheless, HIV-infected patients (HIV-pts) remain at an increased risk of hematologic malignancies for which HCT is considered standard therapy. HAART enables HIV-pts to undergo autologous HCT for lymphoma with comparable results to the general population. However, the outcome of HIV-pts after allogeneic (allo-HCT) is unknown, with only limited case reports and small series. Here, we present the experience of allo-HCT for hematologic indications in HIV-pts reported to the EBMT registry.

Materials (or patients) and methods: This was a retrospective cohort study of HIV-pts who received a first allo-HCT from 1997 to 2014. HIV-pts' outcomes were compared with HIV-negative controls (HIV-neg), including a 1:3 case-control matched analysis for the following criteria: diagnostic indication, disease status at HCT, year and age at HCT (both $+/-3$ years), gender, previous auto-HCT, donor type, cell source, conditioning regimen (myeloablative vs reduced intensity, TBI vs chemotherapy-based) and ex-vivo manipulation.

Results: We identified 111 HIV-pts who received a first alloHCT for AML $(n=42,38 \%)$, CLPD $(n=25,22 \%)$, ALL $(n=18$, $16 \%)$, MDS $(n=17,15 \%)$, SAA $(n=5,4.5 \%)$, and CML $(n=4$ $3.6 \%)$; 68 cases were male (61\%), median age 42 (range, 3-65; 
including 12 pediatric/adolescent cases $<18$ years), 74 with myeloablative conditioning (67\%; $34 \mathrm{TBI}$ and 40 non-TBIbased), 64 from HLA-identical siblings (58\%), and 9 using ex vivo T-cell depletion. In addition to the above matching criteria, cases and controls were also balanced for other factors such as donor gender and gender mismatch, CMV serostatus, in vivo T-cell depletion and Karnofsky's performance status. Compared to HIV-neg, HIV-pts had lower rates of neutrophil engraftment $(92.6 \%$ vs $97.5 \%, P=0.03)$, higher incidence of grade III-IV acute GVHD ( $21 \%$ vs $13 \%, P=0.05)$, higher NRM at day $100(17 \%$ vs $11 \%, P=0.04)$ and 2 years $(33 \%$ vs $23 \%$, $P=0.04)$, and similar incidence of relapse $(29 \%$ vs $25 \%$, $P=0.96)$. Overall, HIV-pts had poorer PFS (39\% vs $52 \%$ $P=0.03 ; \mathrm{HR} 1.42$ [1.04-1.94]) and OS (47\% vs $59 \% ; P=0.004$ HR 1.60 [1.16-2.22]) at 2 years than HIV-neg cases.Outcomes within HIV-pts were comparable for myeloablative vs reduced intensity conditioning and among peripheral blood, bone marrow and cord blood stem cell sources (data not shown). Finally, while HIV-pts' outcomes were comparable for allo-HCT from HLA-identical siblings and alternative donors (OS: $48 \%$ vs $40 \% ; P=0.38$ ), the use of such alternative donors in HIV-pts was less common than in HIV-neg HCT recipients ( $42 \%$ vs $50 \%$, $P=0.02$ ).

Conclusion: This study showed that the outcome of allo-HCT is poorer in HIV-pts than in the general population, primarily driven by higher NRM, and in keeping with their inferior overall life expectancy despite HAART. Even so, allo-HCT is feasible in HIV-pts with hematologic indications, with a $47 \%$ OS at 2 years. In view of the current reduced use of alternative donors despite comparable results to matched sibling donors, HIV-pts requiring an allo-HCT should be granted access to donor search and consideration for transplantation at the same level as HIV-neg counterparts. These data are key to inform allo-HCT strategies in HIV-pts, including its investigational use to eradicate HIV infection.

Disclosure of Interest: None declared.

\section{ORAL SESSIONS \\ Working Party: Lymphoma (Jian-Jian Luan Award Presentation)}

\section{8}

Haploidentical transplantation (HAPLO-HSCT) with

busulfan based Reduced Intensity Conditioning (RIC) regimens and Post-Transplant Cyclophosphamide (PTCy) as GvHD prophylaxis in patients with relapsed/refractory Hodgkin Lymphoma (HL): Spanish Experience

J. Gayoso 1,*, P. Balsalobre ${ }^{1}$, M. J. Pascual ${ }^{2}$, C. Castilla-Llorente ${ }^{3}$ M. Kwon ${ }^{1}$, D. Serrano ${ }^{7}$, D. Caballero ${ }^{4}$, J. L. Piñana ${ }^{5}$, P. Herrera $^{6}$, C. Ferrá' , C. Pascual', I. Heras ${ }^{3}$, E. Olavarría ${ }^{8}$, L. Bento ${ }^{9}$, I. Buño ${ }^{7}$, J. L. Díez-Martín ${ }^{1}$ on behalf of Grupo Español de Trasplante Hemopoyético (GETH) on behalf of Grupo Español de Trasplante Hemopoyético (GETH)

${ }^{1}$ Hematology, HGU Gregorio Marañón, Madrid, ${ }^{2}$ Hematology, CHU Málaga, Málaga, ${ }^{3}$ Hematology, HU Morales Messeguer, Murcia, ${ }^{4}$ Hematology, Hospital Clínico, Salamanca, ${ }^{5}$ Hematology, Hospital Clínico, Valencia, ${ }^{6}$ Hematology, HU Ramón y Cajal, Madrid, ${ }^{7}$ Hematology, ICO Badalona, Barcelona, ${ }^{8}$ Hematology, CH Navarra, Pamplona, ${ }^{9}$ Hematology, Hosp. Son Espasses, Palma Mallorca, Spain

Introduction: HAPLO-HSCT is a therapeutic option for patients with high risk hematologic neoplasms with the advantages of quick availability, easy programation and logistics, and a committed donor. It has shown promissing results in patients diagnosed with relapsed or refractory Hodgkin Lymphoma $(\mathrm{HL})$ at least comparable to allogeneic transplant from siblings or unrelated donors (Burroughs LM et al. Biol Blood Marrow Transplant 2008; 14:1279-1287).

Materials (or patients) and methods: We retrospectively evaluate the results of HAPLO-HSCT with IV Busulfan (BUX) based RIC regimens (Fludarabine $30 \mathrm{mg} / \mathrm{m} 2 \times 5$ days $(-6$ to -2 ), Cyclophosphamide $14,5 \mathrm{mg} / \mathrm{kg} \times 2$ days ( -6 to -5$),$ BUX $3,2 \mathrm{mg} /$ $\mathrm{kg} \times 1$ (BUX1)or 2 days (BUX2) on days -3 to -2 ) and GvHD prophylaxis based on PTCY $(50 \mathrm{mg} / \mathrm{kg}$ on days +3 and +4$)$ and a calcineurin inhibitor plus mycophenolate from day +5 performed in GETH centers to patients diagnosed with relapsed or refractory HL.

Results: From March-2009, 43 HAPLO-HSCT have been performed in patients diagnosed with relapsed or refractory $\mathrm{HL}$ in 11 GETH centers. Median age was 31 years (17-53), 67\% were males and all were in advanced phases of their disease, after a median of 4 prior treatment lines (2-8). Autologous HSCT was previously employed in $79 \%$, and allogeneic HSCT in $7 \%$. Five patients $(11.5 \%)$ have received more than 2 prior transplants. Disease status at HAPLO-HSCT evaluated by PET was complete remission in $14(32 \%)$ and persistent disease in 29 (68\%). Bone marrow was employed in 11 (26\%) and peripheral blood in 32 (74\%), without T-cell depletion in all cases. The haploidentical donor was patient́s mother (20), father (3), siblings (19) or daughter (1). The RIC regimens employed were BUX1 in $14(32.5 \%)$ and BUX2 in 29 patients (67.5\%). Median neutrophils engraftment was day + 18 (1344) and platelets $>20 \mathrm{~K}$ was day +26 (13-150). Graft failure with autologous reconstitution happened only in 1 patient (2.5\%). The day +100 cumulative incidence $(\mathrm{Cl})$ of non-relapse mortality (NRM) was 7\% (3/43) and $16 \%(7 / 43)$ at 1 year posttransplant. The day $+100 \mathrm{Cl}$ of grade II-IV acute GVHD was $43 \%$, and grade III-IV was $14.5 \%$. Chronic GVHD Cl was $26.5 \%$ at 1 year, being extensive in $6 \%$. After a median follow-up for survivors of 13 months (3-60), the event-free survival (EFS) was $59.5 \%$ and overall survival (OS) was $84 \%$. The 1 -year $\mathrm{Cl}$ of relapse or progression was $25 \%$. Factors related with better 1year EFS were CR prior to HAPLO-HSCT ( $93 \%$ vs $45 \% ; P=0.017$ ) and receiving less than 4 treatment lines prior to HAPLO-HSCT (100\% vs $51.5 \% ; P=0.018)$. No significant differences were seen when comparing BUX1 against BUX2 in terms of NRM, EFS or OS.

Conclusion: HAPLO-HSCT with PTCY and BUX based RIC conditioning in relapsed or refractory $\mathrm{HL}$ patients, renders long-lasting remissions with acceptable toxicity and GVHD, obtaining better results in those transplanted in CR and with less than 4 treatment lines prior to HAPLO-HSCT.

Disclosure of Interest: None declared. 
Oral session: Acute leukemia 1

0009

Activity of Azacitidine in Patients who Relapse after Allogeneic Stem Cell Transplantation for Acute Myeloid Leukaemia (AML) and Myelodysplasia (MDS): a survey from the European Society for Blood and Marrow Transplantation (EBMT)

C. Craddock ${ }^{1, *}$, M. Labopin ${ }^{2}$, M. Houhou ${ }^{3}$, M. Robin ${ }^{4}$, J. Finke ${ }^{5}$, P. Chevallier, I. Yakoub-Agha ${ }^{7}$, J.-H. Bourhis ${ }^{8}, H$. Sengeloev ${ }^{9}, D$. Blaise ${ }^{10}$, P. Dreger ${ }^{17}$, M. Hallek ${ }^{12}$, A. Nagler ${ }^{13}$, N. Kroeger ${ }^{14}$, M. Mohty ${ }^{15}$ ${ }^{1}$ Haematology, University of Birmingham, Birmingham, United Kingdom, ${ }^{2}$ Haematology, Faculté de Médecine, ${ }^{3}$ EBMT, ${ }^{4} \mathrm{Haema-}$ tology, Hôpital Saint-Louis, Paris, France, ${ }^{5}$ Haematology, University of Freiburg, Freiburg, Germany, ${ }^{6}$ Haematology, CHU, Nantes, ${ }^{7}$ Service des Maladies du Sang, Lille, ${ }^{8}$ Haematology, Gustave Roussy, Paris, France, ${ }^{9}$ Copenhagen University, Copenhagen, Germany, ${ }^{10}$ Institut Paoli Calmettes, Marseille, France, ${ }^{11}$ Heildelberg University, Heildelberg, ${ }^{12}$ Center of Intergrated oncology Koln Bonn, Cologne, Germany, ${ }^{13}$ Sheba Medical Center, Ramat Gan, Israel, ${ }^{14}$ Hanburg University, Hamburg, Germany, ${ }^{15}$ Hopital St Antoine, Paris, France

Introduction: Disease relapse is the most common cause of treatment failure after allo-SCT for high risk AML and MDS. Treatment options for patients with relapsed disease are extremely limited and re-induction chemotherapy when utilised is often either poorly tolerated or ineffective. Azacitidine (AZA) is a DNMT inhibitor which is well tolerated and clinically active in high risk AML/MDS. Of interest AZA also up-regulates the expression of tumor antigens and plausibly augments a graft-versus-leukemia effect. A number of small studies have suggested clinical activity of AZA in patients who relapse after an allograft for AML/MDS and we now report the first systematic study of the activity and tolerability of AZA in this patient population.

Materials (or patients) and methods: 272 patients who relapsed at a median of 6.5 months (range 1-49) months after an allograft for AML $(n=174)$ or MDS $(n=98)$ were studied. The median age of patients studied was 58 years (range 2276). 64 patients received a myeloablative conditioning (MAC) regimen and 208 a reduced intensity (RIC) regimen. AZA was administered for 5-7 consecutive days every month. The median duration of AZA treatment was 61 days (IQR 31-147) 111 patients received additional donor lymphocyte infusions (DLI).

Results: AZA was well tolerated in the majority of patients. 4 patients developed Grade 3-4 acute GVHD after AZA treatment. 65 patients achieved a complete or partial remission after AZA administration at a median of 114 days after commencement of treatment. The median number of courses of AZA to achieve a clinical response was 3 . In multivariable analysis the only significant factor determining improved response to AZA was presentation karyotype and relapse occurring more than 12 months post-transplant. The 2 year overall survival (OS) for all patients with relapsed disease was $12 \%$ months after the commencement of AZA therapy. In patients who achieved a CR the 2 year OS was $46 \%$ versus $11 \%$ for the whole population $(P=0.001)$. In multivariable analysis OS was determined by disease relapse more than 6 months post-transplant and bone marrow blasts less than the median at the commencement of therapy. Administration of DLI did not influence CR rate or 2 yr OS. A scoring system including time to relapse, presentation karyotype and bone marrow blast percentage was predictive of CR: $(46 \% \vee 15 \%)$ and $2 \mathrm{yr}$ OS (32\% v 9\%) after AZA salvage.

Conclusion: In conclusion, these data confirm the ability of AZA to salvage a proportion of patients with AML or MDS who relapse after an allogeneic SCT and identify prognostic factors for response and survival. In selected patients $C R$ rates achieved with salvage AZA are comparable to those previously reported with either intensive chemotherapy or DLI. We conclude AZA represents an important and well-tolerated salvage option in the management of patients with $A M L$ and MDS who relapse after allo-SCT.

Disclosure of Interest: None declared.

\section{0}

Matched and Mismatched Unrelated Donor versus Unmanipulated Haploidentical donors for hematopoietic cell transplantation in acute leukemia in remission: a pair matched analysis on behalf of the ALWP of the EBMT

S. Piemontese ${ }^{1,2, *}$, F. Ciceri ${ }^{1,2}$, M. Labopin ${ }^{1,3}$, W. Arcese $^{4}$,

S. Kyrcz-Krzemien ${ }^{5}$, S. Santarone ${ }^{6}$, H. Huang ${ }^{7}$, D. Beelen ${ }^{8}$

N. C. Gorin 1,3, C. Craddock ${ }^{9}$, Z. Gulbas ${ }^{10}$ A. Bacigalupo ${ }^{11}$

A. Nagler ${ }^{1,12}$, M. Mohty ${ }^{1,3}$ on behalf of on behalf of the Acute Leukemia Working Party (ALWP) of the European society for Blood and Marrow Transplantation (EBMT)

${ }^{1}$ EBMT Acute Leukemia Working Party and Registry, Saint Antoine Hospital, Paris, France, ${ }^{2}$ Unit of Hematology and Bone Marrow Transplantation, San Raffaele Scientific Institute, Milan, Italy, ${ }^{3} \mathrm{AP}$ HP, Hématologie Clinique et Thérapie Cellulaire, Hôpital SaintAntoine. Sorbonne Université, UPMC Univ Paris 06, UMR_S 938, CDR Saint-Antoine, F-75012, INSERM, MR S 938., Paris, France, ${ }^{4}$ Hematology Division - Stem Cell Transplant Unit, University of Rome Tor Vergata, Rome, Italy, ${ }^{5}$ Hematology and Bone Marrow Transplantation, Medical University of Silesia, Katowice, Poland, ${ }^{6}$ Hematology - Bone Marrow Transplant Center, Spirito Santo Hospital, Pescara, Italy, ${ }^{7} B M$ Transplantation Center, The First Affiliated Hospital, Zhejiang University School of Medicine, Hangzhou, China, ${ }^{8}$ Department of Bone Marrow Transplantation, University Hospital of Essen, Essen, Germany, ${ }^{9}$ Center for Clinical Hematology, Main Drive Queen Elizabeth Hospital, Birmingham, United Kingdom, ${ }^{10}$ Bone Marrow Transplantation Department, Anadolu Medical Center Hospital, Gebze, Turkey,

${ }^{11}$ Division of Hematology II, IRCCS, San Martino University Hospital - IST, Genoa, Italy, ${ }^{12}$ Hematology Division, BMT and Cord Blood Bank, TelAviv University, Chaim Sheba Medical Center, Tel-Hashomer Ramat- Gan, Tel-Aviv, Israel

Introduction: The best donor source for adult patients with acute leukemia lacking an HLA-identical donor still remains to be established. The objective of this study was to compare the outcome after T-cell-replete hematopoietic stem cell transplantation from haploidentical donor (HAPLO) and transplant from Matched Unrelated Donor (10/10 UD) or Mismatched Unrelated Donor at a single HLA-locus (9/10 UD) for patients with acute leukemia in remission.

Materials (or patients) and methods: From January 2007 to December 2012, 313 consecutive HAPLOs were performed as first allogeneic transplants in adult patients with de novo acute leukemia in CR1 or CR2 and reported to the EBMT Registry. We were able to pair match 273 of these 313 patients with 273 patients who received transplant from 10/10 UD and 273 who received a 9/10 UD transplant. The matching factors were as follows: age $(+/-5 y)$, diagnosis (myeloid or lymphoblastic leukemia), disease status at transplant (CR1 or CR2) and interval from diagnosis to transplant $(+/-1 \mathrm{mo})$.

Results: The median follow-up was 22 months. The two-year non-relapse mortality (NRM) and relapse (RI) cumulative incidences were $28 \%$ and $30 \%$ for HAPLO, $21 \%$ and $19 \%$ for $10 / 10$ UD, and $28 \%$ and $27 \%$ for $9 / 10$ UD. The two-year Kaplan-Meier estimates of leukemia-free survival (LFS) and overall survival (OS) were $42 \%$ and $49 \%$ for HAPLO, $59 \%$ and $65 \%$ for $10 / 10$ UD, $45 \%$ and $51 \%$ for $9 / 10$ UD, respectively. In multivariate analysis both NRM and RI were significantly reduced in 10/10 UD compared to HAPLO (NRM, hazard ratio (HR): $0.61, P=0.02,95 \% \mathrm{Cl}: 0.40-0.91$; RI, HR: $0.59, P=0.01$, $95 \% \mathrm{Cl}: 0.39-0.89)$ but there was no statistical difference between HAPLO and 9/10 UD (NRM, $P=0.59$; Rl, $P=0.52$ ). LFS and OS were significantly higher in 10/10 UD compared to HAPLO (LFS HR: $0.61, P=0.001,95 \% \mathrm{Cl}: 0.45-0.81 .0 S$ HR: 0.63 , $P=0.004,95 \% \mathrm{Cl}: 0.46-0.86)$ but not statistically different between $9 / 10$ UD and HAPLO (LFS, $P=0.45$. OS, $P=0.84$ ). Of note, type of donor was neither associated with day-100 grade 
II-IV acute graft-versus-host disease, nor to chronic graftversus-host disease.

Conclusion: These findings suggest that LFS and OS were significantly higher in patients with acute leukemia in remission receiving a 10/10 UD compared to patients with similar age and disease history receiving a HAPLO. We didn't find significant differences between 9/10 UD and HAPLO. In the absence of a 10/10 UD, host/donor characteristics, urgency of transplant and center expertise should drive us towards the best choice between these two alternative donor sources.

Disclosure of Interest: None declared.

\section{1}

Eradication of Minimal Residual Disease in Children with Relapsed and Refractory ALL by a Newly Developed, Fc-optimized CD19 Antibody

U. J. E. Seidel 1, *, L. Grosse-Hovest ${ }^{2,3}$, P. Schlegel ${ }^{1}$, M. Hofmann ${ }^{2,3}$

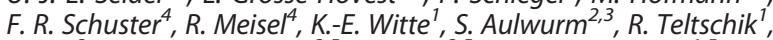
E. Pyz ${ }^{2}$, H.-G. Rammensee ${ }^{2,5}$, G. Jung ${ }^{2,5}$, R. Handgretinger ${ }^{1,5}$, P. Lang ${ }^{1,5}$

${ }^{1}$ Department of General Paediatrics, Oncology/Haematology, University Children's Hospital, ${ }^{2}$ Interfaculty Institute for Cell Biology, Department of Immunology, University of Tübingen, ${ }^{3}$ SYNIMMUNE GmbH, Tübingen, ${ }^{4}$ Department of Paediatric Oncology, Haematology and Immunology, Heinrich Heine University, Düsseldorf, ${ }^{5}$ Partner Site Tübingen, German Cancer Consortium (DKTK) and German Cancer Research Center (DKFZ), Tübingen, Germany

Introduction: B-lineage acute lymphoblastic leukemia (ALL) is the most common childhood cancer. Although this disease can be successfully treated in $80 \%$ of patients, prognosis for primary refractory or relapsed disease is very poor. Even after allogeneic stem cell transplantation (SCT), relapse rates are considerable and correlate significantly with persistent minimal residual disease (MRD) prior to and after SCT. MRD constellations represent favorable effector-target ratios and thus might be optimally suited for immunotherapeutic intervention with therapeutic antibodies.

Materials (or patients) and methods: We developed an Fcoptimized CD19 antibody (4G7SDIE) and produced it in pharmaceutical quality. 4G7SDIE mediates markedly enhanced antibody-dependent cellular cytotoxicity (ADCC) through its improved capability to recruit Fc $\gamma$ RIlla bearing effector cells including NK cells and $\gamma \delta$ T cells.

4G7SDIE was applied on compassionate use to MRD-positive pediatric patients with relapsed or refractory ALL (CR1 $n=3$, $\geq \mathrm{CR} 2, n=11$ ).

Results: Side effects such as headache and fever were negligible. In all patients complete $\mathrm{CD}_{2} \mathrm{O}^{+}$B-cell depletion was observed during therapy. After discontinuation of 4G7SDIE therapy B cell counts recovered rapidly to normal levels. In $9 / 14$ patients MRD was reduced by $\geq 1 \log$ or fell below MRD-detection threshold of $10^{-4}$ over the course of treatment. 2/9 responders were receiving additional treatment. 6 patients relapsed, 1 patient died of CNS chemotherapy associated toxicity and 1 patient died of late posttransplant sepsis. 6 patients have been in sustained remission for 2641115 days (median follow-up 720 days).

In initial cytotoxicity screenings, performed in an allogeneic setting, significantly increased lysis of ALL blasts by PBMC after adding 4G7SDIE was observed. NK cells and $\gamma \delta$ T cells were identified as main effector cell populations.

CD19 expression on patient blasts was confirmed by quantitative flow cytometry (mean $1.71 \times 10^{4}$ molecules/ cell, $\left.\pm 0.54 \times 10^{4}\right)$. Cytotoxicity assays using patient PBMC on autologous blasts confirmed sustained functionality of patient effector cells over the course of 4G7SDIE treatment. Cytotoxicity assays were performed using PBMC from transplanted patients obtained at different time points of 4G7SDIE treatment. Lysis of autologous ALL blasts was increased when 4G7SDIE or autologous patient serum taken after antibody infusion was added. After infusion of $20 \mathrm{mg} / \mathrm{m}^{2}-40 \mathrm{mg} / \mathrm{m}^{2}$
4G7SDIE serum half-life was $20 \mathrm{~h}-43 \mathrm{~h}$. Serum levels of 4G7SDIE remained above saturating concentrations of $\geq 700$ $\mathrm{ng} / \mathrm{ml}\left(\mathrm{EC}_{50}=65 \mathrm{ng} / \mathrm{ml}\right)$ until the following application in the bi-weekly treatment cycle.

Notably, in 2/2 analyzed patients under 4G7SDIE therapy, a down-modulation of CD19 surface expression on the leukemic blasts was observed. In vitro antigenic shift assays on patient blasts showed considerable but very heterogeneous shift of CD19 surface expression. Furthermore, a positive correlation between CD19 surface expression levels and 4G7SDIE mediated lysis was observed. These observations hint at in vivo tumor escape mechanisms and moreover indicate selective pressure exerted by immunotherapy with 4G7SDIE, underlining its therapeutic potential, but also delineating possible limitations.

Conclusion: Promising anti-leukemic effects of the 4G7SDIE antibody have been observed in vitro and in vivo. We are currently preparing a clinical phase I/II trial.

Disclosure of Interest: None declared.

0012

Improving results of allogeneic HCT for adults with acute lymphoblastic leukemia: an analysis from Acute Leukemia Working Party of the EBMT

S. Giebel 1,**, M. Labopin ${ }^{2,3}$, G. Socié, ${ }^{4}$ D. Beelen ${ }^{5}$, P. Brown ${ }^{6}$,

L. Volin ${ }^{7}$, S. Kyrcz-Krzemien ${ }^{8}$, I. Yakoub-Agha ${ }^{9}$, M. Aljurf ${ }^{10}$,

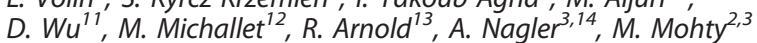

${ }^{7}$ Maria Sklodowska-Curie Memorial Cancer Centter and Institute of Oncology, Gliwice Branch, Gliwice, Poland, ${ }^{2}$ Hospital St. Antoine, ${ }^{3}$ Acute Leukemia Working Party of the EBMT, ${ }^{4}$ Hopital St. Louis, Paris, France, ${ }^{5}$ University Hospital, Essen, Germany, ${ }^{6}$ St. James's Hospital - Trinity College, Dublin, Ireland, ${ }^{7}$ Helsinki University Central Hospital, Helsinki, Finland, ${ }^{8}$ Silesian Medical University, Katowice, Poland, ${ }^{9}$ Hôpital Huriez, Lille, France, ${ }^{10}$ King Faisal Specialist Hospital \& Research Centre, Riyadh, Saudi Arabia, ${ }^{1}$ First Affiliated Hospital of Soochow University, Suzhou, China, ${ }^{12}$ Centre Hospitalier Lyon Sud - Service Hematologie, Lyon, France, ${ }^{13}$ Charité Universitätsmedizin Berlin - Campus Virchow Klinikum, Berlin, Germany, ${ }^{14}$ Chaim Sheba Medical Center, TelHashomer, Israel

Introduction: Allogeneic HCT with myeloablative conditioning is considered a standard of care for adults with high risk acute lymphoblastic leukemia (ALL). However, with improving results of conventional-dose chemotherapy and the introduction of novel agents on one hand, and the improvement in transplantation techniques on the other, the indications for alloHCT require re-evaluation, taking into account patient- and procedure-related factors associated with the risk of nonrelapse mortality (NRM). The aim of this study was to analyze the results of myeloablative alloHCT treatment for patients with ALL according to recipient age and donor type.

Materials (or patients) and methods: 4859 patients treated with alloHCT in first complete remission during the period 1993-2012 were included. The outcomes were analyzed for the periods 1993-2002, 2003-2007 and 2008-2012, in various age groups, separately for HLA matched sibling (MSD, $n=2681$ ) and unrelated donors (URD, $n=2178$ ).

Results: For MSD-alloHCT recipients treated during the period 2008-2012, the following two-year probabilities of OS were obtained: $76 \%$ for the $18-25$ years old (y.o.) group, $69 \%$ for both the $26-35$ y.o. and $36-45$ y.o. groups and $60 \%$ for the $46-55$ y.o. group. The incidence rates of NRM were $12 \%, 11 \%, 15 \%$ and $24 \%$, respectively for those same age groups. In comparison with the 1993-2007 period, significant improvements were observed for all age groups except for the 26-35 y.o. patients. The improved survival rates were a consequence of reduced NRM and a tendency towards a reduced risk of relapse.

Among URD-alloHCT recipients, the OS was $66 \%$ (18-25 y.o.), $70 \%$ (26-35 y.o.), 61\% (36-45 y.o.), and 62\% (46-55 y.o.), while the respective incidence rates of NRM were $21 \%, 20 \%, 21 \%$ and $19 \%$, The improvement of OS over time was documented for $36-45$ y.o. $(P=0.005)$ and $46-55$ y.o. $(0.0007)$ patients due 
to the reduced incidence of NRM with no significant effect on relapse.

In a multivariate analysis adjusted for disease-, patient-, donorand procedure-related factors, transplantations performed for the period 2008-2012, when compared to 1993-2007, were associated with significantly reduced risks of the overall mortality $(\mathrm{HR}=0.72, P=0.0003)$, treatment failure (either relapse or NRM, HR=0.77, $P=0.002)$, and NRM (HR=0.73, $P=0.01)$ and showed a trend towards reduced risk of relapse $(\mathrm{HR}=0.81, P=0.06)$. The overall mortality was reduced for transplants with TBI-based compared to chemotherapy-based conditioning $(\mathrm{HR}=0.71, P=0.005)$ as a result of reduced risk of relapse ( $\mathrm{HR}=0.55, P=0.00004)$. Type of donor (MSD vs. MUD) had no significant effect on survival $(H R=0.9, P=0.24)$. Conclusion: Results of alloHCT for adults with ALL improved significantly over time in all age groups, mainly due to the reduction of NRM. Importantly, results obtained with matched unrelated transplants were comparable to sibling transplants. Total body irradiation should still be considered as the preferable type of myeloablative conditioning for ALL.

Disclosure of Interest: None declared.

\section{3}

CMV seronegativity is associated with a $20 \%$ decrease in 1-year survival in patients undergoing reduced intensity sibling-donor transplantation for treatment of myeloid malignancy

D. J. Lewis ${ }^{1, *}$, C. Holmes ${ }^{1}$, K. Peggs ${ }^{2}$, A. Peniket ${ }^{3}$, M. Nikolousis ${ }^{4}$, S. Nagra ${ }^{5}$, G. Pratt ${ }^{1,4}$ C. Craddock ${ }^{1,5}$, R. Malladi ${ }^{1,5}$, P. Moss ${ }^{1,5}$

${ }^{1}$ School of Cancer Sciences, University of Birmingham, Birmingham, ${ }^{2}$ University College Hospital, London, ${ }^{3}$ Oxford University Hospital, Oxford, ${ }^{4}$ Birmingham Heartlands Hospital, ${ }^{5}$ Centre for Clinical Haematology, University Hospital Birmingham, Birmingham, United Kingdom

Introduction: Reduced intensity (RIC) allogeneic stem cell transplantation is a highly effective treatment for acute myeloid leukaemia and the immunological 'graft versus leukaemia' effect is believed to be a major mechanism of disease control. Cytomegalovirus reactivation has been suggested to reduce the rate of disease relapse in acute myeloid leukaemia (AML). We investigated the influence of CMV serostatus on the clinical outcome of patients who underwent $\mathrm{T}$ cell depleted RIC allografts for myeloid malignancy, and went on to examine reconstitution of lymphoid subsets.

Materials (or patients) and methods: We studied patients who underwent RIC allografts for AML $(n=272)$ and MDS $(n=82)$ from four UK centres, with fludarabine and melphalan conditioning $+/$ - alemtuzumab. Overall survival was calculated. Relapse rate and non-relapse mortality were calculated with competing risk analyses.

Results: The median overall survival was 2.17 years. The relapse rate of the entire cohort was $23 \%$ at 1 year with a nonrelapse mortality of $22 \%$.

The overall survival for the 'CMV at risk' group was $64.7 \%$ at 1 year compared to $57.9 \%$ for CMV (-/-). This difference was most marked in patients transplanted from sibling donors $(n=140)$; the overall survival at 1 year was $79 \%$ in CMV-at-risk patients $(n=99)$ compared to only $59 \%$ in the CMV seronegative group $(n=41)(P=0.027)$. This $20 \%$ increment in survival was due to a $37 \%$ reduction in the rate of disease relapse in patients that were CMV-at-risk (1 year relapse rate of $21 \%$ versus $33 \%$ ). There was a non-significant trend towards improved overall survival in those that experienced CMV reactivation amongst the CMV-seropositive patients (1 year OS $83 \%$ versus $61 \%$, $P=0.19$ ), mainly due to a reduction in the rate of relapse ( 1 year relapse rate $17 \%$ versus $28 \%$ ).

Because of this large difference in relapse risk, we went on to examine the effects of CMV serostatus and alemtuzumab use on reconstitution of lymphocyte subsets at 3 months post transplant. Alemtuzumab led to 5 -fold decrease in the T cell count at 3 months compared to transplants in which $T$ cell depletion was not used. However, within this alemtuzumab group, positive CMV serostatus in the donor or recipient was associated with a relative 7 fold and 35 fold increase in the $\mathrm{CD} 3+$ and CD8 + T cell count compared to CMV seronegative pairs. Indeed, CD8 + T cells were virtually undetectable at this time point in CMV seronegative transplant/donor pairs.

Conclusion: CMV seropositivity is markedly beneficial for patients who undergo a sibling donor reduced intensity allograft for myeloid malignancy and in whom alemtuzumab is used for conditioning. This effect is most likely to be due to the profound influence of chronic viral replication on boosting $T$ cell immune reconstitution in the early post transplant period. CMV seronegative patients with AML should be considered at risk of impaired survival for certain subgroups of stem cell transplant.

Disclosure of Interest: None declared.

\section{4}

Unrelated donor versus matched sibling donor in adults with acute myeloid leukemia in first relapse, an ALWP-

\section{EBMT study}

A. Ruggeri ${ }^{1,2, *}$, M. Labopin ${ }^{2}$, G. Ehninger ${ }^{3}$, D. Beelen ${ }^{4}$, J. Tischer ${ }^{5}$ A. Ganser ${ }^{6}$, R. Schwerdtfeger $^{7}$, B. Glass ${ }^{8}$, J. Finke, M. Michallet ${ }^{10}$ M. Stelljes ${ }^{11}$, P. Jindra ${ }^{12}$, R. Arnold ${ }^{13}$, N. Kröger ${ }^{14}$, M. Mohty ${ }^{2}$,

A. Nagler ${ }^{15}$

${ }^{1}$ Hôpital Saint-Louis AP-HP, Eurocord, ${ }^{2}$ Service d'hématologie clinique et de thérapie cellulaire, Hôpital Saint-Antoine, AP-HP, Paris, France, ${ }^{3}$ Medizinische Klinik und Poliklinik I, Universitaetsklinikum Carl Gustav Carus der TU Dresden, Dresden, ${ }^{4}$ Department of Bone Marrow Transplantation, University Hospital, Essen, ${ }^{5}$ Department of Internal Medicine III, Hematopoietic Cell Transplantation, University Hospital of Munich-Klinikum Grosshadern (LMU), Munich, 'Department of Hematology, Hemostasis, Oncology and Stem Cell Transplantation, Hannover Medical School, Hannover, ${ }^{7} B M T$ Unit, German Clinic for Diagnostic, Wiesbaden, ${ }^{8}$ Asklepios-Klinik St. Georg, Hamburg, ${ }^{9}$ Freiburg University Medical Center, Freiburg, Germany, ${ }^{10}$ Hôpital Edouard Herriot, Lyon, France, ${ }^{11}$ Universitätsklinikum Münster, Medizinische Klinik und Poliklinik A, Münster, Germany, ${ }^{12}$ University Hospital, Pilsen, Czech Republic, ${ }^{13}$ Medical Department, Division of Haematology, Oncology and Tumour Immunology, Charité Campus Virchow Klinikum, Institute of Hygiene and Environmental Medicine, University Medicine, Berlin, ${ }^{14}$ University Medical Center Hamburg-Eppendorf, Hamburg, Germany, ${ }^{15}$ Division of Hematology and Bone Marrow Transplantation, Chaim Sheba Medical Center, Tel-Hashomer, Israel

Introduction: Allogeneic stem cell transplantation is the only curative option for patients with high risk acute myeloid leukemia (AML) and for those experiencing relapse. Either matched sibling donor (MSD) or unrelated donor (UD) is indicated.

Materials (or patients) and methods: With the aim to compare the outcomes of both strategies we have retrospectively analysed 1554 adults with AML receiving either MSD $(n=961)$ or UD $(n=593)$ in EBMT centers from 20002012. For UD, 481 were 10/10 HLA matched and 112 were $9 / 10$. Median follow up was 28 (range 3-157) months. There were statistical differences between the 2 groups. Compared to MSD recipients, UD transplants were older (49 vs 52 years, $P=0.001$ ), were performed more recently (2009 vs 2006, $P=0.001)$, had longer interval between diagnosis to transplant (10 vs 9 months, $P=0.001$ ), had more often secondary AML (13\% vs $19 \%, P=0.002)$ and were transplanted with higher proportion of CMV negative donors ( $38 \%$ vs $37 \%, P=0.001$ ). Peripheral blood stem cells (PBSC) was used as graft source in $90 \%$ of patients in both groups, $P=0.14$. Conditioning regimen was more frequently myeloablative for patients transplanted with a MSD (61\% vs $46 \%, P=0.001)$. MSD received more often busulfan and cyclophosphamide as MAC $(16 \%)$ or a TBI based regimen (18\%). For UD, Bu-fludarabine was the most frequent conditioning used (19\%).

Results: Cumulative incidence $(\mathrm{Cl})$ of neutrophil engraftment was similar ( $93 \%$ vs $92 \%$ for MSD vs UD, respectively, $P=0.07$ ). 
Grade II-IV acute GVHD was $26 \%$ vs $30 \%(P=0.11)$ and $\mathrm{Cl}$ of chronic GVHD was $25 \%$ vs $24 \%(P=0.9)$ for MSD and UD, respectively.

For MSD and UD respectively, $\mathrm{Cl}$ of relapse (RI) was $57 \%$ vs $49 \%, P=0.001 ; \mathrm{Cl}$ of non-relapse mortality (NRM) was $23 \%$ vs $24 \%, P=0.24$. Probability of leukemia-free survival (LFS) at 2 years was $21 \%$ vs $26 \%, P=0.001$, and overall survival (OS) was $26 \%$ vs $33 \% P=0.004$, respectively.

Chronic GVHD as time-dependent variable was associated with lower RI (HR 0.78, $P=0.05)$, higher NRM (HR 1.71, $P=0.001$ ), and higher OS (HR 0.69, $P=0.001)$.

According to HLA-match for MSD, 10/10 UD and 9/10 UD, Cl of relapse (RI) was $57 \%$ vs $50 \%$ vs $45 \% \%, P=0.001 ; \mathrm{Cl}$ NRM was $23 \%$ vs $23 \%$ vs $29 \% \%, P=0.26$ and probability of LFS at 2 years was $21 \%$ vs $27 \%$ vs $25 \%, P=0.003$, respectively.

In a multivariate analysis adjusted for differences between the 2 groups, UD was associated with lower RI (HR 0.76, $P=0.001$ ) and higher LFS (HR 0.83, $P=0.001$ ) compared to MSD. When analyzing according to HLA-match, there was no differences for patients transplanted with an UD 9/10 or a 10/10 for RI (HR $0.77, P=0.10)$, and LFS (HR 0.92, $P=0.53$ ). The other factors independently associated with better outcomes were the interval between diagnosis and transplant (RI HR 0.62, $P<0.001$ ), and LFS (HR 0.67, $P<0.001$ ).

Conclusion: Unrelated donor transplant was associated with better LFS due to lower RI compared to MSD for high-risk patients with AML transplanted in first relapse. There were not differences for the UD $9 / 10$ match probably due the graftversus-leukemia effect in the setting of patients transplanted with active disease.

Disclosure of Interest: None declared.

\section{5}

Prospective trial of RIC transplant versus standard of care in elderly $A M L$ patients

M. Brune ${ }^{1, *}$, P. Ljungman ${ }^{2}$, E. Wallhult ${ }^{3}$, R. Delage ${ }^{4}, H$. Anderson $^{5}$, V. Lazarevic ${ }^{6}$, K. Remes ${ }^{7}$, J. Finke ${ }^{8}$, M. Lalancette', M. Sabloff ${ }^{10}$, R. Spearing ${ }^{11}$, T. Gedde-Dahl ${ }^{12}$, T. Kiss ${ }^{13}$

${ }^{1}$ University of Gothenburg, Göteborg, ${ }^{2}$ Karolinska Insitute, Stockholm, ${ }^{3}$ Department of Hematology, University of Gothenburg, Göteborg, Sweden, ${ }^{4}$ Department of Hematology, Hôpital de I'Enfant-Jésus, Quebec, Canada, ${ }^{5}$ Dpt of Clinical Sciences, ${ }^{6}$ Dpt of Hematology, University of Lund, Lund, Sweden, ${ }^{7}$ Dpt of Hematology, Turku University Hospital, Turku, Finland, ${ }^{8}$ Abtlg. Innere Medizin I, Universitätsklinikum Freiburg, Freiburg, Germany, ${ }^{9} \mathrm{CHU}$ de Québec, Quebec, ${ }^{10}$ Department of Medicine, University of Ottawa, Ottawa, Canada, ${ }^{11}$ Dpt of Hematology, Christchurch Hospital, Christchurch, New Zealand, ${ }^{12}$ Department of Hematology, Oslo University Hospital, Oslo, Norway, ${ }^{13}$ Department of Hematology, Hôpital Maisonneuve Rosemont, Montreal, Canada

Introduction: Allogeneic reduced intensity transplantations (RICT) in elderly patients (pts) with AML in CR1 has become a commonly used treatment modality. However, no prospective studies to date have been reported to support use of this strategy. The aim of this prospective, multicenter study is to compare outcomes of patients receiving RICT with patients being treated with conventional chemotherapy.

In 2012 an amendment allowed also the use of matched unrelated donors. The study is currently ongoing in 8 countries with a total of 250 pts included. Only pts included as per the original protocol are accounted for in this interim analysis which focuses on safety.

Materials (or patients) and methods: The study was designed by the Transatlantic Leukemia Group (TRALG) consisting of centers associated with the Swedish and Canadian BMT Groups, and centers from Germany, Norway, Finland and New Zealand.

Patients should have intermediate or high risk AML in CR1 and not being eligible for a myeloablative transplant due to age or comorbidities, and have at least one potential sibling donor. Date of inclusion was defined as the date of HLA-typing of the first sibling, and pts were allocated to the donor (D) or no-donor group (noD) based on HLA match. Overall survival at $3 \mathrm{yrs}$ is the primary endpoint; secondary endpoints include RFS, GvHD, non-relapse (NRM) and transplant related (TRM) mortality. Conditioning consisted in the vast majority of patients of fludarabine $150 \mathrm{mg} / \mathrm{m}^{2} \mathrm{IV}$ and busulphane $8 \mathrm{mg} /$ $\mathrm{kg}$ PO or $6.4 \mathrm{mg} / \mathrm{kg}$ IV. GvHD prophylaxis was CyA/Methotrexate or CyA/MMF. The study started to accrue patients in 2004. Results were analyzed on an intent to treat basis.

Results: From 2004 to 2011, 146 pts were included in Sweden $(n=62)$, Canada $(n=56)$, Germany (5), Norway (13), New Zealand (5) and Finland (5). 12 patients were excluded from analysis; 3 favorable risk, 9 protocol violations and 134 pts; 60 females, 74 males, median age 62 (51-74) yrs, 21 sAML, were allocated to the $\mathrm{D}$ group $(n=75)$, or the noD group $(n=59)$. In the $D$ group, 16 pts (21\%) did not reach transplant, 15 of these died (12 after relapse, 3 other causes).

Cytogenetic risk groups were categorized as per ELN criteria. The distribution of total disease risk categories was 25\% adverse, $66 \%$ intermediate, $9 \%$ unknown.

Median follow-up of surviving pts at Dec $6^{\text {th }} 2014$ was 4.6 (2.6 $-10.1)$ yrs after inclusion. Ninety-one pts relapsed (75\%). Kaplan-Meier estimates of OS and RFS in the whole group at 3 yrs were $45 \%$ and $37 \%$ respectively. For adverse and intermediate risk pts OS and RFS at 3 yrs were $36 \%$ and $21 \%$, and $51 \%$ and $44 \%$, respectively. In the transplanted pts acute GvHD grade $0-1$ occurred in $73 \%$ of pts, grade $2-4$ in $20 \%$. Extensive GvHD occurred in $42 \%$, limited in $15 \%$.

89 pts (66\%) have died, 17 without and 72 after AML relapse. In the noD group 5 (8\%) pts died from non-relapse causes. In the $\mathrm{D}$ group, 3 pts died before transplant, while the TRM was 9/59 (15\%) with causes of death: 6 GvHD, 3 other.

Conclusion: Selected patients with AML in CR1 tolerate RICT or standard management with low mortality. Disease control remains a major issue. This multicenter prospective protocol will continue to accrue patients until relevant conclusions can be drawn comparing a RICT to standard treatment. The current rapid inclusion of pts and participation of new sites in Australia, Greece and Estonia will help to complete the study. Disclosure of Interest: None declared.

\section{6}

Targeting WT1 reactivation in patients in complete remission after allogeneic haematopoietic stem cell transplantation for AML and MDS: results in 111 patients. E. Sala ${ }^{1, *}$, M. G. Carrabba ${ }^{2}$, C. Messina ${ }^{1}$, L. Vago ${ }^{1}$, C. Tresoldi ${ }^{1}$, F. Pavesi ${ }^{1}$, E. Xue ${ }^{1}$, B. Gentner ${ }^{1}$, A. Assanelli ${ }^{1}$, S. Marktel', M. T. Lupo-Stanghellini ${ }^{1}$, C. Corti ${ }^{1}$, J. Peccatori ${ }^{1}$, F. Ciceri $^{\prime}$, M. Bernardi ${ }^{7}$

${ }^{1}$ Unit of Hematology and Bone Marrow Transplantation, IRCCS San Raffaele Scientific Institute, ${ }^{2}$ Unit of Hematology and Bone Marrow Transplantation, San Raffaele Scientific Institute, Milan, Italy

Introduction: The Wilms' tumor gene 1 (WT1) is overexpressed in $>80 \%$ of acute myeloid leukemias (AML) and myelodysplastic syndromes (MDS) and proved to be a good marker for minimal residual disease (MRD) monitoring. Although allogeneic haematopoietic stem cell transplantation (allo-HSCT) is the most effective treatment for AML/MDS, disease recurrence remains a major problem. After allo-HSCT, quantitative WT1 monitoring can represent a useful tool to detect MRD and tailor immunotherapeutic strategies.

Materials (or patients) and methods: We included in this retrospective analysis 111 pts with AML/MDS (99 and 12 respectively) overexpressing WT1 and allotransplanted in our Institution from $12 / 2007$ to $01 / 2014$. Twenty two pts were transplanted from a matched related donor, 26 from a matched unrelated donor, 57 from a mismatched related donor and 6 from cord blood units. 101/111 pts received a reduced toxicity conditioning treosulfan-based. At the time of transplant, $73 / 111$ pts (66\%) were in CR, while 38/111 (34\%) had active disease. Median follow up (FU) of our cohort was 
599 days (range, 55-2538 days). WT1 MRD monitoring was performed by RQ-PCR and considered positive for values $>250$ copy numbers of WT1 per 10e4 ABL [1]. After allo-HSCT, detection of positive WT1 was followed by immunomodulatory therapeutic interventions according to the time from transplant, the presence of active graft-versus-host disease (GvHD) and the general clinical conditions: tapering and/or discontinuation of immunosuppressive drugs (IS), donor lymphocytes infusions (DLI), administration of hypomethylating agents. Median time to disease relapse was calculated from the time of detection of WT1 value above the threshold. Results: At day 30 post allo-HSCT, 109 out of 111 pts (98\%) were in CR. Forty-five out of 109 (41\%) CR pts had WT1 levels persistently negative during follow up (FU) and 23/45 remained in CR at the last FU. Sixty-four out of 109 pts (59\%) had at least one increase of BM WT1 levels above the threshold during observation and were evaluated for preemptive treatment. In 45/64 (70\%) IS was tapered until suspension and/or DLI +/- azacytidine was administered. Median time from first WT1 positive value to treatment was 30 days (range, $15-45$ days). In 16 out of these 45 pts (35\%) WT1 level normalized, whereas 29 pts $(65 \%)$ progressed to overt disease. All the 16 that normalized WT1 remained in CR. No grade III or IV acute GvHD was observed, while severe chronic GvHD occurred in 2/45 pts (4\%). Median time to disease relapse for the 45 treated pts was 116 days (range, 20-951 days). In 11/64 pts (17\%) with a post allo-HSCT WT1 positivity, the presence of an active form of acute or chronic GvHD prevented from applying further immunotherapy strategies. Among these pts, 5 remained in CR, 6 relapsed. Median time to disease recurrence for these 11 pts with GVHD was 156 days (range, 32-204 days). Finally in 8/64 pts (13\%) concurrent clinical issues (e.g. active infection) did not allow any attempt to prevent relapse and 4/8 relapsed. Median time to disease relapse for untreated pts was 35 days (range, 15-82 days).

Conclusion: In our series, pre-emptive immune-modulatory maneuvers targeting WT1 levels proved to be feasible and safe and of potential clinical benefit to postpone overt relapse in high risk AML/MDS pts post allo-HSCT.

References: [1] Cilloni et al. JCO 2009. Nov 1;27(31): 51955201

Disclosure of Interest: None declared.

\section{Oral session: Conditioning regimens}

\section{7}

Allogeneic Stem-Cell Transplantation In AML with Myeloablative compared to Reduced Intensity Conditioning: 10 Years Later. A report on behalf of the ALWP of EBMT

A. Shimoni ${ }^{1, *}$, M. Labopin ${ }^{2}$, F. Ciceri ${ }^{3}$, A. Bacigalupo ${ }^{4}$, W. Arcese ${ }^{5}$, Y. Koc ${ }^{6}$, D. Beelen ${ }^{7}$, Z. Gülbas ${ }^{8}$, D. Wu ${ }^{9}$, S. Santarone ${ }^{10}$, J. Tischer ${ }^{17}$, B. Afanasyev ${ }^{12}$, M. Mohty', A. Nagler ${ }^{1}$ ${ }^{1}$ CHAIM SHEBA MEDICAL CENTER, Tel-Hashomer, Israel, ${ }^{2}$ Hôpital Saint Antoine, Service d'Hématologie et de Thérapie cellulaire, Paris, France, ${ }^{3}$ Ospedale San Raffaele, Milano, ${ }^{4}$ Ospedale San Martino, Genova, ${ }^{5}$ Rome Transplant Network, Rome, Italy, ${ }^{6}$ Medical Park Hospitals, Antalya, Turkey, ${ }^{7}$ University Hospital, Essen, Germany, ${ }^{8}$ Anadolu Medical Center Hospital, Kocaeli, Turkey, ${ }^{9}$ First Affiliated Hospital of Soochow University, Suzhou, China, ${ }^{10}$ Ospedale Civile, Pescara, Italy, ${ }^{11}$ Ludwig-Maximilians University of Munich-Klinikum Grosshadern, Munich, Germany, ${ }^{12}$ Saint Petersburg State Medical Pavlov University, St. Petersburg, Russian Federation

Introduction: Allogeneic stem cell transplantation (SCT) with both myeloablative (MAC) and reduced intensity conditioning (RIC) is effective therapy in AML. Several studies have shown that leukemia-free survival (LFS) is similar after SCT with MAC and RIC. However, there is paucity of data on the long term outcome (beyond 10 years) following RIC due to the relative recent introduction of this approach. The ALWP of EBMT published in Leukemia 2005 the largest study until that time, comparing the outcome of AML patients (pts) given RIC $(n=315)$ and MAC $(n=407)$. The median follow-up was 13 months. In multivariate analysis, non-relapse mortality (NRM) was significantly lower and relapse rate was significantly higher after RIC resulting in similar LFS.

Materials (or patients) and methods: In order to better predict long-term outcome and late events we have now updated SCT outcomes in a larger cohort of pts, age $\geq 50$ years $(n=1423)$ after SCT from matched sibling donors, in the years 1997-2005 with a median follow up of 8.3 years (0.1-17) Results: 722 pts were given RIC and 701 MAC regimens. The median age at SCT was 57 (50-75) and 54 (50-72) years, respectively $(P<0.001)$. $21 \%$ of RIC recipients had advanced disease at SCT compared to $25 \%$ of MAC recipients. The percentage of pts in CR1 and CR2/ later CR was $62 \%$ and $17 \%$ compared to $63 \%$ and $12 \%$, respectively $(P=0.02)$. RIC recipients were more likely to receive PBSC $(92 \%$ Vs $73 \%$, $P<0.001)$ and in vivo T-cell depletion (33\% Vs $12 \%, P<0.001)$. $16 \%$ and $20 \%$ had poor-risk cytogenetics, respectively $(P=0.19)$. 10 -year NRM was $20 \%(95 \% \mathrm{Cl}, 17-24)$ and $35 \%$ $(95 \% \mathrm{Cl}, 31-39)$ after $\mathrm{RIC}$ and $\mathrm{MAC}$, respectively $(P<0.001)$. Relapse incidence was $48 \%(44-52)$ and $34 \%(31-38), P<0.001$. 10-year LFS was 32\% (28-35) and 31\% (27-35), respectively. Multivariate analysis identified RIC (HR $0.56, P<0.001$ ), age $\geq 55$ (HR 1.5, $P=0.004$ ), advanced disease (HR 1.6, $P=0.02$ ) and female donor to male recipient (HR 1.4, $P=0.01$ ) as factors predicting NRM. Relapse was predicted by RIC (HR 1.2, $P=0.13)$, SCT at CR2/later CR (HR 1.7, $P=0.004)$ or advanced disease (HR 2.7, $P<0.001$ ), in vivo T-cell depletion (HR 1.4 $P=0.01)$, age $\geq 55$ (HR 1.5, $P=0.004$ ) and poor cytogenetics (HR 3.4, $P<0.001$ ). LFS was predicted by SCT at CR2/later CR (HR 1.5, $P=0.001$ ) or advanced disease (HR 2.2, $P<0.001$ ), age $\geq 55$ (HR 1.4, $P<0.001$ ) and poor cytogenetics (HR 1.7, $P<0.005)$. The conditioning regimen did not predict 10 -year LFS. NRM rates were higher after MAC than RIC throughout the late post SCT course, while relapse rates were only mildly decreased at the late phases. In pts surviving leukemia-free 2 years after SCT the subsequent NRM was $15 \%$ and $9 \%$, respectively $(P=0.03)$. Subsequent relapse rates were $14 \%$ and $9 \%(P=0.12)$ and LFS was $71 \%$ and $73 \%$, respectively $(P=0.76)$. In pts surviving leukemia-free 5 years after SCT, subsequent NRM was $9 \%$ and $4 \%$, respectively $(P=0.06)$. Subsequent relapse rates were $5 \%$ and $6 \%(P=0.53)$, and LFS was $86 \%$ and $90 \%$, respectively $(P=0.27)$.

Conclusion: LFS remains similar after RIC and MAC even 10 years after SCT. The trend for excess NRM with MAC remains throughout the late course, however excess relapse after RIC is more obvious in the early phases. Pts remaining leukemia-free 5 years after SCT can expect excellent subsequent outcome with both regimens. Long-term follow-up studies (beyond 10 years) are of significant importance when assessing SCT outcomes.

Disclosure of Interest: None declared.

\section{8}

Treosulfan-based conditioning for Stem Cell

Transplantation in Non-malignant Diseases - The

Experience of four German Centers

M. Sirin 1,* , M. Hönig ${ }^{1}$, I. Müller ${ }^{2}$, K.-W. Sykora ${ }^{3}$, R. Beier ${ }^{3}$, I. Furlan ${ }^{1}$, A. S. Schulz, M. H. Albert ${ }^{4}$

${ }^{1}$ Pediatrics and Adolescent Medicine, University Hospital Ulm, UIm, ${ }^{2}$ Pediatric Hematology and Oncology, University Medical Center Hamburg-Eppendorf, Hamburg, '3 Pediatric Hematology/ Oncology, Hannover Medical School, Hannover, ${ }^{4}$ Pediatric Hematology/Oncology, Dr. von Haunersches Children's Hospital, Munich, Germany

Introduction: Treosulfan (Treo) based conditioning regimens have been increasingly used as less toxic alternatives to 
myeloablative Busulfan based strategies in transplantation particularly of children with non-malignant diseases. A reduction in short term mucosal and hepatic (SOS) toxicity and the absence of long term pulmonary toxicity have been demonstrated. It is unclear, however, whether this reduction of toxicity is accompanied by an equal or inferior myeloablative capacity compared to Busulfan based myeloablative regimens.

Materials (or patients) and methods: We performed a retrospective analysis of consecutive patients with nonmalignant diseases transplanted in 4 German pediatric transplant centers (Hamburg, Hannover, Munich and Ulm) with a Treo based regimen (Treo and Fludarabin (Flu) \pm Thiotepa $(T T) \pm$ Serotherapy) in the period between January 1st 2000 and June 30th 2013.

Results: We identified 153 patients with inborn errors including primary immunodeficiencies, hemoglobinopathies, hemophagocytic lymphohistiocytosis, mucopolysaccharidosis and osteopetrosis. The median age at transplantation was 4.8 years (0.1-22). All pts received Treo/Flu. TT was added in 102 of these pts, serotherapy in 139 pts. The OS after 2 years was $89 \%$, the EFS $81 \%$. The incidence of aGvHD ${ }^{\circ} \|-^{\circ}$ IV was $19 \%$ and $5.4 \%$ for $\mathrm{CGvHD}{ }^{\circ} 1-{ }^{\circ} 3$. Primary engraftment of donor cells was present in $97 \%$ of patients. However, mixed chimerism at any time point was found in 59\% and disease recurrence in $11 \%$ An additional cellular therapy (ACT: stem cell boost, DLI, or 2nd transplant) was applied in $28 \%$ of patients. ACT was more often needed after transplantations from MMFDs and MUDs than from MSD/MFDs $(P=0.08)$. There was a significant difference in patients who received Alemtuzumab as serotherapy early (d-13 to $d-4)$ versus late (d-3 or later) during conditioning, with $32 \%$ ACT in the early and $52 \%$ ACT in the latter group $(P=0.02)$. Cell source (BM or PBSC) and addition of $\Pi$ did not affect the cumulative incidence $(\mathrm{Cl})$ of ACT.

Conclusion: There is an excellent overall survival and EFS with Treo based conditioning in the transplantation of patients with non-malignant diseases. Despite a good primary engraftment, a high rate of mixed chimerism, disease recurrence and need for additional cellular therapy was observed. Interestingly, early administration of serotherapy was correlated with a higher probability of stable donor engraftment and has to be considered as a relevant factor for transplant outcome. A randomized controlled prospective study comparing conditioning regimens with Treo against Busulfan in nonmalignant diseases is needed.

Disclosure of Interest: None declared.

\section{9}

Long-term outcomes of reduced-intensity conditioning allogeneic stem cell transplantation for adult high-risk acute lymphoblastic leukemia in first complete remission S. Lee ${ }^{1, *}, K_{\text {. }}$-S. Eom ${ }^{1}$, Y.-J. Kim ${ }^{1}$, N.-G. Chung ${ }^{1}$, J.-A. Kim ${ }^{1}$, J.-Y. Kwak', S.-K. Park ${ }^{3}$, H.-J. Kim $^{i}$, C.-K. Min ${ }^{1}$, S.-G. Cho', D.-W. Kim ', J.-W. Lee', W.-S. Min

${ }^{1}$ CATHOLIC BMT CENTER, COLLEGE OF MEDICINE, THE CATHOLIC UNIVERSITY OF KOREA, Seoul, ${ }^{2}$ Chonbuk National University Medical School, Chonju, ${ }^{3}$ Soonchunhyang University Medical School, Bucheon, Korea, Republic Of

Introduction: Reduced-intensity conditioning (RIC) allogeneic stem cell transplantation (SCT) has emerged as an option designed to lower nonrelapse mortality (NRM) for older patients and those with comorbid conditions. However, the role of RIC-SCT in adult acute lymphoblastic leukemia (ALL) remains unclear because the interpretation of transplantation outcome is mainly limited by the small sample size, short follow-up duration, various regimens for conditioning and graft-versus-host disease (GVHD) prophylaxis, and the heterogeneity of the criteria used to select patients for RIC-SCT. Previously, we conducted a phase 2 trial of RIC-SCT in adults with high-risk ALL and showed the potential role of this strategy, especially in patients in first complete remission (CR1). Here, we report on the updated results of RIC-SCT by analyzing 93 consecutive adult high-risk ALL transplants in CR1.

Materials (or patients) and methods: During the period between 2000 and 2012, 93 consecutive patients in CR1 (median age, 51 years [range, 15-65 years]) were given an identical RIC regimen consisting of fludarabine $\left(150 \mathrm{mg} / \mathrm{m}^{2}\right.$ in total) and melphalan ( $140 \mathrm{mg} / \mathrm{m}^{2}$ in total). The indications for RIC-SCT were advanced age ( $\geq 50$ years; $n=53 ; 57.0 \%)$ and comorbid conditions ( $n=40 ; 43.0 \%)$. Graft sources were peripheral blood stem cells $(n=91 ; 53$ 8/8-matched sibling donor, 15 8/8-matched unrelated donor, 23 7/8-matched unrelated donor) and bone marrow $(n=2 ; 1$ 8/8-matched unrelated donor, 1 7/8-matched unrelated donor). The median time-to-transplantation was 161 days (range, 106-291 days). GVHD prophylaxis was attempted by administering calcineurin inhibitors (cyclosporine for sibling donor transplants, tacrolimus for unrelated donor transplants) plus methotrexate. Antithymocyte globulin $(2.5 \mathrm{mg} / \mathrm{kg}$ in total) was administered to the patients who received allele-mismatched unrelated donor grafts. If residual leukemia was detected in the absence of GVHD at 3 months after transplantation, calcineurin inhibitors were rapidly discontinued.

Results: Fifty-two patients developed grade II to IV acute GVHD (42 grade II, 6 grade III, 4 grade IV). The cumulative incidence of acute GVHD at 100 days was $55.9 \%$. Of the 87 patients who survived for at least 100 days with sustained engraftment after transplantation, 61 developed chronic GVHD (30 limited, 31 extensive), resulting in a 5-year cumulative incidence of $65.6 \%$. After a median follow-up of 60 months (range, 24-174 months), the cumulative incidence of relapse $(\mathrm{CIR})$ and NRM at 5 years were $28.1 \%$ and $22.9 \%$, respectively, and the 5-year disease-free survival (DFS) and overall survival (OS) rates were $54.4 \%$ and $60.4 \%$, respectively. Within the cohort of Ph-negative ALL transplants $(n=43)$, the 5-year CIR, NRM, DFS, and OS rates were $26.4 \%, 15.5 \%, 61.7 \%$, and $67.4 \%$, respectively. In a subgroup of Ph-positive ALL transplants $(n=50)$, the 5-year CIR, NRM, DFS, and OS rates were $29.7 \%, 28.9 \%, 48.6 \%$, and $55.0 \%$ respectively, and for these patients, minimal residual disease kinetics (early-stable molecular response vs late molecular response vs poor molecular response) and the presence of chronic GVHD were closely related to CIR and DFS.

Conclusion: Our data suggest that RIC can be considered as a reasonable choice for providing a long-term disease control for adult high-risk ALL patients in CR1.

Disclosure of Interest: None declared.

0020

Substitution of TBI by intravenous Busulfan for elderly AML/MDS patients within the FLAMSA-RIC protocol is feasible and yields comparable results

M. Schleuning ${ }^{1, *}$, D. Judith ${ }^{1}$, I. Burlakova ${ }^{1}$, H. Baurmann ${ }^{7}$, R. Schwerdtfeger ${ }^{1}$, G. Stuhler

${ }^{1}$ Centre for hematopoietic cell transplantation, DKD Helios Klinik, Wiesbaden, Germany

Introduction: The FLAMSA-RIC protocol is a highly effective conditioning protocol for high risk myeloid leukaemia patients (pts). However, many of these pts are beyond the age of 60 . In this population the use of total body irradiation (TBI) might be toxic, even with the reduced dose of $400 \mathrm{cGy}$, as described in the original FLAMSA-RIC protocol. In an attempt to further reduce toxicity for elderly $(>60 \mathrm{y}$ ) or comorbid pts we substituted TBI by intravenous Busulfan (ivBu; $8 \times 0.8 \mathrm{mg} / \mathrm{kg}$ $\mathrm{BW}$ ) within the FLAMSA-RIC protocol.

Materials (or patients) and methods: Retrospective study to analyze the results of ivBu in comparison to those achieved in pts receiving the classical FLAMSA-RIC protocol with TBI during the same time period.

Results: From November 2006 to October 2012173 pts with high-risk AML or MDS received an allogeneic stem cell transplant after FLAMSA-RIC conditioning. Eighty-three pts (median age 47y) received TBI and ninety pts (median age 
64y) received ivBu. In the TBI group 76 pts suffered from AML and 7 pts from MDS and in the ivBu group diagnoses were AML in 74 and MDS in 16 pts. Unfavourable cytogenetics or molecular genetics were found in $64 \%$ of TBI and $50 \%$ of ivBu pts, respectively. In the TBI group $43 \%$ were transplanted with active disease as compared to $71 \%$ in the ivBu group. In both groups stem cell grafts from unrelated donors were used in approximately $75 \%$ of pts. All pts engrafted. After a median follow-up of 5.5 y for surviving pts the probability of leukaemia-free survival at $5 \mathrm{y}$ after transplant is $40 \%$ in the $\mathrm{TBI}$ group and $35 \%$ in the ivBu group $(P=0.133)$. Twentyseven relapses occurred in the TBI and 21 in the ivBu group. Non-relapse mortality (NRM) for the TBI cohort was $10 \%$ and for the ivBu group $24 \%$ at day $+100(P=0.052)$ and $33 \%$ and $41 \%$ during the entire observation period $(P=0.004)$. The difference in NRM is probably owing to the older age of the pts in the ivBu group and was due to more infectious complications. No significant difference in survival was observed in MUD or sibling transplants in either group.

Conclusion: In conclusion, substitution of $\mathrm{TBI}$ by ivBu is feasible with no enhanced relapse rates observed and should be further evaluated in prospective clinical trials also for younger pts.

Disclosure of Interest: $M$. Schleuning Funding from: Pierre Fabre Corp, D. Judith: None declared, I. Burlakova: None declared, H. Baurmann: None declared, R. Schwerdtfeger: None declared, G. Stuhler: None declared.

\section{1}

Two chemotherapy-based conditioning regimens compared to TBI-based conditioning secure consistent engraftment of T-cell depleted allogeneic HSCT, similarly low incidences of GVHD and favorable rates of disease-free survival (DFS)

G. Koehne ${ }^{1, *}$, F. Boulad ${ }^{1}$, J. Barker ${ }^{1}$, H. Castro-Malaspina ${ }^{1}$,

K. Curran ${ }^{1}$, S. Giralt ${ }^{1}$, A. Hasan ${ }^{7}$, G. Heller ${ }^{1}$, K. Hsu',

A. Jakubauski', N. Kernan', R. Kobos', E. Papadopoulos ${ }^{1}$

M. Perales ${ }^{1}$, D. Ponce ${ }^{1}$, S. Prockop ${ }^{1}$, C. Sauter ${ }^{1}$, A. Scaradavou', M. van den Brink ${ }^{1}, J$. Young ${ }^{1}, R$. O'Reilly ${ }^{1}$

${ }^{1}$ Memorial Sloan Kettering Cancer Center, New York, United States

Introduction: To ascertain the therapeutic potential of nonTBI-based conditioning for CD34+ HPC-selected, T celldepleted allografts, we conducted a trial comparing our standard regimen, arm (A) $1375 \mathrm{cGy}$ HFTBI + thiotepa, $5 \mathrm{mg} /$ $\mathrm{kg} /$ day $\times 2$ days + cyclophosphamide $60 \mathrm{mg} / \mathrm{kg} /$ day $\times 2$ days vs. arm (B) Busulfex $0.8 \mathrm{mg} / \mathrm{kg} / 6 \mathrm{~h} \times 12$ (dose adjusted) + melphalan $70 \mathrm{mg} / \mathrm{kg} /$ day $\times 2+$ fludarabine $25 \mathrm{mg} / \mathrm{m}^{2} /$ day $\times 5$ and arm (C) Clofarabine $20 \mathrm{mg} / \mathrm{m}^{2} /$ day $\times 5+$ melphalan $70 \mathrm{mg} / \mathrm{m}^{2} /$ day $\times 2$ thiotepa $5 \mathrm{mg} / \mathrm{kg} /$ day $\times 2$, as preparation for T-cell depleted CD34 ${ }^{+}$PBSC transplants from GCSFmobilized leukocytes fractionated with the CliniMACS CD34 + reagent system.

Materials (or patients) and methods: Primary endpoints were engraftment, GVHD, transplant-related mortality (TRM) and $2 \mathrm{yr}$ OS and DFS (Confer Table). Stratification of pts to arms A (standard), B or C was based on the patient's disease, disease stage and clinical factors such as age, prior therapy or comorbidities enhancing risks of TBI. Arm B was the non-TBI arm predominantly used for myeloid and Arm C for lymphoid malignancies. Prior to transplant, recipients of HLA-matched or non-identical transplants received rabbit thymoglobulin at $2.5 \mathrm{mg} / \mathrm{kg} /$ day $\times 2$ or 3 days respectively, to prevent graft failure. No GVHD drug prophylaxis was given post transplant. Results: A total of 215 consecutive patients, accrued between $5 / 13 / 2010$ and 11/20/2014, were analyzed (84 in arm A, 103 in arm B, 28 in arm C). These pts have been followed for a median of 19.7 months. Donors were related or unrelated and HLA-matched for $73 \%$ of the patients and 1-2 HLA alleles disparate for $27 \%$. Median age for the entire group was 47.8 years, with older pts predominating in the non-TBI groups (medians arm A,31.2 yrs; arm B, 58.8 yrs; arm C, 24 yrs). The CD34 + PBSC transplant provided a mean dose of $9.7 \times 10^{6}$
$\mathrm{CD}^{+} 4^{+}$progenitors $/ \mathrm{Kg}$ (range $1.4-89.7$ ) and $4.5 \times 10^{3} \mathrm{CD}^{+}$ T-cells/Kg (range 0.6-25.3). All pts engrafted; but 2 pts (1.9\%) in arm B experienced late graft failure, one of whom was reconstituted after a secondary graft. Overall the incidence of grade II-IV acute GVHD was $6 \%, 6 \%$ and $7 \%$ for arm A, B and C respectively. TRM at 1 year was $9 \%$ in Arm $A$, and $15 \%$ in Arm $B$ and $16 \%$ in Arm C. Two year OS and DFS for each arm are: arm A $-62.2 \%$ and $56 \%$; arm B $-68 \%$ and $59 \%$ and $48 \%$ and $48 \%$ for arm C. For the 115 pts who received standard risk transplants (i.e., pts with high risk forms of $A M L, A L L$ or $\mathrm{NHL}$ in $1^{\circ} \mathrm{CR}$, AML in $2^{\circ} \mathrm{CR}$, MDS RA/RCMD, CML in $1^{\circ} \mathrm{CP}$ or MM in CR1), 2 year OS and DFS are: arm A - 60\% and 59\%; arm B $74 \%$ and $65 \%$; arm C - 50\% and 50\%, with relapse rates at 2 yrs of arm A - 23\%, arm B - $10.4 \%$, and arm C - $11.5 \%$.

Table 1. Overall Results

\begin{tabular}{|c|c|c|c|c|c|c|c|}
\hline & \multirow[b]{2}{*}{ PTs } & \multirow{2}{*}{$\begin{array}{l}\text { Graft } \\
\text { Failure }\end{array}$} & \multirow{2}{*}{$\begin{array}{l}1 \text { Year } \\
\text { TRM }\end{array}$} & \multicolumn{2}{|c|}{1 Year Acute GVHD } & \multicolumn{2}{|c|}{2 Year } \\
\hline & & & & II-IV & III-IV & OS & DFS \\
\hline ARM A & 84 & 0 & $9 \%$ & $6 \%$ & $3 \%$ & $62 \%$ & $56 \%$ \\
\hline ARM B & 103 & 2 & $16 \%$ & $6 \%$ & $3 \%$ & $68 \%$ & $59 \%$ \\
\hline ARM C & 28 & 0 & $18 \%$ & $7 \%$ & $0 \%$ & $48 \%$ & $48 \%$ \\
\hline
\end{tabular}

Conclusion: These results identify two non-TBI-based conditioning regimens that secure consistent engraftment of rigorously T-cell depleted allogeneic HSCT and can yield favorable long-term DFS and OS with low incidences of GVHD and relapse.

Disclosure of Interest: None declared.

0022

Thiotepa-based versus total body irradiation/ cyclophosphamide-based myeloablative conditioning prior to allo-HSCT for acute myeloid leukemia in first complete remission, an update: a retrospective matched-pair analysis from the ALWP of the EBMT

S. Eder ${ }^{1, *}$, M. Labopin ${ }^{1}$, W. Arcese ${ }^{2}$, R. Or $^{3}$, I. Majolino ${ }^{4}$, A. Bacigalupo ${ }^{5}$, G. de Rosa ${ }^{6}$, L. Volin', D. Beelen ${ }^{8}, H$. Veelken $^{9}$, N. Schaap ${ }^{10}$, J. Kuball ${ }^{11}$, J. Cornelissen ${ }^{12}$, A. Nagler ${ }^{1,13}$, M. Mohty ${ }^{1,1^{\prime}}$ on behalf of the ALWP of the EBMT

${ }^{1}$ EBMT Office Paris, HÔPITAL SAINT-ANTOINE, Paris, France, ${ }^{2}$ Rome Transplant Network "Tor Vergata », University of Rome Stem Cell Transplant Unit, Rome, Italy, ${ }^{3}$ Dept. of Bone Marrow Transplantation, Hadassah University Hospital, Jerusalem, Israel, ${ }^{4}$ Haematology \& SCT Unit, Ospedale S. Camillo, Rome, ${ }^{5}$ Department of Haematology II, Ospedale San Martino, Genova, ${ }^{6}$ Division of Hematology, University of Napoli Federico II Medical School, Napoli, Italy, ${ }^{7}$ Department of Medicine, Helsinki University Central Hospital, Helsinki, Finland, ${ }^{8}$ University Hospital Essen, Essen, Germany, ${ }^{9}$ Leiden University Medical Center, Leiden, ${ }^{10}$ Radboud University Medical Centre, Nijmegen, ${ }^{11}$ Dept. of Haematology, University Medical Centre, Utrecht, ${ }^{12}$ Erasmus MC-Daniel den Hoed Cancer Centre, Rotterdam, Netherlands, ${ }^{13}$ Chaim Sheba Medical Center, Tel Hashomer, Israel, ${ }^{14}$ Service d'Hématologie et Thérapie Cellulaire, Hôpital Saint-Antoine, Paris, France

Introduction: Thiotepa is an alkylating compound with an antineoplastic and myeloablative activity and can mimic the effect of radiation. TBI is reported to be associated with undesirable long-term side effects. In this study, we compared thiotepa-based regimens with a conditioning consisting of total body irradiation/cyclophosphamide in a retrospective matched-pair analysis.

Materials (or patients) and methods: We evaluated the outcome of adults with acute myeloid leukemia in first complete remission who received myeloablative conditioning 
either with a thiotepa-based chemotherapy $(n=121)$ or a total body irradiation/cyclophosphamide-based (TBI/Cy; $n=358$ ) regimen for allogeneic hematopoietic stem cell transplantation from an HLA-matched sibling or an unrelated donor.

The median age was 48 years in both groups. Females comprised $54 \%$ of patients in the thiotepa group and $46 \%$ in the $\mathrm{TBI} / \mathrm{Cy}$ group. Donors were male in $64 \%$ and $63 \%$ in the thiotepa and TBI/Cy group, respectively. Bone marrow was the stem cell source in $26 \%$ of patients in the thiotepa group and $32 \%$ in the $\mathrm{TBI} / \mathrm{Cy}$ group, whereas the remaining patients received peripheral blood stem cells. The median dose of TB was $12 \mathrm{~Gy}$. Thiotepa was administered with cyclophosphamide $(n=48 ; 40 \%)$ and fludarabine $(n=70 ; 58 \%)$ with or without busulfan.

Results: With a median follow-up of 44 months, the outcome was similar in both groups. Engraftment occurred in $97 \%$ of patients in the thiotepa group versus $98 \%$ in the TBI/Cy group. Acute graft-versus-host disease grade II-IV was observed in $25 \%$ after thiotepa-containing regimen versus 35\% after TBI/ Cy $(P=0.06)$. The 2 -year cumulative incidence of chronic graftversus-host disease was $40.5 \%$ for thiotepa and $41 \%$ for TBI/Cy, respectively $(P=0.98)$. At 2 years, the cumulative incidences of non-relapse mortality and relapse incidence were $23.9 \%$ with thiotepa versus $22.4 \%$ with $\mathrm{TBI} / \mathrm{Cy}(P=0.66)$ and $17.2 \%$ (thiotepa) versus $23.3 \%(\mathrm{TBI} / \mathrm{Cy} ; P=0.77)$, retrospectively. The probabilities of leukemia-free and overall survival at 2 years were not significantly different between the thiotepa and TBI/ Cy group, at $58.9 \%$ versus $54.2 \%(P=0.95)$ and $61.4 \%$ versus $58 \%(P=0.72)$, respectively.

In a multivariate analysis, adjusted for age, gender matching, interval from diagnosis to transplantation, donor type, cytogenetics, secondary $\mathrm{AML}$, year of transplantation and use of anti-thymocyte globulin, there was no difference in terms of non-relapse mortality in patients receiving thiotepa or TBI/Cy (hazard ratio [HR] 0.88; 95\% confidence interval [CI] $0.55-1.41 ; P=0.60)$. Similarly, relapse incidence was not statistically different between the two groups (HR: 1.12; Cl: 0.68-1.85; $P=0.64$ ). Finally, leukemia-free and overall survival were similar between the thiotepa and TBI/Cy group (LFS HR: 0.99; Cl: $0.70-1.39 ; \quad P=0.96$ ), (OS HR: 0.99; Cl: 0.69-1.42; $P=0.98)$.

Conclusion: In summary, comparing 479 patients with acute myeloid leukemia in first complete remission receiving either a thiotepa-based chemotherapy or traditional TBI/Cy regimen, we could not observe any difference in transplant outcome. Our data are in accordance with other recent publications demonstrating the possibility to deliver effective anti-leukemic chemotherapy-based conditioning omitting TBI and its associated long-term side effects.

Disclosure of Interest: S. Eder: None declared, M. Labopin: None declared, W. Arcese: None declared, R. Or: None declared, I. Majolino: None declared, A. Bacigalupo Conflict with: Adienne, G. de Rosa: None declared, L. Volin: None declared, D. Beelen: None declared, H. Veelken: None declared, N. Schaap: None declared, J. Kuball: None declared, J. Cornelissen: None declared, A. Nagler: None declared, M. Mohty Funding from: Riemser.
0023

Disease Risk as Exclusive Predictor of Relapse after Reduced-Intensity Conditioning Hematopoietic Cell Transplantation in Patients with AML and MDS: Data from the Pooled Interim Analysis of a Prospective Randomized Multicenter Phase III Trial

N. Shayegi $i^{1, *}$, M. Stelljes ${ }^{2}$, P. Dreger ${ }^{3}$, S. Kyrcz-Krzemien ${ }^{4}$, F. Ciceri ${ }^{5}$, W. Bethge ${ }^{6}$, G. U. Grigoleit ${ }^{7}, R$. Trenschel ${ }^{1}$, D. W. Beelen ${ }^{7}$ on behalf of MC-FludT.14/L Study Group

${ }^{1}$ KMT Klinik, Essen, ${ }^{2}$ Universitätsklinikum Münster, Münster, ${ }^{3}$ Universitätsklinikum Heidelberg, Heidelberg, Germany, ${ }_{5}^{4}$ Poznan University of Medical Sciences, Poznan, Poland, "5 Scientific Institute H. San Raffaele, Milan, Italy, ${ }^{6}$ Universität Tübingen, Tübingen, ${ }^{7}$ Klinikum der Universität Würzburg, Würzburg, Germany

Introduction: The concept of allografting relies on a potentially curative graft versus leukemia reaction nonetheless relapse remains the major reason for RIC-HCT failure. Though this approach has become an established treatment option, most published data are retrospective or from phase 2 clinical trials, so that limited conclusions can be drawn. Here, we report on the first pooled interim analysis of a prospective randomized multicenter phase III trial comparing a treosulfan based conditioning regimen with busulfan based RIC in adult patients with AML or MDS considered ineligible to standard myeloablative conditioning regimens.

Materials (or patients) and methods: Event-free survival (EFS) estimate within 1 year after HCT was designated as the primary study endpoint. EFS was defined as the time from HCT to relapse, graft failure, or death. $\mathrm{N}=330$ patients (pts) between 50 and 70 years of age and/or with a pretransplant $\mathrm{HCT}$ comorbidity index $(\mathrm{HCT}-\mathrm{Cl})>2$ were included. Regarding the primary endpoint the first scheduled interim analysis has been performed in $n=320$ evaluable pts.

Results: After a median follow up of 24 months the pooled 1 year-EFS estimate for all 320 pts is $65 \%(95 \% \mathrm{Cl}, 59-70 \%)$. This estimate is $68 \%(95 \% \mathrm{Cl}, 61-74 \%)$ for AML pts in $1^{\text {st }}$ complete remission (CR) and $65 \%(95 \% \mathrm{Cl}, 54-75 \%)$ for pts with MDS. The only factor affecting the 1-year EFS estimate is the predefined unfavorable disease risk (AML: unfavorable karyotype, FLT3-ITD, MLL-PTD, N-RAS, wild-type NPM1 and CEBPA without FLT3-ITD, RPN1-EVI1, DEK-NUP214, MLL rearranged in $1^{\text {st }}$ CR or advanced CR, MDS: > intermediate-II IPSS). For pts with an unfavorable disease risk, the 1-year EFS is $59 \%(95 \% \mathrm{Cl}$, $51-66 \%)$ compared to $70 \%(95 \% \mathrm{Cl}, 62-76 \%)$ for pts with more favorable disease characteristic $(p<0.03)$. The

\section{Cumulative incidence of relapse/progression stratified by Risk group}

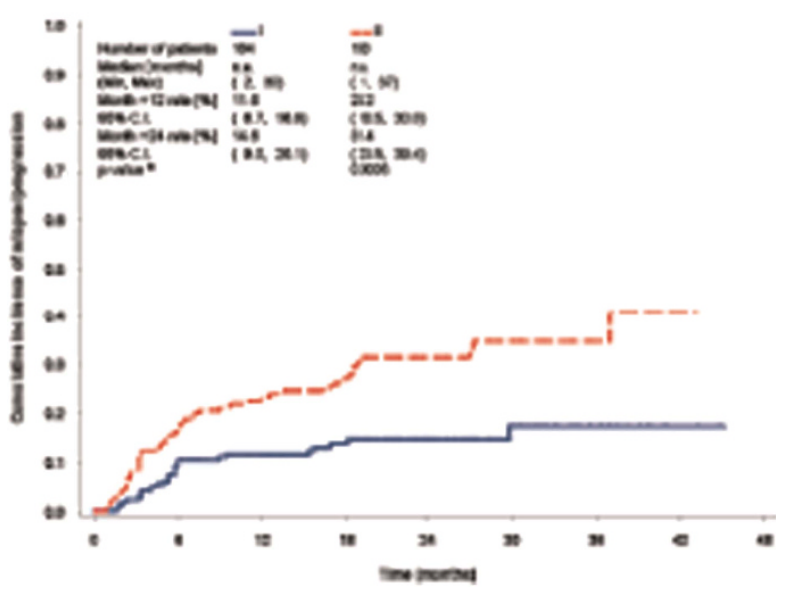


cumulative incidence of relapse (CIR) stratified by risk group reveals that the probability for relapse is significantly higher for the pts with high risk disease $(p<0.0005)$, whereas the cumulative incidence of non-relapse mortality (NRM) is comparable $(p=0.36)$ (Figure 1$)$. The median time to relapse has not been reached in either group. The estimates of relapse at 1 year were $23.2 \%(95 \% \mathrm{Cl}, 16.5-30.0 \%)$ for high risk pts and $11.6 \%(95 \% \mathrm{Cl}, 6.7-16.6 \%)$ for the other group. The estimated 2-year CIR was $31.4 \%(95 \% \mathrm{Cl}, 23.5-39.4 \%)$ for the group at high risk and $14.6 \%(95 \% \mathrm{Cl}, 9.0-20.1 \%)$ for pts at low and intermediate risk. Further univariate analyses for EFS revealed that factors without a significant impact were age, diagnosis of AML or MDS, donor type (HLA related versus HLA unrelated) and $\mathrm{HCT}-\mathrm{Cl}>2$

Conclusion: Our results identify within a prospective trial disease biology as the only parameter determining the risk of relapse after RIC-HCT for pts with AML and MDS. Furthermore, they offer a rationale for studies to test whether strategies preventing relapse such as administration of targeted or epigenetic therapeutics after RIC-HCT as well as immuntherapies benefit pts with AML and MDS at high risk for disease recurrence.

Disclosure of Interest: None declared.

0024

Sequential Regimen of Clofarabine, Cytarabine and Reduced Intensity Conditioning (RIC) Prior to Allogeneic Stem Cell Transplantation (allo-SCT) for Acute Myeloid Leukemia (AML) in Primary Treatment Failure

M. Mohty, , F. Malard ${ }^{2}$, D. Blaise ${ }^{3}$, N. Milpied ${ }^{4}$, G. Socié $^{5}$, A. Huynh ${ }^{6}$, O. Reman ${ }^{7}$, I. Yakoub-Agha ${ }^{8}$, S. Furst ${ }^{3}$, T. Guillaume ${ }^{2}$, R. Tabrizi ${ }^{4}$, J. Delaunay ${ }^{2}$, P. Moreau ${ }^{2}$, M. Labopin ${ }^{1}$, P. Chevallier ${ }^{2}$ ${ }^{1}$ Hematology department, AP-HP, Hopital saint Antoine, Paris, ${ }^{2}$ Hematology department, CHU de Nantes, Nantes, ${ }^{3}$ Hematology department, Institut Paoli-Calmette, Marseilles, ${ }^{4}$ Hematology department, CHU de Bordeaux, Bordeaux, ${ }^{5}$ Hematology department, AP-HP, Hopital Saint Louis, Paris, ${ }^{6}$ Hematology department, CHU de Toulouse, Toulouse, ${ }^{7}$ Hematology department, $\mathrm{CHU}$ de Caen, Caen, ${ }^{8}$ Hematology department, CHU de Lille, Lille, France

Introduction: The prognosis of AML in primary treatment failure remains very poor. Salvage allo-SCT might be a potential therapeutic option in this setting. However, the limited feasibility of standard myeloablative conditioning (MAC) allo-SCT in these patients, lead to the development of the so-called "sequential" transplant approach (e.g. FLAMSA regimen combining both intensive chemotherapy and transplant conditioning within the same procedure). With this background, we conducted a phase 2 prospective trial to test the feasibility of a novel "sequential" approach, using busulfan and clofarabine.

Materials (or patients) and methods: This phase 2 prospective multicenter trial (ClinicalTRials.gov Identifier: NCT01188174) aimed to assess the efficacy (survival) and safety of a new "sequential" conditioning regimen based on Clofarabine $30 \mathrm{mg} / \mathrm{m} 2 / \mathrm{d}$ for 5 days, aracytine $1 \mathrm{~g} / \mathrm{m} 2 / \mathrm{d}$ for 5 days and after a 3 days rest, cyclophosphamide $60 \mathrm{mg} / \mathrm{Kg} / \mathrm{d}$ for 1 day, IV Busulfan $3.2 \mathrm{mg} / \mathrm{Kg} / \mathrm{d}$ for 2 days and ATG (Thymoglobuline $\left.^{*}\right) 2.5 \mathrm{mg} / \mathrm{Kg} / \mathrm{d}$ for 2 days in $\mathrm{AML}$ patients in primary induction failure (i.e. persistent leukemia after 2 courses of induction chemotherapy or persisting bone marrow hypoplasia following induction chemotherapy). For GVHD prophylaxis, patients received cyclosporine alone in case of a family donor, and cyclosporine + mycophenolate mofetil in case of an HLAmatched unrelated donor (MUD: $10 / 10$ or $9 / 10$ ).

Results: Between 2010 and 2013, a total of 24 patients were included. The median age of patients at time of allo-SCT was 47 (range, 20-55) years. At time of conditioning, all patients were confirmed having $A M L$ in primary induction failure. Karyotype was intermediate in 9 patients (38\%) and adverse in 13 patients (54\%), no patients had favorable karyotype and karyotype was missing in 2 patients (8\%). Seven patients (29\%) had secondary AML. The donor was an HLA-identical sibling donor in 15 cases $(62 \%)$ and an HLA MUD in 9 cases (38\%). Per protocol performance status was comprehensively assessed at time of inclusion. The Karnofsky score was 50\%, 60\%, 70\%, $80 \%, 90 \%$, and $100 \%$ in $2(8 \%), 1(4 \%), 2(8 \%), 6(25 \%), 8(33 \%)$ and $5(21 \%)$ patients, respectively.

Conclusion: Results from this phase 2 prospective multicenter trial validated the safety and efficacy of a clofarabine-based sequential reduced-toxicity conditioning regimen. Such sequential regimen appeared to be safe with a $8 \%$ NRM at one year in high risk patients, and exhibits an efficient disease control both in very high risk refractory AML with a LFS of $46 \%$ at 1 year, warranting further investigations as part of phase 3 trials.

Disclosure of Interest: None declared.

\section{Oral session: Myeloma / autologous transplantation}

\section{5}

Donor T-cell responses modulate disease progression patterns of multiple myeloma after T-cell depleted allogeneic stem cell transplantation

M. Eefting ${ }^{1,2}$, L. De Wreede ${ }^{3, *}$, P. Von dem Borne ${ }^{1}$, C. Halkes ${ }^{1}$, S. Kersting ${ }^{1}$, E. Marijt ${ }^{1}$, H. Putter ${ }^{3}$, H. Veelken ${ }^{1}$, J. Schetelig ${ }^{4}$,

F. Falkenburg'

${ }^{1}$ Hematology, Leiden University Medical Center, Leiden, ${ }^{2}$ Hematology, Erasmus MC, Rotterdam, ${ }^{3}$ Medical Statistics and Bioinformatics, Leiden University Medical Center, Leiden, Netherlands, ${ }^{4}$ Medical Department I, University Hospital Carl Gustav Carus, Technische Universität Dresden \& DKMS, German Bone Marrow Donor Center, Dresden, Germany

Introduction: Multiple myeloma is characterized by diffuse marrow plasmacytosis and focal lesions like plasmacytomas and lytic bone lesions. Allogeneic stem cell transplantation (alloSCT) can result in cure or long term control of the disease by the graft-versus-myeloma (GvM) effect mediated by immune-competent donor $\mathrm{T}$ cells. However, directing antigen-specific T-cells to all lesions is essential for a complete GvM effect. Homing of T cells to bone marrow (BM) has been found to occur constitutively, whereas homing to other tissues may need specific homing molecules on $\mathrm{T}$ cells, or an inflammatory environment. Consequently, the strength of immune responses in BM and extra-medullary sites may differ, resulting in differential progression patterns of myeloma after alloSCT and donor lymphocyte infusion (DLI) in the presence or absence of a donor T cell mediated GvM reactivity.

Materials (or patients) and methods: At the Leiden University Medical Center, between May 2002 and July 2010, 43 subsequent patients with multiple myeloma underwent exvivo T-cell depleted reduced-intensity conditioning (TCD-RIC) alloSCT with postponed DLI to prevent severe GVHD. In the absence of GvHD requiring systemic immunosuppression at 6 months after alloSCT, patients were eligible for DLI in case of disease progression or persistence and/or if the patient showed mixed-chimerism. Diffuse bone marrow (BM) progression was defined as an increase of $>10 \%$ patient chimerism fraction with myeloma cells in the BM by morphology or immune phenotyping. Focal progression was defined as development of new soft tissue plasmacytomas or bone lesions and/or definite increase in size of existing plasmacytomas or bone lesions. BM and focal progression patterns before and after DLI were assessed separately.

Results: Median follow-up of surviving patients was 70 months (range 35-93 months). Estimated 2-year overall survival from alloSCT of the entire cohort was $61 \%(95 \% \mathrm{Cl}$ 46-76\%). Cumulative DLI incidences at 12 and 24 months after alloSCT were $56 \% \pm 15 \%$ and $67 \% \pm 15 \%$, respectively. 
As measured from alloSCT prior to DLI BM progression and focal progression patterns were highly similar with cumulative incidences of BM progression of $23 \% \pm 13 \%$ and $23 \% \pm 13 \%$, and of focal progression of $19 \% \pm 12 \%$ and $21 \% \pm 12 \%$, after 12 and 24 months post-transplant, respectively. In contrast, as measured from DLI BM progression and focal progression patterns showed strong dissimilarity: at 12 and 24 months after DLI cumulative incidences of BM progression were $14 \% \pm 13 \%$ and $17 \% \pm 14 \%$ respectively, whereas focal progression was $48 \% \pm 19 \%$ and $62 \% \pm 18 \%$, respectively, illustrating a potent immunological response in BM with only limited effect of DLI on focal lesions.

Conclusion: Disease progression patterns of multiple myeloma after TCD-RIC alloSCT diverged from initially similar BM and focal progression patterns in the absence of alloimmune responses towards disease control in BM with focal progression after DLI. This finding illustrates failure of donor lymphocytes to target extra-medullary/focal disease in multiple myeloma.

Disclosure of Interest: None declared.

\section{6}

Engineered T cells modified to express a CS-1-specific chimeric antigen receptor (CAR) confer anti-myeloma activity in vitro and in pre-clinical in vivo models

T. Gogishvili ${ }^{1}$, S. Danhof ${ }^{1}$, C. Brede ${ }^{1}$, A. Beilhack ${ }^{1}$, V. Kunzmann ${ }^{1}$, H. Einsele ${ }^{1}, M$. Hudecek ${ }^{1,}$

${ }^{7}$ Medicine II - Hematology, UNIVERSITY OF WUERZBURG, Wuerzburg, Germany

Introduction: Adoptive immunotherapy with T cells that were modified by gene-transfer to express tumor-targeting chimeric antigen receptors (CARs) has therapeutic potential in advanced B-cell malignancies. We are pursuing the glycoprotein CS1 (SLAMF7/CD319) as candidate target for CAR T cells in Multiple Myeloma (MM), due to its restricted high level expression on malignant plasma cells in a significant proportion of MM patients. Here, we evaluated the anti-MM function of T cells that we modified with CS1-specific CARs in vitro and pre-clinical in vivo models.

Materials (or patients) and methods: We constructed two CS1-specific CARs with antigen-binding domains derived from the huLuc63 and Luc90 mAbs that target distinct CS1 epitopes, each comprising a signaling module of CD3zeta and a CD28 co-stimulatory domain, and encoded both constructs in lentiviral vectors for gene-transfer.

Results: CD8 + and CD4 + T-cell lines expressing the huLuc63 and Luc90 CS1-CARs could be readily generated from healthy donors and MM patients $(n=5 / 3)$, and propagated and expanded in vitro with similar kinetics as $T$ cells expressing a CD19-specific CAR that we included as a reference. In functional experiments, CD8 $+\mathrm{T}$ cells expressing either of the CS1-CARs conferred specific high level lysis of MM lines (MM1.S, NCl-H929 and OPM-2), primary MM, K562 that had been stably transfected with CS1, but not native CS1-negative K562. We also detected high level production of IFNg and IL-2 $(\mathrm{CD} 4>\mathrm{CD} 8)$, and productive proliferation after stimulation with $\mathrm{CS} 1+$ target cells, with significantly superior anti-MM reactivity mediated by the huLuc63 compared to the Luc90 CS1-CAR construct. We confirmed the anti-MM efficacy of CS1CAR modified T cells using a xenograft model in immunodeficient mice (NSG/MM1.S). Mice were inoculated with fireflyluciferase labeled MM1.S myeloma by tail vein injection and 14 days later, when mice presented with disseminated disease, administered a single dose (i.v.) of a cell product consisting of equal proportions of CD8 + and CD4 + T cells modified with either the optimal CS1-CAR, the CD19-specific CAR or mock transduced. We observed rapid and durable complete rejection of established MM from bone marrow and resolution of extramedullar MM manifestations in all of the mice treated with CS1-CAR T cells $(n=4)$, whereas mice treated with CD19CAR T cells, or control $T$ cells had to sacrificed due to progressive disease $(n=4 / 4)$. Of interest, in this in vivo model, we observed similarly effective anti-MM responses mediated by CS1-CAR T cells that had been derived from healthy donors and MM patients.

Conclusion: Our data suggest the potential of $T$ cells expressing CS1-specific CARs to confer anti-MM activity in clinical settings. The experience with anti-CS1 mAb huLuc63, that as single agent has only minute anti-MM activity, indicates targeting this molecule will be safe and not be associated with toxicity to normal tissues. We observed stronger anti-MM reactivity with CS1-CARs targeting a proximal (huLuc63) rather than a distal (LuC90) epitope on CS1 protein, in line with our previous observation that the targeted epitope on a given antigen affects tumor recognition of CAR T cells. Experiments to analyze the function of our CS1-CARs against panels of primary MM in our NSG model are ongoing to inform our efforts of clinically translating CAR T-cell therapy in this entity. References: none.

Disclosure of Interest: None declared.

\section{7}

T-cell depleted allogeneic hematopoietic stem cell transplantation for patients with relapsed, high-risk multiple myeloma permits long-lasting remissions in the absence of graft-versus-host disease

G. Koehne ${ }^{1, *}$, S. Devlin ${ }^{1}$, H. Landau' ${ }^{1}$, H. Hassoun ${ }^{1}$, A. Lesokhin ${ }^{1}$, N. Lendvai ${ }^{1}$, D. Chung ${ }^{\prime}$, S. Giralt ${ }^{1}$

${ }^{1}$ Memorial Sloan Kettering Cancer Center, New York, United States

Introduction: We proposed to reduce early transplant related mortality associated with conventional allogeneic hematopoietic stem cell transplantation (allo HSCT) and reduce acute or chronic GvHD related to non-myeloablative allo HSCT while maintaining the graft-versus-myeloma effect for patients with high-risk multiple myeloma.

Materials (or patients) and methods: We report results of a phase II trial of 44 pts, using T-cell depleted allo HSCT (allo TCD HSCT) from HLA compatible (matched related $=14$, matched unrelated $=17$, and $9 / 10$ mismatched unrelated $=13$ ) donors. All 44 pts had relapsed myeloma within 15 mos following auto HSCT, and $31 / 44$ pts also had high-risk cytogenetics at diagnosis $[t(4 ; 14), t(14 ; 16)$, del17p by FISH and/or del13q by karyotyping]. All pts had to achieve at least a partial response from preceding salvage chemotherapy $(n=32)$ or second salvage auto HSCT $(n=12)$. Pts underwent allo TCD HSCT with busulfan $(0.8 \mathrm{mg} / \mathrm{kg} \times 10$ doses $)$, melphalan $\left(70 \mathrm{mg} / \mathrm{m}^{2} \times 2\right.$ days), fludarabine $\left(25 \mathrm{mg} / \mathrm{m}^{2} \times 5\right.$ days $)$ and rabbit ATG $(2.5 \mathrm{mg} /$ $\mathrm{kg} \times 2$ days). TCD was performed by positive CD34 selection (Isolex) followed by rosetting with sheep erythrocytes for the initial 13pts (2008-09) and by CD34 + enrichment by the Miltenyi Device in 31 pts thereafter, achieving $<10^{4} \mathrm{CD} 3+/ \mathrm{kg}$ for all grafts. None of these pts received immuno suppressive therapy post TCD HSCT. Pts with 10/10 HLA matched donors were also eligible to receive low doses of DLI $\left(5 \times 10 e^{5}-1 \times 10 e^{6}\right.$ $\mathrm{CD} 3+/ \mathrm{kg}$ ) no earlier than 5 mos post allo HSCT.

Results: 44 pts with a median follow up of 24.8 mos (range: 11.1-81.2 mos) of survivors are reported, median age 56 years (range 32-69). All pts engrafted promptly (median $d+10$, range $+9-+12)$.TRM (grade II-IV) at $12 \operatorname{mos}$ is $18 \%(95 \% \mathrm{Cl}$ : $8 \%-31 \%)$. Acute GvHD was $2 \%(95 \% \mathrm{Cl}: 0 \%-11 \%)$ and chronic GvHD was not observed in any pt. The overall survival (OS) and progression-free survival (PFS) with their 95\% confidence intervals $(\mathrm{Cl})$ are shown in Table 1. Factors 
associated with worse outcome were disease status and number of previous treatments prior to TCD HSCT.

Table 1.

\begin{tabular}{|c|c|c|c|c|}
\hline & $\begin{array}{l}\text { All } \\
\text { patients }\end{array}$ & $\begin{array}{l}\text { 3-4 lines } \\
\text { of } R x\end{array}$ & $\begin{array}{l}5-6 \\
\text { lines }\end{array}$ & $\begin{array}{l}>6 \\
\text { lines }\end{array}$ \\
\hline$N=$ & 44 & 12 & 17 & 15 \\
\hline \multicolumn{5}{|l|}{ Disease status } \\
\hline $\mathrm{CR} / \mathrm{nCR}$ & 4 & 2 & 2 & 0 \\
\hline VGPR & 19 & 6 & 7 & 6 \\
\hline PR & 21 & 4 & 8 & 9 \\
\hline $\begin{array}{l}\text { OS @ } 1 \mathrm{yr}(95 \% \mathrm{Cl}) \text { OS @ } \\
2 \mathrm{yr}(95 \% \mathrm{Cl})\end{array}$ & $\begin{array}{l}0.68 \\
(0.56- \\
0.83) \\
\end{array}$ & $\begin{array}{l}0.67 \\
(0.45- \\
0.99) \\
\end{array}$ & $\begin{array}{l}0.76 \\
(0.59- \\
1.0)\end{array}$ & $\begin{array}{l}0.59 \\
(0.39- \\
0.91)\end{array}$ \\
\hline $0.54(0.41-0.72)$ & $\begin{array}{l}0.67 \\
(0.43- \\
0.99)\end{array}$ & $\begin{array}{l}0.63 \\
(0.43- \\
0.92)\end{array}$ & $\begin{array}{l}0.26 \\
(0.09- \\
0.77)\end{array}$ & \\
\hline PFS @ 1 yr (95\%Cl) & $\begin{array}{l}0.55 \\
(0.42- \\
0.71)\end{array}$ & $\begin{array}{l}0.5 \\
(0.28- \\
0.88)\end{array}$ & $\begin{array}{l}0.71 \\
(0.52- \\
0.96)\end{array}$ & $\begin{array}{l}0.4 \\
(0.22- \\
0.74)\end{array}$ \\
\hline PFS @ 2 yr (95\%Cl) & $\begin{array}{l}0.31 \\
(0.19- \\
0.50)\end{array}$ & $\begin{array}{l}0.33 \\
(0.15- \\
0.74)\end{array}$ & $\begin{array}{l}0.42 \\
(0.23- \\
0.77)\end{array}$ & 0.0 \\
\hline
\end{tabular}

$19 / 44$ pts are alive, $11 / 19$ pts are in complete remission (CR) of which 3 pts are $>6$ years post allo TCD HSCT. 8/19 pts alive have progressed but are responding to salvage chemotherapy and/or DLI. 17/25 pts died of disease progression, 7/8 died of infectious complications and $1 \mathrm{pt}$ died of complications associated with aGvHD.

Conclusion: Long-lasting disease control can be achieved with TCD HSCT in pts with multiply relapsed MM including those with high-risk cytogenetics in the absence of acute and chronic GVHD. Outcome of TCD HSCT is influenced by numbers of regimens administered prior to allo BMT. We are aiming to perform TCD HSCT for pts with MM who have highrisk cytogenetics at an earlier time point and to integrate post transplant immunotherapeutic strategies to further reduce risk of relapse in these high-risk pts.

Disclosure of Interest: None declared.

\section{8}

Multiple Myeloma patients with del(8)(p21) are more resistant to Bortezomib treatment

A. D. Duru ${ }^{1,2, *}$, T. Sutlu ${ }^{1,3}$, A. Wallblom ${ }^{1}$, K. Uttervall ${ }^{1,4}$, J. Lund ${ }^{1,4}$, B. Stellan ${ }^{1}$, G. Gahrton $^{1,4}$, H. Nahi ${ }^{1,4}$, E. Alici ${ }^{1,2,4}$

${ }^{1}$ Center for Hematology and Regenerative Medicine, Karolinska Institutet, Stockholm, Sweden, ${ }^{2}$ Center for Diseases of Aging, Vaccine and Gene Therapy Institute of Florida, Port St. Lucie, United States, ${ }^{3}$ Nanotechnology Research and Application Center, Sabanci University, Istanbul, Turkey, ${ }^{4}$ Haematology Centre, Karolinska University Hospital, Stockholm, Sweden

Introduction: The impact of chromosomal abnormalities on survival in multiple myeloma (MM) is well documented (1). In this study, we have analyzed the molecular consequences of del(8)(p21), an abnormality we and others have previously shown to have an adverse impact on survival of MM patients (2-4).

Materials (or patients) and methods: In a cohort of 140 patients that were diagnosed with MM between 2001 and
2012, we have investigated the clinical impact of del(8)(p21) on time to progression (TTP) and overall survival (OS). Moreover, response rate of 84 patients to $1^{\text {st }}$ line bortezomib treatment was investigated. We have also analyzed the expression profiles of genes located near the 8 p21 region in patients with and without del(8)(p21). Additionally, we have analyzed the in vitro response of primary MM cells with and without the deletion to bortezomib-mediated killing and sensitization to TRAIL/APO2L-triggered apoptosis in an attempt to understand why MM patients carrying $8 \mathrm{p} 21$ deletion respond poorly to bortezomib treatment.

Results: We found that MM patients carrying del(8)(p21) deletion had significantly shorter TTP compared to patients without the deletion $(P=0.011)$ and most importantly these patients had significantly shorter OS compared to patients without the deletion $(P=0.001)$. In a cohort of 84 patients, we observed that patients with $\operatorname{del}(8)(\mathrm{p} 21)(n=24)$ responded poorly to bortezomib, $50 \%$ showing no response while $90 \%$ of patients without the deletion $(n=60)$ responded to bortezomib treatment. In vitro analysis revealed that MM cells from patients with del(8)(p21) show higher resistance to bortezomib treatment possibly due to upregulated expression of genes such as PTK2B, CCDC25, RHOBTB2, NFkB, MYC and BCL2 while showing downregulated levels of TP53 and SCARA3 when compared to MM cells without the deletion. Furthermore, we have observed that MM cells with del(8)(p21) express higher levels of the decoy death receptor, TRAIL-R4 and fail to upregulate the pro-apoptotic death receptors TRAIL-R1 and TRAIL-R2 that are located in the 8p21 region. As a result, $M M$ cells with del(8)(p21) were largely resistant to bortezomib and TRAIL/APO2L-mediated apoptosis.

Conclusion: Substantiating the clinical outcome of the patients, our data provides a potential explanation regarding the poor response of $\mathrm{MM}$ patients with $\operatorname{del}(8)(\mathrm{p} 21)$ to bortezomib treatment. Furthermore, our clinical evaluation suggests that including immunomodulatory agents such as lenalidomide in the treatment regimen may help to overcome this negative effect, providing an alternative thought in planning treatments of patients with del(8)(p21).

References:

1) Nahi $\mathrm{H}$ et al. Clinical impact of chromosomal aberrations in multiple myeloma. Journal of internal medicine 2011 Feb; 269(2): 137-147

2) Sutlu T et al. The prognostic significance of $8 p 21$ deletion in multiple myeloma. British journal of haematology 2009 Jan; 144(2): 266-268.

3) Neben $\mathrm{K}$ et al. Combining information regarding chromosomal aberrations $t(4 ; 14)$ and del(17p13) with the International Staging System classification allows stratification of myeloma patients undergoing autologous stem cell transplantation. Haematologica 2010 Jul; 95(7): 1150-1157.

4) Gmidene A et al. 8p21.3 deletion suggesting a probable role of TRAIL-R1 and TRAIL-R2 as candidate tumor suppressor genes in the pathogenesis of multiple myeloma. Medical oncology 2013 Jun; 30(2): 489.

Disclosure of Interest: A. Duru: None declared, T. Sutlu: None declared, A. Wallblom: None declared, K. Uttervall: None declared, J. Lund: None declared, B. Stellan: None declared, G. Gahrton Funding from: GG received financial support from the Celgene Company, USA, Conflict with: GG is an advisor to Fujimoto Pharmaceutical Company, Japan, H. Nahi: None declared, E. Alici: None declared. 
0029

Blood graft cellular composition and early post-transplant recovery in myeloma patients mobilized with or without low-dose cyclophosphamide - a randomized comparison J. Valtola $a^{1, *}$, R. Silvennoinen ${ }^{1}$, A. Ropponen ${ }^{2}$, T. Siitonen ${ }^{3}$, M. Säily ${ }^{3}$, M. Sankelo ${ }^{4}$, V. Terävä ${ }^{5}$, M. Putkonen ${ }^{6}$, T. Kuittinen ${ }^{1}$, J. Pelkonen ${ }^{2,7}$, P. Mäntymaa ${ }^{7}$, K. Remes ${ }^{6}$, V. Varmavuo ${ }^{1}$, E. Jantunen ${ }^{1}$

${ }^{1}$ Department of Medicine, Kuopio University Hospital, ${ }^{2}$ Department of Clinical Microbiology, University of Eastern Finland, Kuopio, ${ }^{3}$ Department of Medicine, Oulu University Hospital, Oulu, ${ }^{4}$ Department of Medicine, ${ }^{5}$ Tampere University Hospital,

Tampere, ${ }^{6}$ Department of Medicine, Turku University Hospital, Turku, 'Laboratory of Eastern Finland, Kuopio, Finland

Introduction: Autologous stem cell transplantation is the standard treatment in patients with multiple myeloma (MM). However, there is discrepancy over the optimal mobilization regimen. Therefore a randomized study was conducted to compare cellular composition of the collected grafts as well as early hematopoietic and immune recovery in MM patients receiving G-CSF with or without low-dose cyclophosphamide for mobilization of blood grafts after induction with lenalidomide, bortezomib and dexamethasone.

Materials (or patients) and methods: Thirty patients with MM were included into this prospective multicenter study. There were 16 males and 14 females with a median age of 62 years (range 43-70). Fourteen patients were mobilized with cyclophosphamide plus G-CSF (arm A) whereas sixteen patients were mobilized with G-CSF alone (arm B). Melphalan $200 \mathrm{mg} / \mathrm{m}^{2}$ was used as high-dose therapy and patients having graft $\mathrm{CD} 34^{+}$cell counts $<3 \times 10^{6} / \mathrm{kg}$ (measured before freezing) were scheduled to receive G-CSF after the graft infusion. Cryopreserved graft samples were analyzed with a flow cytometry for T and B cells (CD3/CD8/CD45/CD19) as well as for NK cells (CD3/CD16 + CD56/CD45). Also CD34 ${ }^{+}$cell subclasses were analyzed (CD34/CD38/CD133/CD45). Complete blood counts were evaluated on day +15 and one month post-transplant and a flow cytometry for blood lymphocyte subsets ( $T, B, N K)$ was performed one month after the graft infusion.

Results: The blood grafts in arm A contained significantly higher amounts of $\mathrm{CD} 34^{+}$cells and the grafts of the arm B contained significantly higher proportion of primitive CD34 ${ }^{+}$

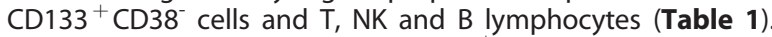
The median amount of infused $\mathrm{CD} 34^{+}$cells was comparable between the arms $\left(3.9 \times 10^{6} / \mathrm{kg}\right.$ in group $A$ vs. $3.1 \times 10^{6} / \mathrm{kg}$ in group $B, P=0.056)$. The number of platelets was slightly lower in the group $B$ at $d+15(P=0.094)$ otherwise the course of early hematological and immune recovery was comparable between the groups.

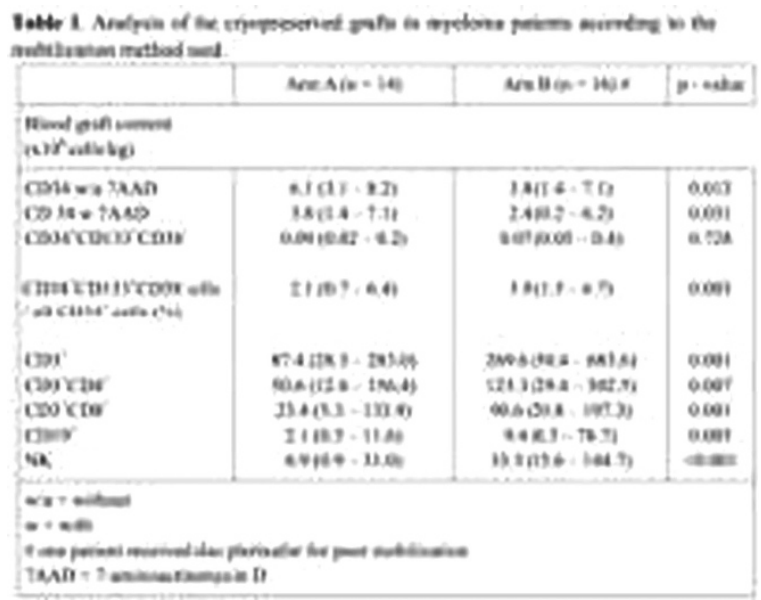

Conclusion: The use of G-CSF alone instead of a combination with cyclophosphamide seems to enrich the blood grafts with significantly higher number of $T$ and $B$ lymphocytes and a higher proportion of more primitive stem cells. The hematological and immune recovery was comparable between the arms. The possible effects of graft composition in long-term patient outcomes will be further evaluated in the ongoing GOA study (Graft and Outcome in Autologous stem cell transplantation).

Disclosure of Interest: None declared.

0030

Upgrade Of FDG-PET Response Following Autologous Stem Cell Transplantation Is Associated With Better Progression Free Survival Among Patients With Multiple Myeloma

M. Beksac ${ }^{1, *}$, P. Topcuoglu' ${ }^{1}$, M. Gunduz ${ }^{1}$, P. Ataca ${ }^{1}$, S. M. Bakanay Ozturk ${ }^{2}$, B. Kirazli , M. Merter ${ }^{1}$, M. Ozen ${ }^{1}$, N. O. Kucuk ${ }^{3}$, E. Ozkan ${ }^{3}$ ${ }^{1}$ ANKARA UNIVERSITY DEPARTMENT OF HEMATOLOGY, ${ }^{2}$ ATATURK HOSPITAL, ${ }^{3}$ ANKARA UNIVERSITY DEPARTMENT OF NUCLEAR MEDICINE, ANKARA, Turkey

Introduction: PET is a useful tool that allows deeper assessment of response beyond that measured by $M$ protein levels. It has been reported to predict outcome following both ASCT. To be able to integrate PET-CT negativity to internationally accepted response criteria the cut-off level needs to be validated by independent investigators. This prospective study was initiated to elucidate the prognostic role of PET-CT in the ASCT setting utilizing the cut-off found in our patients in Ankara University (3.35) comparing with those initially reported by Barlogie et al (3.6) and Zamagni et al (4.2)

Materials (or patients) and methods: 85 consecutive patients diagnosed and transplanted in Ankara University with pre- and post-ASCT FDG- PET-CT imaging were included. Patients were: Median age $56.6+/-8.8$ (M/F: 45/40), ISS I/II/III: 37/33/15, renal impairment $(8,2 \%)$, bone involvement $(94,1 \%)$, del13q $(40.9 \%)$, $\mathrm{t}(4 ; 14)$ and/or p53(26.5\%), LDH high(10,5\%), induction with Bortezomib (72,9\%). Pre-ASCT clinical response ${ }^{3}$ VGPR: $52.9 \%$, post-ASCT clinical response ${ }^{3}$ VGPR:77.5\%. Overall Survival (OS): median: 33 months (4.2-141 months). PASW statistics for Windows program was used for statistical analysis.

Results: As reported previously ROC analysis revealed 3.35 as a significant cut-off level ( $P=0.005 ; \mathrm{OS})$. PET-CR was defined FDG uptake less than 4.2 or 3.35 depending on the analysis. Post-ASCT PET $(>4.2)$ was predictive for PFS $(P=0.05)$ but not OS $(P=0.096)$. However PET $(>3.35)$ was predictive for OS $(P=0.037)$ but not PFS. Depending on the cut-off more (SUV ${ }^{3}$ 4.2: 43/64) or less (SUV 3.35: 29/64) patients met the criteria for PET-negativity (or remission) following ASCT. Expert PET assessment resulted with PET-CR 39/85 similar to the SUV 3.35 frequency. As shown in figure patients to converted to $C R$ after ASCT (positive/negative group) displayed a better PFS than those who had reached CR prior to ASCT. This analysis was significant if cut-off was 3.35 but not 4.2. Expert assessment was also able to differentiate patients with better prognostic features.

Table 1

\begin{tabular}{lllll}
\hline preASCT/ & Total N & event & & \multicolumn{2}{c}{ censored } \\
\cline { 4 - 6 } postASCT PET & & & $\mathrm{N}$ & $\%$ \\
\hline positive /positive & 46 & 23 & 23 & 50,0 \\
positive/negative & 27 & 8 & 19 & 70,4 \\
negative/negative & 12 & 5 & 7 & 58,3 \\
Overall & 85 & 36 & 49 & 57,6 \\
\hline
\end{tabular}

Conclusion: In conclusion is addition to expert assessments both PET $(<4.2)$ and PET $(<3.35)$ recognizes deeper responses as manifested by extension of PFS $(\mathrm{PET}<4.2)$ and OS 

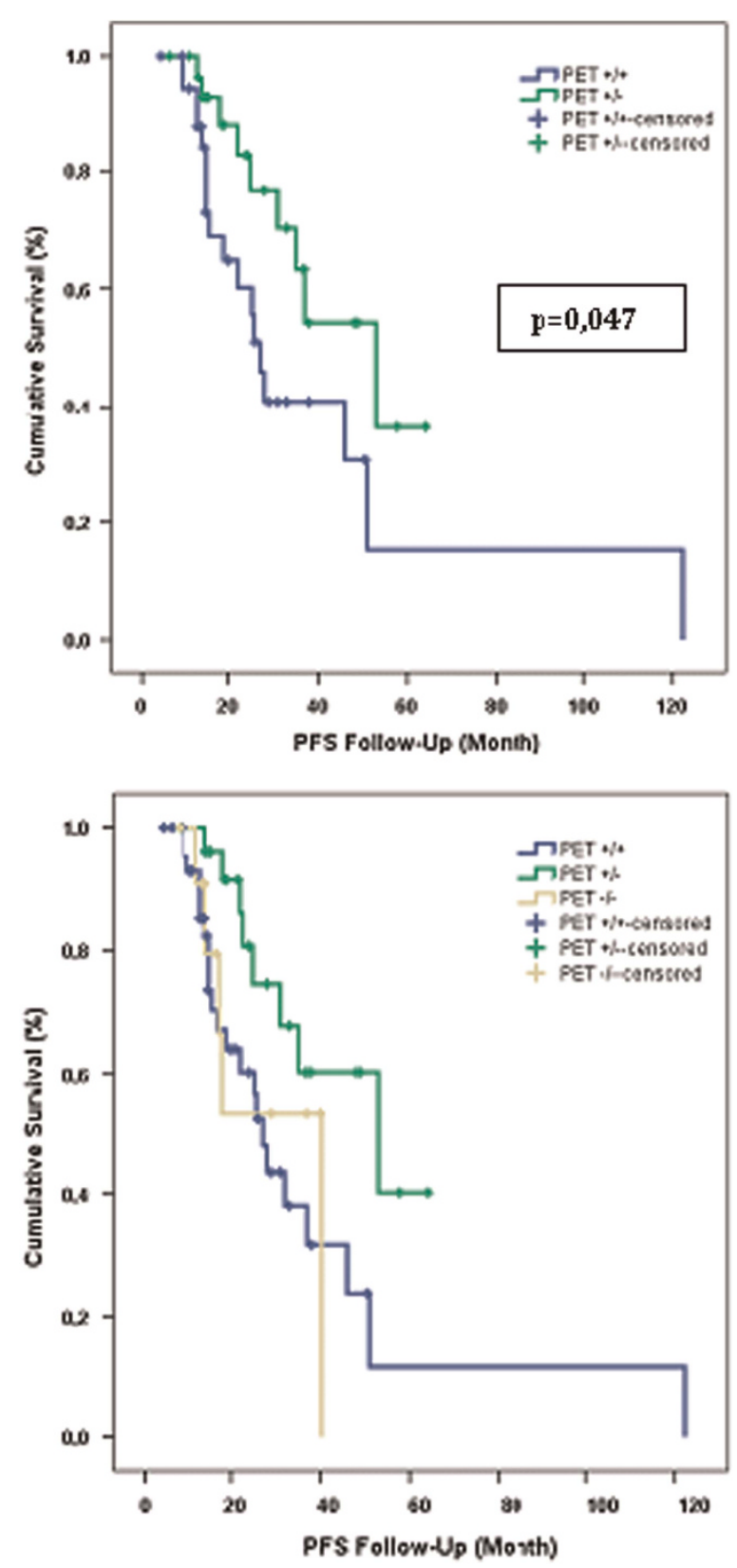

(PET<3.35). Response to high dose Melphalan can be measured by PET response and is a good prognostic feature. Disclosure of Interest: None declared.

0031

Registry based retrospective analysis of bendamustine conditioned autologous hematopoietic stem cell transplants in myeloma

L. Floro ${ }^{1, *}$, R. Benjamin ${ }^{1}$, A. van Biezen ${ }^{2}$, L. Koster ${ }^{2}$, S. lacobelli ${ }^{3}$, S. Schoenland ${ }^{4}$, N. Kroeger ${ }^{5}$, L. Garderet ${ }^{6}$ on behalf of EBMT Chronic Malignancy Working Party

${ }^{1}$ KINGS COLLEGE HOSPITAL, London, United Kingdom, ${ }^{2}$ EBMT Data Office, Leyden, Netherlands, ${ }^{3}$ University of Rome, Rome, Italy, ${ }^{4}$ University Hospital, Heidelberg, ${ }^{5}$ University Hospital, Hamburg, Germany, ${ }^{6}$ Saint Antoine Hospital, Paris, France

Introduction: The current standard of care for myeloma includes consolidation with a high dose melphalan conditioned autologous stem cell transplant (auto SCT), if transplant

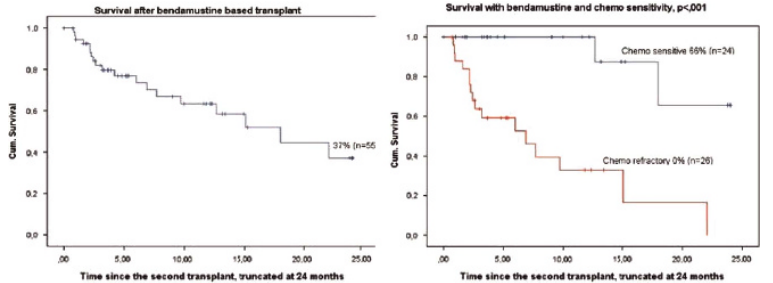

eligible. Second auto SCTs are also performed in relapsed patients, again mainly using melphalan for conditioning, however bendamustine has also been used in this context.

Materials (or patients) and methods: We performed a registry based retrospective analysis using the EBMT database to assess the outcomes of bendamustine conditioned auto SCTs in myeloma.

Results: Altogether 60 cases were identified in the registry during the time period of 2011-2014, of which 5 patients received bendamustine as part of their first auto SCT and 55 during their second auto SCT. No patients received it in both conditioning. Our analysis was performed on those receiving bendamustine in their second transplant. All of these patients received high dose melphalan for their first auto SCT with a median progression-free survival (PFS) of 18.1 months. The median time from first to second transplant was 36.57 (7.13$155)$ months. At the time of the second transplant the median age of the cohort was $62(28-74)$ years and the median Karnofsky score was $80 \%$ (40-100\%). At least a partial response prior their second transplant was achieved in $46 \%$ of patients (CR 2\%, VGPR 26\%, PR 18\%), 14\% had stable disease, and the remaining $40 \%$ had progressive disease. Bendamustine was used as monotherapy in 18 transplants (33\%) and in combination with other chemotherapeutic agents in 37 transplants $(67 \%)$. The most common regimen was high dose melphalan with bortezomibe and bendamustine. The median dose of bendamustine was $200 \mathrm{mg} / \mathrm{m} 2$ (80-450). Engraftment rate was $96 \%$ with median time to neutrophil engraftment of 12 (65-25) days. The 2 year overall survival (OS) of the entire cohort was $37 \%$ (figures). However the subgroup analysis showed that patients with chemotherapy sensitive disease had a 2 year OS of $66 \%$ compared to those with chemo refractory disease where there were no survivors beyond 22 months (see figures, this data was not available in 5 cases).

Conclusion: Bendamustine conditioned salvage auto SCTs result in favourable 2 year overall survival if performed in patients with chemosensitive disease. The final analysis of this cohort will include transplant related mortality and PFS data as well as matched case-control study with patients having high dose melphalan alone for their second auto SCT.

Disclosure of Interest: None declared.

\section{2}

Bortezomib-based PBSC mobilization, followed by ASCT, in 75 Multiple Myeloma elderly patients: a prospective multicenter study

I. Attolico ${ }^{1, *}$, R. Nuccorini ${ }^{1}$, M. Pizzuti ${ }^{1}$, G. Specchia ${ }^{2}$, A. Fragasso ${ }^{3}$, V. Pavone, S. Capalbo ${ }^{5}$, P. Musto ${ }^{6}$, A. Amendola ${ }^{1}$, M. Cimminiello ${ }^{1}$, N. Filardi ${ }^{1}$, A. Matturro ${ }^{1}$, D. Vertone ${ }^{1}$, S. Pascale ${ }^{1}$ S. Coluzzi ${ }^{1}$, A. Olivieri ${ }^{7}$

${ }^{1}$ Ematologia-Azienda Ospedaliera San Carlo, Potenza, Italy, Potenza, ${ }^{2}$ Ematologia con Trapianto, Azienda OspedalieroUniversitaria Policlinico, Bari, ${ }^{3}$ U.O. di Ematologia, Ospedale Madonna delle Grazie, Matera, ${ }^{4}$ U.O. di Ematologia, Ospedale G. Panico, Tricase (LE), ${ }^{5}$ U.O. di Ematologia, Azienda OspedalieroUniversitaria Ospedali Riuniti di Foggia, Foggia, ${ }^{6}$ Direzione Scientifica IRCCS CROB, Rionero in Vulure (PZ), ${ }^{7}$ Clinica di Ematologia-Università Politecnica delle Marche, Ancona, Italy

Introduction: Autologous Stem Cell Transplantation (ASCT) is a mainstream therapy in Multiple Myeloma (MM) young 
patients (pts); however, in the "new drugs era", the role of ASCT in elderly pts is still debated; moreover the impact of Bortezomib combination both with mobilization schedules and with conditioning regimens has never been tested in this setting.

Materials (or patients) and methods: We have enrolled $75 \mathrm{fit}$ elderly pts $(39 \mathrm{~F}, 36 \mathrm{M})$, with a median age of 65 years (range: 52-78), who received a first line therapy with Bortezomib, Cyclophosphamide and Dexamethasone (i.v. BOR $1.3 \mathrm{mg} / \mathrm{m} 2 /$ dose on days $1-4-8-11$, i.v. DEX $40 \mathrm{mg} /$ day on days $1-4-8-11$ and i.v. CY $300 \mathrm{mg} / \mathrm{m} 2 /$ dose on days 1-8-15) for at least 4 courses; pts achieving at least a PR after 4 courses of Cy-BOR, proceeded to mobilization; mobilization schedule consisted in i.v. BOR $1.3 \mathrm{mg} / \mathrm{m} 2 /$ dose (days $1-4-8-11$ ), i.v. DEX $40 \mathrm{mg} /$ day (days 1-4-8-11) and i.v. CY $3000 \mathrm{mg} / \mathrm{m} 2 /$ dose (day 8). G-CSF, at $5 \mathrm{mg} / \mathrm{Kg} / \mathrm{day}$, was administered starting from day 9. Conditioning regimen consisted in: BOR $1.0 \mathrm{mg} / \mathrm{m} 2$ (days -6 ,$3,+1,+4$ ) and Melfalan $200 \mathrm{mg} / \mathrm{m} 2$ (day -1 ).

Results: 62 pts are evaluable for response after induction; 47 achieved $\geq$ PR (76\%) among them $34 \geq$ VGPR (55\%); 43 pts were mobilized ( 3 pts withdrew the consent, 1 is ongoing) and 42 pts (98\%) collected $\geq 2.0 \times 10 \mathrm{e} 6 \mathrm{CD} 34+$ cells $/ \mathrm{Kg}$ (median: $6.4 \times 10$ e6 CD34 + $/ \mathrm{Kg}$, range: $2.75-15.8$ ) in a median number of two aphereses (range1-4). 36 pts (36/43 84\%) collected $\geq 4.0 \times 10 \mathrm{e} 6 \mathrm{CD} 34+/ \mathrm{Kg}$. The mobilization procedure was well tolerated; only one patient did not complete the mobilization procedure, due to a serious adverse event (dyspnea and pleural effusion due to progressive disease). 41 pts underwent ASCT; (1 withdrew consent). Engraftment was achieved at day 12 for neutrophils (range 9-15) and at day 14 for platelets (range 10-26). At day +90 after ASCT, 33 pts are evaluable for response: all achieved $\geq$ VGPR, with $27 \geq \mathrm{nCR}(82 \%)$. 13 pts obtained an improvement of response after ASCT. During the whole program we observed gr.3/4 neutropenia in 3 pts, gr.3/4 thrombocytopenia in 4 pts, gr.3/4 neuropathy in 3 pts.

With a median follow up of 31 months (range 0-80) 51/75 (68\%) pts are alive. Causes of death were: progressive disease in $17 \mathrm{pts}$, acute respiratory failure in $1 \mathrm{pt}$, DIC in $1 \mathrm{pt}$ septic shock in $1 \mathrm{pt}, \mathrm{CMV}$ pneumonia in $1 \mathrm{pt}$, other causes in 1 pt with an overall Treatment Related Mortality of $6 \%$. Flow cytometric evaluation of MRD was performed in 43 pts after induction with a median value of clonal PCs of $0.2 \%$ (range 0.0 89). 27 pts are evaluable at day +90 after ASCT with a median value of $0.07 \%$ (range 0.0-0.49). 23 leukaphereses were analized for MRD: the median plasma cells value was $0,03 \%$ $(0,00-0,7 \%$ ); in 6 pts PCs were $<0,01 \%$ (cut off for MRD negativity).

Conclusion: Mobilization with Cy-BOR-Dx + G-CSF and BORbased ASCT is safe and effective in elderly MM patients. This schedule allows the collection of an adequate dose of CD34 + cells, with a very low rate of mobilization failure (2\%), also in elderly MM pts. A low rate of clonal PCs contamination in the harvest was also observed. This approach allows to perform ASCT in most elderly pts, achieving high response rate and promising outcome with a short term treatment:20 weeks compared to the non-ASCT programs (54 weeks in the VMP program).

Disclosure of Interest: None declared.
Oral session: GvHD 1

0033

MICA genotype, serum and expression level effects on the outcome of HSCT

R. Gam 1,*, J. Norden ${ }^{1}$, R. Crossland ${ }^{1}$, K. Pearce ${ }^{1}$, E. Holler ${ }^{2}$,

R. Dressel ${ }^{3}, A$. M. Dickinson ${ }^{1}$ on behalf of This work was done under the framework of CellEurope project (FP7-People-2012-ITN, No. 315963) coordinated by Professor Anne Dickinson from University of Newcastle upon Tyne

${ }^{1}$ Department of haematological sciences, Newcastle University, Newcastle-Upon-Tyne, United Kingdom, ${ }^{2}$ Department of Haematology and Oncology, University Clinic Regensburg, Regensburg, ${ }^{3}$ Department of Cellular and Molecular Immunology, University of Göttingen, Göttingen, Germany

Introduction: Graft-versus-host disease (GvHD) is a serious complication of allogeneic haematopoietic stem cell transplantation (allo-HSCT). The immunopathology of GvHD involves secretion of proinflammatory cytokines with subsequent expression of danger signals by the affected tissues. MHC class I chain-related gene A (MICA), is a stress-induced molecule that acts as danger signal to alert NK and $\alpha \beta$ or $\gamma \delta$ CD8 T cells through engagement of the activating NKG2D receptor.

We investigated MICA genotype together with serum and mRNA expression levels in the development and progression of GvHD.

Materials (or patients) and methods: We analysed the MICA SNP (rs1051792) Val129Met in $n=1384$ patient-donor pairs. Genotyping was performed by KASP, based on competitive allele specific PCR (LGC Genomics, UK).

MICA expression levels were investigated in $n=180$ gut biopsies with SYBR GREEN ${ }^{\circledR}$ qRT-PCR. Histological grades of the gastrointestinal GvHD (GI GvHD) were determined by the pathology department at the University Clinic, Regensburg and severity was grouped by assigning an apoptotic score ( $0=$ absence of apoptosis, $3=$ maximum apoptosis).

A Protein Biochip Array (Evidence Investigator ${ }^{\mathbb{R}}$, RANDOX) was utilised for measuring MICA serum levels and evaluated in $n=129$ samples from allo-HSCT patients collected at pretransplantation, day-7, day +14 , day +28 and 3 months post transplantation.

Results: Our analysis showed that the Methionine allele in rs 1051792 was associated with an increased risk of relapse $(P=0.029)$. The same allele was also found to be associated with a reduced overall survival $(P=0.041)$ which was more severe for non-T cell-depleted allo-HSCT $(P=0.001)$. Vice, versa, the presence of the Valine allele was associated with the development of aGvHD $(P=0.044)$.

In the gut, MICA expression was investigated in patients treated with low doses of steroids ( $\leq 20 \mathrm{mg} / \mathrm{kg}$ ), as high dose steroid treatment strongly suppressed MICA expression. Higher levels of MICA were associated with an apoptotic score $=0$ (no apoptosis) $(P=0.044)$ and the absence of active Gl GvHD $(P=0.046)$.

Increased soluble MICA levels at 3 months post-transplantation were significantly associated with aGvHD $(P=0.0123)$.

Conclusion: MICA molecules have been shown to play prominent roles in immune processes and therefore are also potential aGVHD biomarkers. In this study, we showed that the Methionine (MICA-129Met) allele was associated with the incidence of relapse while the Valine (MICA-129Val) allele was associated with an increased risk aGvHD. A low overall survival for patients who did not have had the $T$ cell depletion treatment was also associated with the presence of the Methionine (MICA-129Met) allele. In the gut of patients treated with low doses of steroid, MICA gene expression levels were higher with the absence of GvHD. This may indicate that the isoforms are able to meditate NK-cell and T cell inactivation, and down-regulate NKG2D with high levels of soluble MICA contributing to the development of aGvHD. 
Disclosure of Interest: R. Gam Funding from: This work was done under the framework of CellEurope project (FP7-People2012-ITN, No. 315963) coordinated by Professor Anne Dickinson from University of Newcastle upon Tyne, J. Norden: None declared, R. Crossland: None declared, K. Pearce: None declared, E. Holler: None declared, R. Dressel: None declared, A. M. Dickinson: None declared.

0034

Preventing GvHD and high-risk acute leukemia relapse by Treg and Tcon adoptive immunotherapy in HLAhaploidentical transplantation: an update on the clinical trial and insights from murine studies

L. Ruggeri ${ }^{1,{ }^{*}}$, M. Di lanni ${ }^{1}$, F. Falzetti ${ }^{1}$, A. Carotti ${ }^{1}$, A. Terenzi ${ }^{1}$, M. S. Massei ${ }^{1}$, L. Amico ${ }^{1}$, A. Pierini ${ }^{1}$, E. Urbani ${ }^{1}$, B. Falini ${ }^{1}$, M. F. Martelli ${ }^{1}$, A. Velardi ${ }^{\top}$

${ }^{1}$ Hematology and Clinical Immunology Section, University of Perugia, Perugia, Italy

Introduction: Clinical trials showed that conventional $\mathrm{T}$ lymphocytes (Tcons), when co-infused with Tregs, exerted a powerful GvL effect in the absence of GvHD (Di lanni et al., Blood 2011; Martelli et al., Blood 2014). The mechanism by which the GvL effect is exerted in the absence of GvHD is unknown.

Materials (or patients) and methods: 52 patients (median age 39, range 18-65) with high-risk AML (37) and ALL (15) were transplanted. Conditioning included TBI, thiotepa, fludarabine and cyclophosphamide in 29 patients or anti-T antibodies instead of cyclophosphamide in 23 patients. On day -4 , patients received donor Tregs (mean $2.5 / \mathrm{kg} \pm 1$ ) followed on day 0 by CD34 + cells $\left(9.7 \times 10^{6} / \mathrm{kg} \pm 3.1\right)$ and Tcons (mean 1.1 / $\mathrm{kg} \pm 0.6 ;$ ). No post-transplant GvHD prophylaxis was given. Sublethally irradiated NSG mice were engrafted with primary human leukemia $\left(3 \times 10^{6}\right)$ and were given Treg/Tcon combinations $\left(3 \times 10^{6}\right)$. Human T cells were harvested from a GvHD site (liver) and BM and analysed for their phenotypes and regulatory vs GvL functions.

Results: Cinical trial: 50/52 patients achieved primary, sustained full-donor engraftment. Six of $50(12 \%)$ evaluable patients developed $\geq$ grade II acute GvHD ( 3 alive, treated and now off-therapy). One patient developed chronic GvHD. $\mathrm{CD} 4+$ and $\mathrm{CD} 8+\mathrm{T}$ cell counts reached $100 / \mu \mathrm{L}$ on days 40 (range 25-150) and 45 (range 18-100). At a median follow-up of 4 years (range 7-58), 30/52 patients are alive and well with a disease free survival probability of 0.58 . Cumulative incidence of relapse was extremely low (i.e., 0.05 ) as only two (AML) patients relapsed. Murine studies: Mice that received leukemia and Tcons (without Tregs) cleared leukemia but died of GvHD. Human T cells harvested from their BM and liver were CD8 + (90\%) and displayed potent allo-cytotoxicity against leukemia. In mice treated with Tregs alone, human $\mathrm{T}$ cells were recovered from the liver. They displayed a CD4 + /FOXP3phenotype and inhibited MLR. No human T cells were found in the BM. Mice that received human leukemia and Tcons plus Tregs were rescued from leukemia and survived without GvHD. Human T cells harvested from liver were composed of CD8 + T cells (40\%) and CD4 + /FOXP3- T cells (60\%). CD4 + T cells had retained their regulatory function as they inhibited MLR. Purified CD8 + T cells displayed no allo-cytotoxicity against leukemia. In contrast, human $\mathrm{T}$ cells harvested from $\mathrm{BM}$ were still predominantly CD8 + and still displayed potent allo-cytotoxicity against leukemia.

Conclusion: Clinical data reported here show Treg prevent acute GvHD and allow safe co-infusion of Tcons to eradicate minimal residual disease thus eliminating the usual 30-35\% incidence of leukemia relapse. The mechanism by which the GvL effect is exerted in the absence of GvHD is shown by our murine studies. It is known that naïve CD45RA + Tregs express CXCR4 and, therefore, preferentially localize to the BM while memory CD45RO + Tregs express lower CXCR4 levels and, therefore, preferentially home to the periphery (Booth et al., J Immunol. 2010). Peripheral blood T regs used for adoptive immunotherapy were predominantly CD45RO + and when infused in mice, migrated to the periphery (i.e., liver) where they blocked Tcons and prevented GvHD. However they were unable to migrate to the BM thereby allowing Tcons to exert unopposed alloantigen recognition and leukemia killing.

Disclosure of Interest: None declared.

\section{5}

Kinetics of acute and chronic GvHD control by ganciclovir in-vivo HSV-TK suicide gene activation: long-term GvHDfree survival in $\mathbf{5 7}$ patients after haploidentical TK-cells F. Ciceri $i^{1, *}$, M. T. Lupo Stanghellini ${ }^{2}$, R. Greco ${ }^{1}$, A. Forcina', A. Bondanza ${ }^{1}$, L. Vago ${ }^{1}$, J. Peccatori ${ }^{1}$, A. Lambiase ${ }^{3}$, C. Bordignon ${ }^{3}$, C. Bonini ${ }^{T}$

${ }^{1}$ Hematology and BMT Unit, ${ }^{2}$ Hematology, San Raffaele Scientific Institute, ${ }^{3}$ Molmed SPA, MolMed SpA, Milano, Italy

Introduction: Haploidentical SCT (haplo-HSCT) is currently extended to patients affected by high risk hematological diseases. However, platforms based on unmanipulated graft have a significant risk of both acute and chronic GvHD. In the past 20 years we investigated the application of herpes simplex virus thymidine kinase (TK) suicide gene strategy to allow the selective elimination of genetically modified haploidentical donor $\mathrm{T}$ cells during GvHD, while sparing immune-recostitution (IR) with effective graft-versus-leukemia and graft-versus-infectious effects. Here we report the analysis of outcome for both acute (a-) and chronic (c-) GvHD in haploHSCT after TK-cells infusion.

Materials (or patients) and methods: We analyzed 57 adult patients (pts) after haplo-HSCT within 2 trials: a phase TK007 (Ciceri, Bonini et al, Lancet Oncol 2009) and a Phase III (TK008, NCT0091462), between 2002 and 2014 at our Center.

All pts received a graft after selection of peripheral CD34+ cells - CliniMacs. No post-transplant immune-suppression was administered after transplantation as GvHD prophylaxis. In vivo T-cell depletion with ATG (Fresenius) was administered in all pts. Pts median age was 53 (range, 17-66) years.

Donor lymphocytes genetically engineered to express the TK gene were infused in 34/57 pts (median 2 infusion/pts), 25/34 achieved IR (median time from HSCT 84 days - range 18/182 median time from last TK-cells infusion 27 days - range 13/42). Results: Twelve of 25 immune-reconstituted patients developed a-GVHD (grade I-IV; median time of onset 84 days post HSCT - range 20/162; 19 days post last TK-infusion - range 8/ 54) and one developed c-GVHD. Direct association of TK-cells and GVHD was confirmed by vector-encoded protein immunostaining of lymphocytes infiltrating affected lesions.

Eleven patients needed GvHD treatment: 4 pts received ganciclovir iv (GCV $5 \mathrm{mg} / \mathrm{Kg} / 12 \mathrm{~h} / 14$ days), 7 pts valganciclovir per os (VGCV $900 \mathrm{mg} / 12 \mathrm{~h} / 14$ days). Both GCV and VGCV were effective in control clinical manifestations of GVHD in a median of 13 days (range 7-52) and resulted in a significant reduction in numbers of circulating TK-cells, without reduction of CD3 + TK-negative lymphocytes resulting in no effect on long-term immune reconstitution. In five patients additional concomitant treatment with low-dose steroid (prednisone $<0.5 \mathrm{mg} / \mathrm{kg}$ per day for a median of 2 weeks) was given.

A pt who presented severe gut and liver GvHD and one pt who received at transplantation an high dose of unmanipulated lymphocytes $\left(5.4 \times 10^{5} / \mathrm{Kg}\right)$ - were succesfully treated with a combined therapy of prednisone and cyclosporine or rapamicine in association with GCV.

One patient developed a severe classic de novo c-GVHD, with sclerodermatous lichenoid skin and mouth features plus moderate dry-eye symptoms that was successfully treated with VGCV and a transient course of mycophenolate mofetil ( $2 \mathrm{~g}$ per day) over a 2 months period.

No cases of quiescent or progressive c-GvHD was observed after a median follow-up of 679 days (range 139/4035).

Conclusion: In our long-lasting clinical application of haploidentical TK-cells, an effective induction of immune 
reconstitution and a complete control of GvHD, provided a long-term immunosoppressive therapy free survival in absence of GvHD related deaths or longterm complications. Disclosure of Interest: None declared.

\section{6}

High response rates in patients treated with ruxolitinib for corticosteroid-refractory Graft-versus-host disease: analysis including 13 Transplant Centers

R. Zeiser ${ }^{1, *}$, C. Lengerke ${ }^{2}$, S. Spoerl ${ }^{3}$, K. Maas-Bauer ${ }^{1}$, M. Ditschkowski ${ }^{4}, M$. Ecsedi', K. Sockel ${ }^{5}$, F. Ayuk,

F. Sicre-de-Fontbrune ${ }^{7}$, I.-K. Na ${ }^{8}$ S. Ajib ${ }^{9}$, M. Verbeek ${ }^{10}$, H. Nogai ${ }^{8}$, U. Holtick ${ }^{11}$, D. Wolf ${ }^{12}$, E. Schuler ${ }^{13}$, E. Meyer $^{14}$,

P. Apostolova ${ }^{1}$, L. Illert ${ }^{1}$, H. Bertz', R. Marks ${ }^{1}$, R. Wäsch ${ }^{1}$,

B. Hackanson ', C. Scheid', R. Ordemann ${ }^{5}$, G. Bug ${ }^{9}$, G. Kobbe ${ }^{13}$,

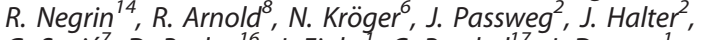

G. Socié7, D. Beelen ${ }^{16}$, J. Finke', C. Peschel ${ }^{17}$, J. Duyster ${ }^{1}$,

N. von Bubnoff'

${ }^{1}$ Hematology, Oncology and Stem Cell Transplantation, Freiburg University Hospital, Freiburg, Germany, ${ }^{2}$ Hematology, University Hospital Basel, Basel, Switzerland, ${ }^{3}$ Hematology, Oncology and Stem Cell Transplantation, Technical University of Munich, Munich, ${ }^{4}$ Department of Bone Marrow Transplantation, West German Cancer Center, University Hospital Essen, Essen, ${ }^{5}$ Hematology, Oncology and Stem Cell Transplantation, Universitätsklinikum Carl Gustav Carus an der Technischen Universität Dresden, Dresden, ${ }^{6}$ Department of Stem Cell Transplantation, University Hospital Hamburg-Eppendorf, Hamburg, Germany, ${ }^{7}$ Hematology Stem cell transplant Unit, Saint Louis Hospital, APHP, Paris, Paris, France, ${ }^{8}$ Department of Stem Cell Transplantation, Charité University Medicine Berlin, Berlin, ${ }^{9}$ Department of Internal Medicine II, University Hospital, Frankfurt/Main, Frankfurt, ${ }^{10}$ Department of Internal Medicine II, Technical University of Munich, Munich, ${ }^{11}$ Department of Internal Medicine I, University Hospital Cologne, Cologne, ${ }^{12}$ Oncology, Hematology and Rheumatology, University Hospital Bonn, Bonn, ${ }^{13}$ Department of Hematology, Oncology and Clinical Immunology, Universitätsklinikum Düsseldorf, Düsseldorf, Germany, ${ }^{14}$ Department of Stem Cell Transplantation, Stanford University Medical Center, Stanford, United States, ${ }^{15}$ Department of Internal Medicine I, University Hospital Cologne, Cologne, ${ }^{16}$ Department of Bone Marrow Transplantation, West German Cancer Center, University Hospital Essen, Essen, ${ }^{17}$ III Department of Internal Medicine, Technical University of Munich, Munich, Germany

Introduction: Despite major improvements in allogeneic hematopoietic cell transplantation (allo-HCT) over the last decades, severe corticosteroid-refractory acute and chronic graft-versus-host-disease (GvHD) still remains a life-threatening complication characterized by high mortality rates (40-70\%). Since preclinical and early clinical evidence indicated anti-inflammatory effects of ruxolitinib, we collected the outcome data from multiple Stem Cell Transplant Centers using the JAK $1 / 2$ inhibitor ruxolitinib as salvage treatment in patients suffering from corticosteroid-refractory GvHD.

Materials (or patients) and methods: A total of 13 Stem Cell Transplant Centers in Germany, France, Switzerland and United States reported outcome data from 52 patients who received ruxolitinib for corticosteroid-refractory GvHD (skin, mucosa, intestine, liver, lung, musculoskeletal) between 01/ 2012 and 12/2014. Patients were classified as having acute $(n=32)$ or chronic $(n=20) \mathrm{GvHD}$. The median number of previous GvHD-therapies was 4 for acute GvHD (range: 1-7) and 3 for chronic GvHD (range: 2-10).

Results: The overall response rate was $84.3 \%(27 / 32)$ in acute GvHD comprising 10 CRs (31.2\%) and 17 PRs (53.1\%). In chronic GvHD the overall response rate was $80 \%(16 / 20)$. Clinical improvement was rapid with a median time to response of 1.5 (1-4) weeks and 1 (1-11) week after initiation of ruxolitinib treatment in acute and chronic GvHD, respectively. All responders were in persistent remission at last follow-up. The median follow-up was 18 (2-58) and 13 (2-70.5) weeks for acute and chronic GvHD patients, respectively. Non-responders (acute GvHD: 5/32, chronic GvHD: 4/20) received other salvage therapies.

Cytopenias (anemia, leukopenia or thrombocytopenia) and CMV reactivation were observed during the time of ruxolitinib treatment in both acute $(18 / 32,56.2 \%$ and respectively $11 / 32$, $34.3 \%)$ and chronic (4/20, 20\% and respectively $3 / 20,15 \%)$ GvHD patients, sometimes however cytopenias already preceded ruxolitinib treatment. Ruxolitinib treatment was stopped or reduced in 2 patients because of cytopenia. CMV was controlled by antiviral therapy in all patients. 3 ruxolitinib responders died, one because of leukemia relapse 4 weeks after ruxolitinib was stopped, and two from GvHD progression. In one of the patients who died, ruxolitinib was stopped due to impossibility of oral drug application.

Conclusion: Overall, these data collected in multiple centers using different strategies for GvHD prophylaxis and treatment suggest that ruxolitinib is a very promising agent in the treatment of corticosteroid-refractory acute or chronic GvHD and may be successfully used to treat a major subset of patients beyond 2 nd line of GvHD treatment. A prospective randomized multicentre clinical trial testing therapeutic JAK1/ 2 inhibition as salvage treatment in GvHD is planned to verify the efficacy of ruxolitinib and to identify potential biomarkers that may be predictive for response.

Disclosure of Interest: None declared.

0037

Transplant-associated renal microangiopathy is associated with a high risk of refractory acute GVHD and characterized by a specific biomarker signature M. Zeisbrich ${ }^{1}$, J. Beimler ${ }^{1}$, M. Zeier', C. S. Falk ${ }^{2}$, A. D. Ho ${ }^{3}$, P. Dreger ${ }^{3}$, T. Luft ${ }^{3, *}$

${ }^{1}$ Kidney Center Heidelberg, UNIVERSITÄTSKLINIKUM HEIDELBERG, Heidelberg, ${ }^{2}$ Institute for Transplant Immunology, IFB-TX, Hannover Medical School, Hannover, ${ }^{3}$ Medicine V, UNIVERSITÄTSKLINIKUM HEIDELBERG, Heidelberg, Germany

Introduction: There is increasing evidence that endothelial damage is involved in the pathogenesis of steroid-refractory graft-versus-host disease (refGVHD). Recently, serum soluble ST2 (suppressor of tumorigenicity, IL-33 receptor), an independent risk factor of cardiovascular death, has been identified as a risk factor of refractory GVHD. However, the pathomechanism of endothelial cell dysfunction which is associated with mortality from GVHD is yet poorly characterized. Renal transplant-associated microangiopathy (TMA) is another endothelial complication of alloSCT, and its association with severe GVHD and with biomarkers of endothelial damage (ST2, SCD141 (soluble thrombomodulin)), and endothelial function (VEGF) is investigated in this study. Materials (or patients) and methods: Evidence for renal TMA was studied in a cohort of 508 patients who underwent alloSCT between 2002 and 2013 at our institution and who have provided informed consent for this observational study. Criteria to diagnose renal TMA included an otherwise unexplained $50 \%$ rise in creatinine and lactate dehydrogenase (LDH) levels (or a pre-existing LDH above $400 \mathrm{U} / \mathrm{L}$ ), a 50\% drop in platelet counts (or a pre-existing platelet count below $50 / \mathrm{nl}$ ) and at least 4\%o schistocytes. Cytokines were measured in sera taken prior to alloSCT and on the indicated days thereafter and stored at minus $80 \mathrm{C}$. Statistical analyses were performed using SPSS19 and included a cumulative incidence analysis of causespecific hazards and the non-parametrical median test of independent probes.

Results: Both renal TMA and refGVHD were rare complications after alloSCT but were significantly associated with each other (TMA only $18 / 508(3.5 \%)$, refGVHD only $26 / 508(5.1 \%)$, both $20 / 508(3.9 \%)$ chi $\left.^{2} 0.000\right)$. Median time intervals from alloSCT to renal TMA and refGVHD were $1.93(0.23-60.16)$ months and $1.23(0.16-12.4)$ months respectively. In the overlap group, GVHD onset usually occurred before renal TMA (-0.69 months, -55.4 to 11.2 ) NRM rates were significantly increased in all 
three cohorts but approached $100 \%$ in patients with both complications.

Serum STM levels as well as soluble ST2 levels increased between transplantation and day 100/day 200 after alloSCT in all cohorts, refGVHD, TMA and both. In contrast, VEGF levels (day 100) were significantly lower specifically in patients with TMA with or without refGVHD, but not in patients with refGVHD without TMA.

Conclusion: This study identifies renal TMA as an endothelial cell dysfunction associated with extremely high mortality rates in the context of GVHD. The absence or presence of renal TMA defines two separate subsets of refGVHD with a different prognosis. Biomarkers of endothelial damage or vulnerability, such as sTM, ST2, and VEGF can help to dissect TMA and refGVHD, and might be useful to identify and guide management of patients with endothelial dysfunction who are at high risk of fatal complications after alloSCT.

Disclosure of Interest: None declared.

0038

Single-Nucleotide Polymorphisms (SNPs) of the NF-кB1 Gene Are Associated with Acute Graft-versus-Host Disease (aGvHD) in Allografted Patients

A. Kuba ${ }^{1, *}$, L. Raida ${ }^{1}$, E. Faber ${ }^{1}$, F. Mrazek ${ }^{2}$, T. Furst ${ }^{3}$, J. Furstova ${ }^{3}$, P. Schneiderova ${ }^{2}$, E. Kriegova ${ }^{2}$, Z. Ambruzova ${ }^{2}$, T. Papajik ${ }^{1}$

${ }^{1}$ Department of Hemato-Oncology, University Hospital and Faculty of Medicine and Dentistry, Palacky University, ${ }^{2}$ Laboratory of Immunogenomics and Proteomics, Department of Immunology, Palacky University and University Hospital, ${ }^{3}$ Department of Mathematical Analysis and Applications of Mathematics, Faculty of Science, Palacky University, Olomouc, Czech Republic

Introduction: SNPs of the key cytokines and chemokines involved in the pathogenesis of aGvHD have become an object of major interest recently. Here we present SNPs rs3774937 CC/CT/TT and rs3774959 AA/AG/GG of the NF- $\kappa B 1$ gene in association with aGvHD.

Materials (or patients) and methods: In our single-center study we analyzed 70 patients allografted for the following hematological malignancies: AML (37\%), ALL (14\%), CLL (13\%), MDS (13\%), CML (6\%), NHL (6\%), IMF (3\%), CMML (4\%), and other hematological disorders (4\%) between 2009-2014. The median age of the study group was 51 (21-62) years. Patients were allografted after myeloablative (11\%), non-myeloablative (14\%) and reduced (intensity/toxicity) conditionings (74\%). GvHD prophylaxis was done by solo cyclosporine-A (80\%), cyclosporine-A with mycophenolate mofetil (19\%) and cyclosporine-A with short-methotrexate (1\%). "In vivo" T-depletion with thymoglobuline was used in $64 \%$ of recipients. Patients were allografted from HLA identical donors (related $46 \%$ ) with median age 37 (18-63) years. The female donor/male recipient combination represented $14 \%$ of all pairs. The grafts contained median $4.6(2.8-8.0) \times 106 \mathrm{CD} 34+$ cells $/ \mathrm{kg}$ and median 6.8 (1.9-27.1) x108 MNC $/ \mathrm{kg}$. SNPs analysis was done from genomic DNA isolated from EDTA-treated peripheral blood. Genotyping was performed with Sequenom MassARRAY platform using allele-specific MALDI-TOF mass spectrometry assay (Sequenom, San Diego, CA, USA). Primers were designed using the Sequenom SNP Assay Design software version 3.0 for iPLEX reactions. Univariate analysis was performed to find significant difference in aGvHD among the patient groups with different NF-KB1 profiles. The asymptotic Pearson's chi-square test was used in cross tabulation with significance level set to 0.05 .

Results: Out of 70 patients 46 patients (66\%) are still alive, 24 patients (34\%) died (12 died of transplant-related complications). The median post-transplant follow-up was 1.6 (0.1-4.5) years. AGvHD developed in 21 patients (30\%), grade III-IV in 7 of them (10\%).

Both NFkB1 SNPs were completely correlated in the sense that knowing the genotype of one fully determined the genotype of the second one (5 patients did not follow this correlation and were excluded from the analysis). Consequently, we defined a common predictor NF- $\mathrm{KB} 1$ which codes the information carried by both NF- $\kappa B 1$ SNPs in the following way: all patients carrying the rs3774937 CC genotype were also positive for rs3774959 AA allele and were marked as NF$\kappa B 1=\mathrm{I}$, all patients carrying the rs3774937 CT genotype were also positive for rs3774959 AG allele and were marked as NF$\kappa B 1=\|$ and finally all patients carrying the rs3774937 $\Pi$ genotype were also positive for rs3774959 GG allele and were

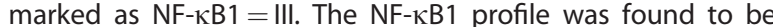
significantly associated $(P=0.002)$ with aGvHD in the following way: Patients in the NF- $\kappa B 1=$ I group are more probable to suffer from aGvHD than in the NF-KB1 = III group.

Conclusion: This is the first report showing the association of $N F-\kappa B 1$ gene SNPs with aGvHD. The transcription factor NF- $\kappa B$ has been implicated in the regulation of cellular stress and inflammatory signals. According to our pilot data patients with inherited genetic abnormalities of the NF- $\kappa B 1$ gene may be prone to aGvHD.

Supported by the project LF-2014-001 of Palacky University in Olomouc.

Disclosure of Interest: None declared.

\section{9}

Grade I acute graft vs host disease: a GITMO randomized trial of prednisolone vs no treatment

A. Bacigalupo, 1,* G. Milone ${ }^{2}$, F. Fagioli ${ }^{3}$, P. Chiusolo ${ }^{4}$, A. Locasciulli ${ }^{5}$, S. Santarone ${ }^{6}$, S. Sica ${ }^{4}$, M. Berger ${ }^{3}$, S. Mammoliti $^{1}$, N. Mordini ${ }^{7}$, D. Massi ${ }^{8}$, F. Galaverna ${ }^{1}$, A. Ghiso ${ }^{1}$, A. Risitano on behalf of for the Gruppo Italiano Trapianto di Midollo Osseo (GITMO)

${ }^{1}$ U.O. Ematologia Trapianto di Midollo, IRCCS - Ospedale San Martino-IST, Genoa, '2U.O. Ematologia Trapianto di Midollo, Ospedale Ferrarotto, Catania, ${ }^{3}$ Centro Trapianti Cellule Staminali, Ospedale Infantile Regina Margherita, Torino, ${ }^{4}$ Istituto di Ematologia, Università Cattolica S Cuore, ${ }^{5}$ Haematology \& SCT Unit, Ospedale San Camillo, Rome, ${ }^{6}$ Divisione di Ematologia, Ospedale Civile, Pescara, ${ }^{7}$ Divisione di Ematologia, Az. Ospedaliera S. Croce e Carle, Cuneo, ${ }^{8}$ Dip. Anatomia Patologica ed Oncologica, Ospedale Careggi, Firenze, ${ }^{9}$ Divisione di Ematologia, Università Federico II, Napoli, Italy

Introduction: In a previous Gruppo Italiano Trapianto di Midollo Osseo (GITMO) study, $25 \%$ of patients with grade I GvHD treated with 6 methylprednisolone (6MPred) $2 \mathrm{mg} / \mathrm{kg}$, progressed to grade II or greater GvHD (Blood 2006).

Materials (or patients) and methods: We hypothesized that treatment of GvHD grade I, with 6 MPred $1 \mathrm{mg} / \mathrm{kg}$ would reduce the risk of progression to grade II $+\mathrm{GvHD}$. The primary end point of the study was acute GvHD grade II. Secondary end points were: transplant related mortality (TRM) and overall survival (OS).

Randomization. 170 patients with grade I GvHD, grafted in 7 GITMO Centers, were randomized between january, 1,2009 and march,23,2014 to a treatment arm $(n=85)$ (6MPred $1 \mathrm{mg} / \mathrm{kg} /$ day i.v. for 5 days, with tapering and discontinuation on day $+30)$ or observation $(n=85)$ (controls). All patients progressing to grade II acute GvHD, were treated with 6MPred $2 \mathrm{mg} /$ $\mathrm{kg} /$ day. 169 patients are evaluable $(n=85$ treatment; $n=84$ controls).

Patients.The two groups -control/treatment - were well balanced in terms of diagnosis $(P=0.9)$, and disease phase $(P=0.4)$ : the most frequent diagnosis was AML $(n=75)$, followed by ALL $(n=38)$ and MDS $(n=10)$. The median age for control/treatment was 46 years (1-69) vs 38 yers $(0,4-68)$, $(P=0.06)$; the proportion of patients over 50 years was $51 \%$ in the control and $49 \%$ in treatment group $(P=0.8)$. The donor type in the control/treatment arms was as follows: HLA identical siblings $n=36 / n=34$, unrelated cord blood (CB) $n=6 / n=2$, unrelated donor (UD) $n=36 / n=42$, and haploidentical family donors (HAPLO) $6 / 7(P=0.4)$.

Skin biopsies.A skin biopsy before randomization, was not mandatory: it was performed in 38 patients. GvHD was diagnosed as proven, probable and possible respectively in 
$21 \%, 32 \%$ and $29 \%$ of the patients.These different reports were equally distributed in treatment and controls $(P=0.7)$.

Results: The cumulative incidence of acute GvHD grade I (primary end point), was 50\% in controls and $35 \%$ in treatment patients $(P=0.02)$. This difference was maintained in different subgroups. The $\mathrm{Cl}$ of transplant related mortality (TRM) was $18 \%$ (control) vs $27 \%$ (treatment) $(P=0.1)$, despite a non significant younger median age in treatment patients. Excess mortality in the treatment arm was due to an excess incidence of infections. Actuarial 1 year survival was $80 \%$ (control) vs $78 \%$ (treatment) $(P=0.1)$. Cases of death in the control/treatment groups were as follows: GvHD $10 \%-13 \%$; infection, $4 \%>8 \%$; interstitial pneumonia, 2\% $-0 \%$; toxicity, $1 \%-2 \%$; leukemia relapse $19 \%-18 \% \quad(P=0.3)$. There was no significant difference in TRM among different Centers $(P=0.5)$

Progression of GvHD and skin biopsies. The proportion of patients progressing to GvHD grade II+ was simlar in proven, probable, possible GvHD $(62 \%, 50 \%, 45 \%)(P=0.7)$. However the proportion of GvHD related deaths was $37 \%$ for proven GvHD, and 8\% for probable/possible GvHD.

Conclusion: This study proves that early treatment of GvHD grade I, reduces the risk of progressing to grade II GvHD. However, there is no beneficial effect on TRM, relapse related death and overall survival. The comparable proportion of GvHD related deaths in the control and treatment arm, confirms that severe GvHD can not be prevented by early steroid treatment, at the onset of the disease. A skin biopsy showing proven GvHD at the onset of skin rash, may predict evolution towards severe GvHD.

Disclosure of Interest: None declared.

\section{0}

Decidual stromal cells as treatment for acute graft versus host disease

M. Solders ${ }^{1,2, *}$, M. Remberger ${ }^{2,3}$, T. Erkers ${ }^{1}$, S. Nava ${ }^{1}$, P. Molldén ${ }^{1}$, J. Mattsson ${ }^{2,3^{\prime}}$ H. Kaipe ${ }^{1,4}$, O. Ringdén ${ }^{1,2}$

${ }^{1}$ Division of Therapeutic Immunology, Karolinska Institutet, ${ }^{2}$ Center for Allogeneic Stem Cell Transplantation, Karolinska University Hospital, ${ }^{3}$ Department of Oncology-Pathology, Karolinska Institutet, ${ }^{4}$ Center for Apheresis and Stem Cell Handling, Karolinska University Hospital, Stockholm, Sweden

Introduction: Graft versus host disease (GvHD) is a common and severe complication after allogeneic stem cell transplantation. During pregnancy, the placenta and the fetal membranes function as an immunological barrier, protecting the fetus from the mother's immune system. We have isolated stromal cells from the decidual layer of term placentas. Decidual stromal cells (DSCs) are of maternal origin and strongly inhibit the alloreactivity of T-cells in vitro. The effect is mainly contact dependent, and decreases the production of several key cytokines involved in the cytokine storm promoting the continuation of GvHD.

Materials (or patients) and methods: To investigate the effect of DSCs on acute GvHD we enrolled 34 patients diagnosed with acute GvHD and clinically non-responsive to standard therapy. The protocol was modified after 17 patients, the DSCs were then thawed and infused in infusion solution with albumin instead of AB-plasma and given repeatedly and earlier upon diagnosis. This led to the formation of two treatment groups (group $1 n=17$ and group $2 n=17$ ), which were compared to matched historical controls $(n=54)$. We also performed a retrospectively corrected analysis of steroid refractivity.

Results: Group 1 received a median of 1 infusion on day 11 after standard treatment compared to group 2, who received a median of 2 infusions $(P=<0,05)$ on day 7 (ns). No adverse events related to the treatment were observed. At 4 weeks after treatment, $60 \%$ of the patients in group 1 hade responded to the treatment. In contrast, all patients in group 2 responded $(P=<0,05)$. All patients in the treatment groups received fungal prophylaxis. The overall cumulative survival (OS) at one year was $28 \%$ for the controls as compared to $47 \%$ for group 1 and $80 \%$ for group $2(P=<0.001)$. When the groups were corrected for steroid-refractivity, the OS was $3 \%$ for the controls, 38\% for group 1 and $70 \%$ for group $2(P=<0.001)$. In the steroid-refractory control group $(n=32)$, the risk of dying from GvHD at one year was $81 \%$, whereas no patients in the steroid-refractory group 2 died from $\operatorname{GvHD}(n=13, P=<0.001)$.

In the last 19 months, no patients have died from acute GvHD at our center.

Conclusion: In conclusion, DSCs might be an effective treatment of acute GvHD. The infusion should be prepared in albumin, given as early as possible and in repeated doses. To confirm these striking findings, we will extend the followup time and enroll more patients in our study.

Disclosure of Interest: None declared.

\section{Oral session: Stem cell source and donor type}

\section{1}

Unmanipulated haploidentical stem cell transplantation after reduced intensity or ablative conditioning regimen for the treatment of acute leukemia- a report from the acute leukemia working party of the EBMT

M. T. Rubio ${ }^{1,2, *}$, B. N. Savani, ${ }^{3,4}$, M. Labopin ${ }^{5}$, E. Polge ${ }^{6}$, F. Ciceri ${ }^{7}$, A. Bacigalupo ${ }^{8}$, W. Arcese ${ }^{9}, Y$. Koc ${ }^{10}$, D. Beelen ${ }^{11}$, Z. Gülbas ${ }^{12}$, D. Wu ${ }^{13}$, P. Di Bartolomeo ${ }^{14}$, J. Tischer ${ }^{15}$, B. Afanasyev ${ }^{16}$, C. Schmid ${ }^{17}$, S. Giebel $^{18}$, M. Mohty ${ }^{2,3,19}$, A. Nagler ${ }^{3,20}$ on behalf of Acute Leukemia Working Party of EBMT

${ }^{1}$ Service d'Hématologie et Thérapie cellulaire, HOPITAL SAINT ANTOINE, PARIS CEDEX 12, ${ }^{2}$ INSERM UMR 938, Université Pierre et Marie Curie, ${ }^{3}$ Acute Leukemia Working Party of EBMT, EBMT Paris study office, Paris, France, ${ }^{4}$ Hematology, Vanderbilt University Medical Center, Nashville, TN, United States, ${ }^{5}$ EBMT Paris study office/CEREST-TC, Paris, France, ${ }^{6} E B M T$ Paris study office / CEREST-TC, Saint Antoine Hospital, Paris, France, ${ }^{7}$ Hematology, Ospedale San Raffaele s.r.l., Milano, ${ }^{8}$ Haematology II, Ospedale San Martino, Genova, ${ }^{9}$ Stem Cell Transplant Unit, Policlinico Universitario Tor Vergata, Rome, Italy, ${ }^{10}$ Stem Cell Transplant Unit, Medical Park Hospitals, Antalya, Turkey, ${ }^{11}$ Dept. of Bone Marrow Transplantation, University Hospital, Essen, Germany, ${ }^{12}$ Bone Marrow Transplantation, Anadolu Medical Center Hospital, Kocaeli, Turkey, ${ }^{13}$ Hematology, First Affiliated Hospital of Soochow University, Suzhou, China, ${ }^{14}$ Hematology, Ospedale Civile, Pescara, Italy, ${ }^{15}$ Hematology, Klinikum Grosshadern, Munich, Germany, ${ }^{16}$ Hematology, SPb State I. Pavlov Medical University, St. Petersburg, Russian Federation, ${ }^{17}$ Klinikum Augsburg, University of Munich, Munich, Germany, ${ }^{18}$ Bone Marrow Transplantation and Hemato-Oncology, Cancer Center, Gliwice, Poland, ${ }^{19}$ Service d'Hématologie et Thérapie cellulaire, Hopital Saint Antoine, Paris, France, ${ }^{20}$ Hematology, Chaim Sheba Medical Center, Tel Hashomer, Israel

Introduction: Increasing numbers of patients are receiving reduced intensity conditioning (RIC) allogeneic hematopoietic stem cell transplantation (allo-SCT). At the same time, the numbers of patients receiving alternative donor allo-SCT using related haploidentical SCT (haplo-SCT) are rapidly rising. RIC regimen is reported to be associated with equivalent outcome compared to ablative regimens (MAC) but it is not known if this applies to haplo-SCT. We therefore performed a retrospective analysis within the EBMT registry to evaluate the impact of conditioning intensity on haplo-SCT outcomes for acute leukemia.

Materials (or patients) and methods: Adult patients who underwent MAC or RIC T-replete haploidentical peripheral blood (PB) or bone marrow (BM) transplantation from 2001 to 2012 were included in the study. All donors were Human Leucocyte Antigens (HLA) mismatched at least at two loci $(\leq 8 / 10)(-A,-B,-C, D R B 1,-D Q B 1)$. Regimens were classified as 
MAC or RIC based on published criteria. The Kaplan-Meier estimator, the cumulative incidence function and Cox proportional hazards regression models were used where appropriate.

Results: 425 patients receiving MAC were compared with 271 RIC followed by haplo-SCT. Median year of MAC haplo-SCT was 2011 vs. 2010 for RIC cohorts. Median follow-up of surviving patients was 22 months. Groups differed by many variables. MAC recipients were significantly younger (median age 38 vs. 53 years; $P<0.0001$ ). Significantly higher numbers of patients had AML in the RIC group ( $80 \%$ vs. $67 \%$ ) and more MAC recipients were documented in ALL cohorts (33 vs. 20\%). There were more patients in CR1 in MAC group (41 vs. 29\%) and higher numbers of patients with active disease in RIC compared to MAC groups (47 vs. $31 \% ; P<0.0001$ ). Majority of patients in RIC group had PB (65\%) compared to similar distribution of $\mathrm{PB}$ and $\mathrm{BM}$ as stem cell source in MAC cohort. $125(32 \%)$ patients in MAC and $66(25 \%)$ in RIC groups received post-transplant cyclophosphamide (PT-CY) based GVHD prophylaxis. Percentage of engraftment (92 vs. 90\%; $P=0.58$ ) and day 100 grade II to IV acute GVHD (29 vs. 24\%) were not different between MAC and RIC groups. In CR1 patients, 2 year LFS in MAC and RIC groups were $49.1 \%$ and $38.9 \%(P=0.42)$ and 2 year OS were $55.4 \%$ and $46.9 \%$ $(P=0.54)$, respectively.

In multivariate analysis, there was a trend towards higher RI in RIC vs. MAC (HR 1.31, $P=0.07$ ) but no differences in NRM (HR $0.95, P=0.76)$, chronic GVHD (HR 0.80, $P=0.21$ ), LFS (HR 1.13, $P=0.27)$ and OS (HR 1.09, $P=0.44)$. Patients in CR2 and with active disease had poor OS and LFS vs. CR1 (HR 1.40, $P=0.01$ and 2.92, $P<0.0001$, respectively); (HR 1.30, $P=0.05$ and 2.94, $P<0.0001$, respectively). Adjusted outcome of ALL was inferior compared to AML (RI 1.39, $P=0.04$, LFS 1.28, $P=0.03$; OS HR $1.3, P=0.03)$. PT-CY based GVHD prophylaxis was independently associated with decreased NRM (HR 0.63, $P=0.02)$ and chronic GVHD was higher with PB graft (HR 1.64, $P=0.005)$. There was no difference in RI between PT-CY and other GVHD prophylaxis regimens.

Conclusion: Despite the need for randomized trials, we conclude that unmanipulated RIC haplo-SCT results in similar outcomes in comparison to MAC haplo-SCT in patients with AL. PT-CY provides a safer transplant platform with lower NRM and similar RI compared to other GVHD prophylaxis regimens. Disclosure of Interest: None declared.
0042

Haploidentical hematopoietic stem cell transplantation in children and adolescents with acute leukemia in progressive disease: MAC vs RIC, PTCY vs conventional prophylaxis of aGVHD, T-cell depleted vs T-cell replete haplo-grafts

O. V. Paina ${ }^{1, *}$, N. Stancheva ${ }^{1}$, E. Semenova ${ }^{1}$, E. Babenko ${ }^{1}$, A. Borovkova', S. Razumova , P. Kozhokar ${ }^{7}$, K. Ekushov', L. Zubarovskaya ${ }^{1}$, B. Afanasyev

${ }^{1}$ R.M.Memorial Institute of children Oncology, Hematology and Transplantation, I.P.Pavlov 1st St.Petersburg State Medical University, St.Petersburg, Russian Federation

Introduction: Haploidentical hematopoietic stem cell transplantation(Haplo-HSCT)is feasible option for patients with acute leukemia(AL)at high risk of relapse who do not have HLA-matched related or unrelated donors. Haplo-HSCT was associated with severe acute graft-versus-host disease (aGVHD) in unmanipulated transplants and a high incidence of graft rejection in T-cell depleted transplants because of the high frequency of T cells that recognized major class I or II HLA disparities between donor and recipient. Two approaches were developed to overcome these problems:megadose of T-cell depleted hematopoietic progenitor cells without any posttransplant immunosuppression and T-cell replete grafts with innovative pharmacological prophylaxis of aGVHD.Posttransplant cyclophosphamide(PTCY)is regarded as a GVHDspecific immunosuppressant in adults but its feasibility is unknown in children. Purpose: to evaluate the feasibility and outcome of haplo-HSCT in children and adolescents with acute leukemia in active disease depending on conditioning regimens and methods of harvesting haplo-graft. Primary end points: overall survival (OS), transplant-related mortality (TRM). Secondary end points: engraftment rate, aGVHD, cGVHD, relapse rate.

Materials (or patients) and methods: 56 patients(range from 1-21y.o. median 9 y.o.)with AL(ALL-32pts, AML-24pts)in progressive disease (PD)(cytoreduction chemotherapy (CTx) prior conditioning regimen-15pts, without-41pts) were analysed.MAC + ATG regimen based on GIAC protocol received 20pts, MAC + PTCY $50 \mathrm{mg} / \mathrm{kg}$ on $\mathrm{D}+3,+4-5 \mathrm{pts}$, $\mathrm{RIC}+$ ATG regimen based on Flu-18pts, RIC + PTCY $50 \mathrm{mg} / \mathrm{kg}$ on $\mathrm{D}+3,+4-13$ pts. All pts received prophylaxis of aGVHD based on CsA-30pts, Tac-8pts, Tac + Sir-18pts. G-CSF-primed T-cell replete BM was used as a graft source in 33pts (median CD34 ${ }^{+}$cells $4,7 \times 10^{6} / \mathrm{kg}$ ), G-CSF mobilized peripheral blood $\left(\mathrm{CD} 34^{+}\right.$selected by CliniMACS, Miltenyi Biotec) and G-CSFprimed BM-23pts (median CD34 ${ }^{+}$cells $11,3 \times 10^{6} / \mathrm{kg}$ ).

[0042]

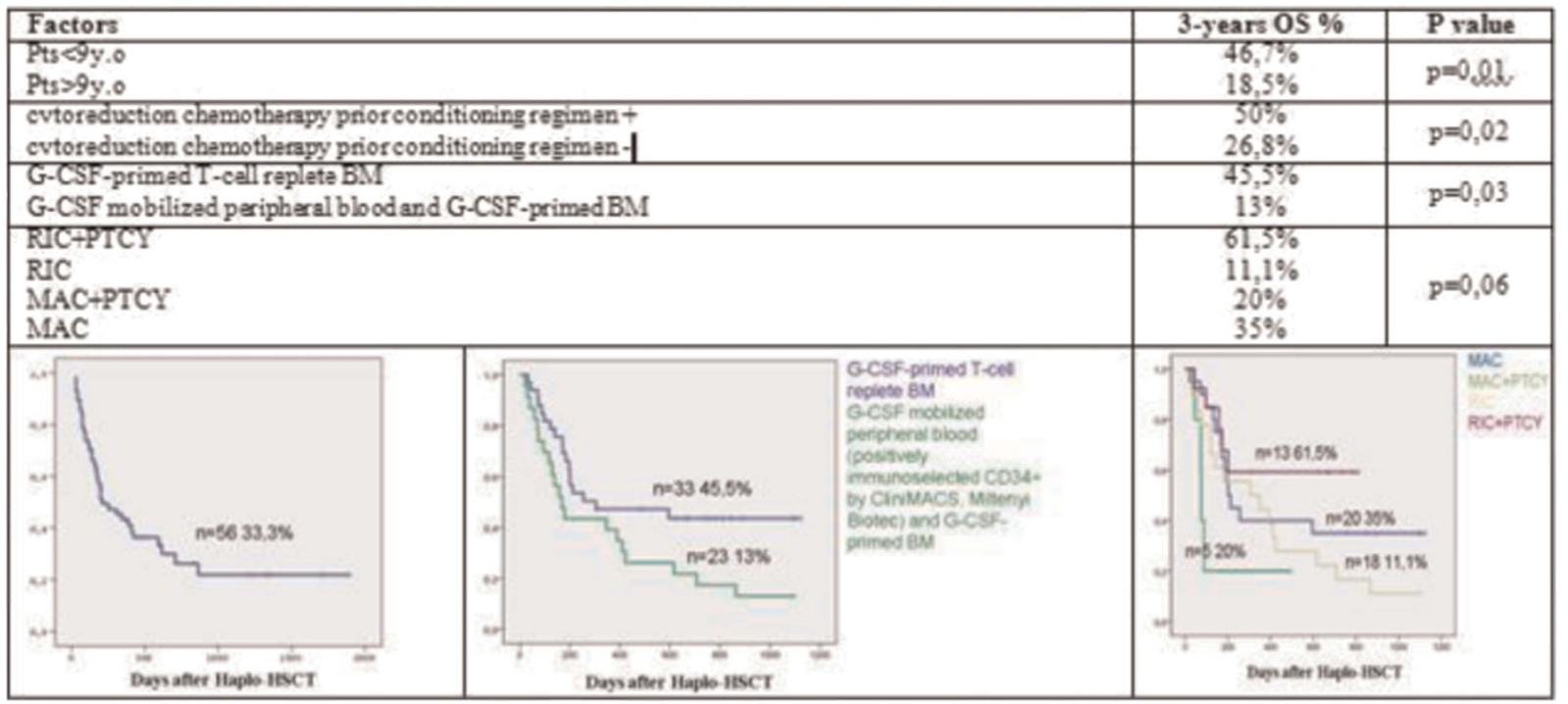


Results: 3-year OS was 33,3\%. Pts $<9 y .0$. had significantly higher OS vs pts $>9 y .0 .46,7 \%$ and $18,5 \%$ respectively $(P=0,01)$. 3-year OS in pts receiving CTx prior conditioning regimen was $50 \%$ vs $26,8 \%$ in pts with leukemic burden $(P=0,02)$. Significantly difference were observed in 3year OS in pts transplanted G-CSF-primed T-cell replete BM $45,5 \%$ vs $13 \%$ in pts after G-CSF mobilized peripheral blood and G-CSF-primed BM $(P=0,03)$. Pts receiving RIC + PTCY had 3-year OS 61,5\%, RIC-11,1\%, MAC-35\%, MAC+PTCY-20\% $(P=0,06)$. TRM after haplo-HSCT in pts with AL in PD was $38 \%$. Engraftment was sustained in $82,1 \%$ pts. Full donor chimerism was achieved in $73,2 \%$ pts on D +30 . Median ANC engraftment $\left(>0,5 \times 10^{9} / \mathrm{L}\right) \quad \mathrm{D}+19, \mathrm{PLT}$ recovery $\left(>20 \times 10^{9} / \mathrm{L}\right)$ $\mathrm{D}+17$. Cumulative incidence of grade $2-4$ aGVHD was $27,3 \%$, cGVHD-23,9\%. Cumulative incidence of relapse was $39,3 \%$.

Conclusion: Haplo-HSCT G-CSF-primed unmanipulated BM is an effective method of achieving remission with good sustained engraftment rate in children and adolescents with resistant disease. RIC regimen followed by T-cell replete haploHSCT with PTCY on D $+3,+4$ was associated with good OS, low incidences of GVHD and TRM. Before any definitive conclusions can drawn, a randomized study is required.

Disclosure of Interest: None declared.

\section{3}

The detection of donor specific anti-HLA antibodies in recipients of unmanipulated haploidentical blood and marrow transplantation is predictive of poor graft function Y.-J. Chang ${ }^{1, *}$, M.-R. Huo ${ }^{1}$, X.-S. Zhao ${ }^{1}$, X.-Y. Zhao ${ }^{1}$, G.-L. He ${ }^{1}$,

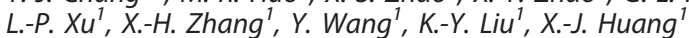
${ }^{1}$ Peking University People's Hospital \& Peking University Institute of Hematology, Beijing, China

Introduction: Our previous study suggest that choosing young, male, non-inherited maternal antigen-mismatched donors is reasonable following unmanipulated haploidentical blood and marrow transplantation (HBMT). Recently, a correlation between the presence of donor-specific anti-HLA antibodies (DSA) and graft failure has been demonstrated in haploidentical transplant settings. In our protocol, approximately $99 \%$ patients can achieve sustained, full donor chimerism. However, poor graft function (PGF) remains one of complications after unmanipulated HBMT. Therefore, we determined the effect of DSA on primary PGF in order to provide further evidence for donor selection.

Materials (or patients) and methods: Three hundreds and fourty-five patients with hematological diseases receiving HBMT were enrolled in this prospective study. The median age of the patients was 26 years (range, 2-58 years). These patients were randomly selected as training group $(n=173)$ and validation group $(n=172)$. Peripheral blood serum were collected pre-conditioning regimens. DSA were determined using the Luminex-based assay.

Results: In all 345 patients, the percentages of DSA positive cases were $11.3 \%$ (39/345). The incidence of DSA in female patients was higher than that of male cases $(16 \%$ vs. $8 \%$, $P=0.020)$. In the training set, a cutoff value of DSA $(\mathrm{MFI}=2000)$ were developed. Multivariate analysis showed that the presence of DSA (patients with $\mathrm{MFI} \geq 2000$ vs. cases with $\mathrm{MFI} \geq 2000$ ) was associated with primary PGF. In the validation set, the association of DSA with primary PGF following transplantation was also confirmed. The association of PGF with inferior overall survival (OS) was demonstrated both in the training group and in the validation group. In all 345 patients, the median time to platelet recovery in DSA positive $(\mathrm{MFI} \geq 2000)$ patients was slower than that of DSA negative ones ( 25 days vs. 18 days, $P=0.004$ ). The incidence of primary PGF and primary graft failure was $5.5 \%$ and $0.9 \%$, respectively. DSA positive patients experenced higher incidence of primary PGF (31\% vs. $3.2 \%, P=0.000)$ and primary graft failure $(10.3 \%$ vs. $0 \%, P=0.000)$ than those of DSA negative cases. Multivariate analysis confirmed the association of DSA with primary PGF (HR 10.186, 95\% Cl: 4.136-20.085, $P=0.000$ ) in all 345 patients. However, the multivariate analysis can not be done to correlate DSA with primary graft failure due to the low incidence $(0.9 \%)$, although univariate analysis showed an association. After a median follow-up of 384 days (range, 25-784 days), the 1 year cumulative incidences of relapse, TRM, DFS, and OS were $8.3 \% \pm 2.4 \%$, $11.8 \pm 2.6 \%, 79.8 \% \pm 3.3 \%$, and $81.3 \% \pm 3.3 \%$, respectively. Multivariate analysis showed that PGF was an independent variable and associated with higher incidence of TRM (HR $6.000,95 \% \mathrm{Cl}: 2.868-12.551, P=0.000$ ) and inferior DFS (HR 3.125, 95\% Cl: 1.564-6.244, $P=0.000$ ) and OS (HR 3.634, 95\% Cl: $1.808-7.302, P=0.000)$.

Conclusion: We for the first time demonstrated that DSA might contribute to the primary PGF after unmanipulated HBMT. The onset of PGF leads to inferior survival. Our results add new evidence that suggest DSA must be considered when choosing among several haploidentical donor sources.

References: 1. Curr Opin Hematol, 2012,19:454.

2. Blood, 2014,124:843.

3. Bone Marrow Transplant, 2012,47:508.

4. Biol Blood Marrow Transplant, 2013, 19:1465.

5. 2013,119(5):978-985

Disclosure of Interest: None declared.

0044

Mother donors confer protection against infectious

mortality after haploidentical $\mathrm{T}$ cell-depleted hematopoietic transplantation

L. Ruggeri ${ }^{1, *}$, A. Tosti ${ }^{1}$, A. Mancusi ${ }^{1}$, E. Urbani ${ }^{1}$, F. Topini ${ }^{1}$, A. Carotti ${ }^{7}$, M. F. Martelli ${ }^{1}$, A. Velardi ${ }^{i}$

${ }^{1}$ University of Perugia, Perugia, Italy

Introduction: Transplacental trafficking of maternal and fetal cells during pregnancy establishes long-term, reciprocal microchimerism in both mother and child because of exposure of the two immune systems to the non-self alloantigens (Maloney et al., J Clin Invest. 1999). Studies show the immune system in the mother is capable of being sensitized by paternal histocompatibility antigens. For example, antibodies directed against paternal HLA-antigens (van Rood et al., Nature. 1958) and T lymphocytes directed against paternal major (van Kampen et al., Hum Immunol. 2001) and minor histocompatibility antigens (Verdijk et al., Blood. 2004) are frequently detected in multiparous women. We previously demonstrated mother/child immune interactions positively influenced the outcome of mother to child HLA haploidentical $T$ cell-depleted hematopoietic transplantation. In a series of adult and pediatric patients we demonstrated mother donors conferred protection against leukemia relapse and improved transplant related mortality (TRM), which was largely due to infection, and improved survival (Stern et al., Blood 2008).

Materials (or patients) and methods: The Kaplan-Meier method evaluated leukemia-free survival. Cumulative incidence estimates were used for relapse and TRM, as they are competing risks. Multivariate analysis assessed the impact of diverse variables on transplantation outcomes.

Results: We analyzed the outcomes of 238 adult acute leukemia patients after $\mathrm{T}$ cell-depleted haploidentical transplantation. When compared with transplantation from all other family members, transplantation from mother donors was associated with significantly lower TRM (largely infectious) (27\% vs $50 \%$ from all other donors, $P=0.01$ ). Multivariate analyses demonstrated transplantation from mother donors was an independent factor predicting improved survival (hazard ratio $0.41,95 \%$ confidence interval 0.12 to $0.95, P=0.03$ ). In an attempt to elucidate the mechanism, we analyzed donor $T$ cell repertoires that were specific for CMV antigens presented by recipient antigen-presenting cells (by ELISPOT and by limiting dilution cloning). Unlike all other donor/recipient pairs, mothers possessed CMV-specific CD8 cell clones that killed child's and father's CMV-pulsed dendritic cells (DCs). Such clones were nonalloreactive as they did not kill the child's or father's non-CMV- 
pulsed DCs. Mothers also possessed CD4 T cell clones that produced IFN-gamma in response to child's and father's CMVloaded DCs. Such clones were non-alloreactive as they did not respond to child's or father's non-CMV-pulsed APCs. Thus, mothers possessed a $T$ cell repertoire that recognized CMV antigens also when presented by the unshared, father's, HLA haplotype. In fact, they showed twice as many T cells that recognized CMV antigens presented by the child's APCs than all other donor/recipient pairs $(P<0.05)$

Conclusion: Therefore, pregnancy resulted in the generation of an additional $\mathrm{T}$ cell repertoire that specifically recognized pathogen antigens presented by the unshared paternal HLA haplotype antigens on the child's APCs. Apparently, upon mother to child T cell-depleted hematopoietic transplantation, such repertoire expands over time and helps reduce infectious mortality. Further studies are needed to elucidate the mechanisms underlying mother $\mathrm{T}$ cell selection/education by paternal HLA haplotype antigens on the child's APCs.

Disclosure of Interest: None declared.

0045

Uni-directional and bi-directional non-permissive HLADPB1 T cell epitope group mismatches have similar risk associations in 10/10 matched unrelated donor HCT K. Fleischhauer, ${ }^{1, *}$, K. W. Ahn ${ }^{2}$, H.-L. Wang ${ }^{2}$, L. Zito ${ }^{3}$, C. Mueller ${ }^{4}$, M. Verneris ${ }^{5}$, J. Pidala ${ }^{6}$, M. Oudshoorn ${ }^{7}$, S. Lee ${ }^{8}$, S. Spellman ${ }^{9}$ on behalf of CIBMTR Immunobiology Working Committee ${ }^{1}$ Institute for Experimental Cellular Therapy, University Hospital Essen, Essen, Germany, ${ }^{2}$ Center for International Blood and Marrow Transplant Research, Medical College of Wisconsin, Milwaukee, United States, 3 Unit of Molecular and Functional Immunogenetics, San Raffaele Hospital, Milan, Italy, ${ }^{4}$ ZKRDZentrales Knochenmarkspender-Register Deutschland, UIm, Germany, ${ }^{5}$ Pediatric Blood and Marrow Transplantation, University of Minnesota, Minneapolis, ${ }^{6}$ Blood and Marrow Transplantation, $H$. Lee Moffitt Cancer Center and Research Institute, Tampa, United States, ${ }^{7}$ Europdonor Foundation, Leiden University Medical Center, Leiden, Netherlands, ${ }^{8}$ Clinical Transplant Research, Fred Hutchinson Cancer Research Center, Seattle, Immunobiology and Observational Research, Center for International Blood and Marrow Transplant Research, Minneapolis, United States

Introduction: In 8/8 HLA-matched unrelated HCT, nonpermissive HLA-DPB1 mismatches across three different $T$ cell epitope (TCE) groups are associated with significantly higher risks of mortality compared to permissive HLA-DPB1 mismatches within the same TCE group 1, 2 however, the relevance of mismatch directionality (host versus graft [unidirectional HvG], graft versus host [uni-directional $\mathrm{GvH}$ ] or both [bi-directional]) in the non-permissive setting is unknown.

Materials (or patients) and methods: A cohort of 3281 patients who underwent myeloablative $\mathrm{HCT}$ for $\mathrm{AML}, \mathrm{ALL}, \mathrm{CML}$ or MDS from an 8/8 HLA-matched unrelated donor for which we previously demonstrated a significant impact of nonpermissive HLA-DPB1 donor-recipient disparity on the risk of mortality (Pidala et al, Blood 2014), was analyzed after separating uni-directional from bi-directional non-permissive mismatches. Non-permissive mismatches were defined as unidirectional HvG when the donor but not the patient carried an HLA-DPB1 allele from a TCE group not present in the patient, and vice versa as uni-directional $\mathrm{GvH}$. Bi-directional nonpermissive mismatches were present when none of the HLADPB1 alleles in patient and donor were from the same TCE group. The associations with clinical endpoints of overall survival (OS), transplant related mortality (TRM), relapse, acute GvHD (aGvHD) and chronic GvHD (cGvHD) were studied using multivariate proportional hazards methods.

Results: The number of transplants with permissive or nonpermissive uni-directional $\mathrm{HvG}$, uni-directional $\mathrm{GvH}$ and bidirectional mismatches was 1537, 527, 529 and 143, respectively. In the TRM analysis, non-permissive uni-directional HvG
(HR 1.32, $P=0.001)$ and GvH mismatches (HR 1.28, $P=0.005)$ had significantly higher relative risks (RR) of TRM compared to the permissive group. The bi-directional group had similar RR (HR 1.35, $P=0.05$ ). In pairwise comparisons, there were no statistical differences between the uni- and the bi-directional non-permissive groups for any of the outcomes tested.

Conclusion: Bi-directional non-permissive HLA-DPB1 mismatches carry similar risks of adverse clinical events including TRM and OS in comparison to uni-directional mismatches. Based on these data, consideration of directionality does not improve the risk stratification defined by non-permissive HLADPB1 mismatches.

References: 1. Fleischhauer et al., Effect of T-cell-epitope matching at HLA-DPB1 in recipients of unrelated-donor haemopoietic-cell transplantation: a retrospective study. Lancet Oncol. 2012 Feb 14.

2. Pidala et al., Non-permissive -DPB1 mismatch among otherwise HLA-matched donor-recipient pairs results in increased overall mortality after myeloablative unrelated allogeneic hematopoietic cell transplantation for hematologic malignancies. Blood. 2014 Aug 26.

Disclosure of Interest: None declared.

\section{6}

Unmanipulated haploidentical transplantation is a valid alternative to identical-sibling donor transplant as post-remission treatment for intermediate- or high-risk acute myeloid leukemia in first complete remission: a multi-centre, prospective study

Y. Wang ${ }^{1, *}$, Q.-F. liu', L.-P. $x u^{1}$, D.-P. $w u^{3}$, X.-J. huang

${ }^{1}$ Peking University People's Hospital, Peking University Institute of Hematology, beijing, ${ }^{2}$ Nanfang Hospital, Southern Medical University, guangzhou, ${ }^{3}$ The first affiliated hospital of Soochow University, suzhou, China

Introduction: The effects of HLA-identical sibling donor (ISDs) hematopoietic stem cell transplantation (HSCT) on adults with intermediate- or high-risk acute myeloid leukemia (AML) in first complete remission (CR1) are well established. Previous single-center studies demonstrated similar survival after unmanipulated haploidentical donor (HIDs) vs ISDs HSCT for hematological malignancies. Therefore, we hypothesized that haploidentical HSCT would be a valid alternative as postremission therapy for AML patients in CR1 lacking a matched donor.

Materials (or patients) and methods: We conducted a disease-specific, prospective, multi-center study. Between July, 2010 and Nov, 2013, we enrolled a total of 450 patients at 3 centers. Patients were assigned to undergo HIDs (231 patients) or ISDs HSCT (219 patients) according to donor availability. The primary end point was 1-year disease free survival (DFS).

Results: The 1-year DFS rate was $84 \%$ and $87 \%$ among recipients from HIDs and ISDs $(P=0.37)$. For patients undergoing HIDs and ISD transplantation, the 1-year probabilities of overall survival were $86 \%$ and $89 \%$ ( $P=0.34$ ); the 1-year cumulative incidences of relapse were $8 \%$ and $8 \%(P=0.99)$ and of non-relapse mortality were $8 \%$ and $5 \%(P=0.20)$, respectively. Multivariate analysis showed that DFS correlated significantly with cytogenetic risk category $(P=.015)$, WBC count at diagnosis $(P=.042)$, grades III to IV aGVHD $(P=.043)$ and extensive chronic GVHD $(\mathrm{P}<.001)$.

Conclusion: Unmanipulated haploidentical transplantation achieves outcomes similar to those of contemporaneous transplantation performed using ISDs for AML patients in CR1. Such transplantation was proved to be a valid alternative option as post-remission treatment for intermediate- or highrisk $A M L$ patients in CR1 who lack an identical donor. (Chictr.org number ChiCTR-OCH-10000940)

References: 1 . Huang XJ, et al. The superiority of haploidentical related stem cell transplantation to chemotherapy alone as postremission treatment for patients with intermediate- or high- risk acute myeloid leukemia in first complete remission. Blood 2012; 119:5584-90. 


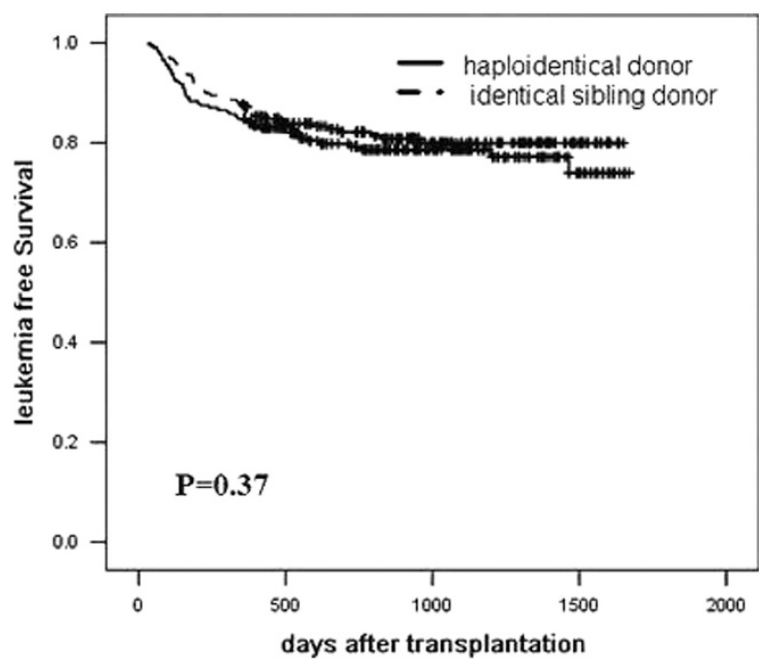

2. Wang $\mathrm{Y}$, et al. Long-term follow-up of haploidentical hematopoietic stem cell transplantation without in-vitro T-cell-depletion for the treatment of leukemia: nine years of experience at a single center. Cancer 2013;119: 978-985.

3. Basara N, et al. Early related or unrelated haematopoietic cell transplantation results in higher overall survival and leukaemia-free survival compared with conventional chemotherapy in high-risk acute myeloid leukaemia patients in first complete remission. Leukemia 2009:23:635-640.

Disclosure of Interest: None declared.

\section{7}

Influence of HLA-matching after reduced intensity conditioning regimen and unrelated donor allo-SCT for acute leukemia in patients older than $\mathbf{5 0}$ years - a report from the acute leukemia working party of the EBMT B. Savani ${ }^{1, *}$, M. Labopin ${ }^{2,3,4,5}$, E. Polge ${ }^{2}$, D. Niederwieser ${ }^{6}$, A. Ganser,' G. Ehninger ${ }^{8}$, M. Bornhäuser ${ }^{8}$, A. Renate, , J.'Finke ${ }^{10}$ N. Kröger ${ }^{11}$, C. Craddock ${ }^{12}$, R. Schwerdtfeger ${ }^{13}$, M. Hallek ${ }^{14}$, P. Jindra ${ }^{15}$, M. Mohty 2,3,4,5, A. Nagler 2,16

${ }^{1}$ VANDERBILT UNIVERSITY, Nashville, United States, ${ }^{2}$ EBMT Paris study office / CEREST-TC, ${ }^{3}$ Department of Haematology, Saint Antoine Hospital, ${ }^{4}$ INSERM UMR 938, ${ }^{5}$ Université Pierre et Marie Curie, Paris, France, ${ }^{6}$ UniversityHospital Leipzig - Div. Hematology, Oncology and Hemostasiology, Leipzig, 'Hannover Medical School - Department of Haematology, Hemostasis, Oncology and Stem Cell Transplantation, Hannover, ${ }^{8}$ UniversitaetsklinikumDresden - MedizinischeKlinikundPoliklinik I, Dresden, ${ }^{9}$ Charité Universitätsmedizin Berlin - Campus Virchow Klinikum - MedizinischeKlinik m. S. Hämatologie/Onkologie, Berlin, ${ }^{10}$ University of Freiburg - Dept. of Medicine -Hematology, Oncology, Freiburg, ${ }^{11}$ UniversityHospitalEppendorf - Department of Stem cell Transplantation, Hamburg, Germany, ${ }^{12}$ Centre for Clinical Haematology - Queen Elizabeth Hospital, Birmingham, United Kingdom, ${ }^{13}$ Deutsche KlinikfürDiagnostik - KMT Zentrum, Wiesbaden, ${ }^{14}$ University of Cologne - I. Dept. ofMedicine, Cologne, Germany, ${ }^{15}$ Charles University Hospital - Dept. of Hematology/Oncology, Pilsen, Czech Republic, ${ }^{16}$ Hematology Division, Chaim Sheba Medical Center, Tel Hashomer, Israel

Introduction: There are several alternative sources of donor stem cells available for patients (pts) who need an allo-SCT and especially for those who lack a HLA- matched donor. Outcomes of mismatched-unrelated-donor (MM-URD) transplant have recently improved, and a comparison between matched and MM-URD sources in a uniform cohort of pts has not been performed after RIC regimens.

Materials (or patients) and methods: Pts, aged $\geq 50$ year, who underwent fully matched or MM-URD RIC PBSCT or BMT from 2000-2012 were included in the study. All donors were HLA-matched $(10 / 10)$ or mismatched at one or two-loci $(9 / 10$ or $8 / 10)$. The Kaplan-Meier-estimator, the cumulative incidence function and Cox proportional hazards regression models were used where appropriate.

Results: In total 3197 pts receiving matched or mismatched RIC-URD allo-SCT were included in the study (AML 2947, ALL 250). $237010 / 10 \mathrm{HLA}$-matched pts were compared with recipients receiving $9 / 10(n=712)$ or $8 / 10(n=115)$ after RIC MM-URD allo-SCT. Median age of 10/10, 9/10 and 8/10 HLAmatched recipients were 60 years. Higher female donor to male recipients were in $8 / 10$ cohorts (20\%) compared to $10 / 10$ (12\%) and 9/10 (14\%) group $(P=0.02)$. More pts with CR2 and advanced-disease were among $9 / 10$ and $8 / 10$ cohort compared to 10/10 matched donor recipients (CR2 24, 24 and 20\%; advanced disease $26,25,24 \%$ respectively; $P=0.04$ ). Also higher percentage of pts with secondary leukemia were in $8 / 10$ cohorts compared $10 / 10$ and $9 / 10$ matched donor $(35,27,25 \% ; P=0.07)$. Percentages of engraftment $(97 \%$, $95 \%, 97 \%, P=0.16)$ were no different between the 3 groups. Acute GVHD grade II-IV was 27\%, 33\%, 33\%, and grade III-IV 10,13 , and $10 \%$, respectively for $10 / 10,9 / 10$ and $8 / 10$ matched donors, respectively $(P=0.003$ and 0.03$)$. In univariate analysis, 2-year survival rate was significantly higher for pts receiving 10/10 donor RIC-URD allo-SCT in CR1 compared to $9 / 10$ or $8 / 10$ MM-URD (OS: $55 \%, 46 \%, 46 \%$, $P=0.01$; LFS: $51 \%, 43 \%, 45 \%, P=0.03$, respectively). However, among the CR2 and advanced disease groups there were no differences in outcome between fully matched or MM-URD (9/ 10 or $8 / 10$ ) donor (CR2 + : OS $49 \%, 43 \%, 48 \%, P=0.30$; LFS $42 \%, 37 \%, 45 \%, P=0.36$; advanced-disease: OS $38 \%, 31 \%$, $31 \%, P=0.39$; LFS $32 \%, 26 \%, 28 \%, P=0.50$, respectively). There was no difference in RI between the 3 groups and NRM was higher after fully matched donor compared to MM-URD $(9 / 10$ or $8 / 10)$ only in pts with CR1 diseases $(P=0.02)$. Multivariate analysis showed higher NRM after 9/10 (HR 1.33, $P=0.001$ ) compared to fully matched donor and no difference in NRM between $9 / 10$ vs. $8 / 10$ MM-URD. There was no difference in RI between $10 / 10$ vs. $9 / 10$ or $9 / 10$ vs. $8 / 10$ MMURD donor. OS and LFS were superior after fully matched donor vs. 9/10 MM-URD (OS: HR 1.24, $P=0.001$; LFS: HR 1.19, $P=0.002)$. However, there was no difference in adjusted OS and LFS between $9 / 10$ vs. 8/10 MM-URD RIC allo-SCT. Chronic GVHD rate was not different between matched or MM-URD allo-SCT groups.

Conclusion: Despite the limitations of a retrospective registrybased study, our analysis shows no significant outcome difference between $9 / 10$ and 8/10 MM-URD allo-SCT after $\mathrm{RIC}$ regimen in patient aged $\geq 50$ year. In the absence of prospective data, we conclude that MM-URD RIC allo-SCT is a therapeutic option for acute leukemia pts not having fully matched donor.

Disclosure of Interest: None declared.

\section{8}

\section{Health-related Quality of Life among Pediatric} Hematopoietic Stem Cell Donors

G. E. Switzer,", J. Bruce , D. M. Kiefer ${ }^{2}$, H. Kobusingye ${ }^{2}$, R. J. Drexler ${ }^{2}$, R. M. Besser ${ }^{2}$, R. J. King ${ }^{2}$, M. M. Horowitz ${ }^{3}$, D. L. Confer', J. W. Varni ${ }^{4}$, M. A. Pulsipher

${ }^{1}$ University of Pittsburgh, Pittsburgh, ${ }^{2}$ CIBMTR (Center for International Blood and Marrow Transplant Research), National Marrow Donor Program/Be The Match, Minneapolis, ${ }^{3}$ CIBMTR (Center for International Blood and Marrow Transplant Research), Medical College of Wisconsin, Milwaukee, ${ }^{4}$ Center for Health Systems \& Design, Department of Pediatrics, College of Medicine, Texas A\&M University, College Station, ${ }^{5}$ Primary Children's Hospital, Division of Hematology/Hematological Malignancies, Huntsman Cancer Institute/University of Utah, Salt Lake City, United States

Introduction: The use of minors as HSC donors is medically and legally accepted and is increasing. However, there is a lack 
of understanding of the physical and psychosocial effects of pediatric HSC donation. The goal of this investigation was to longitudinally investigate $\mathrm{HRQ}$ oL in this group.

Materials (or patients) and methods: Participants were related pediatric donors $(n=105)$ who donated at domestic U.S. centers between $4 / 10$ and 5/13. Data were collected from donors and their parents via structured telephone interviews at pre-donation, and 4 weeks and 1 year post-donation. A healthy age/gender matched pediatric sample was generated from existing data for normative comparisons. Interviews gathered socio-demographics, psychosocial characteristics, and multidimensional HRQoL using the well-validated Pediatric Quality of Life Inventory (PedsQL) which produces a total score and physical, emotional, social, school and psychosocial subscores. T-tests were used to compare HRQoL from donor self-report, parental proxy-report, and the normative sample across the three assessment points. Mixed logistic models were used to examine the effects of pre-donation variables on post-donation HRQoL.

Results: Donors were 5-17 yrs (median $=11$ yrs) and all but one were sibling donors. Most parental respondents were mothers (74\%; median age $40 \mathrm{yrs}$ ), 87\% were married, and $36 \%$ had at least a bachelor's degree. Donor vs Proxy. Across all $\mathrm{HRQoL}$ domains except emotional functioning and at all three assessment time points, donor self-reported HRQoL was significantly lower than that reported by parental proxies. Donor vs Norm. At pre-donation, as compared to the normative sample, donors reported significant HRQoL deficits across multiple subdomains and in total HRQoL $(\mathrm{t}=-$ $2.76, P<.01)$. At 4 weeks post-donation, donors reported deficits in physical functioning $(\mathrm{t}=-5.99, P<.001)$ and total HRQoL $(t=-2.82, P<.01)$. At 1 year post-donation, donors reported deficits in physical $(\mathrm{t}=-2.51, P<.05)$ and school functioning $(\mathrm{t}=-2.12, P<.05)$. Donors at $\mathrm{HRQ}$ oL risk. Across the three assessment time points, $21 \%, 19 \%$, and $17 \%$ of donors respectively had self-reported PedsQL total scores below the standard cutoff indicating significant clinical risk of poor HRQoL - scores below the cutoff are similar to those of chronically ill children. Sixteen percent, 13\%, and 5\% were below the cutoff at only one, two, or all three assessments respectively. The youngest donors (5-7 yrs) were at significantly greater risk of being below the cutoff than were their older counterparts with $37 \%, 39 \%$ and $82 \%$ of this group below cutoff at each of the three assessments respectively. In multivariable analyses, the pre-donation factor most strongly and consistently associated with below cutoff PedsQL scores at 4 weeks and 1 year post-donation was pre-donation donor self-reported PedsQL score (likelihood ratio: 9.17, $P<.01$; $15.54, P<.001)$

Conclusion: These findings suggest that there may be significant $\mathrm{HRQ}$ oL deficits among pediatric HSC donors and that in this particular context, parents are not able to accurately report those deficits. These findings also indicate that research to identify predictors of poor HRQoL and the development of interventions to screen and address HRQoL deficits are urgently needed.

Disclosure of Interest: None declared.
Oral session: Infectious complications 1

\section{9}

Improved Outcomes in Allogeneic Hematopoietic Cell Transplant (allo HCT) Patients Treated with Brincidofovir (CMX001, BCV) for Disseminated Adenovirus (AdV) Disease Compared to Literature: Updated Preliminary Results from the AdVise (CMX001-304) Study

M. Grimley ${ }^{1, *}$, G. Papanicolaou ${ }^{2}$, G. Marón ${ }^{3}$, G. Chittick ${ }^{4}$, T. Brundage, A. Bae , H. Momméja-Marin ${ }^{4}$, W. G. Nichols ${ }^{4}$, V. K. Prasad

${ }^{1}$ Bone Marrow Transplant and Immune Deficiency, Cincinnati Children's Hospital Medical Center, Cincinnati, ${ }^{2}$ Infectious Disease Service, Memorial Sloan Kettering Cancer Center, New York ${ }^{3}$ Infectious Diseases, St. Jude Children's Research Hospital, Memphis, ${ }^{4}$ Chimerix, Inc., ${ }^{5}$ PBMT Division, Duke University Medical Center, Durham, United States

Introduction: In allo HCT patients (pts) with disseminated AdV disease, mortality is reported to be up to $80 \%$. Antiviral treatment (tx) usually consists of IV cidofovir (CDV), which has a significant risk of nephrotoxicity. BCV is an orally-available, lipid-conjugate of CDV with no evidence of nephrotoxicity in clinical trials. The pilot portion of the Phase 3 AdVise (CMX001304) study was initiated in March 2014 to enroll 100 allo HCT and other immunocompromised AdV pts with, or at risk of progression to, disseminated AdV disease, to guide the final study design. As of 10NOV2014, 73 subjects have been enrolled and entered into the database, including 60 allo HCT pts (48 with disseminated AdV disease), 7 solid organ transplant pts and 6 "other" pts. Preliminary safety and virologic results for the 48 allo HCT pts with disseminated disease are described.

Materials (or patients) and methods: All subjects receive open-label BCV $100 \mathrm{mg}$ ( $\geq 50 \mathrm{~kg}$ ) or $2 \mathrm{mg} / \mathrm{kg}$ ( $<50 \mathrm{~kg})$ twiceweekly for $12 \mathrm{wks}$, extendable up to $24 \mathrm{wks}$ for pts at high-risk of relapse, and are followed for 24 wks post-tx. AdV DNA viral load (VL) in plasma is measured using a quantitative PCR test (limit of detection [LOD] $2 \log _{10} \mathrm{c} / \mathrm{mL}$ ).

Results: Baseline (BL) characteristics for the 48 subjects are: median (range) age $12(0.7,69)$ y, 65\% <18 y; 69\% male median (range) plasma AdV VL 4.6 ( $<$ LOD to 7.6$) \log _{10} \mathrm{c} / \mathrm{mL}$ $(n=45) ; 42 \%$ AdV positive by qualitative PCR in respiratory secretions, $60 \%$ in urine, $65 \%$ in stool; $29 \%$ with CMV in plasma, $6 \%$ EBV in plasma and $42 \%$ BKV in urine; $42 \%$ received prior IV CDV. As of 25NOV2014, 5 subjects had completed tx and 21 had discontinued tx prematurely. The most common reasons for tx discontinuation were death $(n=11)$ and adverse event $([\mathrm{AE}] n=4)$. Median (range) tx duration was $38(1,141)$ days $(n=45)$. Virologic response in CDV-naïve and exposed subjects with detectable plasma $A d V V L$ at $B L$ are summarized in the table. In subjects with positive AdV PCR at BL, 65\% (13/ 20) cleared $A d V$ in respiratory secretions, $55 \%(16 / 29)$ in urine and $48 \%(15 / 31)$ in stool. Through 08DEC2014, 40\% (19/48) of allo HCT subjects with disseminated AdV disease had died, with a median 71-day observation period for living subjects. No death was attributed to BCV. AEs leading to permanent tx discontinuation attributed to BCV were vomiting and abdominal pain in 1 subject, and acute GVHD in 1 subject.

\begin{tabular}{lllll}
\hline & $\begin{array}{l}\text { Median (range) Change in AdV VL } \\
\text { from BL (log } 10 \mathrm{c} / \mathrm{mL})\end{array}$ & $\begin{array}{l}\text { Median (range) Time to } \\
\text { Minimum On-tx (days) }\end{array}$ & $\begin{array}{l}\text { Proportion ?3 } \log _{10} \text { Reduction in AdV VL } \\
\text { or to Undetectable at Nadir }\end{array}$ \\
\cline { 2 - 3 } & \multicolumn{2}{c}{ Minimum On-tx } & Last On-tx & \\
\hline CDV-naive $(n=23)$ & $-2.0(-5.1,0.5)$ & $-1.8(-5.1,+2.1)$ & $15(3,106)$ & $57 \%(13 / 23)$ \\
\hline CDV-exposed $(n=18)$ & $-1.5(-5.4,+0.6)$ & $-1.2(-5.4,+0.6)$ & $15(4,77)$ & $72 \%(13 / 18)$ \\
\hline
\end{tabular}


Conclusion: The observed mortality rate was $40 \%$ for allo HCT pts with disseminated AdV disease in AdVise, which is lower than literature rates reported for this pt population $(50-80 \%$; Ison 2006, Sandkovsky 2014). BCV showed potent virologic activity in CDV-naïve and exposed pts with no new safety concerns. These preliminary data support expansion of the pilot portion to a definitive Phase 3 study.

References: Ison M. CID 2006:433;31-39; Sandkovsky U, et al. Curr Infect Dis Rep 2014:16:416-424.

Disclosure of Interest: M. Grimley: None declared, G. Papanicolaou: None declared, G. Marón: None declared, G. Chittick Employee of: Chimerix, Inc., Conflict with: Stockholder in Chimerix, Inc., T. Brundage Employee of: Chimerix, Inc. Conflict with: Stockholder in Chimerix, Inc., A. Bae Employee of Chimerix, Inc., Conflict with: Stockholder in Chimerix, Inc., $\mathrm{H}$. Momméja-Marin Employee of: Chimerix, Inc., Conflict with: Stockholder in Chimerix, Inc., W. G. Nichols Employee of: Chimerix, Inc., Conflict with: Stockholder in Chimerix, Inc., V. K. Prasad: None declared.

\section{0}

Long-term persistence of the immune response to anti-pneumococcal vaccines after allogeneic stem cell transplantation: Ten years later after the EBMT-IDWP01 trial

C. Cordonnier, ${ }^{1, *}$, M. Labopin ${ }^{2}$, C. Robin ${ }^{1}$, P. Ribaud ${ }^{3}$, L. Cabanne ${ }^{1}$ C. Chadelat ${ }^{3}$, S. Cesaro ${ }^{4}$, P. Ljungman ${ }^{5}$ on behalf of IDWP ${ }^{1}$ Hematology, Henri Mondor University Hospital, Créteil, ${ }^{2}$ EBMT Data and Study Office, Saint Antoine University Hospital, ${ }^{3}$ Hematology, Saint-Louis SCT Unit, Paris, France, ${ }^{4}$ Pediatric Hematology, Policlinico GB Rossi, Verona, Italy, ${ }^{5}$ Karolinska University, Karolinska Institutet, Stockholm, France

Introduction: The guidelines for immunization of hematopoietic stem cell transplant (HSCT) recipients recommend 3 doses of anti-pneumococcal conjugate vaccine (PCV) from 3-6 months after transplant, followed by a dose of polysaccharide 23-valent (PPV23) vaccine at 12 months in case of no chronic graft-versus-host disease (GVHD), or an additional PCV dose in case of GVHD. However, due to lack of long-term data, there is no recommendation for boosts after 12 months. Our goal was to assess the retainment of anti-pneumococcal antibodies in allogeneic HSCT recipients vaccinated 10 years ago.

Materials (or patients) and methods: In 2009, the IDWP published the results of the IDWP01 trial that compared the immune response assessed one month after 3 doses of PCV7, started either at 3 , or at 9 months after myeloablative $\mathrm{HSCT}^{1}$. Additionally, all patients received 1 dose of PPV23 at 12 or 18 months after transplant. All surviving patients had been assessed for anti-pneumococcal antibodies against the vaccine-serotypes 24 months after HSCT. This study was the basis of the current guidelines for anti-pneumococcal immunization after allogeneic HSCT. The present study included 30 surviving patients from the IDPW01, who were assessed for antibody levels against the 7 PCV7-antigens and against 2 of the PPV23antigens (pn1 and pn5), between 8.3 and 11 years after transplant, i.e. 6 to 9 years after the last assessment in the initial study. The mean age was 39 y (18-55), and 18/30 had acute leukemia. Only 7 had chronic GVHD (limited: 6 , extensive: 1) and 2 had suffered a leukemia relapse. Eleven (37\%) had received an additional dose of PPV23 at a mean time of 6.5 years (2-11 years) after transplant, according to local procedure.

Results: The rates of persistent responses to all 7 antigens of PCV7 were $65.5 \%$ for an Ab cut-off of $0.15 \mu \mathrm{g} / \mathrm{mL}$, and $40 \%$ for a cut-off of $0.50 \mu \mathrm{g} / \mathrm{mL}$. Compared to the response rate at 24 months after transplant, these rates were not significantly decreased but showed important serotype-specific variability. Similar findings were observed for pn1 and pn5 antibody levels. Neither the recipient or donor age, donor type, source of stem cells, GVHD, nor the administration of an additional dose of PPV23 (given to $11 / 30$ patients) influenced the maintenance of the response. The timing of the initial vaccination was the only parameter influencing the long-term response; patients who were vaccinated lately after HSCT (from 9 months) had a significantly better maintenance of the response than patients vaccinated early (from 3 months) after transplant. The 3 patients who were not responders at 24 months and who received an additional dose of PPV23 at 39, 40 and 72 months after transplant, respectively, did not respond.

Conclusion: In long-term HSCT survivors without severe chronic GVHD vaccinated against $S$ pneumoniae according to the current guidelines, the specific immunity is not fully maintained a decade later. Patients, who received an additional PPV23 dose after 24 months post-transplant, do not seem to benefit from this boost. Boosts with PCV should be explored. So far, the optimal schedule of anti-pneumococcal vaccination in HSCT recipients after 12 months remains to be established.

References: ${ }^{1}$ Cordonnier C et al. Clin Infect Dis 2009.

Disclosure of Interest: C. Cordonnier Funding from: Speaker bureau Pfizer, research grants Pfizer, M. Labopin: None declared, C. Robin: None declared, P. Ribaud Personal Interest: Speaker bureau Pfizer, research grants Pfizer, L. Cabanne: None declared, C. Chadelat: None declared, S. Cesaro: None declared, P. Ljungman: None declared.

0051

Risk factors and outcome of infections by Multidrug

Resistant gram negative bacteria in patients undergoing hematopoietic stem cell transplantation

F. Patriarca ${ }^{1, *}$, M. Dozzo ${ }^{1}$, M. L. Battista ${ }^{1}$, M. Medeot ${ }^{1}$, G. Ventura', A. Sperotto', C. Cigana' ', R. Fanin ${ }^{1}$

${ }^{1}$ DISM, DIVISION OF HEMATOLOGY, UNIVERSITY OF UDINE, Udine, Italy

Introduction: Infection and colonization by gram negative (GN) bacteria showing extended spectrum of resistance to beta-lactam antibiotics including carbapenems represents a challenging problem in recipients of hematopoietic stem cell transplantation (HSCT) for the management of post-transplant complications and also for the eligibility to transplant.

Materials (or patients) and methods: Between January 2013 and September 2014. we prospectively evaluated risk factors and outcome of infections by multidrug resistant (MDR) GN bacteria in 142 consecutive HSCT recipients. Throat, nasal and rectal swabs and urine culture were collected at admission in all patients. They were isolated in single room and protection measures for avoiding any contact with body fluids were adopted in colonized patients. Antibiotic association containing aminoglucosids, with at least one antibiotic active in vitro against the isolate. was instituted in case of fever in colonized patients.

Results: Median age was 56 years (15-71), underlying diseases were acute leukemias (30\%), lymphomas (28\%), multiple myeloma (31\%) and others (11\%). 69 patients (49\%) received allo-HSCT and donors were unrelated or haploidentical in 47 cases $(68 \%)$. Overall, 12 patients $(8 \%)$ had a previous history of infection (8) or colonization (4) by MDRGN bacteria before HSCT. Only 4 patients (3\%) showed colonization at admission in at least one of the samples. Overall, 19 patients (13\%) developed infections by carbapenem-resistant Klebsiella pnueumoniae (3), MDR Pseudomonas aeruginosa (14) or both (2) at a median time of 9 days (0-62) after transplant, occurring during the aplasia period in 11/19 patients (58\%). 16 infections developed after allo-HSCT and 3 after auto-HSCT. MDR infections were not preceded by colonization in 9 of 19 patients $(47 \%)$, while 2 of 12 carriers (17\%) of MDRGN bacteria never developed infection after transplant. In the univariate analysis we found a significant association between infections by MDRGN bacteria and acute leukemias $(P<0.001)$, allogeneic transplant $(P=0.001)$, bone marrow as stem cell source $(P=0.03)$, history of infection or colonization by MDRGN bacteria before transplant $(P=0.004)$ and delayed platelet recovery $(P=0.02)$. We did not observe a correlation between 
colonization in one or more surveillance swabs and the development of infections by MDRGN bacteria. At a median follow of 8 months after transplant the cumulative incidence of NRM after auto- and allo-HSCT was $0 \%$ and $19 \%$, respectively. The overall mortality rate from the diagnosis of MDR infections was $0 \%$ ( 0 out 3 patients) after auto-HSCT and $75 \%$ (12 out 16 patients ) after allo-HSCT. MDR infection was primary cause of death in 6 out of 12 patients, with an infection-related mortality rate of $37 \%$ after allo-HSCT.

Conclusion: We conclude that,even if nearly half of the infections by MDRGN bacteria in HSCT recipients were preceded by an history of previous infection or colonization before transplant, surveillance swabs at admission failed to detect MDRGN carriers. Taking in account a 37\% infectionrelated mortality and 58\% occurrence in the aplasia period, measures to speed engrafment, such as reduced-intensity conditioning and infusion of large numbers of PBSC, are mandatory in patients eligible to allo-HSCT.

Disclosure of Interest: None declared.

0052

Early cytomegalovirus reactivation - a potential factor for early robust $\mathrm{T}$ cell reconstitution and possibly a prognostic factor for aGvHD after HSCT

P. R. Varanasi ${ }^{1,2,3, *}$, J. Ogonek ${ }^{3,4}$, S. Luther-Wolf ${ }^{3}$, P. Schweier ${ }^{3}$, M. Stadler ${ }^{3}$, A. Ganser, 2,3 . Messerle, ${ }^{2,5}$ S. Borchers ${ }^{1,2,3}$,

L. Hambach ${ }^{1,3}$, E. Mischak-Weissinger ${ }^{1,2,3,4}$

${ }^{1}$ Integrated Research and Treatment Centre Transplantation IFB-TX, ${ }^{2}$ German Centre for Infection Research - DZIF, ${ }^{3}$ Department of Hematology, Hemostasis, Oncology and Stem Cell Transplantation, ${ }^{4}$ Marie Curie CellEurope, ${ }^{5}$ Institute of Virology, MEDICAL SCHOOL HANNOVER, Hannover, Germany

Introduction: Cytomegalovirus reactivation (CMV-R) and acute graft versus-host disease (aGvHD) are early complications after hematopoietic stem cell transplantation (HSCT). Many previous studies have shown that aGvHD puts patients at risk of CMV reactivation, most likely due to more intensive immunosuppression. However, recent studies and case reports also show that that CMV-R could be a risk factor for aGvHD. Here, we studied the effect of CMV-R on T cell reconstitution in patients with and without aGvHD.

Materials (or patients) and methods: $106 \mathrm{CMV} R+/ \mathrm{D}+$ patients transplanted 2005-2013 in our institution were included in this study. All the patient samples were monitored for the CMV viral load (CMVpp65 expressing cells/400,000 leukocytes) and T cell reconstitution (CD3, CD4, CD8 and all available HLA specific CMV tetramers for each patient) within the first 100 days after HSCT. Patients were subdivided into five groups: group 1: No aGvHD but CMV-R (no-aGvHD-CMV$\mathrm{R})$, group 2: aGvHD after CMV-R (aGvHD-after-CMV-R), group 3: aGvHD before CMV-R (aGvHD-before-CMV-R), group 4: aGvHD but no CMV-R (aGvHD-no-CMV-R) and group 5: no aGvHD-and no CMV-R (no-aGvHD-no-CMV-R).

Results: The characteristics for onset of CMV reactivation and aGvHD in the different subgroups are provided in Table 1. CD3, CD4, CD8 and CMV specific T cells were analyzed on day $50 \pm 10$ days after HSCT.

In order to investigate the potential influence of CMV-R in the absence of aGvHD on T cell reconstitution, we compared the T cell numbers in the groups CMV-R + /- subsequent aGvHD (i.e. group $1+2$ ) with group 5 (no-aGvHD-no-CMV-R). We found significantly more CD3 $(P=0.021)$ and CD8 T cells $(P=0.0057)$ in groups $1+2$ compared to group 5 . There were no differences in $T$ cells between the groups with CMV-R $+/$ subsequent aGvHD (i.e. groups $1+2$ ) compared to the groups with aGvHD + /- subsequent CMV-R (i.e. groups $3+4$ ). Moreover, there were no differences in $T$ cell numbers between the groups with aGvHD $+/$ - subsequent CMV-R (i.e. groups $3+4$ ) compared to group 5 .

To study the impact of CMV-R on $\mathrm{T}$ cell reconstitution in patients with subsequent aGvHD we compared the $T$ cell numbers in group 2 (aGvHD-after-CMV-R) with group 1

\begin{tabular}{|c|c|c|c|c|}
\hline \multirow[b]{2}{*}{ Group no. } & \multirow[b]{2}{*}{ Groups } & \multirow[b]{2}{*}{ (n) } & \multirow{2}{*}{$\begin{array}{l}\text { mean day } \\
\text { and SD for } \\
\text { CMV-R }\end{array}$} & \multirow{2}{*}{$\begin{array}{c}\text { Mean day } \\
\text { and SD for } \\
\text { aGvHD }\end{array}$} \\
\hline & & & & \\
\hline 1 & no-aGvHD-CMV-R & 37 & $36(S D ; 10.6)$ & n.a. \\
\hline 2 & aGvHD-after-CMV-R & 18 & $38(\mathrm{SD} ; 10.3)$ & $88(S D ; 32)$ \\
\hline 3 & aGvHD-before-CMV-R & 11 & $45(\mathrm{SD} ; 9.4)$ & $25(S D ; 10.9)$ \\
\hline 4 & aGvHD-no-CMV-R & 17 & n.a. & $47(S D ; 20.5)$ \\
\hline 5 & no-aGvHD-no-CMV-R & 23 & n.a. & n.a. \\
\hline
\end{tabular}

(no-aGvHD-after-CMV-R). There were significantly more CD4 T cells $(P=0.0041)$ and a trend for more CD3, CD8 and CMVCTLs in group 2 (aGvHD-after-CMV-R) compared to group 1 (no-aGvHD-after-CMV-R).

Subsequently, we compared the potential influences of CMV-R and of aGvHD on $\mathrm{T}$ cell reconstitution. We found significantly more CD3 $(P=0.0138)$ and CD8 T cells $(P=0.0125)$ in group 2 (aGvHD-after-CMV-R) compared to group 4 (aGvHD-no-CMV$\mathrm{R})$. Moreover, we studied the overall potential influence of CMV-R in the presence of aGvHD on T cell reconstitution. We found a trend for more T cells (CD3, CD4, CD8 and CMV-CTLs) in group 2 (aGvHD-after-CMV-R) compared to group 3 (aGvHDbefore-CMV-R).

Conclusion: In conclusion, patients with aGvHD after CMV-R had considerably more CD4 T-cells on day 50 compared to patients with CMV-R but no aGvHD and significantly more CD3 and CD8 T cells compared to patients with aGvHD but noCMV-R. These results suggest that early CMV-R enhances overall $\mathrm{T}$ cell reconstitution which could be a potential risk factor for developing aGvHD after HSCT.

Disclosure of Interest: None declared.

\section{3}

Diagnostic metagenomics from BAL samples of allogenic HSCT patients with pulmonary complications

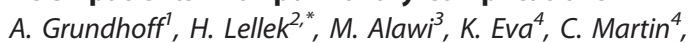

D. Indenbirken ${ }^{1}$, N. Kröger ${ }^{2}, H$. Rohde ${ }^{4}$, N. Fischer ${ }^{4}$

${ }^{1}$ Leibniz Institute for Experimental Virology, Heinrich-PettoInstitut, ${ }^{2}$ Stem Cell Transplantation, ${ }^{3}$ Bioinformatics Service Facility, ${ }^{4}$ Institute of Medical Microbiology, University Medical Center Hamburg-Eppendorf, Hamburg, Germany

Introduction: Despite of improved prophylaxis and conventional microbiological diagnostics the risk for pulmonary complications in of hematopoietic stem cell transplant (HSCT) patients continues to be high. While pneumonia remains a significant cause of mortality after HSCT, detection of an infectious agent fails in more than $50 \%$ of cases. Unbiased, non-targeted metagenomic RNA sequencing (UMERS) represents a novel method which can detect not only known, but also distantly related or even novel pathogens of viral, bacterial or parasitic origin. As such, UMERS has the potential to significantly improve the detection of infectious agents in clinical as well as health settings.

Materials (or patients) and methods: We have previously established a streamlined NGS platform specifically dedicated to the detection and analysis of human pathogens in diagnostic samples (Fischer, Rohde EID 2014). We here apply unbiased metagenomic RNA sequencing (UMERS) to 24 diagnostic respiratory specimens (BAL) of HSCT patients with CT confirmed pulmonary lesions.

The performance of UMERS analysis was systematically compared to conventional diagnostic tests including bacterial/fungal culture together with multiplex PCR for human viruses known to cause respiratory diseases (InflA, InflB, hPIV14, RSV, Rhinoviruses, Enteroviruses, Metapneumovirus, Bocavirus, Adenoviruses and Coronaviruses including HUK1, NL64, $229 \mathrm{E}$ and OC43) and bacteria with the potential to cause pneumonia that are difficult to grow in culture (Bordetella 
pertussis, Bordedella parapertussis, Legionella, Mycoplasma pneumoniae and Chlamydophila pneumoniae).

Results: UMERS detected in 58\% (14/24) of the samples pathogens with the potential to cause respiratory disease. In contrast, conventional diagnostics succeeded in only $37,5 \%$ (9/ 24) of the cases to detect a putative pathogen. All pathogens detected by conventional tests were also faithfully identified by UMERS analysis. Additionally, UMERS revealed a viral infection with human Parainfluenza 3 in one patient: two additional samples showed clear signs of bacterial infection (S. pneumoniae, Pseudomonas simiae) and three samples contained sequences of fungal organisms (Aspergillus fumigatus, Fusarium $s p$.) which can cause fatal pulmonary complications in immunosuppressed patients.

We furthermore report a cluster of severe pneumonia $(n=6)$ in which the index case was tested negative by conventional diagnostic procedures, while UMERS successfully detected hPIV3 viral sequences. Furthermore, the recovery of whole genome sequences from all six cases by UMERS showed low interspecies sequence diversity between these hPIV3 sequences thereby highly suggesting nosocomial infection and spread of the virus between these patients.

Furthermore, we report a case of S. pneumoniae infection unambiguously detected by UMERS but not by bacterial culturing in one patient with suspected post-transplant malignancies as the cause of pulmonary complications.

Conclusion: These results illustrate the potential of metagenomic approaches for the diagnostic detection of viral, bacterial and/or fungal sequences from clinical samples. UMERS can significantly contribute to both the improvement of diagnostics as well as support of epidemiological analysis of putative nosocomial infections and thus improve the level of recognition and treatment of pulmonary complications in HSCT patients.

Disclosure of Interest: None declared.

\section{4}

Early HHV-6 reactivation predicts graft failure in patients receiving myeloablative conditioning for unrelated cord blood transplantation: a SFGM-TC, EUROCORD and CBCCTIWP EBMT study

P. Chevallier ${ }^{1, *}$, M. Labopin ${ }^{2}$, J.-H. Dalle ${ }^{3}$, A. Le Bourgeois', G. Michel ${ }^{4}$, G. Socie ${ }^{5}$, M. Michallet ${ }^{6}$, E. Deconinck ${ }^{7}$, L. Clement $^{8}{ }^{{ }_{13}}$ N. Fegueux ${ }^{9}$, L. Mannone ${ }^{10}$, C. Jubert ${ }^{11}$, Y. Bertrand ${ }^{12}$, N. Raus ${ }^{13}$, F. Giannotti ${ }^{14}, R$. Peffault de Latour ${ }^{5}, R$. Vanderson ${ }^{14}$

E. Gluckman ${ }^{14}$, A. Ruggeri ${ }^{14}$

${ }^{7} \mathrm{CHU}$ Nantes, Nantes, ${ }^{2}$ ALWP, ${ }^{3}$ Robert Debre hospital, Paris, ${ }^{4} \mathrm{CHU}$ La Timone, Marseille, ${ }^{5}$ Saint Louis, Paris, ${ }^{6} \mathrm{CHU}$ Lyon, Lyon, ${ }^{7} \mathrm{CHU}$ Besancon, Besancon, ${ }^{8} \mathrm{CHU}$ Nancy, Nancy, ${ }^{9} \mathrm{CHU}$ Montpellier, Montpellier, ${ }^{10} \mathrm{CHU}$ Nice, Nice, ${ }^{11} \mathrm{CHU}$ Bordeaux, Bordeaux, ${ }^{12} \mathrm{CHU}$,

${ }^{13}$ SFGM-TC, Lyon, ${ }^{14}$ Eurocord, Paris, France

Introduction: HHV-6 reactivation has been described to be significantly associated with the use of cord blood (CB) as stem cell source. However, the impact on outcomes of HHV-6 reactivation after $\mathrm{CB}$ transplantation (CBT) is still discussed. Recently, we have reported higher incidence of graft failure in patients who experienced HHV-6 reactivation after CBT (Le Bourgeois, EJH, 2014). Here, we have evaluated the role of HHV- 6 reactivation on CBT outcomes in a large series of patients.

Materials (or patients) and methods: All patients with haematological disorders who underwent a myeloablative CBT between 2002 and 2012 and assessed for HHV-6 reactivation after CBT were analyzed. Within this period, 448 cases (male $n=268,60 \%)$ were reported to SFGM-TC and EUROCORD. Median age was $17 y$ at transplant $(0.5-66),<18$ years $n=263$ (59\%). The majority of patients had haematological malignancies $(n=431,96 \%)$ including 326 cases with acute myeloid leukemia (73\%), 69 cases with myelodysplastic and/or myeloproliferative disorders and 24 cases with lymphoproliferative disorders. The majority of patients were in complete remission at time of transplant (CR1 $n=146$; CR2 $n=143$ ).
Single and double CBT were used in 289 and 159 cases, respectively. TBI was part of the conditioning regimen in 263 cases (59\%). In vivo T-cell depletion by ATG was used in $61 \%$ of patients. At least one PCR with a viral load $>3 \mathrm{log} / \mathrm{mL}$ of blood was sufficient to define HHV- 6 reactivation after the graft. The impact of HHV- 6 reactivation on CBT outcomes has been studied as a time-dependent variable.

Results: The cumulative incidence of neutrophil engraftment was $87+/-2 \%$, median time 28 days. aGVHD grade II-IV was reported in 162 patients (36\%), (grade II, $n=94$; grade III, $n=42$; grade IV, $n=26$ ). With a median follow-up of 41 months (range: 2-142), 4-year overall survival (OS) was $45+-2 \%, 233$ patients died. Transplant-related mortality was the main cause of death $(n=147$ (64\%): infections $n=59$, GVHD $n=32$, multiple organ failure $n=14$, other causes $n=42$ ), 84 cases died from disease progression and 2 of unknown cause.

Overall, 151 patients (34\%) experienced HHV-6 reactivation, of those, 87 patients had HHV6 reactivation before neutrophil engraftment. HHV6 reactivation occurred in a median time of 21 days after CBT. There was no difference in term of HHV- 6 reactivation between patients receiving a single $(48 \%)$ or a double (52\%) CBT.

In univariate analysis, HHV-6 reactivation was significantly associated with graft failure (HR: 1.44, 95\%Cl: 1.18-1.87, $P=0.005)$ and higher incidence of chronic GVHD (HR: 1.75, $95 \% \mathrm{Cl}$ : $1.18-2.60, P=0.005)$ but had no impact on DFS $(P=0.52)$, OS $(P=0.76)$, NRM $(P=0.40), \mathrm{RI}(P=0.99)$ or acute GVHD $(P=0.09)$. In multivariate analysis, HHV-6 was independently associated with graft failure (HR: $1.44,95 \% \mathrm{Cl}$ : 1.09-1.91, $P=0.009$ ). TNC dose at infusion was the other factor associated with engraftment (HR: 1.03, 95\%Cl: 1.02-1.34, $P=0.03$ )

Conclusion: Our study confirms that HHV-6 reactivation is a risk factor for graft failure in CBT recipients after myeloablative conditioning regimen. This result has to be confirmed prospectively and in the setting of reduced-intensity conditioning CBT. This paves the way also to test prospectively the indication of ganciclovir or foscarnet use as anti-HHV-6 prophylaxis in CBT recipients.

Disclosure of Interest: None declared.

\section{5}

A retrospective IDWP-ALWP study on Candida infections in allogeneic haematopoietic stem cell transplant patients S. Cesaro, ${ }^{1, *}$, G. Tridello ${ }^{1}$, L. Volin ${ }^{2}$, C. Craddock ${ }^{3}$, M. Michallet ${ }^{4}$, N. Schaap ${ }^{5}$, J. Cornelissen ${ }^{6}$, P. Ljungman ${ }^{7}$, A. Hunter ${ }^{8}$,

J. Maertens ${ }^{9}$, N. Russell ${ }^{10}$, J. Snowden ${ }^{11}$, J. Hoek ${ }^{12}$, G. Socié ${ }^{13}$, P. Bordigoni ${ }^{14}$, C. Cordonnier ${ }^{15}$, A. Nagler $^{16}$

'Oncoematologia Pediatrica, Azienda Ospedaliera di Verona, Verona, Italy, ${ }^{2}$ Helsinki University Central Hospital, Helsinki, Finland, ${ }^{3}$ Queen Elizabeth Hospital, Birmingham, United Kingdom, ${ }^{4}$ Centre Hospitalier Lyon Sud, Lyon, France, ${ }^{5}$ Radboud University - Nijmegen Medical Centre, Radboud, ${ }^{6}$ Erasmus MC Daniel den Hoed Cancer Centre, Rotterdam, Netherlands, ${ }^{7}$ Karolinska University Hospital, Stockholm, Sweden, ${ }^{8}$ Leicester Royal Infirmar, Leicester, United Kingdom, ${ }^{9}$ University Hospital Gasthuisberg, Leuven, Belgium, ${ }^{10}$ Nottingham City Hospital, Nottingham, "Sheffield Teaching Hospitals, Sheffield, United Kingdom, ${ }^{12}$ Data Office, EBMT, Leiden, Netherlands, ${ }^{13} \mathrm{Hopital}$ St. Louis, Paris, ${ }^{14}$ Centre Hospitalier Universitaire, Vendoeuvre-lesNancy, ${ }^{15}$ Henri Mondor University Hospital, Créteil, France, ${ }^{16}$ Chaim Sheba Medical Centre, Tel-Hashomer, Israel

Introduction: Candida is the second more frequent cause of invasive fungal infection in haematological immunocompromised hosts, especially in the patients who undergo an haematopoietic stem cell transplantation (HSCT). The aim of this study was to analyse retrospectively the outcome of patients with Candida infections acquired in the first 100 days after allogeneic HSCT.

Materials (or patients) and methods: The analysis included patients of any age, who underwent a first allogeneic HSCT 
from 2000 to 2012 for acute leukaemia, recorded in the EBMT registry. The infections were classified as candidemia, Candida endorgan disease, and Candida infection with site non specified.

A multivariate Cox model was performed in order to detect differences in survival in the three groups. Results were adjusted for confounders, including disease, host and HSCT characteristics.

Results: During the study period, 46630 first allogeneic HSCT were performed in 483 EBMT centres. A total of 941 episodes of Candida infections within 100 days were reported in 136 centres who performed 21997 HSCT.

Deep Candida infections were 84\% (489 candidemia and 306 endorgan diseases), while $16 \%$ were site not specified (146). Underlying patient disease was lymphoblastic, myeloid and biphenotipic leukaemia in $61 \%, 37 \%$ and $2 \%$ of cases, respectively; $55 \%$ were male and $45 \%$ female. Median age at HSCT was 40 years, range 1-73. Stem cell source was peripheral blood in most of the cases (64\%); donor was sibling, mismatched related, matched unrelated and mismatched unrelated in $40 \%, 3 \%, 49 \%$ and $8 \%$ of patients, respectively. Among the three groups, the main difference in baseline characteristics regarded time from diagnosis to HSCT, CMV recipient/donor serostatus, stem cell source and conditioning regimen (TBI, ATG, ex-vivo T-cell depletion, myeloablative vs. reduced intensity).

The strains of Candida were specified in 122 episodes as follows: C. albicans, 38\%; C. non albicans 55\%; C. albicans and C. non albicans (double-strain isolation) $7 \%$.

The 100- day and 1-year overall survival (OS) were $89 \%$ (95\% C.I. 87-91) and 64\% (C.I. 61-67), respectively, the median follow-up being 4.4 years (C.I. 3.9-5.0). One year probability for OS and relapse free survival were: candidemia, 58\% and 49\%; endorgan disease, $66 \%$ and $56 \%$; site not specified, $78 \%$ and $68 \%, P<0.0001$ and $P=0.0002$, respectively.

The difference between groups was confirmed after adjusting for confounders.

Non-relapse mortality (NRM) at 100- day and 1-year was $9 \%$ (C.I. 8-12) and 21\% (C.I. 19-24), respectively. The 100 days and 1 -year NRM in the three groups were: candidemia, $12 \%$ and 26\%; Candida endorgan disease, $8 \%$ and $19 \%$ and Candida site not specified, $3 \%$ and $9 \%$, respectively $(P=0.0001)$.

Conclusion: Despite the introduction of new classes of antifungal drugs from 2000, overall mortality in patients who had Candida infection remained high, especially in patients with candidemia. The role of Candida on the morbidity and attributable-infection-mortality post-HSCT deserves a prospective investigation.

Disclosure of Interest: None declared.

\section{6}

Allogeneic hematopoietic stem cell transplantation in patients with invasive aspergillosis

M. O. Popova, ${ }^{1, *}$, A. Volkova ${ }^{1}$, S. Elias Soulaiman ${ }^{2}$, V. Vavilov ${ }^{1}$ S. Bondarenko', O. Slesarchuk', A. Smirnova ${ }^{1}$, O. Shadrivova ${ }^{3}$ S. Khostelidi', S. Ignatyeva', T. Bogomolova ${ }^{3}$, L. Zubarovskaya', N. Klimko ${ }^{3}$, B. Afanasyev

${ }^{1}$ Raisa Gorbacheva Memorial Institute of Children Oncology, Hematology and Transplantation, First Pavlov State Medical University of Saint-Petersburg, Saint-Petersburg, Russian Federation, ${ }^{2}$ Hematology and Bone Marrow Transplantation Department, Tishreen Hospital, Damascus, Syrian Arab Republic, ${ }^{3}$ Department of Clinical Mycology, I. Mechnikov North-Western State Medical University, Saint-Petersburg, Russian Federation

Introduction: Invasive aspergillosis (IA) is a major cause of morbidity and mortality in hematological patients, but introducing new antifungals and diagnostic procedures has improved recent prognosis of IA. Number of patients with IA who are candidates for allogeneic hematopoietic stem cell transplant (alloHSCT) has increased. This study focuses on clinical characteristics, risk factors and outcomes of IA after alloHSCT in pts who have had IA before transplant.
Materials (or patients) and methods: In prospective study 173 alloHSCT recipients were included from Dec 2012 to Jul 2013. The median age was 34 y.o., males $-54 \%$. Most of pts had high-risk acute leukemia (70\%). AlloHSCT from MUD were performed in $57 \%$, MRD - 24\%, haplo - $11 \%$, MMUD - $8 \%$, predominantly with RIC (80\%). EORTC/MSG 2008 criteria for diagnosis and response to therapy were used. Since 2011 active diagnostic strategy, including bronchoscopy with BAL, in pts with CT-scan lung lesions before alloHSCT has been introduced to the routine practice. "Active $I A^{\prime \prime}$ is the $I A$ diagnosed just before HSCT.

Results: Incidence of IA before alloHSCT was 22,5\% ( $n=39$ / 173). According to EORTC/MSG 2008 criteria $92 \%$ of pts had probable IA and $8 \%$ proven IA. The main sites of infection were lungs $-95 \%$, central nervous system $-3 \%$, and colon $-3 \%$, other localizations were observed mostly in a combination with lung involvement: sinuses - $5 \%$, spleen - 3\%, and liver $3 \%$. Antifungal therapy before alloHSCT was administrated in $69 \%$ pts (Voriconazole - 95\%, other - 5\%) with the median duration of therapy - 2 months. Complete response to antifungal therapy was registered in $10(26 \%)$ pts, partial response or stabilization in $17(43 \%)$, and "active $I A^{\prime \prime}$ in 12 (31\%) pts. After alloHSCT all pts received antifungal therapy with Voriconazole (first line $-31 \%$, continuation of treatment $43 \%$, and secondary prophylaxis - 26\%). Median length of treatment was 166 days (37-394). Cumulative incidence of relapse or progression of IA after alloHSCT was $12,4 \%(n=6)$. Relapse of underlying disease was the main risk factor for the relapse or progression of IA after alloHSCT $(6 \%$ vs $33 \%$, $P=0,007)$. Progression of IA after alloHSCT was treated with Voriconazole $600 \mathrm{mg}$ per day $(n=1)$ and combination Vori + Caspo $(n=3)$. Relapse of the IA after alloHSCT was treated with Voriconazole $400 \mathrm{mg}$ per day $(n=2)$. No toxicity of the antifungal treatment was registered. Complete response was achieved in 4 pts, and stabilization - 2. 12-weeks overall survival (OS) after the start of antifungal therapy was $67 \%$. Two pts died with the progression of the underlying disease. 100days OS after alloHSCT was 70\%, 1-year OS after alloHSCT was $57 \%$. There was no significant difference in OS in pts with or without IA before alloHSCT.

Conclusion: Incidence of the IA before alloHSCT was 22,5\% Cumulative incidence of relapse or progression of IA after alloHSCT in pts with proven and probable IA before alloHSCT was $12,4 \%$. Relapse of the underlying disease was the main risk factor for relapse or progression of IA after alloHSCT. Secondary prophylaxis with Voriconazole should be used in pts with IA before alloHSCT. Relapse or progression of IA after alloHSCT didn't impair OS. IA is not a contraindication for alloHSCT.

Disclosure of Interest: None declared.

\section{Oral session: Early complications 1}

\section{7}

Low pre-transplant 25-hydroxyvitamin D3 predicts relapse and survival in male but not in female patients undergoing allogeneic stem cell transplantation

A. Radujkovic ${ }^{1, *}$, A. Benner ${ }^{2}$, S. Dietrich ${ }^{1}$, U. Hegenbart ${ }^{1}$,

S. Schönland ', A. D. Ho ${ }^{1}$, P. Dreger ${ }^{1}$, T. Luft

${ }^{1}$ University Hospital Heidelberg, '2German Cancer Research Center, Heidelberg, Germany

Introduction: Vitamin D3 (VitD3) deficiency is frequent in patients with hematopoietic malignancies undergoing allogeneic transplantation (alloSCT). However, the prognostic relevance in the transplant context has not been fully delineated. This retrospective study investigated the prognostic impact of pre-transplant VitD3 levels on outcome parameters after alloSCT. 


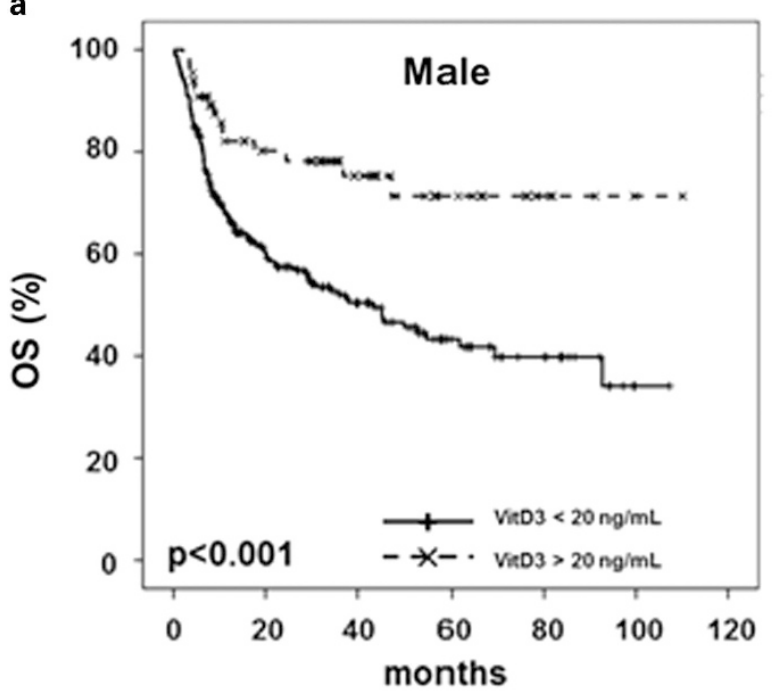

Materials (or patients) and methods: Between 2001 and 2012, a total of 650 patients undergoing alloSCT at our institution (61\% male, 39\% female, median age 53 years) provided informed consent to participate in this observational study. 223 patients $(34 \%)$ received transplants from related donors, 281 patients $(43 \%)$ from matched unrelated donors and $139(21 \%)$ from mismatched unrelated donors. Diagnoses were acute myeloid leukemia in 202 patients (32\%), lymphoid malignancies (lymphoma, chronic and acute lymphoid leukemia) in 228 patients (35\%), multiple myeloma in 110 patients (17\%) and other myeloid malignancies (including myelodysplastic syndrome, myelofibrosis and chronic myeloid leukemia) in 110 patients (17\%). A total of 285 patients (44\%) received statin treatment post alloSCT. Pre-transplant serum samples for VitD3 were collected between 0 and 2 months before alloSCT and cryopreserved at $-80^{\circ} \mathrm{C}$. VitD3 ( $=25$-hydroxyvitamin D3) concentrations were measured by an enzyme-linked immunosorbent assay. A total of 497 patients had serum samples prior to alloSCT available. In order to consider 153 missing VitD3 data sets multiple imputation $(k=20)$ was applied. Cox regression analysis with cause specific hazard models was applied for overall survival (OS), non-relapse mortality (NRM) and time to relapse (TTR). As confounding prognostic factors we included disease entity, statin treatment, VitD3, age, recipient's sex and donor as well as the interaction between VitD3 and donor, sex and statin treatment into the models.

Results: Estimated median follow-up time was 47.1 months. Median pre-transplant 25-hydroxyvitamin D3 level was 11.9 $\mathrm{ng} / \mathrm{mL}$ (range $1-46.3 \mathrm{ng} / \mathrm{mL}$ ). In multivariate analysis of the entire cohort, a significant interaction between recipient's sex and VitD3 level for OS $(P=0.04)$ and TTR $(P=0.03)$ could be observed. There was no interaction effect for NRM. Consequently, separate models for OS and TTR in male and female patients were performed. For female patients, no influence of VitD3 with regard to OS and TTR was observed. In contrast Cox regression analysis of male patients revealed that lower VitD3 levels were significantly associated with inferior OS $(P=0.008)$. In male patients VitD3 was also significantly associated with TTR $(\mathrm{HR}=0.6895 \% \mathrm{Cl} 0.50-0.93, P=0.015)$, i.e. longer time to relapse with higher VitD3 levels. When stratified at $20 \mathrm{ng} / \mathrm{mL}$ (recommended VitD3 level for the normal population), in both sexes lower VitD3 did not affect NRM incidence. However, in male but not in female patients VitD3 levels below $20 \mathrm{ng} / \mathrm{mL}$ resulted in shorter TTR $(P=0.001)$. Accordingly, VitD3 levels lower than $20 \mathrm{ng} / \mathrm{mL}$ were significantly associated with inferior OS in male $(P<0.001)$ (Figure $1 \mathrm{a})$ but not in female patients (Figure $1 \mathrm{~b}$ ).

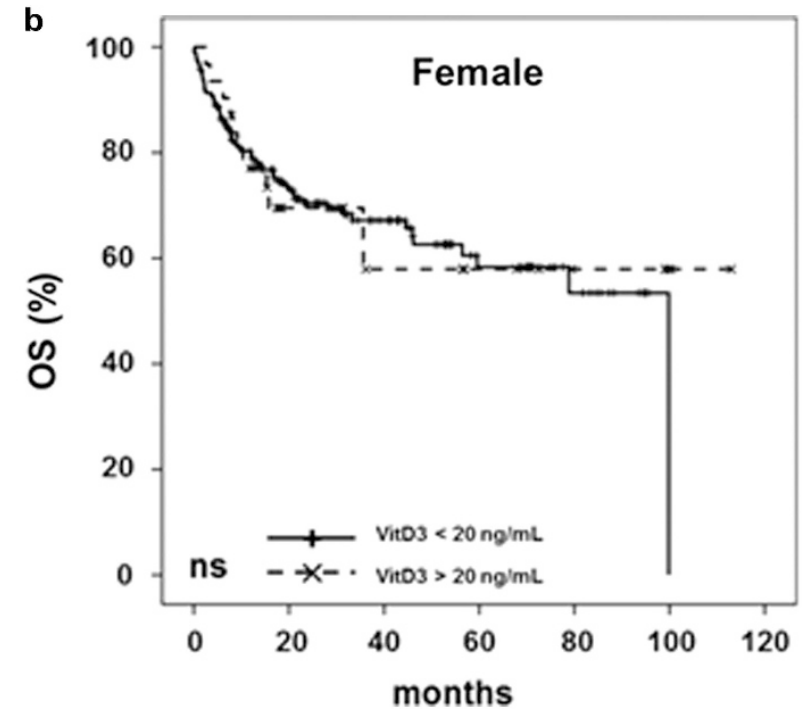

Conclusion: This single center study suggests that male patients with lower pre-transplant VitD3 levels have higher relapse rates translating into a survival disadvantage across all diagnostic entities. Prospective studies investigating VitD3 in the alloSCT setting are highly warranted.

Disclosure of Interest: None declared.

\section{8}

Defibrotide for Hepatic Veno-Occlusive Disease Following Allogeneic or Autologous Hematopoietic Stem Cell Transplant: Interim Subgroup Survival Analysis From an Ongoing US Study

P. G. Richardson 1,*, A. R. Smith ${ }^{2}$, B. M. Triplett ${ }^{3}$, N. A. Kernan ${ }^{4}$, S. A. Grupp ${ }^{5}$, J. H. Antin ${ }^{6}$, L. Lehmann 7 , M. Miloslavsky ${ }^{8}$, R. Hume ${ }^{8}$, A. L. Hannah ${ }^{8}$, B. Nejadnik ${ }^{8}$, R. J. Soiffer ${ }^{6}$

${ }^{7}$ Division of Hematologic Malignancy, Department of Medical Oncology, Jerome Lipper Multiple Myeloma Center, Dana-Farber Cancer Institute, Harvard Medical School, Boston, MA, ${ }^{2}$ Division of Pediatric Blood and Marrow Transplantation, University of Minnesota, Minneapolis, MN ${ }^{3}$ Bone Marrow Transplantation and Cellular Therapy, St. Jude Children's Research Hospital, Memphis, TN, ${ }^{4}$ Pediatric BMT Service, Memorial Sloan-Kettering Cancer Center, New York, NY, ${ }^{5}$ Pediatric Oncology, The Children's Hospital of Philadelphia, Philadelphia, PA, 'Stem Cell/Bone Marrow Transplantation Program, Division of Hematologic Malignancy, Department of Medical Oncology, Dana-Farber Cancer Institute, ${ }^{7}$ Center for Stem Cell Transplantation, Division of Hematologic Malignancy, Department of Medical Oncology, Dana-Farber Cancer Institute, Harvard Medical School, Boston, MA, ${ }^{8}$ Jazz Pharmaceuticals, Inc., Palo Alto, CA, United States

Introduction: Hepatic veno-occlusive disease (VOD, also known as sinusoidal obstruction syndrome) is an important complication of hematopoietic stem cell transplant (HSCT). Severe VOD (sVOD) is clinically defined as VOD with multiorgan failure (MOF). sVOD is life-threatening with a reported mortality rate $>80 \%$. Defibrotide (DF) is thought to have a protective effect on activated microvascular endothelium and restores thrombo-fibrinolytic balance. In a phase 3 SVOD trial, DF significantly improved complete response rates and survival at day (D) +100 post HSCT (vs a rigorous historical control), with a favorable safety profile. In the European Union, DF is approved for the treatment of severe hepatic VOD in HSCT patients (pts). In the US, DF is available through an expanded access, protocol-directed treatment IND (T-IND) collecting data on safety/efficacy of DF in pts with severe and nonsevere VOD post HSCT or post-chemotherapy. 
Materials (or patients) and methods: The original T-IND protocol required a VOD diagnosis by Baltimore criteria (bilirubin $\geq 2.0 \mathrm{mg} / \mathrm{dL}$ with $\geq 2$ of hepatomegaly, ascites, or $5 \%$ weight [wt] gain) with MOF (renal and/or pulmonary) post $\mathrm{HSCT}$; the study was amended to include nonsevere VOD (ie, without MOF) per modified Seattle criteria ( $\geq 2$ of bilirubin $\geq 2.0 \mathrm{mg} / \mathrm{dL}$, hepatomegaly or upper right quadrant pain, ascites, or, in this study, $5 \%$ wt gain) post HSCT or chemotherapy. Exclusion criteria include clinically significant bleeding or need for $\geq 2$ vasopressors. DF was given as a 2-hour infusion at $6.25 \mathrm{mg} / \mathrm{kg}$ IV q6h $(25 \mathrm{mg} / \mathrm{kg} / \mathrm{d})$ for $\geq 21$ days. Assessments included safety and $D+100$ survival.

Results: Of pts enrolled from December 2007 to December 2013 and eligible for analysis, 641 pts received $\geq 1$ DF dose (including post-chemotherapy pts without HSCT). Most of these pts were male (57\%); median age was 13y (range 0-69) with $58 \%(372 / 636) \leq 16 y$. The most common underlying diagnoses included acute myelogenous leukemia (AML; 27\%) and acute lymphocytic leukemia (ALL; 23\%) and most common graft vs host disease (GvHD) agents were tacrolimus (41\%), cyclosporine (30\%), and methotrexate (29\%). In the 336 pts with SVOD and HSCT, $54 \%$ were male; median age was $12 \mathrm{y}$ (range 0-69) with $61 \%(204 / 333) \leq 16 y$. The most common underlying diagnoses in this group were AML (26\%) and ALL (25\%) with most frequently used GvHD agents including tacrolimus (40\%), cyclosporine (32\%), and methotrexate (29\%). Survival data are currently available for 526 post-HSCT pts. Most were allografts $(n=467)$ vs autografts $(n=56)$, with graft type not available in 3 pts. Among allografts and autografts, respectively, $54 \%$ and $48 \%$ had sVOD, while $46 \%$ and $52 \%$ had nonsevere VOD. D 100 survival is shown in the Table.

Table. D + 100 Survival

\begin{tabular}{lll}
\hline$\%(95 \% \mathrm{Cl})$ & Allografts & Autografts \\
\hline Post-HSCT, all pts & $50 \%(46-55)$ & $66 \%(54-79)$ \\
\hline sVOD only & $43 \%(37-49)$ & $59 \%(41-78)$ \\
\hline Non-severe VOD only & $58 \%(52-65)$ & $72 \%(56-89)$ \\
\hline
\end{tabular}

Conclusion: $D+100$ survival in both the allograft and autograft SVOD subsets of this trial, which is the largest of its kind, was substantially improved vs historical controls and highly consistent with prior studies of DF in this setting. The higher survival rate in nonsevere VOD here indicates further study is warranted to determine impact of treatment earlier in the course of VOD.

Support: Jazz Pharmaceuticals.

Disclosure of Interest: P. G. Richardson Funding from: Jazz Pharmaceuticals, Conflict with: has served on advisory committees with Jazz Pharmaceuticals., A. R. Smith: None declared, B. M. Triplett: None declared, N. A. Kernan: None declared, S. A. Grupp: None declared, J. H. Antin Conflict with: is a consultant to Tempera and Enlivex and has served on advisory committees with Jazz Pharmaceuticals, L. Lehmann: None declared, M. Miloslavsky Employee of: Jazz Pharmaceuticals, Conflict with: in the course of employment has received stock options exercisable for, and other stock awards of, ordinary shares of Jazz Pharmaceuticals plc., R. Hume Employee of: Jazz Pharmaceuticals, Conflict with: in the course of employment has received stock options exercisable for, and other stock awards of, ordinary shares of Jazz Pharmaceuticals plc., A. L. Hannah Conflict with: is a consultant to Jazz Pharmaceuticals, B. Nejadnik Employee of: Jazz Pharmaceuticals, Conflict with: in the course of employment has received stock options exercisable for, and other stock awards of, ordinary shares of Jazz Pharmaceuticals plc., R. J. Soiffer Conflict with: has served on advisory committees with Jazz Pharmaceuticals.
0059

A single centre experience in Veno-occlusive disease and defibrotide

R. R. Poll, ${ }^{1, *}$, N. Russell ${ }^{1}$, E. Das-gupta ${ }^{1}$, L. Watson ${ }^{1}$, L. Rachael ${ }^{1}$, J. Byrne

${ }^{1}$ Nottingham University hospital, U.K., Nottingham, United Kingdom

Introduction: Defibrotide is is recommended as the definitive treatment for veno-occlusive disease (sinusoidal obstruction syndrome). We report a single center experience at Nottingham university hospitals NHS trust.

Materials (or patients) and methods: We reviewed all allogeneic transplants performed in our centre from Jan 2011-Sep 2014 with respect to VOD. There were a total of 273 allogeneic transplants performed. None had prior liver disease but 2 patients had mildly raised trasaminases prior to transplantation. 12 patients had an unrelated donor whereas 1 had a sibling donor. All patients had T cell depletion. 1/13 had a prior allograft, 4/13 patients with VOD had myeloablative transplant with $\mathrm{Cy}$-TBI whereas 9 patients had a reduced intensity transplant. Out of the $\mathrm{RIC}^{\prime} \mathrm{s}, 7$ patients received busulphan as part of their conditioning (either Flu-BuATG or FLAMSA-Bu) and 2 patients received Fludarabine, melphalan and alemtuzumab conditioning.

Results: $13 / 273$ patients had VOD as a complication (4.7\%). VOD developed in AML-7/113(6.1\%), MF-2/6(33\%), NHL-1/ 47(2.1\%), CML-1/14(7.1\%) and MDS-2/27(7.4\%).The median age of the patients having VOD was 60.5 years. 12 patients were male and 1 female. All were diagnosed using the modified Seattle criteria but only $6 / 13$ satisfied Baltimore criteria. The mean time to develop VOD was 11.8 days. All received defibrotide therapy within 2 days of diagnosis and aggressive supportive management. The dose of defibrotide was $25 \mathrm{mg} / \mathrm{kg} /$ day. 1 patient's dose was increased to $40 \mathrm{mg} /$ $\mathrm{kg} /$ day in view of progression of VOD. The median duration of defibrotide therapy was 14 days (6-21). 3/13 patients died at day $100(23 \%)$ and a total of $6 / 13$ patients had died at 1 year $(46 \%)$. However none of the cause of the late mortality was due to VOD. The reasons were relapse in 2 , sepsis in 2 , liver GvHD (biopsy proven) in 1 and drug induced liver failure in 1 During this time period VOD occurred in $7 / 20$ (35\%) patients who received IV busulphan as part of their conditioning whereas only $4 / 42$ (9.5\%) patients with a myeloablative transplant (Cy-TBI) and 2/101 (1.9\%) patients conditioned with FMC developed VOD. Busulphan based conditioning was statistically associated with the development of VOD as compared to the other conditioning regimens ( $p$ value $<0.0001)$. At diagnosis of VOD, all patients had a serum bilirubin $>56 \mathrm{umol} / \mathrm{L}$ (range $56-810$ ). $5 / 13$ of the patients had moderately elevated transaminases (range 20-270 U/L). APTT was the earliest and the predominant abnormality compared to PT. 12 patients had acute kidney injury which was managed conservatively without the need for renal replacement therapy.

3/6 survivors have normal LFT's, 2 patients have a residual mild increase in transaminases due to cGvHD, whereas 1 patient has a moderate increase in LFT's due to CGvHD.

Conclusion: Busulphan based conditioning were the most important risk factor for VOD. Myelofibrosis had a strong trend towards causing VOD (P-0.06). Early intervention with defibrotide along with supportive management was able to completely resolve VOD in most of the cases and the 100 day mortality was only $3 / 13$ (23\%). Only 1 death was directly attributed to VOD and 1 death each due to sepsis and biopsy proven drug induced liver failure.

Disclosure of Interest: None declared. 
0060

Validation of two new prognostic scores to predict nonrelapse mortality in patients undergoing reduced-intensity conditioning allogeneic hematopoietic cell transplantation P. Barba ${ }^{1, *}$, R. Martino ${ }^{2}$, G. Orti ${ }^{3}$, A. Esquirol ${ }^{2}$, S. Perez-Hoyos ${ }^{4}$, I. Garcia-Cadenas ${ }^{2}$, J. Sierra ${ }^{2}$, D. Valcarcel ${ }^{3}$

${ }^{1}$ HOSPITAL VALL HEBRON. AUTONOMOUS UNIVERSITY OF BARCELONA, ${ }^{2}$ HEMATOLOGY, HOSPITAL SANT PAU, ${ }^{3}$ HEMATOLOGY, HOSPITAL VALL HEBRON. AUTONOMOUS UNIVERSITY OF BARCELONA, ${ }^{4}$ INFORMATICS AND BIOSTATISTICS, VALL HEBRON INSTITUT DE RECERCA, BARCELONA, Spain

Introduction: In 2014, 2 new pretransplant predictive models of non-relapse mortality (NRM) for patients undergoing allogeneic hematopoietic cell transplantation (all-HCT) have been created based on modifications of the HCT Comorbidity Index (HCT-Cl) and the EBMT score. The first model (HCT-Cl/ age) consisted of the addition of an extrapoint for patients $>40$ years to the HCT-Cl (Sorror et al. JCO.2014). The other model developed by the ALWP of the EBMT combined 16 categories of the HCT-Cl and the EBMT score into an integrated score (Versluis et al. Leukemia. 2014). None of these models have been validated in independent cohorts.

Materials (or patients) and methods: We analyzed the predictive capacity of these new models and compared it with the $\mathrm{HCT}-\mathrm{Cl}$ and the EBMT score in a population of reducedintensity conditioning allo-HCT (allo-RIC) consecutively transplanted patients in 2 Spanish centers during an 11 year period (2000-2011). The scores of all models were calculated by a single investigator as originally defined. Risk-groups stratification was also performed as originally defined, except for the EBMT score in which patients were divided into low- (score 0 3 ), intermediate- (score 4-5) and high-risk (scores 6) according to percentiles 33,66 and 100 . For the $\mathrm{HCT}-\mathrm{Cl} / \mathrm{Age}$ patients scoring 0 points $(n=3)$ were grouped with those scoring 1-2 points. The predictive capacity of all models was calculated by means of the Harrell's c-statistic and were compared by calculating a $z$-score and $p$ values from the estimated standard error.

Results: A total of 232 patients were included. Median age at HCT was 55 years (range 18-71). Most patients received alloHCT from HLA identical sibling donors $(n=170,73 \%)$ mainly for acute myeloid leukemia and myelodisplastic syndromes $(n=73,32 \%)$. Median follow-up for survivors was 5.5 years (range 0.3-11). The median $\mathrm{HCT}-\mathrm{Cl} /$ age and the ALWP model scores were 4 (range 0-14) and 5 (range 1-15), respectively. The median HCT-Cl, EBMT scores were 3 (range $0-13$ ) and 5 (range 1-7), respectively. Risk group distribution of patients according to each model is summarized in Table 1 . The cumulative incidence of 5-year NRM for all patients was 31\% (95\% Cl 28 34). The cumulative incidence of 5-year NRM progressively increased in the low-, intermediate- and high-risk groups according to all models. The predictive capacity of the $\mathrm{HCT}-\mathrm{Cl} /$ age models and the ALWP integrated score for 5-years NRM were $0.617(95 \% \mathrm{Cl} 0.559-0.676)$ and $0.626(95 \% \mathrm{Cl} 0.567-$ $0.686)$, respectively, whereas the predictive capacity of the classical HCT-Cl and the EBMT score were $0.571(95 \% \mathrm{Cl} 0.511$ 0.632 ) and $0.573(95 \% \mathrm{Cl} 0.523-0.623)$, respectively. The HCT$\mathrm{Cl} /$ age c-statistic was significantly better than the HCT-Cl's $(P=0.04)$ while the ALWP model showed a trend $(P=0.1)$. The probability of OS for the whole cohort was $39 \%$ (95\% Cl 36-42) and the cumulative incidence of 5-year relapse was 33\% (95\% Cl 30-36). The predictive capacity of all models for overall survival and relapse was weaker than for NRM (c-statstics $<0.6$ ).

Conclusion: The $\mathrm{HCT}-\mathrm{Cl}$ /age and the ALWP integrated model yielded relatively strong prediction for 5-year NRM in an independent cohort of allo-RIC population with large follow-up.

Disclosure of Interest: None declared.

\begin{tabular}{|c|c|c|c|}
\hline & $N(x)$ & $\begin{array}{c}\text { NKM } \\
\therefore \text { (95\% (1) }\end{array}$ & $\frac{05}{3,5(1)}$ \\
\hline \multicolumn{4}{|l|}{ HCT.CI } \\
\hline $0_{\text {proints }}$ & $24(10)$ & $13(4-25)$ & $57(17-57)$ \\
\hline 1-2. points & $47(2 x)$ & $25(17-33)$ & $4 y(41-57)$ \\
\hline$\geq 3$ potas & $161(69)$ & $36(31-41)$ & $33(29.37)$ \\
\hline Comxisal as & & $\begin{array}{c}0.573 \\
(0.523-0.623)\end{array}$ & $\begin{array}{c}0.5 \% 2 \\
(0.539-0.608)\end{array}$ \\
\hline
\end{tabular}

\begin{tabular}{|c|c|c|c|}
\hline \multicolumn{4}{|l|}{ RBAT seore } \\
\hline 0.3 yolina & 51 (22) & $26(16-39)$ & $50(43.48)$ \\
\hline d-5 points & $139(60)$ & $29(22-38)$ & $37(33-12)$ \\
\hline Q-7 axiltx & 4).(18) & 15 (91-59) & $30(73-37)$ \\
\hline Comx:sal as & & $\begin{array}{c}0.571 \\
(0.510-0.631)\end{array}$ & $\begin{array}{c}0.574 \\
(0.516-0.622)\end{array}$ \\
\hline
\end{tabular}

\begin{tabular}{|c|c|c|c|}
\hline ALWP sonere & & & \\
\hline 0.3 polisa & 42 (18) & $24(14-20)$ & $42(35-51)$ \\
\hline 1.6 poins & $120(60)$ & $25(18-33)$ & $14(40-49)$ \\
\hline$\geq 7$ jxinin & SD (XX) & $57(49-70)$ & $1 x(12-34)$ \\
\hline Comectex & & $\begin{array}{c}0.602 \\
\text { (D)sed }\end{array}$ & $\begin{array}{c}0.566 \\
(0.515-0.007)\end{array}$ \\
\hline
\end{tabular}

\begin{tabular}{|c|c|c|c|}
\hline \multicolumn{4}{|l|}{ HCT.CV/Ag } \\
\hline $0-2$ paiss & 42.(18) & $x 7(19-35)$ & $44(36-5 x)$ \\
\hline $3-4$ youra & $94(11)$ & $31(26-3 \phi)$ & $52(47-58)$ \\
\hline$>9$ poins & 96 (11) & $54(43-60)$ & $24(20-29)$ \\
\hline Correcto & & $\begin{array}{c}0.617 \\
\text { (D.559-0670) }\end{array}$ & $\begin{array}{c}0.552 \\
(0.507-0.500)\end{array}$ \\
\hline
\end{tabular}

0061

The early assessment of the systemic inflammatory response syndrome score is able to predict acute graftversus-host disease and non-relapse mortality in patients undergoing an allogeneic stem cell transplantation C. De Philippis ${ }^{1, *}$, F. Spina ${ }^{1}$, L. Farina ${ }^{1}$, L. Roncari ${ }^{1}$, M. Soldarini ${ }^{1}$, A. Dodero ${ }^{1}$, V. Montefusco ${ }^{7}$, G. Perrone ${ }^{7}$, P. Corradini ${ }^{2}$

${ }^{1}$ Hematology, Fondazione IRCCS Istituto Nazionale dei Tumori, ${ }^{2}$ Hematology, Università degli Studi di Milano, Milan, Italy

Introduction: Systemic inflammatory response syndrome (SIRS) is defined as an inflammatory state induced by infections or toxic damages. SIRS is diagnosed when two or more of the following criteria are met: body temperature $<36$ C or $>38^{\circ} \mathrm{C}$, heart rate $>90$ beats/minute, tachypnea $>20$ breaths/minute or $\mathrm{PaCo} 2<32 \mathrm{mmHg}$, leukocytes $<4000$ cells/ $\mathrm{mm}^{3}$ or $>12000$ cells $/ \mathrm{mm}^{3}$ or presence of $>10 \%$ immature neutrophils. The goal of this study was to assess the incidence of SIRS early after an allogeneic stem cell transplantation (alloSCT) (from day 0 to hematopoietic recovery) and evaluate whether SIRS may influence the occurrence of acute 
graft-versus-host disease (aGVHD) and non-relapse mortality (NRM).

Materials (or patients) and methods: Data from 256 patients with hematological malignancies (83\% lymphoid, $17 \%$ myeloid), who underwent an alloSCT from 2003 to 2012 at our center were retrospectively collected. The median age was 48 (range, 15-68): 48\% were in complete remission (CR) before transplant, $29 \%$ in partial remission (PR) and $23 \%$ in stable/ progressive disease (SD/PD). The median number of previous therapies was 3 (range, $0-8$ ), and $71 \%$ failed an autologous transplant. The hematopoietic-cell-transplantation comorbidity index (HCT-Cl) was $0-1$ in $71 \%$ and $\geq 2$ in $29 \%$ of the patients. Patients received stem cells from a sibling (matched $40 \%$, haploidentical $18 \%$ ) or an unrelated (42\%) donor. Myeloablative or reduced intensity conditioning regimens were used in $25 \%$ and $75 \%$ of alloSCT, respectively.

Results: One hundred eighty nine $(73 \%)$ patients had at least one SIRS episode (score $2 n=98$, score 3-4 $n=91$ ). The causes of SIRS were: infectious (61\%), toxic (10\%), and the remaining cases were judged as not clinically relevant (29\%). The incidence of aGVHD until day 100 was $38 \%$, with $18 \%$ of patients developing grade $\geq 2$ aGVHD. NRM rate was $5.5 \%$ at 100 days and $10 \%$ at one year. Patients who developed SIRS experienced aGVHD more frequently $(44 \%$ vs $21 \%, P<0.001)$. The correlation was maintained even when the analysis was limited to patients with aGVHD $\geq 2$ and/or those with SIRS $\geq 3$ $(P<0.01)$. In particular, aGVHD was correlated with infectious and toxic SIRS $(P<0.001)$. The occurrence of SIRS $\geq 3$ as well as of aGVHD predicted a worse NRM $(P=0.004$ and $P<0.001$, respectively). One hundred-day and 1 -year NRM was $9 \%$ vs $4 \%$ and $15 \%$ vs $7 \%$ in patients with or without $\operatorname{SIRS} \geq 3$, respectively. In multivariate analysis, taking into account age ( $<$ vs $\geq 55$ years), previous autologous transplant, previous therapies ( $<$ vs $\geq 3$ lines), disease status at transplant (CR vs PR vs SD/PD), HCT-Cl (0-1 vs $\geq 2$ ), donor type (sibling vs matched unrelated vs haplo) and conditioning regimen, only transplant from haploidentical donors was correlated with the onset of SIRS $(P<0.01)$. In multivariate analysis that took into account patients characteristics as above and SIRS score, SIRS score $\geq 2$ was an independent variable impacting on the NRM regardless of donor type: in particular every increase in SIRS score was correlated with 1.55 fold increase in NRM $(P=0.04)$.

Conclusion: In our retrospective analysis we observed a significant correlation between the early development of SIRS during the neutropenic phase, the onset of aGVHD and NRM. The SIRS score may therefore represent a simple and sensitive tool able to predict patients at high risk of aGVHD and NRM.

Disclosure of Interest: None declared.

0062

No post-transplant PRCA development in 106 major ABO incompatible cord blood transplantation: A single center analysis

S. Wada ${ }^{1, *}$, Y. Asano-Mori ${ }^{1}$, H. Yamamoto ${ }^{1}$, M. Yuasa $^{1}$ K. Kageyama' ${ }^{1}$ D. Kaji ${ }^{1}, A$. Nishida ${ }^{2}$, K. Ishiwata ${ }^{7}, M$. Tsuji $^{2}$, S. Takagi', G. Yamamoto' , N. Uchida' ${ }^{i}$ K. Izutsu' ', S. Makino ${ }^{3}$, A. Wake ${ }^{2}$, S. Taniguchi ${ }^{1}$

${ }^{7}$ Department of Hematology, Toranomon Hospital, Tokyo, ${ }^{2}$ Department of Hematology, Toranomon Hospital Kajigaya, Kawasaki, ${ }^{3}$ Department of Transfusion Medicine, Toranomon Hospital, Tokyo, Japan

Introduction: Pure red cell aplasia (PRCA) after allogeneic hematopoietic stem cell transplantation (HSCT) is a relatively rare complication after major $A B O$ incompatible allogeneic transplant. Although reduced intensity transplant was a potential risk factor for PRCA, the impact of stem cell source has not been fully evaluated. We conducted a retrospective risk factor analysis for developing PRCA in 163 major ABO incompatible transplant including 106 cord blood transplantation (CBT) and 74 reduced-intensity conditioning.
Materials (or patients) and methods: We reviewed the medical records of 668 adult patients who underwent allogeneic HSCT for the first time at the Toranomon hospital from 2006 to 2013. PRCA after HSCT was defined as anemia with low reticulocyte counts $(<1 \%)$ in peripheral blood for more than 60 days after transplantation in association with neutrophil engraftment and a lack of erythroid precursors in bone marrow.

Results: One-hundred and sixty-three patients with major or bi-directional $A B O$ incompatibility who achieved neutrophil engraftment and survived more than 60 days after HSCT were included in this study. Seventy four patients received reducedintensity conditioning, 106 patients underwent CBT, 39 did bone marrow transplantation (BMT) and 18 did peripheral blood stem cell transplantation (PBSCT). Reticulocyte engrafted (reticulocyte $>=1 \%$ ) in 160 patients with a median time of 29 days after HSCT during this study, which was significantly longer after CBT compared to BMT/PBSCT (31 days vs. 26 days, $P=0.008$ ). In 9 patients, reticulocyte count remained $<1 \%$ beyond 60 days post-transplant, 5 of whom were diagnosed as PRCA with a cumulative incidence of $3.1 \%$. PRCA was not observed in CBT patients, and the cumulative incidence of PRCA was significantly lower after CBT compared to BMT/PBSCT ( $0 \%$ vs. $8.9 \%, P=0.002)$. Four patients had blood group $\mathrm{A} / \mathrm{O}$ donor/recipient pairs. In multivariate analyses, CBT was identified as the only factor which significantly decreased a risk of PRCA (HR 5.8x10e-6, $\mathrm{P}<0.001)$, in contrast that blood group $A / O$ donor/recipient pairs was a significant risk factor of developing PRCA (HR 12.6, $P=0.02$ ). Reduced-intensity conditioning, reported as a significant risk factor in earlier study, did not affect PRCA development in our study $(P=0.51)$. All patients with PRCA achieved reticulocyte engraftment at a median of 261 (85-428) days after HSCT spontaneously or after withdrawal of immunosuppressants. PRCA did not affect non-relapse mortality (PRCA vs. no PRCA HR 0.71, $P=0.72$ ).

Conclusion: Blood group A/O donor/recipient pairs and nonCBT were identified as risk factors for post-transplant PRCA in multivariate analysis. Of note, the fact that no PRCA development was observed in over 100 CBT recipients may suggest different mechanisms of erythroid precursor development unique to $C B T$ recipients.

Disclosure of Interest: None declared.

\section{3}

Incidence, clinical characteristics and treatment of isolated extramedullary relapses after allogeneic hematopoietic stem cell transplantation for acute leukemias: an updated single-center analysis of $\mathbf{5 9 5}$ patients

D. Kata ${ }^{1, *}$, S. Kyrcz-Krzemień ${ }^{1}$, M. Markiewicz ${ }^{1}$, M. Kopera ${ }^{1}$, A. Wacławik', K. Białas ${ }^{7}$

${ }^{1}$ Department of Hematology and BMT, Silesian Medical University, Katowice, Poland

Introduction: Isolated extramedullary (EM) relapses are increasingly observed late complications after allogeneic hematopoietic stem cell transplantation (allo-HSCT) for acute myeloid leukemia (AML) and acute lymphoblastic leukemia (ALL). However, an optimal therapeutic approach to isolated EM relapses following allo-HSCT remains uncertain.

Materials (or patients) and methods: We retrospectively analyzed incidence, clinical features, treatment options and long-term outcome of this pattern of leukemia recurrence in a cohort of 595 consecutive patients (pts) (228 with ALL, 367 with $\mathrm{AML}$ ) who underwent allo-HSCT in our center between June 1993 and December 2012. 95 pts (43 with ALL, 52 with $\mathrm{AML}$ ) relapsed (any site).

Results: 18 (19\%) out of all pts who relapsed ( 5 with high-risk B-line ALL, 2 with T-line ALL, 11 with AML, F/M 9/9, median age 33 years, range $23-60$ years) experienced histologically proven isolated EM relapse after a median time of 11 months (mts) (range, 5-80 mts) following allo-HSCT. 8 pts (5 with ALL, 3 with AML) developed skin and/or subcutaneous tissue infiltrates. 
Other sites of relapse included (No. of cases/diagnosis): central nervous system (2/ $A L L, 1 / A M L)$, paraspinal soft tissues (1/AML), small intestine (1/AML), lymph nodes (1/AML), paranasal sinuses (1/AML), pleura (1/ALL), breast (1/AML), retro-orbital region (1/ALL).

Treatment plans for those isolated EM relapses included (No. of cases/diagnosis): 1/ involved-field radiotherapy (IF-RT) (2/ALL, 2/AML), 2/ IF-RT followed by chemotherapy (CHT) and interferon-alpha (2/ ALL), 3/imatinib $+\mathrm{CHT}+$ steroids and methotrexate intrathecally (1/ ALL), 4/ imatinib + CHT (1/ ALL), 5/ CHT (4/ AML, 3/ALL), 6/ dasatinib (1/ CD117+ $A M L), 7 /$ surgery (1/AML), 8/ CHT and secondary alloHSCT (1/ALL, 2/AML). 13/18 pts died after a median time of $9 \mathrm{mts}$ (range, 1-30 mts) due to resistant systemic relapse and/or infectious complications, 5/18 pts are currently under IF-RT/ CHT or after secondary allo-HSCT.

Conclusion: Our data indicate that EM disease following alloHSCT affects a significant proportion of pts with acute leukemias.

Sites of EM relapses vary widely among the pts with skin and/ or subcutaneous tissue being frequently involved. IF-RT seems to be effective initial treatment option, but it does not prevent from systemic relapse. An aggressive approach combined of local and systemic therapy including secondary allo-HSCT can produce favorable response in a percentage of patients with isolated EM relapses.

Disclosure of Interest: None declared.

\section{4}

Cytotoxic T lymphocyte expansions after allogeneic hematopoietic transplantation: absence of STAT3 mutation and correlation with reduced-intensity conditioning and early post-transplant events

J. Muñoz-Ballester ${ }^{1}$, C. Castilla ${ }^{1}$, T. Chen-Liang ${ }^{1}$, A. M. Hurtado ${ }^{1}$, P. Iniesta ${ }^{1}$, M. D. García-Malo ${ }^{1}$, J. B. Nieto ${ }^{1}$, F. De Arriba ${ }^{\prime}$ F. J. Ortuño ${ }^{1}$, M. L. Lozano ${ }^{1}$, I. Heras ${ }^{1}$, V. Vicente ${ }^{7}$, A. Jerez ${ }^{1, *}$

${ }^{1}$ Hematology and Oncology Department, Hospital Universitario Morales Meseguer. Centro Regional de Hemodonación. IMIB, Murcia, Spain

Introduction: Up to now there are few studies that evaluate cytotoxic T lymphocyte (CTL) expansions development after allogeneic hematopoietic cells transplantation (AHCT) in a large series of patients. The characteristics of these expansions are not well known in the emerging reduced-intensity conditioning (RIC) setting The recent description of somatic STAT3 mutations in $30 \%$ of large granular lymphocyte leukemias (LGL) facilitates the distinction between exacerbated physiological CTL and true neoplasia.

Materials (or patients) and methods: Patients undergoing AHCT in our hospital were included if they met two criteria: Systematic longitudinal determination of lymphocyte subpopulations by flow cytometry and a minimum of 270 days of post-transplant follow-up. We defined persistent CTL expansion as those cases where the CD8 $+/ C D 4+$ ratio was higher than 1.5 for at least 6 months.

Results: Persistent CTL expansion was detected in 73 patients (47\%) out of a total of 154 patients included. In every case the presence of LGL in morphological analysis of peripheral blood smear was observed. Screening for STAT3 mutations was negative in all cases; in $65 \%$ cases a TCR rearrangement was detected and, in eleven of these patients, an expanded immunophenotype analysis showed a NK marker (CD57 and / or CD56). The CTL expansion development was more frequent in patients receiving RIC $(P=0.04)$, presenting HLA disparity $(P=0.06)$, in unrelated donor transplantation $(P=0.004)$, those who developed aGVHD $(P=0.01)$, moderate to severe aGVHD $(P=0.023)$ and those who had CMV reactivation before day $+100(P=0.02)$. In $88 \%$ of patients CTL was already present on day $+100,82 \%$ kept it oneyear later, $41 \%$ kept it at 2 years and in no case it was detected beyond 4 years.

Conclusion: Our study, the first to include a large cohort of allogeneic transplantation with RIC, shows a higher cytotoxic T lymphocyte expansions frequency in this conditioning setting. Both dynamics of expansions and the absence of STAT3 mutations suggest the benign course of the $T$ lymphocyte expansion. These expansions are related to early postransplant events as CMV reactivation and aGVHD development.

Disclosure of Interest: None declared.

\section{Oral Session: Paediatric diesases WP}

\section{5}

Immune Reconstitution of Children with Fanconi Aplastic Anemia After Allogeneic Hematopoietic Stem Cell Transplantation

A. Birbilen ${ }^{1}$, B. Kuşkonmaz ${ }^{1}$, M. Çetin ${ }^{1}$, D. Uçkan ${ }^{1}$, F. Gümrük ${ }^{1}$, B. Tavil ${ }^{1, *}$

${ }^{1}$ Hacettepe University Faculty of Medicine, Pediatric Hematology Unit, Ankara, Turkey

Introduction: Fanconi anemia (FA) is an autosomal recessive or X-linked inherited disorder characterized by bone marrow failure/aplasia. Hematopoietic stem cell transplantation (HSCT) is the only curative treatment in FA. Immune reconstitution after HSCT is increasingly recognized as a critical determinant of morbidity and mortality in HSCT. The aim of the study was to better understand the kinetics of immune reconstitution in children with FA who underwent allogeneic HSCT after a Fludarabine based reduced intensity conditioning regimen.

Materials (or patients) and methods: In this study, lymphocyte subgroups of children who underwent HSCT were evaluated before HSCT and 1, 3, 6, 12, and 24 months after HSCT. Children with FA (n:21) comprised the study group and children with non-malignant diseases ( $n: 36)$ comprised the control group. In addition to classical lymphocyte subgroups; activated $\mathrm{T}$ lymphocyte subgroups including CD8/57(+), CD8/56(+), CD3/HLA-DR $(+), C D 4 / 25(+), C D 4 /$ $28(+)$ T lymphocytes were evaluated in study and control groups.

Results: When absolute levels of lymphocyte subgroups were evaluated in children with FA, CD3 $(+)$ lymphocyte count returned to pre-HSCT levels at 12 months. CD4(+) T lymphocyte count reached to pre-HSCT levels at 24 months. $\mathrm{CD} 8(+)$ T lymphocyte count returned to pre-HSCT levels at 3 months. CD19(+) B lymphocyte count turned to pre-HSCT levels at 3 months. CD4/8 ratio returned to pre-HSCT levels at 12 months. CD16/56(+)CD3(+) NK-T and CD16/56(+)CD3(-) NK lymphocytes returned to pre-HSCT levels within 1 month after HSCT. Among HSCT related complications; acute GvHD developed in $2 / 21$ (9.5\%) children in study group and in 10/36 (27.7\%) children in control group. On the other hand, chronic GvHD developed in $1 / 21$ (4.7\%) children in study group and in $5 / 36(13.8 \%)$ children in control group.

When specific subgroups reflecting lymphocyte activation were evaluated in study and control groups; activated CD8/ $56(+)$ NK lymphocyte and CD8/57(+) T lymphocyte count returned to pre-HSCT levels at 3 months in both groups. While activated CD3/HLA-DR(+) T lymphocyte count returned to pre-HSCT levels at 1 months in both groups; activated CD3/ HLA-DR(+) T lymphocyte count was higher at 1, 6, and 12 months in control group. Activated CD4/25(+ ) T lymphocytes returned to pre-HSCT levels at 12 months in study group and those returned to pre-HSCT levels after 24 months in control groups. CD4/25( + ) activated T lymphocyte count was higher at 24 months in study group $(p \leq 0.05) . C D 4 / 28(+)$ activated T lymphocytes reached pre-HSCT levels at 12 months in control group and at 24 months in study group. Besides, CD4/28(+) activated T lymphocyte count was higher at 6 and 12 months in control group $(p \leq 0.05)$. 
Conclusion: In this study, we show that the kinetics of recovery of the lymphocytes subgroups in children with FA after HSCT follows those patterns also described for children with other diseases: early recovery of NK cells (1 month), followed by effector cytotoxic T cells ( 3 months) and B cells ( 3 months), and finally, CD4( + ) T-helper cells (24 months). High levels of CD3/DR $(+)$ activated T lymphocyte count at 1,6 , and 12 months and high levels of CD4/28( +) T lymphocyte count at 6 and 12 months in control group are attributable to the high frequencies of acute and chronic GvHD in control group than those of study group.

Disclosure of Interest: None declared.

\section{6}

Hematopoietic stem cell transplantation following a Treosulfan based preparative regimen for children with myelodysplastic syndrome: preliminary results of a EWOG-MDS study

B. Strahm ${ }^{1, *}$, M. Albert ${ }^{2}$, P. Bader ${ }^{3}$, M. Dworzak ${ }^{4}$, J. Stary ${ }^{5}$, R. Masetti ${ }^{6}$, S. Matthes-Martin ${ }^{7}$, P. Sedlacek ${ }^{5}$, K. W. Sykora ${ }^{8}$, T. Vraetz ${ }^{1}$, F. Locatelli ${ }^{9}$, C. Niemeyer ${ }^{7}$

${ }^{1}$ Department of Pediatric Hematology and Oncology, University of Freiburg, Freiburg, ${ }^{2}$ Department of Pediatric Hematology and Oncology, Dr. von Haunersches Children's Hospital, LudwigMaximilians-University, München, ${ }^{3}$ Department for Children and Adolescents Medicine, University Hospital Frankfurt, Goethe University Frankfurt am Main, Frankfurt, Germany, ${ }^{4}$ Department of Pediatrics, St. Anna Children's Hospital and Children's Cancer Research Institute, Vienna, Austria, ${ }^{5}$ Department of Pediatric Hematology and Oncology, Charles University and University Hospital Motol, Prague, Czech Republic, ${ }^{6}$ Department of Pediatric Hematology and Oncology, University of Bologna, Bologna, Italy, ${ }^{7}$ Department of Pediatrics, St. Anna Children's Hospital, Vienna, Austria, ${ }^{8}$ Pediatric Hematology and Oncology, Medical School Hannover, Hannover, Germany, ${ }^{9}$ Department of Pediatric Hematology and Oncology, IRCCS Ospedale Bambino Gesu, Rome, Italy

Introduction: Hematopoietic stem cell transplantation (HSCT) remains the only curative treatment for children with myelodysplastic syndrome (MDS). However, HSCT following a myeloablative regimen consisting of Busulfan, Cyclophosphamide +/- Melphalan has historically been associated with considerable transplant related mortality (TRM) ranging from $19 \%$ in children with refractory cytopenia of childhood (RCC) up to $35 \%$ in adolescents with advanced MDS. Therefore, EWOG-MDS recommended the use of a Treosulfan based reduced toxicity regimen for selected patients groups with MDS.

Materials (or patients) and methods: Thirty-five patients (25 males/10 females) were diagnosed RCC (22) or advanced MDS (13). In RCC karyotypes at diagnosis were normal (17), trisomy 8 (1), monosomy 7/del 7q (3) or structural complex (1). Two patients with RCC and normal karyotype progressed to RCC with monosomy 7 or advanced MDS prior to HSCT. Advanced MDS was classified as refractory anemia with excess of blasts (RAEB) (7), RAEB in transformation (RAEBt) (1), MDS related acute myeloid leukemia (MDR-AML) (2) or MDS with myelofibrosis (3). Karyotype aberrations in advanced MDS included monosomy 7 (5), a structural complex karytotype (1), random aberrations (3) or no abnormalities (4). The median age at HSCT was 12.8 years (range 1.2 - 22.1) with an interval from diagnosis to HSCT ranging from 42 days -5.5 years (median 166 days). Patients were grafted from a matched sibling donor (8), an alternative matched family donor (2) or a matched unrelated donor (25). Stem cell source was bone marrow (25) or peripheral blood (10). All patients were prepared with thiotepa $(8-10 \mathrm{mg} / \mathrm{kg})$, treosulfan $42 \mathrm{mg} / / \mathrm{m}^{2}$ and fludarabine $\left(150-160 \mathrm{mg} / \mathrm{m}^{2}\right)$. Patients grafted from an alternative family or an unrelated donor received anti-thymocyte globuline for in vivo T-cell depletion.

Results: All patients experienced prompt engraftment with full donor hematopoiesis. Fifteen out of 35 patients developed
'II-IV acute Graft-versus-Host disease (GvHD) including 6 patients with ${ }^{\circ} \mathrm{III}-\mathrm{IV}$ acute GVHD. Mild and moderate chronic GVHD was observed in 2 and 3 patients, respectively. Four patients with advanced MDS at HSCT relapsed at a median time of 392 days (range 356-634 days). Of these, two are in continuous remission 2 and 2.8 years after a second allograft whereas the other two succumbed to subsequent relapses following a second transplant procedure. Two additional patients with RCC, one each with normal karyotype and a structural complex karyotype, suffered from transplant related mortality. The probability of overall survival (pOS) was analysed according to risk groups defining patients with RCC and normal karyotype or trisomy 8 as low risk group (16) and patients with RCC with chromosome 7 aberrations or a complex karyotype and advanced MDS as high risk group (19). After a median follow up of 1.6 years (0.2-5.3 years) the pOS for the low risk group was 94\% [82-100] compared to $71 \%$ [45-97] for the high risk group.

Conclusion: In summary, children and adolescents with MDS transplanted following a Treosulfan based regimen had an excellent outcome with complete engraftment and low toxicity. However, a longer follow up is needed to assess the efficacy of the regimen in patients at high risk for relapse.

Disclosure of Interest: None declared.

\section{7}

HLA haploidentical hematopietic stem cell transplantation after removal of $\alpha \beta+T$ lymphocytes for children with nonmalignant disorders: a fulfilled promise

A. Bertaina ${ }^{1, *}$, B. Lucarelli ${ }^{1}$, D. Pagliara ${ }^{1}$, P. Merli ${ }^{1}$, G. Palumbo ${ }^{1}$, D. Pende ${ }^{2}$, G. M. Milano', G. Li Pira', M. T. Romano', L. Moretta ${ }^{3}$, F. Locatelli ${ }^{7}$

${ }^{1}$ BAMBINO GESU HOSPITAL, Rome, ${ }^{2}$ Istituto di Ricovero e Cura a Carattere Scientifico, Azienda Ospedaliera Universitaria San Martino - Istituto Nazionale per la Ricerca sul Cancro, ${ }^{3}$ Istituto Giannina Gaslini, Genova, Italy

Introduction: Although HLA haploidentical HSCT has been largely employed in children with life-threatening nonmalignant disorders, the survival of patients given this type of allograft has been reported to be inferior to that of patients transplanted from a compatible unrelated volunteer (UV). We implemented a novel method of ex vivo T- and B-cell depletion based on the selective elimination of $\alpha \beta+T$ cells and B cells. We herein report an update of 31 children with non-malignant disorders who were given this type of allograft.

Materials (or patients) and methods: Twenty-two patients were males and 9 females, median age at HSCT being 3.5 years (range 0.3-13.2). Nine patients had severe combined immunedeficiency (SCID), 8 Fanconi Anemia (FA), 4 Severe Aplastic Anemia (SAA), 2 Thalassemia Major, 2 Hemophagocytic Lymphohistiocytosis (HLH) and 1 each Immunedeficiency with Polyendocrinopathy Enteropaty X-linked (IPEX), Kostmann Syndrome, Hyper IgE Syndrome, Osteopetrosis, SwachmannDiamond Syndrome and Congenital Amegakaryocytic Thrombocytopenia (CAMT). All patients were transplanted from 1 of the 2 parents ( 21 from the mother and 10 from the father), the median number of $\mathrm{CD}_{3} 4^{+}$and $\alpha \beta+\mathrm{T}$ cells infused being $22.57 \times 10^{6} / \mathrm{kg}$ and $4 \times 10^{4} / \mathrm{kg}$. The original conditioning regimen consisted of treosulphan and fludarabine (FLU) + thiotepa in 13 (9 SCID, 1 IPEX, 1 CAMT, 1 Kostmann Syndrome and 1 Swachmann-Diamond Syndrome), FLU and cyclophosphamide + single dose TBI in 12 (8 FA and 4 SAA) and busulphan, FLU and thiotepa in 6 (2 Thalassemia, 2 HLH, 1 Osteopetrosis and 1 Hyper IgE Syndrome). No patient received immunosuppression after HSCT. All patients received Fresenius rabbit ATG $(4 \mathrm{mg} / \mathrm{kg} /$ day) on days -5 through -3 before allografting and rituximab $\left(200 \mathrm{mg} / \mathrm{m}^{2}\right)$ to prevent EBV-related PTLD on day -1 .

Results: All patients but 6 engrafted, the median time to reach neutrophil and platelet recovery being 13 days (range 9-23) and 9 days (range 7-40), respectively. The 6 patients ( 2 with SAA and 1 each with thalassemia, FA, HLH and Osteopetrosis) 
who had primary graft failure were successfully re-transplanted ( 2 from the same parent, 3 from the other relative and 1 from an 1-HLA locus disparate UV). Grade I/II skin-only acute GVHD occurred in 4 patients, while no patient had chronic GVHD. Three patients died due to multi-organ failure secondary to cytomegalovirus (1 SAA) and adenovirus infection (1 CAMT and 1 SCID). No patient developed EBVrelated PTLD. With a median follow-up of 17.6 months (range 4-46), 28 out of the 31 patients are alive and disease-free, the 3-year DFS probability being $90.3 \%$ (Standard Error 5.3). Recovery of $\gamma \delta+\mathrm{T}$ cells was prompt, but $\alpha \beta+\mathrm{T}$ cells progressively ensued over time.

Conclusion: These data confirm that infusion of B-cell and $\alpha \beta+$ T-cell-depleted hematopoietic progenitors from an HLAhaploidentical parent is an effective option for children with either congenital or acquired non-malignant disorders. Acute GVHD involved only the skin and the absence of chronic GVHD is particularly noteworthy, considering the detrimental impact of this complication on the quality of life of children with nonmalignant disorders. Infusion of titrated numbers of donorderived $\alpha \beta+$ T cells, transduced with a suicide gene for controlling possible alloreactive reactions, could further optimize this type of allograft by accelerating adaptive immunity recovery.

Disclosure of Interest: None declared.

\section{8}

Genetic susceptibility to transplant-associated thrombotic microangiopathy

S. Jodele ${ }^{1, *}$, B. Laskin ${ }^{2}$, K. Zhang ${ }^{3}$, F. Zou ${ }^{4}$, C. Dandoy ${ }^{5}$, K. Myers $^{5}$, J. El-Bietar ${ }^{5}$, G. Wallace, J. Goebel ${ }^{6}$, A. Lane ${ }^{5}$, S. Davies ${ }^{5}$

${ }^{1}$ Bone Marrow Transplantation and Immune Deficiency, ${ }^{2}$ Nephrology, ${ }^{3}$ Human Genetics, Cincinnati Children's Hospital Medical Center, ${ }^{4}$ Human Genetics, Cincinnati Children's Hospital, ${ }^{5}$ Bone Marrow Transplantation and Immune Deficiency, ${ }^{6} \mathrm{Neoh}-$ rology, Cincinnati Children's Hospital Medical Center, Cincinnati, United States

Introduction: Transplant associated thrombotic microangiopathy (TMA) is frequent after HSCT, and in severe cases causes significant morbidity and mortality. Currently there are no data addressing individual susceptibility to transplant associated TMA. Frequent genetic variants in ADAMTS13 and complement genes have been described in other microangiopathies such as aHUS and TTP. We hypothesized that polymorphic variants in complement genes increase susceptibility to transplant associated TMA.

Materials (or patients) and methods: Genomic DNA was isolated from recipient blood pre-HSCT (recipient DNA, $n=77$ ) and at 100 days after transplant with documented full donor engraftment (donor DNA, $n=75$ ). Twelve candidate genes (ADAMTS13, CFH, CFI, CFB, MCB, THBD, C3, C5, CFD, CFHR1, CFHR3 and CFHR5 ) likely to play a role in terminal complement activation and vascular damage in TMA were sequenced using next generation sequencing technology. Observed variants were compared against dbSNP (NCBI). Novel variants were further evaluated using laboratory developed bioinformatic tools. Results were compared in patients with and without TMA. TMA was diagnosed using rigorous diagnostic criteria (Jodele et al, Blood 2014)

Results: DNA samples of 77 recipients and 75 corresponding donors were tested. Twenty three of $34(68 \%)$ subjects with TMA had complement gene variants as compared to 4 of 43 (9\%) controls without TMA $(P<0.0001)$. The median number of gene variants (of known or unknown significance) seen in recipients with TMA was $1(0-7)$, and $0(0-2)$ in those without TMA $(P<0.0001)$. Gene variants were more frequent in nonCaucasian than Caucasian recipients, $(3.1$ vs $0.8, P<0.0001)$. More than 3 variants were observed only non-Caucasian recipients with 5 of 6 dead due to severe TMA. Likely pathogenic variants previously described in other microangiopathies, were seen in ADAMTS13, CFH, CFB, CFHR5 and CFI, all in recipients with TMA. No known pathogenic variants were

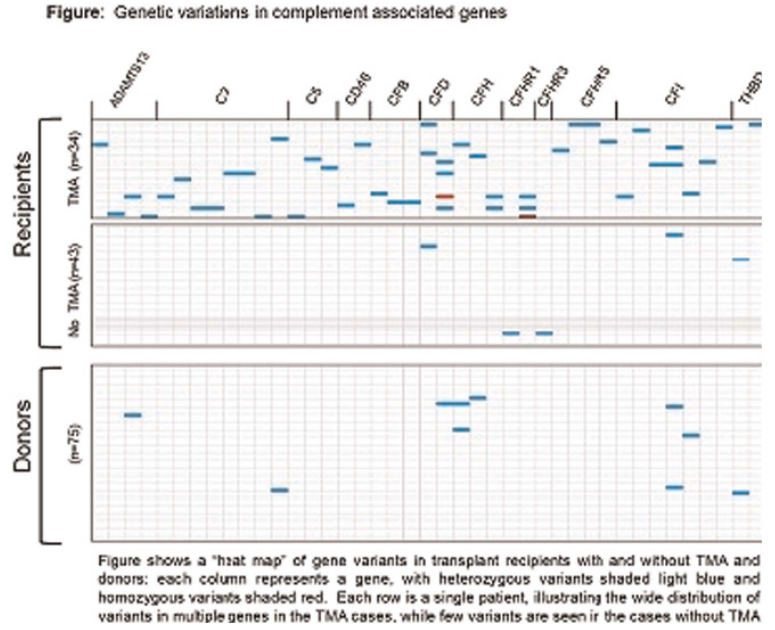

seen in recipients without TMA. Two recipients with TMA had homozygous deletion of CFHR3/R1, which was not seen in the recipients without TMA. Overall survival in non-Caucasian recipients with TMA was inferior as compared to Caucasian recipients with TMA $(P<0.04)$. Gene variants were infrequent in 75 healthy marrow donors, with a single gene variant found in 8 donors, and 2 variants found in one donor. Overall, donors, even non-Caucasian, had significantly less gene variants than HSCT recipients ( 0.18 vs $0.69, P=0.007$ ).

Conclusion: The incidence of complement gene variants is much higher in HSCT recipients with TMA, especially nonCaucasians, as compared to recipients without TMA and healthy donors. Transplant outcomes in non-Caucasian recipients with TMA are significantly inferior as compared to Caucasian recipients, indicating that genetic susceptibility importantly alters risk of TMA and may in part explain racial disparities in HSCT outcomes. Additional larger studies are needed to define the mechanistic importance of variants of unknown clinical significance.

Disclosure of Interest: S. Jodele Funding from: Research support by NIH P50DK096418 grant (Pediatric Center of Excellence in Nephrology: Critical Translational Studies in Pediatric Nephrology; Proteomics for early diagnosis of TATMA) and unrestricted research grant from Alexion Pharmaceuticals for complement genes in TA-TMA., Conflict with: Patent application pending: "Compositions and Methods for Treatment of HSCT-Associated Thrombotic Microangiopathy" (application number PCT/US2014/055922), B. Laskin Funding from: New Investigator Award from the American Society for Blood and Marrow Transplantation., Conflict with: Patent application pending: "Compositions and Methods for Treatment of HSCT-Associated Thrombotic Microangiopathy" (application number PCT/US2014/055922), K. Zhang: None declared, F. Zou: None declared, C. Dandoy: None declared, K. Myers: None declared, J. El-Bietar: None declared, G. Wallace: None declared, J. Goebel: None declared, A. Lane: None declared, S. Davies: None declared.

\section{9}

Methotrexate prophylaxis prevents severe aGvHD after HLA-identical sibling transplantation for pediatric ALL without compromising relapse risk

G. Lugthart, ${ }^{1, *}$, C. M. Jol-van der Zijde ${ }^{1}$, A. G. Van Halteren ${ }^{1}$, R. G. Bredius', M. J. van Tol', M. W. Schilham ', A. C. Lankester ${ }^{1}$ ${ }^{1}$ Dept. of Pediatrics, Leiden University Medical Center, Leiden, Netherlands

Introduction: Methotrexate (MTX) can be combined with ciclosporin to prevent the occurrence of acute graft-versushost disease (aGvHD) after hematopoietic stem cell transplantation (HSCT). In pediatric acute lymphoblastic leukemia (ALL) 
and myeloid leukemias, the omission of MTX is often recommended to favor a graft versus leukemia effect. However, the beneficial effect of this strategy after $T$ cell replete HLA-identical sibling transplantation for pediatric ALL is a subject of debate.

Materials (or patients) and methods: Between 2002 and 2013, 25 pediatric ALL patients received an unmanipulated bone marrow graft from an HLA-identical sibling after myeloablative (total body irradiation based) conditioning without serotherapy in the Leiden University Medical Center. In all children, aGvHD prophylaxis consisted of ciclosporin A (i.v., targeted plasma level 100-150 $\mu \mathrm{g} / \mathrm{L}$ ). Fourteen patients, transplanted before 2007 and in 2013, additionally received MTX $\left(10 \mathrm{mg} / \mathrm{m}^{2}\right.$ i.v. $)$ on day $+1,+3$ and +6 .

Results: Neutrophil engraftment occurred median 22 (range 834) days after HSCT and did not differ between non-MTX recipients and MTX recipients. However, non-MTX recipients had a more rapid lymphocyte reconstitution, reaching $>100$ lymphocytes/ $\mu \mathrm{L}$ at day 11-15 (median 15) after HSCT compared to day 15-28 (median 17) in MTX recipients $(P=0.003$, Mann-Whitney test). Three weeks after HSCT, T lymphocyte numbers did not differ significantly between the groups but within the T cell compartment, a skewing towards $\mathrm{CD}^{+}{ }^{+} \mathrm{T}$ cells was observed in non-MTX recipients (CD4/CD8 ratio 0.4 vs. $1.1, P=0.02$ ).

Grade II-IV aGvHD was observed in 1/14 MTX-recipients (onset day 41, grade III, steroid responsive) compared to $6 / 11$ nonMTX recipients $(P=0.007$, log rank test, Figure $1 \mathrm{a})$. In the latter group, aGVHD started median 21 days after HSCT and was $\geq$ grade III and steroid refractory in $3 / 6$ cases. A higher aGvHD rate was also observed in male patients who received a graft from a female donor ( $\mathrm{F}->\mathrm{M}$ mismatch) compared to all other sex-matches $(5 / 9$ vs. $2 / 16, P=0.02)$. In multivariate cox regression analysis, both omission of $\mathrm{MTX}$ and $\mathrm{F}->\mathrm{M}$ mismatch were associated with aGvHD occurrence. In line

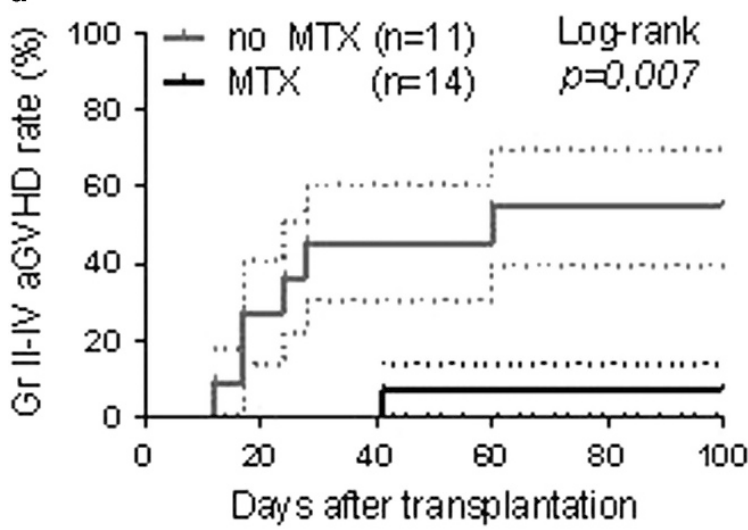

b

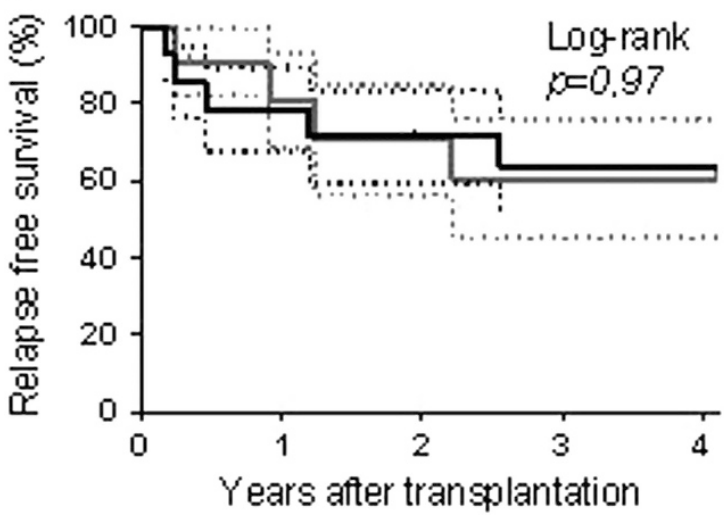

with this, the protective effect of MTX was most pronounced in the subcohort of patients with a F- $>\mathrm{M}$ mismatch: grade II-IV aGvHD occurred in 5/5 F- $>M$ mismatched non-MTX recipients whereas $0 / 4 \mathrm{~F}->M$ mismatched MTX recipients developed aGvHD $(P=0.005)$

ALL relapse occurred in $9 / 25$ patients (36\%). We did not observe differences in relapse rate between non-MTX recipients and MTX recipients (4/11 vs. 5/14, $P=0.97$, Figure $1 \mathrm{~b}$ ). Although non-MTX recipients were more often transplanted for ALL in $2^{\text {nd }}$ remission, pre-transplantation minimal residual disease (MRD) status did not differ between the groups. In line with earlier reports, MRD positivity was the most important risk factor for ALL recurrence. In MRD positive patients, the omission of MTX and aGvHD occurrence did not prevent relapse after HSCT.

Conclusion: MTX prophylaxis reduced the occurrence of severe aGvHD, without compromising relapse free survival in pediatric ALL patients after T cell replete HLA-identical bone marrow transplantation. Prevention of aGvHD reduces morbidity and the need for high-dose immunosuppressive agents, allowing for alternative immunotherapy-based therapeutic interventions in individuals at high risk for disease recurrence. Disclosure of Interest: None declared.

\section{0}

Outcome after Relapse or Progression following First Allogeneic Hematopoietic Stem Cell Transplantation in Children with Acute leukemia. A Retrospective Analysis from the French society of Bone Marrow Transplantation and Cell Therapies (SFGM-TC)

C. Roux ${ }^{1, *}$, K. Tifratene ${ }^{2}$, N. Raus ${ }^{3}$, G. S. Gerard Socie ${ }^{4}$,

C. Galambrun ${ }^{5}$, Y. Bertrand ${ }^{6}$, F. Rialland ${ }^{7}$, C. Jubert ${ }^{8}$, C. Pochon ${ }^{9}$, C. Paillard ${ }^{10}, A$. Sirvent ${ }^{17}$, B. Nelken ${ }^{12}, Y$. Beguin $^{13}$

I. Yakoub-Agha ${ }^{14}$, M. Mohty ${ }^{15}$, J.-H. Dalle ${ }^{16}$, G. Michel' ${ }^{17}$,

C. Pradier ${ }^{2}$, R. Peffault de latour ${ }^{18}$, P. S. Rohrlich ${ }^{1}$

${ }^{1}$ Hematology Dept, CHU Archet NICE, ${ }^{2}$ University CHU Nice, University CHU Nice, nice, ${ }^{3}$ Lyon, France, Société Française de Greffe de Moelle et de Thérapie Cellulaire (SFGM-TC), lyon, ${ }^{4}$ Hematology / Transplantation, Hematology / Transplantation, Saint Louis Hospital, Paris, France, paris, ${ }^{5}$ Pediatric Hematology Department, Hopital de La Timone, Marseille, France, Marseille, France, marseille, ${ }^{6}$ nstitute of Hematology and Oncology Paediatrics, Hospices Civils de Lyon, Lyon, France, Iyon, ${ }^{7}$ Pediatric Hematology, CHU de Nantes, nantes, ${ }^{8}$ Pediatric Hematology, Hôpital des Enfants, bordeaux, ${ }^{9}$ Pediatric Hematology, CHU Nancy-Brabois, Vandoeuvre les Nancy,, Vandoeuvre les Nancy, ${ }^{10}$ Centre de cancérologie et thérapie cellulaire pédiatrique INSERM CIC501, Centre de cancérologie et thérapie cellulaire pédiatrique INSERM CIC501, Clermont-ferrand " ${ }^{11}$ Pediatric Hematology, CHU montpellier, montpellier, ${ }^{12}$ Pediatric Hematology, eanne de Flandre Hospital, CHRU, Lille, Lille, France, ${ }^{13}$ Pediatric Hematology, CHU of Liège and University of Liège, liege, Belgium, ${ }^{14}$ Pediatric Hematology, CHRU de Lille, lille, ${ }^{15}$ Hematology Dept, Centre Hospitalier et Universitaire, Nantes, nantes, ${ }^{16}$ Pediatric Hematology, Hopital Robert Debré, paris, ${ }^{17}$ Service de Pédiatre et Hématologie Pédiatrique, Hôpital de la Timone Enfants, marseille, ${ }^{18}$ Hematology Dept, hôpital Saint Louis, paris, France

Introduction: Hematopoietic stem cell transplantation (HSCT) has contributed to improved outcome in childhood acute leukemia (AL). However, post-HSCT relapse is associated with a dismal prognosis and its optimal treatment remains unclear. We aimed to compare patients' related factors and treatment strategy, in case of relapse or progression post-allogeneic HSCT in children with AL in a recent ten-year period.

Materials (or patients) and methods: A total of 334 children who received a first allogeneic HSCT for ALL or AML from January 2000 to December 2009 experienced a relapse or progression thereafter. They were treated in the 33 centers of the SFGM-TC, among them 279 cases were analysable. Primary endpoint was overall survival (OS) after diagnosis of relapse or progression post first HSCT whatever the treatment post relapse was. 
Results: 279 patients (110 females and 169 males) with a median age of 8.8 years at transplantation (range: $0.52-17.99$ ) were included. Diseases were 149 ALL (B-ALL, $n=120$; T-ALL $n=29), 122$ AML. At transplantation, 233 patients were in complete remission (CR) (CR1 $n=121$, CR2 $n=94$, CR3 $n=18$ ) Donors were matched related siblings (36\%), mismatched related $(4 \%)$, matched unrelated $(18 \%)$, mismatched unrelated $(24 \%)$, unrelated without precision $(16 \%)$ or syngenic $(2 \%)$. Stem cell source was bone marrow, peripheral stem cells, umbilical cord blood in $65 \%, 12 \%, 23 \%$ of cases, respectively. Median time from diagnosis to first HSCT was 263 days. 91\% children received myeloablative conditioning and 55\% received TBI. Acute GVHD after first HSCT occurred in 157 patients (grade I $n=62$ grade II to IV $n=94$ ). Median delay from first HSCT to failure was 182 days. Treatments for relapse after first HSCT consisted in chemotherapy alone $(n=103)$, chemotherapy followed by second HSCT $(n=70)$, supportive and palliative care $(n=66)$, combination of chemotherapy and DLI $(n=27)$, or DLI only $(n=13)$. With a median follow up of 1315 days (range 58; 4182), median OS duration after relapse was 149 days. Median survival duration in days according to therapy was as follows [confidence interval]: DLI after chemotherapy $=407[156 ; 658]$, Second allograft: $391[264 ;$ 518], chemotherapy: 180 [121; 239], DLI alone: 140 [10; 270], Palliative care: 43 [33;53]. Cox model analysis showed the following hazard ratio, when comparing to the best line of treatment, i.e. chemotherapy + DLI; second allograft HR 1.41, $(P=0.20)$, chemotherapy alone; HR $2.05,(P=.008)$, DLI HR 2.00 $(P=0.08)$, palliative care $\mathrm{HR}=5,7,(P<.001)$. The time interval from transplantation to relapse was also associated with prognosis: when comparing to relapses occuring within the first 90 days, day $90-179$, day $180-269$, and $\geq 270$ days yielded hazard ratios of respectively.549,.479 and .293 ( $\mathrm{p} \leq .003$ ) There was no impact on outcome from the following variables: gender, age at transplant, leukemia subtype,type of conditioning, TBI, GVHD occurrence after the first transplant, stem cell source.

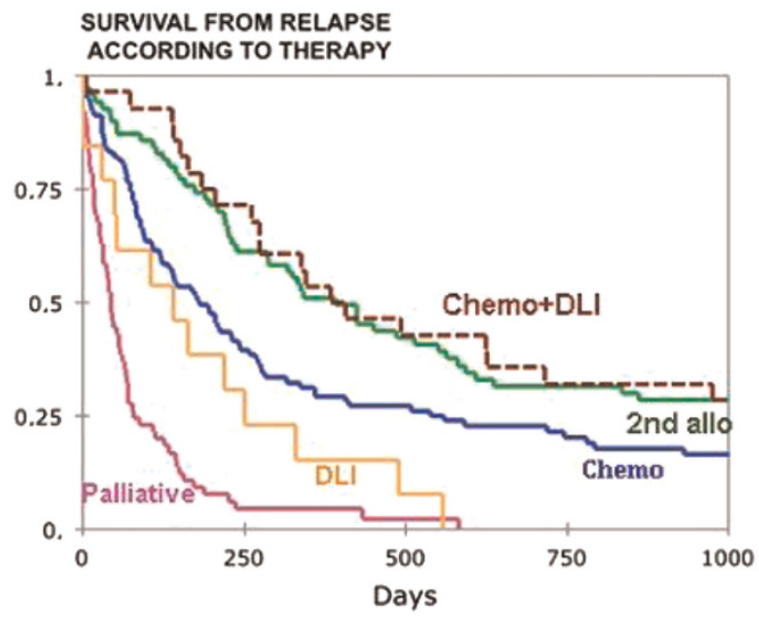

Conclusion: The therapeutic choice after post transplant relapse is a sensitive decision influenced by several factors. However, this study highlights the positive role of immunotherapy: the longest survival was achieved either through a second HSCT or through a combination of chemotherapy and DLI, both yielding similar long-term results.

Disclosure of Interest: None declared.
0071

NK-cell recovery after allogeneic HSCT occurs in the presence of circulating Thymoglobulin (ATG) or Alemtuzumab

E. Jol-van der Zijde, ${ }^{1, *}$, A. Jansen-Hoogendijk ${ }^{1}$, G. Hale ${ }^{2}$,

A. Lankester ${ }^{1}$, R. Bredius' ${ }^{7}$, M. van Tol ${ }^{1}$

${ }^{1}$ Pediatrics, LEIDEN UNIVERSITY MEDICAL CENTER, Leiden, Netherlands, ${ }^{2}$ Science and Technology Centre, University of Reading, Reading, United Kingdom

Introduction: Serotherapy with lymphocyte depleting antibodies, like anti-thymocyte globulin (ATG) and alemtuzumab, is frequently used in allogeneic stem cell transplantation (HSCT) to prevent GvHD and rejection. ATG recognizes many different antigens on all lymphocyte (sub)populations. Alemtuzumab depletes cells by targeting the CD52 antigen, which is expressed at different expression levels on $\mathrm{T}_{-}, \mathrm{B}-$-, and NK cells. ATG and especially alemtuzumab have a long half-life, and are detectable in the circulation for many weeks post HSCT.

It is known that especially T-cell recovery is delayed in alemtuzumab based conditioning regimens in comparison with ATG (Willemsen, BBMT 2014). However, the lower threshold of serum concentration of ATG or alemtuzumab at which different lymphocyte (sub)populations can recover is not defined.

Materials (or patients) and methods: The concentration of active ATG and alemtuzumab was longitudinally measured in sera of 198 and 63 children, respectively, transplanted between 2003 and 2014. Samples (mean 15 samples per patient) were tested by quantitative flow cytometry (active ATG) or by ELISA with an anti-idiotypic antibody (alemtuzumab). Lymphocyte (sub)populations were analysed frequently post transplantation by flow cytometry. CD3 + T-cell recovery was defined as appearance of $\geq 100$ cells $/ \mu \mathrm{L}$, B-cell and NK cells recovery as $\geq 50$ cells $/ \mu \mathrm{L}$.

Results: The median active ATG serum concentration at time of T-cell or B-cell reappearance was $0.03 \mathrm{AU} / \mathrm{mL}$, the maximum level was $1 \mathrm{AU} / \mathrm{mL}$. In $25 \%$ of the patients, NK cells re-appeared when the median active ATG level was higher $(0.16 \mathrm{AU} / \mathrm{mL})$ up to a maximum level of $7 \mathrm{AU} / \mathrm{mL}$ (see Figure). For alemtuzumab the median concentration at T-cell recovery was $0.008 \mu \mathrm{g} / \mathrm{mL}$, max. $0.2 \mu \mathrm{g} / \mathrm{mL}$, but in $80 \%$ of the patients NK cells reappeared at a higher concentration up to $2.2 \mu \mathrm{g} / \mathrm{mL}$. For both drugs, T-cell recovery was significantly correlated with the serum level of the drug $(P<0.001$ for ATG, and $P<0.01$ for $C 1 \mathrm{H})$, whereas a significant correlation was absent for NK cells.

Conclusion: ATG and alemtuzumab are both able to deplete $\mathrm{T}_{-}, \mathrm{B}-$, and NK cells. For both drugs, the exposure is highly

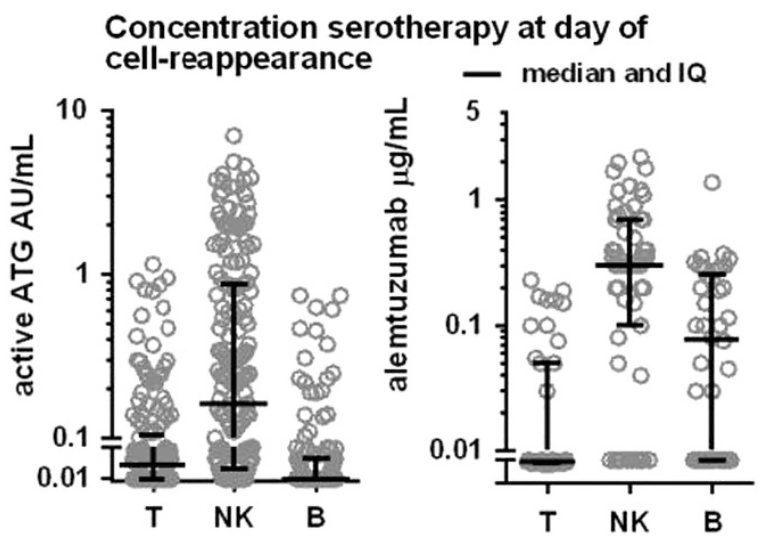


variable even in patients with an equal weight receiving the same dose. Here, we report that T-cell recovery is closely related with serotherapy exposure. NK-cells are the first cells that re-appear post HSCT. In this study we demonstrate for the first time that NK cell recovery post HSCT occurs in the presence of relatively high levels of circulating active ATG or alemtuzumab.

The expansion of NK cells in the presence of both alemtuzumab and ATG might explain the comparable overall incidence of viral reactivations between ATG and alemtuzumab treated patients despite the delayed T-cell reconstitution in alemtuzumab recipients.

References: Willemsen, L. et al. BBMT 2014 doi:10.1016/ j.bbmt.2014.11.674.

Disclosure of Interest: None declared.

0072

Hematopoietic stem cell transplantation with a reducedintensity conditioning regimen in pediatric patients with primary hemophagocytic lymphohistiocytosis

A. A. Hamidieh ${ }^{1,1, *}$, Z. Purpak ${ }^{2}$, M. Behfar ${ }^{1}$, M. R. Fazlollahi' ${ }^{2}$, A.-S. Hosseini ${ }^{1}$, M. Moin ${ }^{2}$, A. Ghavamzadeh ${ }^{1}$

${ }^{1}$ Hematology, Oncology and Stem Cell Transplantation Research Center, ${ }^{2}$ Immunology, Asthma \& Allergy Research Institute, Tehran University of Medical Sciences, Tehran, Iran, Islamic Republic Of

Introduction: All patients with Primary hemophagocytic lymphohistiocytosis $(\mathrm{HLH})$ experience severe crisis of $\mathrm{HLH}$, a life-threatening state of hyper inflammation. Hematopoietic stem cell transplantation (HSCT) remains the only curative treatment of choice for patients with primary HLH. Conditioning regimens used for HSCT in this group of patients is still a controversial issue.

Materials (or patients) and methods: Since 2007, in a prospective trial, reduced-intensity conditioning (RIC) regimen have been developed for 19 consecutive pediatric patients (9 female, 10 male) with primary HLH who were referred to our center. Seven patients were diagnosed with Familial HLH and 6 patients had Chediak-Higashi syndrome. Six other patients had Griscelli syndrome. Before transplantation all patients had experienced at least one $\mathrm{HLH}$ crises and were treated with $\mathrm{HLH}$ 2004 chemotherapy protocols. The median age at transplantation was 12 months (range: 4-72). All of the patients received the same RIC regimen based on the use of fludarabine in combination with melphalan and antithymocyte globulin (ATG). Prophylaxis against GvHD was achieved via cyclosporine and methylprednisolone.

Results: All patients were engrafted. The median times to neutrophil and platelet engraftments were 11 days (range: 8-33), and 22 days (range: 10-67), respectively. Patients underwent HSCT from HLA matched sibling donors $(n=9)$, full matched other related donors $(n=6)$, unrelated matched donor $(n=1)$ and unrelated mismatched donor $(n=3)$. The source of graft were peripheral blood $(n=11)$, bone marrow $(n=6)$ and cord blood $(n=2)$. The 24-month overall survival rate was $78.9 \%(S E=9.4 \%)$. 11 of 14 alive patients have full chimerism and the rest have mixed chimerism. All of them are disease free without any sign and symptom of underline disorder. 6 patients developed acute GvHD grade II-IV and 3 patients developed chronic GvHD. The causes of death were GvHD and infection.

Conclusion: With regard to severe and serious comorbidities in this group of patients, our recommendation is to use RIC regimen in primary HLH. It is suggested to carry out HSCT in these patients at lower ages, before presentations with different infections and HLH crisis. Recipients of RIC transplant, those with either full or mixed chimerism, had a long-term survival rate with no manifestation of primary HLH symptoms. Disclosure of Interest: None declared.
Oral Session: Inborn errors WP

\section{3}

Improved Outcome of HSCT in Infantile Osteopetrosis the Paris and UIm experience

A. S. Schulz ${ }^{1, *}$ on behalf of Inborn Erro Working Party,

M.-L. Fremond ${ }^{2,3}$, I. Furlan ${ }^{1}$, C. Schuetz ${ }^{1}$, M. Hoenig ${ }^{4}$, B. Neven ${ }^{3,5}$ S. Blanche ${ }^{2,3}$, W. Friedrich $^{7}$, A. Fischer ${ }^{2,3,6}$, D. Moshous ${ }^{2,3}$

${ }^{1}$ Department of Pediatrics, University Medical Center UIm, Ulm, Germany, ${ }^{2}$ Unité d'Immunologie, Hématologie et Rhumatologie Pédiatriques, Assistance Publique-Hôpitaux de Paris, Hôpital Necker-Enfants Malades, ${ }^{3}$ Institut Imagine, Université Paris Descartes-Sorbonne Paris Cité, Paris, France, ${ }^{4}$ Department of Pediatrics, University Medical Center UIm, Ulm, Guernsey,

${ }^{5}$ Assistance Publique-Hôpitaux de Paris, Hôpital Necker-Enfants Malades, Unité d'Immunologie, Hématologie et Rhumatologie Pédiatriques, ${ }^{6}$ Collège de France, Collège de France, Paris, France

Introduction: HSCT is a curative approach for patients with Malignant Infantile Osteopetrosis (MIOP), but is associated with high risk of complications such as SOS, pulmonary hypertension, rejection, autologous and delayed hematopoietic reconstitution. As a consequence, probabilities of 5 year disease free survival of transplanted MIOP patients using myeloablative Busulfan and Cyclophosphamid based conditioning regimens were reported to be relatively low with $73 \%$ for recipients of a graft from MSD, 40\% for recipients of a graft from MUD and $24 \%$ for patients, who received a graft from HLA haploidentical donors (EBMT report, 2003). In order to reduce treatment related toxicity, Cyclophosphamide was substituted by Fludarabine in the conditioning regimens. Here we report on the results of MIOP patients receiving HSCT after Busulfan and Fludarabine based conditioning in two large European centers.

Materials (or patients) and methods: Twenty-nine patients with MIOP were treated by HSCT at the University childreńs hospitals in Paris $(n=9$, since 2008) and UIm $(n=20$, since 1998). MIOP was caused by mutations in TCIRG1 $(n=20)$, CLCN7 $(n=5), \operatorname{SNX10}(n=1), \operatorname{RANK}(n=2)$, and FERMT3 $(n=1)$; age at transplant was between 2 and 72 months (median 6 months); donors were haploidentical family donors $(n=16), \operatorname{MUD}(n=7)$, phenoidentical relatives $(n=4)$, and MSD $(n=2)$. Thiotepa and serotherapy was added to the Busulfan and Fludarabine based regimen in all patients with donors other than MSD.

Results: All but 6 patients showed a primary and sustained engraftment; 4 of 6 patient, who rejected their haploidentical graft, could be rescued by a second graft from the second parent. Severe VOD, which had to be treated by ascites puncture, was seen in 2 patients only. Only one case of GvHD > 2 and no case of chronic GvHD was observed. Cause of death in 4 patients were liver toxicity in conjunction with CMV and fungal infection after prolonged aplasia (MUD transplant at age of 1 month) and complications in conjunction with engraftment failure ( 3 haplo transplants at age of 72 , 16 and 4.5 months, respectively). Twenty-five of 19 patients $(86 \%)$ survive and are free of disease with a follow up of 2 to 191 months (median 53 months).

Conclusion: In conclusion, patients with MIOP can be treated successfully by HSCT using a Busulfan and Fludarabine based conditioning regimen. In comparison to historical data using Cyclophosphamid containing regimens, the outcome improved significantly particularly in patients, who received grafts from matched unrelated or HLA haploidentical donors.

Disclosure of Interest: None declared. 
0074

Efficacy and Safety of Hematopoietic Stem Cell Therapy in Adrenoleukodystrophy: a Multi-Institutional Study

A. Paker, ${ }^{1, *}$, J.-H. Dalle, ${ }^{2}$ J. Balser ${ }^{3}$, S. Paedre ${ }^{3}$, M. Gérard ${ }^{4}$, M. Escolar ${ }^{5}$, P. Aubourg ${ }^{6}$, A. Fischer ${ }^{7}$, J. Kurtzberg ${ }^{8}$, A. Baruchel ${ }^{2}$, G. Raymond ${ }^{9}$, P. Orchard ${ }^{9}$

${ }^{1}$ bluebird bio Inc, Cambridge, United States, ${ }^{2}$ Hôpital RobertDebré, Paris, France, ${ }^{3}$ Veristat, Holliston, United States, ${ }^{4}$ Hôpital de la Timone, Marseille, France, ${ }^{5}$ University of Pittsburgh, Pittsburgh, United States, ${ }^{6}$ Hôpital Bicêtre, ${ }^{7}$ Hôpital NeckerEnfants Malades, Paris, France, ${ }^{8}$ Duke University, Durham, ${ }^{9}$ University of Minnesota, Minneapolis, United States

Introduction: Hematopoietic stem cell transplant (HCT) is the only therapy for childhood cerebral adrenoleukodystrophy (CCALD), but there is limited information about its outcome.

Materials (or patients) and methods: We conducted a retrospective study to characterize CCALD in untreated subjects and collect efficacy and safety data from HCT treated subjects. Data was collected from 5 centers (4 in the US and 1 in France) on 136 cases (72 untreated/65 HCT) from diagnosis until either 2 years post-diagnosis or death. Established measures of neurologic function (Neurological Functional Score $\left[N^{2} S^{1}\right]$ ) and MRI (Loes score ${ }^{2}$ ) were used. Severe disability of each functional domain of NFS that was identified as critical for independent functioning was labeled as a major functional disability (MFD; i.e., loss of communication, cortical blindness, requirement for tube feeding, total incontinence, wheelchair dependence \& complete loss of voluntary movement). Early CCALD was defined as NFS $\leq 1$, Loes $\leq 9$.

Results: In the Untreated Cohort, 70 of 72 (97\%) subjects had at least one NFS and MRI. 30 (42\%) had MRIs with contrast and 21 of these 30 subjects had contrast enhancing lesions on MRI $(\mathrm{GdE}+) . \mathrm{GdE}+$ was highly predictive of rapid progression. The majority of the GdE + subjects showed significant decline in NFS and Loes scores during follow-up period. Although overall survival rate for $\mathrm{GdE}+$ untreated subjects 2 years after diagnosis was $64 \%$, only $21 \%$ of subjects $(90 \% \mathrm{Cl} 7.5 \%>42 \%)$ were alive and MFD-free.

In the HCT cohort, 62/65 (95\%) subjects had at least one NFS and MRI; 52 (80\%) had MRIs with contrast (45 GdE + / 7GdE-). Engraftment failure occurred in 12 (18.5\%) subjects, with 10 $(18.8 \%)$ of these subjects receiving full myeloablation and 2 $(16.6 \%)$ receiving reduced intensity conditioning. $60 \%$ of subjects with engraftment failure had early CCALD.

Resolution of GdE + was seen in 40 (93\%) HCT subjects. Among the $27 \mathrm{GdE}+$ subjects with early CCALD, GdE + resolved in all subjects with a median time to resolution of 3.4 months (95\% Cl 2.3, 4.4 months).

Five subjects $(7.7 \%)$ died within first 100 days post-HCT. The 2year overall survival rate post-HCT was $82 \%$ and 2 -year MFDfree survival rate was $56 \%$. The 2-year MFD-free survival of the early CCALD GdE + subjects was $84 \%$.

The highest incidence of death occurred in subjects with a mismatched unrelated donor compared to all other donor types $(37.5 \%, P=0.041)$. These subjects also had lowest 2 -year MFD-free survival compared to all others $(38 \%, P=0.01)$. The 2-year MFD-free survival rate was $40 \%$ for mismatched related donors, $69 \%$ for matched related donors, and $82 \%$ for matched unrelated donors. A similar trend was also seen for subjects with early CCALD.

For all HCT subjects, the cumulative incidence of acute graftversus-host disease (GVHD) was $45 \%$ (26/58), with grade $3-4$ acute GVHD being $10.3 \%$. Subjects with a mismatched donor had the highest incidence of grade 3-4 acute GVHD 18.5\%. Incidence of chronic GVHD was $21 \%$ in all HCT subjects and $18.5 \%$ in subjects with mismatched donors.

Conclusion: We demonstrated that MRI enhancement is predictive of progression of CCALD, and that it rapidly resolves following HCT. Successful HCT improved survival and maintained function of subjects with early CCALD. There is a clear unmet need for novel therapies for patients without a well matched donor.
References: ${ }^{1}$ Moser HW, et al. Neuropediatrics 2000;31(5):227239.

2 Loes DJ, et al. Am J Neuroradiol 1994;15(9):1761-176.

Disclosure of Interest: A. Paker Employee of: bluebird bio (full time), J.-H. Dalle: None declared, J. Balser: None declared, S. Paedre: None declared, M. Gérard: None declared, M. Escolar: None declared, P. Aubourg: None declared, A. Fischer: None declared, J. Kurtzberg: None declared, A. Baruchel: None declared, G. Raymond: None declared, P. Orchard: None declared.

0075

Delivering optimal care for sickle cell patients on regular red blood cell exchange programme: the role of Vortex Ports, the Imperial College Healthcare NHS Trust experience

R. Haynes ${ }^{1, *}$, R. Eio ${ }^{1}$, L. Alexander ${ }^{1}$, O. Brokka ${ }^{1}$, K. Bradley ${ }^{7}$

${ }^{1}$ Haematology, IMPERIAL COLLEGE HEALTHCARE NHS TRUST, London, United Kingdom

Introduction: The centralisation of the haemoglobinopathy service at Hammersmith Hospital $(\mathrm{HH})$ resulted in increased numbers of sickle cell patients on regular red blood cell exchange (RBCX) programme. In order to address the increasing numbers of patients and to have an efficient RBCX service, the issue of central venous access devices (CVAD) came to the fore.

Materials (or patients) and methods: RBCX is a procedure to remove sickle cells and replace them with donated red blood cells to prevent further complications. RBCX increases oxygen carrying capacity and reducing thickening of the blood. In 2012, basing on the data of the efficacy of RBCXs via vortex ports (VP), a consensus decision was made to try VP in our apheresis practice. VP is an implantable venous access system placed under the skin on the chest or below the collar bone. A criteria was formulated for insertion of dual portacath Vortex port (VP) for sickle cell patients on RBCX programme. It is inserted in angiography unit or in theatre as a day case. To date we have 14 patients with VPs, and we have performed 120 RBCXs via VPs. In July 2013 a clinical audit was initiated on 13 patients with VPs. The audit requirements were to improve clinical outcomes and efficiency of VPs with RBCXs. The summary of the audit was positive and encouraging.

Results: The use of VP has doubly increased slots for RBCXs from 3 days to 5 days. It has also decreased in-patient admission. It shows the overall capability of VPs with RBCX and the benefit it has on sickle cell patient.

Conclusion: The use of VPs with RBCXs is relatively new. We do not have hard data on the overall efficacy for long term use of VP on RBCX especially on the issue of infection, and it is still too early to say what the long-term disadvantages might be. At present VP has improved and doubly increased RBCXs which in turn has decreased sickle cell inpatient admission at $\mathrm{HH}$. Our future plan is to do another audit on the infection rates and disadvantages of long term use of VPs on RBCXs and a survey satisfaction and quality of life of sickle cell patients with VPs.

Disclosure of Interest: None declared. 
0076

Parental Haploidentical HSCT with a Post-Infusion of Stem Cells Cyclophosphamide Approach is Feasible and Leads to a High Rate of Donor Engraftment in Haemoglobinopathies Allowing Universal Application of Transplantation

J. de la Fuente ${ }^{1,2, *}$, Y. Harrington ${ }^{2}$, A. Bradshaw ${ }^{1}$, B. Inusa ${ }^{3}$, P. Telfer 4

${ }^{1}$ Department of Haematology, ${ }^{2}$ Department of Paediatrics, IMPERIAL COLLEGE LONDON, ${ }^{3}$ Department of Paediatrics, Evelina Children's Hospital, ${ }^{4}$ Department of Paediatrics, Royal London Hospital, London, United Kingdom

Introduction: BMT is the only proven curative treatment available for haemoglobinopathies. However, the number of patients who can benefit is seriously restricted by the lack of HLA-matched related donors not suffering from the condition and the limited number of unrelated donors available for the ethnic groups in which these conditions are prevalent. In order to expand the donor pool, haploidentical transplantation with a post-infusion of stem cells cyclophosphamide approach has been developed for young adults, but whilst well tolerated it has resulted in relatively high rates of rejection and the need for a prolonged period of immunosuppression ${ }^{1}$.

Materials (or patients) and methods: 12 consecutive parental haploidentical transplants (11 for sickle cell disease and one for $\beta$ halassaemia major) were performed at St. Mary's Hospital, London, from June 2013 to November 2014. The median age was 9.5 years of age (range 3 to 14). All patients lacked a suitable HLA-matched related donor and an unrelated search had not identified a 10/10 or 9/10 donor. Endogenous haemopoieis was suppressed with hypertransfusions, hydroxycarbamide $30 \mathrm{mg} / \mathrm{kg}$ and azathioprine $3 \mathrm{mg} / \mathrm{kg}$ for at least two months pre-transplantation. The conditioning included fludarabine $150 \mathrm{mg} / \mathrm{m}^{2}$, thiotepa $10 \mathrm{mg} / \mathrm{kg}$ was added, cyclophosphamide $29 \mathrm{mg} / \mathrm{kg}, \mathrm{TBI} 2 \mathrm{~Gy}$ and ATG (Thymoglobulin) $4.5 \mathrm{mg} / \mathrm{kg}$. GvHD prophylaxis was provided with cyclophosphamide $50 \mathrm{mg} / \mathrm{kg}$ on days +3 and +4 , MMF and sirolimus. The minimum follow-up was 40 days post-transplantation and half of the patients are $>150$ days post-transplantation and have completed all treatment. The source of stem cells was G-CSF primed bone marrow in all cases, aiming $>8 \times 10^{8} \mathrm{TNC} / \mathrm{kg}$.

Results: All patients engrafted, though one patient subsequently suffered secondary graft failure following macrophage activation syndrome and died. The median neutrophil engraftment was 17 days (range 16 to 19). All 11 surviving patients are cured from the manifestations of the original disease. None of the patients suffered VOD, though infectious complications occurred at a higher rate than seen for related transplants for the same conditions at our institution. Five patients had no evidence of acute or chronic GvHD. Five patients developed stage 1 acute GvHD (median presentation day +36 , range 21 to 66 ) responding to topical steroids; one patient suffered skin GvHD stage 3 on day +35 and one patient gut GvHD stage 4 on day +24 , both treated with MSC. All patients responded to first line treatment with no recurrence of disease. All patients but one achieved $>90 \%$ donor fraction both in whole blood and T cells. One patient requires immunosuppression beyond day +180 with stable $59 \%$ donor fraction in whole blood and $10 \%$ in T cells. Conclusion: Parental haploidentical transplantation providing sufficient myelosuppression to avoid rejection is feasible, leading to outcomes which approach the results for related transplantation and allowing all patients with haemoglobinopathies requiring a transplant to benefit fromm such treatment.

References: 1. Bolaños-Meade J, Fuchs EJ, Luznik L, Lanzkron SM, Gamper CJ, Jones RJ, Brodsky RA. HLA-haploidentical bone marrow transplantation with posttransplant cyclophosphamide expands the donor pool for patients with sickle cell disease. Blood. 2012 Nov 22;120(22):4285-4291.

Disclosure of Interest: None declared.

\section{7}

Haematopoietic stem cell transplantation for CD40 ligand deficiency: results from an EBMT Inborn Errors Working Party (IEWP) study

F. Ferrua ${ }^{1,2, *}$, V. Courteille ${ }^{3}$, A. Janda ${ }^{4}$, M. Slatter ${ }^{5}$, M. H. Albert ${ }^{6}$, H. Al-Mousa", B. Al-Saud", D. Balashov', Y. Bertrand ${ }^{9}$, C. Booth ${ }^{10}$ V. Bordon ${ }^{11}$, W. Czogala ${ }^{12}$, F. Dogu ${ }^{13}$, A. Fasth ${ }^{14}$,

R. Formankova ${ }^{15}$, J. Gozdzik ${ }^{12}$, C. Heilmann ${ }^{16}$, M. Hönig ${ }^{17}$

A. Ikinciogullari ${ }^{13}$, K. Kałwak ${ }^{18}$, S. J. Keogh ${ }^{19}{ }^{\prime}$ 'G. Kriván ${ }^{20}$,

A. Lange ${ }^{21}$, A. C. Lankester 22 , P. Ljungman ${ }^{23}$, I. Meyts ${ }^{24}$,

B. Neven ${ }^{25}$, E. Soncini ${ }^{26}$, F. Suarez ${ }^{27}$, N. Mahlaoui ${ }^{3}$, A. Fischer ${ }^{28}$,

E. G. Davies ${ }^{29}$, A. R. Gennery ${ }^{5}$ on behalf of EBMT IEWP

${ }^{1}$ Department of Paediatric Immunology and HSCT, Great North Children's Hospital, Newcastle upon Tyne, United Kingdom, ${ }^{2}$ Pediatric Immunohematology and Bone Marrow Transplantation Unit, IRCCS San Raffaele Scientific Institute, Milan, Italy, ${ }^{3}$ Stem Cell Transplantation for ImmunoDeficiencies in Europe (SCETIDE) Registry, Necker Enfants Malades University Hospital, Assistance Publique-Hôpitaux de Paris and Paris Descartes University, Sorbonne Paris Cité, Imagine Institute, Paris, France, ${ }^{4}$ Center for Paediatrics and Adolescent Medicine, University Medical Center Freiburg, Freiburg, Germany, ${ }^{5}$ Institute of Cellular Medicine, Newcastle University, Newcastle upon Tyne, United Kingdom, ${ }^{6}$ Pediatric Hematology/Oncology, Dr. von Hauner University Children's Hospital, Munich, Germany, ${ }^{7}$ Department of Pediatrics, King Faisal Specialist Hospital \& Research Center, Riyadh, Saudi Arabia, ${ }^{8}$ Department of Hematopoietic Stem Cell Transplantation, Federal Research and Clinical Centre of Paediatric Haematology, Oncology and Immunology named after Dmitry Rogachev, Moscow, Russian Federation, 9 Institut d'Hematologie et d'Oncologie Pediatrique, Hospices Civils de Lyon, Lyon, France, ${ }^{10}$ Department of Paediatric Immunology, Great Ormond Street Hospital NHS Foundation Trust, London, United Kingdom, ${ }^{11}$ Pediatric Hematology and Oncology, Ghent University Hospital, Ghent, Belgium, ${ }^{12}$ Department of Transplantation, University Children's Hospital in Krakow, Krakow, Poland, ${ }^{13}$ Department of Pediatric Immunology and Allergy, Ankara University School of Medicine, Ankara, Turkey, ${ }^{14}$ Dept of Pediatrics, University of Gothenburg, Gothenburg, Sweden, ${ }^{15}$ Department of Pediatric Hematology and Oncology, University Hospital Motol, Prague, Czech Republic, ${ }^{16}$ Paediatric Clinic, Rigshospitalet, Copenhagen, Denmark, ${ }^{17}$ Department of Pediatrics and Adolescent Medicine, University Medical Center, Ulm, Germany, ${ }^{18}$ Department of Pediatric Hematology/Oncology and BMT, Wroclaw Medical University, Wroclaw, Poland, ${ }^{19}$ Oncology Unit, The Children's Hospital Westmead, Westmead, New South Wales, Australia, ${ }^{20}$ Dept. of Pediatric Hematology and Stem Cell Transplantation, United St. Istvan and St Laszlo Hospital, Budapest, Hungary, ${ }^{21}$ Institute of Immunology and Exp Therapy, Lower Silesia Centre for Cellular Transplantation, Wrocław, Poland, ${ }^{22}$ Dep of Pediatrics/Div of Stem Cell Transplantation, Leiden University Medical Center, Leiden, Netherlands, ${ }^{23}$ Department of Hematology, Karolinska University Hospital, Stockholm, Sweden, ${ }^{24}$ Department of Immunology and Microbiology, Department of Pediatrics, University Hospitals Leuven, Leuven, Belgium, ${ }^{25}$ Unité d'immuno-hématologie pédiatrique, Hôpital Necker-Enfant Malades, Assistance Publique des Hôpitaux de Paris (APHP), Paris, France, ${ }^{26}$ Pediatric Oncohematology and BMT Unit, Spedali Civili, Brescia, Italy, ${ }^{27}$ Hématologie Adulte, Hôpital Necker, AP-HP, ${ }^{28}$ Scientific Director, Institut Imagine, Paris, France, ${ }^{29}$ Centre for Immunodeficiency, Great Ormond Street Hospital \& Institute of Child Health, London, United Kingdom

Introduction: CD40 ligand (CD40L) deficiency is a rare X-linked primary immunodeficiency, which causes recurrent sinopulmonary infections, Pneumocystis jirovecii and Cryptosporidium spp. infections, and autoimmunity. Long-term survival with supportive therapy only is poor, with hepatic disease representing the major cause of death. Currently, the only curative treatment is haematopoietic stem cell transplantation (HSCT).

Materials (or patients) and methods: We retrospectively collected data on outcome of HSCT to improve future patients' 
a

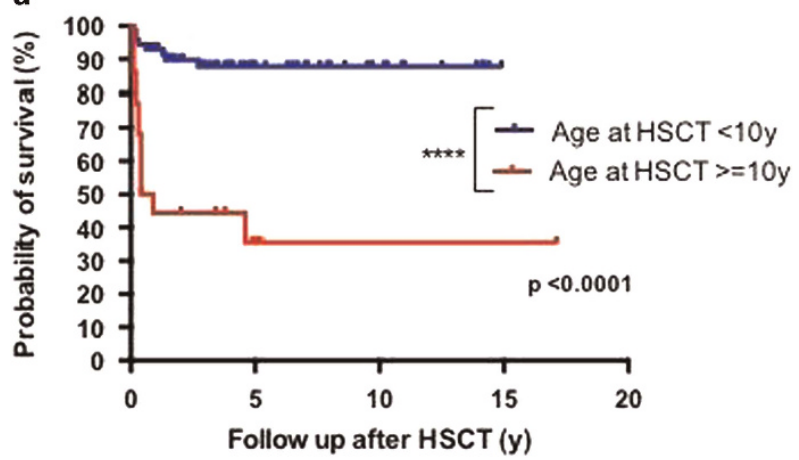

management. We present the preliminary analysis of 93 patients undergoing HSCT for CD40L deficiency between 1993-2014, reported in the EBMT and SCETIDE registries from 23 different centres, in 15 countries.

Results: Overall survival (OS) after HSCT was $77.4 \%$; results were better in transplants performed after year 2000 (OS 83\%) and in children $<10$ years of age (OS $88.7 \%$ - Figure $1 \mathrm{a}$ ) [median follow up (FU): 4.3 years (yrs), range: 0.4-17.1 yrs; median age at HSCT: 4.2 yrs, range: 0.6-38.3 yrs]. Moreover, survival reached $96.1 \%$ in patients with no chronic lung disease and/or liver alterations before HSCT (Figure 1b). Preexisting organ damage and severe GVHD resulted in poor outcome: incidence of chronic lung disease, Cryptosporidium infection and protracted diarrhoea before HSCT was higher in non-survivors $(P<0.02, P<0.02$ and $P<0.003$, respectively). In this group, liver disease was also significantly more frequent $(P<0.0005)$, especially sclerosing cholangitis $(P<0.0001)$ which was the most important adverse risk factor before transplant. Acute GVHD rate was higher in non-survivors $(P<0.05)$, in particular its severe form (grade $3-4, P<0.0001$ ) Donors included $27 \mathrm{HLA}$-identical siblings, 76 unrelated volunteers and 3 haploidentical parents, for a total of 106 procedures ( 8 second and 1 third HSCT, 4 boosts), of which 22 were T-cell depleted. In $41.6 \%$ of the transplants reducedintensity conditioning (RIC) was used. Most deaths occurred within 4 months of HSCT, predominantly from infections (47.6\%). Twelve patients (13\%) rejected their first transplant, eight of whom (66.7\%) received RIC. Donor chimerism $>50 \%$ was observed in $73.1 \%$ of survivors at last FU. In some of them decreasing chimerism was detected over time. Among those with FU >1 year, immunoglobulin replacement and prophylactic antibiotics were stopped in $78 \%$ and $69.2 \%$ of patients respectively.

Conclusion: Our preliminary results show that HSCT can be curative in CD40L deficiency, with improved survival if performed early, before the onset of organ damage, particularly sclerosing cholangitis. Further studies are required to determine the optimal conditioning regimen that ensures good rates of long-term engraftment and full immune reconstitution without increasing toxicity.

References: [1] Gennery A.R. et al. Treatment of CD40 ligand deficiency by hematopoietic stem cell transplantation: a survey of the European experience, 1993-2002. Blood. 2004 Feb 1;103(3):1152-1157. Epub 2003 Oct 2.

Disclosure of Interest: None declared. b

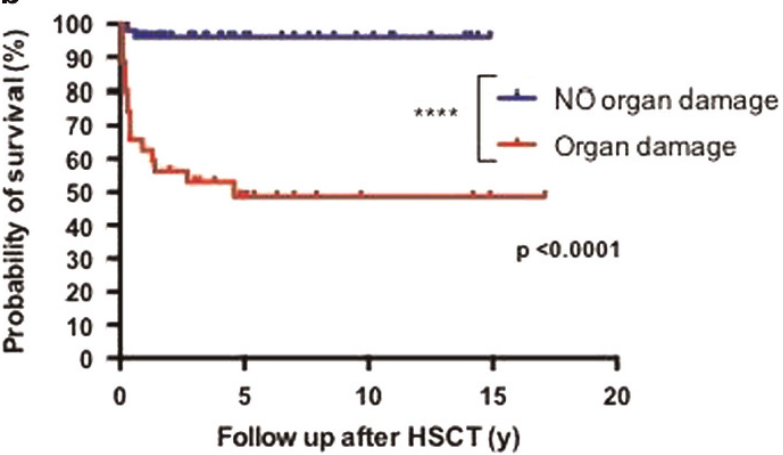

0078

Hematopoietic Stem Cell Transplantation in Sickle Cell Anemia children patients

A. Isgro ${ }^{1, *}$, K. Paciaroni ${ }^{1}$, J. Gaziev ${ }^{1}$, P. Sodani ${ }^{1}$, C. Gallucci ${ }^{1}$, M. Marziali ${ }^{1}$, G. De Angelis ${ }^{1}, C$. Alfieri ${ }^{1}, M$. Ribersani ${ }^{1}$,

O. O. Akinyanju ${ }^{2}$, T. T. Wakama ${ }^{3}$, F. O. Olowoselu ${ }^{4}$, A. Morrone ${ }^{1}$, G. Lucarelli ${ }^{1}$

${ }^{1}$ International Center for Transplantation in Thalassemia and Sickle Cell Anemia. Mediterranean Institute of Hematology, Policlinic University of Roma "Tor Vergata", Roma, Italy, 'Sickle Cell Foundation Nigeria, Lagos, ${ }^{3}$ National Hospital, Abuja, Abuja,

${ }^{4}$ Lagos University Teaching Hospital, Lagos, Nigeria

Introduction: Sickle cell anemia (SCA) remains associated with high risks of morbidity and early death. Children with SCA are at high risk for ischemic stroke and transient ischemic attacks, secondary to intracranial arteriopathy involving carotid and cerebral arteries. Allogeneic hematopoietic stem cell transplantation (HSCT) is the only curative treatment for SCA. We report our experience with transplantation in a group of children affected by SCA.

Materials (or patients) and methods: This study included 33 consecutive SCA patients who underwent bone marrow transplantation from human leukocyte antigen (HLA)-identical sibling donors between 2010 and 2014 following a myeloablative-conditioning regimen. Patients received fludarabine (30 $\mathrm{mg} / \mathrm{m}^{2} /$ day) for 5 days and a conditioning regimen including targeted intravenous busulfan ( $14 \mathrm{mg} / \mathrm{kg}$ total dose) and cyclophosphamide ( $200 \mathrm{mg} / \mathrm{kg}$ total dose).

Results: The median patient age was 10 years (range 2-17 years). Before transplantation, 16 patients had recurrent, painful, vaso-occlusive crisis; ten patients had recurrent painful crisis in association with acute chest syndrome; three patients experienced ischemic stroke and recurrent vaso-occlusive crisis; two patients experienced ischemic stroke; one patient exhibited leucocytosis; and one patient exhibited priapism. Of the 33 patients, 30 survived without sickle cell disease, with Lansky/Karnofsky scores of 100, following a myeloablativeconditioning regimen. The probabilities of survival, SCA-free survival, and transplant-related mortality after transplant were $91 \%, 91 \%$, and $9 \%$, respectively. All surviving patients remained free of any SCA-related events after transplantation. Conclusion: The protocols used for the preparation to the transplant in thalassemia are very effective also in the other severe hemoglobinopathy as in the sickle cell anemia with more than $90 \%$ disease free survival. Today, if a SCA patient has a HLA identical family member, the cellular gene therapy through the transplantation of the allogeneic hemopoietic cell should be performed. Tomorrow, hopefully, the autologous genetically corrected stem cell will break down the wall of the immunological incompatibility.

Disclosure of Interest: None declared. 
0079

ATG vs. Thiotepa in addition to BuCy for low-risk BMT in severe thalassemia

L. Faulkner ${ }^{1, *}$, P. Marwah ${ }^{2}$, N. Yaqub ${ }^{3}$, R. Soni ${ }^{2}$, S. Khalid ${ }^{4}$, I. Fatima ${ }^{4}$, S. Khan Gilani ${ }^{4}$, T. Zahra ${ }^{4}$, L. Gooneratne, R. Dissanayake ${ }^{5}$, M. El Missiry ${ }^{7}$, C. Zuanelli Brambilla ${ }^{7}$, 'N. Manna', C. Uderzo

${ }^{1}$ Cure2Children Foundation, Firenze, Italy, ${ }^{2}$ South East Asia Institute for Thalassemia, Jaipur, India, ${ }^{3}$ Pediatrics, Shaheed Zulfikar Ali Bhutto Medical University, ${ }^{4}$ Cure2Children Foundation, Islamabad, Pakistan, ${ }^{5}$ The Central Hospital, Colombo, Sri Lanka

Introduction: Bone marrow transplantation (BMT) offers a definitive cure for thalassemia in over $90 \%$ of low-risk children with a matched related donor. Many centers currently incorporate thiotepa in busulfan- or treosulfan-based BMT regimens for thalassemia. This combination, however, may permanently impair fertilty in most patients. In the era of increasingly effective supportive care in which many thalassemia patients may have children, this is concerning. Very longterm follow up studies have shown how the standard Bu-Cy regimen may be associated with birth rates comparable to the control population (La Nasa et al. Blood 2013). This study retrospectively compares BMT outcomes in two groups of low risk patients (defined as liver $<2 \mathrm{~cm}$ and age $<12 \mathrm{y}$ ) with severe thalassemia (ST) (defined as a thalassemia syndrome with spontaneous hemoglobin $<7 \mathrm{~g} / \mathrm{dL}$ ), receiving oral busulfan $(14 \mathrm{mg} / \mathrm{kg})$, cyclophosphamide $(200 \mathrm{mg} / \mathrm{kg})$ and either thiotepa $(10 \mathrm{mg} / \mathrm{kg})($ TT-Bu-Cy) or rabbit ATG (Fresenius $16 \mathrm{mg} / \mathrm{kg}$ or Thymoglobulin $4 \mathrm{mg} / \mathrm{kg}$ total doses from day -12 to -10 ) (ATG-Bu-Cy) as preparative regimen. Standard cyclosporine and short-term methotrexate plus low dose methylprednisolone were used for GvHD prophylaxis.

Materials (or patients) and methods: This is a retrospective multicentre comparative study of the safety and efficacy of substituting $\Pi$ with ATG in low-risk ST patients undergoing matched-related BMT. Between January 2009 and July 2013, a group of 35 patients were transplanted after conditioning with TT-Bu-Cy, while between August 2013 and July 2014, 30 patients were conditioned with ATG-Bu-Cy.

Results: The actuarial overall survival in the TT-Bu-Cy and ATGBu-Cy groups is $91.3 \%$ and $93.4 \%$; thalassemia-free survival is $88.4 \%$ and $93.4 \%$ at a median follow up of 23.5 and 3.1 months respectively, with no statistically significant difference by logrank test.

Conclusion: Substituting thiotepa with ATG in the standard Bu-Cy context seems safe and effective. Higher fertility rates are expected for patients on the ATG-Bu-Cy regime.

Disclosure of Interest: None declared.

\section{0}

Clinical Features and HSCT Outcomes for SCID in Turkey

A. Ikinciogulları, ${ }^{1,}$, D. Cagdas ${ }^{2}$, F. Dogu ${ }^{1}$, T. Tugrul ${ }^{2}$, G. Karasu ${ }^{3}$, S. Haskologlu', S. Aksoylar ${ }^{4}$, V. Uygun ${ }^{3}$, A. Küpesiz', A. Yıldıran ${ }^{6}$,

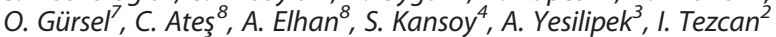
on behalf of Inborn Error Working Party (IEWP)

${ }^{1}$ Department of Pediatric Immunology-Allergy, BMT Unit, Ankara University, Medicine School, '2Department of Immunology, Hacettepe Medical School, Ankara, ${ }^{3}$ Department of Pediatric Hematology, BMT Unit, Bahçeşehir University, Istanbul, ${ }^{4}$ Pediatric BMT Center, Ege University, Izmir, ${ }^{5}$ Akdeniz University, Medical School, Department of Oncology and BMT, Antalya, ${ }^{6}$ Ondokuz Mayıs University, Department of Pediatric Immunology-Allergy, Samsun, ${ }^{7}$ Gülhane Military Medical School, Department of Pediatric Hematology, ${ }^{8}$ Ankara University Medical School, Department of Biostatistics, Ankara, Turkey

Introduction: Severe combined immunodeficiency (SCID) is the most serious PID, characterized by T-cell lymphocytopenia and lack of antigen-specific T-cell and B-cell immune responses. It is a genetically heterogeneous disorder of infancy, normally leading to death within the first year if hematopoietic stem cell transplantation (HSCT) is not performed.

Materials (or patients) and methods: Data has been collected retrospectively from 234 infants with SCID who received transplants at 8 different centers over a 20-year period (1994-2014). The differences between groups were compared by using Chi-Square or Fisher's Exact test, where appropriate. A p value of less than 0.05 was considered statistically significant.

Results: 145 boys (62\%) and 89 (\%38) girls with SCID whose ages ranged between $0.25-176$ months (median 5 months) at the time of diagnosis were transplanted. Parental consanguinity was identified in 171 (76\%) of 224 infants. $72 \%$ of the patients had received BCG vaccination before diagnosis. B+ and B-phenotypes were detected in $41.8 \%(n=97)$ and $51.3 \%$ $(n=119)$ respectively, while ADA deficiency was recognized in $4.7 \%$, RD (reticular dysgenesis) in $2.2 \%$ of the cases. RAG1, JAK3, RAG2 and Artemis defects were the leading genotypes among the patients with molecular diagnosis $(42.3 \% ; n=101)$. Out of 234, 114 patients (48.7\%) had either a matched sibling or a family donor, while $81(34.6 \%)$ and 39 (16.7\%) children received haploidentical (MMFD) and MUD transplants respectively. The HSCT source was bone marrow in $113(48.3 \%)$ peripheral blood in $89(38 \%)$ and cord blood in $32(13.7 \%)$ of the patients. Among a total of $24(10.25 \%)$ retransplants, 18 received a second transplant while 6 children received a boost only. 153 children survived, 80 died and 1 was lost to followup. The overall survival rate was $65.7 \%$ over a 20 years period. It increased from 54\% (1994-2004) to 69\% $(P=0.052)$ during the latter 10 years (2005-2014) and even to $72,9 \%$ during the last 4 years (2010-2014). The survival rates with relation to donor types were as follows: MSD $=85.7 \% \quad(n=77)$ $\mathrm{MRD}=70.3 \% \quad(n=37)$, haploidentical $47.5 \% \quad(n=80)$ and MUD 59\% $(n=39)$. Age at diagnosis significantly ( $\leq 5$ months or $>5$ months) influenced the survival rate of the patients $(P=0.002)$. Immunophenotype did not seem to have an effect on survival rate and immunoglobulin $(\mathrm{lg})$ requirement following HSCT did not differ between B + vs B- phenotypes $(P>$ 0.05).

Conclusion: This is the first multicenter study with the largest data obtained from SCID patients transplanted in Turkey. The median age at diagnosis was 5 months, B- phenotype and RAG were the most common among other defects. Age at diagnosis ( $>5$ months), and donor type (haploidentical) $(P<0.01)$ were two major factors significantly related to poor outcome. Expanded donor availability, advances in intensive care facilities, diversity of transplantation centers and specialized teams are among major factors contributing to the longterm outcomes of HSCT. However, Newborn Screening is of paramount importance in ensuring early diagnosis and timely transplantation thus improving the survival of SCID patients in Turkey.

Disclosure of Interest: None declared. 


\section{Oral session: Stem cell mobilization \& regenerative medicine}

\section{1}

Cryopreserved amniotic membrane improves healing of chronic wounds

M. Rodríguez ${ }^{1, *}$, A. M. García-Hernández ${ }^{1}$, M. Blanquer ${ }^{1}$, F. Iniesta ${ }^{1}$, S. Valiente ${ }^{1}$, C. L. García de Insausti ${ }^{1}$, S. Almansa ${ }^{2}$, G. Castellanos ${ }^{2}$, P. Alcaraz ', L. Lucas ${ }^{1}$, D. Sanchez', C. Alguero', M. Punzano', M. M. Molina ${ }^{3}$, C. Asin ${ }^{3}$, J. M. Moraleda ${ }^{1}$

${ }^{1}$ Haematology, BMT Unit, Hospital Clínico Universitario Virgen de la Arrixaca, IMIB, University of Murcia, ${ }^{2}$ Surgery, Hospital Clínico Universitario Virgen de la Arrixaca, ${ }^{3}$ Haematology, BMT Unit, Hospital Clínico Universitario Virgen de la Arrixaca, IMIB, University of Murcia, Murcia, Spain

Introduction: Amniotic membrane (AM) is a non-tumorigenic tissue attributed with various biological properties (low immunogenicity, anti-inflammatory, anti-fibrotic and antimicrobial effects) related to its ability to synthesize and release cytokines and growth factors. AM, that is usually discarded after birth, is in our experience an easily obtained tissue which processing, storage and management can be included in the daily routine of the Cryobiology Laboratory. MA can be used as a "biologic bandage" for healing management of chronic wounds in diabetic and non-diabetic patients. In our Hospital there is an ongoing clinical trial to study the use of AM to improve epithelialization (NCT01824381). Here, we describe the results obtained prospectively after the compassionate use of cryopreserved human amniotic membrane allografts in 6 patients with chronic diabetic foot ulcers.

Materials (or patients) and methods: AM was obtained from healthy mothers who had programmed an elective caesarean operation for obstetric reasons after they signed the informed consent. Donors were screened by reviewing their medical records and by performing laboratory test to discard transmissible disease agents. AM processing was done under sterile conditions in the GMP facility; the process involves: washing the AM to eliminate blood traces, cut AM into several fragments, sew each fragment on an impregnated dressing sheet and introduce them on cryopreservation bags adding the cryoprotectant solution based on human albumin, TC-199 medium and $9 \%$ DMSO. The AM fragments were storaged at $196^{\circ} \mathrm{C}$ and delivered once we the negative viral serology of the donor was confirmed 3 months later.

Cryopreserved AM was applied to six consecutive patients with diabetic foot ulcers under a compassionate use program of the Diabetic Foot Unit from May to November 2014. Wound size reduction and rates of complete healing were evaluated. Results: Patients were aged between 45 and 65 years. They were affected by grade II diabetic foot ulcers in the Wagner Ulcer Classification Scale that had failed previous treatments for periods between 2 months and 4 years. AM was applied weekly or every ten days until complete healing or partial reepithelialization of the ulcers. A median of 5 (3-8) cryopreserved AM fragments were applied for an average treatment period of 45 days. The mean size of the ulcer was reduced by $76 \%$.l wreduced in size by $76 \%$. At last follow-up, 4 out 6 patients have total epithelialization of the ulcer. No adverse events related to its application were observed.

Conclusion: Our results show that the application of cryopreserved amniotic membrane is a feasible and safe treatment in complex diabetic foot ulcers. More rapid healing may decrease clinical operational costs and prevent long-term medical complications.

Furthermore, the treatment achieves re-epithelialization of long evolution wounds that were not reached with conventional therapies.

Disclosure of Interest: None declared.
0082

Bone as a regulator of human hematopoietic stem cell (HSC) trafficking: Study of biochemical markers of bone remodeling and angiogenic cytokines during HSC mobilization, in patients with lymphoma and myeloma P. Tsirkinidis ${ }^{1, *}$, E. TERPOS ${ }^{2}$, G. BOUTSIKAS ${ }^{1}$, A. PAPATHEO-

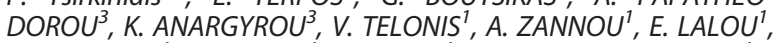
A. MELETIOU ${ }^{1}$, Z. GALANI ${ }^{\prime}$, K. PETEVI ${ }^{1}$, A. DIMITRAKOPOULOU ${ }^{4}$, M. MOSCHOGIANNIS ${ }^{5}, K$ KONSTANTOPOULOS ${ }^{1}$, C. KALPADAKIS $^{6}$, M. SIAKANTARIS ${ }^{7}$, P. PANAYIOTIDIS ${ }^{8}$, G. PANGALIS ${ }^{5}$,

M.-C. KYRTSONIS ${ }^{8}$, T. VASSILAKOPOULOS ${ }^{1}$, M. Angelopoulou ${ }^{1}$ ${ }^{1}$ DEPARTMENT OF HEMATOLOGY, ${ }^{2}$ Department of Clinical Therapeutics, NATIONAL AND KAPODISTRIAN UNIVERSITY OF ATHENS, ${ }^{3}$ Department of Hematology and Medical Research, 251 General Air Force Hospital, ${ }^{4}$ Department of Immunology Research and Flow Cytometry, 'Laikon' General Hospital of Athens, ${ }^{5}$ DEPARTMENT OF HEMATOLOGY, ATHENS MEDICAL CENTER, PSYCHIKON BRANCH, ATHENS, ${ }^{6}$ DEPARTMENT OF HEMATOLOGY, UNIVERSITY OF CRETE, HERAKLION, ${ }^{7} 1$ TST DEPARTMENT OF INTERNAL MEDICINE, ${ }^{8} 1$ st Propedeutic Department of Internal Medicine, NATIONAL AND KAPODISTRIAN UNIVERSITY OF ATHENS, ATHENS, Greece

Introduction: Bone is not considered just a structural, supportive tissue of bone marrow, but an HSC-niche regulator. Data regarding the role of bone turnover in HSC mobilization in humans are scarce. The aim of the present study was to study bone remodeling and vessel equilibrium during HSC mobilization in lymphoma and multiple myeloma patients.

Materials (or patients) and methods: Forty-six patients (32 lymphoma and 14 multiple myeloma) were studied. Serum samples were collected at two time points: before mobilization (pre-mobilization sample) and on the day of HSC collection, which coincided with the peak circulating CD34 counts (collection sample). In 19/46 patients, 3 additional serum samples were collected, between mobilization and collection. The following molecules were measured by ELISA in patients' sera: 1) bone resorption markers: carboxyterminal telopeptide of collagen type 1 (CTX), aminoterminal telopeptide of collagen type 1 (NTX), tartrate resistant acid phosphatase isoform 5b (TRACP-5b), 2) bone formation markers: bone alkaline phosphatase (BALP), osteocalcin (OSC), osteopontin (OPN), 3) the osteoblastogenesis inhibitor dickkopf-1 (DKK-1) 4) the osteoclastic regulators: receptor activator NF-kB ligand (RANKL), osteoprotegerin (OPG), 5) angiogenic cytokines: angiopoietin-1 (ANGP1), angiopoietin-2 (ANGP2), angiogenin (ANG). Values were compared with non-parametric methods. Patients who had either a collection of CD34+ cells $<2.0 \times 10^{6} / \mathrm{kg}$, or a circulating CD34 count peak $<20 / \mu \mathrm{L}$ were considered poor mobilizers.

Results: The comparison of the aforementioned molecules between the pre-mobilization and collection samples revealed: BALP $(P=0.000)$ and OPN $(P=0.049)$ increased significantly, while OSC, a marker of bone turnover, and DKK-1 decreased significantly $(P=0.000$ and $P=0.041$, respectively). These findings reveal a significant increase of bone formation during mobilization. RANKL $(P=0.000)$ and OPG $(P=0.000)$ increased significantly, leading to an increase of RANKL/OPG ratio $(P=0.000)$, consistent with osteoclastic activation. However, there was no evidence of increased osteoclastic activity, as CTX decreased significantly $(P=0.026)$, while both NTX and TRACP-5b did not change. ANGP-1 showed a dramatic reduction $(P=0.000)$, while ANGP-2 increased $(P=0.000)$, resulting in a significant decrease of the ANGP-1/ANGP-2 ratio, a finding indicating vessel destabilization during mobilization. These results were further supported by the intermediate measurements, which showed a straightforward alteration of bone metabolism early in HSC mobilization. Poor mobilizers had significantly higher CTX levels both at premobilization $(P=0.004)$ and collection samples $(P=0.001)$, higher NTX levels at collection $(P=0.02)$, lower ANGP-1 premobilization $(P=0.004)$ and higher OSC at collection $(P=0.000)$ compared to good mobilizers. Thus, CTX, NTX 
and ANGP-1 pre-mobilization levels may serve as reliable predictors of poor mobilization.

Conclusion: Our study showed for the first time in humans, that bone plays a dynamic role during HSC mobilization: Bone formation and vessel destabilization are the two major events and osteoblasts seem to be the orchestrating cells during this process. Osteoclasts are stimulated, but not fully active. Moreover, some of these markers may identify poor mobilizers.

Disclosure of Interest: None declared.

0083

Plerixafor Alone Effectively Mobilizes CD56bright NKp46 regulatory NK cells in Peripheral Blood Providing an Allograft Predicted to be Protective Against Graft-versusHost Disease

P. Wong ${ }^{1}$, A. Kariminia ${ }^{2}$, S. Ivison ${ }^{3}$, J. Rozmus ${ }^{3}$, C. Eaves ${ }^{4}$,

S. Couban ${ }^{5}$, K. Schultz

${ }^{\prime}$ Pediatric Hematology/Oncology/BMT, BC Children's Hospital, ${ }^{2}$ Pediatric hematology/Oncolcogy/BMT, BC Children's/Child and Family Research Institute, ${ }^{3} B C$ Children's Hospital/CFRI, ${ }^{4} B C$ Cancer Research Institute, Vancouver, ${ }^{5}$. Capital District Health Authority and Dalhousie University, Halifax, ${ }^{6}$ Pediatric Hematology/Oncology/BMT, BC Children's Hospital/Univ. of BC, Vancouver, Canada

Introduction: Allogeneic bone marrow transplantation is a curative treatment for leukemia and genetic disorders. Although some patients are cured of their underlying illness, they are at risk of developing potentially fatal graft-versus-host disease (GvHD). A recent phase 3 randomized trial conducted by the Canadian BMT Group ( $\mathrm{N}=230)$ comparing the impact of G-CSF mobilized peripheral blood (PB) to bone marrow (BM). A representative group of 85 donor samples were evaluated and identified that high concentrations of CD56 bright NK cells in the donor product is strongly associated with a lack of development of both acute and chronic GvHD (odds ratio $0.11 ; P=0.0002)$ in G-CSF stimulated BM. We found that the CD56 ${ }^{\text {bright }}$ NK population was CD335 (NKp46) positive with comparable expression of CD337 in both sub-populations consistent with regulatory $\mathrm{NK}$ cells $\left(\mathrm{NK}_{\mathrm{reg}}\right)$. We hypothesized that alternate strategies utilizing the mobilizing agent, Plerixafor, may enrich for CD56 bright $\mathrm{NKp46}$ cells in PB thus reducing the risk of GvHD and circumventing the need of harvesting donor cells from the BM.

Materials (or patients) and methods: We performed a pilot study to examine the impact of Plerixafor, that blocks the SDF1 CXCR4 interaction, in mobilizing CD56 $6^{\text {bright }} \mathrm{NK}_{\text {reg }}$ cells in the $\mathrm{PB}$ and $\mathrm{BM}$, to determine the whether we can optimize a donor source with the highest potential for post transplant tolerance, resulting in a lack of both aGvHD and cGvHD. We recruited 9 healthy adult human volunteers. Five subjects received one dose of Plerixafor at 240 micrograms $/ \mathrm{kg} /$ day and PB and BM samples were harvested prior to, at 4, and 24 hours after Plerixafor administration. The subsequent 4 participants each received 4 daily doses of granulocyte colony stimulating factor (G-CSF) at 5 micrograms/kg/day for 4 days, followed by a dose of Plerixafor on day 5. PB and $\mathrm{BM}$ samples were collected prior to G-CSF, after G-CSF, and at 4 and 24 hours after Plerixafor administration. We used multi-parametric flow cytometry to identify CD56 bright $\mathrm{NK}$ cells. The phenotype of CD56 bright $\mathrm{NK}$ cells was $\mathrm{CD}^{\text {bright }} \mathrm{CD}^{\text {b35 }}{ }^{+}\left(\mathrm{NK}^{\circ} 6^{+}\right)$ $\mathrm{CD}^{-} \mathrm{CD} 16$ perforin granzyme $\mathrm{B}^{-}$.

Results: We observed a significant rise in PB nucleated cells at both 4 and 24 hours after Plerixafor administration with a peak increase at 4 hours. Plerixafor alone induced a significantly higher proportion of CD56 $6^{\text {bright }} \mathrm{NK}_{\text {reg }}$ cells in $\mathrm{PB}$ when compared to G-CSF for 4 days ( 2.5 vs. 0.94 fold, $P=0.041$ ) and G-CSF for 4 days followed by Plerixafor treatment ( 2.5 vs. 0.43 fold, $P=0.011$ ). Moreover, cells collected 4 hours after Plerixafor alone had a significant increase in the proportion of CD56 bright $\mathrm{NK}_{\text {reg }}$ cells (2.5 vs. 0.89 fold, $\left.P=0.031\right)$ in $\mathrm{PB}$ compared to BM.
Conclusion: This study suggests that Plerixafor alone is able to mobilize a high number of CD56 ${ }^{\text {bright }} \mathrm{NK}_{\text {reg }}$ cells in PB harvested after 4 hours. We were not able to increase that population in a similar manner by using G-CSF alone or G-CSF plus Plerixafor. These findings suggest that allogeneic donor mobilization of peripheral blood may give a product with a low rate of GvHD potentially superior to G-CSF stimulated marrow and warrants further testing in a randomized clinical trial.

Disclosure of Interest: None declared.

\section{4}

Dual center retrospective performance analysis of three apheresis systems (COM.TEC; Cobe Spectra; Spectra Optia) used in $\mathbf{5 2 8 8}$ allogeneic stem cell collections

J. M. Rox ${ }^{1, *}$, A. Kozlova ${ }^{2}$, H. Schmidt ${ }^{2}$, K. Buhrmann ${ }^{2}$, J. C. Fischer ${ }^{1}$, G. Ehninger 2,3 , M. Punzel ${ }^{2}$

${ }^{1}$ Insitute for Transplantation Diagnostics and Cell Therapeutics, Heinriche-Heine-University, Düsseldorf, ${ }^{2}$ MediaPark Klinik Cologne, Cellex Cologne, Cologne, ${ }^{3}$ Medizinische Klinik 1, Technische Universität, Dresden, Germany

Introduction: Only small studies have compared performance of the novel Spectra Optia apheresis system (Terumo BCT) with the widely used COM.TEC (Fresenius Healthcare) and the late Cobe Spectra (Terumo BCT) device in allogeneic stem cell collections. Our collaborative working group compared performance data of all 3 devices from two collection centers to analyze device- as well as center-specific performance parameters.

Materials (or patients) and methods: We analyzed 5288 firstday apheresis collections in G-CSF-stimulated healthy donors that were performed in Cologne (CGN) using Spectra Optia MNC $(n=1818)$, Cobe Spectra MNC $(n=877)$, or COM.TEC $(n=1657)$ and in Düsseldorf (DUS) using Spectra Optia MNC $(n=63)$ or Cobe Spectra MNC (873). Peripheral blood and product samples were analyzed in center-specific laboratories. Results: In both centers, irrespective of the apheresis device, similar yields of CD34 + cells per kg donor bodyweight were reached. In CGN, collection rates (collected CD34 + cells per $\mathrm{kg}$ donor bodyweight per CD34 + cell count in peripheral blood before apheresis) were similar between the three apheresis systems. The continuously collecting Cobe Spectra yielded more CD34 + cells over time (CR per h: 0,034 $\pm 0,009)$ than the discontinuously collecting apheresis systems COM.TEC $(0,030 \pm 0,009, P<0,001)$ and Spectra Optia MNC $(0,025 \pm 0,008, P<0,001)$. The COM.TEC collected CD34 + cells with highest efficiency (CE2: $63 \pm 15 \%$ vs $61 \pm 16 \%, P<0,001$ and $55 \pm 15 \%, P<0,001$ for Spectra Optia MNC and Cobe Spectra, respectively). In comparison to Cobe Spectra (178 $\pm 49 \mathrm{~min})$, procedure times were longer when using COM.TEC $(199 \pm 50 \mathrm{~min}, P<0,001)$ or Spectra Optia MNC $(237 \pm 58 \mathrm{~min}, P<0,001)$ systems. The product purity, measured as percentage of MNC was highest in products collected with the Spectra Optia MNC $(79 \pm 15 \%$ vs $76 \pm 10 \%, P<0,001$ vs $75 \pm 14 \%, P<0,001$ for Cobe Spectra and COM.TEC, respectively). The relative differences between Cobe Spectra and Spectra Optia collection performance parameters were similar in the DUS apheresis center: shorter procedures with higher CR per h for Cobe Spectra, higher MNC purity in Spectra Optia MNC products. Absolute results differed between the two centers.

Conclusion: The highest collection efficiency (CE2) was seen when the COM.TEC device was used. However, this was accompanied by low product purity. Compared to any other apheresis device that has been analyzed in this study the novel Spectra Optia allows collection of higher MNC purity apheresis products from allogeneic donors. However, this was associated with a significant prolongation of procedure time the major disadvantage of this device. The Cobe Spectra collects more cells over time by allowing higher mean inlet flow rates despite inferior collection efficiency. Therefore, a continuous collection procedure on the Spectra Optia device that allows high inlet flow rates would be an ideal collection 
setting in terms of collection efficiency and collection rate per unit of time.

Disclosure of Interest: None declared.

\section{5}

Automated DMSO removal for cryopreserved peripheral blood hematopoietic progenitors with Sepax. Comparison of two different washing solutions

C. Cava ${ }^{1}$, M. Blanquer ${ }^{1, *}$, A. M. García-Hernández ${ }^{7}$, M. J. Majado $^{7}$, P. Menchón ${ }^{1}$, A. Marín ${ }^{1}$, J. Monserrat ${ }^{1}$, A. Sánchez-Salinas',

J. M. Moraleda

${ }^{1}$ Hospital Virgen de la Arrixaca, University of Murcia. IMIB., Murcia, Spain

Introduction: Our group has previously established the safety and effectivity in terms of CD34 + cell recovery and viability of the automated washing of cryopreserved hematopoietic progenitors (HP) with the Sepaxâ device (Biosafe) using normal saline supplemented with $2.5 \%$ albumin (NSA), as well as the absence of infusion-related events and of a negative impact on engraftment dynamics (Sánchez-Salinas et al. 2012). In our present study we compare this solution with a ready-to-use free of human derived components solution: $6 \%$ hydroxyethyl starch $130 / 0.4$ in $0.9 \%$ sodium chloride injection (Voluven ${ }^{\hat{a}}$, Fresenius Kabi).

Materials (or patients) and methods:: 411 peripheral blood HP units apheresis cryopreserved using autologous plasma plus 9\% DMSO corresponding to 158 autologous peripheral blood HP transplants were studied. After rapid thawing in a water bath at $37^{\circ} \mathrm{C}$, an automatic wash with the Sepax (2 washes cycle) was performed using either NSA (229 units) or Voluven (182 units). Nucleated cell levels determined by an hematology analyzer, flow cytometry CD34 + cell counts, Trypan Blue cell viability and granulocyte macrophage (GMCFU) and erythroid (E-BFU) colony forming cell cultures were performed on aliquots collected prior to and after the washing technique. Statistical analysis was performed using descriptive statistics and a simple-measures ANOVA.

Results: The mean total nucleated cell (TNC) and CD34 + cell recovery was $75,12 \% \pm 14,66$, and $113,18 \% \pm 57,76$ respectively for NSA and $79,08 \% \pm 14,75$ and $110,02 \% \pm 44,02$ for Voluven. The mean GM-CFU and E-BFU cell recovery was $163,80 \% \pm 152,64$ and $144,62 \% \pm 176,61$ respectively for NSA and $187,59 \% \pm 232,48$ and $141,82 \% \pm 148,75$ for Voluven. The mean viability recovery was $102.02 \pm 17,79$ for NSA and $101,59 \pm 15,04$ for Voluven without differences between both solutions ( $p, 795)$. There were no significant differences between both solutions in none of this parameters In spite of the TNC significant loss ( $p<0,001)$, there were no significant differences between the pre and post-washing CD34+ cell numbers $(P=0,146), \mathrm{GM}$-CFU (p 0,051), E-BFU $(p 0,952)$ or viability $(p \quad 0,537)$. In contrast with the $40 \%$ of untoward reactions recorded in our historical data of 226 DMSO containing cell infusions, we observed just three adverse effect with the washed cells with Voluven $(1,6 \%)$ and none with NSA. One patient experienced a epileptic fit related to the infusion speed, another two suffered grade 1 nausea and transient hipotension respectively. Median time to neutrophil engraftment $(>500$ cells $/ \mathrm{mL})$ and platelet engraftment ( $>20.000$ cells $/ \mathrm{mL}$ ) for NSA were 11,29 $\pm 3,32$ and 13,19 $\pm 6,8$ days respectively and $12,02 \pm 3,01$ and 14,77 $\pm 14,87$ for Voluven. When comparing both solutions, there were no significant differences in neutrophil engraftment $(p 0,169)$ or in platelet engraftment ( $p$ 0,376).

Conclusion: Both NSA and Voluven are equally effective for washing cryopreserved HP, ensuring a good CD34 + cell recovery and preserving their viability and engraftment potential. Both solutions avoid the DMSO infusion related adverse events. As so, Voluven constitutes an excellent alternative free of human-derived products and ready-to-use solution to our previous NSA standard washing solution.

References: Sánchez-Salinas A. et al. Transfusion. 2012 Nov;52(11):2382-2386

Disclosure of Interest: None declared.
Oral session: GvHD 2

\section{6}

The JAK inhibitor ruxolitinib impairs dendritic cell migration via off-target inhibition of ROCK activation J. Rudolph ${ }^{1, *}$, A. Heine ${ }^{1}$, T. Quast ${ }^{2}$, W. Kolanus ${ }^{2}$, P. Brossart ${ }^{1}$, D. Wolf

${ }^{1}$ University Clinic Bonn (UKB), Department of Internal Medicine III, Oncology, Hematology and Rheumatology, '2University of Bonn, Molecular Immunology \& Cell Biology, Life and Medical Sciences Institute (LIMES), Bonn, Germany

Introduction: Ruxolitinib is a JAK inhibitor that was recently approved for treatment of primary and secondary MF and shows impressive symptom control by suppression of inflammation. Ruxolitinib is also a promising drug for treatment of acute and chronic GvHD. The immune-modulatory effects of ruxolitinib are at least in part due to an inhibitory effect on dendritic cell biology (Heine et al., Blood 2014). Dendritic cells (DCs) are important antigen-presenting cells. Upon antigen contact they migrate into the draining lymph nodes to prime T cells. The aim of this study was to define the impact of the JAK inhibitor ruxolitinib on DC migration.

Materials (or patients) and methods: $\mathrm{CD}_{14}{ }^{+}$cells isolated from human buffy coats were differentiated for seven days in the presence of GM-CSF and IL-4 to moDCs and finally matured with LPS. Murine bone marrow-derived DCs (bmDCs) were generated by flushing bone marrow from femur and tibia of mice, plating the cell in medium containing GM-CSF and maturing the cells with LPS. Migration of DCs was analyzed in Transwell assays or dynamically by time-lapse microscopy within three dimensional collagen gels towards CCL-19 gradients. Signaling events were analyzed by Western Blot to evaluate changes in phosphorylation levels.

Results: 2D migration of ruxolitinib-exposed DC is dosedependently reduced in vitro. By analyzing the migratory phenotype of human moDCs within three dimensional collagen gels, ruxolitinib-exposed DCs are still able to sense chemokine gradients and form lamellipodia at the leading edge of the cell, whereas the retraction of the uropod is inhibited. Additional in vivo studies could show that the JAK inhibitor potently reduces homing of bmDCs into draining lymph nodes in mice. siRNA knockdown experiments revealed that this inhibitory effect is JAK1- and JAK2-independent. On a molecular level we could show a reduced phosphorylation of Rho-associated protein kinase (ROCK) in ruxolitinib-treated moDC upon CCL-19 stimulation. Finally, the migration phenotype of moDC exposed to ruxolitinib could be mimicked by the ROCK inhibitor Y-27632.

Conclusion: RhoA family members are key proteins controlling important steps of cell migration, such as protrusion formation at the front of the cell and consequently cell polarization. ROCK is a downstream effector of RhoA and leads to stabilization of the actin cytoskeleton via cofilin and actomyosin II contraction by the myosin light chain. The observed reduction of ROCK phosphorylation may reveal an important mechanism of ruxolitinib-induced inhibition of DC migration. Our current efforts focus on the validation of ROCK as offtarget JAK1/2-independent target kinase of ruxolitinib as potential mediator of the observed effects, which may at least in part also explain the potential therapeutic impact of ruxolitinib for therapy of GvHD.

Disclosure of Interest: J. Rudolph: None declared, A. Heine: None declared, T. Quast: None declared, W. Kolanus: None declared, P. Brossart: None declared, D. Wolf Funding from: Research funding from Novartis. 
0087

Acute GvHD after Alemtuzumab based conditioning regimen correlates with persistence of $\mathrm{GPI}$-anchor negative, functionally altered regulatory $\mathbf{T}$ cells

K. Epp ${ }^{1, *}$, L. Schäfer ${ }^{1}$, M. Theobald ${ }^{1}$, T. Bopp ${ }^{2}$, R. Meyer ${ }^{1}$, E. M. Wagner ${ }^{7}$

${ }^{1}$ Department of Heamatology, Medical Oncology, and Pneumology, ${ }^{2}$ Department of Immunology, University Medical Centre Mainz, Mainz, Germany

Introduction: Alemtuzumab, a monoclonal CD52-antibody, is used for T-cell depletion (TCD) in the context of allogeneic hematopoietic stem cell transplantation (HSCT) to prevent graft versus host disease (GvHD). When we applied this approach in a clinical trial, nearly half of our patients developed acute GvHD (aGVHD) overall Grade I-II ${ }^{\circ}$. Since regulatory $T$ cells (Treg) play a major role in controlling GvHD, we hypothesized that they might be functionally impaired in our patients after Alemtuzumab based treatment and further investigated on these cells.

Materials (or patients) and methods: We analyzed peripheral blood samples of 20 patients with aGvHD, 10 patients with chronic GvHD, and 12 patients who never developed GvHD after Alemtuzumab-mediated TCD. Treg were identified as $\mathrm{CD} 3+\mathrm{CD} 4+\mathrm{CD} 25+\mathrm{CD} 127-$ or $\mathrm{CD} 3+\mathrm{CD} 4+\mathrm{CD} 25+$ FoxP3 + and subsets described by expression of CD52. Since CD52 is linked to the membrane by a glycosylphosphatidylinositol (GPI)-anchor, we used FLAER to stain for GPI-anchors themselves. To further investigate Treg activation, we stained additional markers: CD39, CD44, GITR, CXCR3, CCR5, CTLA-4, GARP and Granzyme.

To observe how Treg reconstitute after HSCT, we analysed samples from patients with aGvHD at different time points after transplantation and correlated our findings with the clinical course of GvHD.

Treg function was evaluated in CFSE-suppression-assays: patient derived ex vivo Treg were FACS-sorted according to their CD52 expression and incubated with proliferating CFSEstained CD4-effector T cells from healthy donors. Reduced CD4-proliferation was used as indirect marker for the suppressive function of the CD52 positive and negative Treg. Results: Patients with aGvHD showed significantly elevated percentages of CD52 negative Treg: mean 55.3\% (range 34.4$79.7 \%$ ) in comparison to only $10.1 \%$ (range $1-21.3 \%$ ) in patients with chronic our without GvHD. By FLAER-staining we confirmed that loss of CD52 correlates with the absence of GPI-anchors on the cell surface, these cells do also lack other GPI-anchored proteins (e.g. CD56, CD59).

Patients who overcame aGvHD over time reconstituted GPIanchor positive Treg, whereas GPI-anchor negative Treg remained the dominant population in patients with ongoing aGvHD.

The fraction of activated GARP positive Treg was mainly detected among the GPI-anchor positive Treg population. All other markers showed a heterogeneous expression profile with a tendency towards lower expression on GPI-anchor negative Treg.

Suppression-assays showed a higher suppressive capacity of GPI-anchor positive ex vivo Treg in contrast to GPI-anchor negative Treg of the same patient.

Conclusion: CD52 negative / GPI-anchor negative Treg reconstituted in patients after Alemtuzumab mediated TCD and mainly persisted in patients with acute GvHD. These Treg were less likely to express the activation marker GARP. Functional assays demonstrated that GPI-negative Treg were functionally impaired - in patients developing acute GVHD, these impaired Treg represented the major Treg-population. Our preliminary data suggest that GPI-anchor negative Treg, like other GPI-anchor negative T-cell subpopulations (previously shown), are functionally altered. We hypothesize that this might promote acute GvHD. These GPI-anchor negative Treg could be useful to diagnose and guide immunosuppressive therapy in patients with acute GVHD.

Disclosure of Interest: None declared.

\section{8}

Human CD4- invariant NKT lymphocytes can regulate human $\mathrm{T}$ cell alloreactivity in a xenogeneic mouse model of graft versus host disease

T. Coman 1,*, J. Rossignol ${ }^{1}$, M. D'Aveni ${ }^{2}$, B. Fabiani ${ }^{3}$, F. Coté ${ }^{1}$, B. Marie ${ }^{1}$, I. Moura', O. Hermine ${ }^{4}$, M. T. Rubio ${ }^{3}$

${ }^{1}$ INSERM U1163, Institut Imagine, Paris, ${ }^{2} \mathrm{CHU}$, Nancy, ${ }^{3}$ Hôpital Saint Antoine, ${ }^{4}$ Hôpital Necker, Paris, France

Introduction: We previously showed that enhanced human invariant NKT (hiNKT) lymphocytes recovery after allogeneic stem cell transplantation was associated with a reduced risk of acute graft versus host disease (aGVHD). Using a humanized mouse model of GVHD, we aimed to determine whether hiNKT cell subsets could be involved in the regulation of allogeneic immune response and to elucidate their mechanisms of action.

Materials (or patients) and methods: Human iNKT were obtained by in vitro expansion of total peripheral blood mononuclear cells (PBMC) from healthy donors followed by electronic sorting of $\mathrm{CD}^{+}{ }^{+}$and $\mathrm{CD}^{-}$iNKT subsets among the $\mathrm{CD}^{+} \mathrm{CD} 1 \mathrm{~d}$-tetramer positive total iNKT population. Xenogeneic GVHD was assessed in $2 \mathrm{~Gy}$ irradiated NOD/SCID/ gamma-c $\mathrm{c}^{-/}$(NSG) mice previously injected with $\mathrm{hPBMC}$ alone or hPBMC enriched with $\mathrm{CD}^{-}$(PBMC $+\mathrm{iNKT4}^{-}$) or $\mathrm{CD}^{+}$ $\left(\mathrm{PBMC}+\mathrm{iNKT}^{+}{ }^{+}\right)$hiNKT cells. The effects of hiNKT lymphocytes on the survival and phenotype of human monocyte derived dendritic cells (hMo-DC) were assessed by flow cytometry (on the expression of Annexine $\mathrm{V}$, propidium iodide, and that of CD86, CD80, CD40, respectively). The effects of $\mathrm{CD}^{-}$or $\mathrm{CD}^{+}{ }^{+}$hiNKT cell subsets on the expression of the $\mathrm{T}$ cell activation marker CD25 and on the cytokine expression profile of CD4 $+\mathrm{T}$ lymphocytes was assessed in vivo in the NSG model and in vitro, during the allogeneic mixed lymphocyte reaction (MLR).

Results: In vivo, NSG mice transplanted with PBMC + iNKT4 cells showed a significantly prolonged overall survival in comparison to NSG mice transplanted with PBMC alone $(P=0.001)$ or with PBMC + iNKT4 $^{+}$iNKT cells $(P=0.01)$. Improved survival observed with hPBMC + iNKT4 cells was associated with lower clinical and histological GVHD scores. In vitro, at 2 iNKT/ $1 \mathrm{DC}(2 \mathrm{~N})$ ratio, hCD4 ${ }^{-}$iNKT cells significantly increased the apoptosis of mature hMo-DC compared to the $\mathrm{CD}^{+}$iNKT subset $(P=0.001)$. hMo-DC apoptosis in the presence of $\mathrm{CD}^{-}$iNKT cells was contact dependent $(21 \%$ in contact vs. $3 \%$ in transwell, $P<0.0001$ ), occurred as early as 4 hours after cell-contact and was associated to the degranulation of CD4 iNKT cells as shown by CD107 expression. While $h$ iNKT CD4- lymphocytes could inhibit the maturation of hMo$\mathrm{DC}$ in vitro, their $\mathrm{CD} 4{ }^{+}$counterpart strongly induced high levels of expression of CD80, CD86 and CD40 on Mo-DC in a contact dependent way.

When allogeneic MLR was performed, in the presence of CD4 iNKT lymphocytes, proliferating alloreactive T CD4 ${ }^{+}$lymphocytes showed a lower median fluorescence intensity of CD25 $(P=0.02)$, and intracellular INF-g $(P=0.005) \mathrm{IL}-17(P=0.002)$ and IL-21 $(P=0.021)$ in comparison to MLR without addition of iNKT cells. Compared to NSG mice injected with PBMC alone, mice injected with PBMC + iNKT4 cells showed lower CD25 expression on circulating T CD8 lymphocytes $(P=0.001)$, and reduced intracellular expression of IL-17 on circulating T CD4 lymphocytes $(P=0.01)$.

Conclusion: These results are in line with our clinical observations and suggest that $\mathrm{h} \mathrm{CD}^{-}$iNKT cell subset could directly modulate the allogeneic immune response by downregulating antigenic presentation and subsequently $\mathrm{T}$ cell activation and Th1/Th17 cytokine production. The NSG preclinical mouse model suggest that developing a cellular therapy based on iNKT CD4- lymphocytes might be interesting for the prevention of human aGVHD.

Disclosure of Interest: None declared. 
0089

In vivo expansion of host type regulatory $\mathrm{T}$ cells via a selective TNFR2 agonist protects from acute GvHD

A. Beilhack ${ }^{1, *}$, M. Chopra ${ }^{1}$, M. Biehl ${ }^{1}$, A. Brandl ${ }^{1}$, J. Amich ${ }^{1}$, A.-L. Jordán Garrote', C. Baeuerlein ${ }^{1}$, M. Vaeth ${ }^{2}$, J. Findeis ${ }^{2}$, C. Brede ${ }^{1}$, S. Schwinn ${ }^{1}$, A. Mottok ${ }^{2}$, H. Einsele ${ }^{1}$,

F. Berberich-Siebelt ${ }^{2}, H$. Wajant ${ }^{1}$

${ }^{7}$ Medicine II, Wuerzburg University Hospital, ${ }^{2}$ Pathology, Wuerzburg University, Wuerzburg, Germany

Introduction: $\mathrm{CD}^{+}{ }^{+} \mathrm{Foxp}^{+}$regulatory $\mathrm{T}$ cells (Tregs) suppress graft-versus-host disease (GvHD) after allogeneic hematopoietic stem cell transplantation (allo-HCT). By controlling the magnitude of the alloresponse Tregs still allow for efficient anti-leukemia (GvL) effects of transplanted conventional T cells in preclinical mouse models. Current clinical study protocols for donor Tregs in the treatment or prophylaxis of GvHD rely on their ex vivo expansion and infusion in high numbers. Here we present a fundamentally novel strategy for inhibiting GvHD based on the in vivo expansion of recipient Tregs prior to allo$\mathrm{HCT}$, exploiting the crucial role of tumor necrosis factor receptor 2 (TNFR2) in Treg biology.

Materials (or patients) and methods: To expand Tregs in vivo we developed a highly selective TNFR2 agonist (that would not bind to TNFR1) and treated allo-HCT recipients two weeks before allo-HCT. Expansion of radioresistant host type Tregs, alloresponses of conventional $\mathrm{T}$ cells, and tumor progression were monitored with bioluminescence imaging fluorescence microscopy, and flow cytometry in different $\mathrm{MHC}$ major mismatch mouse models $\left(\mathrm{C} 57 \mathrm{BL} / 6, \mathrm{H}-2^{\mathrm{b}}>\mathrm{Balb} / \mathrm{c}, \mathrm{H}-2^{\mathrm{d}}\right.$ and $\mathrm{FVB} / \mathrm{N}, \mathrm{H}-2^{\mathrm{q}}>\mathrm{C} 57 \mathrm{BL} / 6, \mathrm{H}-2^{\mathrm{b}}$ ) of GvHD and $\mathrm{GvL}$.

Results: In vitro, this new TNFR2-agonist expanded natural Tregs from wild type but not from TNFR2-deficient mice. Accordingly, a human variant of this TNFR2-specific agonist expanded human Tregs in vitro. In vivo treatment of healthy mice with the murine TNFR2-agonist significantly increased Treg numbers in secondary lymphoid organs and peripheral tissues, particularly in the gastrointestinal tract, a prime target of acute GvHD.

Consequently, pre-treatment of recipient mice with this novel TNFR2-agonist expanded host-type radiation resistant Tregs prior to allo-HCT in two models across MHC barriers. TNFR2agonist pre-treatment resulted in significantly prolonged survival and reduced GvHD severity when compared to TNFR2-deficient recipients or untreated allo-HCT recipients. This was accompanied by reduced donor $\mathrm{T}$ cell proliferation and infiltration into GvHD target organs. In vivo depletion of host derived Tregs completely abrogated the protective effect of TNFR2-agonist pre-treatment. While in vivo TNFR2-agonist pre-treatment protected allo-HCT recipients from GvHD, antitumor effects of transplanted $T$ cells remained unaffected in two different murine B cell lymphoma models.

Conclusion: Our novel strategy demonstrates that the expansion of host Tregs by selective in vivo TNFR2-activation significantly improves the outcome after allo-HCT and results in prolonged tumor-free survival.

Disclosure of Interest: None declared.

\section{0}

miR-155 affects T cell migration in acute Graft Versus Host Disease (aGVHD)

P. Ranganathan ${ }^{1}$, P. A. Taylor ${ }^{2}$, N. Apollinaire', Y. Efebera', S. Devine", B. R. Blazar', R. Garzon",

${ }^{1}$ Medicine, The ohio State University, Columbus, ${ }^{2}$ Pediatric Blood and Marrow Transplantation Program, University of Minnesota, Minneapolis, United States

Introduction: We reported that miR-155 expression is upregulated in donor T cells during aGVHD and mice receiving miR-155 knock-out (KO) donor splenocytes do not exhibit lethal GVHD and have improved survival as compared to mice receiving wild type (WT) splenocytes. While we showed that miR-155 does not affect the alloreactive or homeostatic proliferative potential of $\mathrm{T}$ cells, a significant decrease in the expression of the homing receptors CCR5, CXCR4, and S1P1 were found on miR-155-KO T cells, suggesting that the loss of miR-155 could impair the migration of these cells to the peripheral target organs resulting in less aGVHD. Here, we further investigate the impact of miR-155 expression in T cell migration and elucidate the $\mathrm{T}$ cell population responsible for aGVHD modulation.

Materials (or patients) and methods: Lethally irradiated $\mathrm{BALB} / \mathrm{C}$ or $\mathrm{B} 6 \mathrm{D} 2 \mathrm{~F} 1$ recipients were infused with $\mathrm{T}$ cell depleted bone marrow (BM) cells $\left(5 \times 10^{6}\right)$ and GFP expressing miR-155 KO or GFP-B6 WT T cells $\left(1 \times 10^{6}\right)$. Transplants with CD4 + $\left(2 \times 10^{6}\right)$ only $T$ cell subsets were performed to identify the lymphocyte population that contributes to the miR-155 mediated effects on aGVHD.

Results: On days 7, 14 and 21 post transplant, recipient mice were sacrificed, and tissues harvested in order to study the kinetics of miR-155 KO T cell migration following allogeneic HSCT. There was a dramatic decrease in T cell infiltration of peripheral organs (Peyer's patches, liver, lung and skin) in recipients of miR-155-KO T cells as compared to WT T cells as evidenced by confocal microscopy of GFP labeled donor cells. We reasoned that these effects could be due to the modulation of CCR5 and other chemokine receptors by miR155 . We found that a recently described long noncoding RNA (LincR-Ccr2-5'AS) located in the vicinity of several chemokine receptor encoding genes including CCR1, CCR2 and CCR5 and that is involved in chemokine regulation and $\mathrm{T}$ cell migration has 3 conserved potential miR-155 binding sites. We then set out to determine if miR-155 regulates the expression of this IncRNA in relationship with CCR5 expression. There was a significant decrease in CCR5 mRNA expression in miR-155-KO versus WT donor T cells obtained from recipient mice at the time of aGVHD, but no significant difference in the levels of LincR-Ccr2-5'AS. A luciferase reporter assay, however indicate that there was an interaction between miR-155 and LincRCcr2-5'AS. Our result does not exclude the possibility that miR155 might influence the activity rather than LincR-Ccr2-5'AS levels, a hypothesis that is currently being tested. To identify the lymphocyte population involved in miR-155 mediated modulation of aGVHD we performed a B6 into F1 transplant using CD4 $+\mathrm{T}$ cells alone as the source of donor T cells. Median survival of recipients of T cell depleted BM + WT CD4 + T cells $(n=12)$ was 48 days as compared to $100 \%$ survival of all recipient mice of miR-155 KO CD4 + T cells $(n=12)$ on day 100, $(P=0.02)$. Recipients of miR-155 KO CD4 + T cells exhibited also significant lower aGVHD clinical $(P<0.01)$ and pathological scores $(P<0.01)$ than WT recipients. Conclusion: Our data suggest that miR-155 may exert its modulating effects in aGVHD by affecting $T$ cell migration. Our results also point to the CD4 $+\mathrm{T}$ cell subset seems to play an important role in the miR-155 regulation of aGVHD. Experiments are currently underway to determine the role of miR155 in regulatory $\mathrm{T}$ cells and CD8 $+\mathrm{T}$ cell subsets in the modulation of aGVHD.

Disclosure of Interest: None declared. 
0091

Impact of response to induction chemotherapy in patients with germ cell tumors (GCT) receiving salvage high-dose chemotherapy (HDCT): an EBMT Solid Tumors Working Party (STWP)-sponsored retrospective study

A. Necchi ${ }^{1, *}$, R. Miceli ${ }^{2}$, M. Bregni ${ }^{3}$, L. A. Berger ${ }^{4}, K$. Oechsle ${ }^{4}$, K. Schumacher ${ }^{5}$, J. H. Bourhis ${ }^{6}$, D. Laszlo ${ }^{7}$, A. Flechon ${ }^{8}$, F. Arpaci', S. Secondino ${ }^{10}$, D. Raggi ${ }^{1}$, P. Dreger ${ }^{17}$,W. Krüger ${ }^{12}$, M. Ringhoffer ${ }^{13}$, A. Unal ${ }^{14}, A$ A Nagler ${ }^{15}$, Campos $^{16}$, Wahlin $^{17}$, I. Donnini ${ }^{18}$, M. Badoglio ${ }^{19}$, M. Martino ${ }^{20}$, P. Pedrazzoli ${ }^{10}$, F. Lanza ${ }^{21}$ on behalf of EBMT Solid Tumors Working Party ${ }^{1}$ Medical Oncology, ${ }^{2}$ Clinical Epidemiology and Trials Organization Unit, Fondazione IRCCS Istituto Nazionale dei Tumori, Milano, ${ }^{3}$ Medical Oncology, Azienda Ospedaliera di Busto Arsizio, Busto Arsizio, Italy, ${ }^{4}$ Medical Oncology, Eppendorf University, Hamburg, ${ }^{5}$ Medical Oncology, UKGM- Marburg Klinik for Hematology and Oncology, Marburg, Germany, 'Hematology, Gustave Roussy, institut de cancérologie, Villejuif, France, ${ }^{7}$ Hematology, European Institute of Oncology, Milano, Italy, ${ }^{8}$ Hematology, Centre Léon Bérard, Lyon, France, ${ }^{9} B M T$ Center, Gülhane Military Medical Academy, Ankara, Turkey, ${ }^{10}$ Medical Oncology, Fondazione IRCCS Policlinico San Matteo, Pavia, Italy, ${ }^{11}$ Medizinische Klinik, Universitätsklinikum Heidelberg, Heidelberg, ${ }^{12}$ Hämat./Onkol., Universitätsmedizin - Klinik Innere Medizin C, Greifswal, ${ }^{13}$ Haematologie, Onkologie, Klinikum Karlsruhe gGmbH- III. Med. Klinik, Karlsruhe, Germany, ${ }^{14}$ Dept. of Hematology - Oncology, Kapadokya (Cappadocia) BMT Center, Kayseri, Turkey, ${ }^{15}$ BMT Center, Chaim Sheba Medical Center, Tel-Hashomer, Israel, ${ }^{16}$ BMT Unit, Inst. Português de Oncologia do Porto, Porto, Portugal, ${ }^{17}$ BMT Unit, Umea University Hospital, Umeå, Sweden, ${ }^{18} B M T$ Unit, University Hospital of Careggi, Firenze, Italy, ${ }^{19}$ EBMT Study Office, EBMT, Paris, France, ${ }^{20}$ Hematology and Bone Marrow Transplant Unit, Azienda Ospedaliera BMM, Reggio Calabria, ${ }^{2}$ Hematology, Istituti Ospitalieri di Cremona, Cremona, Italy

Introduction: $\mathrm{HDCT}$ has a recognized indication in the salvage setting of advanced GCT and is steadily utilized worldwide. While the prognostic impact of response to prior lines of CT (i.e. definition of chemoresistance) is ascertained, that of response to induction/mobilization $\mathrm{CT}$ preceding single or multiple HDCT cycles is unknown. We present the results of a retrospective study of the EBMT-STWP.

Materials (or patients) and methods: Data have been collected from EBMT registry, including 23 European centers. Eligibility included adult male patients (pts) with GCT, and treatment with second or further-line HDCT between the years 2002 and 2012. Multivariable logistic model evaluated the association between response to induction $C T$ and line of HDCT by adjusting for known confounding factors. Multivariable Cox regression analyses were undertaken to evaluate the association of prespecified factors (site of tumor primary, IGCCCG category, response to induction CT, response to prior lines [chemosensitive vs chemorefractory], line of HDCT, year of HDCT) with progression-free (PFS) and overall survival (OS). Cox analyses excluded missing data.

Results: Since 10/2013, 442 pts have been registered, 344 of them were suitable for present analysis. 22 mobilization regimens were used, including 15 platinum-based $C T$. HDCT regimens were as follows: 210 (61\%) HD-CBDCA-VP16 (CE), 41 (12\%) adding ifosfamide (CEI), 32 (9\%) CarboPEC, 46 (13\%) were taxane-containing, $15(4 \%)$ other various regimens. HDCT was administered as single $(n=142)$, double $(n=95)$, or multiple $(n=106)$ courses ( 1 missing). Median follow up was 30 months (IQR: 15-54).

59 pts (17\%) had a progression to induction, $250(73 \%)$ a response (35 [10\%] missing or GCSF only). Progression was significantly associated to the transplant setting (Chi squared test $P=0.007$ ).
In the multivariable model for PFS, tumor primary (overall $p$ value $=0.039$ ), IGCCCG category (overall $p$ value $=0.033$ ), and progression to induction (HR: $2.02,95 \% \mathrm{Cl}: 1.31-3.12, P=0.001$ ) were significantly prognostic. The latter was also significantly prognostic for OS (HR: 2.36, 95\%Cl, 1.44-3.85, $P<0.001$ ) together with the transplant setting (overall $\mathrm{p}$ value $=0.014$ ) Results for response to induction CT were confirmed in the models that included missing data. The c-index of the model was 0.62 for PFS and 0.63 for OS.

Conclusion: Progression to induction CT prior to HDCT was independently and significantly associated with shorter PFS and OS, while response or progression to prior $\mathrm{CT}$ lines was not. This information could have important implications to refine patient eligibility to transplantation and enhance the prognostic risk grouping. These data need external validation. Disclosure of Interest: None declared.

\section{2}

KIR-ligand incompatibility in the graft-versus-host

direction improves progression-free survival in patients with primary high risk neuroblastoma after umbilical cord blood transplantation with nonmyeloablative conditioning

Y. Takahashi, ${ }^{1, *}$, A. Narita ${ }^{1}$, N. Kawashima ${ }^{1}$, Y. Sekiya ${ }^{1}$, K. Suzuki $^{1}$, N. Murakami ${ }^{1}$, D. Kojima ${ }^{1}$, S. Dosisaki ${ }^{1},{ }^{1}$ M. Kamei ${ }^{1},{ }^{1}$ M. Irie ${ }^{7}$,

Y. Okuno ${ }^{1}$, H. Muramatsu', N. Nishio ${ }^{1}$, A. Hama ${ }^{1}$, K. Horibe ${ }^{2}$,

K. Kato ${ }^{3}$, S. Kojima

${ }^{1}$ Pediatrics, Nagoya University Graduate School of Medicine, ${ }^{2}$ Clinical Research Center, National Hospital Organization Nagoya Medical Center, ${ }^{3}$ Department of Hematology and Oncology, Children's Medical Center, Japanese Red Cross Nagoya First Hospital, Nagoya, Japan

Introduction: Donor NK cells expressing inhibitory killer cell immunoglobulin-like receptors (KIRs), which do not recognize their cognate ligands ('KIR ligands') on recipient targets, are free from HLA inhibition, resulting in a decreased incidence of relapse and improved the outcome after HLA haploidentical stem cell transplantation (HSCT) (Ruggeri, Blood 2007) or umbilical cord blood transplantation (UCBT) (Willemze, Leukemia 2009) in leukemia patients. Venstrom reported that significant association between KIR/HLA genotypes predictive of missing KIR ligands have a better outcome without allogeneic HSCT following anti GD2 monoclonal antibody therapy in patients with high risk neuroblastoma (Venstrom, Clin Cancer Res 2009). These observations led us to start the clinical trial of allogeneic UCBT from KIR ligand incompatible donor for patients with high risk neuroblastoma in 2008.

Materials (or patients) and methods: Eligibility criteria of this study were newly diagnosed stage IV neuroblastoma patients with one of the following 1) Chemo-resistant disease defined as MIBG positive score after 4 courses of induction chemotherapy, 2) Older age defined as 10 years old and older at diagnosis or 3) MYCN amplification defined as greater than 10 copies per tumor cell. KIR ligand mismatched UCBT donor was prospectively selected from the Japan Cord Blood Bank Network according to the genotyping of HLA-C or B as previously reported (Willemze, R. Leukemia 2009). We scheduled UCBT with reduced intensity conditioning regimen about three months after conventional high dose chemotherapy followed by autologous peripheral blood stem cell transplantation. Reduced intensity conditioning regimen for CBT was Flu, L-PAM $140 \mathrm{mg} / \mathrm{m} 2$ and TBI $2 \mathrm{~Gy}$. Tacrolimus and methotrexate were used for GVHD prophylaxis. Single inhibitory KIR expressed NK cells with no corresponding recipient's HLA were monitored by flowcytometry before and after UCBT to access the expansion of alloreactive NK cells in vivo. We retrospectively analyzed the outcome of 82 patients with high risk neuroblastoma treated in Nagoya University hospital between April 1982 and June 2014.

Results: Fifty four patients were treated before Dec. 2007. After this study started in Jan. 2008, 15 patients underwent KIR ligand incompatible CBT who match eligibility criteria (7 
Cumulative incidence of relapse in high risk neuroblastoma after stem cell transplantation in different donor sources

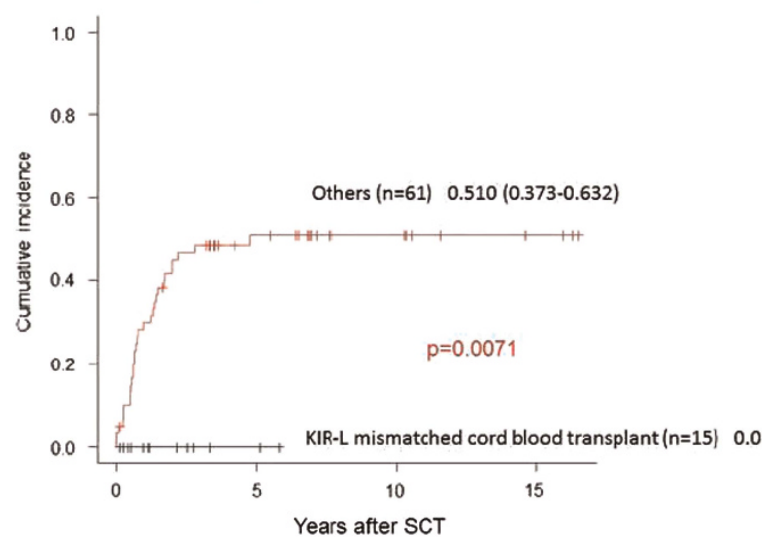

chemo-resistant, 6 MYCN amplification and 2 older age) and 13 patients received standard treatment. All patients achieved engraftment in UCBT group and no patients developed grade III or more acute GVHD and chronic GVHD. Only two patients died in this group because of BU related lung toxicity. Surprisingly, no patient in this group relapsed with the median follow-up period of 51 (6-71) months. On the other hand, cumulative incidence of relapse in others was $51.0 \%$. 3year progression free survival in CBT group was significantly better than in others $(83.6 \%$ vs $40.7 \%, P=0.048)$. Single inhibitory KIR expressed NK cells significantly expanded after $\mathrm{CBT}(P=0.0009)$. Finally, multivariate analysis revealed only KIR ligand incompatible CBT and stage III were significant better covariate factors for relapse.

Conclusion: This is the first report that KIR ligand incompatible allogeneic UCBT significantly reduced the incidence of relapse in high risk neuroblastoma.

Disclosure of Interest: None declared.

0093

Better prognosis for BRCA-mutated breast cancers treated with high-dose chemotherapy and autologous hematopoietic progenitor cell transplantation. A singleinstitution retrospective study

L. BOUDIN ${ }^{1,2,3, *}$, A. GONCALVES ${ }^{1,2,4}$, J. M. EXTRA ${ }^{1,2}$, R. SABATIER ${ }^{1,2}$, H. SOBOL ${ }^{2,5}$, F. EISINGER ${ }^{2,5}$, J. MORETTA ${ }^{2,5}$, C. TARPIN ${ }^{1,2}$,

J. CAMERLO' ${ }^{1,2}$, B. CALMELS $S^{2,6}$, C. LEMARIE ${ }^{2,6}$, A. GRANATA ${ }^{2,6}$ E. CHARAFE-JAUFFRET ${ }^{2,4,7}$, F. BERTUCCI, ${ }^{1,2,4}$, A. MADROSZYK ${ }^{1,2}$, P. VIENS ${ }^{1,2,4}$, C. CHABANNON ${ }^{2,4,6}$

${ }^{1}$ Medical Oncology, Institut Paoli Calmettes, ${ }^{2}$ Centre de Recherches en Cancérologie de Marseille (CRCM), Inserm UMR 1068, CNRS 7258, Marseille, ${ }^{3}$ Medical Oncology, Hôpital d'Instruction des Armées Sainte Anne, Toulon, ${ }^{4}$ Aix Marseille Université, ${ }^{5}$ Cancer Genetics, ${ }^{6}$ Cell therapy, ${ }^{7}$ Pathology, Institut Paoli Calmettes, Marseille, France

Introduction: Hereditary BRCA genetic mutation is responsible for approximately $2-3 \%$ of breast cancers (BC).The objective of this study was to compare the outcome of BRCA-mutated (BRCA ${ }^{\text {mut }}$ ) versus BRCA wild type or unknown $\left(B R C A^{\text {wt/uk }}\right) B C$ following treatment with high dose chemotherapy (HDC) and autologous hematopoietic progenitor cell transplantation (ACT).

Materials (or patients) and methods: All female patients treated for breast cancer (BC) with HDC and ACT at Institut Paoli-Calmettes between 2003 and 2012 were included. Patients were divided into 2 groups depending on the indication of HDC with ACT: Metastatic breast cancer (MBC) or high risk breast cancer (HRBC) including inflammatory breast cancer (IBC) and/or massive lymph node involvement (LNI). All patients were examined for the presence of known
BRCA 1 or 2 mutations. Information regarding patient, tumor characteristics and treatment was also collected. The main objectives were the analysis of overall survival (OS) and progression-free survival (PFS) according to the BRCA mutation status in the two groups. Survival curves were generated using Kaplan-Meier method and compared using Log-rank test.

Results: A total of 377 patients were included, $235 \mathrm{MBC}$ and $142 \mathrm{HRBC}$ (73 LNI and $69 \mathrm{IBC}$ ). Among MBC and HRBC, 10 (6 BRCA1, 4 BRCA2) and 5 (3 BRCA1, 2 BRCA2) patients were $\mathrm{BRCA}^{\text {mut }}$, respectively. In $\mathrm{MBC}$, median age was respectively 36 (range 29-53) and 42 years (range 24-61) for BRCA ${ }^{\text {mut }}$ and BRCA $^{\text {wt } / \text { uk }} ; 70 \%$ of BRCA ${ }^{\text {mut }}$ patients had triple negative (TN) BC subtype (HER2-negative and Hormonal receptor-negative) versus only $21 \%$ in BRCA $^{\text {wt/ }} / \mathrm{uk}_{\text {individuals. In }}$ BRCA $^{\text {mut }}$ and $\mathrm{BRCA}^{\text {wt/uk }}$ median number of metastatic sites was 2 and 1 , with $90 \%$ and $69 \%$ visceral metastases, respectively. In HRBC, median age for BRCA ${ }^{\text {mut }}$ and BRCA ${ }^{\text {wt/uk }}$ was 36 (range 28-58) and 49 (range 20-62), respectively; $60 \%$ of BRCA ${ }^{\text {mut }}$ had TN BC subtypes versus $24,6 \%$ of BRCA ${ }^{\text {wt } / u k}$. IBC represented $49 \%$ of HRBC in BRCA ${ }^{\text {wt } / 4 k}$ and $40 \%$ in BRCA ${ }^{\text {mut }}$. In MBC, $90 \%$ (all except one) of BRCA ${ }^{\text {mut }}$ remained alive at 5 years; the median overall survival (OS) was not reached, compared with a median OS of 3.62 years for BRCA ${ }^{\text {wt }}(C \mid 95 \%=3.07-4.68), \quad(P=0.048$ Log-rang test); median progression free survivals (PFS) were 26,4 months $(C \mid 95 \%=0.46-N R)$ in $B^{2} C^{\text {mut }}$ patients versus 15,6 months $(\mathrm{Cl} 95 \%=11.64-18.48)$, and in $\mathrm{BRCA}^{\mathrm{wt} / \mathrm{uk}}$ patients $(P=0.073$ Log-rank test). In HRBC, 8 years probabilities of OS were respectively 1 (all patients alive) vs $0.7(\mathrm{Cl} 95 \%=0.69$ 0.78 ) for BRCA $^{\text {mut }}$ and BRCA ${ }^{\text {wt/uk }}$ individuals, while 8 years probabilities of disease free survival (DFS) were 0.8 (Cl $95 \%=0.20-0.97)$ and $0.64(\mathrm{Cl} 95 \%=0.54-0.72)$, respectively $(P=0.374$ Log-rank test)

Conclusion: The prognostic impact of BRCA mutation in BC remains controversial. In this series of $M B C$ and $H R B C$, we have reported an outstanding survival outcome in a small subset of patients with documented BRCA mutation. In spite of a higher proportion of TN and more aggressive features, BRCA ${ }^{\text {mut }} B C$ had a better outcome than their BRCA ${ }^{\text {wt/uk }}$ counterpart, the difference reaching statistical significance in MBC population. Hypersensitivity to DNA-damaging agent, possibly due to defect in homologous recombination associated with BRCA mutation, could explain these results. Despite the low numbers of cases in our series, HDC with ACT, may be considered as an option for $\mathrm{BRCA}^{\text {mut }}$, women with advanced BC.

Disclosure of Interest: None declared. 
0094

Impact of prior paclitaxel-based chemotherapy on the outcome of salvage high-dose chemotherapy (HDCT) for relapsed germ-cell tumors (GCT): EBMT Solid Tumors Working Party (STWP)-sponsored retrospective study A. Necchi ${ }^{1, *}$, R. Miceli ${ }^{2}$, M. Bregni ${ }^{3}$, L. A. Berger ${ }^{4}$, K. Oechsle ${ }^{4}$ K. Schumacher ${ }^{5}$, J. Apperley ${ }^{6}$, J. H. Bourhis', D. Laszlo ${ }^{8}$, A. Flechon', F. Arpaci ${ }^{10}$, S. Secondino ${ }^{11}$, P. Dreger ${ }^{12}$, M. Crysandt ${ }^{13}$, K. H. Plüger ${ }^{14}$, N. Worel ${ }^{15}$, W. Krüger ${ }^{16}$, M. Ringhoffer ${ }^{17}$, A. Unall ${ }^{18}$, A. 'Nagler ${ }^{19}$, M. Michieli' $^{20}$, A. Campos ${ }^{21}$ A. Wahlin ${ }^{22}$, G. Sucak ${ }^{23}$, R. Schots ${ }^{24}$, I. Donnini ${ }^{25}$, D. Raggi ${ }^{1}$, P. Giannatempo ${ }^{1}$, N. Ifrah ${ }^{26}$, M. Badoglio ${ }^{27}$, M. Martino ${ }^{28}$, P. Pedrazzoli ${ }^{17}$, F. Lanza ${ }^{29^{\prime}}$ on behalf of EBMT Solid 'Tumors Working Party

${ }^{1}$ Medical Oncology, ${ }^{2}$ Clinical Epidemiology and Trials Organization Unit, Fondazione IRCCS Istituto Nazionale dei Tumori, Milano, ${ }^{3}$ Medical Oncology, Azienda Ospedaliera di Busto Arsizio, Busto Arsizio, Italy, ${ }^{4}$ Medical Oncology, KMT- Universitätsklinikum Hamburg-Eppendorf, Hamburg, ${ }^{5}$ Medical Oncology, UKGMMarburg Klinik for Hematology and Oncology, Marburg, Germany, ${ }^{6}$ Hematology, Imperial college, London, United Kingdom, 'Hematology-BMT, Gustave Roussy, Institut de cancérologie, Villejuif, France, ${ }^{8}$ Hematology-BMT, European Institute of Oncology, Milano, Italy, ${ }^{9}$ Hematology, Centre Léon Bérard, Lyon, France, ${ }^{10}$ GATA BMT Center, Gülhane Military Medical Academy, Ankara, Turkey, ${ }^{11}$ Medical Oncology, Fondazione IRCCS Policlinico San Matteo, Pavia, Italy, ${ }^{12}$ Hematology, Universitätsklinikum Heidelberg, Heidelberg, ${ }^{13}$ Dept. of Oncology, Hematology and SCTMedizinische Klinik IV, University Hospital Aachen, Aachen, ${ }^{14}$ Hematology, EV. Diakonie-Krankenhaus gemeinnitzige $\mathrm{GmbH}$, Bremen, Germany, ${ }^{15}$ BMT Unit, Medical University of Vienna, Vienna, Austria, ${ }^{16}$ Klinik Innere Medizin C - Hämat./Onkol, Universitätsmedizin, Greifswal, ${ }^{17}$ III. Med. Klinik -Haematologie, Onkologi, Klinikum Karlsruhe gGmbH, Karlsruhe, Germany, ${ }^{18}$ Dept. of Hematology - Oncology, Erciyes Medical SchoolKapadokya (Cappadocia) BMT Center, Kayseri, Turkey, ${ }^{19}$ BMT Unit, Chaim Sheba Medical Center, Tel-Hashomer, Israel, ${ }^{20}$ Hematology, Centro di Riferimento Oncologico- C.R.O. IRCCS Aviano, Aviano, Italy, ${ }^{21}$ BMT Unit, Inst. Português de Oncologia do Porto, Porto, Portugal, ${ }^{22}$ Hematology, Umea University Hospital, Umeå, Sweden, ${ }^{23}$ Department of Hematology \& Stem Cell Transplantation, Gazi University of Medicine, Ankara, Turkey, ${ }^{24}$ Division of Clinical Hematology, Universitair Ziekenhuis Brussel, Brussels, Belgium, ${ }^{25}$ BMT Unit, University Hospital of Careggi, Firenze, Italy, ${ }^{26}$ BMT Unit, CHRU Angers, Angers, ${ }^{27} E B M T$ Study Office, EBMT, Paris, France, ${ }^{28}$ Hematology and Bone Marrow Transplant Unit, Azienda Ospedaliera BMM, Reggio Calabria, ${ }^{29}$ Hematology and Bone Marrow Transplant Unit, Istituti Ospitalieri di Cremona, Cremona, Italy

Introduction: Paclitaxel-based regimens are now commonly employed for second or third-line salvage therapy for GCT. This might have an impact on the results with subsequent salvage HDCT in these patients (pts). The EBMT-STWP is sponsoring a retrospective study on the outcomes of HDCT administered in the last 10 years. Hence, we aimed to study outcomes with HDCT after relapse to paclitaxel-CT to identify the level of chemoresistance in these pts.

Materials (or patients) and methods: Data have been collected from EBMT registry, including 24 European centers. Eligibility included adult male patients (pts) with GCT, and treatment with second or further-line HDCT between the years 2002 and 2012. Both paclitaxel used in prior CT lines of therapy and in induction-mobilization regimens pre-HDCT were considered. Multivariable Cox regression analyses (MVA) were undertaken to evaluate the association of prespecified factors (site of tumor primary, IGCCCG category, response to induction $\mathrm{CT}$, response to prior lines [chemosensitive vs chemorefractory], line of HDCT, year of HDCT) including prior-paclitaxel therapy with progression-free (PFS) and overall survival (OS).

Results: Since 10/2013, 442 pts have been registered, 324 of them were suitable for present analysis. 165 pts (51\%) received HDCT in second-line, 102 (31\%) in third and 57 (18\%) beyond the third-line. HDCT regimens were as follows: 177 (55\%) HDCBDCA-VP16 (CE), 41 (12\%) adding ifosfamide (CEI), 106 (33\%) other mixed regimens. 76 (23\%) were taxane-containing. 120 pts received a single HDCT course, 99 pts double and 104 multiple courses (1 missing). Median follow up was 36 months (IQR: 19-70). Prior paclitaxel was significantly associated with shorter OS in the univariable model $(P=0.032)$. However, on MVA prior paclitaxel-therapy was not significantly prognostic for both PFS and OS, as shown in the Table.

A separate model evaluated the interaction between prior paclitaxel-therapy and taxane-containing HDCT: no significant interaction was found $(P=0.221$ and $P=0.077$ for $P F S$ and $O S$, respectively).

\begin{tabular}{|c|c|c|c|c|c|c|}
\hline & PFS & OS & & & & \\
\hline Variable & $H R$ & $95 \% \mathrm{Cl}$ & $p$ & HR & $95 \% \mathrm{Cl}$ & $\mathrm{p}$ \\
\hline $\begin{array}{l}\text { Tumor primary } \\
\text { site }\end{array}$ & & & 0.043 & & & 0.142 \\
\hline $\begin{array}{l}\text { Gonadal vs } \\
\text { Retroperitoneal }\end{array}$ & 1.44 & $0.83-2.49$ & & 1.58 & $0.79-3.20$ & \\
\hline $\begin{array}{l}\text { Mediastinal vs } \\
\text { Retroperitoneal }\end{array}$ & 2.70 & $1.33-5.49$ & & 2.43 & $1.00-5.94$ & \\
\hline Other vs & 1.19 & $0.32-4.52$ & & 0.43 & $0.05-3.64$ & \\
\hline $\begin{array}{l}\text { IGCCCG category } \\
\text { Intermediate } \\
\text { vs Good }\end{array}$ & 1.22 & $0.68-2.19$ & 0.048 & 1.07 & $0.54-2.11$ & 0.255 \\
\hline $\begin{array}{l}\text { Poor vs Good } \\
\text { Response to } \\
\text { prior CT lines }\end{array}$ & 1.76 & $1.08-2.86$ & & 1.51 & $0.86-2.67$ & 0.953 \\
\hline $\begin{array}{l}\text { Chemorefractory } \\
\text { vs Sensitive }\end{array}$ & 0.92 & $0.63-1.34$ & 0.668 & 1.31 & $0.84-2.04$ & \\
\hline $\begin{array}{l}\text { Transplant setting } \\
>3^{\text {rd }} \text { line vs } \\
2^{\text {nd }} \text { line }\end{array}$ & 1.35 & $0.72-2.50$ & 0.338 & 1.65 & $0.80-3.40$ & 0.340 \\
\hline $\begin{array}{l}3^{\text {rd }} \text { line vs } \\
2^{\text {nd }} \text { line }\end{array}$ & 1.38 & $0.89-2.14$ & & 1.39 & $0.81-2.38$ & \\
\hline $\begin{array}{l}\text { Progression to } \\
\text { induction } C T\end{array}$ & & & & & & 0.003 \\
\hline $\begin{array}{l}\text { Yes vs No } \\
\text { Year of transplant }\end{array}$ & 1.92 & $1.24-2.98$ & 0.003 & 2.09 & $1.27-3.42$ & 0.276 \\
\hline $\begin{array}{l}<2008 \text { vs } \geq 2008 \\
\text { Prior paclitaxel CT }\end{array}$ & 0.77 & $0.47-1.25$ & 0.289 & 1.45 & $0.74-2.81$ & 0.739 \\
\hline $\begin{array}{l}\text { Yes vs No } \\
\text { Tax-containing } \\
\text { HDCT }\end{array}$ & 1.10 & $0.70-1.73$ & 0.674 & 1.09 & $0.64-1.86$ & 0 \\
\hline Yes vs No & 1.07 & $0.55-2.07$ & 0.840 & 2.61 & $1.18-5.76$ & .017 \\
\hline
\end{tabular}

Conclusion: The administration of paclitaxel-based regimens before HDCT did not affect PFS/OS. Results were confirmed when excluding pts who were administered taxane-containing HDCT. Line of HDCT was not significantly prognostic too. There is no evidence to disallow patients who have been treated with taxanes in second or third line to receive HDCT as futher salvage therapy. These data need external validation.

Disclosure of Interest: None declared.

\section{5}

Favorable outcome of a cohort of metastatic breast cancer patients treated with high dose chemotherapy and autologous transplantation at a single institution does not change the prognostic significance of histopathological subtypes L. BOUDIN ${ }^{1,2,3, *}$, A. GONCALVES ${ }^{2,3,4}$, J. M. EXTRA ${ }^{2,3}$, R. SABATIER ${ }^{2,3}$, C. TARPIN ${ }^{2,3}$, J. CAMERLO ${ }^{2,3}$, B. CALMELS ${ }^{2,5}$, C. LEMARIE LEM $^{2,5}$ A. GRANATA ${ }^{2,5}$, E. CHARAFE-JAUFFRET ${ }^{2,4,6}$, 'F. BERTUCCl $^{2,3,4}$, A. MADROSZYK' ${ }^{2,3}$, P. VIENS ${ }^{2,3,4}$, C. CHABANNON ${ }^{2,4,5}$

${ }^{1}$ Medical Oncology, Hôpital d'Instruction des Armées Sainte Anne, Toulon, ${ }^{2}$ Centre de Recherches en Cancérologie de Marseille (CRCM), Inserm UMR 1068, CNRS 7258, ${ }^{3}$ Medical Oncology, Institut Paoli Calmettes, ${ }^{4}$ Aix Marseille Université, ${ }^{5}$ Cell therapy, ${ }^{6}$ Pathology, Institut Paoli Calmettes, Marseille, France

Introduction: Studies of high dose chemotherapy (HDC) in breast cancer (BC) often lack of biomarker information, notably 
the human epidermal growth factor receptor 2 (HER2) status. The objective of this study was to evaluate the outcome of patients affected with different subtypes of BC following treatment with HDC and autologous hematopoietic progenitor cell transplantation (ACT)

Materials (or patients) and methods: All female patients treated for metastatic breast cancer (MBC) with HDC and ACT at Institut Paoli-Calmettes between 2003 and 2012 were included. Patient, tumor and treatment characteristics were collected. Patients were categorized in three subtypes based on hormonal receptor (HR) and HER2 status of the primary tumor: Luminal (L), (HR + /HER2-), HER2 (HER2 + , any HR), and triple negative (TN) (HER2- and HR-). Main study endpoints were overall survival (OS) and progression-free survival (PFS) categorized by BC subtypes. Treatment related mortality (TRM) was also evaluated. Survival curves were generated using Kaplan-Meier method and compared using Log-rank test.

Results: A total of $231 \mathrm{MBC}$ patients are included; Median age was 47 (range 24-61); Metachronous and synchronous MBC were $64 \%$ and $36 \%$, respectively. $96 \%$ patients received HDC with ACT as part of their first-line treatment following diagnosis of metastases. Median number of metastatic sites was 1 (range 1-6), including $69 \%$ of visceral metastases. L, HER2 and TN subtypes were 61,18 and $21 \%$ of patients, respectively. All but one HER 2 patients (98\%) received trastuzumab during the metastatic phase of the disease before $(88 \%)$ and/or after (91\%) HDC. Median follow-up was 53.3 months. For the entire population, the median OS and PFS were $44,4(\mathrm{Cl} 95 \%=39,1-62,4)$ and $15,7(\mathrm{Cl} 95 \%=11,4-19,7)$ months, respectively. The longest survival was observed for the HER2 subtype with $53 \%$ patients remaining alive at 5 years (median OS not reached) compared with L (median OS $=44,7$ months; $\mathrm{Cl} 95 \%=40,3-67,6)$ and TN (median OS $=19.7$ months; $\mathrm{Cl} 95 \%=11,8-44,4) \quad(P<0.01$, Log-rank test). Median PFS were 16,3 (Cl95\%=8,8-24), 19,7 (Cl95\%=15,6-22,6) and $7,44(\mathrm{Cl} 95 \%=4,56-10,6)$ months for the HER2, L and TN subtypes, respectively. TRM occured in only three patients $(1,28 \%) ; 72 \%$ patients experienced no grade 3 or 4 adverse effect, except hematological toxicity. A median number of 3 lines of treatment (range 0-8) were administered after progression and $14,9 \%$ of progressive patients were included in clinical studies after HDC with ACT.

Conclusion: This is the first study of HDC with $A C T$ in $M B C$ in which BC subtype status is available for all patients. Overall, PFS and OS are in the highest range of current results in MBC population. Consistent with data from the literature, in the era of anti-HER2 targeted therapies, the HER2 subtype is associated with a better OS while the TN subtype confers a significantly worse prognosis. Thus, the prognostic impact of BC cancer subtypes is maintained in the context of HDC with ACT. Recent progress in transplant administration help reduce extra hematological toxicities, and result in low TRM. Finally, HDC with ACT does not compromise the delivery of postprogression treatment including investigational therapies. We conclude HDC with ACT may still have a place in the management of selected MBC patients.

Disclosure of Interest: None declared.
Oral session: Acute leukemia 2

0096

Comparison of RIC-alloHCT and autoHCT for $>55$ years old patients with acute lymphoblastic leukemia: an analysis from Acute Leukemia Working Party of the EBMT

S. Giebel ${ }^{1, *}$, M. Labopin ${ }^{2,3}$, N. C. Gorin ${ }^{2}$, N. Milpied ${ }^{4}$, E. Petersen ${ }^{5}$, M. Potter ${ }^{6}$, J. Cornelissen ${ }^{7}$, D. Niederwieser ${ }^{8}$, M. Rovira ${ }^{9}$,

P. Jindra ${ }^{10}$, E. Meijer ${ }^{11}$, J. Maertens ${ }^{12}$, A. Rambaldi ${ }^{13}$, A. Nagler ${ }^{3,14}$, M. Mohty ${ }^{2,3}$

${ }^{7}$ Maria Sklodowska-Curie Memorial Cancer Centter and Institute of Oncology, Gliwice Branch, Gliwice, Poland, ${ }^{2}$ Hospital St. Antoine, ${ }^{3}$ Acute Leukemia Working Party of the EBMT, Paris, ${ }^{4} \mathrm{CHU}$ Bordeaux, Hôpital Haut-Leveque, Bordeaux, France, ${ }^{5}$ University Medical Centre, Utrecht, Netherlands, ${ }^{6}$ Royal Marsden Hospital, London, United Kingdom, "'Erasmus MC-Daniel den Hoed Cancer Centre, Rotterdam, Netherlands, " University Hospital Leipzig, Leipzig, Germany, ${ }^{9}$ Hospital Clinic Institute of Hematology \& Oncology, Barcelona, Spain, ${ }^{10}$ Charles University Hospital,, Pilsen, Czech Republic, ${ }^{11}$ VU University Medical Center, Amsterdam, ${ }^{12}$ University Hospital Gasthuisberg, Leuven, Netherlands, ${ }^{13}$ Azienda Ospedaliera Papa Giovanni XXIII, Bergamo, Italy, ${ }^{14}$ Chaim Sheba Medical Center, Tel-Hashomer, Israel

Introduction: The outcome of patients $>55$ years old with ALL is poor with no clear recommendations regarding the indications for HCT. These patients are usually considered ineligible for myeloablative alloHCT and offered transplantation with reduced intensity conditioning (RIC). Autologous HCT is an alternative. However, the role of both treatment options has not been established so far. The aim of this study was to retrospectively compare results of RIC-alloHCT and autoHCT in ALL > 55 years old and to identify factors affecting outcome. Materials (or patients) and methods: 267 patients treated with RIC-alloHCT from either HLA-identical sibling $(n=154)$ or matched unrelated donor $(n=113)$ and 179 treated with autoHCT in first complete remission between 2000 and 2011 have been included in this analysis. Median age in both groups was 60 (55-74)y and $60(55-76) y$, while median interval from diagnosis to HCT was 5.9 months and 6.6 months, respectively. The proportion of $\mathrm{Ph}(+) \mathrm{ALL}$ among those with reported cytogenetics was $71 \%$ and $66 \%$, respectively.

Results: With a median follow-up of 33 months, the probability of OS at two years was $44 \%$ for RIC-alloHCT and $57 \%$ for autoHCT $(P=0.02)$, while LFS rates were $34 \%$ and $41 \%$, respectively $(P=0.06)$. Relapse incidence at two years was comparable for RIC-alloHCT and autoHCT (42\% vs. $48 \%$, $P=0.39)$ while non-relapse mortality was significantly reduced for autoHCT ( $23 \%$ vs. $11 \%$, respectively, $P=0.002)$.

The advantage in favor of autoHCT was significant for $\mathrm{Ph}(-)$ ALL (OS: $61 \%$ vs. $38 \%, P=0.02$; LFS: $54 \%$ vs. $21 \%, P=0.005$ ) while no significant differences could be observed for $\mathrm{Ph}(+)$ ALL (OS: $55 \%$ vs. $47 \%, P=0.6$; LFS: $42 \%$ vs. $35 \%, P=0.4$ ).

In a multivariate analysis adjusted for recipient age and gender as well as interval from diagnosis to transplantation the use of autoHSCT was independently associated with reduced risk of mortality $(H R=0.69, P=0.01)$, treatment failure $(H R=0.76$, $P=0.03)$ and non-relapse mortality $(\mathrm{HR}=0.39 ; P=0.0004)$ with no effect on relapse incidence ( $\mathrm{HR}=0.98, P=0.88)$.

In the RIC-alloHSCT subgroup LFS was negatively affected by female donor/male recipient combination $(H R=1.64$, $P=0.01)$. LFS rates for both sibling and MUD transplants were comparable ( $32 \%$ vs. $35 \%, P=0.18)$. The use of peripheral blood cells compared to bone marrow was associated with reduced risk of relapse $(\mathrm{HR}=0.5, P=0.03)$. In the autoHSCT setting there was a tendency to higher risk of treatment failure with increasing recipient age $(\mathrm{HR}=1.05, P=0.06)$. Other variables including type of conditioning (TBI-based vs. chemotherapy-based) did not affect survival in any of the study cohorts.

Conclusion: Considering poor overall prognosis of ALL patients $>55$ years old, results of both RIC-alloHCT and 
autoHCT appear enhancing and both types of transplantation may be considered valuable treatment options. Potential advantage of autoHCT as suggested by results of our analysis should be further explored including data on disease-related prognostic factors and the status of minimal residual disease. Disclosure of Interest: None declared.

0097

Cytogenetic risk stratification system for adult patients with AML undergoing allogeneic hematopoietic cell transplantation: A nationwide retrospective study from the adult AML Working Group of the Japan Society for Hematopoietic Cell Transplantation

T. Yamashita ${ }^{1, *}$, N. Uchida ${ }^{2}$, T. Fukuda ${ }^{1}$, K. Iwato $^{3}$, K. Ohashi ${ }^{4}$, T. Etoh ${ }^{5}, H$. Ogawa ${ }^{6}$, T. Nagamura-Inoue 7,8 , Y. Morishima ${ }^{9,10}$, T. Ichinohe ${ }^{11,12}, Y$. Atsuta ${ }^{13,14}$, A. Takami ${ }^{15}$ on behalf of the adult AML Working Group of the Japan Society for Hematopoietic Cell Transplantation

${ }^{1}$ Department of Hematopoietic Stem Cell Transplantation, National Cancer Center Hospital, '2Department of Hematology, Federation of National Public Service Personnel Mutual Aid Associations Toranomon Hospital, Tokyo, ${ }^{3}$ Department of Hematology, Hiroshima Red Cross Hospital \& Atomic-bomb Survivors Hospital, Hiroshima, ${ }^{4}$ Hematology Division, Tokyo Metropolitan Cancer and Infectious Diseases Center, Komagome Hospital, Tokyo, ${ }^{5}$ Department of Hematology, Federation of National Public Service Personnel Mutual Aid Associations Hamanomachi Hospital, Fukuoka, ${ }^{6}$ Division of Hematology, Hyogo College of Medicine, Nishinomiya, 'Department of Cell Processing and Transfusion, Research Hospital, The Institute of Medical Science, The University of Tokyo, ${ }^{8} J a p a n$ Cord Blood Bank Network, Tokyo, ${ }^{9}$ Division of Epidemiology and Prevention, Aichi Cancer Center Research Institute, Nagoya, ${ }^{10}$ Japan Marrow Donor Program, Tokyo, ${ }^{11}$ Department of Hematology and Oncology, Hiroshima University Hospital, Hiroshima, ${ }^{12}$ The Japan Society for Hematopoietic Cell Transplantation, ${ }^{13}$ Department of Healthcare Administration, Nagoya University Graduate School of Medicine, ${ }^{14}$ Japanese Data Center for Hematopoietic Cell Transplantation, ${ }^{15}$ Department of Hematology, Aichi Medical University Hospital, Nagoya, Japan

Introduction: The karyotype of the leukemic cells at diagnosis is one of the strongest prognostic factors in acute myeloid leukemia (AML). But the major cytogenetic risk categorizations were based on the large clinical studies of conventional chemotherapy for AML. In this retrospective study, we analyzed the influence of the cytogenetics of AML at diagnosis on the outcomes of allogeneic hematopoietic cell transplantation $(\mathrm{HCT})$.

Materials (or patients) and methods: From the database of JSHCT, we extracted the data of adult patients with AML who receive first HCT between 2006 and 2010. A total of 4,241 recipients were included. Additional survey for the recipients who had been reported to have cytogenetic abnormalities at diagnosis $(n=2,384)$ confirmed the data of karyotype of 1,360 cases.

Results: Cytogenetics at diagnosis were categorized into (A) normal karyotype $(41.5 \%),(B) \operatorname{inv}(16)$ or $t(16 ; 16)(2.9 \%),(C)$ $\mathrm{t}(8 ; 21)(9.1 \%),($ D) $11 \mathrm{q} 23$ abnormality $(4.9 \%)$, (E) complex karyotype ( $>=3$ abnormalities) (12.3\%), (F) monosomal karyotype (MK) (6.3\%), and others. Overall survival (OS) and cumulative incidence of relapse at 2 years of all cases were $48.2 \%$ and $37.5 \%$, respectively. These recipients were classified into 4 groups: favorable (Fav) included (B) $(n=121)$; intermediate (Int), (A), (C), t $(9 ; 11)$ and others $(n=3,169)$; unfavorable-1 (Unf-1), (D) except $\mathrm{t}(9 ; 11)$, and (E) except (F) $(n=404)$; unfavorable-2 (Unf-2), (F) $(n=265)$. Adjusted OS at 2 years were $65.2 \%$ in Fav, 53.2\% in Int, 37.8\% in Unf- 1 and $24.2 \%$ in Unf-2 (Figure). Risk of overall mortality (ROM) and risk of relapse (RR) were compared among these four groups, adjusted by age, sex, disease status at transplantation and stem cell source. In Fav group, ROM (HR 0.71, 95\% Cl 0.51-0.98, $P=0.0038)$ and $\operatorname{RR}(0.44,0.27-0.71, \quad P=0.0007)$ were

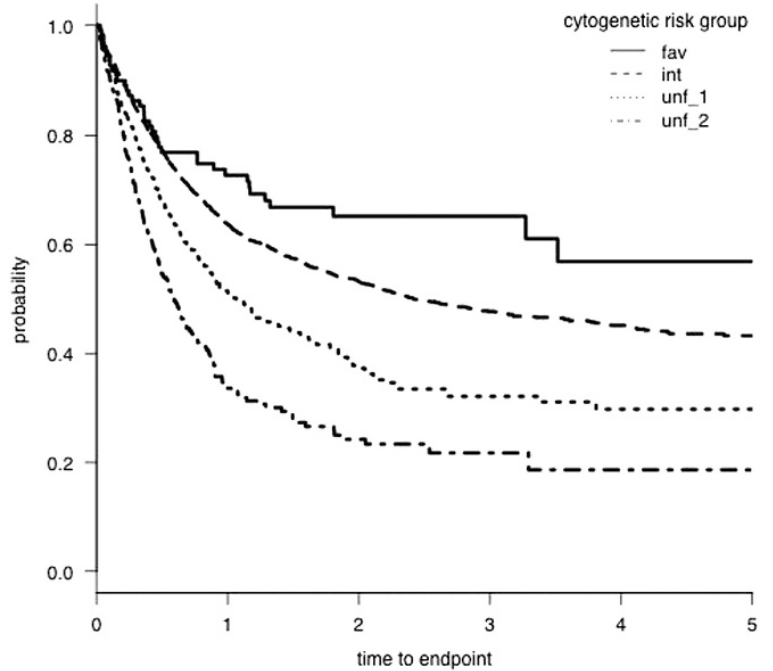

significantly lower than in Int group. On the other hand, compared with Int group, recipients in Unf-1 and Unf-2 group had significantly $(P<0.001)$ higher ROM (Unf-1 1.48, 1.29-1.70; Unf-2 2.22 1.93-2.57) and RR (Unf-1 1.65, 1.41-1.92; Unf-2 2.35, 1.94-2.73)

Conclusion: These results showed that the karyotype of leukemic cells at diagnosis was also one of the powerful prognostic factors in HCT for AML. But the impact of each cytogenetic presentation on the outcomes of transplantation may have some differences from that of chemotherapy. To improve this risk stratification system, molecular abnormalities of $A M L$ at diagnosis are also considered for the influence on outcomes of HCT.

Disclosure of Interest: None declared.

\section{8}

Late intensification with autologous HSCT after nonmyeloablative BEAM conditioning has shown to be effective approach in adults with T-cell acute lymphoblastic leukemia treated by non-intensive protocol: results of the RALL study group

E. Parovichnikova ${ }^{1, *}$, L. Kuzmina ${ }^{1}$, L. Mendeleeva ${ }^{1}$,

Z. Akhmerzaeva ${ }^{2}$, S. Bondarenko ${ }^{3}$, O. Baranova ${ }^{4}$, G. Kliasova $^{5}, \mathrm{~V}$. Troitskaya ${ }^{2}$, A. Sokolov ${ }^{2}$, S. Kravchenko ${ }^{6}$, T. Ryltzova ${ }^{7}$,

L. Gavrilova ${ }^{8}$, A. Pristupa ${ }^{9}$, T. Kaporskaya ${ }^{10}$, E. Zotina ${ }^{11}$,

E. Zinina ${ }^{12}$, V. Lapin ${ }^{13}$, I. Kruchkova ${ }^{14}$, M. Rusinov ${ }^{15}$,

S. Kulikov ${ }^{15}$, V. Savchenko ${ }^{1}$ on behalf of RALL

${ }^{1}$ Bone Marrow Transplant Department, ${ }^{2}$ Acute leukemia department, National Research Center For Hematology, Moscow, ${ }^{3}$ Acute leukemia department, Raisa's Gorbacheva Transplant Center, St.Petersburg, ${ }^{4}$ Hematology department, National Research Center For oncology, Bacteriology department, ${ }^{6}$ Lymphoma High Dose Therapy, National Research Center for Hematology, Moscow, ${ }^{7}$ Hematology Department, Tula's OKB, Tula, ${ }^{8}$ Hematology department, Sarnsk's GKB No. 4, Saransk, ${ }^{9}$ Hematology department, Ryazan's OKB, Ryazan, ${ }^{10}$ Hematology department, Irkutsk's OKB, Irkutsk, ${ }^{11}$ Hematology department, Kirov's National Resears Center for Hematology, Kirov, ${ }^{12} \mathrm{Hema}$ tology Department, Surgut's OKB, Surgut, ${ }^{13}$ Hematology Department, National Research Center For Hematology, Yaroslavl, ${ }^{14}$ Hematology department, Novosibirsk's Clinical Immunology institute, Novosibirsk, ${ }^{15}$ Biostatistic Department, National Research Center For Hematology, Moscow, Russian Federation

Introduction: No data is available in the literature regarding the autologous hematopoietic stem cell transplantation (HSCT) after non-myeloablative BEAM conditioning in patients with acute lymphoblastic leukemia. Since 2009 the RALL study 
group is conducting the ALL-2009 trial where the main principle is non-intensive but non-interruption treatment and autologous HSCT after BEAM followed by maintenance in T-cell ALL pts without HLA-identical donors (EBMT 2014, OP009). The autologous HSCT was planned for all patients as late high dose consolidation (on the 20-22 weeks of the protocol). In patients with early and late T-cell immunophenotype having HLA-identical siblings allogeneic BMT was an option.

Materials (or patients) and methods: From Nov, 2008, till Nov, 2014, 30 centers enrolled 264 ALL patients. In 6 phenotype was unknown (2,2\%). B-cell precursor phenotype was diagnosed in $62,6 \%(n=166)$, T-cell precursor phenotype in $34,8 \%(n=91)$; biphenotypic AL - in 0,4\% $(n=1)$. T-cell ALL patients were young - 28 y (16-56); male gender prevailed $33 \mathrm{f} / 58 \mathrm{~m}$. T-ALL subtypes distribution in our study was as follows: 44 (47,8\%) patients had early T-ALL (T-I/II), 36 (40,1\%) thymic (T-III), $11(11,1 \%)$ - mature (T-IV). For the whole group medians were: $\mathrm{Hb}-112 \mathrm{~g} / \mathrm{l}(42-180), \mathrm{L}-22,3^{*} 10 /{ }^{9} \mathrm{I}(0,5-313)$, plt - $90^{*} 10 /^{9}(5-943), \mathrm{b} / \mathrm{m}$ blasts - 74,9\% (0-99), LDH - $995 \mathrm{IU}$ (13112 000). No born marrow involvement was detected in 4 pts, $\mathrm{b} / \mathrm{m}$ blasts $5-25 \%$ - in 10 (so T-LBL - 14,5\%). Cytogenetics was available in $63,2 \%$ of pts $(n=55 / 87), 45,5 \%$ of them $(n=25 / 55)$ had normal (NK), 25 pts - abnormal karyotype, 5 pts (9\%) had no mitosis. Mediastinum involvement was registered in $48 / 87$ $(54,5 \%)$ and CNS disease - in 11/87 (12,5\%).

Results: Induction and follow-up data were available in 87 pts. CR was achieved in 78/87 (89,8\%): after prephase $=12$, after $1^{\text {st }}$ ind.phase $=45$, after $2^{\text {nd }}$ ind.phase $=21$. Induction death occurred in $n=5(5,7 \%)$. Primary resistance and even progression during induction was registered in $n=4(4,5 \%)$. 28 pts proceeded to autologous HSCT, almost all $(n=26)$ were successfully harvested at a median time of 20 weeks. 2 pts were poor mobilizers and bone marrow exfusion was carried out. No data on MRD level at time of harvesting is available so far. Auto-HSCT was applied at a median 6 months from CR Allogeneic HSCT from sibling donors was performed in $6 \mathrm{CR}$ pts (4 with TI/II and 2 with T-IV). No deaths in allo-HSCT group have occurred.

The land mark analysis at 6 months for chemotherapy group $(n=27)$ and at day of HSCT for transplanted groups was done. Disease free survival constituted for chemotherapy - 55\% at 5 years, and $100 \%$ - in both transplanted groups. None of the transplanted patients relapsed so far. The overall and diseasefree survival in the whole cohort of T-cell ALL patients was $58 \%$ and $68 \%$. In a multivariate analysis only autologous HSCT influenced DFS.

Conclusion: Our study demonstrates that T-ALL may be treated by non-intensive, but non-interruption approach. Even without high dose consolidation 5-years OS and DFS (land mark analysis) constituted 55\%. Autologous HSCT with BEAM conditioning after mild induction/consolidation followed by prolonged maintenance seems to add benefit to the overall optimistic results decreasing the relapse rate from $34 \%$ to $0 \%$ within 5 years.

Disclosure of Interest: None declared.
0099

Allogeneic Transplant in Acute Myeloid Leukemia (AML) According to the Policy of the Rome Transplant Network (RTN): An Intention-to-Treat Analysis

W. Arcese ${ }^{1, *}$, S. Amadori ${ }^{2}$, R. Cerretti ${ }^{1}$, L. Cudillo ${ }^{1}$, G. De Angelis ${ }^{1}$, A. Venditti ${ }^{2}$, F. Buccisano ${ }^{2}$, M. Cantonetti ${ }^{2}$, I. Mangione ${ }^{1}$, M. Andreani ${ }^{3}$, M. Testi ${ }^{3}$, M. C. Rapanotti ${ }^{1}$, P. De Fabritiis', T. Dentamaro ${ }^{4}$, L. Cupelli ${ }^{4}$, A. Tendas ${ }^{4}, M$. C. Tirindelli ${ }^{5}$, O. Annibali ${ }^{5}$, F. Saltarelli $i^{5}$, A. Mengarelli ${ }^{6}$, F. Marchesi ${ }^{6}$, S. Gumenyuk ${ }^{6}, A$. Chierichini, ${ }^{7}$ B. Anaclerico ${ }^{7}, A$. Tafuri ${ }^{8}$, E. Montefusco ${ }^{8}$, A. Ferrari ${ }^{8}$, V. Naso ${ }^{8}$, F. Di Piazza ${ }^{7}$, A. Picardi ${ }^{1}$ on behalf of on behalf of the Rome Transplant Network

${ }^{1}$ Hematology Division - Stem Cell Transplant Unit, ${ }^{2}$ Hematology Division, ${ }^{3}$ Laboratory of Immunogenetics and Transplant Biology, IME Foundation, TOR VERGATA UNIVERSITY OF ROME, ${ }^{4}$ Hematology Division, S.Eugenio Hospital, ${ }^{5}$ Hematology Division, University Campus Bio Medico, ${ }^{6}$ Hematology Division, Regina Elena National Cancer Institute, ${ }^{7}$ Hematology Division, S.Giovanni Addolorata Hospital, ${ }^{8}$ Hematology Division, S.Andrea Hospital, Sapienza University, Rome, Italy

Introduction: Depending on the general policy of each single Center, the number of patients definitively transplanted is variable and does not reflect the true impact of the transplant strategy on all patients considered eligible for the procedure. The present study is an intention-to-treat (ITT) analysis of survival of all adult patients with AML referred to RTN and eligible to an allogeneic transplant.

Materials (or patients) and methods: The first aim of the RTN is the identification of a suitable donor for patients with highrisk haematological malignancy in order to perform transplant in adequate timing. For patients eligible to an allogeneic hematopoietic stem cell transplant (HSCT) and lacking an HLA identical sibling (Id-Sib), the algorithm of choice for an alternative donor is based on a hierarchy according to the following selection criteria: 1) Matched Unrelated Donor (MUD): $\geq 8 / 10 \mathrm{HLA}$ antigen matching tested at high resolution for both class I and class II HLA loci; 2) Cord Blood (CB): single unit selected on the base of cell dose and number of HLA mismatched antigens; 3) Haploidentical Related Donor (HRD), as GCS-F primed, unmanipulated bone marrow.

Results: From January 2006 to December 2013, of 303 patients candidate to an allogeneic HSCT for high-risk AML 254 were adults, of whom $225(89 \%)$ were considered eligible to the procedure. An Id-Sib was available in $72(32 \%)$ cases and a search for an alternative donor was activated for 153 (68\%) patients. During the search process, $10(7 \%)$ patients died early, $6(4 \%)$ were ongoing at time of the analysis and no alternative donor could be identified for $12(9 \%)$ patients. Of the remaining 137 evaluable patients, an alternative donor was definitively identified for $125(91 \%)$ patients. Overall, a donor was available for 197 (94\%) of 209 (137 alternative +72 Id-Sib) evaluable patients. At time of the analysis, of these 197 patients $18(9 \%)$ had lost the transplant eligibility and 7 were scheduled for transplant, so 172 of 225 eligible (80\%) or 209 evaluable $(86 \%)$ patients have been definitively transplanted from Id-Sib $(n=72)$, MUD $(n=35), \mathrm{CB}(n=16)$ or $\operatorname{HRD}(n=49)$. With a median follow up from time of HLA typing of 14 months (range, 0.3-97) for all patients and 30 months (range, 3-97) for survivors, the 1- and 8-year probability of survival for all 225 eligible AML patients was $68 \pm 3 \%$ and $40 \pm 4 \%$, respectively. At the same time points, for the 172 transplanted patients the probability of survival from time of graft was $60 \pm 4 \%$ and $43 \pm 4 \%$, respectively. The probability of survival was particularly dismal for the 37 patients transplanted in advanced disease phase ( $4 \pm 4 \%$ at 4 yrs), whereas for the 135 patients transplanted in early $(C R 1+C R 2)$ phase the 8-yrs probability of survival was $54 \pm 5 \%$ and, by excluding the low number of CB recipients, it was $55 \pm 7 \%$ for 56 HLA ID Sib, $58 \pm 8 \%$ for $40 \mathrm{HRD}$ and $68 \pm 9 \%$ for 28 MUD recipients $(P=\mathrm{ns})$.

Conclusion: RTN policy allows a donor identification in $94 \%$ of all evaluable AML patients and provides an allogeneic transplant to $86 \%$ of them with no substantial differences in 
terms of long-term survival between initially eligible and definitively transplanted patients or by comparing the different donor stem cell sources. Transplant results should be given following information on the specific transplant policy and only the ITT analysis allows to know the real impact of each transplant program.

Disclosure of Interest: None declared.

0100

Panobinostat as maintenance therapy after allogeneic stem cell transplantation in patients with high-risk MDS or AML: Results from the dose-escalation part of the PANOBEST trial

G. Bug 1,* , A. Burchert ${ }^{2}$, E. M. Wagner ${ }^{3}$, N. Kröger ${ }^{4}$, Z. Jedlickova ${ }^{1}$, S. Güller ${ }^{1}$, A. Wolf ${ }^{1}$, S. Hünecke ${ }^{5}$, P. Bader ${ }^{5}$, H. Serve ${ }^{7}$,

O. G. Ottmann ${ }^{1}$

${ }^{1}$ Department of Medicine II, Hematology and Oncology, University Hospital Frankfurt, Frankfurt/Main, ${ }^{2}$ Department of Medicine, Hematology, Oncology and Immunology, University Hospital of Giessen and Marburg, Marburg, ${ }^{3}$ Third Department of Medicine, Hematology, Oncology and Pneumology, University Medical Center of the Johannes Gutenberg-University, Mainz, ${ }^{4}$ Department of Stem Cell Transplantation, University Medical Center Hamburg, Hamburg, ${ }^{5}$ Department for Stem Cell Transplantation and Immunology, University Children's Hospital for Children and Adolescence Medicine, Frankfurt/Main, Germany

Introduction: After allogeneic stem cell transplantation (HSCT), relapse and graft-versus-host disease (GvHD) remain major obstacles. Deacetylase inhibitors (DACi) possess antileukemic and immunomodulatory effects. In this phase I/II trial, we explored whether the DACi panobinostat (PAN) can prevent relapse after HSCT for high-risk (HR) myeloid malignancies while simultaneously reducing GvHD. Primary objectives were to determine the dose-limiting toxicity (DLT), maximum tolerated dose (MTD) and/or recommended phase 2 dose (RP2D) of PAN in adult pts with HR AML or MDS in complete hematologic remission (CR) after reduced-intensity (RIC) HSCT. Secondary objectives were evaluation of safety and tolerability of PAN as well as overall (OS) and disease-free survival (DFS).

Materials (or patients) and methods: Oral PAN was administered in two sequential schedules, either thrice weekly (TIW) every week (A) or every other week (B). Arm A, in which PAN was started at a dose of $10 \mathrm{mg}$ TIW and escalated to $30 \mathrm{mg}$ TIW using a $3+3$ design was followed by arm $B$, in which PAN doses increasing from $20 \mathrm{mg}$ TIW to $40 \mathrm{mg}$ TIW were investigated. PAN was initiated between day +60 and +150 after HSCT and given for up to 1 year. Eligibility criteria included: ANC $\geq 1,000 / \mu \mathrm{L}$, platelets $>75,000 / \mu \mathrm{L}$, adequate organ function and no severe GVHD. DLT was defined as prolonged G4 hematologic toxicity or any non-hematologic toxicity $\geq \mathrm{G} 3$ unrelated to disease progression within 28 days of the first PAN dose.

Results: 24 pts (21 AML, $3 \mathrm{MDS}$ ), median age 52 yrs (range, 2171), are evaluable for MTD. Cytogenetics were classified as low $(n=2)$, intermediate-1/2 $(n=11)$ or adverse risk $(n=11)$ according to ELN criteria. PAN was started a median of 98 days (range, 60-147) after HSCT from a MRD $(n=8)$, MUD $(n=10)$ or mismatched donor $(n=6)$ which was performed in active disease ( $n=17$, median bone marrow blasts $23 \%$, range, 8-77), CR1 $(n=5)$ or CR2 $(n=2)$. The RP2D was $20 \mathrm{mg}$ TIW in arm A and $30 \mathrm{mg}$ TIW every other week in arm B based on 5 DLTs: fatigue $\mathrm{G} 3$ at $20 \mathrm{mg}$, colitis and nausea/emesis G3 each at $30 \mathrm{mg}(\operatorname{arm~A})$, diarrhea and headache G3 at $40 \mathrm{mg}$ each (arm B). PAN-related or unrelated G3/4 AEs were reported in 20 of 24 pts $(83 \%)$ and included hematologic toxicity (50\%), laboratory alterations (42\%), infections $(29 \%)$, constitutional (29\%) and gastrointestinal symptoms (25\%) and neurologic, pulmonary, pancreatic/hepatobiliary or vasculary toxicity (4-8\% each). AEs were rapidly reversible after interruption and/or dose reduction $(n=6)$ and no study-related deaths occurred. Fifteen pts (63\%) have completed one year of PAN, 9 pts discontinued treatment prematurely after 7-217 days due to AEs $(n=8)$ or relapse $(n=1)$. Prophylactic or preemptive DLls (1-6) were administered to 15 pts (63\%). Eleven pts (45\%) developed mild $(n=6)$, moderate $(n=3)$ or severe $(n=2)$ chronic GvHD. Patients died of AML $(n=3)$ or severe chronic $\operatorname{GvHD}(n=1)$. With a median follow up of 24 mos (13-48) after HSCT, 20/24 pts are alive and in continuous CR, resulting in an OS and DFS of $92 \pm 6 \%$ at 2 yrs. The median OS and DFS have not been reached.

Conclusion: PAN maintenance following HSCT is feasible with a RP2D dose of $20 \mathrm{mg}$ TIW weekly or $30 \mathrm{mg}$ TIW every other week. Results of this first prospective trial combining epigenetic therapy with DLI as post-HSCT maintenance for HR myeloid malignancies indicate a remarkably low relapse rate.

Disclosure of Interest: G. Bug Funding from: Novartis Oncology, A. Burchert: None declared, E. M. Wagner: None declared, N. Kröger: None declared, Z. Jedlickova: None declared, S. Güller: None declared, A. Wolf Funding from: Novartis Oncology, S. Hünecke: None declared, P. Bader: None declared, H. Serve: None declared, O. G. Ottmann Funding from: Novartis Oncology, Deutsche Jose Carreras LeukämieStiftung.

\section{Oral session: Autoimmune diseases}

\section{1}

Resetting the immune system in severe autoimmune diseases with immunoablation followed by autologous stem cell transplantation using enriched CD34 + cells is associated with long-term remissions - getting closer to cure

T. Alexander ${ }^{1,2, *}$, A. Sattler ${ }^{3}$, A. Thiel $^{3}$, G. R. Burmester ${ }^{1}$, A. Radbruch ${ }^{4}$, F. Hiepe ${ }^{1,2}$, R. Arnold ${ }^{5}$

${ }^{1}$ Department of Rheumatology and Clinical Immunology, CHARITè - UNIVERSITY MEDICINE BERLIN, ${ }^{2}$ Autoimmunology Group, German Rheumatism Research Center (DRFZ), ${ }^{3}$ BerlinBrandenburg Center for Regenerative Therapies (BCRT), CHARITè - UNIVERSITY MEDICINE BERLIN, ${ }^{4}$ Cell Biology Group, German Rheumatism Research Center (DRFZ), ${ }^{5}$ Department of Hematology, Oncology and Tumor Immunology, CHARITè - UNIVERSITY MEDICINE BERLIN, Berlin, Germany

Introduction: Our previous research has provided the evidence that an autoreactive immune system can be ?reset? into a healthy, tolerant state by immunoablative treatment to eradicate pathogenic effector cells, followed by transplantation of hematopoietic progenitor cells (HSCT). Here, we present the clinical and serologic responses and long-term immune reconstitution in 20 patients with severe ADs for up to 15 years after receiving immunoablation and ASCT.

Materials (or patients) and methods: Since 1998, 20 patients with refractory autoimmune diseases (SLE, $n=10 ; S S C, n=4, M S$, $n=2$; polychondritis, $n=1$; panniculitis, $n=1$, granulomatosis with polyangiitis, $n=1$, and chronic inflammatory demyelinating polyneuropathy, $n=1$ ) received a $\mathrm{CD} 34^{+}$-selected autologous stem cell transplantation after immunoablation with ATG (Neovii, formerly Fresenius) and cyclophosphamide as part of a prospective monocentric phase I/II clinical trial. Autoantibody titers were evaluated with ELISA, peripheral Tand $B$ lymphocyte subsets immunophenotyped using multicolor flow cytometry.

Results: With a median follow-up of 12 years after immune reset (range, 24 months to 16 years), 15 of 20 patients (75\%) achieved a progression-free survival (PFS), defined as survival without major organ failure. $50 \%$ of these patients showed durable clinical remissions for up to 15 years despite discontinuation of immunosuppressive treatment, while 25\% of patients had a stabilization of their underlying disease under maintenance therapy. Disease relapse occurred in three 
SLE patients (at 18, 36, and 80 months, respectively), two of whom died later from uncontrolled disease and infection, respectively. 3 of 20 patients died from infection $(n=2)$ or cardiac failure $(n=1)$. Anti-dsDNA antibodies completely normalized in all SLE patients and ANA significantly declined from a median titer of 1:5120 at baseline to 1:160 six months after transplantation in patients with connective tissue diseases. Recovery of recent thymic emigrants (RTEs) was completed between 12 and 24 months after immune reset, reaching on average 3.6 to 5.1 times the baseline levels. Recurring Foxp $3^{+}$Tregs had significantly higher expression levels for CD31 and CD45RA compared to age-matched healthy controls, indicating their recent thymic origin. Numeric recovery of the naÿve B cell compartment was completed within 1 year after immune reset in responding patients.

Conclusion: These data confirm our assumption that the reprogramming of an autoreactive immune system into a juvenile and self-tolerant immune system is feasible and associated with long-term remissions. Our findings propose that chronic autoimmunity is not an end point depending on continuous treatment with specific anti-inflammatory agents, but may be cured by combining specific targeting of autoreactive memory and effector cells with a reactivation of thymic activity. A future challenge is to make this therapeutic approach attractive for a larger number of patients by reducing the rate of severe infections. This may be achieved by either using a more selective graft purging, e.g., depletion of T cell receptor alpha/beta and CD19+ cells from apheresis products with the CliniMACS Device, or an adoptive transfer of microbe- or virus-specific memory T and/or B cells.

Disclosure of Interest: None declared.

\section{2}

Long term effects of immunoablation and hematopoietic stem cell transplantation in new-onset type 1 diabetes

E. Snarski ${ }^{1, *}$, K. Hataburda ${ }^{1}$, A. Milczarczyk ${ }^{2}$, T. Torosian ${ }^{1}$,

M. Paluszewska', E. Urbanowska', M. Król ${ }^{1}$, E. Franek ${ }^{2,3}$,

W. Wiktor - Jedrzejczak ${ }^{7}$

${ }^{1}$ Hematology, Oncology and Internal Diseaes, Medical University of Warsaw, ${ }^{2}$ Department of Internal Diseases, Diabetology and Endocrinology, Central Hospital, Ministry of Interior Affairs and Administration, ${ }^{3}$ Mossakowski Medical Research Centre, Polish Academy of Sciences, Warszawa, Poland

Introduction: In May 2008 we performed first autologous hematopoietic stem cell transplantation in patient with newonset type 1 diabetes (T1DM). Since then other 23 patients have been transplanted and majority achieved remission of diabetes. In the present report we summarize the long term effects of transplantation.

Materials (or patients) and methods: The mobilization protocol included cyclophosphamide and G-CSF. The conditioning consisted of cyclophosphamide $(50 \mathrm{mg} / \mathrm{kg} / \mathrm{day}$ on days $-5,-4,-3,-2$ prior to transplantation) and antithymocyte globulin (Thymoglobulin - of $0.5 \mathrm{mg} / \mathrm{kg} /$ day given on day -5 , and $1.0 \mathrm{mg} / \mathrm{kg} /$ day given on days $-4,-3,-2$ and -1 ) except for three patients, who received ATG Fresenius at adjusted doses.

Results: One of the transplanted patients died during neutropenia due to the sepsis and its complications. The mean time of observation of remaining 23 patients as of December 2014 was 57 months (range 33 - 80 months). The independence of exogenous insulin after the transplantation was achieved in 20 of them. Three patients were lost to follow up. The median time without exogenous insulin for 20 patients in follow up was 30 months (range $0-80$ months). Four out of $20(20 \%)$ remain in remission of diabetes (exogenous insulin free) with median follow up of 54 months (range 34-80 months). Notably, three patients in the series were treated with ATG Fresenius (due to transient problems with Thymoglobulin availability) - the median follow-up of these patients is 65 months with 58 months of remission of diabetes. The treatment led to significant reduction of $\mathrm{HbA1C}$ and good glycemic control in patients. No attempt was made to repeat procedure in relapsing patients.

Conclusion: The HSCT leads to remission of new onset T1DM in majority of patients but the response in most of them is limited to average of 30 months. Patients receiving ATG Fresenius tended to have longer remission but small number prevented drawing conclusions. There is a need to develop procedure to either prolong response or to effectively treat the relapse of diabetes.

Disclosure of Interest: None declared.

\section{3}

Hematopoietic stem cell transplantation for refractory crohn's disease: feasibility and toxicity

J.-A. $A^{1},{ }^{\prime}$ R. $M^{2,}{ }^{*}$, M. $P^{3}{ }^{3}$, S. $A^{1}$, P.-D. S. ${ }^{1}$, F. F. ${ }^{1}$, E. J.I. ${ }^{1}$, F.-A. F. ${ }^{4}$, M. $C^{4}$, , G. G. ${ }^{4}$, R. L. ${ }^{4}{ }^{\prime}$ C. $E^{4}{ }^{4}$, U. A. ${ }^{4}$, L. M. ${ }^{5}$, C. J. ${ }^{5},{ }^{3}$ S.L. M. ${ }^{4}$,

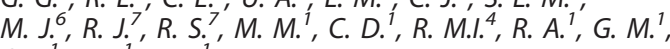
O. I. ${ }^{1}$, P. J. ${ }^{1}, R . E^{1}$

${ }^{1}$ Gastroenterology Department, HOSPITAL CLINIC, ${ }^{2}$ Member of Autoinmune Working Party, EBMT, ${ }^{3}$ Hemotherapy and Hemostasis Department, ${ }^{4}$ Hematology Department, ${ }^{5}$ Hemotherapy and Hemostasis, ${ }^{6}$ Infectious Diseases Department, ${ }^{7}$ Radiology Department, HOSPITAL CLINIC, Barcelona, Spain

Introduction: Autologous hematopoietic stem cell transplantation (HSCT) is a salvage treatment for severe refractory Crohńs disease (CD) patients. We evaluated feasibility and toxicity of autologous HSCT for refractory Crohn's disease (CD). Materials (or patients) and methods: In this prospective study, refractory CD patients with an aggressive course despite medical treatment, impaired quality of life and no surgical options were included. Hematopoietic stem cells were mobilized with cyclophosphamide and G-CSF and collected by leukapheresis from pheripheral blood. In a second step, conditioning regimen with cyclophosphamide and rabbit antithymocite globulin (rATG) was used and previously collected stem cells were infused. Toxicity and complications during the procedure and within first-year after transplantation were evaluated, along with the impact on safety after the introduction of supportive measures over the study.

Results: Twenty-six patients were enrolled. During mobilization, 16 patients presented febrile neutropenia, including 1 bacteremia and 2 septic shocks. Neutropenia median time after mobilization was 5 days. Five patients withdrew from the study after mobilization and 21 patients entered into the conditioning phase. Hematopoietic recovery median time for neutrophils $\left(>0.5 \times 10^{9} / \mathrm{L}\right)$ was 11 days and for platelets $\left(>20 \times 10^{9} / L\right)$ was 4 days. Ninety-five percent of patients suffered febrile neutropenia and 3 patients presented worsening of perianal CD activity during conditioning. Among non-infectious complications, 6 patients presented antithymocyte globulin reaction, 12 patients developed mucositis and 2 patients had hemorrhagic complications. Changes in supportive measures over the study, particularly antibiotic prophylaxis regimes during mobilization and conditioning, markedly diminished the incidence of severe complications. During the first 12-month follow-up, viral infections were the most common observed complications, and one patient died due to systemic cytomegalovirus infection.

Conclusion: Autologous HSCT for refractory CD patients is feasible but extraordinary supportive measures need to be implemented. We consider that this procedure should only be performed in highly-experienced centers.

Disclosure of Interest: None declared. 
0104

Autologous haematopoietic stem cell transplantation in aggressive multiple sclerosis: a UK cohort from two centres

J. Clay 1,* P. Kelsey ${ }^{2}$, S. Bell ${ }^{2}$, C. Lim', J. Helen ${ }^{2}$, R. Nicholas ${ }^{3}$, E. Silber ${ }^{4}$, O. Malik ${ }^{3}$, P. Brex ${ }^{4}$, P. Muraro ${ }^{3}$, B. Sharrack,

J. Snowden ${ }^{2}$, M. Kazmi ${ }^{6}$

${ }^{1}$ Haematology, King's College Hospital, London, ${ }^{2}$ Haematology, Sheffield Teaching Hospitals NHS Foundation Trust, Sheffield, ${ }^{3}$ Neurology, Imperial College Healthcare NHS Trust, ${ }^{4}$ Neurology, King's College Hospital, London, ${ }^{5}$ Neurology, Sheffield Teaching Hospitals NHS Foundation Trust, Sheffield, ${ }^{6}$ Haematology, Guys and St Thomas' Hospital, London, United Kingdom

Introduction: Despite the emergence of disease modifying therapies (DMT) for multiple sclerosis (MS) many patients have ongoing progression/relapse associated with progressive disability. Autologous Haematopoetic Stem Cell Transplantation (AHSCT) has been recommended for aggressive MS with inflammatory changes on MRI ${ }^{1}$, although uptake in the UK has previously been low compared with the rest of Europe ${ }^{2}$. We report our experience across 2 UK transplant centres (Kings and Sheffield).

Materials (or patients) and methods: A retrospective evaluation of AHSCT performed for MS in 2 UK centres from 2009-2014 was undertaken. Patients were selected based on persistent clinical relapses (Relapse-Remitting) or secondary progressive neurological disability with MRI lesion activity despite use of at least 1 disease modifying treatment (DMT). Follow-up included clinical evaluation, EDSS assessment and MRI scanning. We report our preliminary findings.

Results: Follow-up was for a median of 517 days (range 371992), with 1 death at 5.5 years due to progressive MS. There was no transplant related mortality. Whilst conditioning and stem cell infusion were well tolerated 19/21 patients experienced infectious complications. Of patients with a 100 day assessment $(n=19) 7$ patients had a stable EDSS, 8 had an improved EDSS score with a median improvement of 0.5 (range $0.5-4.5$ ), whilst the remaining 4 had a deterioration of $0.5-1.0$ in EDSS.

Conclusion: AHSCT was feasible and well tolerated in this UK cohort, with the majority of patients showing an improvement in EDSS. There is a need for randomised controlled trials to compare AHSCT against DMT.

References: 1. Snowden JA. Bone Marrow Transplantation. 2012. Jun;47(6):770-790

2. Snowden JA et al. British Journal of Haematology. 2012. 157(6):742-746

Disclosure of Interest: None declared.

0105

Hematopoietic stem cell transplantation for pediatric patients with multiple sclerosis - Russian project, 5 years after beginning

K. Kirgizov ${ }^{1,2, *}$, E. Volkova ${ }^{2}$, E. Pristanskova ${ }^{2}$, N. Sidorova ${ }^{2}$,

V. Konstantinova ${ }^{2}$, O. Blagonravova ${ }^{2}$, S. Piliya ${ }^{2}$,

A. Bologov', R. Bembeeva ${ }^{3}$, E. Skorobogatova',

A. Maschan

${ }^{7}$ Dmitry Rogachev Research Center of Pediatric Hematology, Oncology and Immunology, 'The Russian Children's Research Hospital, ${ }^{3}$ Pirogov Russian National Research Medical University, Moscow, Russian Federation

Introduction: Auto-HSCT for pediatric pts. with refractory multiple sclerosis showed effectiveness in earlier post-transplant period. Long-time follow-up required.
EDSS (J. Kurtzke, 1983)

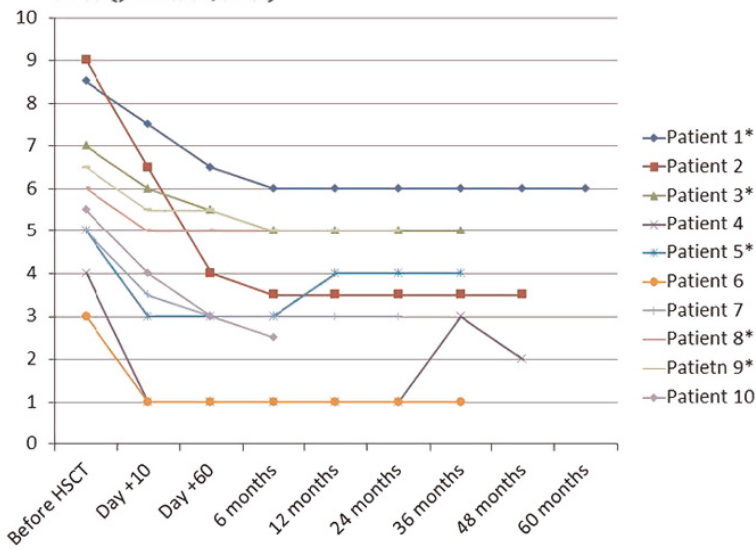

Materials (or patients) and methods: 10 pts. were included in the analysis. Gender: female $-80 \%(n=8)$, male $-20 \%$ $(n=2)$. Median age $-16,7 \pm 1,7$. Median length of MS prior to HSCT - 15,5 $\pm 4,1$. Age of MS debut: 4-14 y.o. (12,3 $\pm 1,7$ y.o.). Median EDSS 6,16 $\pm 0,2$. All patients had severe refractory MS treated with corticosteroids, interferons, plasmapheresis and mitoxantron with negative results. All pts. had inflammation signs. Procedures: PBSC mobilization - Cph $60 \mathrm{mg} / \mathrm{kg}$ followed by G-CSF. Conditioning: Cph $200 \mathrm{mg} / \mathrm{kg}$ and ATGAM $160 \mathrm{mg} /$ $\mathrm{kg}$. PBSC reinfusion - on day 0 . G-SCF stimulation from day +5 .

Results: All pts. showed the fast improvement (EDSS improvement: first 60 d. $-2,5 \pm 0,21$, after 60 d. $-0,2 \pm 0,02$ ) Maximal EDSS improvement - 5,5. EDSS at pts. 4 and 6 improved to 1. Median follow-up 21,1 $\pm 0,9$ months (4-60 months). Pts. 1, 3, 5, 8 and 9 (marked ${ }^{*}$ in scheme) had severe refractory secondary-progressive MS with the long duration of ineffective treatment (late transplant). These pts. showed the minor improvement and required the additional immunomodulation after Auto-HSCT. Pts 4 and 5 relapsed (clinical and $\mathrm{MRI})$. No severe complications were registered.

Conclusion: Auto-HSCT is an effective way of autoimmune inflammation reduction and successful approach to the treatment of severe refractory pediatric MS. In-time AutoHSCT can significantly minimize the disability level and improve the outcome of MS. Most pts. remain in remission during the long time of follow-up.

Disclosure of Interest: None declared. 


\section{Oral session: Stem cell source and graft composition}

\author{
0106 \\ Modification of standard ISHAGE methodology for CD34 + \\ cells count on thawed Cord Blood Units: results from a \\ multi-center Eurocord/Netcord study \\ R. Saccardi ${ }^{1, *}$, C. Azqueta ${ }^{2}$, C. Ballerini ${ }^{3}$, L. Ballerini ${ }^{1}$, E. Baudoux ${ }^{4}$, \\ R. Caporale, S. Dal Pozzo', S. Gomez', G. Kogler', F. Lanza ${ }^{5}$, \\ M. L. Angelotti ${ }^{3}$, A. Madrigal ${ }^{9}$, M. Ongari ${ }^{8}$, S. Querol ${ }^{2}$, T. Radke ${ }^{7}$, \\ V. Rocha ${ }^{10}$, A. Ruggeri ${ }^{11}$, S. Urbani ${ }^{1}$, E. Gluckman ${ }^{11}$ on behalf of \\ Eurocord/Netcord \\ ${ }^{1}$ Hematology, AOU Careggi, FLORENCE, Italy, ${ }^{2}$ Barcelona Cord \\ Blood Bank, Barcelona, Spain, ${ }^{3}$ University of Florence, FLORENCE, \\ Italy, ${ }^{4}$ University of Liege, Liege Cord Blood Bank, Liege, France, \\ ${ }^{5}$ AOU Careggi, FLORENCE, Italy, ${ }^{6}$ Anthony Nolan Cell Therapy \\ Centre, Nottingham, United Kingdom, ${ }^{7}$ University of Dusseldorf, \\ Dusseldorf Cord Blood Bank, Dusseldorf, Germany, ${ }^{8}$ Section of \\ Hematology \& BMT Unit, Hospital of Cremona, Cremona, Italy, \\ ${ }^{9}$ Anthony Nolan Research Institute, Nottingham, ${ }^{10}$ Churchill \\ Hospital, Oxford, United Kingdom, ${ }^{11}$ Hospital Saint Louis, \\ $A P-H P$, and IUH University Paris VII, Eurocord, Paris, France
}

Introduction: CD34 + cells counting technique in Cord Blood (CB) is well standardized on fresh samples, whereas the flowcytometry (FC) analysis of thawed samples is still controversial, and no validated techniques are yet available. We report an exercise carried out in 5 European Banks (Barcelona, Cremona Dusseldorf, Florence, Nottingham) on behalf of Eurocord and Netcord, aimed to standardize and improve the FC assessment of thawed CB Units (CBU).

Materials (or patients) and methods: $25 \mathrm{CB}$ Units (CBU) were frozen and thawed across the participating Banks, according to local SOPs; pre-freezing and post-thaw samples were assessed for clonogenic potential (CFU) and CD34 + recovery and viability through standard ISHAGE protocol (SI) (Brocklebank AM 2001). A modified ISHAGE gating strategy (MI) was developed by reduction of the debris gate and by extension of Lympho-monocytes gate in order to detect CD34 + events with abnormal physical parameters (CD34+ gate). FACS sorting was performed to collect CD45 + /CD34 + events included inside (P8) and outside(P9) the SI CD34 gate. Sorted cells were then characterized by FC, confocal microscopy analysis and CFU assay in order to confirm that CD34 + cells detected out of the SI CD34 gate are not an artifact. Confocal microscopy analysis was performed by labeling with a CD34 $A b$ recognizing a different epitope and marked with a nuclear counterstain.

Results: SI and MI strategies showed no significant discrepancies when determining CD34 + absolute number and viability in pre-freezing CBUs. The recovery of viable CD34+ cells assessed by SI or MI in the thawed products did not show any differences statistically significant, whilst the recovery of total CD34 + cells was significantly lower in SI (58,3 $\pm 18 \%)$ than in $\mathrm{MI}(80,1 \pm 19.7 \%)$-analyzed samples (mean $\pm S D$, $P<0,05$ with pair $T$ test). This difference resulted in a lower CD34 + viability with MI than SI $(79,5 \% \pm 15.9$ vs $96,7 \% \pm 3 \%$, respectively, $P<0.02$ ).

FC post-sorting analysis showed that the sorted P8 (SI CD34 gate) population was $\mathrm{CD} 45^{\mathrm{dim}} \mathrm{CD} 34^{+} \mathrm{CD} 133^{+} \mathrm{CD} 5^{-}$7-AAD-, whereas P9 (MI extended CD34 gate) showed the same markers expression but a 7-AAD + staining, therefore determining such events as CD34+ dead cells. This data was confirmed by confocal microscopy analysis. Finally, P8 events showed a $67 \%$ clonogenic efficiency, whereas P9 events were not able to generate any colony.

Conclusion: A CD34 + count after CBU thawing lower than the fresh sample is often reported, usually with a high rate of viability. We showed that the standard methodology for CD34 + counting leads to the exclusion of a number of dead $\mathrm{CD} 34+$ cells, thus resulting in a viability overestimation. We propose here a modification of the ISHAGE counting standard for thawed samples, which provides a more reliable assessment of CD34 + viability. If validated, this methodology might improve the reliability of quality controls on stored CBU and reduce discrepancies between CD34 + counting carried out at Banks and Transplant Units, respectively.

References: Brocklebank AM, Sparrow RL. Enumeration of CD34 + cells in cord blood: a variation on a single-platform flow cytometric method based on the ISHAGE gating strategy. Cytometry, 2001 Aug 15;46(4):254-261.

Disclosure of Interest: None declared.

\section{7}

The peripheral blood stem cell CD34 + and CD3 + compositions do not influence outcome after allogeneic transplantation prepared with ATG-included reduced intensity conditioning regimen

A. Collignon ${ }^{1, *}$, R. devillier ${ }^{1}$, S. furst ${ }^{1}$, S. harbi ${ }^{1}$, A. granata ${ }^{1}$,

J. el cheikh ${ }^{1}$, B. mohty ${ }^{7}$, B. calmels ${ }^{1}$, C. lemarie ${ }^{1}$, C. chabannon ${ }^{1}$, D. blaise

${ }^{1}$ HEMATOLOGY, Institut Paoli Calmettes, marseille, France

Introduction: We previously reported that a high CD34 + and $\mathrm{CD} 3+$ cell dose in peripheral blood stem cell (PBSC) graft increased the incidence of GVHD, impairing survival. This issue is presently debated in the setting of reduced intensity conditioning regimen using in-vivo T-cell depletion. We investigated the impact of CD34 + and CD3 + cells graft composition on clinical outcome after Allo-HSCT in patients receiving a homogeneous $\mathrm{RIC}$ regimen with fixed dose of rabbit antithymocyte globulin (ATG) at a single institution.

Materials (or patients) and methods: Consecutive patients transplanted from 2005 to 2013 with following selection criteria were included: 1) Reduced intensity conditioning (RIC) regimen combining fludarabine $(30 \mathrm{mg} / \mathrm{m} 2 / \mathrm{d}$ during 5 days), intravenous busulfan ( $3.2 \mathrm{mg} / \mathrm{kg} / \mathrm{d}$ during 2 days) and ATG ( $2.5 \mathrm{mg} / \mathrm{kg} / \mathrm{d}$ during 2 days); 2) Allo-HSCT using PBSC from matched related or 10/10 matched unrelated donor; 3) CSA alone as GVHD prophylaxis.

We analyzed the impact of CD34 + and CD3 + cell doses (low vs. high with cut-off at the median value) on non-relapse mortality (NRM), cumulative incidence of relapse (CIR), progression free (PFS) and overall survival (OS) in both univariate and multivariate analyses adjusted for age, hematopoietic cell transplantation comorbidity index (HCT-Cl), revised disease risk index (DRI) and donor type.

Results: We analyzed 246 patients with a median age of 59 years (19-71).Among them, $114(46 \%)$ and $132(54 \%)$ patients had myeloid and lymphoid disease, respectively. HCT-Cl was $>=3$ in 110 patients (46.4\%) and DRI was low, intermediate and high/very high in $44(18 \%), 152(52 \%)$ and $50(20 \%)$ patients, respectively. Median CD34 + and CD3 + cell dose was $6.5 \times 10 \mathrm{e} 6 / \mathrm{kg}(1-14.2)$ and $278 \times 10 \mathrm{e} 6 / \mathrm{kg}$ (61-1919), respectively.

Cumulative incidences of grade 2-4 acute GVHD and all grade chronic GVHD was $38 \%$ and $22 \%$. With a median follow-up of 23 months (5-76), 2-year NRM, CIR, PFS and OS in the overall population was $21 \%, 26 \%, 54 \%$ and $65 \%$, respectively.

In univariate analyses, no difference in terms of GVHD (acute and chronic), NRM, CIR, PFS and OS was found between low and high CD34 + cell doses, as well as according to the CD3 + cells. The multivariate model confirmed the absence of impact of both CD34 + and CD3 + cell doses on acute GVHD (CD34: $H R=0.8, C D 3: H R=0.9)$, chronic GVHD (CD3: $H R=1.0, C D 34:$ $\mathrm{HR}=1.1)$, NRM (CD34: $\mathrm{HR}=1.0, \mathrm{CD} 3: \mathrm{HR}=0.8), \mathrm{CIR}(\mathrm{CD} 34:$ $\mathrm{HR}=0.7, \mathrm{CD} 3: \mathrm{HR}=0.8), \mathrm{PFS}(\mathrm{CD} 34: \mathrm{HR}=0.8, \mathrm{CD} 3: \mathrm{HR}=0.8)$ and OS (CD34: $\mathrm{HR}=0.7, \mathrm{CD} 3: \mathrm{HR}=0.8$ ) [all $P>0.20$ ]. High or very high DRI was the only predictor for shorter $\mathrm{OS}(\mathrm{HR}=2.6$, $P=0.009)$ while the use of a MUD was associated with lower CIR (HR $=0.55, P=0.043)$ but also with higher acute GVHD $(\mathrm{HR}=3.2, P<0.001)$, leading to similar $\mathrm{OS}(\mathrm{HR}=1.2, P=0.415)$. We found no difference according to age and HCT-Cl, for none of the end points. 
Conclusion: We found no impact of CD34 + and CD3 + cell doses on outcome after Allo-HSCT following RIC regimens containing intermediate ATG dose. The use of the latter could explain in part these results, notably the low incidence of chronic GVHD. Although the presence of inherent bias due to its retrospective nature, our study suggest that there is no rational for limiting the maximal amount of CD34 + or CD3 + cell dose in PBSC graft in this situation.

Disclosure of Interest: None declared.

0108

Plasmacytoid dendritic cells (pDC) from different allograft sources differ in expression of genes that regulate innate and adaptive immunity after allogeneic stem cell transplantation: implications for selection of the source of the stem cell graft

E. K. Waller ${ }^{1, *}$, W. Harris ${ }^{2}$, R. Pauly ${ }^{3}$, J. Kowalski ${ }^{3}$, B. Logan ${ }^{4}$

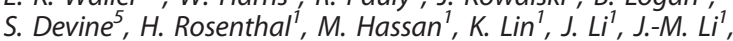
C. Giver ${ }^{7}$, S. Hosoba

${ }^{1}$ Dept. Hematology/Medical Oncology Winship Cancer Institute, ${ }^{2}$ EMORY UNIVERSITY, Atlanta, United States, ${ }^{3}$ Biostatistics and Bioinformatics Shared Resource, Winship Cancer Institute, EMORY UNIVERSITY, Atlanta, ${ }^{4}$ Center for International Blood and Marrow Transplant Research, Department of Medicine, Medical College of Wisconsin, Milwaukee, ${ }^{5}$ Blood and Marrow Transplant Program, Ohio State University, Columbus, United States

Introduction: BMTCTN0201 randomized leukemia or MDS patients and their unrelated donors between bone marrow (BM) or GCSF-mobilized blood stem cell (GPB) transplantation. The content of $\mathrm{pDC}$ in BM grafts had a significant beneficial effect on post-transplant survival, an association not seen in GPB grafts ${ }^{1}$. The $20 \%$ improved survival seen in recipients of more donor BM pDC was due to decreased deaths from graft rejection and acute and chronic GvHD. Donor BM pDCs in murine BMT favorably modulated T-cell activation, decreasing GvHD through gamma-Interferon-dependent upregulation of IDO, while preserving GVL effects ${ }^{2}$. The present study tested whether the biological activity of pDC from different graft sources are explained by the differential expression of homing receptor molecules or immuneregulatory pathway genes.

Materials (or patients) and methods: FACS isolated $\mathrm{pDC}$ from allogeneic stem cell grafts obtained from 10 BM, 8 GPB, 4 umbilical cord blood (UCB) and 2 following mobilization with plerixafor (PLXPB) samples. Expression of chemokine receptor integrin and selectin were measured by flow cytometry. Gene expression on flow cytometry-sorted pDC was analyzed with Illumina chips. Differentially expressed genes using a 2-sided t-test comparing groups of replicate samples with $P<0.05$ were selected for exploratory analyses of regulatory pathways. $\mathrm{MHC}$ mismatched mouse $\mathrm{H} 2 \mathrm{~b} \rightarrow \mathrm{H} 2 \mathrm{k}$ transplants compared outcomes with pDC from CCR7 KO vs WT donor. Indirect presentation of allogeneic peptides by $\mathrm{pDC}$ and classical DC was studied using $\mathrm{H} 2 \mathrm{~b} \rightarrow \mathrm{H} 2$ b/d transplants with $\mathrm{H} 2 \mathrm{Kd}$ peptide recognition by TEA transgenic donor T-cells.

Results: $\mathrm{pDC}$ from BM and PLXPB grafts had higher CD62L expression but lower CCR7 and CXCR3 expression than pDC from GPB and UCB grafts. High CXCR4 and low CCR9 expression were seen in $\mathrm{pDC}$ from all graft sources. While differences in CCR7 expression between pDC from BM vs GPB grafts suggested homing differences might explain observed differences in TRM associated with the pDC content of the allograft, donor PDC from CCR7 KO mice had equal ability to modulate GvHD as pDC from WT mice. pDC in grafts from both human and mice were phenotypically immature and were relatively ineffective in activating T-cells through indirect presentation of alloantigen peptide, indicating that immune regulatory effects might be more important than antigen presentation. Supporting this hypothesis, gene expression patterns of human pDC from BM showed lower expression levels of genes related to adaptive immunity, and higher

\section{Figure 1. Non-supervised hierarchical gene cluster analysis of gene expression of FACS isolated pDC from BM $(n=2)$ versus G-PB $(n=4)$ grafts.}

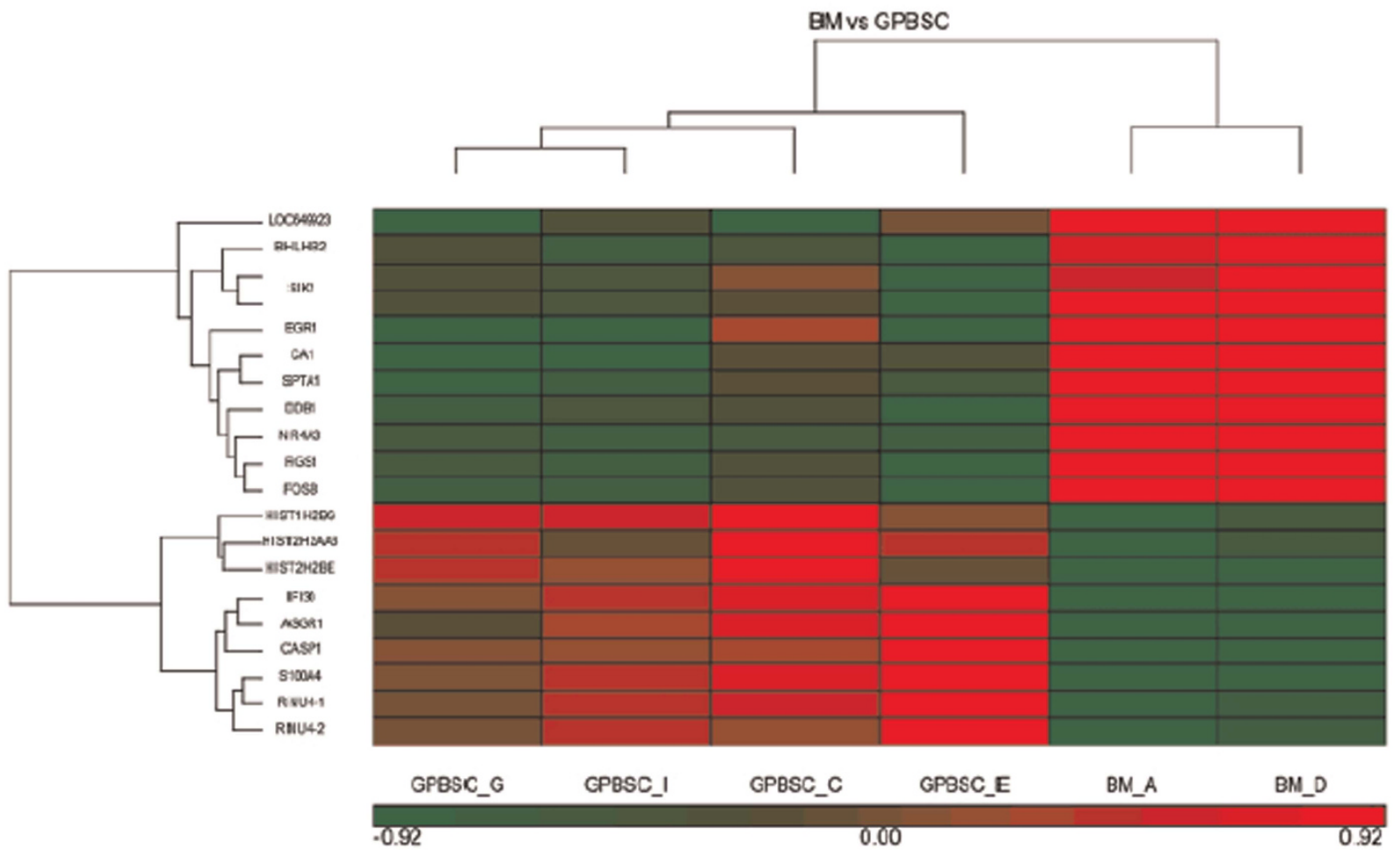


expression of genes associated with innate immunity, than pDC from GPB, UCB, or PLXPB grafts.

Conclusion: Functional differences in immuneregulatory genes explain some of the observed differences in transplant outcome when stratifying recipients based upon between the content and source of donor pDC. BM pDC appear to be polarized towards activation of innate immunity while $\mathrm{pDC}$ from other graft sources are more polarized towards antigen presentation. Novel approaches of stem cell mobilization, such as plerixafor, may increase the content of immature pDC enriched for expression of that might be effective in recapitulating the beneficial effects of $\mathrm{BM}$ pDC with respect to preventing early posttransplant transplant related mortality.

References: ${ }^{1}$ Waller EK. JCO, 2014

${ }^{2}$ Lu Y. Blood, 2012

Disclosure of Interest: None declared.

\section{9}

Effect of graft source on unrelated donor hemopoietic stem cell transplantation in adults with acute myeloid leukaemia after RIC or NMA conditioning: a study from the Société Francaise de Greffe de Moelle et de Thérapie Cellulaire

F. Malard, ${ }^{1, *}$, N. Milpied ${ }^{2}$, D. Blaise ${ }^{3}$, P. Chevallier ${ }^{1}$, M. Michallet ${ }^{4}$, B. Lioure $^{5}$, L. Clement ${ }^{6}, Y$. Hicheri ${ }^{7}$, C. Coordonnier ${ }^{8}$,

R. Peffault de Latour ${ }^{9}, M$. Mohty ${ }^{10}$

${ }^{1}$ Hematology department, CHU de Nantes, Nantes, ${ }^{2}$ Hematology department, $\mathrm{CHU}$ de Bordeaux, Bordeaux, ${ }^{3}$ Hematology department, Institut Paoli-Calmette, Marseilles, ${ }^{4}$ Hematology department, CHU de Lyon, Lyon, ${ }^{5}$ Hematology department, CHU de Strasbourg, Strasbourg, ${ }^{6}$ Hematology department, $\mathrm{CHU}$ de Nancy, Nancy, ${ }^{7}$ Hematology department, CHU de Montpellier, Montpellier, ${ }^{8}$ Hematology department, AP-HP, Hôpital Henri Mondor, Créteil, ${ }^{9}$ Hematology department, AP-HP, Hôpital Saint Louis, ${ }^{10}$ Hematology department, AP-HP, Hôpital Saint Antoine, Paris, France

Introduction: Allo-SCT is a well-established therapy for adult AML patients. Previous studies found a similar outcome between HLA 4-6/6 matched UCB, 7/8 mismatched unrelated donors (URD) and $8 / 8$ matched URD. However, it is more common practice in many centers worldwide to search for 10/ 10 or $9 / 10$ URD.

Materials (or patients) and methods: This retrospective analysis assessed the outcome of 651 patients over 18 years at time of transplant with an AML who received a reduced intensity conditioning $(\mathrm{RIC})$ or non myeloablative conditioning (NMA) allo-SCT from UCB $(n=205)$ or G-CSF PBSC 9/10 URD $(n=99)$ or $10 / 10$ URD $(n=347)$ between 2002 and 2010 and who were reported to the registry SFGM-TC. AML cytogenetic risk was favorable in 77 patients, intermediate in 373 and high risk in 113 cases; and unknown in 88 patients. Cytogenetic risk groups were comparable between all three groups. Patients' median age was 55 years (19-70), 540 patients (83\%) were in CR and 97 (17\%) were in relapse/progression. In UCB, 99 patients received a single UCB unit and 106 patients received 2 UCB units.

Results: With a median follow-up of 43,43 and 49 months in the 10/10 URD, 9/10 URD and UCB, hematopoietic recovery was slower in UCB compared to URD recipient: the cumulative incidence of neutrophils recovery at day 28 and platelet recovery $>50 \times 10^{9} / \mathrm{L}$ at day 180 were respectively $73 \%$ and $56 \%$ in UCB against $96 \%$ and $85 \%$ in $10 / 10$ URD and $95 \%$ and $75 \%$ in $9 / 10$ URD $(P<0.0001)$. While there was no significant difference in the day 100 cumulative incidence of grade 2-4 aGVHD: $31 \%$ in 10/10 URD, 39\% in 9/10 URD and 32\% in UCB, the 2 years cumulative incidence of CGVHD was significantly lower in UCB recipients: $16 \%$ against $37 \%$ in $10 / 10$ URD $(P<0.0001)$ and $29 \%$ in $9 / 10$ URD $(P=0.004)$. In multivariate analysis, there was no statistically significant difference in NRM between UCB recipients and $9 / 10$ recipients (HR $1.58, P=0.13$ ) or $10 / 10$ recipients (HR $1.35, P=0.25$ ). In multivariate analysis, the cumulative incidence of relapse/progression was significantly lower in 10/10 URD recipients compared to UCB recipients (HR 0.60, $P=0.02$ ) and there was also a trend towards a decreased incidence of relapse in the 9/10 URD recipients (HR 0.62, $P=0.07$ ). Compared to UCB transplants there was no significant difference in PFS after 9/10 URD (HR $1.17, P=0.29)$ and $10 / 10$ URD (HR $1.10, P=0.49$ ). For OS, there was no significant difference between the 9/10 URD group and UCB transplants (HR 0.98, $P=0.92$ ), but there was a trend toward a better OS in the 10/10 URD group (HR 0.74, $P=0.08$ ). Disease status at transplant (patients not in $C R$ versus $C R$ ) was the only parameter with a significant impact on OS in multivariate analysis (HR 3.2, $\mathrm{P}<0.0001$ ).

Conclusion: These data suggest that UCB is a valid alternative graft source compared to 9/10 URD in patients who lack a 10/ 10 URD. The increased incidence of relapse/progression in UCB transplant was counterbalance by a decreased NRM incidence. Furthermore this study highlighted the significantly lower cumulative incidence of cGVHD in UCB transplant recipients, an important factor for patients' quality of life after transplant. This should encourage us to pursue our effort to use UCB RIC/ NMA allo-SCT with the development of new strategies to prevent relapse after transplant.

Disclosure of Interest: None declared.

\section{0}

Single Umbilical Cord Blood Transplanation with CoInfusion of CD34 + Cells from a Third Party Donor Compared to Unmanipulated Haploidentical transplantation (Haplo-SCT) with Post-Transplant Cyclophosphamide in Patients with Acute Leukemia M. Kwon ${ }^{1, *}$, J. Gayoso ${ }^{1}$, P. Balsalobre ${ }^{1}$, I. Buño ${ }^{1}$, D. Serrano ${ }^{1}$, J. Anguita ${ }^{1}$, J. L. Diez Martin ${ }^{1}$

${ }^{1}$ Hematology, Hospital Gral. Univ. Gregorio Marañon, Madrid, Spain

Introduction: In the absence of an HLA matched donor, umbilical cord blood (CB) and haploidentical donors are alternative sources. We analyze outcomes of patients with acute leukemia and myelodysplastic syndrome who underwent both procedures in a single centre.

Materials (or patients) and methods: Between 2004 and 07/ 2014, 27 consecutive patients underwent single CB myeloablative transplantation with the co-infusion of CD34+ cells from a third party HLA- mismatched donor (haplo-cord SCT), and between 2012 and 07/2014, 17 patients recieved a myeloablative unmanipulated haploidentical transplantation (haplo-SCT) with post-transplant cyclophosphamide as GvHD prophylaxis in our centre (Table 1).

Results: There were no significant differences in pre-transplant characteristics between both groups (Table 1). The 30day cumulative incidence of neutrophil engraftment was similar in both groups ( $93 \%$ vs $100 \%)$, with a median time of 16 and 17 days, respectively $(P=0.28)$. Similarly, the 60 - day cumulative incidence of platelet engraftment was $70 \%$ vs $76 \%$, in a median time of 39 and 35 days, respectively $(P=0.11)$. Four cases among the haplo-cord group showed primary $C B$

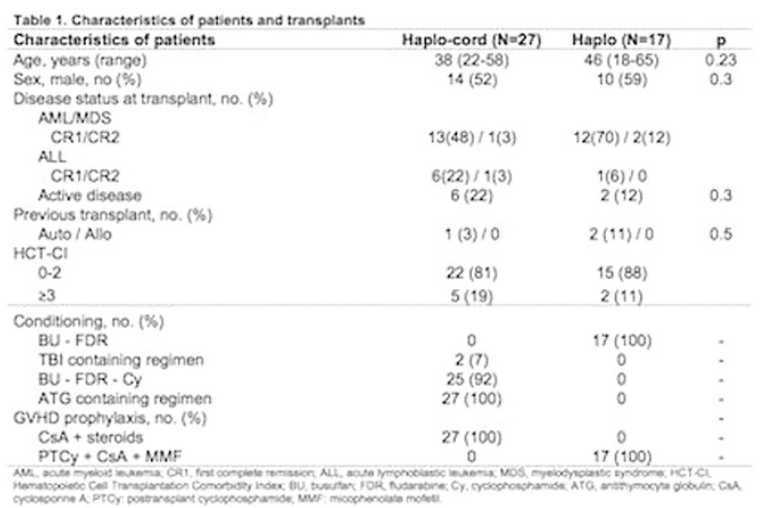


graft failure (3 of them showing third party donor cells engraftment) who were rescued by a second CB transplant in two cases and haplo-SCT in two cases. There were no graft failures in the haplo-SCT group. Grade II-IV acute GvHD was more frequent in the haplo-SCT group ( $20 \%$ vs $41 \%, P=0.06$ ) and chronic GvHD showed a higher tendency in the haplo group ( $18 \%$ vs $35 \%, P=0.24)$. With a median follow-up of 51 months (10-122) in the haplo-cord group and 19 months (6$34)$ in the haplo-SCT group, OS was $52 \%$ and $78 \%(P=0.15)$, respectively. EFS was $41 \%$ vs $72 \%(P=0.06)$, relapse rate was $22 \%$ vs $20 \%(P=0.71)$, and TRM was $22 \%$ vs $8 \%(P=0.16)$, respectively.

Conclusion: In our experience, albeit differences in follow-up and limited number of patients, myeloablative single cord supported by HLA-mismatched cells and haploidentical transplantation with post-transplant cyclophosphamide both offer valid alternatives for patients with acute leukemia. Engraftment rates are similar in both groups as well as relapse rate. Early toxic mortality is higher in the haplo-cord group while GvHD rates seem to be higher in the haplo-SCT patients. Further analysis including larger series and longer follow-up are needed to confirm these observations.

Disclosure of Interest: None declared.

\section{Oral session: Lymphoma}

0111

Benda-BEAM conditioning prior to auto-SCT is effective in resistant DLBCL specific subset: a phase II multicenter study

G. Visani $i^{1, *}$, A. Olivieri', E. Angelucci ${ }^{3}$, S. Guidi ${ }^{4}$, P. Scalzulli, S. Capria ${ }^{6}$, F. Patriarca ${ }^{7}$, B. Castagnari ${ }^{8}$, F. Angrilli ${ }^{9}$, D. Vallisa $^{10}$ G. Musuraca ${ }^{11}$, A. Mengarelli $i^{12}$, P. Galieni ${ }^{13}$, F. Gaudio ${ }^{14}$, P. Tosi ${ }^{15}$, P. Zinzani ${ }^{16}$, A. Bosi $^{4}$, M. Montanari ${ }^{2}$, D. Baronciani ${ }^{3}$,

N. Cascavilla ${ }^{5}$, F. Loscocco ${ }^{1}$, M. Rocchi ${ }^{17}$, A. Isidori ${ }^{1}$

${ }^{1}$ AORMN Hospital, Hematology and Stem Cell Transplant Center, Pesaro, ${ }^{2}$ Hematology, Ospedali Riuniti, Ancona, ${ }^{3}$ Hematology, Armando Businco Hospital, Cagliari, ${ }^{4}$ Azienda ospedaliera Universitaria Careggi, Hematology and Stem Cell Transplant Center, Firenze, ${ }^{5}$ IRCCS-Casa Sollievo della Sofferenza Hospital, Hematology, San Giovanni Rotondo, ${ }^{6}$ Department of Biotechnologies and Hematology, Università "La Sapienza", Roma, 'Santa Maria della Misericordia Hospital, Hematology, Udine, ${ }^{8}$ Oncohematology, Santa Maria delle Croci Hospital, Ravenna, ${ }^{9}$ Hematology, Spirito Santo Hospital, Pescara, ${ }^{10}$ Hematology, Guglielmo da Saliceto Hospital, Piacenza, ${ }^{11}$ Oncohematology, IRST, Meldola, ${ }^{12}$ Hematology and Stem Cells Unit, IRCCS, Regina Elena Institution, Roma, ${ }^{13}$ C.G. Mazzoni Hospital, Hematology, Ascoli Piceno, ${ }^{14}$ University of Bari Medical School, Division of Hematology, Bari, ${ }^{15}$ Infermi Hospital, Hematology, Rimini, ${ }^{16}$ Azienda Ospedaliera "Sant'Orsola-Malpighi", Insitute of Hematology "L\&A Seragnoli", Bologna, ${ }^{17}$ Università di Urbino, Institute of Biomathematics, Urbino, Italy

Introduction: Studies on conditioning regimens prior to autologous stem cell transplant (ASCT) in lymphomas generally present a major pitfall, the miscellaneous mix of histologies composing the series, resulting in a reduced statistical power when focusing on a specific subset.

We previously demonstrated (Visani et al, Blood 2011) the safety of a new conditioning regimen with bendamustine, etoposide, cytarabine, and melphalan (BeEAM) prior to ASCT in resistant/relapsed lymphoma patients (EUDRACTnumber2008002736-15). The regimen showed long-lasting significant antilymphoma activity, with a 3-year PFS of $75 \%$.

Materials (or patients) and methods: We thus designed a single histology, phase II study to evaluate the efficacy of the BeEAM conditioning in resistant/relapsed diffuse large B-cell non-Hodgkin lymphoma (DLBCL) patients. The study was registered at at European Union Drug Regulating Authorities Clinical Trials (EudraCT) N. 2011-001246-14.The primary endpoint of the study is to evaluate the 1-year complete remission rate. Fixing the lowest acceptable rate as $55 \%$ and the successful rate as $70 \%$, with a significance level $a=0.05$ and a power $1-b=0.90$, the sample size was estimated in 88 patients.

Results: Until now, 53 patients (median age 54 years, range 19-69) were enrolled. At the time of writing, we have data available for 44 patients with resistant/relapsed diffuse large $B$ cell non-Hodgkin lymphoma were consecutively enrolled in the study. Briefly, 33/44 patients had advanced stage disease (III-IV), 14 were primary refractory and 30 had relapsed after a median number of 2 lines of therapy (range: 2-3). Eleven patients had 1 or more relevant comorbidities (range: 1-5). 22 patients were in II or subsequent CR after salvage therapy, whereas 19 were in PR and 3 had progressive disease.

A median number of $5.90 \times 10^{6} \mathrm{CD} 34^{+} / \mathrm{kg}$ cells (range $2,8-9,42$ ) collected from peripheral blood was reinfused to patients. All patients engrafted, with a median time to ANC $>0.5 \times 10^{9} / \mathrm{L}$ of 10 days. Median times to achieve a platelet count $>20 \times 10^{9} / \mathrm{L}$ and $>50 \times 10^{9} / \mathrm{L}$ were 12 and 16 days respectively. Ten out of 44 patients presented a fever of unknown origin (23\%), whereas 21 patients $(47 \%)$ presented a clinically documented infection. All patients received G-CSF after transplant for a median time of 8 days (range: 8-13). One patient died due to an incomplete hematological recovery after transplant, producing an overall transplant related mortality of $2.7 \%$. Thirtyeight out of 44 patients are evaluable up to now for response to treatment. $31 / 38$ (81.5\%) obtained a CR, $3 / 38$ a PR, whereas $4 / 38$ did not respond to therapy. After a median follow-up of 12 months after transplant (range 2-30), 4/38 patients were refractory, $7 / 38$ relapsed, and $27 / 38(71 \%)$ are still alive, in continuous $\mathrm{CR}$.

Conclusion: In conclusion, this data provide evidence that the BeEAM regimen is safe and has promising efficacy in resistantrelapsed aggressive diffuse large B cell lymphoma.

Acknowledgements: The study was supported in part by AlL Pesaro Onlus.

Disclosure of Interest: None declared.

\section{2}

Subgroup Analysis of Patients Participating in The AETHERA Trial: A Randomized, Double-Blind, PlaceboControlled Phase 3 Study of Brentuximab Vedotin in Patients at Risk of Progression Following Autologous Stem Cell Transplant for Hodgkin Lymphoma

A. Sureda ${ }^{1, *}$, J. Walewski ${ }^{2}$, A. Nadamanee ${ }^{3}$, T. Masszi ${ }^{4}$, E. Agura ${ }^{5}$, J. Holowiecki ${ }^{6}$, M. Abidi ${ }^{7}$, A. Chen ${ }^{8}$, P. Stiff, S. Viviani ${ }^{10}$,

A. Carella ${ }^{11}$, D. Osmanov ${ }^{12}$, V. Bachanova ${ }^{13}$, J. Sweetenham ${ }^{14}$, S.-Y. Lee ${ }^{15}$, E. Larsen ${ }^{16}$, N. Hunder ${ }^{16}$, C. H. Moskowitz ${ }^{17}$

${ }^{1}$ Institut Català d'Oncologia, Hospital Duran i Reynals, Barcelona Spain, ${ }^{2}$ Maria Sklodowska-Curie Institute and Oncology Center, Warszawa, Poland, ${ }^{3}$ City of Hope National Medical Center, Duarte, United States, ${ }^{4}$ Szent Istvan \& Szent Laszlo Corporate Hospital Hematology \& Stem Cell Dept., Budapest, Hungary, ${ }^{5}$ Baylor University Medical Center, Dallas, United States, ${ }^{6}$ Department of Bone Marrow Transplantation \& Oncohematology, Maria Sklodowska-Curie Institute of Oncology, Gliwice, Poland, ${ }^{7}$ Karmanos Cancer Institute, Detroit, ${ }^{8}$ Oregon Health and Science University, Portland, ${ }^{9}$ Loyola University Medical Center, Maywood, United States, ${ }^{10}$ Istituto Nazionale dei Tumori, Milano, ${ }^{11}$ IRCCS Azienda Ospedaliera Universitaria San Martino-Ist, Genova, Italy, ${ }^{12}$ Blokhin Cancer Research Center under the Russian Academy of Medical Sciences, Moscow, Russian Federation, ${ }^{13}$ University of Minnesota Medical Center, Minneapolis, ${ }^{14}$ Huntsman Cancer Institute, University of Utah, Salt Lake City,

${ }^{15}$ Takeda Pharmaceuticals International Company, Cambridge,

${ }^{16}$ Seattle Genetics, Inc., Bothell, ${ }^{17}$ Memorial Sloan-Kettering Cancer Center, New York, United States

Introduction: The AETHERA trial was initiated to evaluate whether brentuximab vedotin (BV) can prevent progression in 
pts with $\mathrm{HL}$ at risk of relapse post-ASCT (ClinicalTrials. gov \#NCT01100502). The primary results showed that BV significantly improved progression-free survival (PFS) per independent review vs. placebo. Efficacy analyses by investigator review were performed in subgroups as these data may provide useful information for making treatment decisions.

Materials (or patients) and methods: The AETHERA trial is a phase 3, randomized, double-blind, placebo-controlled study. After ASCT, pts received BV $1.8 \mathrm{mg} / \mathrm{kg}$ q3wk or placebo for up to 16 cycles. Pts were enrolled in 1 of 3 high-risk categories: refractory to frontline $(\mathrm{FL})$ therapy, relapse $<12$ mos after FL therapy, and relapse $\geq 12$ mos after FL therapy with extranodal disease. The primary endpoint was PFS per independent review. PFS by investigator was also evaluated.

Results: 329 pts were randomized at 78 sites in the US and Europe. Median age was 32 yrs (range, 18-76) and 53\% were male. ASCT conditioning regimens were BEAM: $61 \%$, CBV: $11 \%$, or other regimens: $28 \% .6 \%$ of pts received radiation as part of their transplant regimen. All pts had completed the treatment phase as of Aug 2013.

Subgroup analyses by demographics, stratification factors, pt characteristics, and number of risk factors showed that PFS by investigator was improved across all groups; the hazard ratio comparing BV to placebo was $<1$ for all analyses (Table 1). In an ad hoc analysis, pts with increasing numbers of risk factors for progression had progressively greater PFS benefit with BV vs. placebo.

The most common adverse events (AEs) in the BV arm (vs. placebo) were peripheral sensory neuropathy (56\% vs. $16 \%$ ) and neutropenia (35\% vs. $12 \%)$. A higher incidence of herpes simplex and zoster infections were observed with BV (11\%) vs. placebo (3\%); otherwise, opportunistic infections were rare and balanced between the arms. The incidence of pulmonary toxicity (by MedDRA SMQ) was low, but slightly higher with BV vs. placebo ( $5 \%$ vs. $3 \%$ ). Two pts died $\leq 40$ days of last dose of $\mathrm{BV}$; cause of death was acute respiratory distress syndrome.

Table 1: Univariate subgroup analyses of PFS by investigator assessment

\begin{tabular}{lll}
\hline Subgroup & $\mathrm{N}$ & $\begin{array}{l}\text { PFS Hazard Ratio } \\
(95 \% \mathrm{CI})\end{array}$ \\
\hline $\begin{array}{l}\text { Response to salvage } \\
\text { therapy pre-ASCT }\end{array}$ & & \\
$\quad$ CR & & \\
PR & 123 & $0.78(0.42,1.46)$ \\
SD & 113 & $0.44(0.26,0.76)$ \\
& 93 & $0.40(0.23,0.71)$ \\
HL status after frontline therapy & & \\
$\quad$ Refractory & 196 & $0.55(0.37,0.83)$ \\
$\quad$ Relapse $<12$ months & 107 & $0.45(0.27,0.93)$ \\
$\quad$ Relapse $\geq 12$ months with & 26 & $0.30(0.08,1.16)$ \\
$\quad$ extranodal disease & & \\
$\quad>2$ treatments pre-ASCT & 149 & $0.32(0.19,0.54)$ \\
$\quad$ B symptoms after failure & 87 & $0.29(0.15,0.55)$ \\
of frontline therapy & & \\
$\quad$ Extranodal disease at time of & 107 & $0.37(0.20,0.68)$ \\
pre-ASCT relapse & & \\
Risk Factors & & \\
$\quad \geq 1$ & & \\
$\geq 2$ & 329 & $0.50(0.36,0.70)$ \\
$\geq 3$ & 280 & $0.40(0.28,0.57)$ \\
\hline
\end{tabular}

a Risk factors $=$ Best response of $P R$ or SD to salvage therapy pre-ASCT, refractory to FL therapy, relapsed $<12$ mos after FL therapy, $>2$ treatments pre-ASCT, B symptoms after failure of FL therapy, extranodal disease at pre-ASCT relapse
Conclusion: BV improved post-ASCT PFS across all subgroups of pts. Pts with more risk factors had the most benefit from BV. AEs were consistent with the known safety profile of BV.

Disclosure of Interest: A. Sureda Funding from: Seattle Genetics, Inc., Conflict with: Takeda Pharmaceuticals International Co., J. Walewski Funding from: Seattle Genetics, Inc., Conflict with: Takeda Poland, A. Nadamanee Funding from: Seattle Genetics, Inc; Spectrum, T. Masszi Funding from: Seattle Genetics, Inc., E. Agura Funding from: Seattle Genetics, Inc., J. Holowiecki Funding from: Seattle Genetics, Inc., M. Abidi Funding from: Seattle Genetics, Inc., Conflict with: Seattle Genetics, Inc., A. Chen Funding from: Seattle Genetics, Inc., P. Stiff Funding from: Seattle Genetics, Inc., Conflict with: Seattle Genetics, Inc., S. Viviani Funding from: Seattle Genetics, Inc., A. Carella Funding from: Seattle Genetics, Inc., D. Osmanov Funding from: Seattle Genetics, Inc., V. Bachanova Funding from: Seattle Genetics, Inc., Conflict with: Seattle Genetics, Inc., J. Sweetenham Funding from: Seattle Genetics, Inc., Conflict with: Seattle Genetics, Inc., S.-Y. Lee Employee of: Takeda Pharmaceuticals International Co., E. Larsen Employee of: Seattle Genetics, Inc., Personal Interest: Seattle Genetics, Inc., N. Hunder Employee of: Seattle Genetics, Inc., Personal Interest: Seattle Genetics, Inc., C. H. Moskowitz Funding from: Seattle Genetics, Inc., Conflict with: Seattle Genetics, Inc.

\section{3}

Allogeneic haematopoietic cell transplantation as curative therapy for non-transformed follicular lymphomas: longterm follow-up data of the German Registry for Stem Cell Transplantation (DRST)

F. Heinzelmann 1,*, W. Bethge ${ }^{1}$, D. W. Beelen ${ }^{2}$, M. Engelhard ${ }^{2}$, N. Kroeger ${ }^{3}$, P. Dreger ${ }^{4}$ D. Niederwieser ${ }^{5}, J$. Finke ${ }^{6}$ D. Bunjes, J. Tischer ${ }^{8}$, G. Kobbe ${ }^{9}$, E. Holler ${ }^{10}$, M. Bornhaeuser ${ }^{17}$, M. Stelljes ${ }^{12}$, I. Haubitz ${ }^{13}$, H. Schrezenmeier ${ }^{7}$, C. Mueller ${ }^{7}, H$. Ottinger ${ }^{2}$

${ }^{1}$ University of Tuebingen, Tuebingen, ${ }^{2}$ University of Essen, Essen, ${ }^{3}$ University of Hamburg, Hamburg, ${ }^{4}$ University of Heidelberg, Heidelberg, ${ }^{5}$ University of Leipzig, Leipzig, ${ }^{6}$ University of Freiburg, Freiburg, 'University of Ulm, Ulm, "University of Muenchen, Muenchen, ${ }^{9}$ University of Duesseldorf, Duesseldorf, ${ }^{10}$ University of Regensburg, Regensburg, "11University of Dresden, Dresden, ${ }^{12}$ University of Muenster, Muenster, ${ }^{13}$ University of Wuerzburg, Wuerzburg, Germany

Introduction: Allogeneic hematopoietic cell transplantation (HCT) offers the chance of cure for patients with nontransformed follicular lymphoma (FL) but is associated with the risk of non-relapse mortality (NRM). The aim of this study was to identify subgroups of FL patients who benefit from HCT.

Materials (or patients) and methods: The Minimum Essential A Data of 146 consecutive patients who received HCT for FL between 1998-2008 were extracted from the database of the German Registry "DRST". Diagnosis of FL was verified by contact with reference pathologists.

Results: The median patient age at time of transplantation was 48 years (range 29-71). Prior to allogeneic HCT 90/146 patients $(62 \%)$ had undergone autologous HCT. At time of HCT, 110 patients (77\%) had sensitive disease while 33 patients (23\%) had chemorefractory disease (RD). Engraftment was achieved in $99 \%$ of evaluable patients. Day 100 NRM was $16 \%$. The median follow-up of surviving patients was 9.1 years (range 3.6-15.7). Estimated 1,2,5,10-year overall survival (OS) was $67 \%, 60 \%, 53 \%$, and $48 \%$, respectively. The corresponding estimates for $\mathrm{EFS}$ were $63 \%, 53 \%, 47 \%$, and $40 \%$, respectively. $40 \%$ of the 33 patients with RD at time of transplantation survived long-term. Of the $n=116$ patients with documented CR after HCT only $n=17$ (15\%) relapsed. Only two late relapses (beyond year 3 ) were diagnosed among the 77 patients with a follow-uP $>5$ years. Patients with chronic GvHD (irrespective of stage) had a lower risk of relapse, if transplanted in $C R$, and a higher chance to achieve $C R$, if transplanted in $P R$ or with $P D$ (no chronic GvHD: 19/48, chronic GvHD: 11/75, $P=0.0019$ ). 
Univariate statistical analysis suggested limited chronic GvHD, donor age $\leq 42$ years, and TBI-based conditioning in treatment-refractory patients to be correlated with favorable OS. Multivariate analysis revealed treatment-sensitive disease, limited chronic GVHD, and TBI-based conditioning in treatment-refractory patients as independent beneficial prognostic factors for OS. In contrast, patient age $\geq 55$ years had no impact on outcome.

Conclusion: This study states that a substantial number of patients with non-transformed FL can be cured by HCT, even if presenting with RD and/or advanced age ( $\geq 55$ years), and suggests that donors aged $\leq 42$ years are preferred, if available, and might encourage clinicians to use TBI-containing conditioning regimen in patients with RD.

Disclosure of Interest: None declared.

\section{4}

Feasibility and Efficacy of Tandem Autologous-Allogeneic Transplantation in a Cohort of 111 Patients Affected By High-Risk Hodgkin's and Non-Hodgkin's Lymphoma

R. Crocchiolo 1,*, L. Castagna ${ }^{1}$, S. Furst ${ }^{2}$, J. El-Cheikh ${ }^{3}$, B. Sarina ${ }^{1}$, S. Bramanti' ${ }^{1}$ A. Granata', S. Harbi ${ }^{2}$, L. Morabito ${ }^{1}$, B. Mohty ${ }^{2}$, L. Giordano', R. Devillier ', S. Garciaz ', D. Coso' ${ }^{2}$, M. Balzarotti ${ }^{1}$, C. Chabannon ${ }^{2}$, C. Carlo-Stella ${ }^{1}$, A. Santoro ${ }^{1}$, R. Bouabdallah ${ }^{2}$, D. Blaise ${ }^{2}$

${ }^{1}$ Istituto Clinico Humanitas, Rozzano, Italy, ${ }^{2}$ Institut PaoliCalmettes, Marseille, France, ${ }^{3}$ Institut Paoli-Calmettes, Institut Paoli-Calmettes, Marseille, France, ${ }^{4}$ Institut Paoli-Calmettes, Rozzano, Italy

Introduction: although high-dose chemotherapy (HDC) is the gold standard for the treatment of many relapsed or refractory lymphomas, the outcome is still unsatisfactory in some subsets of patients with adverse prognostic features. We treated 111 high-risk patients with a tandem strategy associating debulking with HDC followed by autologous stem cell transplantation (ASCT) and subsequent allogeneic SCT (tandem auto-allo) Materials (or patients) and methods: adult patients consecutively treated at two centers were included. Criteria for receiving tandem auto-allo were: $\mathrm{HL}$ and $\mathrm{NHL}$ refractory to first-line therapy, less than CR after first salvage treatment, relapse after prior ASCT, multiple relapses, histology of transformed follicular, mantle-, T- and NK-cell lymphoma Results: 111 consecutive patients with $\mathrm{HL}(n=44)$ and $\mathrm{NHL}$ ( $n=67$ ) transplanted from June 2002 to September 2013 were retrospectively identified. Median interval between auto and allo was 85 days (36-235). Sixty-two patients received (B)EAM and 45 high-dose Melphalan (HD-Mel, $140-200 \mathrm{mg} / \mathrm{m} 2$ ); 1 patient received Mel $100 \mathrm{mg} / \mathrm{mq}, 3$ patients received other regimens. Grade 3-4 mucositis occurred in 49 patients (44\%)

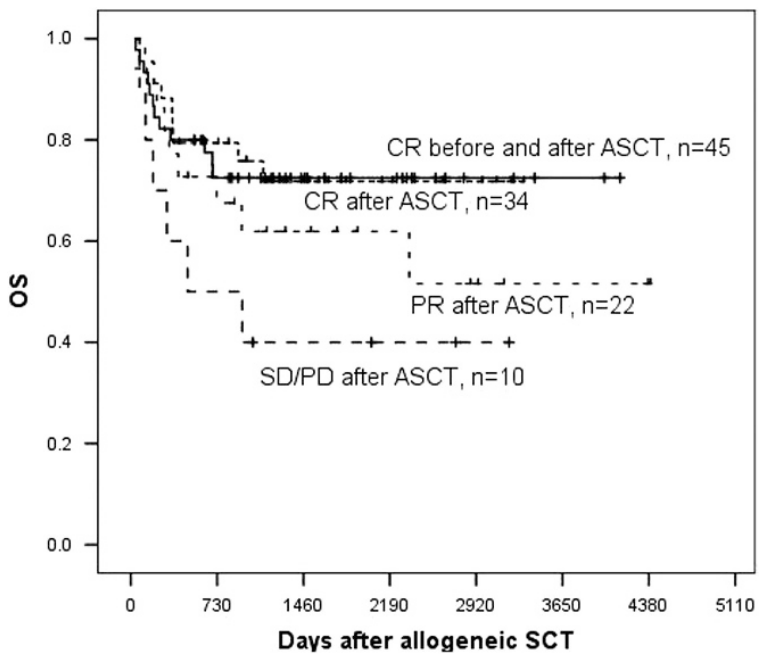

and documented infections during hospital stay in 30 (27\%), without significant differences between BEAM and HD-Mel groups. Among the 65 patients with active disease before ASCT, the overall response rate was $86 \%$ ( $n=56$ responders) and those in CR were 34 (52\%). No difference was observed in terms of response in the BEAM and HD-Mel groups $(P=0.28)$. Allogeneic SCT donor was either HLA-identical $(n=86)$, MUD $9 / 10(n=2)$, haploidentical $(n=20)$ or cord blood $(n=3) .3-y$ OS of entire cohort was $68 \%$ (95\% Cl: $59-77)$, 3-y PFS was $61 \%$ (52-70), rates of acute GvHD grade 2-4 and chronic GvHD were $28 \%$ and $38 \%$ respectively. TRM rate was $18 \%(n=20)$. No difference between BEAM and HD-Mel group was observed for OS $(73 \%$ and $64 \%$ respectively, $P=0.40)$ or TRM $(19 \%$ and $13 \%, P=0.44)$. Of note, OS of patients in CR before and after ASCT and OS of those obtaining CR after ASCT was superimposable (Figure 1). In a time-dependent Cox model, CR after ASCT conferred a significant survival advantage vs. lack of response: $\mathrm{HR}=0.27(95 \% \mathrm{Cl}: 0.09-0.80), P=0.02$. Auto-allo interval and donor type did not significantly impact on survival $(P=0.26$ and $P=0.68)$. No survival difference was observed between $\mathrm{HL}$ and NHL $(P=0.53)$

Conclusion: tandem auto-allo confirms to be a feasible and effective therapeutic strategy in those lymphoma patients whose prognosis is expected to be unsatisfactory with ASCT alone. BEAM vs. HD-Mel appeared to be similar in terms of extrahematological toxicity, disease response and survival. Patients converted to CR after ASCT had similar OS than those in CR before ASCT, underlying the importance of high-dose chemotherapy followed by ASCT as further tumor shrinking before allogeneic immunotherapy in this subset of high-risk patients.

Disclosure of Interest: None declared.

\section{5}

Early allogeneic hematopoietic stem cell transplantation from a related donor might improve the clinical outcome in patients with adult $\mathbf{T}$ Cell leukemia/lymphoma

S. Fuji ${ }^{1, *}$, H. Fujiwara ${ }^{2}$, N. Nakano ${ }^{3}$, A. Wake ${ }^{4}$, Y. Inoue ${ }^{1}$

T. Fukuda ${ }^{1}$, M. Hidaka ${ }^{5}$, Y. Moriuchi ${ }^{6}$, T. Ichinohe, Y. Atsuta ${ }^{8,9}$,

A. Utsunomiya ${ }^{3}$ on behalf of ATL Working Group of the Japan Society for Hematopoietic Cell Transplantation

${ }^{1}$ Division of Hematopoietic Stem Cell Transplantation, National Cancer Center Hospital, Tokyo, ${ }^{2}$ First Department of Internal Medicine, Ehime University Hospital, Ehime, ${ }^{3}$ Department of Hematology, Imamura Bun-in Hospital, Kagoshima, ${ }^{4}$ Department of Hematology, Toranomon Hospital Kajigaya, Kanagawa, ${ }^{5}$ Department of Hematology, National Hospital Organization Kumamoto Medical Center, Kumamoto, ${ }^{6}$ Department of Hematology, Sasebo City General Hospital, Nagasaki, ${ }^{7}$ Department of Hematology and Oncology, Hiroshima University Hospital, Hiroshima, ${ }^{8}$ Japanese Data Center for Hematopoietic Cell Transplantation, ${ }^{9}$ Department of Healthcare Administration, Nagoya University Graduate School of Medicine, Nagoya, Japan

Introduction: Allogeneic hematopoietic stem cell transplantation (allo-HSCT) is an only curative treatment for aggressive adult $T$ cell leukemia/lymphoma (ATLL). Considering the dismal prognosis using any conventional chemotherapy, an early application of allo-HSCT might be beneficial for patients with ATLL when a related donor is available. However, no previous study addressed an optimal timing of allo-HSCT from related donors.

Materials (or patients) and methods: To assess the impact of timing of allo-HSCT for patients with ATLL, we retrospectively analyzed data of 500 patients with ATLL who received the first allo-HSCT from a related donor using database of Japan Society for Hematopoietic Cell Transplantation.

Results: Median age was 52 years. Patients were grouped according to the interval from diagnosis to allo-HSCT; early transplant group, $<100$ days, $n=72$, late transplant group, $\geq 100$ days, $n=428$. Corresponding constituents of disease status between two groups were not statistically different $(P=0.11)$ The probability of overall survival (OS) in the early 


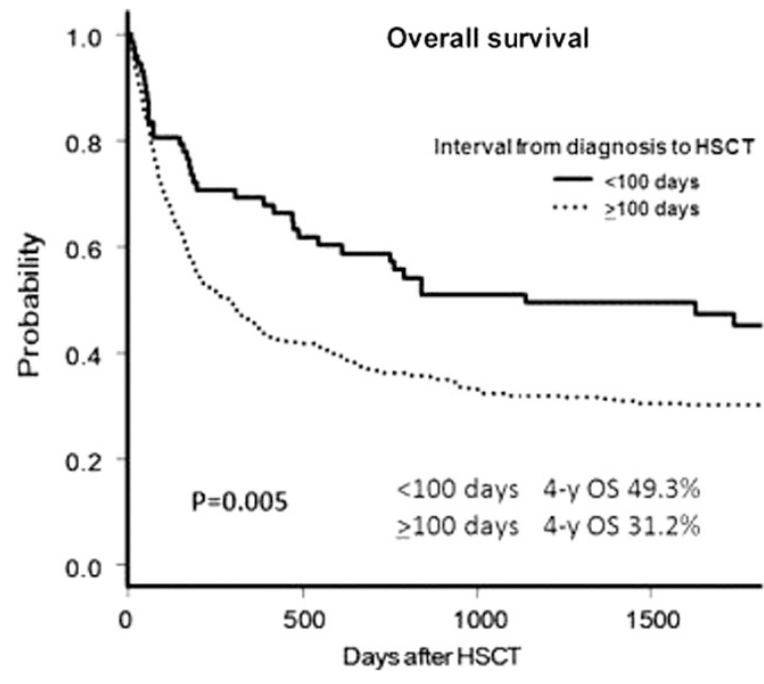

transplant group was significantly higher than that in the late transplant group (4-year OS $49.3 \%$ vs $31.2 \%$, Figure). A multivariate analysis revealed that late allo-HSCT was a unfavorable prognostic factor for OS with a statistical significance (hazard ratio, 1.46; $95 \% \mathrm{Cl}, 1.01-2.11 ; \mathrm{P}=0.04$ ). The cumulative incidence of non-relapse mortality in the early transplant group tended to be lower than that in the late transplant group (4-year NRM $18.6 \%$ vs $30.7 \%, P=0.07$ ), however the interval from diagnosis to allo-HSCT was not a significant variable in a multivariate analysis. Interval from diagnosis to allo-HSCT did not affect the risk of relapse.

Conclusion: In conclusion, we showed that the early application of allo-HSCT from related donors in patients with ATLL was associated with a better clinical outcome. Despite of limitations of a retrospective study, it would be acceptable to recommend an early application of allo-HSCT for ATLL, considering a favorable outcome in the early transplant group. Disclosure of Interest: None declared.

\section{6}

Allogeneic stem cell transplantation in relapsed/refractory Hodgkin's lymphoma: the timing of relapse after autologous transplant and the refractoriness of disease do not impact the survival outcomes

F. Spina ${ }^{1, *}$, S. Dalto ${ }^{2}$, C. De Philippis ${ }^{2}$, S. Viviani ${ }^{1}$, P. Corradini ${ }^{2}$ ${ }^{1}$ Hematology, FONDAZIONE IRCCS ISTITUTO NAZIONALE DEI TUMORI, ${ }^{2}$ Hematology, Università degli Studi di Milano, Milan, Italy

Introduction: Relapsed/refractory Hodgkin's lymphoma (RR-HL) is a challenging disease because it has very few options of cure.

Materials (or patients) and methods: We analyzed the RR-HL patients referred to our tertiary cancer center, the National Tumors Institute of Milan, Italy, to understand whether the refractoriness of disease and the timing of relapse after autologous stem cell transplant (autoSCT) may change the survival outcomes of allogeneic stem cell transplantation (alloSCT).

Multivariate analysis of survival outcomes included as covariates the disease pre-transplant status (CR vs PR vs SD or PD), donor (HLA identical sibling, matched unrelated, or haploidentical), and timing of relapse after autoSCT/refractoriness (relapse $<12 \mathrm{mts}$ after autoSCT or primary refractory vs relapse $>12 \mathrm{mts}$ after autoSCT).

Of 221 patients referred to our center with $\mathrm{HL}, 105$ patients were RR-HL. Sixty-three (60\%) RR-HL patients were treated with alloSCT, 41 patients $(40 \%)$ did not receive alloSCT for progressive disease (29), advanced age (3) or complete response after autoSCT after a third line of chemotherapy (9 pts). Donors of alloSCT were HLA identical siblings in 38\% of patients, matched unrelated in 35\% and haploidentical donors in $27 \%$ of patients. Median age at transplant was 33 (range, 1760). At alloSCT, $43 \%$ of patients were in $C R, 30 \%$ were in $P R$, $27 \%$ in SD or PD. $83 \%$ patients performed alloSCT having relapsed $<12$ months from autoSCT or with primary refractory disease, $17 \%$ having relapsed $>12$ months after autoSCT.

Results: Median follow-up of surviving patients was 4.8 years (range, 0.5-10.5). Overall survival (OS) was 76\% at 1 year, 59\% at 3 years and $55 \%$ at 5 years of follow-up. In multivariate analysis, only disease status before alloSCT significantly impacted the OS $(P=0.003, \mathrm{HR} 1.6, \mathrm{Cl} 95 \% 1.2-2.2)$, whereas donor and timing or relapse/refractoriness did not change OS $(P=0.149, \mathrm{HR}=1.3, \mathrm{Cl} 95 \% 0.7-1.9$, and $P=0.501, \mathrm{HR}=1.3$, C195\% 0.6-2.6, respectively). Progression free survival (PFS) was $71 \%$ at 1 year, $42 \%$ at 3 years and $42 \%$ at 5 years. Only pre transplant disease status impacted the PFS $(P<0.001, \mathrm{HR}=1.6$, Cl95\% 1.2-2.1), which was not influenced by donor $(P=0.387$, $\mathrm{HR}=0.8, \mathrm{Cl} 95 \% 0.6-1.2)$ and timing of relapse/refractoriness $(P=0.929, \mathrm{HR}=1.0, \mathrm{Cl} 195 \% 0.5-1.9)$. Relapse incidence was $31 \%$ at 1 year, $38 \%$ at 3 years and $38 \%$ at 5 years of follow-up. In multivariate analysis, relapse was impacted by donor $(0.036$, reduced risk for MUD donors, $\mathrm{HR}=0.5, \mathrm{Cl} 95 \% 0.3-0.9)$, and pre-transplant disease status $(P=0.003, \mathrm{HR}=1.7, \mathrm{Cl} 95 \% 1.2-$ 2.4), whereas timing of relapse/refractoriness did not change relapse incidence $(P=0.572, \mathrm{HR}=0.8, \mathrm{Cl} 95 \% 0.3-1.8)$. NRM was $10 \%$ at 100 days, $13 \%$ at 6 months, $18 \%$ at 1 year and reached a plateau of $20 \%$ at 2 years of follow-up. NRM was not impacted by the factors analyzed $(P=0.150, P=0.147$, $P=0.328$, respectively).

Conclusion: Long term PFS and OS for relapsed/refractory $\mathrm{HL}$ treated with alloSCT is $42 \%$ and $55 \%$, respectively. Only the disease pre-transplant status, and not the timing of relapse after autoSCT or refractoriness of disease appear to impact the OS and PFS after alloSCT. All the efforts should be made to obtain the deepest disease response before alloSCT in these patients, irrespective of the previous timing of relapse after autoSCT or refractoriness, in order to achieve the best results from alloSCT.

Disclosure of Interest: None declared.

0117

Feasibility and Efficacy of ASCT for Relapsed/Refractory

Hodgkin Lymphoma in Adults Beyond 50 Years of Age:

Results from the Hodgkin Lymphoma Subcommittee of the Spanish Group of Lymphoma and Bone Marrow Transplantation (GELTAMO)

A. A. Jorge ${ }^{1, *}$, M. Moreno ${ }^{2}$, J. Nuñez ${ }^{3}$, P. Balsalobre ${ }^{4}$, A. Sampol ${ }^{5}$, J. Lopéz ${ }^{6}, F$. de la Cruz', E. Pérez-Ceballos', M. J. RodriguezSalazar ${ }^{9}$, I. Krsnik ${ }^{10}$, J. Moraleda ${ }^{11}$, J. Pérez Oteyza ${ }^{12}$, C. Martinez ${ }^{1}$ ${ }^{7}$ Hospital Clínic Barcelona, ${ }^{2}$ Hospital Germans Trias i Pujol, Barcelona, ${ }^{3}$ Hospital Marques de Valdecilla, Santander, ${ }^{4}$ Hospital Marañon, Madrid, ${ }^{5}$ Hospital Son Espasas, Mallorca, ${ }^{6}$ Hospital Ramón y Cajal, Madrid, 'Hospital Virgen del Rocio, Sevilla, ${ }^{8}$ Hospital Morales Meseguer, Murcia, ${ }^{9}$ Hospital Universitario de Canarias, Tenerife, ${ }^{10}$ Hospital Puerta de Hierro, Madrid, ${ }^{11}$ Hospital Virgen de la Arrixaca, Murcia, ${ }^{12}$ Hospital Madrid Sanchinarro, Madrid, Spain

Introduction: Current data support the use of autologous stem cell transplantation (ASCT) in the treatment of young patients with relapsed/refractory (R/R) Hodgkin lymphoma $(\mathrm{HL})$. However, there is currently limited experience on its efficacy and feasibility in older patients.

Materials (or patients) and methods: We retrospectively analyzed the characteristics and outcome of 83 patients $\geq 50 y$ with R/R HL who underwent ASCT between May/2000 and December/2013 at 12 Spanish institutions. Twenty-eight patients (34\%) were $\geq 60 y$ at the time of the transplant (median, 64y). A comparison between these two cohorts of patients $(50-59 \mathrm{y}$ vs. $\geq 60 \mathrm{y})$ was performed. 
Results: Median age at the time of ASCT was 56 years (range $50-74$ ), and $63 \%$ of patients were male. Nodular sclerosis was the predominant histological subtype $(56.6 \%)$, followed by mixed cellularity (21.7\%). Most patients presented with advanced stage disease (57.8\%), and $47 \%$ had B symptoms at the time of diagnosis. Only $12 \%$ had bulky disease. Median number of treatment lines prior to ASCT was 2 (range 2-5), and $60 \%$ of patients received cisplatinum-based regimens as salvage therapy. Most patients had achieved complete or partial response at the time of the transplant $(65.1 \%$ and $25.3 \%$, respectively). There were no statistical differences between the two cohorts of patients regarding sex, stage of disease, B symptoms or bulky disease. More patients at the $\geq 60 y$ group presented mixed cellularity histology (30.8\% vs. $19.6 \%, P=$ NS). Median number of treatment lines, type of conditioning regimens, time from relapse to ASCT and number of early relapses ( $\leq 12$ months) where similar between both groups. There were no differences in comorbidity indexes of Charlson and Sorror regarding both groups. Median number of leukapheresis procedures required for peripheral blood stem cell collection was 1 for both groups, although older patients more often needed 2 procedures in order to obtain adequate CD34 + counts $(29.2 \%$ vs. $17.4 \%$, respectively, $P=\mathrm{NS}$ ). Median time to achieve unsupported platelets $>20 \times 10^{9} / \mathrm{L}$ was 14 days in the older group and 12 days in the younger group $(P=0.07)$. No differences were found regarding transfusion requirements or days of hospitalization. Older patients had more frequently severe nonhematological toxicity, including gastrointestinal (45.4\% vs. $27.3 \%, P=0.09)$, renal $(4.8 \%$ vs $0 \%, P=0.16)$ and infection ( $22.7 \%$ vs. $7.1 \%, P=\mathrm{NS}$ ). Death due to infectious complications was more frequent among the older group ( $14.3 \%$ vs. $3.6 \%$, $P=0.08$ ). After a median follow-up of 45 months for the $50-59 y$ group and 53 months for the $\geq 60$ y group, PFS at 5 years after ASCT was $50.5 \%$ and $68.1 \%$, respectively $(P=0.1)$. OS at 5 years was $58.7 \%$ for the younger group and $58.8 \%$ for the older group $(P=0.88)$. No differences were found regardingTRM at 100 days (5.45\% vs. $3.57 \%)$ and 1 year $(7.27 \%$ vs. $3.57 \%)$ for the $50-59 \mathrm{y}$ and $\geq 60 \mathrm{y}$ groups, respectively $(P=1.0)$.

Conclusion: ASCT can be performed in patients with R/R HL older than 50 years with acceptable outcome and toxicity. Selected patients aged 60 years or older appear to have similar results, although a trend to slower hematopoietic recovery, higher non-hematological toxicity and higher rate of infectious complications was observed. Larger studies focusing on the older R/R HL patients and ASCT are required.

Disclosure of Interest: None declared.

\section{Oral session: MDS / chronic leukemia}

\section{8}

Fludarabine and Treosulfan Conditioning is Associated with a More Favorable Outcome after Allogeneic Stem Cell Transplantation in Myelodysplastic Syndrome. A Survey on Behalf of the Chronic Malignancies Working Party of EBMT

A. Shimoni ${ }^{1, *}$, A. Nagler ${ }^{1}$, S. lacobelli ${ }^{2}$, A. van Biezen ${ }^{3}$, D. Beelen ${ }^{4}$, G. Mufti ${ }^{5}$, F. Ciceri ${ }^{6}$, L. Kanz ' L. Volin ${ }^{8}$ C. Richard ${ }^{9}$, D. Blaise ${ }^{10}$ A. Ganser ${ }^{11}, P$. Dreger ${ }^{12}, P$. Chevallier ${ }^{13}, R$. Schwerdtfeger ${ }^{14}$, T. de Witte ${ }^{15}, M$. Robin ${ }^{16}$, N. Kröger ${ }^{17}$ on behalf of CMWP ${ }^{1}$ hematology and BMT, CHAIM SHEBA MEDICAL CENTER, Tel-Hashomer, Israel, ${ }^{2}$ Centro Interdipartimentale di Biostatistica e Bioinformatica, Rome, Italy, ${ }^{3} E B M T$ Data Office, Leiden, Netherlands, ${ }^{4}$ University Hospital, Essen, Germany, ${ }^{5}$ GKT School of Medicine, London, United Kingdom, 'Ospedale San Raffaele, Milano, Italy, ${ }^{7}$ Universität Tübingen, Tübingen, Germany, ${ }^{8} \mathrm{Hel}-$ sinki University Central Hospital, Helsinki, Finland, ${ }^{9}$ Hospital U. Marqués de Valdecilla, Santander, Spain, ${ }^{10}$ Institut Paoli
Calmettes, Marseille, France, ${ }^{11}$ Hannover Medical School, Hannover, ${ }^{12}$ University of Heidelberg, Heidelberg, Germany, ${ }^{13} \mathrm{CHU}$ Nantes, Nantes, France, ${ }^{14}$ Deutsche Klinik für Diagnostik, Wiesbaden, Germany, ${ }^{15}$ Radboud University - Nijmegen Medical Centre, Nijmegen, Netherlands, ${ }^{16}$ Hopital St. Louis, Paris, France,

${ }^{17}$ University Hospital Eppendorf, Hamburg, Hamburg, Germany

Introduction: Allogeneic stem cell transplantation (SCT) is potentially curative therapy for patients (pts) with myelodysplastic syndrome (MDS). There is no proven advantage for any conditioning regimen over the others. Reduced-intensity conditioning (RIC) is associated with lower non-relapse mortality (NRM) compared with myeloablative conditioning (MAC), however, relapse rates are increased, resulting in similar survival. Fludarabine combined with treosulfan (FT) has been shown to be a reduced-toxicity regimen with intense anti leukemia activity in pts with myeloid malignancies.

Materials (or patients) and methods: We performed a retrospective analysis of all SCTs for MDS performed between 2000 and 2011 and reported to the chronic malignancies working party of EBMT $(n=2516)$. We identified 480 pts given FT and compared their outcomes to pts given various MAC $(n=1090)$ and RIC $(n=946)$ regimens.

Results: FT and RIC recipients were older than MAC recipients, median age 59, 60 and 50 years, respectively $(P=0.001)$. They were more likely to have an unrelated donor $(P=0.001)$ and peripheral blood stem cell grafts $(P=0.001)$. More FT recipients had untreated MDS at SCT, 33\%, 20\% and $24 \%$ while less had chemosensitive disease, $42 \%, 51 \%$ and $51 \%$, respectively $(P=0.001)$. More FT recipients had prior transformation to AML, $15 \%, 9 \%$ and $9 \%$, respectively $(P=0.03)$. The proportion of pts with high or intermediate-risk cytogenetics was $13 \%, 21 \%$ and $18 \%$, respectively $(P=0.01)$. With a median follow-up of 21 months (1-150), 1364 pts are alive, 456 died of relapse and 696 had NRM. The 5 year overall (OS) and diseasefree (DFS) rates for the entire group were $40 \%(95 \% \mathrm{Cl}, 37-43)$ and $35 \%(95 \% \mathrm{Cl}, 33-38)$, respectively. The 5 -year OS rate of FT recipients was $47 \%$ (41-52). OS after RIC and MAC was similar, $39 \%$ (34-43) and 38\% (33-42), respectively, significantly shorter than after FT $(P=0.02)$. DFS rates were $42 \%(37-47), 31 \%(27-$ $36)$ and $35 \%(30-39)$, respectively $(P=0.002)$. Relapse rates were similar after FT and MAC, 25\% (21-30) and 29\% (25-33), respectively, significantly lower than after RIC, 37\% (33-41) $(P=0.001)$. NRM was lower after FT and RIC than after MAC $33 \%$ (28-38), 32\% (28-35) and 36\% (33-40), respectively $(P=0.14)$. Multivariate analysis identified age $>55$ years (HR 1.7, $P=0.01)$, marrow blasts at SCT $>10 \%(\mathrm{HR} 1.5, P=0.02)$, prior transformation to AML (HR 1.7, $P=0.01$ ) and chemorefractory disease (HR 1.8, $P=0.001$ ) as poor prognostic factors for OS, while FT conditioning was protective (HR 0.6, $P=0.02$ ). Cytogenetics, donor type and stem cell source were not predictive. The predicting factors for relapse were RIC (HR 1.6, $P=0.03)$, chemorefractory disease (HR 1.6, $P=0.05)$, marrow blasts at SCT $>10 \%$ (HR 1.6, $P=0.04$ ), poor cytogenetics (HR $2.2, P=0.003)$ and prior transformation to AML (HR 1.8 $P=0.0001)$. The predicting factors for NRM were MAC (HR 1.6, $P=0.004)$, age $>55$ years (HR $1.5, P=0.008$ ), unrelated donor (HR 1.4, $P=0.004)$, chemorefractory disease (HR 1.5, $P=0.05$ ), marrow blasts $>10 \%(\mathrm{HR} 1.6, P=0.01)$ and positive pt CMV serology (HR 1.4, $P=0.04)$.

Conclusion: $\mathrm{FT}$ is associated with similar low relapse rates as MAC and similar low NRM as RIC, resulting in improved outcome over both RIC and MAC. FT might be the preferred regimen for SCT in MDS. These observations merit further study in randomized prospective trials.

Disclosure of Interest: None declared. 
0119

What is an acceptably low transplant related mortality after allogeneic stem cell transplantation for Myelodysplastic Syndromes? The important value of the EBMT score

S. Lozano ${ }^{1, *}$, E. Olavarria ${ }^{2}$, S. Schönland ${ }^{3}$, T. de Witte ${ }^{4}$, N. Kröger ${ }^{5}$, M. Robin ${ }^{6}$ on behalf of EBMT Chronic Malignancies Working Party

${ }^{1}$ Haematology, Complejo Hospitalario de Navarra, Pamplona, Spain, ${ }^{2}$ Haematology, Hammersmith Hospital, London, United Kingdom, ${ }^{3}$ Haematology, Universitätsklinikum Heidelberg, Heidelberg, Germany, ${ }^{4}$ Haematology, Radboud University - Nijmegen Medical Centre, Nijmegen, Netherlands, ${ }^{5}$ Haematology, Universitätsklinikum Hamburg-Eppendorf, Hamburg, Germany, ${ }^{6}$ Haematology, Hopital St. Louis, Paris, France

Introduction: Allogeneic Stem Cell Transplantation (alloSCT) is the only curative treatment for Myelodysplastic Syndromes (MDS). However, the so far perceived high TRM limits the number of patients referred for alloSCT.

Materials (or patients) and methods: Patient's characteristics: We have analyzed the TRM of 5943 consecutive adult MDS patients transplanted at EBMT centres from 1997 to 2011 Median age at time of transplant was 53.5 years (18-76) with 3492 patients $(59 \%)$ being male. WHO classification at transplant (available from 2009) showed: 245 (15\%) patients with RA/RARS/del5q, 228 (14\%) with RCMD ( + /-RS), 412 (25\%) with RAEB-1, 743 (45\%) with RAEB-2. A total of 868 (14.6\%) patients had secondary MDS.

Transplant characteristics: The conditioning was reduced intensity in 2912 (49\%) and myeloablative in 2383 (46\%). The reason for RIC included institutional protocol $(27 \%)$, patient age (21.6\%) and co-morbidities (8\%). The donor was an unrelated donor in 3031 (51\%) patients and an HLA-identical sibling in 2912 (49\%). GvHD prophylaxis consisted of Cyclosporine + MTX with (832 patients - 14\%) or without (1188-20\%) in vivo T-cell depletion with Campath or ATG. In addition, MMF was used instead of MTX in 549 (10\%) and 475 (8\%) patients respectively. The EBMT score separated patients into 3 groups: low risk (0-2 points, $n=1482,26 \%)$, intermediate risk (3-4 points, $n=2718,47 \%)$ and poor risk (5-7 points, $n=1562,27 \%$ )

Results: $96 \%$ of patients engrafted with only $76(1 \%)$ and 40 $(0.6 \%)$ patients experiencing primary or secondary graft failure

\section{[0119]}
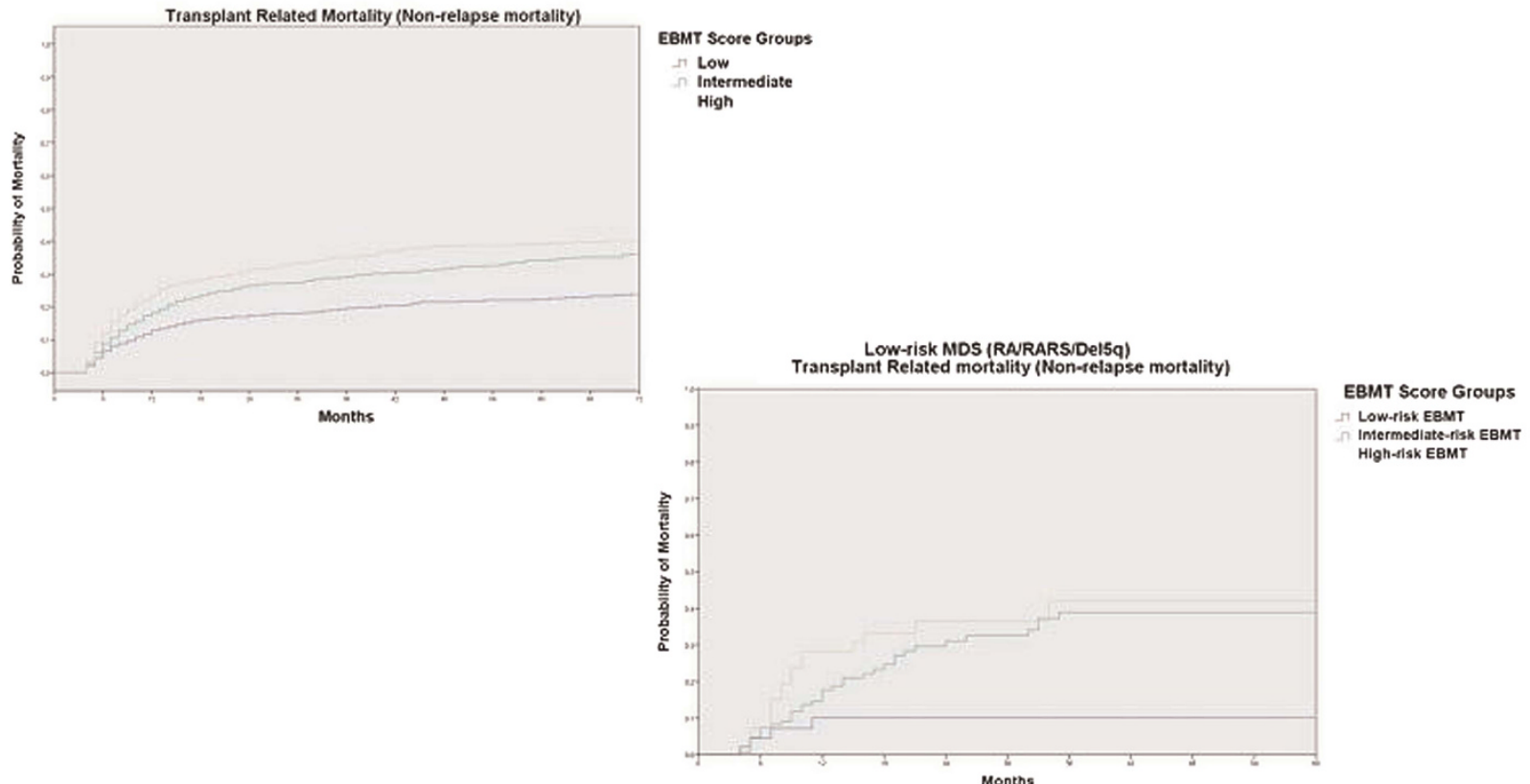

respectively. Grade-I GvHD developed in 1248 (21\%), grade-II in 1037 (17\%), grade-III in 431 (7\%) and grade-IV in 195 (3\%) patients. 2720 patients experienced no acute GVHD (46\%). Relapse of their MDS was detected in 1591 (27\%) patients. At time of analysis, 3027 (51\%) patients were alive without relapse and 2585 (43\%) of patients had died, of whom 1325 (22\%) never relapsed. We analyzed the caused of death of this latter population: 471 (36\%) died of GvHD, 362 (27\%) due to infections and 398 (30\%) from other causes.

The overall survival was $72 \%$ at 12 months and $49 \%$ at 5 years. The EBMT score strongly separated patients with good $(57 \%)$, intermediate (49\%) and poor (42\%) survival at 5 years $(P<0.001)$. TRM was $18 \%$ at 12 months and $32 \%$ at 5 years. TRM for patients with a low EBMT score was $13 \%$ at 12 months compared with $18 \%$ and $23 \%$ for intermediate and poor-risk EBMT scores respectively ( $P<0.001)$. For younger ( $<55$ years) patients with RA/RARS/del5q and low-risk EBMT score, the TRM was $10 \%$ at 5 years.

No differences in TRM at any stage were detected for patients who received reduced intensity or myeloablative conditionings or the different WHO classification. In addition, the overall TRM remained stable during the three different time periods: 1997-2011, 2002-2006 and 2007-2011. However, the TRM for low-risk EBMT score patients was 11\% in the period 2007-2011 showing an improvement in the most recent era.

Conclusion: The TRM for MDS patients after alloSCT is reasonably low and this should not be an obstacle for referring patients for transplantation, especially for patients with low EBMT score where the improvement in TRM has been most significant. Patients with low risk MDS (RA/RARS/del5q) who are otherwise candidates for alloSCT, should expect a very low TRM of $10 \%$ at 5 years from transplant if their EBMT score is low.

Disclosure of Interest: None declared. 
0120

Decision-finding for allogeneic stem cell Transplantation in PMF according to DIPSS

N. Kröger, ${ }^{1, *}, T$. Giorgino ${ }^{2}$, B. L. Scott ${ }^{3}$, M. Ditschkowski ${ }^{4}$, H. Alchalby ${ }^{7}$ F. Cervantes ${ }^{5}, A$. Vannucchi $^{6}$, M. Cazzola ${ }^{7}$, E. Morra $^{8}$, T. Zabelina ${ }^{7}$, M. Maffioli ${ }^{9}$, A. Pereira ${ }^{5}$, D. Beelen ${ }^{4}$, H. J. Deeg ${ }^{3}$, F. Passamonti $i^{9}$ on behalf of Chronic Malignancies Working Party of the EBMT (CMWP)

${ }^{1}$ Department of Stem Cell Transplantation, University Medical Center Hamburg, Hamburg, Germany, ${ }^{2}$ Institute of Biomedical Engineering, NAtional Research Council of Italy, Padova, Italy, ${ }^{3}$ Fred Hutchinson Cancer Research Center \& University $f$ Washington, Seattle, United States, ${ }^{4}$ Department of Stem Cell Transplantation, University of Essen, Essen, Germany, ${ }^{5}$ Hematology, University of Barcelona, Barcelona, Spain, ${ }^{6}$ University of Florence, Florence, ${ }^{7}$ Hematology, University of Pavia, Pavia, ${ }^{8}$ Hematology, Niguarda Ca Granda Hospital, Milan, ${ }^{9}$ Hematology, University Hospital, Varese, Italy

Introduction: Primary myelofibrosis (PMF) is a clonal myeloproliferative neoplasm with a high risk of clonal evolution and mortality. Allogeneic stem cell transplantation (ASCT) is currently the only curative treatment approach for myelofibrosis. Due to the inherent complications, careful patient selection is mandatory.

Materials (or patients) and methods: With the goal of refining patient selection for ASCT we compared results of ASCT with non-transplant approaches in 673 patients with PMF stratified according the Dynamic International Prognostic Scoring System (DIPSS), which assigns a time-dependent risk on a scale of low, intermediate (int)- 1 , int-2, and high. Considering a commonly used age-threshold for indication of ASCT, we restricted the analysis to patients aged 65 years or younger. This included 190 PMF patients (median 50 years at diagnosis) who received ASCT (HLA-matched related, $n=88$ [46\%] HLA-matched unrelated and mismatched related, $n=$ 102 [54\%] donors) after reduced intensity $(n=93)$, or high intensity conditioning $(n=97)$, and 248 patients, median age 55 years, from the DIPSS database.

In line with the dynamic nature of the DIPSS, risk scores in the non-transplanted cohort change with time and, accordingly, were accounted for as time-dependent covariates. The date of diagnosis was considered as origin of the time scale, and patients entered the analysis when receiving ASCT (ASCT cohort) or at the time of acquisition of a specific DIPSS category of a non-transplant cohort. Left truncation (begin of observation) for the ASCT cohort was the time to ASCT after diagnosis, while for the non-transplant cohort it was the time of achieving the respective DIPSS risk category.

Results: The relative risk (RR) of dying among patients receiving ASCT relative to those receiving conventional therapies was $5.6(95 \% \mathrm{Cl}: 1.7-19, P=0.0051)$ for low risk DIPSS, $1.6(95 \% \mathrm{Cl}: 0.79-3.2, P=0.19)$ for int- 1 risk DIPSS, 0.55 (95\%Cl: $0.36-0.83, P=0.005)$ for int-2, and 0.37 (95\%Cl: $0.21-$ $0.66, P=0.0007)$ for high risk DIPSS patients. The proportions of patients with low risk DPSS surviving at 1, 5 and 10 years were $100 \%, 69 \%$, and $60 \%$, respectively, for the ASCT cohort, and $98 \%, 95 \%$, and $92 \%$ for the non-transplant cohort. The corresponding figures for patients with int- 1 risk were $78 \%$, $52 \%$, and $41 \%$, respectively, after ASCT, and $97 \%, 77 \%$, and $63 \%$ for non-transplanted patients. Among patients with int-2 risk survival after ASCT was $82 \%, 50 \%$, and $32 \%$, respectively, and $77 \%, 41 \%$, and $11 \%$ among non-transplanted patients. The corresponding figures for high risk patients were $65 \%$, $37 \%$, and $27 \%$ for the ASCT cohort, and $67 \%, 11 \%$, and $1 \%$, respectively, for the non-transplant cohort. It should be noted that hazard ratios are not constant over time, and summarizing the risk in a single "average" RR ignores the specifics of the survival trends. Survival differences became pronounced beyond 5 years following diagnosis for int- 2 and high risk patients.

Conclusion: Patients with PMF who, at any age $\leq 65$ years, have int-2 or high risk disease by DIPSS have superior survival if receiving ASCT during their disease history. Conversely, conventional therapy appears to result in superior outcome in low risk patients, while intermediate-1 risk patients represent a group in whom individual counseling may be particularly important.

Disclosure of Interest: N. Kröger: None declared, T. Giorgino: None declared, B. L. Scott: None declared, M. Ditschkowski: None declared, H. Alchalby: None declared, F. Cervantes: None declared, A. Vannucchi Funding from: Novartis, Conflict with: Novartis, M. Cazzola: None declared, E. Morra: None declared, T. Zabelina: None declared, M. Maffioli: None declared, A Pereira: None declared, D. Beelen: None declared, H. J. Deeg: None declared, F. Passamonti: None declared.

0121

Improved outcome of alternative donor transplants in patients with myelofibrosis

S. Bregante ${ }^{1}$, A. Dominietto ${ }^{1}$, A. Ghiso ${ }^{1}$, A. M. Raiola ${ }^{1}$

F. Gualandi ${ }^{1}$, R. Varaldo ${ }^{1}$, C. Di Grazia ${ }^{1}$, T. Lamparelli ${ }^{1}$,

S. Luchetti ${ }^{1}$, S. Geroldi ${ }^{1}$, E. Tedone ${ }^{1}$, M. T. van Lint ${ }^{1}$,

A. Bacigalupo,"

${ }^{1}$ U.O. EMATOLOGIA TRAPIANTO di MIDOLLO, IRCCS - OSPEDALE SAN MARTINO-IST, Genoa, Italy

Introduction: The outcome of alternative donor transplants in patients with myelofibrosis (MF) has been reported to be significantly inferior, when compared to identical sibling grafts (Rondelli et al, 2013).

Materials (or patients) and methods: This study analyzes the outcome of alternative donor grafts for MF in our Unit betweeen 2000 and 2014.

Patients. 49 patients with myelofibrosis, underwent an alternative donor transplant between the year 2000 and 2014. The donor was an unrelated donor (UD) $(n=25)$ or a family mismatched (Fmm) donor $(n=24)$. Patients were divided in 2 groups according to the median year of transplant (2010): 23 patients 2000-2010 and 26 patients grafted 2011-2014.

Patients in the recent transplant period (2011-2014), differed from patients grafted in the earlier period (2000-2010)by the following clinical characteristics: older age (median 60 years vs 53 years, $P=0.004)$, more family mismatched donors (76\% vs $13 \%, P=0.0001)$ and conversely less unrelated donors ( $24 \%$ vs $97 \%)$, lower DIPSS $(P=0.04)$, smaller spleen size $(20 \mathrm{~cm}$ vs 25 $\mathrm{cm}, P=0.01$ ), the introduction of the TBF regimen (thiotepa fludarabine busulfan) ( $84 \%$ vs $0 \%, P<0.0001)$. The conditioning regimen other than TBF was total body irradiation 3.3Gyx3 $(n=4)$ or a thiotepa based RIC regimen $(n=23)$. GvHD prophylaxis for UD consisted of cyclosporine methotrexate and ATG, whereas for the family mismacthed, it was high dose post-transplant cyclophosphamide (PT-CY) cyclosporine and mycophenolate. We have splenectomized less patients in the recent period, because the spleens were smaller (we consider splenectomy with spleens $>22 \mathrm{~cm}$ ).

Results: Acute and chronic GvHD were comparable in the 2 time periods. There has been a reduction of TRM from $49 \%$ (2000-2010) to $23 \%(2011-2014)(P=0.06)$, a highly significant reduction of relapse related death (RRD) (39\% vs 4\% respectively, $P=0.001$ ), resulting in a significant improvement of actuarial 2 year survival from $30 \%$ to $70 \%(P=0.01)$. The other variable associated with better OS was a DIPSS $(0,03)$ Donor recipient age were not predictive.

Causes of death were different in the 2 periods: infections with or without interstitial pneumonia and toxicity occurred in 34\% of grafted patients in $2000-2010$ and in $11 \%$ of patients transplanted in 2011-2014. Rejections was seen in 9\% vs 4\% of patients in the 2 time periods. When the recent 26 alternative donor patients, were compared with $26 \mathrm{MF}$ patients grafted from HLA idenitcal siblings in 2011-2014, there was no difference in overall 2 year actuarial survival (70\% for both).

Conclusion: The outcome of transplants from alternative donors in MF has improved significantly with time, such that 2 year survival is now comparable to what is seen with sibling grafts. Improvement is due to reduced TRM, but mostly to 
better control of the disease: this has occurred together with changes in donor type (from unrelated to family haploidentical), in GvHD prophylaxis (from MTX CyA ATG to post transplant cyclophosphamide), and in the conditioning regimen, combining thiotepa and intravenous busulfan; in addition DIPSS was somewhat lower in more recent patients, and also spleens were smaller. We believe these data suggest that alternative donor grafts are currently a therapeutic option for patients with MF.

Disclosure of Interest: None declared.

\section{2}

Long follow-up data show that survival rate after

allogeneic stem cell transplantation in patients with CLL is higher for younger patients because of significantly lower NRM during the whole follow-up period: a retrospective study of the CMWP

M. Van Gelder, ${ }^{1, *}$ on behalf of CMWP

${ }^{1}$ INTERNAL MEDICINE, MAASTRICHT UNIVERSITY MEDICAL

CENTER, Maastricht, Netherlands

Introduction: We aimed at describing long-term survival outcomes after allogeneic HCT in CLL patients, estimating excess mortality compared to the general population (GP) and the relative contribution to death of relapse and of NRM in various time periods after HCT for different age cohorts.

Materials (or patients) and methods: Data from patients with CLL and a first allo-HCT between January 2000 and December 2010 registered with the EBMT database were analyzed. Survival probabilities were calculated by means of the Kaplan-Meier estimator. Excess mortality compared to the age-, sex-, country- and calendar year-matched GP was estimated with a Cox regression model for relative survival using the R-package "relsurv".

Results: 2589 patients were included. The median follow-up of patients alive at the end of follow-up was 4 years, the median age at HCT 55 years, 74\% were male. Fifty-one percent had a HLA-matched sibling donor and $77 \%$ received reducedintensity conditioning. For the whole cohort 5- and 10-year overall survival (OS), event-free survival (EFS) were 45\%, 35\%, and $35 \%, 28 \%$ respectively. The cumulative incidence of relapse within the next 12 months declined from $7 \%$ in patients who passed the 2-year landmark (LM) without relapse of CLL to $5 \%$ in patients who passed the 5-year LM, respectively. During the first year after HCT, excess mortality was highest for all age groups; NRM contributed most (ca $85 \%)$. In patients who had survived the $1^{\text {st }}$ year the contribution of "death after relapse" to death increased gradually with longer follow-up. Vice versa, the contribution of NRM decreased gradually. These trends were most pronounced in younger patients. For patients younger than 45 years, the relative contribution to death of NRM was $54 \%$ during the second year after HCT and 19\% during the 3 years thereafter. For patients older than 65 these figures were $64 \%$ and $44 \%$ respectively. The hazard of mortality remained exceeded compared to the GP even in patients who passed the 2-year LM after HCT without progression $(n=1023)$. The extent of excess hazard was similar in male and female patients. However, the excess hazard was higher in older patients. As an example, the model-based estimate of oneyear mortality for a man aged 45 at HCT who passed the 2year LM of $4.1 \%$ contrasts with $0.4 \%$ in the matched GP. In a man aged 65 at HCT the corresponding figures are $9.3 \%$ oneyear mortality compared to $2.2 \%$ in the matched GP. Notably, the models suggest that the adverse impact of higher age on the excess hazard of mortality increases when more time after HCT has elapsed. Thus, the hazard ratio for 10 years age difference at HCT is 1.43 for the 2-year LM population versus 2.88 for the 5-year LM population.

Conclusion: Long-term follow-up data derived from the EBMT registry show that excess mortality compared to the GP remains significant even after longer follow-up. This excess hazard is higher for older patients. The contribution of "NRM" to mortality remains relatively higher for older patients when compared to younger patients. Thorough investigation of causes of late mortality in elderly patients will provide further insights and could provide a clue for better care for elderly long-term survivors. The extent of contribution of "death after relapse" to death seems to be ageindependent.

Disclosure of Interest: None declared.

0123

Outcomes of allogeneic stem cell transplantation in pediatric patients with myelodysplastic syndrome and bone marrow failure disorders due to GATA2 mutation I. Hofmann ${ }^{1,2, *}$, A. Stetson ${ }^{1}$, M. Lee ${ }^{1}$, D. A. Williams ${ }^{1}$, M. D. Fleming ${ }^{2}$, L. Lehmann ${ }^{1}$ on behalf of Pediatric Myelodysplastic Syndrome and Bone Marrow Failure Registry ${ }^{1}$ Pediatric Hematology/Oncology, Dana-Farber/Boston Children's Cancer and Blood Disorders Center, ${ }^{2}$ Department of Pathology, Boston Children's Hospital, Boston, United States

Introduction: Pediatric myelodysplastic syndromes (pMDS) are clonal hematopoietic stem cell disorders with risk for leukemic transformation. Germline mutations in GATA2 lead to familial MDS/AML, Emberger- and MonoMac syndrome. Hematopoietic stem cell transplantation (HSCT) is the only curative therapy for pMDS, but studies on outcomes of patients with GATA2 mutations are limited. Here we characterize the unique phenotype of pMDS due to GATA2 mutations and describe outcomes after HSCT.

Materials (or patients) and methods: We performed Sanger sequencing of GATA2 on DNA samples of 103 patients with molecularly undefined MDS and bone marrow failure (BMF). First-degree relatives were assessed to determine if the disease was inherited or de novo and if others, in particular matched sibling donors (MSD), were affected. Rigorous phenotype analysis included clinical and laboratory data, and standardized pathology. Data on pre-HSCT therapies, organ dysfunction, conditioning regimen, GVHD prophylaxis, HSCT outcomes including transplant related toxicity (TRT) and mortality (TRM) and relapse were obtained.

Results: We identified pathogenic GATA2 mutations in 18 individuals (14 patients and 4 relatives). The median age at diagnosis was 15 years. There was a male predominance $(n=11)$. The diagnoses were refractory cytopenia of childhood $(n=7)$, refractory anemia with excess blasts/AML $(n=4)$ and acute lymphoblastic leukemia $(n=1)$. We observed a spectrum of disorders with features of familial MDS, Emberger-, and MonoMac syndrome. Other pathologies included: hydroceles, polyneuropathy, thrombosis, kidney abnormalities and tinea versicolor. A distinctive megakaryocytic dysplasia was a characteristic finding on histopathology. Monosomy 7 was the most common cytogenetic abnormalities $(n=7)$. Twelve out of the 14 pediatric patients underwent myeloablative HSCT at a median age of 15.9 years with a median follow up of 4 years. Three patients received AML therapy prior to HSCT. Conditioning regimens consisted of cyclophosphamide (CY)/ total body irradiation (TBI) $(n=7), \mathrm{CY} / \mathrm{TBI} / \mathrm{ATG}(n=2)$, fludarabine/CY/TBI $(n=2)$ and busulfan/CY $(n=1)$. Donors included $\operatorname{MSD}(n=1)$ and matched unrelated donor (MUD) $(n=11)$ with cell sources from marrow $(n=8)$ or umbilical cord blood $(n=4)$. GVHD prophylaxis consisted of cyclosporine A (CSA)/ MTX +/- prednisone $(n=9)$ or CSA/ mycophenolate mofetil $(n=3)$. Seven out of 12 patients are alive. One patient died from relapse and 4 had TRM including respiratory failure, infections and chronic GVHD. Eight patients developed GVHD. Post-HSCT complications were necrotizing fasciitis, recurrent clostridium difficile, leukencephalopathy, severe gastrointestinal bleeding, squamous cell carcinoma, desmoid tumor, chronic lung disease requiring lung transplant.

Conclusion: MDS due to GATA2 mutations occurs frequently, shows a heterogeneous presentation, unique histopathology and is associated with monosomy 7. HSCT is a curative treatment with a low relapse rate and moderate TRM, 
therefore a reduced intensity conditioning approach might be considered in future prospective trials. HSCT is most successful prior to leukemic transformation. Given implications for treatment decisions and donor selection GATA2 mutation screening should be performed on all patients with molecularly undefined MDS and BMF disorders and potential related donors.

Disclosure of Interest: None declared.

0124

Pre-transplant weight loss predicts non-relapse mortality and relapse rates in patients with myelodysplastic syndrome after allogeneic stem cell transplantation

A. Radujkovic ${ }^{1, *}$, N. Becker ${ }^{2}$, A. Benner ${ }^{2}$, S. Dietrich ${ }^{3}$, O. Penack ${ }^{4}$, U. Platzbecker , F. Stölzel', M. Bornhäuser, U. Hegenbart ${ }^{5}$,

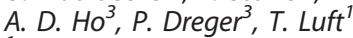

${ }^{1}$ Department of Internal Medicine V, University Hospital Heidelberg, ${ }^{2}$ Division of Biostatistics, German Cancer Research Center, ${ }^{3}$ University Hospital Heidelberg, Heidelberg, ${ }^{4}$ Charité University Medicine Berlin, Berlin, ${ }^{5}$ Universitätsklinikum Carl Gustav Carus der TU Dresden, Dresden, Germany

Introduction: We have recently provided evidence that weight loss and minor metabolic changes prior to alloSCT were able to predict relapse and death of acute myeloid leukemia patients using data from two independent patient cohorts. This retrospective study investigated the influence of pre-transplant weight loss on the outcome of MDS patients after alloSCT in three independent cohorts.

Materials (or patients) and methods: A total of 111 patients (59\% male) with a median age of 52 years were included into the analysis. Patients have been diagnosed with MDS according to WHO criteria and received an alloSCT between 2000 and 2012 in three different German referral centers (Heidelberg, Dresden and Berlin). Weight data were raised from medical records by three independent researchers in three independent institutions. Weight loss (expressed in percent) was calculated on the basis of recorded weight data at the time of alloSCT and the maximum weight in the time period of 3-6 months prior to alloSCT. The MDS WHO subtype was RA(RS)/RCMD in 31 patients (28\%), RAEB1 in 30 patients (27\%) and RAEB2 in 49 patients (45\%). According to IPSS 34\%, $45 \%$ and $21 \%$ of the patients were in the risk groups intermediate-1, intermediate- 2 and high, respectively. The majority of the patients $(n=72,65 \%)$ was previously untreated. Nineteen patients (17\%) and 14 patients (13\%) received hypomethylating agents and induction type chemotherapy prior to alloSCT, respectively. Thirty-one patients $(28 \%)$ received transplants from related donors, 59 patients (53\%) from matched unrelated donors and 21 (19\%) from mismatched unrelated donors. Ninety-three patients (84\%) received reduced intensity conditioning and 18 patients (16\%) received standard myeloablative conditioning. Survival times were measured from date of alloSCT. Overall survival (OS), relapse-free survival (RFS), relapse incidence and non-relapse mortality (NRM) were calculated from date of alloSCT to the appropriate endpoint. Cox regression analysis was applied for OS, RFS, relapse and NRM. Relapse and NRM were considered as competing risks.

Results: Estimated median follow-up at the time of analysis of surviving patients was 36 months. A total of $34(31 \%)$ patients experienced weight loss $>2 \%$ with 17 (15\%) patients losing more than $5 \%$ weight in the period of 3-6 months prior to alloSCT. Patient, disease and transplant characteristics did not differ between patients with weight loss $(>2 \%, n=34)$ and those without $(n=77)$. In multivariate analysis, weight loss and donor type were independently associated with shorter OS and RFS $(P<0.001$ and $P<0.05$, respectively). NRM was predicted by donor type $(P=0.006)$, IPSS $(P=0.015)$ and pre-transplant weight loss $(P=0.014)$ in multivariate analysis. Furthermore, weight loss was also an independent predictor of relapse (cause-specific HR $11.5295 \% \mathrm{Cl}$ 3.23-41.06, $P<0.001)$. In a mixed effect model with weight loss as outcome only IPSS prior to alloSCT had significant impact on weight loss $(P=0.046)$.

Conclusion: Our retrospective study suggests that in MDS patients pre-transplant weight loss was associated with both higher disease recurrence and higher NRM resulting in survival disadvantage after alloSCT. Prospective studies addressing pre-transplant nutritional interventions in order to improve the outcome of MDS patients are highly warranted.

Disclosure of Interest: None declared.

\section{Oral session: Early complications 2}

\section{5}

Effect of HLA-C allele matching in the context of patients HLA-C encoded KIR ligand grouping (C1 or C2) on outcomes of unrelated hematopoietic stem cell transplantation

J. C. Fischer ${ }^{1, *}$, T. Wang ${ }^{2}$, M. Haagenson ${ }^{3}$, S. J. Lee ${ }^{4}$,

S. R. Spellman ${ }^{5}$, M. Uhrberg

${ }^{1}$ Institute for Transplantation Diagnostics and Cell Therapeutics, Heinrich-Heine-University Düsseldorf, Medical Faculty, Düsseldorf, Germany, ${ }^{2}$ Center for International Blood and Marrow Transplant Research, Department of Medicine, Medical College of Wisconsin, Milwaukee, WI, ${ }^{3}$ Center for International Blood and Marrow Transplant Research, CIBMTR, Minneapolis, MN, ${ }^{4}$ Clinical Transplant Research, Fred Hutchinson Cancer Research Center, Seattle, WA, ${ }^{5}$ Center for International Blood and Marrow Transplant Research, NMDP, Minneapolis, MN, United States

Introduction: HLA-C-encoded KIR ligands (C1/C2) have been identified as important factors for the outcome of unrelated allogeneic HSCT: In a previous retrospective study CML recipients bearing at least one C2 ligand showed worse survival when compared to $\mathrm{C} 1 \mathrm{C} 1$ recipients (HR 5.9, $P<0.01$ ), especially when peripheral blood progenitor cells (PBPC) were used or in advanced disease stages. These initial findings were confirmed in a second cohort in advanced AML/CML, (but not in MDS or ALL/NHL) receiving PBPC. Notably, HLA-C allele matching contributed differentially to the transplantation outcome: it was beneficial in $\mathrm{C} 1$ patients, but was detrimental in $C 2$ recipients (increased TRM, HR 3.5, $P<0.012$; increased relapse, HR 2.7, $P=0.06$ ).

We hypothesized that C1 patients have a high frequency of immuno-competent NK cells (icNK) enabling eradication of residual disease due to the genetically hard-wired sequential acquisition of KIR receptors during early reconstitution phase post HSCT, with C1-specific NK cells emerging first. Alloreactive $T$ cells - resulting from HLA-C mismatch- might thus not have an additional beneficial effect in $\mathrm{C} 1$ patients and might even be detrimental (e.g. increased GvHD), but may serve an important function in relapse control in C2 patients. The lack of disease control in HLA-C-matched C2 patients would thus be explained by a combination of insufficient numbers of icNK cells in the early phase and the lack of alloreactive T cells later post HSCT. Consequently, this group exhibited poorest clinical outcome of all four groups defined by recipients KIR ligands and HLA-C allele matching in the investigated cohorts.

Materials (or patients) and methods: The aim of the present retrospective study was to determine the influence of HLA-C allele matching on the background of HLA-C encoded KIR ligand status in a large patient cohort $(n=7327$, provided by CIBMTR). Statistical analysis was performed by CIBMTR. Patients received unrelated allografts between 1988 and 2009 , with the majority of patients after 2000 (70\%). 30\% of the recipients were younger than $30 y, 67 \%$ younger than $40 y$. $70 \%$ of patients had early, $5 \%$ intermediate, and $25 \%$ advanced disease (AML 40\%, ALL 21\%, CML 22\%, MDS 17\%). $56 \%$ received bone marrow, $44 \%$ PBPC. $80 \%$ received a myeloablative conditioning. 
Endpoints were OS, aGVHD II-IV and III-IV, extensive chronic GVHD, relapse, DFS, and NRM. Due to multiple comparisons and multiple endpoints, $P<0.01$ was considered as significant. All models were adjusted for significant clinical covariates. Stratification was used in cases of non-proportional hazards. Patient-donor pairs were classified according the recipient HLA-C KIR ligand expression (C1C1, or C2 $(=\mathrm{C} 1 \mathrm{C} 2$ or $\mathrm{C} 2 \mathrm{C} 2))$ and the degree of the HLA-C allele match:

Results:

\begin{tabular}{lll}
\hline & C1C1 & C1C2 or C2C2 \\
\hline $8 / 8(n)$ & 2224 & 2861 \\
\hline $7 / 8$, HLA-C matched $(n)$ & Good risk (459 & Poor risk (790) \\
\hline 7/8, HLA-C mismatched $(n)$ & Poor risk (405) & Good risk (588) \\
\hline
\end{tabular}

No difference in clinical outcome was found between $\mathrm{C} 1 \mathrm{C} 1 \mathrm{vs}$. C2- patients receiving 7/8-loci matched donors, whether HLA$C$ was matched (all $P>0.33$ ) or mismatched (all $P>0.36$ ). There were too few events in a subset limited to $A M L$ and $C M L$ with advanced disease receiving PBSC to perform a subset analysis that recapitulated the original cohort.

Conclusion: Our results did not confirm the previous observation that recipient C2 KIR ligand status is detrimental when HLA-C is matched.

Disclosure of Interest: None declared.

\section{6}

Enhanced hepatic toxicity in patients with myelofibrosis undergoing allogeneic hematopoietic stem cell transplantation and anti-thymocyte globulin treatment

M. Ecsedi 1, ${ }^{1,}$, J. Halter ${ }^{1}$, M. Heim ${ }^{2}$, J. Passweg ${ }^{1}$, C. Lengerke ${ }^{1,3}$

${ }^{1}$ Clinic for Hematology, ${ }^{2}$ Clinic for Hepatology, ${ }^{3} 3$ Departement for Biomedicine, University Hospital Basel, Basel, Switzerland

Introduction: Primary myelofibrosis or myelofibrosis secondary to other myeloproliferative neoplasms (here collectively referred to as MF) is a devastating disease associated with severe cytopenias, constitutional symptoms, hepatosplenomegaly and progression to acute leukemia. Although JAK2 inhibitors alleviate disease symptoms, allogeneic hematopoietic stem cell transplantation (allo-HSCT) remains the only curative treatment for MF. Widespread application of alloHSCT in MF patients is often restricted by treatment-related risks associated with advanced age and comorbidities. Inclusion of anti-thymocyte globulin (ATG) in conditioning regimens reduces the occurrence of acute graft-versus-host disease (aGVHD), a major complication of allo-HSCT. ATG typically leads to cytokine release manifested by fever, chills and inflammatory blood parameters. Here, we explore whether MF patients who typically present with active extramedullary hematopoiesis in the liver, are more prone to develop ATG-induced hepatic toxicity.

Materials (or patients) and methods: Liver function parameters were evaluated during conditioning (until day 2 posttransplantation) with or without ATG, in patients undergoing allo-HSCT for MF at the University Hospital Basel between 2001 and $2014(n=24)$ and as a control in patients receiving alloHSCT for other indications at our center between January 1st 2013 and September 30th $2014(n=114)$. Mean values were compared using two-tailed t-test for independent samples, contingency tables using Fisher's exact test.

Results: Peak bilirubin levels were higher in MF compared to control patients (mean: $55.42 \mu \mathrm{mol}$ vs. $19.20 \mu \mathrm{mol}, P=0.004$ ), whereas levels of ALAT, ASAT, alkaline phosphatase and GGT did not differ significantly. Addition of ATG to the conditioning regimen enhanced bilirubin in both patient MF and control patients (ATG $+=34,29 \mu \mathrm{mol}$, ATG- $=11,83 \mu \mathrm{mol}, P<10^{-6}$ ) while leaving ALAT, ASAT, alkaline phosphatase and GGT grossly unaltered. However, MF patients were significantly more susceptible to bilirubin increase upon ATG treatment, with $12 / 12(100 \%)$ of MF patients versus $37 / 71$ (52\%) control group patients showing elevation of bilirubin $(P=0.0051)$. We observed similar differences when restricted our analysis to a subgroup of patients receiving myeloablative conditioning $(P=0.0095)$. In 7 cases of myelofibrosis patients receiving ATG, bilirubin differentiation was available, showing elevation of conjugated but not of unconjugated bilirubin. Liver ultrasonography carried out in 5 patients did not indicate visible cholestasis. In $50 \%$ of cases, bilirubin levels normalized within 7 days, in the remaining cases mild elevation persisted over longer periods of time. Despite this benign course, bilirubin increase might be clinically relevant, as it might impair hepatic drug metabolism.

Conclusion: Our findings indicate that MF patients have a higher risk for cholestatic liver injury, which is further enhanced by treatment with ATG during allo-HSCT conditioning. Pathogenetically, ATG may target extramedullary liver hematopoiesis and lead to hepatotoxicity via enhanced local cytokine (e.g. TNF- $\propto$ ) release. The ATG-induced hepatic injury is self-limiting but may alter clearance of concomitantly applied drugs. Thus, careful monitoring of drug plasma concentrations and toxicities is warranted in this patient group.

Disclosure of Interest: None declared.

\section{7}

Defibrotide for Pediatric and Adult Patients with Hepatic Veno-occlusive Disease: Interim Age Subgroup Survival Analysis From an Ongoing Expanded Access Program in the US

P. G. Richardson 1,*, A. R. Smith ${ }^{2}$, B. M. Triplett ${ }^{3}$, N. A. Kernan ${ }^{4}$, S. A. Grupp ${ }^{5}$, J. H. Antin ${ }^{6}$, L. Lehmann ${ }^{7}$, M. Miloslavsky ${ }^{8}$, R. Hume ${ }^{8}$, A. L. Hannah ${ }^{8}$, B. Nejadnik ${ }^{8}$, R. J. Soiffer ${ }^{6}$

${ }^{1}$ Division of Hematologic Malignancy, Department of Medical Oncology, Jerome Lipper Multiple Myeloma Center, Dana-Farber Cancer Institute, Harvard Medical School, Boston, MA, ${ }^{2}$ Division of Pediatric Blood and Marrow Transplantation, University of Minnesota, Minneapolis, MN, ${ }^{3}$ Bone Marrow Transplantation and Cellular Therapy, St. Jude Children's Research Hospital, Memphis, TN, ${ }^{4}$ Pediatric BMT Service, Memorial Sloan-Kettering Cancer Center, New York, NY, ${ }^{5}$ Pediatric Oncology, The Children's Hospital of Philadelphia, Philadelphia, PA, 'Stem Cell/Bone Marrow Transplantation Program, Division of Hematologic Malignancy, Department of Medical Oncology, Dana-Farber Cancer Institute, ${ }^{7}$ Division of Hematologic Malignancy, Department of Medical Oncology, Center for Stem Cell Transplantation, Dana-Farber Cancer Institute, Harvard Medical School, Boston, MA, ${ }^{8} \mathrm{Jazz}$ Pharmaceuticals, Inc., Palo Alto, CA, United States

Introduction: Hepatic veno-occlusive disease (VOD), also called sinusoidal obstruction syndrome, is a potentially fatal complication of hematopoietic stem cell transplantation (HSCT). Severe VOD (sVOD), clinically characterized by multiorgan failure (MOF), has been associated with a $>80 \%$ mortality rate; it may develop in a substantial number of highrisk patients (pts). Defibrotide (DF), a sodium salt of complex single-stranded oligodeoxyribonucleotides, is thought to protect injured endothelium and to restore thrombo-fibrinolytic balance. In a phase 3 trial in sVOD, DF improved complete response rate and survival at day +100 post HSCT vs historical controls, with a favorable safety profile. In the European Union, DF is approved for treatment of severe hepatic VOD in HSCT therapy in adults and children. In the US, DF is available through an expanded access, protocol-directed treatment IND (T-IND) collecting data on safety/efficacy in children and adults with SVOD and non-severe VOD post HSCT or post chemotherapy (CT).

Materials (or patients) and methods: The original T-IND protocol required VOD diagnosed by Baltimore criteria (total bilirubin $\geq 2.0 \mathrm{mg} / \mathrm{dL}$ with $\geq 2$ of hepatomegaly, ascites, or $5 \%$ weight gain) plus MOF (renal and/or pulmonary) following HSCT; the study was amended to include non-severe VOD (ie, 
without MOF) per modified Seattle criteria ( $\geq 2$ of total bilirubin $\geq 2.0 \mathrm{mg} / \mathrm{dL}$, hepatomegaly or pain in upper right quadrant, ascites, or-in this study- $5 \%$ weight gain) post HSCT or post CT. Exclusion criteria include clinically significant bleeding or need for $\geq 2$ vasopressors. DF was given as a $2 \mathrm{~h}$ infusion at $6.25 \mathrm{mg} / \mathrm{kg}$ IV q6h $(25 \mathrm{mg} / \mathrm{kg} / \mathrm{d})$ for a recommended $\geq 21$ days. Assessments included survival at day +100 and safety.

Results: Of pts enrolled between December 2007 and December 2013, 641 received $\geq 1$ DF dose. Of 636 pts with available age, $58 \%$ were aged $\leq 16 \mathrm{y}$, and $42 \%$ were adults ( $>16 \mathrm{y}$ ). Among pediatric pts, $28 \%$ were aged $0-23$ months, $52 \%$ were aged $2-11 y$, and $20 \%$ were aged $12-16 y$.

Day +100 survival data by age group are available for 526 pts post-HSCT and 62 pts post-CT (Table), with rates of sVOD post HSCT of $55 \%$ in pediatric pts and $50 \%$ in adults.

Table 1: Day + 100 Survival

\begin{tabular}{lll}
\hline$\%(\mathrm{n} / \mathrm{N})$ & Pediatric & Adult \\
\hline Post-HSCT, all pts & $58 \%(163 / 283)$ & $45 \%(109 / 243)$ \\
\hline sVOD only & $50 \%(79 / 157)$ & $38 \%(46 / 122)$ \\
\hline Non-severe VOD only & $67 \%(84 / 126)$ & $52 \%(63 / 121)$ \\
\hline Post-CT, all pts & $83 \%(39 / 47)$ & $60 \%(9 / 15)$ \\
\hline sVOD only & $77 \%(20 / 26)$ & $67 \%(4 / 6)$ \\
\hline
\end{tabular}

Adverse events (AEs) occurred in $61 \%$ of (227/372) pediatric pts and $76 \%$ of $(200 / 264)$ adults; treatment-related AEs occurred in only $19 \%$ and $24 \%$, respectively. Serious AEs occurred in $45 \%$ of pediatric pts and $53 \%$ of adults. Other than VOD and MOF, the most common serious AE for pediatric pts was pulmonary hemorrhage ( $8 \%$ [ $2 \%$ in adults]) and for adult pts the most common was hypotension (5\% [ $4 \%$ in children]). Conclusion: Both pediatric and adult pts with sVOD showed potentially favorable survival rates at day +100 . Moreover, survival rates were especially promising among pediatric and adult subgroups with non-severe VOD (no MOF), which may reflect an advantage of earlier treatment before sVOD develops. DF was generally well tolerated in both pediatric and adult groups, with manageable toxicity. Enrollment to the T-IND study continues.

Support: Jazz Pharmaceuticals.

Disclosure of Interest: P. G. Richardson Funding from: Jazz Pharmaceuticals, Conflict with: has served on advisory committees with Jazz Pharmaceuticals, A. R. Smith: None declared, B. M. Triplett: None declared, N. A. Kernan: None declared, S. A. Grupp: None declared, J. H. Antin Conflict with: is a consultant to Tempera and Enlivex and has served on advisory committees with Jazz Pharmaceuticals, L. Lehmann: None declared, M. Miloslavsky Employee of: Jazz Pharmaceuticals, Conflict with: in the course of employment has received stock options exercisable for, and other stock awards of, ordinary shares of Jazz Pharmaceuticals plc., R. Hume Employee of: Jazz Pharmaceuticals, Conflict with: in the course of employment has received stock options exercisable for, and other stock awards of, ordinary shares of Jazz Pharmaceuticals plc., A. L. Hannah Conflict with: is a consultant to Jazz Pharmaceuticals, B. Nejadnik Employee of: Jazz Pharmaceuticals, Conflict with: in the course of employment has received stock options exercisable for, and other stock awards of, ordinary shares of Jazz Pharmaceuticals plc., R. J. Soiffer Conflict with: has served on advisory committees with Jazz Pharmaceuticals.

\section{8}

Supportive care and infectious complications in cord blood and haploidentical stem cell transplantation in adults with high risk hematologic diseases

J. Elcheikh ${ }^{1, *}$, R. crocchiolo ${ }^{2}$, A. vai ${ }^{2}$, S. furst ${ }^{1}$, S. bramanti ${ }^{2}$,

B. sarina ${ }^{2}$, A. granata ${ }^{1}$, C. faucher ${ }^{1}$, B. mohty ${ }^{1}$, S. harbi ${ }^{1}$,

R. devillier ${ }^{1}$, R. bouabdallah ${ }^{3}, N$. vey ${ }^{3}$, P. J. weiller ${ }^{3}$, A. santoro $^{4}$,

C. chabannon ${ }^{3}$, L. castagna ${ }^{2}, D$. blaise ${ }^{7}$

${ }^{1}$ transplantation, institut paoli calmettes, marseille, France, ${ }^{2}$ transplantation, humanitas, rozzano milano, Italy, ${ }^{3}$ hematology, institut paoli calmettes, marseille, France, ${ }^{4}$ hematology, humanitas, rozzano milano, Italy

Introduction: Infections due to post transplant immune deficiency are a major problem following allogeneic stem cell transplantation, particularly in patients receiving cord blood (CBT) or haploidentical transplants (haplo-SCT). To our knowledge, there are only some heterogeneous studies comparing this important issue in this setting.

Materials (or patients) and methods: We evaluated the incidence and type of infectious complications that occurred in these two types of transplant for 150 patients, 81 cord blood and 69 haploidentical, who received the same conditioning regimen: fludarabine (Flu), cyclophosphamide (Cy) and low dose TBI (2Gy) combination in the two groups. The GVHD prophylaxis consisted of Cyclosporine A (CsA) and MMF in all patients in the two groups. In the Haplo group all patients received also $50 \mathrm{mg} / \mathrm{kg}$ Cy at day 3 and 4 post transplant. Of note, supportive care was the same during the whole study period. CMV infection management was also homogeneous. Results: The median times to neutrophil and platelet recovery were $20 \mathrm{~d}$ (14-39) and $29 \mathrm{~d}$ (14-50) after Haplo and $22 \mathrm{~d}$ (6$67)$ and $41 \mathrm{~d}(18-80)$ after CBT. All supportive care measures included red blood cell, platelet transfusions, antibiotics days, parenteral nutrition days and hospitalization time were significantly increased in CBT group. $(P=0.0001)$ While incidence of infections appeared similar in both types of transplant, viral infections were more frequent than bacterial or fungal infections and were the most common cause of death in both groups. During the first year after stem cell transplant, the CBT group had 154 episodes of infection versus 125 of the haplo patients. Viral infections were most common in both groups ( 83 vs 60 episodes) in CBT vs haplo group respectively. Patients in the haplo group were 1.2 times $(95 \%$ $\mathrm{Cl}$ : 1.1 to 2.5$)$ more likely to have a viral infection $(P=0.07)$ Pneumonia was the most common clinical syndrome and was higher in the haplo group $28 \%$ vs $11 \%$ in CBT. Survival analysis showed that transplant source (CBT versus Haplo) did not have a significant effect on the probability to have an infection episode. $(P=0.10)$ Bacterial, fungal and CMV infections still quite frequent and contributed to higher mortality in CBT vs haplo $(P=0.06)$. The first cause of mortality was the viral infection (12 (15\%) $3(4 \%) P=0.060)$ followed by the bacterial infection cause $(4(5 \%)$ vs $3(4 \%))$ and the fungal infection (1 (1\%) vs 0 ) in CBT and haplo group respectively.

Conclusion: Our study is unique in comparing adult patients with advanced hematologic malignancies who received the same reduced-intensity conditioning regimen, which offers a common base for comparing the infections in these two types of transplant. In our hands, outcome is better for haplo donor decreasing the need of supportive measures and facilitating to perform the transplant in time. Haploidentical transplants are a good and promising alternative option for high risk hematologic patients who lack an HLA-matched donor. Collectively, the impact of infectious complications after haplo-SCT was different from that in CBT patients, suggesting that a more intensive strategy for infection control in haplo patients is required to reduce infectious mortality.

Disclosure of Interest: None declared. 
0129

Effectiveness of preventive parenteral nutrition enriched with high glutamine and omega-3 fatty acids in allogeneic stem cell transplantation

M. Kucher ${ }^{1, *}$, B. Afanasyev ${ }^{1}$

${ }^{1}$ Raisa Gorbacheva Memorial Institute of Children Oncology, Hematology and Transplantation, First State Pavlov Medical University of Saint-Petersburg, Russia, Saint-Petersburg, Russian Federation

Introduction: The benefit of nutritional status and routine nutrition support (NS) in allogeneic stem cell transplantation (HSCT) is still controversial. But it seems to be clear that adequate supplementation of nutrients is an important part of supportive care during HSCT: improves therapy tolerance, reduces frequency and severity of various complications. In this study we estimated influence of standard and modified NS regimes on therapy tolerance, occurrence, severity of posttransplant complications, overall survival.

Materials (or patients) and methods: We analyzed data from 400 allogeneic SCT recipients, $129(32,2 \%)$ of them underwent allogeneic relative HSCT, 223 (55,8\%) allogeneic unrelative HSCT and $48(12 \%)$ haploidentical HSCT. $149(37,2 \%)$ of the patients had acute myeloid leukemia, $177(44,2 \%)$ acute lymphoblastic leukemia, aplastic anemia - 22 (5,6\%), chronic myeloid leukemia - 16 (4\%), myelodysplastic syndrome - 25 $(6,2 \%)$, Hodgkin and non-Hodgkin's lymphoma - 11 (2,8\%). Median age was 25,2 (14-54) years. Conditioning regimens and graft versus host disease (GVHD) prophylaxis were comparable in all groups of observations. Control group $(n=150)$ included patients with symptoms of gastrointestinal toxicity and caloric intake lower, then $70 \%$ for 3 or more consecutive days. These patients received standard NS regimen consisting of enteral low-bacterial diet and parenteral nutrition. The second group $(n=150)$ received standard parenteral nutrition $(30-35 \mathrm{kcal} /$ $\mathrm{kg} /$ day) starting $\mathrm{D}+1$ after HSCT. The third group $(n=100)$ received modified NS regimen consisting of enteral lowbacterial diet with parenteral nutrition with additional glutamine $(0,43 \mathrm{~g} / \mathrm{kg} /$ day iv) and omega- 3 fatty acids $(1,5 \mathrm{~g} /$ $\mathrm{kg} /$ day iv) starting D +1 after HSCT. In all groups we evaluated nutritional status using anthropometrical indicators (height weight, body mass index, arm circumference, thickness of skinfat folds over triceps, shoulder muscle circumference) and laboratory data (total protein, albumin, macronutrients, copper, zinc).

Results: In group of patients who received modified NS decrease in frequency of mucositis (84\% vs. 94\%) $(P<0,05)$ and severity of III-IV grade mucositis ( $21 \%$ vs. $40 \%)(P<0,05)$, decrease of grade III-IV acute GVHD $(9,1 \%$ vs. $20,3 \%)(P<0,05)$, less significant decrease of anthropometrical and laboratory values was observed. Between all groups there were no difference in time of engraftment and 1 year overall survival. Conclusion: Patients receiving modified NS tolerate the highdose conditioning regimens and immunological complications in early post-transplant period better, then patients on standard NS regimens.

Disclosure of Interest: None declared.

\section{0}

Association of heparanase gene single nucleotide polymorphisms with veno-occlusive disease after allogeneic hematopoietic stem cell transplantation in childhood

B. Gruhn ${ }^{1, *}$, C. Arndt ${ }^{1}$, S. Wittig ${ }^{1}$, C. Seifert ${ }^{1}$

${ }^{1}$ Department of Pediatrics, Jena University Hospital, Jena, Germany

Introduction: Veno-occlusive disease (VOD) of the liver is a life-threatening early complication after hematopoietic stem cell transplantation (HSCT). Until now, examinations about the influence of genetic risk factors are extremely rare. Heparanase (HPSE), a pivotal endoglycosidase responsible for heparan sulfate degradation, is expressed by activated endothelial cells.
HPSE has been shown to be involved in inflammation and may therefore play an important role in the pathogenesis of VOD of the liver. The purpose of this study was to identify an association between HPSE gene single nucleotide polymorphisms (SNPs) and VOD of the liver after allogeneic HSCT in childhood.

Materials (or patients) and methods: 160 children (median age, 14 years) who underwent allogeneic bone marrow $(n=91)$ or peripheral blood stem cell transplantation $(n=$ 69) in a single center and their respective donors were genotyped of HPSE for rs4693608 and rs4364254 using TaqMan real-time polymerase chain reaction. The donor was HLA-matched unrelated in $63 \%$ of transplants and HLAidentical related in $25 \%$ of transplants. Conditioning regimen was myeloablative in all cases and based on busulfan in $46 \%$ of transplants or total body irradiation in 33\% of transplants. Two forms of post-transplant immunosuppression predominated, cyclosporine A and methotrexate in $50 \%$ of transplants and cyclosporine A alone in 30\% of transplants.

Results: 160 donor/patient pairs were analyzed. Cell samples from the patient were available in 155 cases and from the donor in 153 cases. Genotype AG of HPSE rs4693608 SNP was found in 82 patients (53\%), AA in 49 patients (32\%), and 24 patients were homozygous for GG $(15 \%)$. Analysis of HPSE rs4364254 SNP revealed a similar distribution for TC $(n=69$, $44 \%)$ and $\Pi(n=68,44 \%)$ and a frequency of 18 patients (12\%) for CC. VOD of the liver was observed in $12 / 160$ patients $(8 \%)$. If VOD of the liver was diagnosed all of our patients were treated with defibrotide as early as possible. Altogether, 4/12 patients with VOD of the liver (33\%) died of VOD, whereas $8 /$ 12 patients with VOD of the liver (67\%) survived VOD. Early medical intervention is most probably the reason for this high cure rate. Patients with HPSE genotypes GG or AG of rs4693608 (G>A) had a significantly reduced incidence of VOD of the liver on day 100 after HSCT compared to patients with genotype AA (5\% vs. $14 \%, P=0.038)$. In addition, incidence of VOD in patients with genotype CC or CT of rs4364254 (C>T) was significantly decreased in comparison to patients with genotype $\Pi$ ( $2 \%$ vs. $15 \%, P=0.004)$. Interestingly, no patient with genotype CC developed VOD. Because both SNPs co-occur in vivo, we generated subsets: AA-TT, GGCC and a group with remaining SNP combinations. We found significant differences between all three patient groups $(P=0.035)$. Patients with AA-TT showed the highest incidence of VOD of the liver (17\%), while VOD was not observed in patients with GG-CC (0\%) and residual combinations were numerically in-between (5\%). An impact caused by main patient and donor characteristics, established risk factors for VOD of the liver, and conditioning regimen could be excluded in multivariate analyses.

Conclusion: This study provides the first evidence that HPSE gene SNPs are significant independent risk factors $(P=0.030)$ for the development of VOD of the liver and should be evaluated in further trials.

Disclosure of Interest: None declared.

0131

Genetic polymorphism of cytochrome P450 1B1 (C432G) is associated with lower overall survival in patients undergoing allogeneic hematopoietic stem cell transplantation

N. Stute ${ }^{1, *}$, D. Beelen ${ }^{1}$, M. Koldehoff ${ }^{1}$

${ }^{1}$ Department of Bone Marrow Transplantation, University of Duisburg-Essen, Essen, Germany

Introduction: The human cytochrome P450 1B1 (CYP 1B1) is a key enzyme involved in the production of reactive metabolites and in the activation of environmental carcinogens. Several polymorphisms were identified in CYP 1B1 gene; four of them are single nucleotide polymorphisms and give rise to amino acidic substitutions. The CYP 1B1 codon 432 polymorphism leads to a three-fold higher 4-hydroxylase activity for the variant CYP 1B1 isozymes than the wild types. 


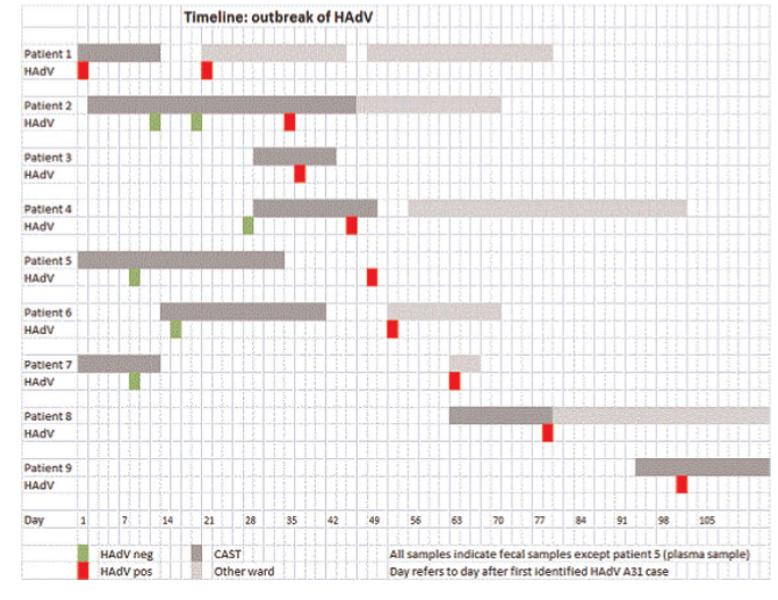

Materials (or patients) and methods: In a single center retrospective study 382 patients who underwent allogeneic hematopoietic stem cell transplantation (HSCT) for various diseases (51\% acute leukemia) were genotyped for CYP 1B1 (C432G) expression and their influence on outcome was analyzed. Genotyping of CYP 1B1 (C432G) was performed by real-time PCR.

Results: 169 patients (44\%) were genotyped as homozygous wild-type (wt) gene C/C, 157 (41\%) as heterozygous genotype C/G and 56 (15\%) as homozygous gene mutated G/G. Calculated genotype frequencies did not differ from that reported earlier by other studies for Caucasians. Patients' demographic and treatment characteristics showed no difference between the three groups except that CYP 1B1 CC was more common in females $52 \%$ than in males $38 \%(P<0.02)$. Five-year estimate for overall survival (OS) was $58+4 \%$ for the CC group and $48+3 \%$ for the $\mathrm{C} / \mathrm{G}-$ and $\mathrm{G} / \mathrm{G}$ groups $(P<0.036)$. Surprisingly, this difference was primarily evident in males $(P<0.009)$, where the group with CYP gene mutations did significantly worse (3-year estimate for OS: $65+5 \%$ vs. $47+4 \%)$, whereas it was virtually absent in females $(P=0.99)$. TRM and RR were higher for the group with mutated genes in regard to the group with wt gene (although not significant). One-year estimate for TRM $15+3 \%$ vs. $22+3 \%$, (3-year TRM $22+3 \%$ vs. $30+3 \%)(P<0.06)$. Oneyear estimate for relapse rate (RR) was $18+3 \%$ vs. $21+3 \%$ (5year RR: $31+4 \%$ vs. $37+4 \%)(P<0.27)$. No statistic differences were found in the incidence of acute GvHD grade 2-4 $(P<0.68)$ and chronic GvHD limited $(P<0.46)$ or extensive $(P<0.74)$ with variant CYP 1B1 $(C 432 \mathrm{G})$ polymorphisms. Multivariate logistic regression revealed no significant association between CYP $1 \mathrm{~B} 1$ genotypes $(P<0.06)$ and OS (adjusted for age, gender constellation, HLA-match, donor-type, grafttype, disease stage, relapse, and occurrence of acute or chronic GVHD). However, when focusing on factors available before transplant, two factors show in multivariate analysis for OS in males: SNP relative risk (RR) $1.5(\mathrm{Cl}: 1.0-2.1), P<0.04$ and advanced disease RR 2.7 (Cl: 1.8-3.9), $P<0.01$.

Conclusion: These results suggest that recipients with genetic polymorphism of CYP 1B1 and in particular male recipients have a lower OS after HSCT. Genotyping for CYP 1B1 (C432G) might help to identify patients with higher risk for allogeneic HSCT and may help explain gender differences in outcome. Disclosure of Interest: None declared.
Oral session: Infectious complications 2

0132

Prolonged Outbreak of Adenovirus A31 Among Allogeneic Stem Cell Transplant Recipients

L. P. Swartling ${ }^{1, *}$, A. Allard ${ }^{2}$, J. Törlen ${ }^{3}$, P. Ljungman ${ }^{4}$, J. Mattsson ${ }^{3}$, E. Sparrelid ${ }^{5}$

${ }^{1}$ Division of Infectious Diseases, Department of Medicine Huddinge, Karolinska Institutet, Karolinska University Hospital, Stockholm, ${ }^{2}$ Department of Clinical Microbiology/Virology, Umeå University, Umeå, ${ }^{3}$ Center for Allogeneic Stem Cell Transplantation, ${ }^{4}$ Department of Hematology and Center for Allogeneic Stem Cell Transplantation, Karolinska Institutet, Karolinska University Hospital, ${ }^{5}$ Division of Infectious Diseases, Department of Medicine Huddinge, Karolinska Instituet, Karolinska University Hospital, Stockholm, Sweden

Introduction: Adenoviruses (HAdV) can cause severe disease after allogeneic stem cell transplantation (HSCT). An outbreak of HAdV A31 occurred from December 2011 to March 2012 at the Center for Allogeneic Stem Cell Transplantation (CAST), Karolinska University Hospital. We analyzed the outbreak, the routes of transmission and report the medical consequences. Materials (or patients) and methods: Nine cases of HAdV A31 were documented. The medical records of all patients admitted to CAST during the outbreak period were studied. For detection of HAdV a Taqman real-time PCR targeting the Hexon gene was used. The HAdV type was determined by sequencing a fragment of the hexon gene. Phylogenetic analysis of the hexon gene was performed using primers and PCR programs according to Leruez-Ville et al. 2006. In phylogenetic analysis of the E3 gene, a two-step PCR amplification of almost the entire adenovirus E3 gene was performed using primers designed from the known sequence of the HAdV A31 reference strain (AM749299.1).

Results: All 9 patients had been admitted to the ward, but the two last patients (patient 8 and 9) had no timely connection to other known HAdV A31 cases (Figure). In addition, four of the patients $(1,5,6$ and 7$)$ made visits to the out-patient clinic on the same day as one or several other HAdV positive patients. Sequencing the hexon gene resulted in 100\% homology between the patient samples but also to the reference strains of HAdV A31 (Accession number AB330112.1 and AM749299.1). Further analysis of E3, a more divergent HAdV gene, showed $100 \%$ homology between the patient strains and contained 5 mutations not present in the reference strain HAdV A31 (AM749299.1).

Transplantation was permanently postponed in one transplant candidate because of the HAdV infection. Among the eight HSCT recipients, none developed proven HAdV disease. However, seven had concomitant GVHD grade II-IV, complicating the clinical picture with difficulty to distinguish gastrointestinal symptoms of GVHD from those possibly due to HAdV infection. Five patients were treated with cidofovir due to high level viremia.

Conclusion: Nosocomial transmission was strongly indicated by DNA sequencing of the patient strains. Unidentified cases with sparse symptoms may have caused continuous transmission within the unit. Although no patient had severe disease, the medical consequences were significant. In conclusion, an outbreak of HAdV among HSCT recipients can be difficult to control. Regular testing of all patients several weeks beyond the last identified case may be an important measure to control transmission.

Disclosure of Interest: None declared. 
0133

HLA matching influences the risk of recurrent reactivations and reconstitution of $\mathrm{CMV}$ immunity after allogeneic stem cell transplantation

J. Ogonek, ${ }^{1, *}$, P. Varanasi ${ }^{1}$, S. Luther ${ }^{1}$, P. Schweier ${ }^{1}$, E. Dammann ${ }^{1}$, B. Woelk ${ }^{2}$, A. Heim ${ }^{2}$, M. Stadler ${ }^{7}$, A. Ganser ${ }^{7}$, L. Hambach',

S. Borchers ${ }^{1}$, E. Mischak-Weissinger ${ }^{1}$

${ }^{1}$ Hematology, Hemostasis, Oncology and Stem Cell Transplantation, ${ }^{2}$ Institute of Virology, Hannover Medical School, Hannover, Germany

Introduction: Cytomegalovirus infection (CMV) is an important cause of morbidity after allogeneic hematopoietic stem cell transplantation (HSCT). The latent virus reactivates in immune-compromised patients, due to both post-HSCT immunosuppressive therapy and impaired T-cell reconstitution/function. Here we report the impact of mismatches in HLA-molecules between donor and recipient on CMV-reactivation and CMV-specific immune reconstitution.

Materials (or patients) and methods: This retrospective study included 752 patients, who received a transplant from a matched related donor (MRD $n=234$ ), matched unrelated donor (MUD $n=384$ ), mismatched unrelated donor (MMUD $n=115$ ) or mismatched related donor (MMRD $n=19$ ). HLAtyping $(10 / 10)$ of patients and donors was conducted via highresolution multiplexed PCR. Blood samples were routinely monitored for CMV pp65 antigen expressing cells per 400,000 leukocytes. CMV-cytotoxic lymphocytes (CMV-CTL) reconstitution was analyzed in the blood from 246 patients at days +50 , $+100,+200,+300$ after HSCT, using 6 HLA-CMV tetramers. Results: The Fisher's exact test was used to analyze the data. The outcome was that HLA mismatch (class I or II) showed significant influence on recurrent (multiple) CMV reactivations (mCMV-R) $(P<0.001)$. We analyzed the relative risk (RR) in the subgroups with different levels of HLA-matching for 1CMV-R and mCMV-R. The group transplanted from MRD served as reference (ref.). Shortly, we found significantly higher risk for mCMV-R in the MMUD group (RR 2.6, 95\% Cl 1.63-4.15, $P=0.0001)$. In the MRD $24(10 \%)$ patients had mCMV-R, while in the MUD 59 (15\%), in the MMRD $3(16 \%)$ and in the MMUD group $31(27 \%)$ had mCMV-R.

Furthermore, we investigated the mean numbers of CMV-CTL/ $\mu \mathrm{l}$ of blood in the groups with different levels of HLA-match. We divided CMV-CTL levels into 3 ranges: $<1,1-10$ and $>10$ $\mathrm{CMV}-\mathrm{CTL} / \mu \mathrm{l}$. In the MMUD group we observed a trend for an increased risk for the lack of CMV-CTL (25 (48\%) patients; RR $1.5,95 \% \mathrm{Cl} 0.96$ to $2.38, P=0.07)$ compared to 21 (32\%) patients from the MRD group. Significantly less patients $(17$ $33 \%$ ) had more than 10 CMV-CTL from the MMUD group (RR $0.6,95 \% \mathrm{Cl} 0.39$ to $0.95, P=0.04)$ when compared to the reference MRD group (35; 53\%) patients.

Table1:

\begin{tabular}{lllll}
\hline mismatch & No & HLA I & HLA II & HLA I \& II \\
\hline $\begin{array}{l}\text { Number } \\
\text { of patients }\end{array}$ & 618 & 62 & 49 & 18 \\
\hline no CMV-R & $65 \%(403)$ & $55 \%(34)$ & $47 \%(23)$ & $61 \%(11)$ \\
\hline 1 CMV-R & $\begin{array}{l}21 \%(131) \\
\text { ref. }\end{array}$ & $\begin{array}{l}19 \%(12) \\
P=0.74\end{array}$ & $\begin{array}{l}24 \%(12) \\
P=0.58\end{array}$ & $\begin{array}{l}22 \%(4) \\
P=0.92\end{array}$ \\
\hline mCMV-R & $\begin{array}{l}14 \%(84) \\
\text { ref. }\end{array}$ & $\begin{array}{l}26 \%(16) \\
P=\mathbf{0 . 0 0 7}\end{array}$ & $\begin{array}{l}29 \%(14) \\
P=\mathbf{0 . 0 0 3}\end{array}$ & $\begin{array}{l}17 \%(3) \\
P=0.70\end{array}$ \\
\hline
\end{tabular}

Conclusion: Donor-recipient mismatches (even minor) increases the risk of recurrent reactivations and impairs the reconstitution of CMV specific immunity. Interestingly, mismatches in class I and class II both led to an increase in mCMV$\mathrm{R}$ (Table 1), indicating miscommunication between dendritic cells (early after HSCT: recipient) and donor immune cells. Our results suggest that HLA incompatibilities between donor and recipient could be the reason for diminished CMV-CTL proliferation and therefore put patients at higher risk for recurrent $C M V$ reactivations.

Disclosure of Interest: None declared.

0134

CMV resistance in patients after allogeneic haematopoietic stem cell transplantation

P. Hubacek ${ }^{1, *}$, M. Kouba ${ }^{2}$, D. Boutolleau ${ }^{3}$, L. Pliskova ${ }^{4}$, E. Vejrazkova ${ }^{5}$, P. Keslova ${ }^{6}$, P. Sedlacek', P. Cetkovsky ${ }^{2}$

${ }^{1}$ Department of PAediatric Haematology and oncology, 2ND MEDICAL FACULTY OF CHARLES UNIVERSITY AND MOTOL UNIVERISTY HOSPITAL, 2 Institute of Haematology and Blood transfusion, Prague, Czech Republic, ${ }^{3}$ Virology Department, Groupe Hospitalier Pitié-Salpêtrière, Paris, France, ${ }^{4}$ Department of clinical biochemistry and diagnostics, ${ }^{5}$ Department of hematology and internal medicine, University Hospital Hradec Kralove, Hradec Kralove, ${ }^{6}$ Department of Paediatric Haematology and oncology, Motol University Hospital, Prague, Czech Republic

Introduction: Human cytomegalovirus (CMV) reactivation can cause a life threatening disease in allogeneic haematopoietic stem cell transplant (HSCT) recipients. Increasing number of $\mathrm{HSCT}$ recipients experiencing the virostatic treatment brings a necessity of better understanding of consequences of CMV infection, including the presence of development of CMV resistance to therapy in UL97 and UL54 CMV genes.

Materials (or patients) and methods: Between January 2002 and January 2013, we obtained 11,657 whole blood samples from 355 children and 18,989 from 700 adult patients drawn weekly during 3 months after HSCT, less frequently thereafter (median 25/patient) for prospective testing of viral infections. Additional 3,509 biological samples from these patients were obtained during this period. DNA was isolated from whole blood samples using Qiagen DNA Blood Mini Kits and Qiagen DNA Mini Kits in case of most of the rest samples. Viral loads were tested using quantitative real-time PCR technology and CMV quantity was normalized to 100,000 human genomic equivalents assessed by quantification of albumine gene in the samples.

Results: CMV DNA was detected in 7,094 samples from 193 children and 448 adult patients. Viral load of load $>1,000$ normalised viral copies (NCVs) which is a threshold for treatment was detected 92 children and 210 adults. Clinical resistance due to non-decrease or increase of viral load after 2 weeks of well conducted virostatic treatment was suspected in 71 adults (33.8\%) and 30 (32.6\%) children patients. Sequence analysis proved CMV resistence in 7 children $(7.6 \%$ of treated pts.; 4 boys and 3 girls) and 13 adult patients (6.1\% of treated; 7 men and 6 women). Most frequently detected resistance coding mutation in UL97 L959S in 6 pts., A594V in 6 pts., M460V in 2 pts., and A591V, L595F, G598S, C592G and del597599 in 5 pts. and in 6 pts. mutations del981-982, N408K, V715M, P522S, L545S, T700A. We detected mutations in both UL97 and UL54 genes in 3 patients. Among these, genotypes $\mathrm{gB} 1 \mathrm{gH} 1$ was observed in 5 pts., $\mathrm{gB} 3 \mathrm{gH} 1$ and $\mathrm{gB} 1 \mathrm{gH} 2$ in 4 pts., $\mathrm{gB} 4 \mathrm{gH} 2$ in 2 pts., gB2gH1 and $\mathrm{gB} 2 \mathrm{gH} 2$ in 2 pts. and infection with more then $1 \mathrm{CMV}$ strain $(\mathrm{gB} 1, \mathrm{gH} 1,2, \mathrm{gB} 2, \mathrm{gH} 1,2$ and $\mathrm{gB} 1,3 \mathrm{gH} 1,2)$ in 3 patients. Resistance was detected with median of 190 days after HSCT (range 53-2105 days) and viral load of 4,245 NVCs. $75 \%$ of patients with detected resistance deceased during the observation period; one of them due to histologically proved florid CMV pneumonia.

Conclusion: Resistance to virostatic treatment is an important phenomenon of increasing importance in consequence of different viral infections including CMV. Impact of such changes has undoubtful impact on the longterm outcome of the patients.

References: Supported by grant of Internal Grant Agency of Ministry of Health of Czech Republic NT/13691-4.

Disclosure of Interest: None declared. 
0135

Selection of adenovirus and Epstein-Barr-virus-specific T-cells with MHC class I streptamers under good manufacturing practice (GMP)-compliant conditions

C. Freimüller ${ }^{1}$, J. Stemberger ${ }^{2}$, M. Artwohl ${ }^{3}$, L. Germeroth ${ }^{4}$ V. Witt' ${ }^{5}$, G. Fischer ${ }^{6}$, S. Tischer ${ }^{7}$, B. Eiz-Vesper ${ }^{7}$, T. Lion ${ }^{8}$, G. Fritsch ${ }^{7}$, R. Geyeregger, ${ }^{1, *}$

${ }^{1}$ Department of Clinical Cell Biology and FACS Core Unit, ${ }^{2}$ CHILDREŃS CANCER RESEARCH INSTITUTE, Vienna, Austria, ${ }^{3}$ Department of Quality Management, CHILDREN'S CANCER RESEARCH INSTITUTE, Vienna, Austria, ${ }^{4}$ Stage Cell Therapeutics, Göttingern, Germany, ${ }^{5}$ St. Anna Childreńs Hospital, ${ }^{6}$ Department of Blood Group Serology and Transfusion Medicine, Medical University of Vienna, Vienna, Austria, 'Institute of Transfusion Medicine, Hannover Medical School, Hannover, Germany, ${ }^{8}$ Department of Molecular Microbiology, CHILDREN'S CANCER RESEARCH INSTITUTE, Vienna, Austria

Introduction: Human adenovirus (HAdV), cytomegalovirus (CMV) and Epstein-Barr virus (EBV) infections still contribute substantially to transplant-related mortality of patients after hematopoietic stem cell transplantation (HSCT) despite antiviral drug therapies. Earlier clinical studies have demonstrated successful adoptive transfer of magnetically selected CMVspecific T-cells via removable GMP-compliant streptamers. Thus the focus of the present study was the selection of HAdVstreptamer + T-cells and EBV-streptamer + T-cells.

Materials (or patients) and methods: Cells from leukapheresis healthy donors were prepared in large $\left(1-6 \times 10^{9}\right)$ and small $(25 \times 106)$ cell batches. Whereas the larger batch was directly labelled with streptamers to select HAdV and/or EBVspecific T-cells (large-scale), the smaller batch was used to generate in vitro virus-specific T-cell lines before streptamerlabelling for streptamer selection (small-scale). Isolation of HAdV- and/or EBV-specific T-cells was performed using the CliniMACS device.

Results: The purity of HAdV-streptamer + T-cells among $\mathrm{CD} 3+$ cells, obtained from large-scale selection was only $7.6 \%$, but reached up to $56 \%$ when HAdV- and EBVstreptamers were applied simultaneously. A further increase in purity of HAdV-specific T-cells reaching up to $98 \%$ was achieved by small-scale selection. All final products fulfilled the microbiological and chemical release criteria. IFN- $\gamma$-response indicating functional activity was seen in 6/9 HAdV and 2/3 EBV large-scale selections and in $2 / 3$ HAdV small-scale selections.

Conclusion: The use of HAdV-streptamers for clinical applications is feasible particularly when combined with other streptamers or when performed after a previous in vitro expansion period.

References: 1. Lion T. Adenovirus Infections in Immunocompetent and Immunocompromised Patients. Clin Microbiol Rev 2014; 27:441-462.

2. Matthes-Martin $\mathrm{S}$ et al. European guidelines for diagnosis and treatment of adenovirus infection in leukemia and stem cell transplantation: summary of ECIL-4 (2011). Transpl Infect Dis 2012; 14:555-563.

3. Schmitt $A$ et al. Adoptive transfer and selective reconstitution of streptamer-selected cytomegalovirus-specific CD8 + T cells leads to virus clearance in patients after allogeneic peripheral blood stem cell transplantation. Transfusion 2011; 51:591-599.

4. Stemberger $C$, et al. Lowest numbers of primary CD8 $+\mathrm{T}$ cells can reconstitute protective immunity upon adoptive immunotherapy. Blood 2014; 124:628-637.

Disclosure of Interest: None declared.
0136

Donor EBV status has impact on the incidence of acute and chronic GVHD

J. Styczynski $i^{1, *}$, G. Tridello ${ }^{2}$, L. Gil ${ }^{3}$, P. Ljungman ${ }^{4}$, J. Hoek ${ }^{5}$,

S. lacobelli ${ }^{6}$, C. Cordonnier ${ }^{7}, H$. Einsele ${ }^{8}, K^{\prime}$. Ward ${ }^{9}$, G. Socie $^{10}$

N. Milpied ${ }^{11}$, J. H. Veelken ${ }^{12}$, P. Chevallier ${ }^{13}$, I. Yakoub-Agha ${ }^{14}$

J. Maertens ${ }^{15}$, D. Blaise ${ }^{16}$, J. Cornelissen ${ }^{17}$, M. Michallet ${ }^{18}$,

E. Deconinck ${ }^{19}$. E. Petersen ${ }^{20}$, J. Passweg ${ }^{21}$, H. Greinix ${ }^{22}$,

R. Duarte ${ }^{23}, N_{\text {. Kröger }}^{24}$, P. Dreger ${ }^{25}$, A. Nagler ${ }^{26}$, S. Cesaro ${ }^{27}$

${ }^{1}$ Pediatric Hematology and Oncology, Collegium Medicum,

Nicolaus Copernicus University, Bydgoszcz, Poland, ${ }^{2}$ Policlinico G.B. Rossi, Verona, Italy, ${ }^{3}$ Medical University, Poznan, Poland, ${ }^{4}$ Karolinska University Hospital, Stockholm, Sweden, ${ }^{5}$ EBMT Data Office, Leiden, Netherlands, "Università di Roma "Tor Vergata", Roma, Italy, ${ }^{7}$ Hôpital Henri Mondor, Creteil, France, ${ }^{8}$ University Hospital, Wurzburg, Germany, ${ }^{9}$ University College Hospital, London, United Kingdom, ${ }^{10}$ Hopital St. Louis, Paris, ${ }^{11} \mathrm{CHU}$ Bordeaux, Pessac, France, ${ }^{12}$ University Medical Centre, Leiden, Netherlands, ${ }^{13} \mathrm{CHU}$, Nantes, ${ }^{14} \mathrm{CHRU}$, Lille, France, ${ }^{15}$ University Hospital Gasthuisberg, Leuven, Belgium, ${ }^{16}$ Centre de Recherche en Cancerologie, Marseille, France, ${ }^{17}$ Erasmus MC - Daniel den Hoed Cancer Centre, Rotterdam, Netherlands, ${ }^{18}$ Centre Hospitalier, Lyon Sud, ${ }^{19}$ Hopital Jean Minjoz, Besanco, France, ${ }^{20}$ University Medical Centre, Utrecht, Netherlands, ${ }^{21}$ University Hospital, Basel, Switzerland, ${ }^{22}$ Medical University, Vienna, Austria, ${ }^{23}$ Catalan Institute of Oncology, Barcelona, Spain, ${ }^{24}$ University Hospital Eppendorf, Hamburg, ${ }^{25}$ University of Heidelberg, Heidelberg, Germany, ${ }^{26}$ Chaim Sheba Medical Center, Tel-Hashomer, Israel, ${ }^{27}$ Azienda Ospedaliera Universitaria Integrata, Verona, Italy

Introduction: Epstein-Barr virus (EBV) has been a major cause of post-transplant lymphoproliferative disorder after allogeneic stem cell transplantation (HSCT). The impact of the donor (D) and recipient (R) serologic status on survival, relapse-free survival, relapse incidence, non-relapse mortality and incidence of graft-versus-host disease was unknown so far. Objective: We analyzed the influence of the donor's and recipient's EBV status on allo-HSCT transplant outcomes.

Materials (or patients) and methods: 11,364 allo-HSCTs performed due to acute leukemia, between 1997 and 2012, with $\mathrm{PB}$ or $\mathrm{BM}$ as a stem cell source were included to the study.

Results: Patients receiving grafts from EBV-seropositive donors had the same overall survival as patients grafted from seronegative donors (hazard ratio [HR], 1.05, 95\% Cl, 0.97-1.12 $\mathrm{P}=0.22$ ). EBV-seropositive donors also had no influence on relapse-free survival $(\mathrm{HR}=1.04,95 \% \mathrm{Cl}, 0.97-1.11 ; \mathrm{P}=0.31)$, relapse incidence $(\mathrm{HR}=1.03,95 \% \mathrm{Cl}, 0.94-1.12 ; \mathrm{P}=0.57)$, and non-relapse mortality $(\mathrm{HR}=1.05,95 \% \mathrm{Cl}, 0.94-1.17 ; \mathrm{P}=0.36)$ However, HSCT recipients receiving grafts from EBV-seropositive donors had higher risk of acute GVHD [32.8 (95\% C.I. 31.8, 33.8 ) vs $30.7 \%$ (95\% C.I. 28.7, 32.8) $\mathrm{P}=0.04 ; \mathrm{HR}=1.07 ; 95 \% \mathrm{Cl}$ $0.98-1.17]$, and chronic GVHD [42.3 (95\% C.I. 41.2, 43.5) vs $33.3 \%(95 \%$ C.I. $31.0,35.7), \mathrm{P}<0.0001 ; \mathrm{HR}=1.42,95 \% \mathrm{Cl}, 1.30$ 1.56]. After adjusting for confounders (donor type, conditioning, stem cell cource, patient age, gender match, T-cell depletion, year of transplant), D + serostatus had an impact on development of acute GVHD [HR 1.12 (95\% C.I. 1.02, 1.24), $P=0.017$ ] and chronic GvHD [HR 1.35 (95\%C.I. 1.22, 1.49), $P<0.0001]$. Also, when adjusting for confounders, in comparison to $\mathrm{D}$-/R- patients, the risk in $\mathrm{D}+/ \mathrm{R}+$ patients for aGVHD was: $\mathrm{HR}=1.22 ; \mathrm{P}=0.02$, and for $\mathrm{CGVHD}: \mathrm{HR}=1.46 ; \mathrm{P}<0.0001$, while in $D+/ R$ - patients for aGVHD it was: $H R=1.23 ; P=0.09$, and for CGVHD: $H R=1.37 ; P=0.005$.

Conclusion: Our data suggest that donor EBV status significantly influences development of acute and chronic GVHD after allo-HSCT.

Disclosure of Interest: None declared. 
0137

Viral isolates with lamivudine resistance and characteristics of immune escape drive $\mathrm{HBV}$ reactivation in a population of hematopoietic stem cell transplant recipients

C. Cerva ${ }^{1, *}$, G. Maffongelli ${ }^{1}$, V. Malagnino ${ }^{1}$, A. Ricciardi ${ }^{1}$, A. Bianchi ${ }^{1}$, E. Fortunato ${ }^{1}$, V. Svicher ${ }^{2}$, R. Salpini ${ }^{2}$, L. Colagrossi ${ }^{2}$ M. Pollicita ${ }^{2}$, A. M. Battisti ${ }^{2}$, G. Lombardi ${ }^{2}$, A. Picardi ${ }^{3}$, L. Cudillo ${ }^{3}$, R. Cerretti $i^{3}$, G. De Angelis ${ }^{3}, M$. Cantonetti ${ }^{3}, M$. Andreoni $^{1}$, C. F. Perno ${ }^{2}$,W. Arcese $^{3}$, M. Aragri ${ }^{2}$, L. Sarmati ${ }^{1}$

${ }^{1}$ Clinical Infectious Disease, ${ }^{2}$ Department of Experimental Medicine and Surgery, ${ }^{3}$ Department of Hematology, Tor Vergata University, Rome, Italy

Introduction: A variable rate (from $6 \%$ to $50 \%$ ) of Hepatitis B virus (HBV) reactivation has been reported in hematopoietic stem cell transplant (HSCT) recipients. The current international guidelines on HBV prophylaxis for HSCT patients are limited, particularly if referred to HSCT donor or to occult HBV infection, which represents a frequent condition in hematological patients. Our policy considers the use of lamivudine prophylaxis also for patients transplanted from a $\mathrm{HbcAb}$ and/ or HBsAb positive donor. The aim of this study was to evaluate the rate of HBV reactivation in HSCT recipients and to study the characteristics of viral isolates driving this phenomenon.

Materials (or patients) and methods: Data of 340 patients undergoing HSCT from 2008 to 2013 at Hematology Stem Cell Transplant Unit of Tor Vergata University were retrospectively collected. One hundred and four (30,5\%) patients received an autologous and $236(69,5 \%)$ an allogeneic transplants. HBV reactivation was defined by the HBV-DNA increasing to more than 2 log compared to the baseline level or by $>100 \mathrm{UI} \mathrm{HBV}$ DNA appearance in the serum.

Results: Out of 340 patients, 58 (17\%) (autologous, $n=16$; allogeneic, $n=42$ ) were considered at risk of HBV reactivation for the following criteria of positivity: 1) donor and/or recipient anti-HBC and/or 2) anti-HBe and/or 3) anti $\mathrm{HBs}+/-$ Lamivudine prophylaxis was given to 51 out of 58 patients (88\%). Overall, 4 patients, 1 autologous and 3 allogeneic recipients, developed HBV reactivation at a median time of 40 months (range, 28-53) after HSCT. Of these 4 patients, 3 were at risk of reactivation and 2 of them were not receiving prophylaxis. One was HBV-negative at the time of pretransplant screening. The cumulative incidence of $\mathrm{HBV}$ reactivation was $4,9 \%$ for patients at risk and $1 \%$.for the entire HSCT population studied. One patient reactivated HBV infection during lamivudine prophylaxis. HBV isolate genotype was studied in all reactivated patients. Two isolates showed HbsAg escape mutations (123 N, 124Y, 126I, 145 K, $145 \mathrm{R}, 144 \mathrm{E}$ $\mathrm{R} 122 \mathrm{~K}, \mathrm{~T} 140 \mathrm{~S})$ and in 1 lamivudine drug resistance mutations (181 S and L801L), 3 of them resulted HBV genotype D and one resulted genotype $\mathrm{F}$.

Conclusion: The low rate of reactivation in our cohort is probably due to the accuracy of pre-transplant donor/ recipients screening and to the extensive prophylaxis program. However, a case of HBV reactivation occurred in a patient negative at serological screening (sero-negative HBV occult infection). The detection of immune-escape $\mathrm{HBsAg}$ mutations, associated with lack of recognition by neutralizing immune activity and by diagnostic assay in most HBVreactivated patients, supports the role of these mutations in the process of immune-suppression driven HBV reactivation. Disclosure of Interest: None declared.
0138

Polyomaviruses-Associated Hemorrhagic Cystitis after Haploidentical Hematopoietic Stem Cell Transplantation with Post-Infusion Cyclophosphamide

A. Vai ${ }^{1, *}$, R. Crocchiolo ${ }^{1}$, J. El-Cheikh ${ }^{2}$, S. Furst ${ }^{2}$, S. Bramanti ${ }^{1}$, B. Sarina ${ }^{1}$, L. Morabito , S. Harbi ${ }^{2}$, A. Granata ${ }^{2}$, B. Mohty ${ }^{2}$, R. Devillier', C. Faucher', C. Chabannon ${ }^{2}$, A. Santoro', L. Castagna? D. Blaise ${ }^{2}$

${ }^{1}$ Humanitas Cancer Center, Rozzano (MI), Italy, ${ }^{2}$ Institut Paoli Calmettes, Marseille, France

Introduction: Polyomavirus (PV) hemorrhagic cystitis $(\mathrm{HC})$ is a severe complication after haploidentical hematopoietic stem cell transplantation (haplo HSCT). In the setting of solid organ transplantation, the use of Tacrolimus (FK) has been associated with higher risk of PV-HC compared with cyclosporine A (CsA). The aim of our study was to investigate risk factors of PV-HC in haplo-HSCT recipients, with particular focus on immunosuppressive agent used as graft-versus-host disease (GVHD) prophylaxis. Materials (or patients) and methods: We retrospectively analyzed the incidence, risk factors and outcome of PV-HC in 149 consecutive adult patients (pts) undergoing haplo HSCT due to hematological malignancies between 2009 and 2014 at our two institutions. All HSCTs were T-cell replete and included post-transplant high-dose cyclophosphamide as part of GVHD prophylaxis. PV-HC was defined as PV detection in urine by PCR testing in association with clinical symptoms of $\mathrm{HC}$ without other concurrent genitourinary conditions. Variables tested as potential risk factors to develop a PV-HC were: conditioning regimen, age, diagnosis, stem cell source, levofloxacin prophylaxis, acute GVHD, interval between diagnosis and HSCT, pre-HSCT therapy lines, previous subdiaphragmatic radiotherapy, n. of CD34+ cells infused, neutrophil and platelet engraftment, FK vs CsA-based immunosuppressive regimen.

Results: Main pts' characteristics are shown in Table 1. After a median follow-up time of 13 months from transplantation, ten (7\%) pts developed a PV-HC. PV-HC occurred in 6/52 (12\%) pts receiving $\mathrm{FK}$ and $4 / 97(4 \%)$ pts receiving $\mathrm{CsA}(P=0.10)$. Etiology was BK virus in $9(90 \%)$ cases and JC virus in one $(10 \%)$ case. Median time of PV-HC diagnosis was 30 (7-68) days post-HSCT. In the multivariate analysis, myeloablative (MYA) or reduced-intensity conditioning $(\mathrm{RIC})$ regimens $(\mathrm{HR}=4.25,95 \%$ $\mathrm{Cl}: 1.18-15.33 ; P=0.03$ ) and $\mathrm{FK}$-based immunosuppression $(\mathrm{HR}=3.78,95 \% \mathrm{Cl}: 1.05-13.64 ; P=0.04)$ were the only two independent factors associated with PV-HC. Regarding treatment, two pts received Cidofovir therapy, one pt benefited from immunosuppression tapering, whereas only supportive therapy was required for the remaining 7 pts. Notably, all pts achieved complete remission of symptoms.

Table 1. Main pts' characteristics.

\begin{tabular}{|c|c|}
\hline & $n=149$ \\
\hline Median age at haplo-HSCT (range) & $52(19-73)$ \\
\hline Gender M/F & $87 / 62$ \\
\hline \multicolumn{2}{|l|}{ Diagnosis } \\
\hline Hodgkin's lymphoma & $48(32 \%)$ \\
\hline Non-Hodgkin's lymphoma & $39(26 \%)$ \\
\hline Acute leukemia & $36(24 \%)$ \\
\hline Multiple myeloma & $11(7 \%)$ \\
\hline Myelodisplastic syndrome & $8(5 \%)$ \\
\hline Chronic leukemia & $6(4 \%)$ \\
\hline Myelofibrosis & $1(1 \%)$ \\
\hline \multicolumn{2}{|l|}{ Disease status at HSCT } \\
\hline Complete/partial remission & $113(76 \%)$ \\
\hline Stable/progressive & $36(24 \%)$ \\
\hline \multicolumn{2}{|l|}{ Stem cell source } \\
\hline Bone Marrow & $83(56 \%)$ \\
\hline Peripheral Blood Stem Cells & $66(44 \%)$ \\
\hline \multicolumn{2}{|l|}{ Conditioning regimen } \\
\hline MYA or RIC & $48(32 \%)$ \\
\hline Non-MYA & $101(68 \%)$ \\
\hline Median CD34 + cell dose $\left(\times 10^{6} / \mathrm{kg}\right)$ & $3.9(0.8-14.0)$ \\
\hline
\end{tabular}


Conclusion: In this cohort of $149 \mathrm{~T}$-cell replete haplo HSCTs using post-transplant high-dose cyclophosphamide, we found that a higher intensity of conditioning (MYA or RIC vs nonMYA) as well as the use of more immunosuppressive calcineurin inhibitor (FK vs CsA) were both significantly associated with a higher incidence of PV-HC.

Disclosure of Interest: None declared.

\section{Oral session: Experimental transplantation / MRD}

\section{9 \\ Transcriptome analysis of circulating lymphoid progenitors after allogeneic stem cell transplantation in humans reveals profound alterations of cellular metabolism S. Glauzy ${ }^{1}$, I. André-Schmutz ${ }^{2}$, J. Lachuer ${ }^{3}$, R. Peffault de Latour ${ }^{4}$, S. Servais ${ }^{4}$, G. Socié $e^{4}$, E. Clave ${ }^{1, *}$, A. Toubert ${ }^{1}$ \\ ${ }^{1}$ INSERM UMRS1160, IUH Univ Paris Diderot, ${ }^{2}$ INSERM U768, Institut Imagine, PARIS, ${ }^{3}$ ProfileXpert, Univ UMR1052, CRCL, UCBL, Lyon, ${ }^{4}$ Service d'hématologie Greffe de Moelle, AP-HP, Hôpital St Louis, PARIS, France}

Introduction: Acute Graft-Versus-Host Disease (aGVHD) results in a delayed T-cell reconstitution after allogeneic hematopoietic stem cell transplantation (allo-HSCT) due to defects in T-cell differentiation. We have shown a strong recovery of circulating lymphoid committed progenitors defined as CD34 Lin ${ }^{-C D} 10^{+} \mathrm{CD}_{24}^{-}$(Glauzy et al., PLoS ONE 2014), 3 months after HSCT, that was abolished in patients who developed aGVHD. Here, we analyzed transcriptional programs of cellsorted circulating lymphoid progenitors after allo-HSCT in patients having or not developed aGVHD.

Materials (or patients) and methods: We sorted (FACS ARIA, $\mathrm{BD})$, rare populations of lymphoid $\mathrm{CD}^{+} 4^{+} \mathrm{Lin}^{-} \mathrm{CD} 10^{+} \mathrm{CD} 24$ and of $\mathrm{CD}_{34}{ }^{+} \mathrm{Lin}^{-} \mathrm{CD} 10^{-}$non-lymphoid progenitors from peripheral blood of 12 adult patients 3 months after a non T-cell depleted allo-HSCT and 4 healthy age-matched donors. Eight patients did not develop aGVHD and 4 had a grade 2 aGVHD. Gene expression was quantified on the GeneChip HG u133 plus 2.0. Data were analyzed with algorithm Maximum Rank Sum (MAXRS) and normalized with global normalization methods. Pathway analyses were performed with KEGG (Kyoto Encyclopedia of Genes and Genomes), GSEA (Gene Set Enrichment Analysis) and DAVID (Database for Annotation, Visualization and Integrated Discovery).

Results: In CD34 ${ }^{+}$Lin $^{-} \mathrm{CD} 10^{-}$cells, 1609 probes were deregulated between patients without aGVHD and patients with aGVHD (1560 of this probe were up-regulated and 49 were down-regulated, $P<0.05$, Fold Change $>1.5$ ). In $\mathrm{CD}_{4}{ }^{+}$ Lin $\mathrm{CD}_{10}{ }^{+} \mathrm{CD}_{24}$ progenitors, 987 probes were deregulated between patients without aGVHD and patients with aGVHD (941 of this probe were up-regulated and 46 were downregulated, $P<0.05$, Fold Change $>1.5$ ). 273 probes were deregulated in both $\mathrm{CD}_{3} 4^{+} \mathrm{Lin}^{-} \mathrm{CD} 10^{+} \mathrm{CD} 24^{-}$and $\mathrm{CD}_{3}{ }^{+}$ Lin ${ }^{-} \mathrm{CD} 10^{-}$populations.

Genes from ribosome protein biogenesis, translation machinery (EEF1D, EEF1G, EIF3K) and cell cycle (CCND1, CDK6) were over-expressed in $\mathrm{CD}_{3} 4^{+} \mathrm{Lin}^{-} \mathrm{CD} 10^{+} \mathrm{CD} 24^{-}$and in $\mathrm{CD}_{34}{ }^{+} \mathrm{Lin}^{-} \mathrm{CD} 10^{-}$populations from patients without aGVHD compared with those from patients affected by aGVHD and from healthy donors. Expressions of genes from the oxidative phosphorylation metabolic pathway (NDUFS2, SDHA, ATP5A1) and genes involved in stress resistance (BTG2, MGST3, $H P X)$ were specifically increased in $\mathrm{CD}_{34}{ }^{+} \mathrm{Lin}^{-} \mathrm{CD} 10^{+} \mathrm{CD} 24$ lymphoid progenitors and not in $\mathrm{CD}^{-} 4^{+} \mathrm{Lin}^{-} \mathrm{CD} 10^{-}$ non-lymphoid progenitors from patients without aGVHD compared with patients suffering from aGVHD and from healthy donors.
Conclusion: We show for the first time that circulating lymphoid T-cell progenitors undergo profound changes in metabolism favoring energy production and response to stress after alloHSCT in Humans. These mechanisms are abolished in case of aGVHD, indicating a persistent cell-intrinsic defect in addition to the impact of aGVHD on the bone marrow environment.

Disclosure of Interest: None declared.

\section{0}

Post Transplant Cyclophosphamide (PTCy) and Sirolimus for Haploidentical PBSC HSCT: favorable Immune Reconstitution and Low Rates of GvHD in $\mathbf{4 0}$ Patients N. Cieri ${ }^{1, *}$, R. Greco ${ }^{1}$, L. Crucitti ${ }^{1}$, M. Morelli ${ }^{1}$, F. Giglio ${ }^{1}$, G. Levati ${ }^{1}$, A. Assanelli ${ }^{1}$, F. Lorentino ${ }^{1}$, M. T. Lupo Stanghellini ${ }^{1}$, L. Vago ${ }^{1}$, T. Freitas ${ }^{1}$, S. Marktel' ${ }^{1}$, M. Bernardi ${ }^{1}$, C. Corti ${ }^{1}$, C. Bonini ${ }^{1}$, F. Ciceri ${ }^{1}$, J. Peccatori ${ }^{1}$

${ }^{1}$ San Raffaele Scientific Institute, Milano, Italy

Introduction: Haploidentical hematopoietic stem cell transplantation (HSCT) performed using BM grafts and post-transplantation cyclophosphamide (PTCy) has gained much interest for the excellent toxicity profile, but has been limited by relatively high relapse rates. We investigated whether the use of myeloablative conditioning, PBSC graft and sirolimus instead of tacrolimus might help increasing the therapeutic index of the procedure.

Materials (or patients) and methods: Forty patients were treated between Nov 2012 and July 2014. Median age was 55 years (range, 21-78). Diagnoses included AML (22), ALL (5), CML-BC (1), MDS (1), NHL (5), and HD (6). The majority of patients $(63 \%)$ were not in remission at time of HSCT. Nine patients $(22 \%)$ received a prior alloHSCT. For the remaining patients who underwent haploPTCy as first HSCT, 19 patients (61\%) scored high/very high according to CIBMTR disease score, $12(39 \%)$ scored intermediate, while no patient scored low. Median HCT-comorbidity index was 3 (range 0-9). Conditioning consisted of treosulfan $(14 \mathrm{~g} / \mathrm{m} 2 /$ day) on days 6 to -4 , fludarabine $(30 \mathrm{mg} / \mathrm{m} 2 /$ day) on days -6 to -2 , and melphalan $(70 \mathrm{mg} / \mathrm{m} 2 /$ day) on days -2 and -1 , followed by T-replete G-mobilized PBSC. Postgrafting immunosuppression consisted of PT-Cy $(50 \mathrm{mg} / \mathrm{kg} /$ day) on days 3 and 4 , followed by MMF for 30 days, and sirolimus for at least 90 days.

Results: PBSC grafts contained a median of CD34/kg of 6 e 6 (4.1-8) and CD3/kg 2.2 e8 (1-4.8). All patients engrafted, with a median time to neutrophil and platelet recovery of 18 days (13-45) and 16 days (9-100), respectively. Post-HSCT recovery of lymphocyte subsets was broad and fast: median time to CD3 $>100 / \mathrm{ml}$ was 28 days, to $C D 4>200 / \mathrm{ml} 41$ days and to CD19>0/ml 41 days. Circulating $T$ cells comprised naïve and memory subsets, with a recovery of CD31 recent thymic emigrants (RTEs) from day 30. All patients had a significantly higher proportion of RTEs at day 30 and 180 compared to their pre-HSCT levels, suggesting an improvement in their thymic function after HSCT. With a median follow-up for living patients of 15 months (5-24), the 1-year cumulative $\mathrm{Cl}$ of NRM and relapse were $17 \%$ and $35 \%$. Three of the 11 acute leukemia relapses were HLA-loss variants. Notably, one was observed for the first time in ALL. Cl of aGvHD grade II-IV and III-IV at 6 months were $17 \%$ and $7 \%$, while 1 -year $\mathrm{Cl}$ of cGvHD was $20 \%$. Low GvHD incidence might be in part due to a significant early increase in circulating Tregs at day 15 post HSCT, with values $<5 \%$ being predictive of subsequent GvHD occurrence. Estimated 1-year OS and DFS were $56 \%$ and $48 \%$. Outcomes were more favorable in patients transplanted in CR (1-year DFS $71 \%$ ) vs. patients transplanted with active disease (DFS $34 \%$ $P=.01)$. Stratification according to CIBMTR score held similar trends: 1 -year DFS was $64 \%$ in patients with intermediate score vs. $41 \%$ in patients scoring high/very high $(P=.07)$.

Conclusion: Myeloablative haploHSCT with PBSC, PT-Cy and sirolimus is a valid option for patients with aggressive/ advanced disease. The acceptable rates of GvHD and NRM as well as the favorable immune reconstitution profile open the way for combining it with novel immunomodulatory/ cellular therapies to improve DFS in patients at high risk for 
relapse who would benefit from further treatment intensification early after transplant.

Disclosure of Interest: None declared.

0141

Interim results of a multicenter phase I/lb study of CTLA4 blockade with ipilimumab for relapsed hematologic malignancies after allogeneic hematopoietic cell transplantation

M. S. Davids ${ }^{1}$, H. Kim ${ }^{1}$, C. Costello ${ }^{2}$, D. Avigan ${ }^{3}$, Y. B. Chen ${ }^{4}$, P. Armand ${ }^{1}$, E. P. Alyea ${ }^{7}$, J. Hedlund ${ }^{5}$, P. McSweeney ${ }^{6}$, R. Liguori ${ }^{1}$, J. Ritz ${ }^{1}, E$. Ball ${ }^{2}$, A. Bashey ${ }^{7}, R$. Soiffer ${ }^{1, *}$ on behalf of Blood Cancer Research Partnership of the Leukemia \& Lymphoma Society

${ }^{1}$ Medical Oncology, Dana Farber Cancer Institute, Boston, ${ }^{2}$ Division of BMT, University of California, San Diego Moores Cancer Center, La Jolla, ${ }^{3}$ BMT Program, Beth Israel Deaconess Medical Center, ${ }^{4}$ BMT Program, Massachusetts General Hospital Cancer Center, Boston, ${ }^{5}$ New England Cancer Specialists, Maine Center for Cancer Medicine, Scarborough, ${ }^{6}$ Colorado Blood Cancer Institute, Denver, ${ }^{7}$ Blood and Marrow Transplant Group of Georgia at Northside Hospital, Atlanta, United States

Introduction: The prognosis for patients with hematologic malignancies (HM) who relapse after alloHCT is dismal, and immune checkpoint modulation with CTLA4 blockade is a novel strategy to enhance the graft versus tumor (GVT) effect. Materials (or patients) and methods: This is a phase $\mathrm{I} / \mathrm{lb}$ study of the CTLA4 blocking antibody ipilimumab to treat patients with any HM histology who relapse after alloHCT. The primary objectives are to determine the MTD and evaluate safety. Secondary objectives include a preliminary evaluation of efficacy and changes in immune cell phenotype. Ipilimumab was given at $3 \mathrm{mg} / \mathrm{kg}$ or $10 \mathrm{mg} / \mathrm{kg} \mathrm{IV} \mathrm{q3}$ weeks for 4 cycles of induction, followed by maintenance dosing q12 weeks up to 1 year. Disease-specific response criteria were assessed at the mid-point (7 weeks), end of induction (13 weeks), and throughout maintenance. Immunophenotyping was performed by 8-color flow cytometry and analyzed by FACSDiva. Results: Twenty-three patients are enrolled to date. In the phase I portion, 6 patients were treated at $3 \mathrm{mg} / \mathrm{kg}$ and 7 patients were treated at $10 \mathrm{mg} / \mathrm{kg}$. An MTD was not reached, and 10 patients subsequently enrolled in the phase $\mathrm{lb}$ expansion cohort at $10 \mathrm{mg} / \mathrm{kg}$. The median number of prior therapies excluding transplant was 3 (range 2-11), and 14 patients had received prior therapy for their post transplant relapse. Histologies included $\mathrm{cHL}(n=7), \mathrm{AML}(n=7), \mathrm{NHL}$ $(n=4)$, and MDS $(n=2)$, and 1 patient each had MM, MPN, and ALL. The median age at enrollment was 55 (range 22-75). Immune-related adverse events (irAEs) were observed in 4 patients, and included ITP $(n=1)$, diarrhea $(n=2)$, pneumonitis $(n=3)$, and colitis $(n=1)$, all of which were generally reversible with steroids with most patients resuming ipilimumab dosing. Three DLTs have been observed, including 2 cases of chronic GVHD (both liver, mild) and one patient death due to presumed sepsis in the context of severe irAEs, including grade 3 colitis and grade 4 pneumonitis. Nine patients remain on treatment, and 11 patients discontinued due to progressive disease, 2 patients due to CGVHD, and 1 patient with TRM. In an interim efficacy analysis, 7/19 (36.8\%) patients evaluable for response had anti-tumor activity with clinical benefit. Four patients achieved formal responses by disease-specific criteria, including a cHL patient with PR with dramatic reduction in nodal and extranodal disease with a complete marrow response at 7 weeks (baseline 90\% involvement), a MM patient with a PR with near resolution of a lung plasmacytoma, and two patients with AML who achieved CR by 7 weeks. The median follow-up time among survivors is 5.4 months, and 6 month overall survival is currently $57 \%$. Immunophenotyping studies revealed that the ratio of regulatory $T$ cells to conventional $T$ cells decreased between $24 \%$ and $41 \%$ after ipilimumab treatment.

Conclusion: Multiple doses of ipilimumab given to patients with relapsed HM after alloHCT were generally well-tolerated, with CGVHD and irAEs observed in a small number of patients. Anti-tumor activity was observed, both in lymphoid malignanices and for the first time in myeloid malignancies, including 2 patients with AML who achieved CR. The ratio of regulatory to conventional $\mathrm{T}$ cells decreased with CTLA4 blockade. The phase $\mathrm{lb}$ expansion cohort at $10 \mathrm{mg} / \mathrm{kg}$ continues to accrue and will provide additional efficacy, safety, and correlative data.

Disclosure of Interest: None declared.

\section{2}

Factors determining the Kinetics of Disease Relapse after Allogeneic Stem Cell Transplantation (allo-SCT) for Acute Myeloid Leukaemia (AML): a survey from the Acute Leukaemia Working Party of the EBMT

C. Craddock, ${ }^{1, *}$, M. Labopin ${ }^{2}$, G. Socie ${ }^{3}$, A. Huyn ${ }^{4}$, E. Deconinck ${ }^{5}$, L. Volin ${ }^{6}$, N. Milpied ${ }^{7}$, J.-H. Bourhis ${ }^{8}$, A. Rambaldi', P. Chevallier ${ }^{10}$, J. Cornelissen ${ }^{11}$, D. Blaise ${ }^{22}$, J. Maertens ${ }^{13}$, C. Schmid ${ }^{14}$,

A. Nagler ${ }^{15}$, M. Mohty ${ }^{16}$

${ }^{7}$ Haematology, Universtiy of Birmingham, Birmingham, United Kingdom, ${ }^{2}$ Hôpital Saint Antoine, ${ }^{3}$ Saint Louis Hospital, Paris, ${ }^{4}$ Institut Universitaire du Cancer de Toulouse, Toulouse, ${ }^{5}$ Hôpital Jean Minjoz, Besançon, France, ${ }^{6}$ Helsinki University Central Hospital, Helsinki, Finland, ${ }^{7}$ University Hospital and university of Bordeaux, Bordeaux, ${ }^{8}$ Gustave Roussy, Paris, France, ${ }^{9}$ Ospedali Riuniti Bergamo, Bergamo, Italy, ${ }^{10}$ Centre Hospitalier Universitaire, Nantes, France, ${ }^{11}$ Erasmus, Netherlands, Netherlands, ${ }^{12}$ Institut Paoli Calmettes, Marseille, France, ${ }^{13}$ University Hospital Gasthuisberg, Leuven, Belgium, ${ }^{14}$ University of Munich, Munich, Germany, ${ }^{15}$ Sheba Center, Ramat Gan, Israel, ${ }^{16}$ University of Nantes, Nantes, France

Introduction: Post transplant interventions such as donor lymphocyte infusion (DLI) or administration of pharmacological agents, represent important novel strategies with the potential to reduce the risk of disease relapse after allo-SCT in Acute Myeloid Leukaemia (AML). Such approaches are critically dependent on timely intervention post-transplant but despite this the factors determining the kinetics of disease relapse in patients allografted for AML have not been defined.

Materials (or patients) and methods: 1052 adults who received an allo-SCT for AML in first complete remission (CR1) between 2000 and 2012 were studied. 544 patients were transplanted using a sibling donor and 508 from an adult matched-unrelated donor. 538 patients received a myeloablative conditioning (MAC) regimen and 514 a reduced intensity (RIC) regimen. A series of landmark analyses were performed at 3,6 and 12 months in order to identify prognostic factors of relapse for patients alive and well at the beginning of each time interval. The probabilities of relapse were calculated by using the cumulative incidence estimator to accommodate for death as a competing risk. Factors predicting relapse were studied using Cox regression model including time dependent variables. A backward stepwise procedure was used for variable selection.

Results: With a median follow-up of 26 months, 244 patients relapsed. The 3 year cumulative incidence of relapse was $26 \%$ [95\%Cl: $23-28$ ]. Overall $84 \%$ of patients destined to relapse did so within the first year post-transplant. The overall factors predicting disease relapse for the whole population were more than one course of chemotherapy to achieve CR1, FLT3 ITD positivity, adverse risk cytogenetics, shorter interval from CR1 to transplant. The occurrence of acute GVHD grade II or greater $(P=0.05)$ and chronic GVHD $(P=0.03)$ were both associated with a lower risk of relapse.

Using landmark analyses the factors determining relapse at different stages post transplant were observed to differ. In the first 3 months post-transplant the significant factors determining relapse risk were: patient age $(P=0.03)$, prolonged interval from diagnosis to CR1 $(P=0.05)$, flt3 ITD positivity $(P=0.002)$, adverse risk cytogenetics $(P=0.02)$ and use of in vivo T cell depletion $(P=0.003)$. The only factors observed to determine relapse risk between 3 and 6 months post-transplant were 
adverse risk cytogenetic $(P=0.04)$. In patients who relapsed between 6 and 12 months post-transplant more than one course of chemotherapy to achieve CR1 $(P=0.02)$, adverse risk cytogenetics $(P=0.05)$ and FLT3 ITD positivity $(P=0.00002)$ all predicted for relapse. Finally only CMV positivity predicted for relapse risk for relapse more than 12 months post-transplant $(P=0.05)$.

Conclusion: This study demonstrates for the first time that a complex interaction of disease specific and transplant factors determine the kinetics of relapse post-transplant. In addition to identifying that heterogenous factors related to transplant characteristics and disease biology determine the timing of disease relapse these data confirm the importance of early intervention post-transplant in patients allografted for AML and and will assist in the design of novel therapeutic strategies.

Disclosure of Interest: None declared.

0143

A novel quantitative PCR approach targeting insertion/ deletion polymorphisms (indel-PCR) for chimerism quantification: finally high sensitivity and quantification capacity together

A. Navarro-Bailón ${ }^{1, *}$, C. Martínez-Laperche ${ }^{1,2}$, D. Carbonell ${ }^{1}$ E. Buces ${ }^{1,2}$, M. González-Rivera ${ }^{2}$, M. Bastos ${ }^{1,2}$, P. Balsalobre ${ }^{1,2}$, M. Kwon ${ }^{1,2}$, D. Serrano ${ }^{1,2}, J$. Anguita ${ }^{1,2}, J_{\text {. Gayoso }}^{1,2}$,

J. L. Díez-Martín ${ }^{1,2}$, I. Buñoo ${ }^{1,2}$

${ }^{1}$ Hospital General Universitario Gregorio Marañón, ${ }^{2}$ Instituto de Investigación Sanitaria Gregorio Marañón, Madrid, Spain

Introduction: Post-hematopoietic stem cell transplantation (SCT) chimerism monitoring is important to assess engraftment, anticipate relapse and provide information on the development of graft versus host disease, facilitating therapeutic intervention. The aim of this study was to test the technical efficacy and clinical utility of a novel quantitative PCR approach targeting insertion/deletion polymorphisms (indel-PCR).
Materials (or patients) and methods: This study included 104 samples (52 bone marrow, 37 peripheral blood (PB), 10 Tcells, 2 myeloid cells, 2 CD34-cells, 1 NK-cells) of 21 patients (5 samples each) who underwent SCT for hematological malignancies. Additionally, 2 sets of 11 artificial mixtures were created using PB of two healthy subjects (a male and a female) with known percentages of male leukocytes (putative recipient): $100,75,50,25,10,5,3,1,0.1,0.01$, 0 . Chimerism analysis was performed by the gold-standard STR-PCR (AmpFISTR SGM Plus ${ }^{\circledR}$, Life Technologies, USA) and by indelPCR (Mentype ${ }^{\mathbb{R}}$ DIPscreen, Mentype ${ }^{\mathbb{R}}$ DIPquant, Biotype, Germany). Concordance between both methods was calculated with SPSS using intraclass correlation and Cohen's kappa coefficients (samples classified in 3 groups: $0-1 \%, 1-10 \%, 10$ $100 \%)$.

Results: The number of informative loci identified with indelPCR (>3/patient) was higher than with STR-PCR (Figure 1). Concordance between both methods for the 104 patient samples and 9 artificial mixtures was "very good" (kappa coefficient $=0.71$, intraclass correlation coefficient $=0.96$ ). Of note, analysis of artificial mixtures provides evidence of significantly ( $\geq 2$ logs) higher sensitivity by indel-PCR $(0.01 \%)$ than by STR-PCR ( $1 \%$, Table 1). Moreover, indel-PCR shows unprecedented quantification capacity (Table 1). Out of the 113 samples analyzed, 29 were positive and 6 negative by both methods, while 78 were positive only by indel-PCR $(95 \%$ with $<1 \%$ recipient). Hematological relapse occurred in 5 patients, molecular relapse/persistence in 2 patients. All of them presented a positive indel-PCR (with increasing \%R in 4/ $5)$ and a negative STR-PCR result in the sample before relapse (Table 2). The 12 patients in complete remission, although presented positive indel-PCR, showed stable or decreasing $\% \mathrm{R}$ chimerism dynamics in 10/12 (data not shown).

Conclusion: This novel indel-PCR is a simple and accurate technique that, in comparison with the current gold standard STR-PCR, shows very good concordance and provides higher rates of informative loci per patient, as well as unprecedented combined sensitivity and quantification capacity. Such

[0143]

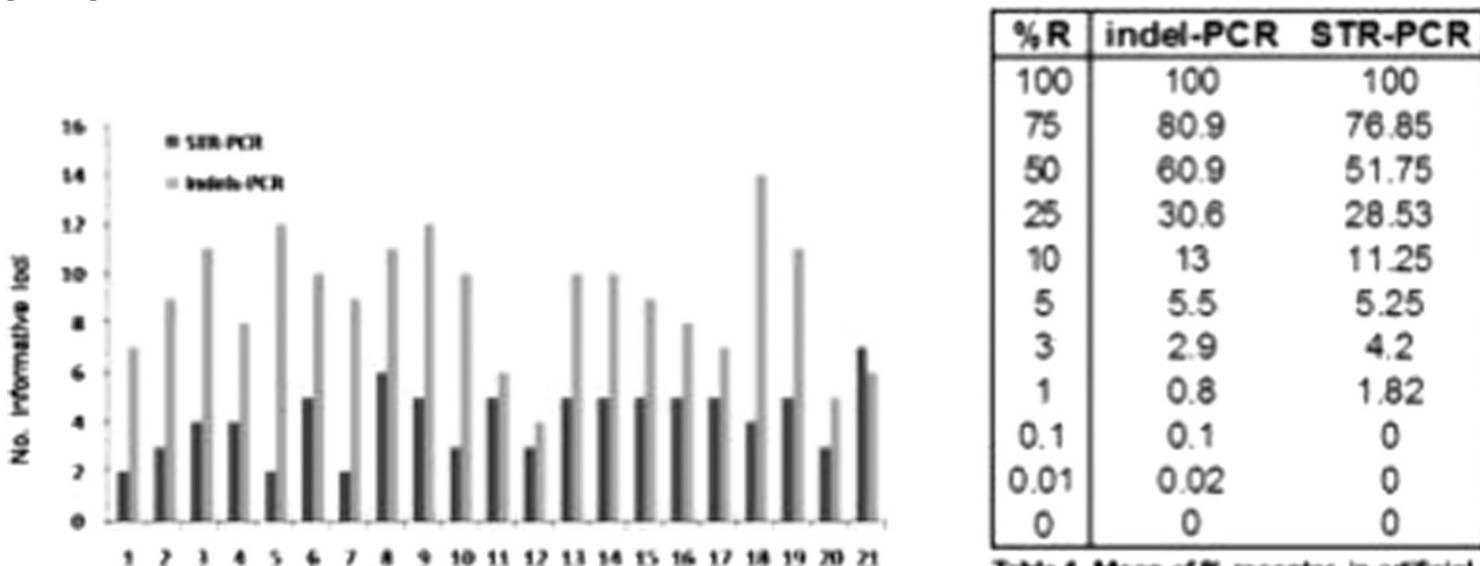

Tatbe 1. Man of \$ receptor in articial mintures detected by indel PCR and STR PCR

\begin{tabular}{|c|c|c|c|c|c|c|}
\hline$=$ & Redacse (dyy) & Pretelapse sarncle (day) & STR-PCR (\$,R) & indel $P C R(W R)$ & Dnames & MPO molocula makes \\
\hline 1 & +202 & .76 & 0 & 02 & 282 & no \\
\hline 2 & +315 & -120 & 0 & 0.2 & $\hat{\tau}$ ya & no \\
\hline 3 & +400 & -218 & 0 & 013 & 个 we & no \\
\hline 4 & +300 & .150 & 0 & 04 & ND & no \\
\hline 5 & +410 & .200 & 0 & 03 & 个 sal & no \\
\hline $6^{\circ}$ & +481 & -360 & 0 & 03 & $\uparrow+2$ & BCR-ABL D 190 \\
\hline $7^{*}$ & persistence & NA & 0 & 005 & stable & BCA AB, 0210 \\
\hline
\end{tabular}

Table 2. Chimerism and clrical characterisibcs of relapsed pabents. "molecular relapse, R: recipient: MFO minimal residual disease, NA: not applicable, ND: not done. 
features allow minutely monitoring chimerism dynamics and improving clinical management of transplanted patients.

Disclosure of Interest: A. Navarro-Bailón: None declared, C. Martínez-Laperche: None declared, D. Carbonell: None declared, E. Buces: None declared, M. González-Rivera: None declared, M. Bastos: None declared, P. Balsalobre: None declared, M. Kwon: None declared, D. Serrano: None declared, J. Anguita: None declared, J. Gayoso: None declared, J. L. DíezMartín: None declared, I. Buño Funding from: Diagnostica Longwood. Biotype.

\section{4}

Haploidentical Stem Cell Transplantation after Depletion of T Cells Expressing the $\alpha \beta$ Chain of the T-Cell Receptor (TCR) and CD19 + B cells for Adults with Hematological Malignancies

L. Prezioso ${ }^{1, *}$, S. Bonomini ${ }^{1}$, G. Sammarelli ${ }^{1}$, C. Schifano ${ }^{1}$,

E. Rossetti ${ }^{1}$, A. Monti ${ }^{1}$, G. Todaro ${ }^{1}$, L. Craviotto ${ }^{1}$, I. Manfra ${ }^{1}$, A. Spolzino' ${ }^{1}$, B. Cambò ${ }^{7}$, M. Sassi ${ }^{2}$, C. Caramatti ${ }^{1}$, F. Aversa ${ }^{1}$ ${ }^{1}$ Department of Hematology and Bone Marrow Unit, ${ }^{2}$ Transfusion and Apheresis Unit, Parma, Italy

Introduction: The immune recovery after CD34 + cell selection is slow and patients tend to remain susceptible to opportunistic infections for several months after HSCT. To hasten and improve post-transplant immune reconstitution broad repertoire various strategies of adoptive donor $\mathrm{T}$ cel immunotherapy (e.g. engineering with a suicide gene; depleting alloreactivity by means of photodynamic purging or through the use of freshly purified regulatory $T$ cells) have been investigated over the past years. Recently, selective elimination of $\alpha \beta^{+}$T cells has been performed to achieve a 4.5-5 log TCD and to retain in the graft NK, dendritic cells, monocytes and $\gamma \delta \mathrm{T}$ lymphocytes. Under this approach, a rapid immunological reconstitution and very promising outcome have been reported in pediatric patients. With the aims of confirming these results even in adults, we have recently launched this programme and here we report our preliminar clinical data in 22 leukemia patients we have so far treated over the past 26 months.

Materials (or patients) and methods: Twenty-two patients, median age 44 years (range 19-67), with AML $(n=16)$, ALL $(n=5), \operatorname{MDS}(n=1)$ entered the study. Eight patients were in CR1, 3 in CR2, and 11 in advanced-stage disease at transplant. Conditioning consisted of ATG $1,5 \mathrm{mg} / \mathrm{kg}$ from day -13 to day 10 , Treosulfan $12 \mathrm{gr} / \mathrm{sqm}$ from -9 to -7 , Fludarabine $30 \mathrm{mg} / \mathrm{sqm}$ from -6 to -2 and Thiotepa $5 \mathrm{mg} / \mathrm{Kg}$ on days -5 and -4 . No patient received any post transplantation pharmacologic prophylaxis for GVHD. Ten $\mu \mathrm{g} / \mathrm{kg}$ G-CSF was used to mobilize PBPCs from one-haplotype mismatched donors (3 mothers, 5 brothers, 2 sisters, 4 son, 3 daughter and 5 cousin). Depletion was performed using the CliniMACS.

Results: Grafts contained a median of $11 \times 10^{6} / \mathrm{kg}$ (range5-19) CD34 + cells, $4.3 \times 10^{6} \mathrm{CD}^{+}$Tcells/kg(range $1-35$ ) with $4.8 \times 10^{4} /$ $\mathrm{kg}$ (range 0.4-62) $\alpha \beta^{+} \mathrm{T}$ cells and $4 \times 10^{6} \gamma \delta^{+}$Tcells/kg(range 134 ), $4 \times 10^{4} \mathrm{~B}$ cells $/ \mathrm{kg}$ (range $1.5-32$ ) and $30 \times 10^{8} \mathrm{CD} 56^{+}$NKcells/ $\mathrm{kg}$ (range 8-91). All but one patient, who required a second graft from the same donor to boost hematopoietic reconstitution, achieved a full donor sustained engraftment. Median time to reach 500 neutrophils and 20,000 platelets was 12 (range 9-18) and 11 days (range 6-16), respectively. Only one patients, who had received the highest dose of $\alpha \beta+$ T cells $\left(37 \times 10^{4} / \mathrm{kg}\right)$, developed and died from severe aGVHD. Eight patients had skin grade II aGVHD and required treatment with short course steroids. Only one patient has so far developed chronic GvHD which recovered completely after steroid and cyclocporin treatment. Median CD4 + cell/ $\mu \mathrm{L}$ counts at 30, 60 and 90 days since the transplant were 36,80 and 110 , respectively. CMV antigenemia reactivation occurred in 6 cases (in 2, CMV serology was unfavourable). No patients has so far developed CMV disease. Invasive fungal disease was prevented in all cases using L-AmB-based prophylaxis over the neutropenic phase. Overall,10 patients have so far died
Fig. 1 Overall Survival

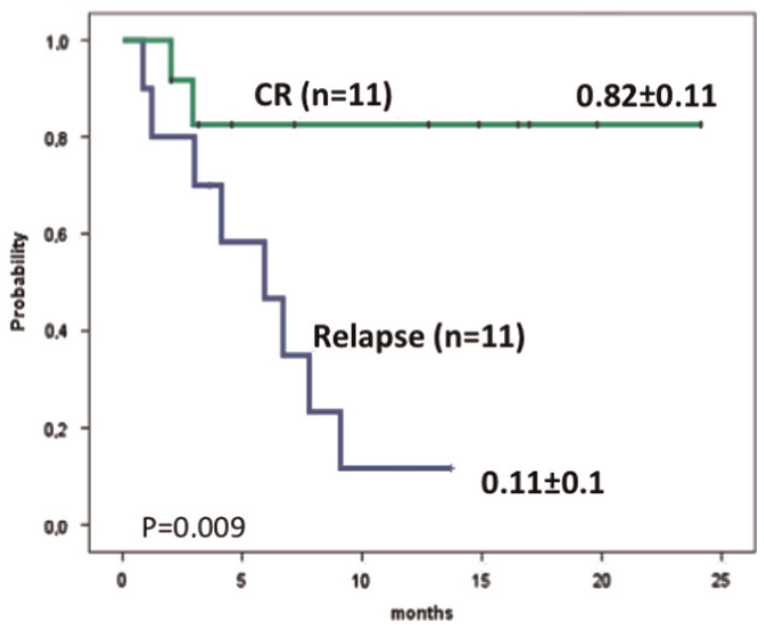

(7 relapse, 3 non-hematologic causes). 12 survive (10 diseasefree, 2 in early relapse) at a median follow-up of 13 months (range 2-24) (Fig.1).

Conclusion: The infusion of $\alpha \beta / C D 19-$ depleted grafts was safe and effective also in adult setting, resulting into rapid engraftment and fast immunological reconstitution, without life-threatening infectious complications. Relapse is still a major concern in patients already in relapse at transplantation. Disclosure of Interest: None declared.

\section{5}

Haploidentical Stem Cell Transplantation In Very Poor Risk Cytogenetics Acute Myeloid Leukemia: results in $\mathbf{4 0}$ consecutive patients.

R. S. Mohammed, $M D^{1,2^{*},}$, E. Sala, $M D^{1}$, M. Cabbrabba, $M D^{1}$, C. Messina, $M D^{1}, M$. T. lupo Stanghellini, $M D^{1}$,

L. Vago, MD, PhD ${ }^{1,3}$, F. Pavesi, $M D^{1}$, M. Morelli, $M D^{1}$,

S. Marktel, $M D^{1}, C^{\prime}$. Corti, $M D^{1}$, J. Peccatori, $M D^{1}$, F. Ciceri, $M D^{7}$, M. Bernardi, $M D^{1}$

${ }^{1}$ Hematology and Bone Marrow Transplantation, IRCCS San Raffael scientific institute, Milan, Italy, ${ }^{2}$ Clinical oncology department, Cairo university, Cairo, Egypt, ${ }^{3}$ Unit of Molecular and Functional Immunogenetics,Division of Regeneration Medicine,Stem Cell and Gene Therapy, San Raffael scientific institute, Milan, Italy

Introduction: Allogenic hematopoietic stem cell transplantation (allo-HSCT) is the most effective post remission-treatment for prevention of relapse in poor risk AML in first complete remission (CR1). For patients (pts) lacking an HLA -matched related or unrelated donor (MRD), haploidentical stem cell transplant (haplo-HSCT) is increasingly offered.Within the poor risk group of AML,complex karyotype (CK), abnormality of the chromosome 7 or 5, monosomal karyotype defined as the presence of two or more monosomies or one monosomy plus one or more somatic abnormalties, are associated with the worst outcome.Allogenic stem cell transplantation (allo-HSCT) can be considered the only potentially curative option for those patients. Till now there is no available data evaluating the role of haplo-SCT in those high risk patients. We reviewed our result to address this issue.

Materials (or patients) and methods: 40 adult patients with very poor risk AML were offered an haplo-HSCT at San Raffaele scientific Institute peroid November 2001 to April 2014. Primary end point of the study included 3-year overall survival (OS), leukemia-free survival (LFS) and cumulative incidence of relapse and non relapse mortality(NRM). Cytogenetic prognostic risk was defined according to the revised Medical Research Counsil (MRC) classification, from diagnostic bone 
marrow samples with standard methods and in accordance with International System of Human cytogenetics guidelines. OS and Disease Free Survival (DFS) were calculated using the Kaplan-Meier methods.

Results: Median age of the patients at time of transplant was 50 years (range 28 to 67).Cytogenetics:chromosome 7 abnormalities 16 pts, monosomal karyotype 3 pts and complex karyotyping $21 \mathrm{pts}$. At the time of haplo-SCT,12 patients (30\%) were in complete remission (9 in CR1) while 28 pts (75\%) had active disease.All patients received a conditioning regimen based on myeloabative doses of treosulan. 24 pts received an ex vivo T-cell depleted haplo-SCT. In 16 pts cyclosporin/ rapamycin/mycophenolate were used as GVHD prophylaxis (Peccatori J, 2014).

Median follow-up after haplo-SCT was 304 days (range 5 to 2897). 30 pts (75\%) were in CR at day +30 after transplant, 1 pt died of sepsis before disease evaluation, 2 pts had persistance disease and 7 pts had early molecular relapse. At the last follow-up 18 out of 40 pts (45\%) were alive,and in clinical remission while 22 pts died from the following causes: progressive disease and relapse (33\%) or infection (12\%).

Non relapse mortality (NRM) was $17 \%$ at 1 year after transplant.Estimated LFS from day +30 after transplant was $34.5 \%$ and $28 \%$ at 3 years.

Conclusion: Haploidentical stem cell transplant (haplo-SCT) is a valid treatment option for the patients with very poor risk AML. References: 1. Bhamidipati PK, DiPersio JF, Stokerl-Goldstein K, Rashidi A. Haploidentical transplantation using G-CSF-mobilized T cell replete. Bone marrow transplant. 2014; 49: $1124-1126$

2. Di Bartolomeo P, Santatarone S, De Angelis G. Haploidentical, unmaipulated, primed bone marrow for pts with high risk hematologic malignancies. Blood 2013; 121: 849-857.

3.Solomon SR, Sizemore CA, Sanacore M, Zhang. Haploidentical transplantation using $\mathrm{T}$ cell replete PBSC and myeloablative conditioning in pts with high risk hematologic malignancies who lack conventional donors. Biol Blood Mawrrow Transplant 2012; 18: 1859-1866.

Disclosure of Interest: None declared.

\section{Oral session: Aplastic anemia \& haemoglobionpathies}

\section{6}

Hematopoietic Stem Cell Transplantation for Bone Marrow Failure and Use of Stem Cell Source: Time for Concerted Actions

A. Yoshimi ${ }^{1,2, *}$, H. Baldomero ${ }^{3}$, J. Passweg ${ }^{4}$, P. Nöllke ${ }^{1}$, M. Aljurf , M. Pasquini, ${ }^{6}$ Y. Atsuta ${ }^{2}$, M. lida ${ }^{7}$, L. F. Bouzas ${ }^{8}$, J. Szer ${ }^{9}$, J. Lipton ${ }^{10}$, C. Dufour ${ }^{11}$, M. Gratwohl ${ }^{12}$, M. Horowitz D. Niederwieser ${ }^{13}, A$. Gratwoh ${ }^{4}, Y$. Kodera ${ }^{7}$ on behalf of the Worldwide Network of Blood and Marrow Transplantation ${ }^{1}$ Department of Pediatrics and Adolescent Medicine, University of Freiburg, Freiburg, Germany, ${ }^{2}$ The Asia Pacific Blood and Marrow Transplant Group Data Center, Nagoya University, Nagoya, Japan, ${ }^{3}$ The European Group for Blood and Marrow Transplantation Transplant Activity Survey Office, University Hospital, Basel, ${ }^{4}$ The European Group for Blood and Marrow Transplantation Transplant Activity Survey Office, University Hospital, Basel, Switzerland, ${ }^{5}$ The Eastern Mediterranean Blood and Marrow Transplant Group, King Faisal Specialist Hospital \& Research Centre, Riyadh, Saudi Arabia, ${ }^{6}$ The Center for International Blood and Marrow Transplant Research, Medical College of Wisconsin, Milwaukee, United States, ${ }^{7}$ The Asia Pacific Blood and Marrow Transplant Group Data Centre, Aichi Medical University, School of Medicine, Negakute, Japan, ${ }^{8}$ The Sociedade Brasileira de Transplante de Medula Ossea, Instituto Nacional de Cancer, Rio de Janeiro, Brazil, ${ }^{9}$ The Australasian BM Transplant Recipient Registry, Royal Melbourne Hospital, Victoria, Australia, ${ }^{10}$ The
Canadian Blood and Marrow Transplant Group, Princess Margaret Hospital, Toronto, Canada, ${ }^{11}$ The European Group for Blood and Marrow Transplantation Severe Aplastic Anemia Working Party, Clinical and Experimental Hematology Unit. G Gaslini Childrens' Hospital, Genova, Italy, ${ }^{12}$ Institute for Operations Research and Computational Finances, University of St. Gallen, St. Gallen, Switzerland, ${ }^{13}$ Hematology-Oncology Department, University Hospital, Leipzig, Germany

Introduction: Bone marrow (BM) is the recommended stem cell source in hematopoietic stem cell transplantation (HSCT) for bone marrow failure (BMF). Recent large studies in aplastic anemia showed that the use of peripheral blood stem cells (PBSC) increased the risk for chronic graft-versus-host disease without reducing graft failures and lead to an inferior survival after HSCT. However, a substantial proportion of HSCT is still performed with PBSC. Ease of collection and a more rapid engraftment might favor PBSC in the short term and more background information to clarify the situation is warranted.

Materials (or patients) and methods: The current global practice of HSCT for BMF was studied for potential macroeconomic factors associated with the selection of stem cell source. Data were used from the worldwide HSCT survey in the years 2009 and 2010 collected by the Worldwide Network for Blood and Marrow Transplantation (WBMT). They were supplemented by data from the European Group for Blood and Marrow Transplantation (EBMT) activity survey between 1990 and 2012.

A total 3282 HSCT for BMF from matched siblings ( $n=1805$ $55 \%)$, mismatched family members $(n=224 ; 7 \%)$, identical twins $(n=25 ; 1 \%)$, or unrelated donors $(n=1228 ; 37 \%)$ during the years 2009 and 2010 were reported from 1482 teams in 82 countries. Of these, 180 patients with cord blood transplantation were excluded, hence 3102 HSCT were analyzed.

Results: BM was used in $57 \%$ of all HSCT. The proportion of BM varied significantly between countries (0-100\%) and regions: use of $\mathrm{BM}$ was highest in the Americas (75\%), followed by Europe (60\%), Eastern Mediterranean /Africa (46\%) and Asia (41\%). The proportion of BM increased from $20.1 \%$ in middle-low/low-income countries to $50.1 \%$ in middle-high and $64.5 \%$ in high income countries $(P<0.01)$ with a clear association with Gross National Income per capita (GNI/cap) $\left(R^{2}: 0.2, P<0.01\right)$. Looking at individual countries, the proportions of BM were similar for HSCT from a family or an unrelated donor $\left(R^{2}: 0.551, P<0 \cdot 01\right)$. According to the EBMT activity survey the use of BM for BMF rapidly decreased in Europe after the introduction of PBSC in the late 1990ies, stabilized between 2000 and 2009 around $60 \%$ and slightly increased since to $70 \%$ in 2012. In contrast, the proportion of BM continuously decreased to a low level of $20 \%$ in acute leukemia.

Conclusion: This study confirms that PBSC is still used for a large number of patients with BMF despite reported inferior outcomes compared to bone marrow. It also demonstrated wide variation in use of stem cell source worldwide. Factors associated with use could be identified. PBSC was preferred in countries with limited resources. BM was more frequently used in countries with higher GNI/cap. The results provide a basis for actions. National and international transplant organizations should foster regional accredited BM harvest centers for patients with BMF. Competent authorities should provide regulatory incentives and resources to establish such infrastructures. Unrelated donor registries should be aware of the necessity of BM donation for BMF and inform early in the donor search.

Disclosure of Interest: None declared. 


\section{7}

Immunosuppressive therapy versus alternative donor hematopoietic stem cell transplantation for children with severe aplastic anemia who lack an HLA-matched familial donor

K. H. Yoo ${ }^{1, *}$, Y. B. Choi ${ }^{1}$, N. H. Lee ${ }^{1}$, S. H. Lee ${ }^{1}$, J. Y. Lee ${ }^{2}$, S. K. Choi ${ }^{3}$ K.W. Sung ${ }^{1}$, H. H. Koo ${ }^{1}$

${ }^{1}$ Pediatrics, ${ }^{2}$ Nurse, ${ }^{3}$ Pharmacy, Samsung Medical Center,

Sungkyunkwan University School of Medicine, Seoul,

Korea, Republic Of

Introduction: The outcome of alternative donor hematopoietic stem cell transplantation (HSCT) for the patients with severe aplastic anemia (SAA) who lack an HLA-matched familial donor has improved recently. This study was aimed to compare the treatment outcome of immunosuppressive therapy (IST) with alternative donor HSCT in children with SAA.

Materials (or patients) and methods: Medical records of SAA patients who received IST and/or alternative donor HSCT from umbilical cord blood (UCB), haploidentical peripheral blood stem cells (PBSC), unrelated bone marrow (UBM), or unrelated PBSC (UPBSC) between June 1996 and December 2012 were reviewed, and data were analyzed retrospectively. The KaplanMeier method was used to calculate the estimated survival rates which were compared with log-rank test.

Results: Of 59 patients with SAA, 42 patients received IST and/ or alternative donor HSCT. Nineteen patients received IST as an initial treatment modality, of whom 13 patients failed to respond at 6 months from the initiation of treatment. Thirtyfour patients received alternative donor HSCT either following IST $(n=11)$ or as frontline therapy $(n=23 ; 11$ UBMT, 11 UPBSCT, 8 UCBT, 4 haploidentical PBSCT). The failure-free survival rate (FFS) of IST group was $31.6 \%$, while that of frontline HSCT group was $91.3 \%(P<0.001)$. Patients who received HSCT following IST showed an inferior event-free survival rate as compared with those who received frontline HSCT $(50.9 \%$ vs $91.3 \% ; P=0.015)$.

Conclusion: The outcome of alternative donor HSCT in our cohort was higher than usually expected, especially in those who received HSCT without prior IST. These results suggest that frontline alternative donor SCT might be a better treatment option than IST for children with SAA who lack an HLA-matched familial donor.

Disclosure of Interest: None declared.
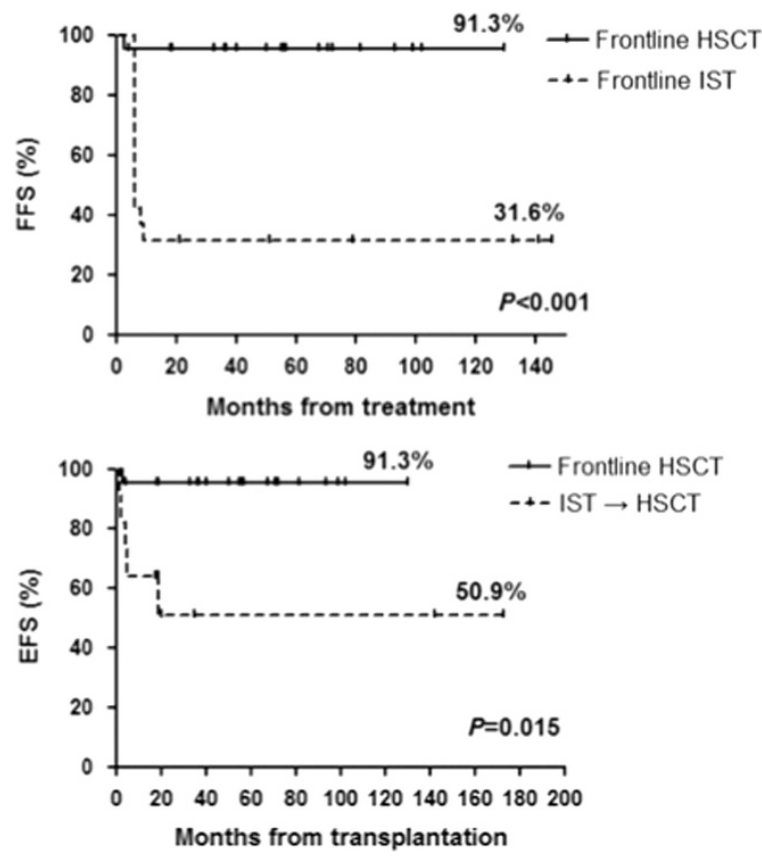

0148

Increased risk of development of malignancies in adult patients treated with antithymocyte globulin as first line treatment for aplastic anaemia during a follow-up period of thirty years.

J. V. D. Hem ${ }^{1, *}$, L. D. Wreede ${ }^{2}$, A. Brand ${ }^{1}, H$. Veelken $^{1}$, F. Falkenburg $^{1}$, C. Halkes ${ }^{1}$

${ }^{1}$ Hematology, ${ }^{2}$ Medical Statistics and Bioinformatics, Leiden University Medical Centre, Leiden, Netherlands

Introduction: Adult aplastic anemia (AA) is considered to be an immune-mediated disease with bone marrow aplasia and pancytopenia. Patients can be treated with either hematopoietic stem cell transplantation (HSCT) or immunosuppressive therapy (IST) with antithymocyte globulin (ATG) and achieve long-term survival. Treatment related long term toxicity and outcomes should guide the decisions about first line therapy. A long-term toxicity of both treatments is the development of malignancies. Follow-up studies until 15 years after IST in AA patients showed cancer development in up to $25 \%$ of patients. Analysis of cohorts with a longer duration of follow up are lacking and the incidence of malignancies in AA patients was never compared with a control population and the contribution of second line HSCT to this risk is not clear. We assessed the long-term cumulative incidence of malignancies in a cohort of adult patients with AA who were treated with ATG as first line treatment at Leiden University Hospital between 1980 and 2008 with HSCT and death as competing risks and compared this incidence to cancer development in the general Dutch population.

Materials (or patients) and methods: 93 Patients treated with first line ATG between 1980 and 2008 were entered in this single-centre retrospective cohort study. Primary endpoint was the cumulative incidence of malignancies with death and second line treatment with HSCT as competing risks. Secondly, the cumulative incidence in our cohort was compared to that of the sex- and age matched general Dutch population derived from the Dutch cancer registration, using standardized incidence ratio (SIR). Furthermore, several patient-dependent (age), disease-dependent (severity of disease) and treatmentdependent (addition of ciclosporin to ATG) variables were assessed as possible prognostic factors for cancer development, using cumulative incidence curves, Gray's test and the Cox proportional hazard model for the cause-specific hazard. SPSS Statistics 20.0 and R were used for all data analyses. Ethical approval for data collection and data analysis was obtained.

Results: Median length of follow-up of surviving patients without HSCT was 17.4 years (range 0.2-32.2 years), with a cumulative malignancy rate of $29 \%$ after 30 years. The incidence of malignancies was increased compared with the general Dutch population (SIR: 3.35 (95\% Cl: 1.98-5.08)). The cumulative incidence of developing MDS/AML was $6 \%$ after 30 years (occurring at 5-17 years after start of treatment). In the multivariate analysis, age at time of IST was significantly associated with a higher malignancy rate (HR: 1.52 per 10 years; $P=0.01$ ). HR for addition of ciclosporin was1.87 $(P=0.25)$ and HR for NSAA vs (V)SAA HR was 1.65 $(P=0.32))$.

Conclusion: The risk of the development of malignancies in AA patients treated with ATG is increased compared to the risk in the age and sex matched Dutch population for both solid and hematologic malignancies. We are not able to discern whether the increased risk is due to the treatment with IST or due to the biology of the disease. Long follow up studies in age matched $A A$ patients receiving HSCT are needed to compare this long term risk between both treatments. These results underscore the recommendation in international Guidelines to follow all patients with AA after initial IST lifelong.

Disclosure of Interest: None declared. 


\section{9}

Graft depletion of TCRalpha/beta and CD19 in matched unrelated and haploidentical transplantation for severe aplastic anemia: high survival with low incidence of graftversus-host disease and transplant-related mortality M. Maschan ${ }^{1, *}$, L. Shelikhova ${ }^{7}$, D. Balashov ' ${ }^{1}$, J. Skvortsova ${ }^{1}$, S. Blagov' ${ }^{1}$, O. Tatarinova' ${ }^{1}$ E. Kurnikova', E. Boyakova', V. Kalinina ${ }^{2}$, E. Gutovskaya ${ }^{1}$, I. Schipitsina ${ }^{1}$, D. Shasheleva ${ }^{1}$ M. Persiantseva ${ }^{1}$, G. Novichkova' ${ }^{7}$, A. Maschan ${ }^{7}$

${ }^{1}$ Hematopoietic stem cell transplantation, ${ }^{2}$ Molecular Biology, DMITRIY ROGACHEV CENTER FOR PEDIATRIC HEMATOLOGY, ONCOLOGY AND IMMUNOLOGY, Moscow, Russian Federation

Introduction: Results of matched unrelated and, to a lesser extent, haploidentical transplantation in severe aplastic anemia have improved over the last decade. The major factors behind this improvement are high-resolution HLA-typing, intensified immune suppression with fludarabine and alemtuzumab and modern supportive care. We implemented a new graft manipulation method, TCR-alpha/beta depletion, to further improve GVHD control and overall results of transplantation in a group of SAA patients, refractory to two courses of ATG/CsA immune suppression.

Materials (or patients) and methods: Twenty six patients with SAA, median age $11,4(2,5-22)$ years, 16 male/ 10 female, received MUD (20) or haploidentical (6) transplantation with TCR alpha/beta and CD19 depletion between 01.09.2012 and 01.09.2014. Patients received a median of 2 ATG/CsA courses. Core preparative regimen included cyclophosphamide $25 \mathrm{mg} /$ $\mathrm{kg} \mathrm{x4}$, fludarabine $30 \mathrm{mg} / \mathrm{kg} \times 5$, ATG (horse) $25 \mathrm{mg} / \mathrm{kg} \times 4$ and total lymphoid irradiation $6 \mathrm{~Gy}$ total dose. One patient received alemtuzumab instead of ATG due to anaphylaxis. In all cases G-CSF- mobilized PBSC were used as graft source. TCR-alpha/beta and CD19 depletion were performed with CliniMACS Plus according to manufacturer's recommendations. Final graft contained 10x106 of CD34+/kg and 17x103 of TCRa/b $+/ \mathrm{kg}$. Post-transplant immune suppression included short methotrexate and tacrolimus till day $+45-60$.

Results: Primary engraftment was registered in all patients, median 15 days for neutrophils and 14 for platelets. Secondary graft failure/rejection developed in 2 patients, both transplanted from MUD. Both patients were salvaged with a second MUD transplantation. Acute GVHD was registered in 4 patients, grade 2 skin in 2 and grade 3 skin + gut in 2 patients. Cumulative incidence of aGVHD grade $2-3$ was $15 \%(95 \% \mathrm{Cl}: 6-$ 38). Chronic GVHD was registered in 1 patient, cumulative incidence 4\%(95\% Cl:0,7-30). Two patients (1 from each cohort) died of CMV pneumonia at 4 and 8 months respectively. In both cases CMV sero status was $\mathrm{D}-/ \mathrm{R}+$. At $2-$ year follow-up cumulative incidence or TRM is $12 \%(95 \% \mathrm{Cl}: 0,3-$ $46)$, KM estimte of overall survival is $88 \%(95 \% \mathrm{Cl}: 72-100)$, $92 \%(95 \% \mathrm{Cl}: 76-100)$ in the MUD group, $67 \%(95 \% \mathrm{Cl}: 13-100)$ in the haplo group.

Conclusion: TCR alpha/beta depletion is a highly effective method of GVHD prophylaxis in SAA patients. Use of this platform makes alternative donor transplantation in SAA a safe therapeutic option. Further improvement will depend on new strategies to control viral infections.

Disclosure of Interest: None declared.
0150

Results of the French National ProspectiveTrial "Drepagreffe" comparing the Cerebral Vasculopathy Outcome following HSCT and Transfusion Program (TP) (AP-HP N : NCT 01340404)

F. Bernaudin ${ }^{1, *}$, S. Verlhac ${ }^{2}$, R. Peffault Delatour ${ }^{3}$, J.-H. Dalle,

E. Petras ${ }^{5}$, V. Brousse 6 , I. Thuret ${ }^{7}$, C. Paillard ${ }^{8}$, B. Neven ${ }^{9}$,

C. Galambrun', L. Lutz ${ }^{10}$, M. Etienne-Julian ${ }^{11}$, C. Pondarré C. Guitton ${ }^{12}$, F. Missud ${ }^{13}$, C. Runel ${ }^{14}$, C. Jubert ${ }^{14}$, G. Elana ${ }^{15}$, M. Elmaleh ${ }^{\prime 6}$, C. Arnaud ', A. Kamdem ${ }^{i}$, G. Socié ${ }^{\prime}$

${ }^{1}$ Pediatrics Referral Center for SCD, ${ }^{2}$ Radiology Referral Center for SCD, Hôpital Intercommunal de Créteil, Créteil, ${ }^{3}$ Hematology, Hôpital St-Louis, ${ }^{4}$ Hematology, Hôpital Debré, Paris, France, ${ }^{5}$ Referral Center for Sickle Cell Disease, CHU Pointe à Pitre, Pointe à Pitre, Guadeloupe, ${ }^{6}$ Referral Center for Sickle Cell Disease, Hôpital Necker, Paris, '7Hemato-Pediatrics, La Timone, Marseille, ${ }^{8}$ Hemato-Pediatrics, CHU-Strasbourg, Strasbourg, ${ }^{9}$ HematoPediatrics, Hôpital Necker, Paris, ${ }^{10}$ Pediatrics, CHU-Strasbourg, Strasbourg, France, ${ }^{11}$ Pediatrics Referral Center for SCD, CHU Pointe à Pitre, Pointe à Pitre, Guadeloupe, ${ }^{12}$ Referral Center for Sickle Cell Disease, CHU-Bicêtre, Bicêtre, ${ }^{13}$ Hemato-Pediatrics, Hôpital Debré, Paris, ${ }^{14}$ Hemato-Pediatrics, CHU-Bordeaux, Bordeaux, France, ${ }^{15}$ Referral Center for Sickle Cell Disease, CHU-Fort de France, Fort de France, Martinique, ${ }^{16}$ Radiology, Hôpital Debré, Paris, France

Introduction: No prospective trial has to date compared the extent of cerebral vasculopathy following TP or HSCT. The premise of the French National Trial "Drepagreffe" is that cerebral velocities will be reduced to a greater extent after HSCT than under TP.

Materials (or patients) and methods: This prospective trial with 2 arms (TP/HSCT) was defined by the random-availability of a genoidentical donor. Inclusion criteria were SCA (SS/Sb0) children younger than 15 years with a history of abnormal cerebral arterial velocities (TAMMX $\geq 200 \mathrm{~cm} / \mathrm{s}$ ), placed on long-term TP, with at least one non-SCA sibling and parents accepting HLA-typing and HSCT if a genoidentical donor was available. Conditioning consisted of Busilvex-CY $200 \mathrm{mg} / \mathrm{kg}$ and $20 \mathrm{mg} / \mathrm{kg}$ rabbit Thymoglobulin with CSA and short MTX or MMF for GVHD prophylaxis. At enrollment and 12 months post-enrollment, blood screening, Doppler, cerebral MRI/MRA were performed along with cognitive performance testing, the latter done in parallel in the control sibling. Primary endpoints were the significantly greater reduction of velocities in the HSCT than in the TP arm and the Doppler normalization (defined by velocities $<170 \mathrm{~cm} / \mathrm{s}$ in all arteries) more often obtained after HSCT than on TP.

Results:: SCA-children $(n=67 ; 35$ F-32M) from 10 French SCAcenters were enrolled between $12 / 2010$ and $6 / 2013$ at the mean (SD) age of 8.1 (3.1) years. History of stroke was present in 7 patients ( 4 in HSCT and 3 in TP). At enrollment all patients were on TP and paired analysis showed that mean (SD) maximum velocities had significantly decreased $(P<0.001)$ under TP:169 (46) cm/s vs 219 (26) $\mathrm{cm} / \mathrm{s}$ at TP initiation. Following HLA-typing, 35 without genoidentical donor were included in the transfusion arm and 32 with genoidentical donor were transplanted in 6 HSCT-centers. During the 12 months follow-up, no stroke was observed but one patient in the TP arm experienced a hyperammonemic reversible coma, without MRI/MRA alteration. In the HSCT arm, all patients successfully engrafted, one grade II and two grade III acute GVHD, and no chronic GVHD were observed. Complications were seizures $(n=2), \mathrm{CMV}(n=9)$ or EBV replications $(n=5)$, hemorrhagic cystitis $(n=4)$, aspergillosis $(n=1)$, prolonged but reversible thrombopenia $(n=2)$, transitory hemolytic anemia $(n=1)$. At 12 months, all patients were alive, $\mathrm{Hb}$ was significantly higher: $12.0(1.0)$ vs $9.0(0.8) \mathrm{g} / \mathrm{dL}$ and ferritin lower: 861 (630) vs $2756(1572)$ in the HSCT arm $(P<0.001)$. In the HSCT arm, mean \% donor chimerism was $86.7 \%(11.5)$ (range:61-100\%). All transplanted patients had the same $\mathrm{Hb}$ electrophoresis than their donor. Mean (SD) maximum velocities were significantly lower post-HSCT than under 
TP:129 (34) vs $173(36) \mathrm{cm} / \mathrm{s}$, respectively; $(P<0.001)$, and were decreased more significantly following HSCT than on TP: mean(SD) $\Delta:-42$ (31) vs +6 (34), respectively. The percentage of patients with normal velocities was significantly higher post-HSCT (29/32) than in the TP arm (16/32) $(P=0.001)$

Conclusion: This prospective national trial comparing TP vs. HSCT in SCA-patients with a history of abnormal velocities shows for the first time that HSCT significantly results in a greater decrease in velocities than TP, and has very little toxicity. These results suggest HSCT is the treatment of choice for SCA-children with a history of abnormal-TCD and genoidentical donor.

Disclosure of Interest: None declared.

\section{1}

New insights into the pharmacokinetics of intravenous busulfan in children with sickle cell anemia undergoing bone marrow transplantation: a novel dose adjustment strategy

J. Gaziev ${ }^{1, *}$, A. Isgrò ${ }^{1}$, A. F. Mozzi ${ }^{2}$, A. Petain ${ }^{3}$, L. Nguyen ${ }^{3}$

C. Ialongo ${ }^{2}$, V. Dinallo ${ }^{2}$, P. Sodani ${ }^{1}$, M. Marziali ${ }^{1}$, M. Andreani ${ }^{1}$, M. Testi ${ }^{1}$, K. Paciaroni ${ }^{1}$, C. Gallucci ${ }^{1}$, G. De Angelis ${ }^{1}$, C. Alfieri ${ }^{7}$,

M. Ribersani ${ }^{1}$, A. Morrone ${ }^{7}$, G. Lucarelli ${ }^{1}$

${ }^{1}$ International Center for Transplantation in Thalassemia and Sickle cell anemia, Mediterranean Institute of Hematology, ${ }^{2}$ Laboratory Medicine, Policlinico Tor Vergata, Rome, Italy, ${ }^{3}$ Istitute de Recherche Pierre Fabre, Oncology Pharmacokinetic Department, Toulouse, France

Introduction: Busulfan $(\mathrm{Bu})$ is the backbone of the conditioning regimen for patients with sickle cell anemia (SCA) undergoing BMT. Patients with SCA might predispose to transplant-related neurological and pulmonary toxicities due to pre-existing disease-related cerebrovascular and lung injury. Bu therapy appears to be an important contributing factor in this context. To date, no studies have evaluated the pharmacokinetic (PK) parameters of intravenous $\mathrm{Bu}$ (IVBu) for subsequent doses in patients with SCA.

Materials (or patients) and methods: We studied IVBu PK parameters and clinical outcomes of 36 children with SCA undergoing BMT from HLA matched siblings. The median age of patients was 10.4 (range, 1.7-17.1) years. Conditioning regimen consisted of Bu/Cy200/ATG $(n=12)$ or Flu/Bu/CY200 $(n=24)$. Six patients $(17 \%)$ had stroke, $11(31 \%)$ exhibited gliosis due to previous brain injury, $13(36 \%)$ had repeated acute chest syndrome, and $8(22 \%)$ patients were on chronic transfusions. IVBu was administered every $6 \mathrm{~h}$ for 4 days with PK-guided dose adjustment to target a conservative area under the concentration versus time curve (AUC) range of 900-1350 $\mu \mathrm{Mol}^{*} \mathrm{~min}$. The role of glutathione S-transferase (GST) polymorphisms has also been investigated.

Results: All patients had sustained engraftment. A repeated measures ANOVA showed that the first-dose Bu clearance $(4.24 \mathrm{ml} / \mathrm{min} / \mathrm{kg})$ was significantly higher than after dose 5 $(3.70 \mathrm{ml} / \mathrm{min} / \mathrm{kg} ; \quad P<0.0005)$, dose $9 \quad(3.58 \mathrm{ml} / \mathrm{min} / \mathrm{kg}$; $P<0.0005)$, and dose $13(3.42 \mathrm{ml} / \mathrm{min} / \mathrm{kg} ; P<0.0005)$. Such differences in clearance have never been described in patients with SCA. After the first-dose, $69 \%$ of patients achieved the target range.None of the patients developed hepatic VOD. No patient developed grade $\geq 3$ toxicity. There was no association between GST polymorphism and the PK of Bu. We adapted a new dose-adjustment strategy targeting exposures to the lower end ( $\left.900 \mu \mathrm{Mol}^{*} \mathrm{~min}\right)$ of the AUC range after the first dose of Bu to avoid unnecessary dose increases on subsequent days due to differences in clearance. This strategy enabled most patients $(90 \%)$ to maintain the AUC within therapeutic range following dose adjustments. A 3-year probability of OS and sickle-cell free survival were $91 \%(95 \% \mathrm{Cl}, 75-97 \%)$. There was no correlation between any Bu PK parameters and survival, toxicity, acute GVHD, chronic GVHD, and transplant-related mortality.

Conclusion: This study found that the PK behavior of IVBu in children with SCA is characterized by significantly higher $\mathrm{Bu}$ clearance after the first-dose compared with subsequent daily doses. This finding allowed us to adapt a new dose adjustment strategy after the first dose of Bu, which effectively prevented subsequent dose readjustments. Conservative AUC range and targeting exposures to the lower end of the range after the first dose was associated with negligible toxicity, and high engraftment and sickle cell-free survival rates.

Disclosure of Interest: None declared.

\section{2}

Haploidentical Hematopoietic Stem Cell Transplantation (Haplo-SCT) with Pre-Transplant Immunosuppression (PTIS) Followed by Reduced Toxicity Regimen and PostTransplant Cyclophosphamide (Post-Cy) in Severe Thalassemia

S. Hongeng ${ }^{1}$, U. Anurathapan 1,* , S. Pakakasama ${ }^{1}$, B. Andersson ${ }^{2}$ ${ }^{1}$ Pediatrics, Ramathibodi hospital, Bangkok, Thailand, ${ }^{2}$ Stem cell transplantationa and cellular therapy, The university of Texas MD Anderson Cancer Center, Houston, TX, United States

Introduction: Currently, the thalassemia free survival rate after hematopoietic stem cell transplant (HSCT) is about $80-90 \%$ with either matched related (MRD) or unrelated (MRD) donor. However, the probability to find a suitable door is only $25-50 \%$. Since most of these patients (pts) could not find the potential donor, we would like to investigate the haploidentical donor HSCT (Hapo-SCT) in thalassemia.

Materials (or patients) and methods: Between Jan 2013 and December 2014, a total of 116 severe thalassemia patients (pts) underwent HSCT in our center. Sixty five pts underwent MRD-HSCT, 33 pts underwent MUD-HSCT and 18 pts underwent haplo-HSCT. For haplo-HSCT, 10 subjects were male and 8 were female. The median age was 16 yrs (range; 2-22). Thirteen of 18 received stem cells from mother and 5 from father. Ten of 18 were high risk class 3 . These high risk class 3 pts received hydroxyurea $20 \mathrm{mg} / \mathrm{kg} / \mathrm{d}$ for at least 3 months prior to HSCT. All pts received 2 courses of pre-transplant immunosuppression (PTIS) consisting of fludarabine (Flu) $40 \mathrm{mg} / \mathrm{m}^{2} / \mathrm{d}$ together with dexamethasone (dex) $25 \mathrm{mg} / \mathrm{m}^{2} / \mathrm{d}$ for 5 days. After 2 courses of PTIS, all pts received a reducedtoxicity conditioning (RTC) regimen consisting of thymoglobulin $1.5 \mathrm{mg} / \mathrm{kg} / \mathrm{d}$ (d-11 to $\mathrm{d}-9$ ), Flu $35 \mathrm{mg} / \mathrm{m}^{2} / \mathrm{d}$ i.v. $(-7$ to -2$)$ each dose immediately followed by busulfan (Bu) $130 \mathrm{mg} /$ $\mathrm{m}^{2}$ once daily i.v. (d-7 to $\left.\mathrm{d}-4\right) \mathrm{GVHD}$ prophylaxis consisted of cyclophosphamide (Cy) $50 \mathrm{mg} / \mathrm{kg} / \mathrm{d}$ (d +3 to $\mathrm{d}+4)$. Tacrolimus or sirolimus was given for 6 months to $1 \mathrm{yr}$ started together with mycophenolate mofetil on $d+5$, the latter was quickly tapered after 2 months. T-cell repleted peripheral blood stem cells (PBSC) were given to all pts, targeting a CD34 + dose of 7-16 x $10^{6}$ cells $/ \mathrm{kg}$.

Results: Sixteen of 18 were engrafted with full donor chimerism (100\%) while 2 pts suffered graft failure. However, these 2 pts received second transplant on day +30 with minimal conditioning regimen and additional PBSC after which they achieved full donor chimerism. The median time to neutrophil engraftment was 18 days (range; $14-22$ ). Six pts had acute GVHD gr I, 3 grade II and 1 gr IV. Only one had extensive chronic GVHD. At this time, 17 of 18 pts survive thalassemia-free and have sustained full donor chimerism (100\%). Event free survival (EFS) and overall survival (OS) rates are $95 \%$. The median follow up time is 12 months (range 4-20). The EFS rates among MRD, MUD and Haplo-HSCT in our center are $88 \%, 82 \%$ and $95 \%$ respectively $(P=0.46)$.

Conclusion: Haploidentical HSCT for high risk thalassemia pts with our novel approach is safe and should be considered as modality to secure thalassemia-free survival with a low risk for graft rejection and treatment-related mortality. In view of our results, we suggest that all thalassemia pts even those with high risk class 3 features should be offered the chance for cure with HSCT.

Disclosure of Interest: None declared. 


\section{Oral session: Late complications \& quality of life}

0153

25-35 year outcomes of allogeneic stem cell

transplantation for haematological malignancies: a single centre experience

A. Khoder ${ }^{1, *}$, R. Szydlo ${ }^{1}$, J. Apperley ${ }^{1}$, J. Todd ${ }^{2}$, N. Salooja ${ }^{1}$

${ }^{1}$ Haematology, ${ }^{2}$ Endocrinology, Hammersmith hospital, London, United Kingdom

Introduction: The number of haematopoietic stem cell transplants being performed worldwide is increasing as is interest in side effects. Despite the increase in published data on late effects in the last decade, data on very long term survivors ( $>25$ years) is lacking. In this study we describe the outcome of all the patients transplanted at our centre more than 25 years ago.

Materials (or patients) and methods: Between June 1979January 1990, 216 patients had received allogeneic SCT for haematological malignancies at the Hammersmith hospital. Most patients (180/216) were transplanted for CML with CY/ $\mathrm{TBI}$ conditioning and the majority were in chronic phase at SCT $(n=140)$. At the time of analysis in December 2014, 151/216 (70\%) patients had died. Of 65 presumed survivors, 14 patients had moved abroad and an additional 13 patients were considered lost to follow up as they had had no contact with our centre within 5 years of the study. Of the remaining 41 patients, detailed follow up information was available for 34 . Results: The majority of deaths (94/151) occurred within 2 years of SCT. A further 27 patients died between 2-10 years after SCT the most frequent cause of death being relapse (17/ 27) and infection (5/27). Between 10-20 years after SCT there were 18 deaths; the most frequent causes were relapse $(n=4)$, second malignancy $(n=4)$ and GVHD $(n=4)$. Between 20-35 years there have been 9 deaths and the most frequent causes were second malignancy $(n=3)$ and respiratory $(n=2)$. The latest recorded relapse was at 14.8 years.

For 34 survivors for whom we had detailed follow up information the median follow up time was 29y 10 months (range 25 y 8 months - 35 years 7 months). The median age at follow up was 61y (range 45-80). 11/34 patients had had a diagnosis of cancer at the following sites: skin (BCC or melanoma) $n=5$, oral or tongue $n=3$, oesophagus $n=1$, breast $n=1$ and a further patient had had testicular and bladder cancer. 16/34 patients had dyslipidaemia and 14/34 were being treated for hypertension. $5 / 34$ were diabetic and $12 / 34$ were hypothyroid. Of 15 male patients, 3 had low testosterone levels requiring treatment. $8 / 34$ had vascular complications including three with ischaemic heart disease one of whom also had PVD, two with venous thrombosis, one with a TIA and two patients with renovascular disease. DXA data was available for 25/34 of these patients and BMD was recorded as low (osteopenia or osteoporosis) in 12/25.

Conclusion: We conclude that late deaths more than $20 \mathrm{y}$ after SCT are more likely to be due to second malignancy than relapse. Appropriate screening identifies a large number of abnormalities in the surviving patients most of which are amenable to treatment.

Disclosure of Interest: None declared.
0154

Metabolic syndrome is common following haematopoietic cell transplantation (HCT) and is associated with increased cardiovascular disease: an EBMT cross-sectional noninterventional study

D. Greenfield ${ }^{1, *}$, J. Snowden ${ }^{1}$, H. Schoemans ${ }^{2}$, K. Hill $^{3}$,

C. Annaloro ${ }^{4}$, M. T. Lupo-Stanghellini ${ }^{5}, Z$. Aki ${ }^{6}, M$. Arat $^{7}$,

B. Metzner ${ }^{8}$ 'P. Turlure T. Han $^{10}$, A. Rovo ${ }^{11}$, G. Socie'

A. Nagler ${ }^{13}$, N. Kröger ${ }^{14}$, P. Dreger ${ }^{15}$, M. Labopin ${ }^{16}$,

S. van_der'Werf ${ }^{17}, G$. Basak ${ }^{18}$, A. Tichelli' ${ }^{19}$, N. Salooja ${ }^{20}$

R. Duarte ${ }^{27}$

${ }^{1}$ Sheffield Teaching Hospitals NHS FT, Sheffield, United Kingdom,

${ }^{2}$ University Hospital Gasthuisberg, Leuven, Belgium, ${ }^{3}$ University Hospital Southampton NHS Foundation Trust, Southampton, United Kingdom, ${ }^{4}$ Fondazione IRCCS $\mathrm{Ca}^{\prime}$ Granda Ospedale Maggiore Policlinico, ${ }^{5}$ Hematology and Bone Marrow Transplantation Unit, San Raffaele Scientific Institute, IRCCS, Milano, Italy, ${ }^{6}$ Gazi Universitesi Tip Fakültesi Hastanesi, Ankara, ${ }^{7}$ Sisli Florence Nightingale Hospital, Istanbul, Turkey, ${ }^{8}$ Klinikum Oldenburg, Oldenburg, Germany, ${ }^{9}$ Service d'hématologie clinique et thérapie cellulaire, Chu Limoges, Limoges, France, ${ }^{10}$ Ashford and St Peter's NHS Foundation Trust, Surrey, United Kingdom, ${ }^{11}$ University of Bern, Bern, Switzerland, ${ }^{12}$ Hospital St. Louis, Paris, France,

${ }^{13}$ Hematology Division, Tel Aviv University, Tel-Hashomer, Israel, ${ }^{14}$ Department of Stem Cell Transplantation, University Hospital Hamburg-Eppendorf, Hamburg, ${ }^{15}$ University of Heidelberg, Heidelberg, Germany, ${ }^{16}$ EBMT Paris Study Office, Paris, France, ${ }^{17}$ EBMT Data Office, Leiden, Netherlands, ${ }^{18}$ The Medical University of Warsaw, Warsaw, Poland, ${ }^{19}$ University of Basel, Basel, Switzerland, ${ }^{20}$ Imperial College, London, United Kingdom, ${ }^{21}$ Institut Català d'Oncologia, Hospital Duran i Reynals, Barcelona, Spain

Introduction: Metabolic syndrome (MS) is defined as a clustering of five factors including (1) hyperglycaemia (2) hypertriglyceridaemia; (3) low HDL cholesterol; (4) hypertension; (5) obesity (high waist circumference or body mass index (BMI)). It is associated with raised risk of cardiovascular disease (CVD) and is increasingly recognised in patients after HCT and revised guidelines for long-term HCT survivors recommend screening for MS. Previous studies have been small and the definition of MS variable, although harmonised criteria are now agreed (Alberti et al Circulation 2009). To determine the prevalence of MS and associated risk factors, we performed a large cross-sectional service evaluation of HCT survivors in centres working in accordance with international screening guidelines (Majhail et al. Bone Marrow Transplant, 2012).

Materials (or patients) and methods: This was an EBMT approved cross-sectional, non-interventional study of consecutive HCT patients aged $18+$ years and a minimum of 2 year post-transplant attending routine follow-up HCT and/or late effects clinics in 9 centres. Centres completed MED C forms incorporating routine recording of the MS parameters (given above) as well as performance status (ECOG); evidence of cardiovascular events (Tichelli et al. Haematologica 2008); family history of premature CVD; and relevant drug history. Statistical analyses were performed using SPSS. Comparison of patients and HCT characteristics between the 2 groups (MS vs no MS) was performed using non-parametric Mann-Witney U test for continuous variables and Chi-square test for dichotomous variables. All tests were two-sided.

Results: Preliminary analyses of 326 patients (188 men and 138 women) are given $(263,80.7 \%$ allogeneic and $63,19.3 \%$ autologous HCT). Median age at diagnosis: 41y (0.5-76); at transplant: 44y (8-76); at follow-up: 52y (21-79); time from HCT: 6.2 y (2-29). Using the harmonised definition of MS (at least $3 / 5$ factors), the prevalence of MS was $47.2 \%$. There was no difference in time since $\mathrm{HCT}$ but there was a significant difference in prevalence by age at diagnosis, HCT, follow-up (all $P<0.001$ with increasing age). As expected, statistical differences $(P<0.001)$ between patients with and without MS were observed for BMI $\left(27.2\right.$ vs $\left.23.3 \mathrm{~kg} / \mathrm{m}^{2}\right)$, HDL-cholesterol levels (1.21 vs $1.5 \mathrm{mmol} / \mathrm{L}$ ); triglyceride levels (2.03 vs 1.24 
$\mathrm{mmol} / \mathrm{L}$ ), and ECOG status $>0$ (31.1 vs $20.2 \%)$. Patients with MS more frequently used anti-hypertensive, anti-diabetic and anti-lipid drugs $(P<0.001)$. Notably, there was a significantly higher frequency of existing cardiovascular events (cerebrovascular accident, coronary heart disease and peripheral vascular disease) in those with MS than those without (18.1 vs. $4.8 \%, \mathrm{P}<0.001)$.

Conclusion: This study in HCT survivors confirms high prevalence of metabolic syndrome (47.2\%) and strong association with cardiovascular events. Routine screening and early intervention may reduce the risk of cardiovascular events in HCT survivors, and should ideally be tested in a randomised controlled trial setting. Meanwhile, screening and management of reversible features of the metabolic syndrome should be robustly integrated within routine HCT long-term follow-up care.

References:

Disclosure of Interest: None declared.

\section{5}

Long-term outcomes of autologous stem cell transplantation with BEAM conditioning in relapsed non-Hodgkin lymphoma: survival and late toxicities F. Spina ${ }^{1, *}$, L. Devizzi ${ }^{1}$, S. Dalto ${ }^{2}$, D. P. Chiara ${ }^{2}$, A. Dodero ${ }^{1}$, L. Farina ${ }^{7}$, V. Montefusco ${ }^{1}$, P. Corradini ${ }^{2}$

${ }^{1}$ Hematology, FONDAZIONE IRCCS ISTITUTO NAZIONALE DEI TUMORI, ${ }^{2}$ Hematology, Università degli Studi di Milano, Milan, Italy

Introduction: Chemotherapy with BCNU, Etoposide, Ara-C, and Melphalan (BEAM), is a common conditioning regimen for autologous stem cell transplantation (ASCT) in relapsed nonHodgkin lymphoma (NHL).

Materials (or patients) and methods: We analyzed a retrospective cohort of 165 consecutive patients with $\mathrm{NHL}$ who received an BEAM ASCT at the National Tumor Institute of Milan in 2000-2012. The aim was to evaluate the survival and late toxicities, defined as any disease condition other than lymphoma occurring after at least 6 months after ASCT. The median patient age at ASCT was 52 years (range, 20-70). All patients relapsed after at least one chemotherapy line (previous treatments range 1-8), 26\% of them received radiotherapy. At ASCT, $76 \%$ of patients were in CR, $15 \%$ in $\mathrm{PR}, 1 \%$ stable, $8 \%$ in progression. Full dose BEAM was given to $87 \%$ of patients while $13 \%$ received dose reductions for comorbidities.

Results: The median follow-up was 5.4 years (range $0.5-12.2$ ). The 5-year OS and PFS were 81 and 69\% (median not reached for both). The non-lymphoma-associated mortality was $5 \%, 8 \%$ and $9 \%$ at 3, 5 and 7 years of follow-up. The OS was impacted in multivariate analysis by disease status before ASCT $(P=0.001, \quad$ HR 2.2, Cl95\% 1.4-3.6), and radiotherapy $(P=0.017$, HR $6.5, \mathrm{Cl} 95 \% 1.4-30.7)$. PFS was impacted by female gender $(P=0.029$, HR 0.4, Cl95\% 0.2-0.9), pre-transplant disease status $(P=0.001, \mathrm{HR} 1.9, \mathrm{Cl} 95 \% 1.3-2.9)$, and radiotherapy (trend, $P=0.064$, HR 2.7, Cl95\% 0.9-7.5). None of the factors analyzed impacted late non-lymphoma-associated mortality, except for a trend given by age $(P=0.075)$. Late toxicities after BEAM ASCT occurred in $61 \%$ of patients, and included infection ( $32 \%$ - most frequently pulmonary), hypogammaglobulinemia (30\%), pulmonary complications (21\% - mostly reduction of pulmonary function tests scores), metabolic syndrome (17\%), cardiovascular complications $(12 \%)$, second tumors $(10 \%)$, hypothyroidism $(8 \%)$, diabetes $(5 \%)$, chronic kidney failure $(4 \%)$, hepatitis B reverse seroconversion (2\%) and ocular complications (1\%). The cumulative incidence of second tumors was 1,6 , and $10 \%$ at 3,5, and 7 years of follow-up, and reached a plateau of $16 \%$ at 10 years of follow-up. 17 patients had a second cancer, of whom 12 had a solid tumor (skin [4], colorectal, prostate, lung [2 each], breast and oropharingeal [1 each]), 5 a hematologic tumor (secondary MDS or AML [4] or NHL [1]). Age ( $P=0.013, \mathrm{HR} 1.5$ per year, CI95\% 1.0-2.1), and male gender $(P=0.043$, HR 0.05 favorable for female sex, Cl95\% 0.0-0.9), increased risk of a second tumor. Of 13 patients who died without lymphoma, 6 died of second tumors, 2 died of cardiovascular complications, 2 of late infections, and 3 for other causes. In multivariate logistic regression, the incidence of second tumors was associated with age $(P=0.04)$, and there was a trend for patients receiving radiotherapy for late cardiovascular complications $(P=0.07)$.

Conclusion: BEAM conditioning is associated with a $61 \%$ crude incidence of late effects, mostly infections, hypogammaglobulinemia, and pulmonary complications. The most important preventive measures for late mortality could be the screening for cancer, especially for older patients, screening for heart disease particularly for patients receiving radiotherapy, and prompt and aggressive treatment of late infections.

Disclosure of Interest: None declared.

0156

Post-transplantation long-term events in a cohort of HIV-positive patients affected by relapsed/refractory lymphoma

E. Zanet ${ }^{1, *}$, C. Durante ${ }^{2}$, A. Lleshi ${ }^{1}$, M. Taborelli ${ }^{3}$, D. Serraino ${ }^{3}$, U. Tirelli, 'M. Michieli' ${ }^{1}$, M. Rupolo ${ }^{1}$, M. Mazzucato ${ }^{2}$, S. Zanussi ${ }^{5}$ P. De Paoli ${ }^{6}$

${ }^{1}$ Unit of Cell Therapy and High Dose Chemotherapy, ${ }^{2}$ Unit of Stem Cells Collection and Processing, ${ }^{3}$ Epidemiology and Biostatistics Unit, ${ }^{4}$ Division of Medical Oncology A, ${ }^{5}$ Microbiology-Immunology and Virology Unit, ${ }^{6}$ Scientific Directorate, National Cancer Institute CRO Aviano, Aviano (PN), Italy

Introduction: The advent of highly active antiretroviral therapy (HAART) in 1996 had led to a suppression of HIV viral load, to an improved immune function resulting in a significant reduction of opportunistic infections and HIV related morbidity and mortality. Consequently, more intensive treatments, including autologous stem cell transplantation (ASCT), have been extended also to the HIV-positive population. However, in the literature data are scarce concerning the long-term events (incidence of lymphoma relapse, of second cancers and AIDSdefining conditions) in HIV-positive patients (pts) affected by relapsed lymphoma who underwent ASCT.

Materials (or patients) and methods: We treated 36 HIVpositive pts affected by relapsed/refractory lymphomas with ASCT consecutively in our cancer center. Ten pts died during or early after ASCT due to progressive disease (4 pts), chemotherapy toxicity (1 pt) and infection (5 pts). We analyzed the post-transplantation long-term data of 26 HIV-positive lymphoma pts, reaching a complete response after ASCT. Eighteen pts were male (69\%) and 8 pts were female. Our cohort of pts included 17 non-Hodgkin's lymphomas (NHL) and 9 Hodgkin's lymphomas (HL), respectively. Twenty-two pts (85\%) received one, and 4 pts received two second-line chemotherapy regimen before ASCT, respectively. The majority of the pts were submitted to a single (BEAM conditioning regimen) and only two pts to a tandem ASCT procedure (high dose Melphalan followed by BEAM conditioning regimen). All pts received HAART concomitantly to cancer treatment.

Results: Two pts experienced a lymphoma relapse, after 4.27 and 3.08 years from ASCT, respectively. Three pts presented with a secondary malignancy ( $1 \mathrm{pt}$ an anal squamous cell cancer, $1 \mathrm{pt}$ a squamous cell carcinoma of the larynx and $1 \mathrm{pt}$ a CIN2, respectively), with a median time of 3.01 years from ASCT. Eight pts had opportunistic infections (Ol): 2 pts developed a Pneumocystis Carinii pneumonia, 1 pt a Cytomegalovirus pneumonia, 1 pt a Mycobacterium Avium Complex pneumonia, 1 pt a Herpes Simplex Chronic Ulcer, 3 pts cutaneous relapsing Herpes Zoster, respectively. The median time of Ol appearance was 0.25 years (IQR: $0.11-2.33$ ). Two pts died: one of lymphoma relapse, the other of car accident. With a median of 6-years follow up (IQR: 4.55-9.87) the OS and PFS of the entire sample of pts were $91 \%$ and $36 \%$ at 10 years, respectively. 
Conclusion: Our results may be summarized as follows: 1) 24 out of 26 pts are still alive and in long-term complete remission after ASCT. These data confirm the long-term efficacy of ASCT in HIV-positive pts affected by relapse/ refractory lymphoma. 2) The appearance of $\mathrm{OI}$ is earlier than that of second malignancies after ASCT. 3) The secondary malignancies developed by our pts are non-AIDS-defining cancers, in agreement with the increased incidence reported by the literature in the HAART-era and at least two cases are linked to a viral pathogenesis (HPV for both anal cancer and cervical cancer precursor lesion). 4) Both OI and second malignancies in our pts series were successfully managed and cured and the only long-term death occurred due to lymphoma relapse.

Disclosure of Interest: None declared.

0157

Standardized care of long-term survivors after allogeneic stem cell transplantation: a cross-sectional 1-year evaluation in $\mathbf{2 4 9}$ adults

M. T. Lupo-Stanghellini ${ }^{1, *}$, A. Orsini ${ }^{1}$, S. Mastaglio ${ }^{1}$, S. Girlanda ${ }^{1}$, T. Perini ${ }^{1}$, M. Brambilla ${ }^{1}$, M. Tassara ${ }^{7}$, F. Lorentino ${ }^{1}$,

S. Piemontese ${ }^{1}$, A. Forcina ${ }^{1}$, L. Vago ${ }^{1}$, F. Pavesi ${ }^{1}$, E. Sala ${ }^{1}$,

A. Malerba ${ }^{7}$, M. Marcatti ${ }^{1}$, E. Guggiari ${ }^{1}$, S. Marktel', A. Assanelli ${ }^{1}$, M. Carrabba ${ }^{1}$, F. Lunghi ${ }^{1}$, M. Bernardi ${ }^{1}$, J. Peccatori ${ }^{1}$, C. Corti ${ }^{1}$, F. Ciceri $^{1}$

${ }^{7}$ Unit of Hematology and Bone Marrow Transplantation, IRCCS San Raffaele Scientific Institute, Milan, Italy

Introduction: Allogeneic hematopoietic cell transplantation (HCT) is an effective therapeutic option for high risk hematological malignancies; $80 \%$ of those who survive the first 2 years are expected to become long-term survivors. The prevalence of chronic health conditions approaches 75\% among HCT survivors and that for severe or life-threatening conditions exceeds $20 \%{ }^{1}$

Materials (or patients) and methods: A standardized followup of HCT survivors is applied at our center, according to Jacie Standards. Here we report the analysis of data collected between Nov 2013 and Nov 2014 in 249 adult patients (pts median age at follow-up 54y - r19-81) who underwent an HCT between 1992 and 2013 at our Institution. Data on 7 items were prospectively collected in an Institutional database. A written consent was given by pts allowing the use of medical records for research in accordance with the Declaration of Helsinki.

Results: Overall $40 \%$ of pts received an haplo, $30 \%$ a MUD and $30 \%$ a match related HCT; 13 pts deceased in the last year $(7$ because of disease relapse, 4 of late major infectious complication, 2 of second cancer).

At a median follow-up of $4 y(r 1-22$; cumulative follow-up 1277y) we observed:

- chronic Graft-versus-Host-Disease (c-GvHD): at a median follow-up of 3y (r1-21) 61 (25\%) pts are presenting c-GvHD features. According to NIH 2004 consensus criteria 15 cases were classified as mild, 25 moderate, 21 severe. Median number of involved organs 3 ( $r 1-4), 32$ pts were experiencing skin lesions, 38 eyes impairment, 23 mouth alterations.

- Late infectious manifestation: 54 (22\%) pts present late infections, 4 pts deceased. Pneumonia was reported in 22 pts, Varicella Zoster virus reactivation in 12 , encephalitis in 5 (3 virus related, 2 Toxoplasma related), hepatitis in 4, EBV reactivation in 2 .

- Second cancers: second malignancies were diagnosed in 32 (13\%) pts, 5 pts are actually under work-up for diagnosis. Nonmelanoma skin cancer was the most frequent diagnosis (17 cases); 3 pts were diagnosed with cervix cancer, 2 with prostate cancer, 2 with lung cancer and 2 with bladder cancer. Single cases of thyroid, parathyroid, colon, gastric, kidney, larynx, endometrial and breast malignancies were also reported. All pts were treated according to standard policy for general population, 30/32 are alive.
- Thyroid dysfunction: 38 (15\%) pts presented overt hypothyroidism.

- Cardiovascular diseases: arterial diseases were reported in 17 pts, atrial fibrillation in 5 and cardiomyopathy in 2 pts - overall $10 \%$. - Metabolic syndrome (MS): 84 (34\%) pts were presenting features of MS ( $3 / 5$ features among hypertension, dyslipidemia -raised triglycerides and lowered high-density lipoprotein cholesterol-, raised fasting glucose and central obesity).

- Secondary hemosiderosis: iron overload was documented (with MRI and blood parameters) and treated in 38 pts (15\%). According to donor source no difference were observed (Chisquare test - $p$ ns) except for higher incidence of moderate/ severe GvHD incidence in match related HCT ( $p$ 0.0097) as compared to alternative HCT.

Conclusion: HCT survivors are at a defined relevant risk of developing long-term complications that have a direct impact on quality of life, morbidity and mortality. ${ }^{1} \mathrm{~A}$ multidisciplinary active screening within routine HCT long-term follow-up care is mandatory to enhance early diagnosis/treatment and overall outcome.

References: 1 Bhatia S. Hematology 2014:495-503.

Disclosure of Interest: None declared.

\section{8}

Provision of Long-term Monitoring and Late Effects

Services Following Allogeneic HSCT in Adults: A British

Society of Blood and Marrow Transplantation (BSBMT)

Survey of UK NHS-Based Programmes

A. Hamblin ${ }^{1, *}$, D. Greenfield ${ }^{2}$, M. Gilleece ${ }^{3}$, N. Salooja ${ }^{4}$

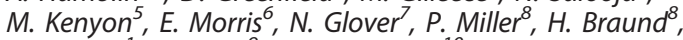

A. Peniket ${ }^{7}$, B. Shaw ${ }^{9}$, J. A. Snowden ${ }^{10}$ on behalf of BSBMT

Clinical Trials Committee

${ }^{1}$ Department of Clinical Haematology, Oxford University Hospitals NHS Trust, Oxford, '2Department of Clinical Oncology, Sheffield Teaching Hospitals NHS Foundation Trust, Sheffield, ${ }^{3}$ Department of Clinical Haematology, Leeds Teaching Hospitals NHS Trust, Leeds, ${ }^{4}$ Department of Haematology, Hammersmith Hospital, ${ }^{5}$ Department of Haematological Medicine, King's College Hospital, ${ }^{6}$ Department of Immunology, Royal Free London NHS Foundation Trust and UCL Medical School, ${ }^{7}$ London Cancer Alliance, ${ }^{8}$ Anthony Nolan, ${ }^{9}$ British Society of Blood and Marrow Transplantation, London, ${ }^{10}$ Department of Haematology, Sheffield Teaching Hospitals NHS Foundation Trust, Sheffield, United Kingdom

Introduction: Long-term survivors of allogeneic HSCT now form an expanding and unique patient population with often complex physical and psychological late effects (LE) and associated unmet needs. Despite international guidelines ${ }^{1}$, optimal delivery models of LE services are unclear from clinical, organisational and economic viewpoints.

Materials (or patients) and methods: In order to scope current models of care for LE service delivery within the UK, we undertook a survey of the 27 NHS adult allogeneic HSCT centres during 2014. Centres were invited to participate in an online survey composed of 30 questions examining service organisation, multi-disciplinary team (MDT) provision, access to other specialist services and patient engagement.

Results: A 100\% response rate was achieved from programme directors or delegated specialist staff. Around half of centres also treated patients $\leq 18$ years and all centres had achieved or were working towards JACIE accreditation. In $>80 \%$ of centres, the LE service was led, coordinated and delivered by consultant medical staff, with the remainder being nurse-led. Most centres (>90\%) provided follow-up in a dedicated allograft or LE clinic for the first year, but thereafter attrition resulted in $\sim 50 \%$ of patients being followed after 5 years, and $\sim 30 \%$ after 10 years. Most centres had easy access to medical specialities necessary for LE management, but specialist interest in long-term HSCT complications was uncommon. Only $18 \%$ of centres had access to a LE MDT, often limited to patients $<25$ years. Despite specific JACIE competency 
standards, a third of centres had held no LE educational event in the previous three years. Most centres (70\%) had an SOP for long-term monitoring and LE management, with the focus predominantly on physical LE with only $28 \%$ including a formal psychological screening assessment. Only 39\% of centres had audited the performance of the SOP. Screening for endocrinopathies, iron overload and cardiovascular complications was near universal, but access to mammography and cervical smear testing was more limited. Revaccination rates were high, but only $23 \%$ of centres routinely tested antibody responses. Despite recommendations, most $(59 \%)$ centres never used standard templates ${ }^{2}$ to communicate LE risk to GPs or referring consultants. Only $41 \%$ of centres had a patient support group accessible to HSCT survivors with equivalent numbers having undertaken patient satisfaction surveys related to LE service provision. The most commonly perceived barriers to implementation of LE services were funding of psychological and other clinical staff and extra investigation costs.

Conclusion: This survey has demonstrated variation and limitations in the provision of long-term follow up of allogeneic HSCT survivors within the UK NHS. Although patients are seen in specialist clinics and have access to other specialities, there are limitations in sustaining long-term screening, MDT working, education, audit and patient engagement, as well as perceived barriers to resourcing staff and investigations. Further work is warranted to optimise effective, sustainable and affordable models of care for delivery of LE services in this expanding specialised patient population.

References: 1. Majhail et al. Biol Blood Marrow Transplant 2012;18:348

2. http://www.ncsi.org.uk/wp-content/uploads/Treatment-

Summary-Template1.doc

Disclosure of Interest: None declared.

\section{9}

Quality of life and emotional wellbeing during the early post-hematopoietic cell transplantation period: the role of the conditioning regimen

A. Barata ${ }^{1,2,}$, R. Martino ${ }^{1,2}$, P. Barba ${ }^{3}$, A. Esquirol, ${ }^{1,2}$, G. Artaza $^{3}$, N. Castillo ${ }^{3}$, I. Gich ${ }^{4}$, G. Ballarin ${ }^{2}$, S. Cortes' ${ }^{2}$, F. Garcia-Pallarols's, I. Garcia-Cadenas ${ }^{2}$, A. Garrido ${ }^{2}$, I. Mensa ${ }^{3}$, J. Romans ${ }^{3}$,

D. Valcárcel ${ }^{3}$, S. Brunet ${ }^{2}$, J. Sierra ${ }^{1,2}$

${ }^{1}$ IIB Sant Pau and Jose Carreras Leukemia Research Institute, ${ }^{2}$ Hematology Department, Hospital Sant Pau, ${ }^{3}$ Hematology Department, Hospital Vall d'Hebrón, ${ }^{4}$ Epidemiology Department, Hospital de Sant Pau, ${ }^{5}$ Hematology Department, Hospital del Mar, Barcelona, Spain

Introduction: The introduction of less toxic conditioning regimens for hematopoietic cell transplantation (HCT) has led to an increase in eligible patients, although their benefit on patient's perceived wellbeing remains unclear. We aimed to prospectively study patients' quality of life (QoL) and emotional wellbeing (EW) in consecutive HCT recipients, with the primary aim of analyzing the impact of type of HCT (Auto/ Allo) and conditioning regimen intensity (AlloRIC/MAC).

Materials (or patients) and methods: This prospective multicenter study assessed QoL (FACT), depression and anxiety (HADS), and sleep quality (PSQI) at pre-HCT, at hospital discharge (HD) and at 3 months post-HCT.

Results: Out of 223 transplanted patients, 191 (86\%) consented to participate. Those who refused $(n=32,14 \%)$ more frequently had active disease at HCT (31\% vs. $21 \%$ for $\mathrm{PR} / \mathrm{CR}, P=0.032)$ and/or had a prior HCT $(28 \%$ vs. $12 \%$, $P=0.027$ )

Among included patients ( $57 \%$ men; median age 53 [range 1971]), 83 received an Auto-HCT $(n=79$ at $\mathrm{HD} ; n=52$ at $+3 \mathrm{~m})$ 55 received an allo RIC-HCT $(n=41$ at HD; $n=33$ at $+3 \mathrm{~m})$; and 53 an allo MAC-HCT ( $n=40$ at HD; $n=28$ at $+3 \mathrm{~m})$. Of note, baseline QoL and EW did not differ between groups.
QoL parameters significantly decreased during the hospitalization and improved afterwards $(P=0.002)$. There was a strong interaction between QoL and HCT groups during the early post-HCT phase $(P=0.003)$. Patients receiving a MAC-HCT reported more QoL impairments than Auto-HCT $(P=0.015)$ whereas there were no differences when comparing Auto-HCT and RIC-HCT $(P=0.135)$ or between MAC- and RIC-HCT $(P=0.586)$

At baseline, clinically significant depressive symptoms were reported by $5 \%$ of the patients, with a slight increase $(7 \%)$ at $\mathrm{HD}$ and at $+3 \mathrm{~m} \quad(P=0.058)$. Again, there was a strong interaction between depressive symptoms and HCT groups in the early post-HCT phase $(P=0.002)$; depression decreased in Auto-HCT after HD and, on the contrary, it increased in the same time-point in MAC-HCT $(P=0.041)$. Borderline differences were seen between Auto- and RIC-HCT $(P=0.062)$ but not between RIC- and MAC-HCT $(P=0.827)$.

Clinically significant anxiety was observed at baseline in $14 \%$ of the patients and significantly decreased at the time of HD $(6 \%)$ and afterwards (5\%). Sleep impairments were referred by $38 \%$ of the patients at baseline, $56 \%$ at HD and $36 \%$ at $+3 \mathrm{~m}$. Both disorders changed significantly over time $(P<.001)$, but no differences were found between HCT groups.

Remarkably, women were more vulnerable to depression, anxiety and worse QoL at any time-point $(P<.05)$ and older patients showed worse baseline EW and QoL $(P<.05)$. Multivariate analyses showed that baseline deficits in EW and QoL were the only predictive variables for later deficits in both EW and QoL $(P<0.05)$.

Conclusion: MAC-HCT recipients reported the greatest impairment in the parameters studied; other variables such as gender, age and baseline EW/QoL should be considered for specific psychological/clinical follow-up and eventual intervention.

Disclosure of Interest: None declared.

\section{Oral session: Cell and gene therapy}

0160

Long-term immunological profile of patients treated with haploidentical HSCT and TK-cells to unravel the requirements of memory $\mathrm{T}$ cell persistence

G. Oliveira ${ }^{1,2,3, *}$, M. T. Lupo Stanghellini ${ }^{4}$, N. Cieri ${ }^{1}$,

M. D'Agostino", E. Ruggiero ${ }^{5}$, R. Greco ${ }^{4}$, S. Mastaglio ${ }^{1,4}$, M. Noviello ${ }^{7}$, L. Vago ${ }^{3,6}$, L. Biasco ${ }^{7}$, A. Bondanza ${ }^{8}$, A. Aiuti' ${ }^{7}$, A. Lambiase ${ }^{9}$, M. Schmidt ${ }^{5}$, C. Traversari ${ }^{9}$, C. Von Kalle ${ }^{5}$, F. Ciceri ${ }^{4}$, C. Bordignon ${ }^{2,9}$, C. Bonini ${ }^{1}$

${ }^{1}$ Experimental Hematology Unit, IRCCS San Raffaele Scientific Institute, ${ }^{2}$ Vita-Salute San Raffaele University, ${ }^{3}$ Unit of molecular and functional immunogenetics, ${ }^{4}$ Unit of Hematology and Bone Marrow Transplantation, IRCCS San Raffaele Scientific Institute, Milan, Italy, ${ }^{5}$ Department of Translational Oncology, National Center for Tumor Diseases and German Cancer Research Center, Heidelberg, Germany, ${ }^{6}$ Hematology and Bone Marrow Transplantation Unit, ${ }^{7}$ Telethon Institute for Gene Therapy (HSRTIGET), 8 Innovative immunotherapy unit, IRCCS San Raffaele Scientific Institute, ${ }^{9}$ MolMed S.p.A., Milan, Italy

Introduction: The immunological memory relies on the capability of $\mathrm{T}$ cells to persist for years after primary response in order eliminate pathogens upon antigen re-challenge. Memory T cells, generated following immunization, are key players for the maintenance of a long-lasting immune protection. While the existence of immunological memory has been recognized for over 2000 years, little is known about how it is maintained over the years, especially in humans. In the present work we exploited Thymidine Kinase (TK) suicide gene marking to shed light into memory $\mathrm{T}$ cell dynamics 
in vivo and to unravel the requirements for their long-term persistence directly in humans.

Materials (or patients) and methods: We studied the immune system of 10 patients who underwent haploidentical HSCT and infusion of donor lymphocytes transduced to express TK suicide gene (median dose: $1.9 \times 10^{7}$ cells $/ \mathrm{kg}$ ) for high-risk hematologic malignancies. In case GvHD, proliferating TK-cells were promptly eliminated upon ganciclor (GCV) administration with complete resolution of the adverse reaction without immunosuppressive treatments.

Results: At a median follow-up of 7 years after HSCT (range 212.3), a complete recovery of NK cells, B lymphocytes and $\alpha \beta$ or $\gamma \delta \mathrm{T}$ cells was observed. The CD8 + and CD4 $+\mathrm{T}$ cell compartment of TK patients were characterized by level of naïve and memory cell comparable to age and sex matched healthy controls.

TK-cells were detected in all patients, at low levels (median $=4$ cells/uL), even in patients treated with GCV. Ex vivo selection of pure TK-cells confirmed the presence of functional transduced cells, thus directly demonstrating the ability of memory $T$ cells to persist for years. Importantly, GCV sensitivity was preserved in long-term persisting TK-cells, independently from their differentiation phenotype. Longitudinal follow up revealed that TK-cells circulated in patients at stable levels and displayed a conserved phenotype comprising effector memory $\left(T_{E M}\right)$, central memory $\left(T_{C M}\right)$ and stem memory $\left(T_{S C M}\right) T$ cells. The low level of Ki-67 positivity suggested the maintenance of a pool of gene-modified memory cells through homeostatic proliferation. Polyclonality was demonstrated by sequencing among TK-cells of thousands of diverse TCRs with a broad usage of $\mathrm{V}$ and $\mathrm{J}$ alpha and beta genes. The number of TK-cells persisting at the longest follow-up did not correlate with the amount of infused cells, but instead with the peak of TK-cells measured within the first months after infusion, suggesting that antigen recognition is dominant in driving in vivo expansion and persistence of memory $\mathrm{T}$ cells. Accordingly, we documented the persistence of CMV and Fluspecific TK-cells only after post-transplant CMV reactivation or after Flu infection. We observed that the number of infused $\mathrm{T}_{\mathrm{SCM}}$ cells positively correlated with early TK-cell expansion and with their long-term persistence, suggesting that $\mathrm{T}_{\mathrm{SCM}}$ might play a privileged role in the generation of a long-lasting immunological memory.

Conclusion: After infusion, gene-modified memory $\mathrm{T}$ cells persist for up to 12 years within a physiological immune system. Antigen exposure and a $\mathrm{T}_{\mathrm{SCM}}$ phenotype were associated with long-term persistence of infused TK-cells. Further studies on TK-cell TCR repertoire and vector integrations are currently being performed to elucidate the in vivo dynamics of infused memory T cells.

Disclosure of Interest: G. Oliveira: None declared, M. T. Lupo Stanghellini: None declared, N. Cieri: None declared, M. D'Agostino: None declared, E. Ruggiero: None declared, R. Greco: None declared, S. Mastaglio: None declared, M. Noviello: None declared, L. Vago: None declared, L. Biasco: None declared, A. Bondanza: None declared, A. Aiuti: None declared, A. Lambiase Employee of: MolMed S.p.A, M. Schmidt: None declared, C. Traversari Employee of: MolMed S.p.A, C. Von Kalle: None declared, F. Ciceri: None declared, C. Bordignon Employee of: MolMed S.p.A, C. Bonini Conflict with: Scientific consultant of MolMed S.p.A.
0161

ErbB2-chimeric antigen receptor (CAR) modified cytokineinduced killer (CIK) cell intervention for refractory solid tumors after allogeneic stem cell transplantation

M. Merker ${ }^{1,}$, E. Rettinger ${ }^{1}$, S. Oelsner ${ }^{1,2}$, V. Pfirrmann ${ }^{1}$, E. Ullrich ${ }^{1}$, W. Wels ${ }^{2}$, T. Klingebiel ${ }^{1}$, P. Bader ${ }^{1}$

${ }^{1}$ University Hospital Frankfurt, Goethe University, Department for Children and Adolescents, Division for Stem Cell Transplantation and Immunology, ${ }^{2}$ Georg-Speyer Haus, Institute for Tumor Biology and Experimental Therapy, Frankfurt/Main, Germany

Introduction: Allogeneic stem cell transplantation (SCT) offered as ultimate therapeutic option to patients with solid malignancies refractory to conventional therapy showed limited beneficial effects in affected patients. Chimeric antigen receptor (CAR) modified cytokine-induced killer (CIK) cell infusions with tumor antigen specificity may enhance graft versus tumor effect in patients after allogeneic SCT. Soft tissue sarcoma cells, unlike hematopoietic cells, express ErbB2. ErbB2 might therefore represent an attractive tumor antigen for targeted CIK cell therapy.

Materials (or patients) and methods: CIK cells were generated from peripheral blood mononuclear cells (PBMC) in the presence of interferon- $\gamma$, anti-CD3 antibody, interleukin (IL)-2 and IL-15. ErbB2-CAR modified and control-vector CIK cells were generated from conventional CIK cells by lentiviral gene transduction on day 4 of culture. Phenotypic analysis and cytotoxic activity were assessed on days $0,7,10$ and between days 12-21 of culture, respectively.

Results: Culture conditions supported a 8.5-fold expansion of CIK cells within ten days. Furthermore, with this protocol CIK cells were generated that contained $8.5-51.3 \%$ ErbB2-CAR vector (eGFP marker gene, $n=17$ ). Already at day 10 of culture, up to $90 \%$ of transduced cells showed surface expression of the ErbB2-CAR $(n=4)$. There were no significant phenotypic differences $\left(\mathrm{CD} 3^{+} / \mathrm{CD} 6^{+} / \mathrm{CD} 4^{+} / \mathrm{CD} 8^{+} / \mathrm{TCR}-\alpha / \beta\right.$ and TCR- $\gamma / \delta$ ) between unmodified, empty control vector and ErbB2-CAR transduced CIK cells. ErbB2-CAR CIK cells efficiently lysed ErbB2-overexpressing breast carcinoma cells (MDA-MB 453) in a 3 hour short-term cytotoxicity (europium release) assay in vitro. Compared with unmodified and empty-vector transduced CIK cells, specific lysis was $73.2 \%, 7.9 \%$ and $8.8 \%$ respectively $(P<0.0005,20: 1$ effector to target ratio (E:T), $n=4)$. There was no difference in cytotoxicity against ErbB2negative target cells (e.g. AML cell line THP1, $n=3$ ) in vitro. The surface expression of ErbB2 on rhabdomyosarcoma (RMS) cell lines (RH30, RH18, RH41, TE671, A204) differed between target cells, but was detectable in all cases. Accordingly, specific lysis of tumor cells was target cell-dependent and correlated with the percentage and degree of ErbB2 expression on tumors. MAbs-blocking of NKG2D receptor, the most important molecule in non-major histocompatibility complex (MHC)restricted cytotoxicity of unmodified CIK cells, demonstrated ErbB2-CAR-modification additionally enhanced cytotoxicity of CIK cells in vitro. As one representative example, specific lysis of established RH30 alveolar RMS cells was increased by 2.5 times during short-term cytotoxicity assay using ErbB2-CAR compared with unmodified CIK cells (20:1 E:T; $46.0 \%$ vs. $18.1 \%$ specific lysis, $n=4 ; P<0.019)$. Long-term cytotoxicity analysis (16h, brightfield imaging cytometry) demonstrated comparable results even at low effector to target ratios of 1:1 (36.3\% vs. $11.8 \%$ specific lysis, $n=3$ ). Comparable results for shortand long time cytotoxicity could be obtained for all other tested rhabdomyosaroma cell lines in vitro.

Conclusion: ErbB2-CAR engineered CIK cells are highly specific and efficient against ErbB2-antigen expressing tumor cell lines in vitro. Our experiments may help to develop an approach for improved treatment of patients with high-risk solid malignancies refractory to conventional therapy.

Disclosure of Interest: None declared. 
0162

Human T Regulatory Cells Expressing a Chimeric Antigen Receptor Specific for HLA-A2 Prevent Graft-Versus-Host Disease

R. Broady ${ }^{1, *}$, K. McDonald ${ }^{2}$, R. Hoeppli ${ }^{2}$, Q. Huang ${ }^{1}$, P. Orban ${ }^{2}$, M. Levings ${ }^{2}$

${ }^{1}$ Medicine, ${ }^{2}$ Surgery, UNIVERSITY OF BRITISH COLUMBIA, VANCOUVER, Canada

Introduction: Allogeneic hematopoietic stem cell transplantation (HSCT) is a curative option for many patients with hematological malignancies. Graft versus host disease (GVHD) mediated by donor alloreactive $\mathrm{T}$ cells remains a major obstacle and limits its wider application. It is now well established that $T$ regulatory cells (Tregs) are critical for the maintenance of self-tolerance, and a deficiency or dysfunction in these cells is thought to be a key factor in the development and progression of GVHD. Numerous murine studies have shown the benefit of adoptive transfer of Tregs in the setting of HSCT. Notably, the success of this approach is improved if the transferred Tregs are specific for antigens expressed by the host. A robust method to generate homogeneous populations of alloantigen specific Tregs in humans would be a major step towards realizing the goal of using these cells to suppress GVHD.

Traditional approaches to generate antigen specific $T$ cells include repetitive cycles of re-stimulation with antigen in vitro, sorting of tetramer positive cells, or over-expression of a T cell receptor for a specific antigen. These methods produce limited cell numbers and require modification for each individual patient depending on their own tissue type and that of the transplanted patient. We hypothesized that a more efficient approach to generating antigen specific Tregs would be to genetically engineer them to express alloantigen-specific chimeric antigen receptors (CARs).

Materials (or patients) and methods: We generated an HLAA2 specific CAR by cloning the immunoglobulin heavy and light chain variable regions from an anti-HLA-A2 antibodysecreting hybridoma. These regions were fused into a single chain antibody, which was then linked to intracellular signalling domains from human CD28 and CD3zeta. Surface expression and antigen-specificity of the A2-CAR was confirmed by flow cytometry to detect expression of a myc epitope tag on the extracellular portion of the CAR and binding to an HLA-A2 tetramer, respectively. CD4 ${ }^{+}$ $\mathrm{CD}_{25}{ }^{\mathrm{hi}} \mathrm{CD} 45 \mathrm{RA}^{+}$naïve Tregs (nTregs) and $\mathrm{CD}^{+}$ $\mathrm{CD} 25^{\mathrm{lo}} \mathrm{CD} 45 \mathrm{RA}{ }^{+}$naïve Tconv (nTconv) cells were sorted from the blood of HLA-A2 donors, stimulated with artificia APCs and transduced with lentivirus encoding the A2-CAR or a control CAR specific for HER2.

Results: Transduced Tregs maintained their expected phenotype, including high levels of FOXP3, CTLA-4, CD25 and Helios, and low levels of CD127 and IL-2 compared to Tconv cells. To test antigen specificity, the transduced $T$ cell lines were stimulated with K562 cells expressing HLA-A2 or HER2: only K562 cells expressing HLA-A2 stimulated proliferation of A2CAR expressing Tregs and Tconv cells. CAR-stimulated Tregs also suppressed the in vitro proliferation of CAR-stimulated Tconv cells. To demonstrate the in-vivo suppressive capacity of A2-CAR Tregs, NSG mice were reconstituted with HLA-A2 ${ }^{+}$ PBMCs in the absence or presence of different ratios of control-transduced or A2-CAR transduced Tregs. Preliminary results suggest that A2-CAR Tregs have a superior ability to delay the onset of GVHD compared to polyclonal Tregs.

Conclusion: Human Tregs can be efficiently transduced to express functional, alloantigen-specific CARs that confer antigen-specific specific suppression of effector $T$ cells both in-vitro and in-vivo. These data supports the feasibility of this strategy to re-direct Tregs for transplant-related therapies.

Disclosure of Interest: None declared.
0163

CAR spacers including NGFR domains allow efficient T-cell tracking and mediate superior antitumor effects

M. Casucci ${ }^{1, *}$, L. Falcone ${ }^{1}$, B. Camisa ${ }^{2}$, F. Ciceri ${ }^{3}$, C. Bonini', A. Bondanza ${ }^{7}$

${ }^{1}$ Innovative Immunotherapies Unit, ${ }^{2}$ Experimental Hematology Unit, ${ }^{3}$ Clinical Hematology and Bone Marrow Transplantation Unit, San Raffaele Hospital Scientific Institute, Milano, Italy

Introduction: Chimeric antigen receptors (CARs) frequently include an IgG1-CH2CH3 spacer conferring optimal flexibility for antigen engagement and allowing the selection and tracking of CAR-expressing $T$ cells. A serious drawback of $\mathrm{CH} 2 \mathrm{CH} 3$-spaced CARs is however their interaction with $\mathrm{Fc} \gamma$ receptors ( $\mathrm{Fc} \gamma \mathrm{Rs})$. Indeed, this antigen-independent binding may lead to the unintended elimination of cells expressing these receptors (mainly phagocytes), foster the development of non-specific immune reactions and drastically decrease the efficacy of the strategy due to the premature clearance of transduced T cells in vivo.

Materials (or patients) and methods: We designed and constructed novel CAR backbones by substituting the IgG1$\mathrm{CH} 2 \mathrm{CH} 3$ spacer with regions from the extracellular portion of the low-affinity nerve-growth-factor receptor (LNGFR), differing for the length and potential binding to NGF. In particular, we used our recently developed CD44v6-specific CAR as a model for comparing the antitumor activity of the different LNGFR-based designs both in vitro and in vivo.

Results: After transduction, all constructs could be identified on the T-cell surface using anti-LNGFR antibodies, indicating that they were correctly processed, mounted on the cell membrane and still recognized by anti-NGFR antibodies. As a consequence, all the LNGFR-based spacers allowed selecting CAR-T cells with immune-magnetic beads coupled to antiNGFR antibodies, without interfering with their expansion and functional differentiation after activation with CD3/CD28 beads plus IL-7 and IL-15. Most importantly, LNGFR-spaced CAR-T cells maintained potent cytotoxic, proliferative and cytokine-release activity in response to CD44v6-expressing leukemia and myeloma cells, while lacking antigen-independent recognition through the Fc $\gamma$ Rs. Noticeably, even at supraphysiological NGF concentrations, the LNGFR-spaced CD44v6CAR.28z CAR T cells were not induced to proliferate, indicating the absence of signaling via soluble NGF. Strikingly, LNGFRspaced CAR-T cells better expanded and persisted in vivo compared to $\mathrm{CH} 2 \mathrm{CH} 3$-spaced CAR-T cells and mediated superior antitumor effects in a well-established tumor disease model. Interestingly, we demonstrated that the premature disappearance of $\mathrm{CH}_{2} \mathrm{CH} 3$-spaced CAR-T cells was due to engulfment by mouse phagocytes, a phenomenon not occurring with LNGFR-spaced T cells.

Conclusion: In conclusion, we have demonstrated that the incorporation of the LNGFR marker gene directly in the CAR sequence allows for a single molecule to work as a therapeutic and as a selection/tracking gene and shows an increased efficacy/safety profile compared to the IgG1- $\mathrm{CH} 2 \mathrm{CH} 3$ spacer. Disclosure of Interest: None declared. 
0164

Adoptive T-cell immunotherapy with hexon-specific THELPER-1 cells as a treatment for refractory adenovirus infection after allo-SCT - safety and efficacy results from a GCP study

J. Feucht ${ }^{1, *}$, K. Opherk ${ }^{1}$, P. Lang ${ }^{1}$, S. Kayser ${ }^{1}$, L. Joachim ${ }^{1}$, W. Bethge ${ }^{2}$, S. Matthes-Martin ${ }^{3}$, P. Bader ${ }^{4}$, M. Albert ${ }^{5}$ B. Maecker-Kolhoff ${ }^{6}$ J. Greil', H. Einsele, P.-G. Schlegel ${ }^{8}$,

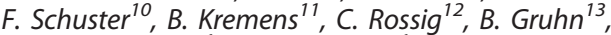
R. Handgretinger ${ }^{1}, T$. Feuchtinger ${ }^{?}$

${ }^{1}$ University Children's Hospital Tübingen, ${ }^{2}$ University Hospital, Tübingen, Germany, ${ }^{3}$ St. Anna Children's Hospital, Wien, Austria, ${ }^{4}$ University Children's Hospital, Frankfurt, ${ }^{5}$ Dr. von Haunersches Kinderspital, München, 'Medizinische Hochschule, Hannover, ${ }^{7}$ University Hospital, Heidelberg, ${ }^{8}$ University Hospital Würzburg, ${ }^{9}$ University Children's Hospital, Würzburg, ${ }^{10}$ Clinic of Pediatric Oncology, Heinrich-Heine-University Düsseldorf ${ }^{11}$ University Children's Hospital, Essen, ${ }^{12}$ University Children's Hospital, Münster, ${ }^{13}$ Department of Pediatrics, Jena University Hospital, Germany

Introduction: Viral infections represent an important cause of morbidity and mortality in immunosuppressed patients post hematopoietic stem cell transplantation (HSCT). As viral infections often remain refractory to pharmacologic treatment, alternative treatment strategies such as immunotherapy are required. Adenovirus (AdV) is the predominant diseasecausing pathogen in pediatric HSCT.

Materials (or patients) and methods: In a clinical trial we analyzed safety and efficacy of ex vivo adoptive T-cell transfer (ACT) with hexon-specific T cells, predominantly of $\mathrm{T}_{\text {HELPER }}{ }^{-1}$ phenotype. Thirty patients suffering from chemo-refractory AdV disease or viremia after HSCT were treated with AdVspecific $\mathrm{T}$ cells generated by IFN- $\gamma$ capture technique.

\section{[0164]}

a

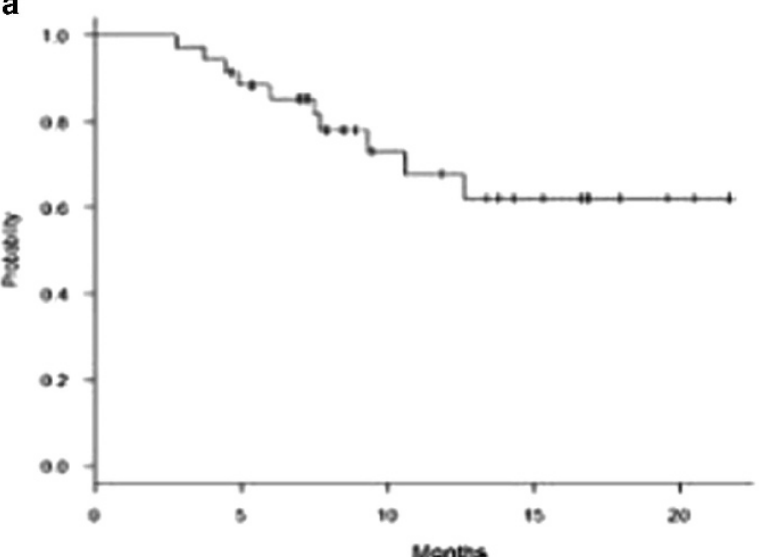

C

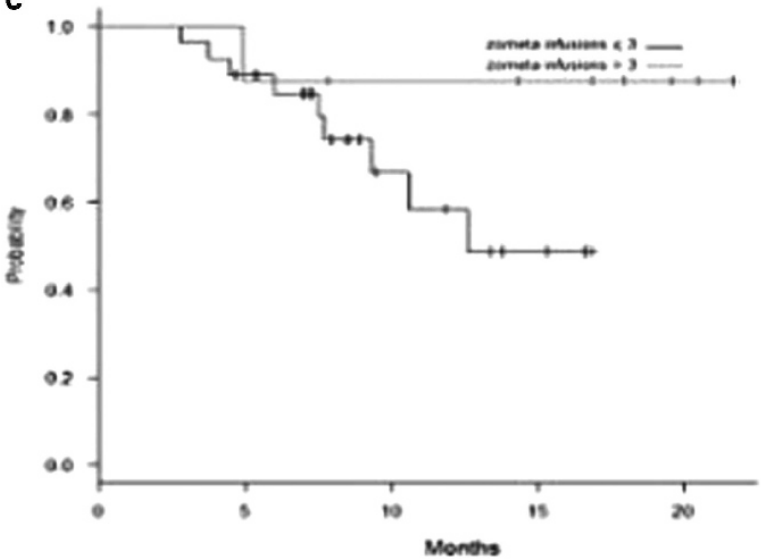

Results: AdV-specific T cells were successfully isolated in 100\% of cases using the adenoviral hexon antigen and were directly infused into the patients without further ex vivo expansion steps. AdV-specific T-cell grafts were composed of a mixture of naïve, central memory, effector memory and effector T-cell populations with a predominance of late effector stages, indicating proliferation and effector potential of the transferred T cells.

In all thirty patients, ACT was feasible without acute toxicities or significant onset of graft-versus-host disease. ACT led to antiviral immunity in vivo up to 6 months with viral control, resulting in complete clearance of viremia in $86 \%$ of patients with antigen-specific T-cell responses. Efficacy of adoptive T-cell transfer was independent of the initial T-cell dose. After a follow up of 6 months post ACT, overall survival was markedly increased in responders (mean: 122 days, 15 survivors) as compared to non-responders who all died shortly after ACT (mean: 24 days, no survivors). AdV-related mortality was $100 \%$ in non-responders compared to $9.5 \%$ in responders ( $\geq 1$ log reduction of DNA copies/ml post ACT), indicating a strong correlation between virus-specific immunity and virus control.

Conclusion: In conclusion, ex vivo ACT of AdV-specific $\mathrm{T}_{\text {HELPER }}$ 1 cells was well tolerated and led to successful and sustained restoration of T-cell immunity, correlated with virological response and protection from virus-related mortality. This cellular immunotherapy is a short-term available and broadly applicable treatment.

Disclosure of Interest: None declared.

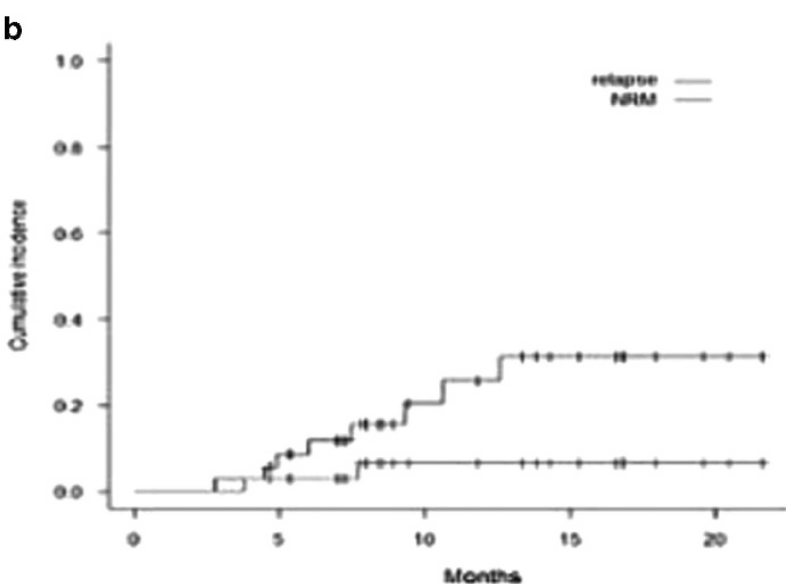

d

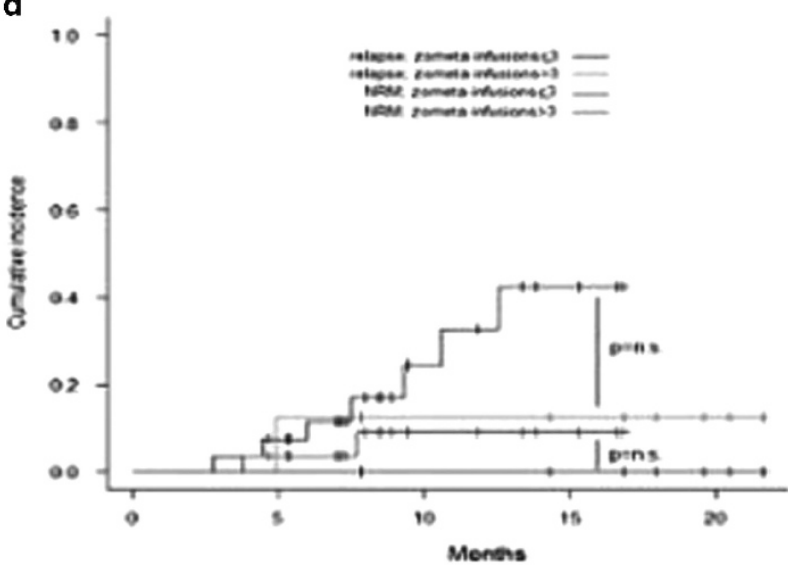


0165

Use of zoledronic acid after TcR $\alpha \beta / C D 19-d e p l e t e d$

haploidentical transplantation to enhance $\gamma \delta$ T cells anti-leukemia effect

P. Merli, ${ }^{1,}$, B. Lucarelli ${ }^{1}$, G. M. Milano ${ }^{1}$, D. Pende ${ }^{2}$, I. Airoldi ${ }^{3}$, G. Li Pira ${ }^{1}$, L. P. Brescia ${ }^{1}$, D. Pagliara ${ }^{1}$, V. Bertaina ${ }^{1}$, S. Ceccarelli ${ }^{1}$ L. Moretta ${ }^{3}$, A. Bertaina ${ }^{7}$, F. Locatelli ${ }^{1}$

${ }^{1}$ BAMBINO GESU HOSPITAL, Rome, ${ }^{2}$ Istituto di Ricovero e Cura a Carattere Scientifico, Azienda Ospedaliera Universitaria San Martino - Istituto Nazionale per la Ricerca sul Cancro, ${ }^{3}$ Istituto Giannina Gaslini, Genova, Italy

Introduction: HSCT is a potentially curative option for a number of malignant disorders; however, up to $30 \%$ of patients lack a suitable HLA-matched either related or unrelated donor. In order to optimize haploidentical transplantation, we recently developed a new method of graft manipulation (i.e. TCR $\alpha \beta / C D 19$ negative selection), which retains in the final product large numbers of effector cells, namely NK and TCR $\gamma \delta$ lymphocytes. Relapse remains the main cause of treatment failure. Based on preclinical data showing bisphosphonates-mediated improvement of TCR $\gamma \delta$ cells-blast killing through accumulation of phosphoantigens, we started a prospective trial based on post-transplant infusion of zoledronic acid, with the aim of enhancing TCR $\gamma \delta$ cells anti-tumor effect.

Materials (or patients) and methods: Enrolled in the study were 35 pediatric patients (median age at transplantation 10.3 years, range 1-18) affected by either ALL and AML (26 and 9 patients, respectively) at very-high risk for relapse/TRM due to disease status (cytogenetic/molecular characteristics, lack of remission or previously failed HSCT). All of them underwent a TCR $\alpha \beta / C D 19-$ depleted HSCT from an HLA-haploidentical donor (one of the two parents). According to the model of $\mathrm{KIR} / \mathrm{KIR}$ ligand mismatch, 13 patients were transplanted from an NK-alloreactive donor. The median number of infused $\gamma \delta+$ T cells was $7.9 \times 10^{6} / \mathrm{kg}$ (range 0.9-42.7). Zoledronic acid was administered monthly at the dose of $0.05 \mathrm{mg} / \mathrm{kg}$ per dose (maximum dose $4 \mathrm{mg}$ ), starting from day +30 after transplantation. Patients underwent zoledronic acid infusions, together with oral calcitriol and calcium supplementation, in the outpatient unit.

Results: A total of 102 infusions were administered with a mean of 2.9 infusions per patient (range 1-5); only one episode of symptomatic hypocalcemia (at first administration) occurred and was rapidly corrected with parenteral calcium supplementation. None of the patients experienced de novo onset or worsening of previously developed acute GVHD, this finding supporting the observation that $\gamma \delta$ T-lymphocytes do not cause GVHD. In the study period, six patients relapsed and 2 died due to infectious complications. With a median follow up of 9 months (range 4-22) the 2-year Kaplan-Meyer estimate of OS and LFS were $88.1 \%$ (SE 6.6) and 62.2 (SE 10.1), respectively (Figure 1a). The cumulative incidence of relapse and TRM were $31.3 \%$ and $6.6 \%$, respectively (Figure $1 \mathrm{~b}$ ). Repeated infusions of zoledronic acid (i.e. more than 3 ) seem to offer an advantage in terms of DFS $(87.5 \%$ vs $48.6 \%$, $P=0.13)$, although the difference was not statistically significant (Figure 1c and d).

Conclusion: These data suggest that the infusion of zoledronic acid after TCR $\alpha \beta / C D 19-d e p l e t e d$ haplo HSCT is safe. Repeated infusions of zoledronic acid after haploidentical HSCT seems to be more effective in preventing leukemia recurrence. More patients and a longer follow-up are needed to establish the efficacy of this approach.

Disclosure of Interest: None declared.
0166

Inducible T-cell receptor expression in precursor T-cells for leukemia control

S. Hoseini ${ }^{1}$, M. Hapke ${ }^{1}$, J. Herbst ${ }^{1}$, D. Wedekind ${ }^{2}$, R. Baumann ${ }^{3}$, N. Heinz ${ }^{4}$, D. Vignali ${ }^{5}, M$. van den Brink ${ }^{6}$, A. Schambach ${ }^{7}$, B. Blazar, M. Sauer ${ }^{7, * *}$

${ }^{1}$ Pediatric Hematology and Oncology, ${ }^{2}$ Central Animal Laboratory, MEDIZINISCHE HOCHSCHULE HANNOVER, ${ }^{3}$ Clinic for Radia-

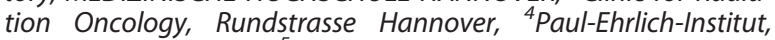
Hannover, Germany, ${ }^{5}$ Department of Immunology, St. Jude Children's Research Hospital, Memphis, ${ }^{6}$ Department of Immunology and medicine, Memorial Sloan Kettering Cancer Center, New York, United States, '7Experimental Hematology, Medizinische Hochschule Hannover, Hannover, Germany, ${ }^{8}$ University of Minnesota Cancer Center and Department of Pediatrics, Division of Blood and Marrow Transplantation, Minneapolis, United States

Introduction: The co-transplantation of hematopoietic stem cells (HS) with those that have been engineered to express tumor-reactive T cell receptors (TCRs) and differentiated into precursor T cells (preTs) may optimize tumor reduction. Since expression of potentially self-(tumor-) reactive TCRs will lead to negative selection upon thymic maturation, we investigated whether preTs forced to express a leukemia-reactive TCR under the control of a tetracycline-inducible promoter would allow timely controlled TCR expression thereby avoiding thymic negative selection.

Materials (or patients) and methods: Using lentiviral vectors, murine LSK cells were engineered to express a Tetracyclineinducible TCR directed against a surrogate leukemia antigen. TCR-transduced LSK cells were co-cultured on T cell development-supporting OP9-DL1 cells to produce preTs. Lethallyirradiated $\mathrm{B} 6 / \mathrm{NCrl}$ recipients received syngeneic $\mathrm{T}$ celldepleted bone marrow and $8 \times 10^{6}$ syngeneic or allogeneic (B10.A) TCR-engineered preTs. An otherwise lethal leukemia cell (C1498) challenge was given 28 days later.

Results: After in vivo maturation and gene induction up to $70 \%$ leukemia free survival was achieved in recipients of syngeneic TCR-transduced preTs $(P<0.001)$ as shown in figure $1 \mathrm{~A}$. Importantly, transfer of allogeneic gene-manipulated preTs increased the survival of recipients $(P<0.05)$ without inducing graft versus host disease (GVHD). Nontransduced preTs provided significantly lower leukemia protection being not significantly superior to the PBS controls. The progenies of engineered preTs gave rise to effector and central memory cells providing protection even after repeated leukemia challenge. In vitro transduction and consecutive expansion of mature $\mathrm{T}$ cells required at least $40 \times 10^{6}$ cells/ recipient to mediate similar anti-leukemia efficacy, risking the development of severe GVHD if of mismatched origin, and providing no long-term protection. Importantly, while transgene induction starting immediately after transplant forced $\mathrm{CD}^{+} \mathrm{T}$ cell development and was required to obtain a mature $T$ cell subset of targeted specificity, late induction favored CD4 differentiation and failed to produce a leukemiareactive population due to missing thymic positive selection. Conclusion: Co-transplanting TCR gene-engineered preTs is of high clinical relevance since small numbers of even mismatched HS can be transduced at a reasonable cost, expanded in vitro, stored if needed, and provide potent and long lasting leukemia protection.

Disclosure of Interest: None declared. 